Copyright

by

Stacy McBride Edgar

2003 


\section{The Dissertation Committee for Stacy McBride Edgar}

certifies that this is the approved version of the following dissertation:

\section{Phylogeny of Aulacoseira (Bacillariophyta)}

\section{Committee:}

Edward C. Theriot, Supervisor

David C. Cannatella

Robert K. Jansen

John W. La Claire, II

Beryl B. Simpson 
Phylogeny of Aulacoseira (Bacillariophyta)

by

Stacy McBride Edgar, B.S., M.S.

\author{
Dissertation \\ Presented to the Faculty of the Graduate School of \\ the University of Texas at Austin \\ in Partial Fulfillment \\ of the Requirements \\ for the Degree of \\ Doctor of Philosophy
}

The University of Texas at Austin

May, 2003 
Dedication

For my husband, Robert:

You were always on my mind and in my heart. 


\section{Acknowledgements}

I would like to thank many people for their help and support over the last five years that has made my experience here at the University of Texas at Austin both prosperous and memorable. I was pleasantly surprised when I was accepted into the Graduate Program in Botany in 1998. And I am still quite grateful to my advisor, Ed Theriot, and what was the Botany Department at that time, for opening their doors to me. I would also like to thank Ed for his support and all the time he was able to devote to discussing diatom systematics and whatnot, despite the competing demands of the TMM. Thanks also go out to all of my committee members and the time and help they so generously gave at every request. I have to thank Bob Jansen in particular for serving as a surrogate advisor and taking us "Theriot kids" under his wing since the day we began arriving at UT. It certainly has become a home away from home.

It has been a privilege and great pleasure to work side by side with many of the members of the Jansen and Theriot labs over the years. I must extend a special debt of gratitude to Les Goertzen who always enthusiastically responded to my pleas for help in the molecular lab. He not only helped me to hone my molecular skills, but he always was able to provide fascinating discussion concerning many areas of science, diatom biology, and plant systematics equally well.

I would like to thank my parents for instilling confidence in me since before I can remember, and always encouraging me to challenge myself. Without this, I surely would have shied away from pursuing this degree and would have missed out on many 
of the enjoyments life has to offer. And I am especially grateful that they are both here to share this with me.

I don't think I will ever be able to thank my husband enough for the sacrifice he has made for me to pursue my dreams. His constant love, support, and encouragement were easily an integral part of my success in completing this work. 


\title{
Phylogeny of Aulacoseira (Bacillariophyta)
}

\author{
Publication No.
}

Stacy McBride Edgar, Ph.D

The University of Texas at Austin, 2003

Supervisor: Edward C. Theriot

The phylogeny of 67 populations representing 45 species of Aulacoseira is estimated by maximum parsimony methods using a combination of nucleotide sequence data and qualitative and quantitative morphological characteristics of the silica cell wall gathered primarily from original observation by LM and SEM. A new type of character employing continuous quantitative variables that describe the ontogenetic-allometric trajectories of cell wall characteristics over the life cycle (size range) of diatoms is introduced. In addition to the 45 Aulacoseira species, the phylogeny also incorporates one Alveolophora species, and two outgroup species (Melosira varians and Stephanopyxis cf. broschii). Fifteen species, represented by 24 populations, also contain molecular data from the chloroplast genome $(r b c \mathrm{~L})$ as well as the nuclear genome $(18 \mathrm{~S})$, which were sequenced or downloaded from GenBank. 
The phylogeny of Aulacoseira is composed of five major clades: 1) an $A$. crenulata and A. italica clade, which is the most basal, 2) an A. subarctica and $A$. distans clade, 3) an A. granulata complex clade, 4) an A. ambigua clade, and 5) an A. islandica, A. skvorzowii, A. baicalensis, clade that also contains Alveolophora and many extinct Aulacoseira taxa. Monophyly of Aulacoseira is only achieved if Alveolophora, originally identified as Aulacoseira, is no longer given separate generic status.

The choice of morphological characters, recognition of character states and explicit consideration of the states in coding are of great import in any phylogenetic study utilizing morphological data. Sensitivity of the Aulacoseira phylogeny to different coding methods was explored. Results indicate that use of step-matrix gap weighting utilizing the maximal number of character states allowable by phylogenetic software has two major advantages over other coding methods. Data are not manipulated in an effort to recognize gaps, i.e., the data remain in as raw a form as possible within the constraints of the requirements of phylogenetic software that they be in integer form. The maximal amount of potential phylogenetic signal contained within the data can contribute to the phylogenetic estimation. An additional methodological step is proposed in this study to allow large data sets to utilize the step-matrix gap weighting method. 


\section{Table of Contents}

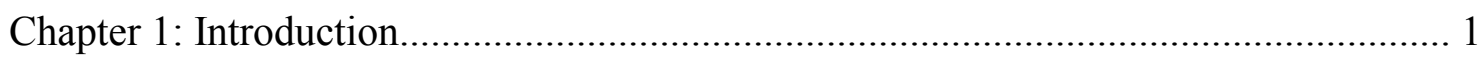

Chapter 2: Phylogeny of Aulacoseira (Bacillariophyta).......................................... 19

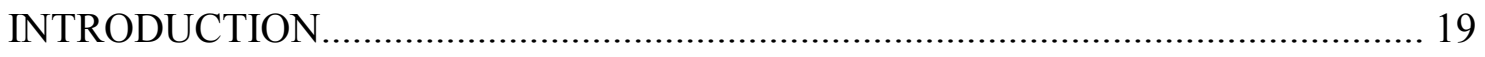

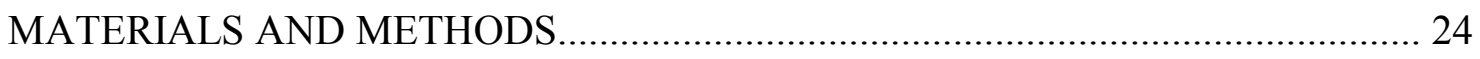

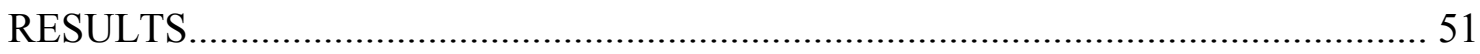

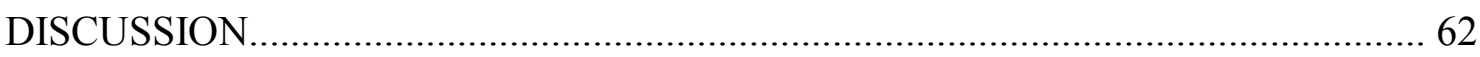

Chapter 3: Phylogenetic Sensitivity to Coding of Overlapping Continuous

Morphometric Characters: an example from Aulacoseira (Bacillariophyta)............... 74

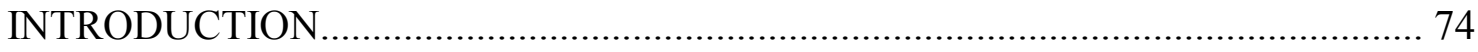

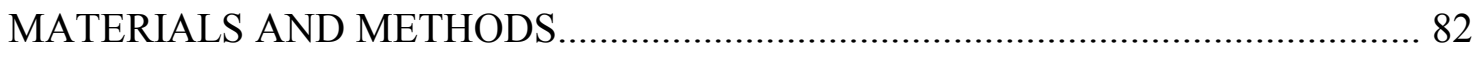

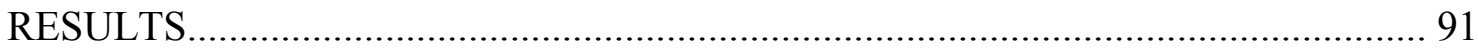

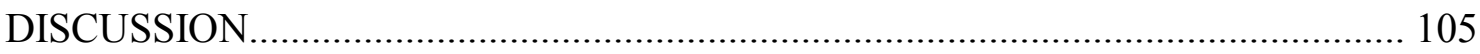

Appendix A. Specimens Examined................................................................... 112

Appendix B. Species data gathered from literature.......................................... 116

Appendix C. Molecular characters: 18S rDNA.................................................. 119

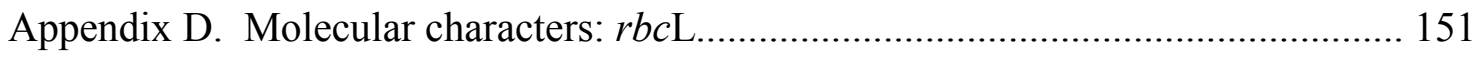

Appendix E. Morphological characters: Morphometric Character Data Coded via a Modified Step-Matrix Gap Weighting Method (32 Character State Limit)............... 167 Appendix F: Step-Matrices Used to Weight Morphometric Characters Coded via a Modified Step-Matrix Gap Weighting Method (32 Character State Limit)............... 170 
Appendix G. Morphological characters: Morphometric Character Data Coded via a Modified Step-Matrix Gap Weighting Method (26 Character State Limit).

Appendix H. Step-Matrices Used to Weight Morphometric Characters Coded via a Modified Step-Matrix Gap Weighting Method (26 Character State Limit).

Appendix I. Morphological Character States as Scored for Gap Weighting Without Step-Matrices Limited to 32 Character States.

Appendix J. Morphological Character States as Scored for Gap Weighting Without Step-Matrices Limited to 26 Character States........................................................... 264

Appendix K. Morphological Character States as Scored for Gap Weighting Without Step-Matrices Limited to 10 Character States......................................................... 267 Appendix L. Morphological Character States as Scored via Quasi-Statistical Student's T-test Gap Identification Method..................................................................... 270 Appendix M. Morphological Character States as Scored Using Statistical Homogeneous Subset Coding Method............................................................. 273

Appendix N. Images of Species Examined...................................................... 286

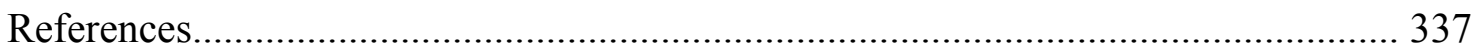

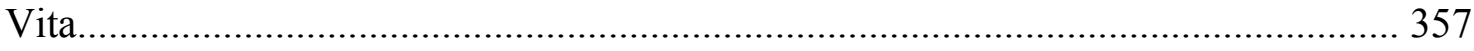




\section{CHAPTER 1. Introduction}

Aulacoseira Thwaites is an ecologically important and ubiquitous diatom genus, with cosmopolitan and endemic species found in ecologically diverse lacustrine and

lotic waters. About $20 \%$ of the described species in this genus are extinct. Aulacoseira is routinely encountered and used in ecological and paleoecological studies, but it has been described as a character-poor and thus a "difficult" genus, which would be more utilizable if more were known about its biology and systematics (Crawford and Likhoshway 2002).

Production of a phylogenetic estimate using morphological characters includes the following steps: 1) character selection or identification, 2) character coding or character state identification (postulates of homology), 3) parsimony analysis, 4) evaluation of the phylogeny via measures of support (non-parametric bootstrap, Bremer support, congruence with phylogenies derived from other types of data, etc.), and 5) evaluation of characters as putative homologies via tests of character congruence. Sometimes sensitivity analyses are also utilized to evaluate the data and the sensitivity of the phylogenetic estimate to some factor in its production, such as type of phylogenetic analysis applied (e.g., distance vs. parsimony methods), and treatments of, or coding of, characters (e.g., gaps as fifth character states vs. missing data in gene sequence data). 
Because Aulacoseira is comprised of extant and extinct taxa, use of multiple kinds of characters for estimating its phylogeny has been a major focus of this study, especially characters derivable for all of the taxa. A fossil record for this genus has been reported to go back at least 45 million years (Khursevich 1995, Krebs 1994). Because the identification and descriptions of taxa have historically focused on the ornamentation of the siliceous cell walls, the great majority of morphological characters used or described are equally available for examination in fossil as well as extant taxa. However, the characters and their states cannot simply be uncritically extracted out of the literature and incorporated into data matrices, as will be discussed below. In addition to examination of morphological characters in Aulacoseira, partial gene sequences from the chloroplast $(r b c \mathrm{~L})$ and the nuclear (rDNA) genomes have also been examined for extant taxa where available.

Among the few taxonomic studies of Aulacoseira, there have been some disagreements about terminology used for various characteristics of the species in the genus. For this study, terminology used is summarized in Figures 1 through 6 below. Extraction of characters solely from the literature for this genus and insertion into data matrices is unadvisable because of: 1) poor or insufficient character descriptions, 2) common misinterpretation of characters, and 3) omission of many useful characters. 

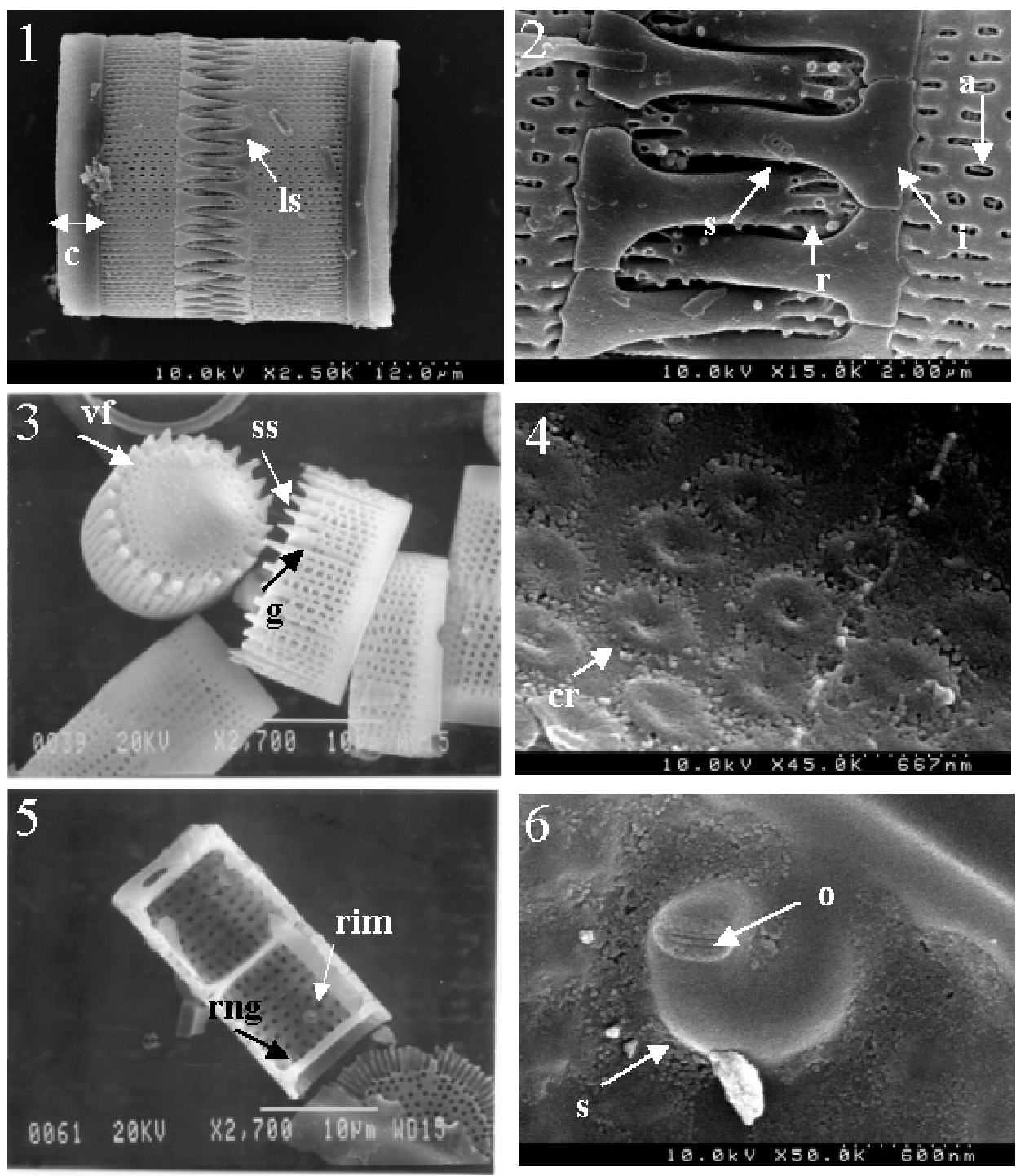

Figures 1-6. Terminology for cell wall structure in Aulacoseira: Fig. 1. Two sibling valves of $A$. crenulata are shown in mantle-view displaying the linking spines (ls) and the collum (c). Fig. 2. A close-up of linking spines in A. italica showing the stalk (s), the inflation (i), and the roots (r) of the linking spine. Areolae (a) and their internal structure (vela) can be seen clearly here as well. Fig. 3. A. agassizii valves with views of the valve face (vf) as well as the mantle, where separation spines (ss) and the grooves on the mantle associated with the separation spines (g) are shown. Fig. 4. Close up of cribra (c) covering areolae on the internal mantle wall of $A$. italica. Fig. 5. Two sibling valves of $A$. granulata are broken open to reveal the internal cell wall structure including the ringleist (rng) and a spiraled rimoportula (rim). Fig. 6. Close up of the rimoportula in A. granulata var. jonensis showing its internal slit-like opening (o) and its spiraled stalk (s). 
The first of these, poor or insufficient character descriptions, can lead to poor coding of characters as well as difficulty in the identification of Aulacoseira species (Crawford and Likhoshway 2002, Siver and Kling 1997). Many attempts to describe or differentiate species, or groups of species, have employed a character describing the shape of a valve as seen in girdle view in comparison to a square: those with mantleheight-to-diameters ratios $\geq 1$ and those with the ratios $<1$ (Hustedt 1942, Krammer and Lange-Bertalot 1991). Based on this, why not code this character as a binary character, $<1$ and $>1$ ? When Siver and Kling (1997) examined populations in the SEM and reported the ratios of mantle-height-to-valve-diameter for 19 North American Aulacoseira species, no simple dichotomy among species for this character was evident. When this character is examined over the life history (i.e., size range) of many Aulacoseira species, the source of the problem with dichotomizing this character becomes apparent. For a great many species (but not all) the valves originating after sexual reproduction have low mantle-height-to-valve-diameter ratios $(\leq 1)$, but as they proceed through the asexual phase of their life cycle, there is a dramatic narrowing of the diameter of the valves and a lengthening of the mantle height (> 1; fig. 7). If the population were sampled at the beginning of its asexual reproductive phase it would be identified differently than if it were sampled towards the end of its asexual phase. Mantle height versus valve diameter is a continuous character and therefore not readily or accurately coded in a binary fashion. Krammer (1991a) noted failure to consider the 
whole life history as a source of problems in understanding Aulacoseira. And characters seemingly facilitating identification can have little or no systematic value.

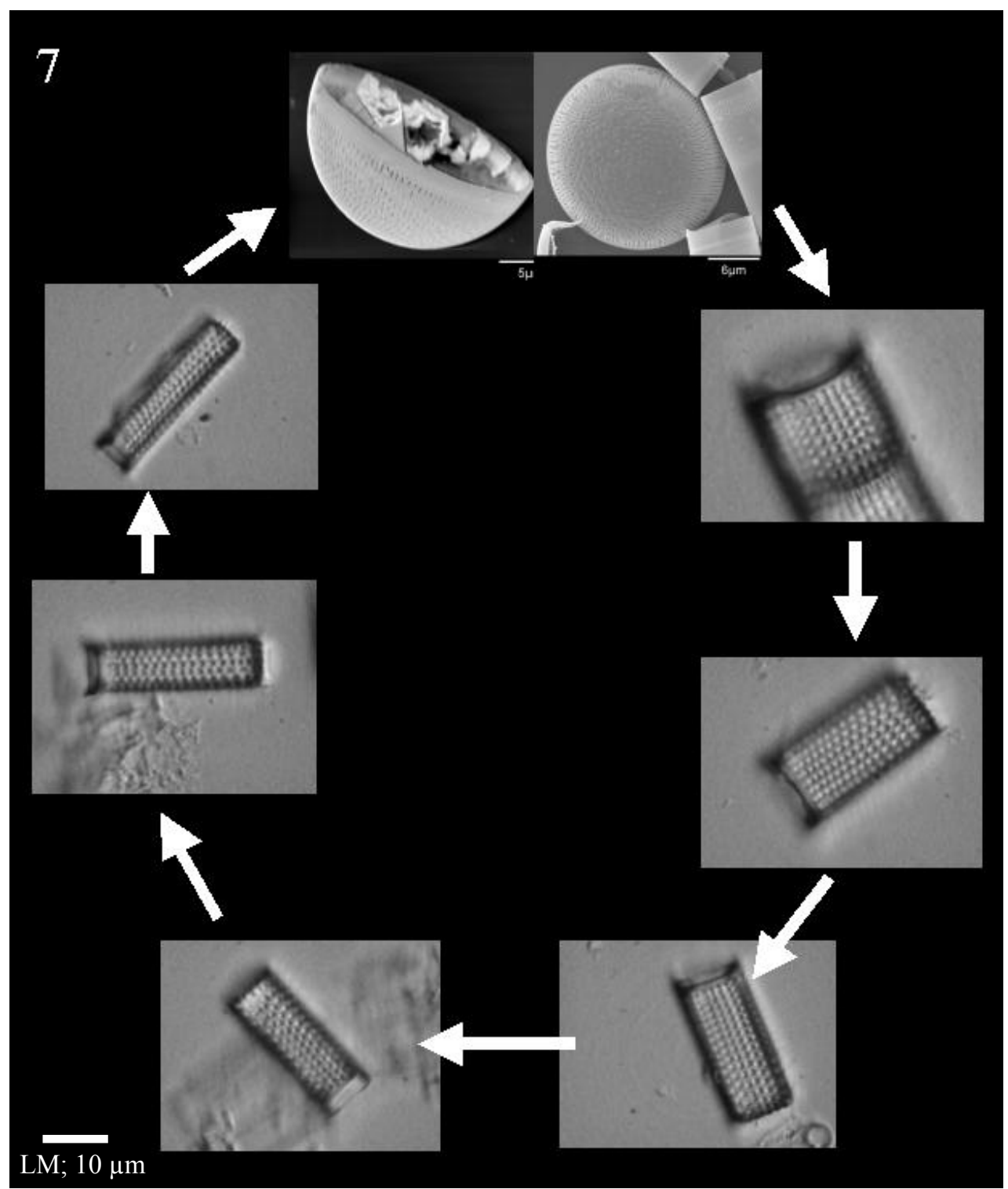

Figure 7. A simplified depiction of the life cycle in $A$. granulata fide Van Heurk emphasizing the asexual portion and characteristic changes of the silica cell wall that occur with diminution in diameter (LMs; scale bar $=10 \mu \mathrm{m}$ ). The zygote, or auxospore, resulting from sexual reproduction that sometimes occurs after a minimum size for the species is reached is also shown (spherical cells; SEMs). 
Other examples of poor or insufficient character descriptions lie in continuous characters typically used for species' descriptions and diagnoses: number of striae in 10 $\mu \mathrm{m}$, number of areolae within a stria in $10 \mu \mathrm{m}$, range of mantle height, and range of valve diameter. The first pair of characters is problematic because, as Theriot (1988) and Theriot and Serieyssol (1994) have pointed out, ratios are not an effective means of removing size effects from a character. When a character for two samples containing different size ranges is described using ratios, the comparisons between these samples still contain additional elements of variability due to their differences in size that are confounding. The second pair of characters is problematic, because simply independently reporting univariate ranges and ignoring any relationship between the variables omits useful and more precise information about the species. For example, when information about diameter and mantle height reported for $A$. granulata by Hustedt (1942) and by Krammer and Lange-Bertalot (1991) are represented in a graph, their potential covariation results in a large area of morphometric space (fig. 8; rectangle). However, when individuals in any of many samples of $A$. granulata are measured for these two variables, the actual covariation is confined to a much smaller area in morphometric space (fig. 8; individual points). Variation in mantle height versus valve diameter in A. granulata is constrained along a trend of increasing mantle height with decreasing mantle diameter, which is reduced during the asexual phase of its life cycle. Among Aulacoseira species, this trend varies in intensity as well as direction, indicating that these bivariate descriptions (e.g., correlation and regression) in 
themselves provide a basis for species descriptions and diagnoses. Measurements were made on Aulacoseira species for this study with specific intention of describing bivariate correlations of all cell wall continuous characteristics with reduction in diameter (size) over the asexual life cycle.

The second problem preventing use of characters from the literature is common misinterpretation of characters. Multiple studies propose that polymorphisms exist within diatom species (e.g., Klee and Houk 1996, McBride and Edgar 1998, Siver and Kling 1997, Stoermer 1967, Teubner 1995). Only one study has tested these claims by utilizing morphometrics to gather information on possible intermediates between two proposed morphotypes in a population (McBride and Edgar 1998). This study on a population composed of $\sim 25 \%$ Janus cells (where valves within cells are visually distinct from one another) in Gomphonema angustatum, it was clearly demonstrated that there were not simply two morphs present (coarsely striated and finely striated), but rather a continuum of variation in striae densities where each of its two ends were being recognized as distinct by eye and the subtle variations connecting the two were overlooked.

Data collected in this study show several proposed polymorphisms in Aulacoseira to be misperceptions and misnomers. For instance, Siver and Kling (1997) reported that ringleist thickness and ringleist penetration were not very good characters for diagnosing taxa, because $A$. subarctica possessed a thick, obvious ringleist in cells with low mantle-height-to-valve-diameter ratios, and a thin, shallow ringleist in cells 
with high mantle-height-to-valve-diameter ratios. This observation was based on SEM examination of many A. subarctica populations but without measurement and statistical analysis. Data from this study (Figure 9) for both of these ringleist characteristics versus cell diameter show a clear trend in the covariation of ringleist thickness and depth of penetration with size in A. subarctica. Two other examples of proposed polymorphisms within Aulacoseira are also not supported (Figures 10 and 11). Previously perceived morphotypes of curved striae and straight striae in A. subarctica are clearly connected by continuous variation in the degree of curvature of striae related to cell size (Figure 10). Two morphs of thickly silicified and thinly silicified cell walls in A. islandica are shown to be two ends of a continuum of wall thickness correlated with cell size (Figure 11). Many cell wall characteristics are related to the reduction in size that accompanies asexual cell division. Again, misinterpretation of the difference between the two ends of the trend and the absence of a quantitative assessment with respect to size often lead to the misperception of polymorphism. 


\section{Aulacoseira granulata}

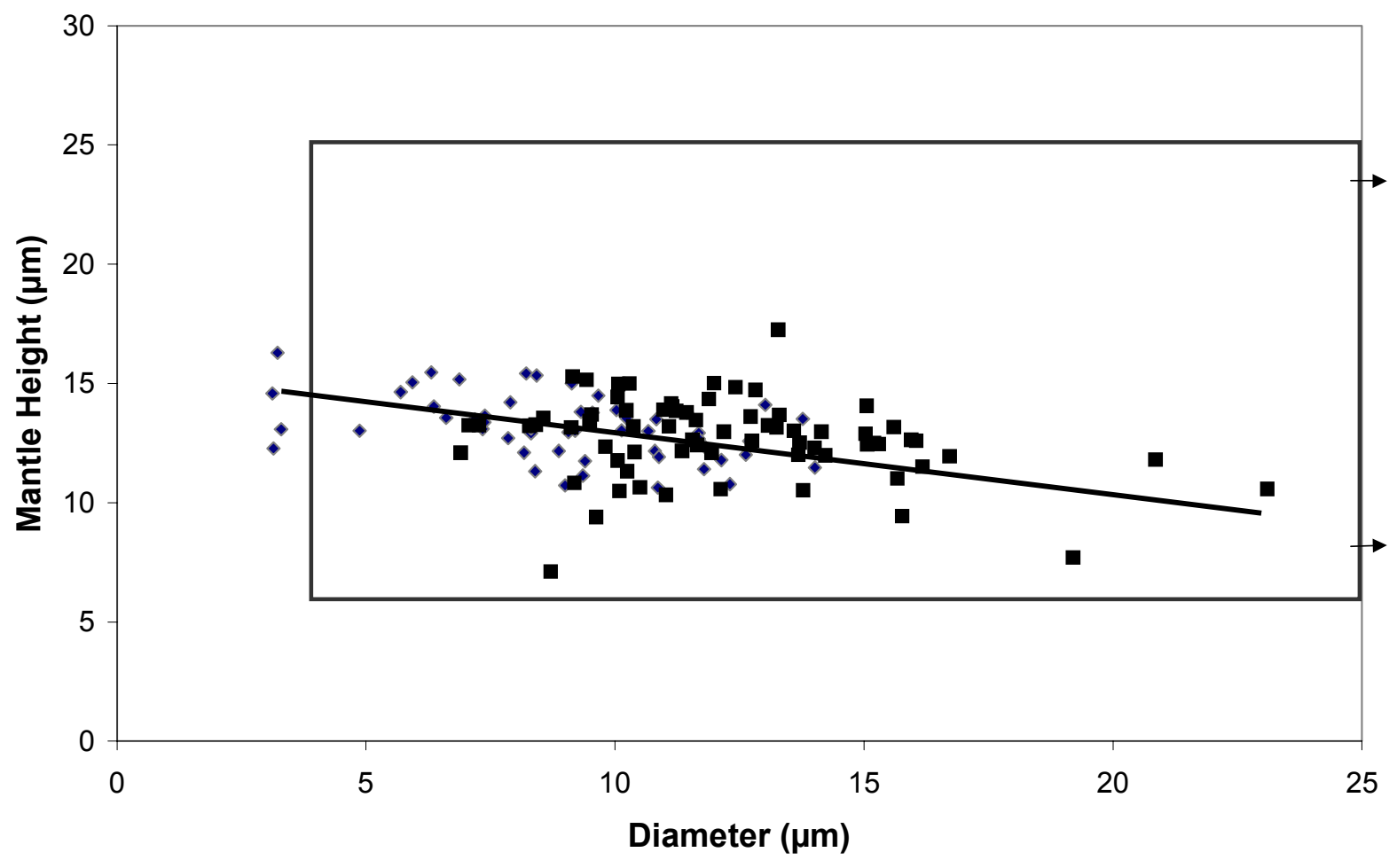

Figure 8. A graphical comparison between reports in the literature of the ranges of mantle height and valve diameter found in A. granulata (large black rectangle; arrows at right indicate values for maximum diameter are greater than $25 \mu \mathrm{m})$ and actual measurements made for two populations of $A$. granulata showing the higher level of information contained in the bivariate relationship. Gray diamonds represent individual valve measurements in Tilden's American Algae No. 367; black squares represent valves in Rabenhorst's Algal Europa No. 2212. The line represents the principal axis regression. 


\section{A. subarctica Ringleist Characteristics versus Valve Diameter}

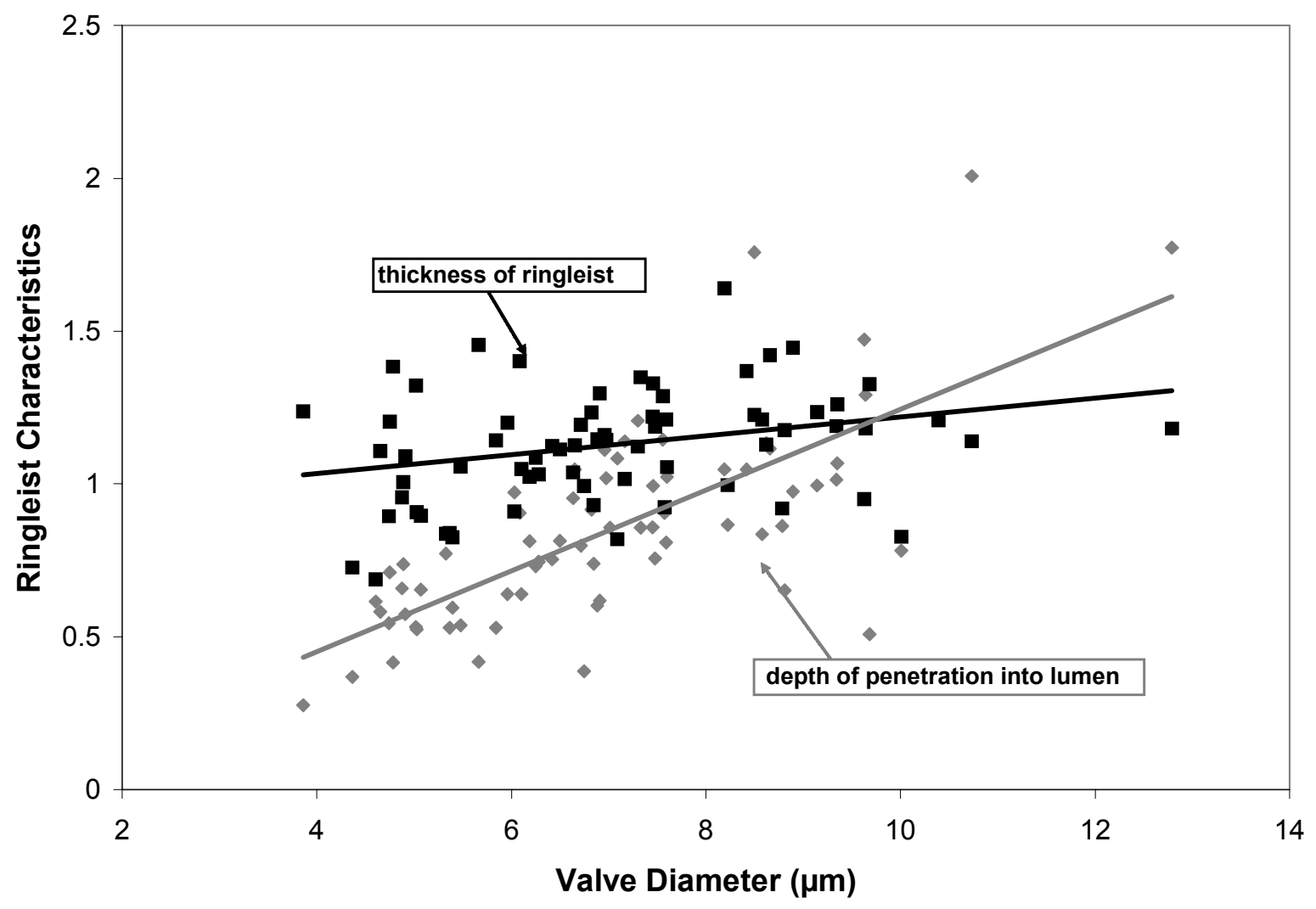

Figure 9. Changes in two ringleist characteristics relative to valve diameter: ringleist thickness (squares) and its depth of penetration into the lumen (diamonds) with the diminution of diameter are shown for a population of $A$. subarctica. The lines represent the principal axis regressions for each of the bivariate comparisons shown. 


\section{A. subarctica Mean Curvature of Striae versus Valve Diameter}

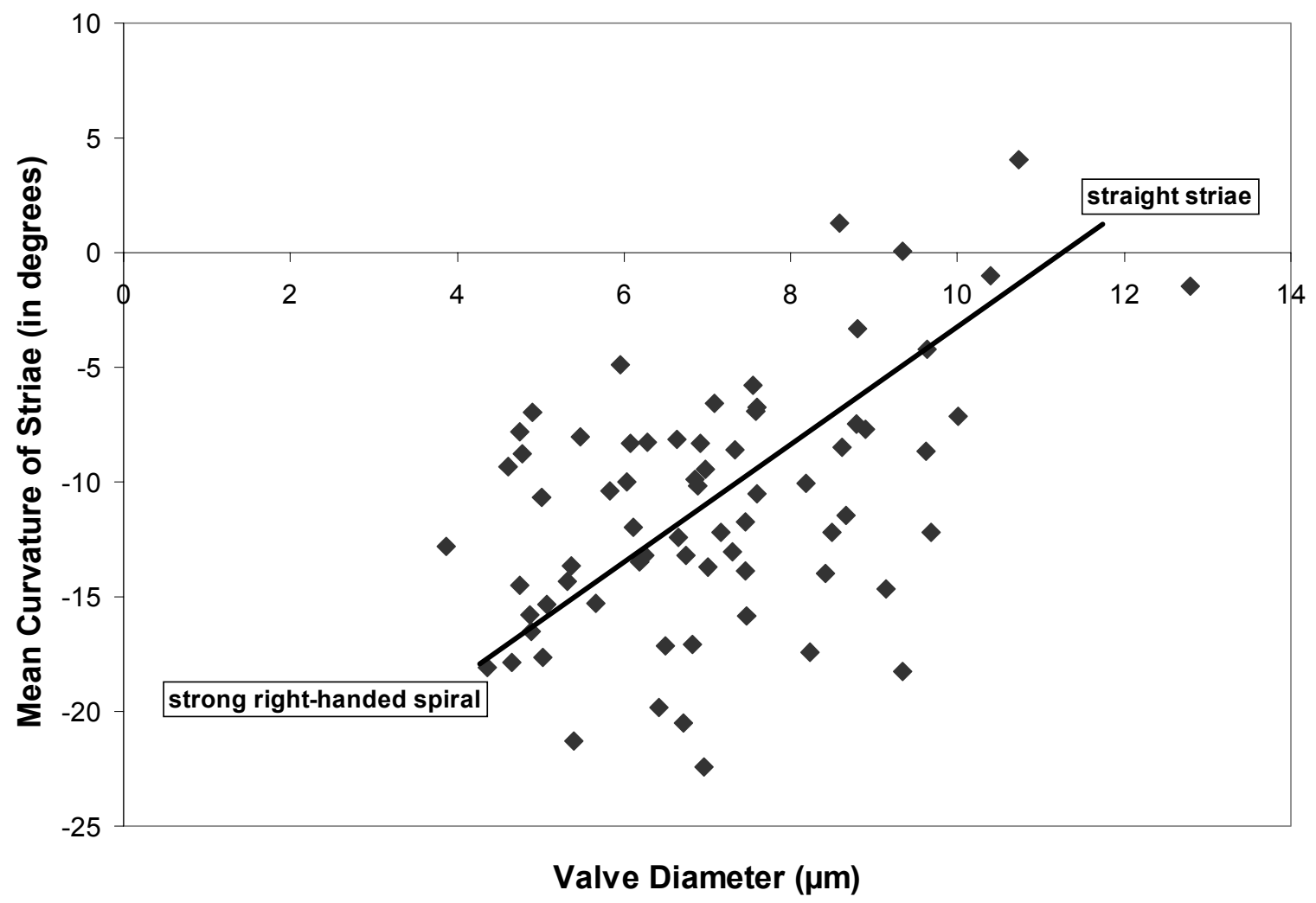

Figure 10. A gradual shift in the curvature of the striae is demonstrated in a population of $A$. subarctica as valve diameter is decreased over the asexual life cycle. The line represents the principal axis regression. 


\section{A. islandica Mantle Thickness versus Valve Diameter}

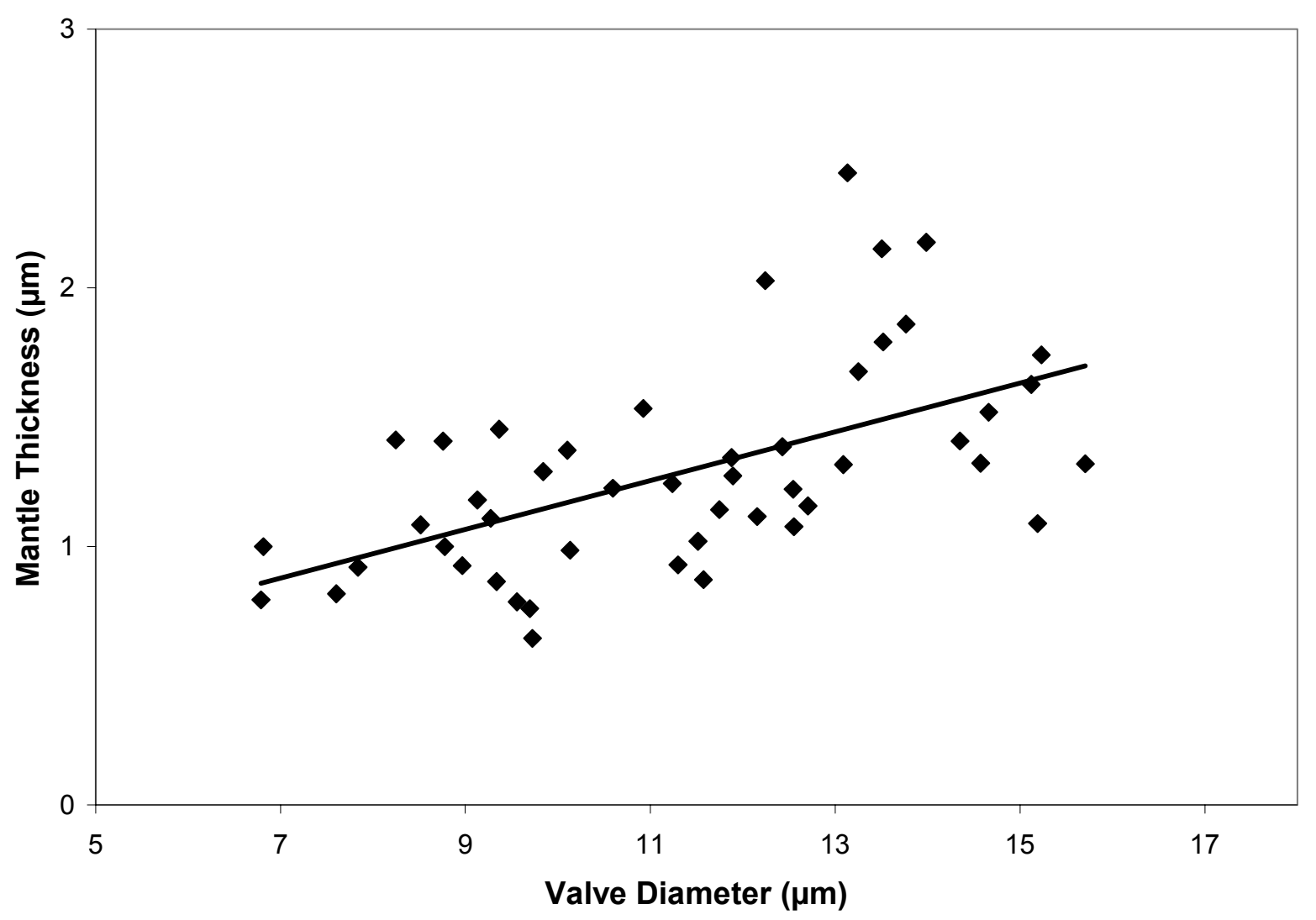

Figure 11. A gradual shift occurs in the thickness of the mantle wall with valve diameter in a population of $A$. islandica. The line represents the principal axis regression. 
Lastly, many useful characters are simply omitted from species descriptions in the literature. Some workers examine internal cell wall structure, such as rimoportula position and structure, and other workers overlook these characters entirely. Also, ontogenetic-allometric trajectories as descriptors are useful in species descriptions and diagnoses and critical to any systematic study in diatoms. To ignore them in diatoms is comparable to ignoring age or stage of growth in morphological studies of an angiosperm or a vertebrate.

However, in order for ontogenetic-allometric characters to be considered as potentially homologous, and therefore useful in phylogenetic reconstruction, they must contain some level of similarity among species and be heritable (Theriot and Serieyssol 1994). Although the similarity criterion is a requirement of phylogenetic studies, and characters are examined for independence among species, heritability is not often actually measured. Heritability studies are arduous and, in some organisms, not even possible. Darwin (1859) initially advanced, in explaining natural selection, the assumption that most variable characters were heritable:

Any variation which is not inherited is unimportant for us. ... No breeder doubts how strong is the tendency to inheritance: like produces like is his fundamental belief: ... Every one must have heard of cases of albinism, prickly skin, hairy bodies, \&c., appearing in several members of the same family. If strange and rare deviations of structure are truly inherited, less strange and commoner deviations may be freely admitted to be inheritable. Perhaps the correct way of viewing the whole subject, would be, to look at the inheritance of every character whatever as the rule, and non-inheritance as the anomaly. 
Subsequent work has justified Darwin's (1859) assumption as many characters have been shown to be heritable, although in most studies of natural selection it remains an untested assumption (Falconer 1981, Hartl and Clark 1989, Stearns 1992). There have been two heritability studies on cell wall characteristics in diatoms that support this assumption, one in Aulacoseira (Edgar \& Theriot 2003, ms accepted) and one in Thalassiosira (Wood et al. 1987).

Independence of ontogenetic-allometric trajectories among species can be examined graphically. These plots can then be examined to see if differences in relationships of character $x$ with size exist among species, or if they all share similar relationships of characters with size, indicating that the relationship is not independently evolving among species but instead may be expressions of developmental constraints. All nine ontogenetic-allometric characters were examined and evidence was found for their independent evolution. An example of typical trajectories for mantle height versus cell diameter in four populations of Aulacoseira is shown in Figure 12. Shared similarity in trajectories for this ontogenetic-allometric character between A. granulata fide Van Heurck and A. granulata fide H. L. Smith are apparent, but there is wide variation among species in intensity and direction of the relationship.

Taxonomic studies on Aulacoseira have leaned heavily toward use of 'qualitative' characteristics of the silica cell wall, such as linking spine shape (Siver and Kling 1997), pattern of areolae on the valve face (Camburn and Charles 2000, Camburn and Kingston 1986), and the structure and location of rimoportula internally on the 
mantle (Likhoshway and Crawford 2001). In Chapter 2, 18 qualitative characters, including rimoportula position, are used in phylogenetic reconstruction along with 21 morphometric (or 'quantitative') characters (12 central tendencies, as measured by means, and 9 ontogenetic-allometric trajectory characters, as measured by principal axis slopes). Sequence data from the nuclear genome (18S rDNA) and the chloroplast genome $(r b c \mathrm{~L})$ are also analyzed. Each data type was also examined independently to assess its contribution in terms of phylogenetic signal and congruence and/or potential conflicts with the other data sets. It is shown in Chapter 2 that both 'types' of morphological data ('qualitative' and 'quantitative') play an integral role in inferring the best working estimate of Aulacoseira phylogeny.

Because the majority of diatom taxa are subject to reduction in cell diameter through their life cycle, ontogenetic-allometric trajectories, introduced in this study, provide a quantitative, repeatable, and unbiased source of information amenable to statistical analyses, that are a useful means of describing character-size covariation in species descriptions and diagnoses and providing useful characters for phylogenetic inference for all systematic studies of diatoms. Continuous overlapping morphometric characters have been shown to be useful in phylogenetic inference, at least in species and population-level studies.

Most diatom phylogenies have been inferred from a single gene sequence (Kooistra and Medlin 1996, Medlin et al. 1993, 1996a, b, Sorhannus et al. 1995), or morphology and/or cytological data alone (Cox and Williams 2000, Julius 2000, 
Kociolek and Stoermer 1988, Williams 1990, Williams and Round 1988). However, Chapter 2 demonstrates a combination of four 'types' of data produced the best working hypothesis of Aulacoseira phylogeny, and so it is strongly recommended that future systematic studies on diatoms evaluate and incorporate as many sources of data as possible.

The quantitative morphometric characters (means and ontogenetic-allometric trajectories) used in Chapter 2 are explored further in Chapter 3 in regards to the coding. Coding of morphological characters, whether 'qualitative' or 'quantitative', has been rarely discussed by the studies that have employed them, and many consider this the 'black box', or 'bête noire', of phylogenetic systematics (Hawkins 2000, Poe and Wiens 2000, Pogue and Mickevich 1990, Reid and Sidwell 2002, Stevens 1991). However, since differences in coding of characters can greatly affect phylogenetic estimates, addressing this part of cladistic methodology is important in any study using morphological data (Stevens 2000, Thiele 1993, Wiens 2001).

In Chapter 3, the Aulacoseira phylogeny estimated from qualitative and quantitative morphological data and $18 \mathrm{~S}$ rDNA and $r b c \mathrm{~L}$ gene sequence data combined (total evidence) is examined for sensitivity to different methodologies for coding of the morphometric data (means and ontogenetic-allometric trajectories). Recent debates and suggested methodologies for coding of continuous overlapping quantitative characters are reviewed. Four coding methods are evaluated for their performance in recovery of species monophyly in tree topology. A novel methodological step is introduced in 
Chapter 3 to permit the step-matrix gap weighting method (Wiens 2001) to be applied to data sets containing larger numbers of taxa possessing unique means for quantitative characters than the previously restrictive number of 32 .

Analysis of the sensitivity of phylogenetic estimates to character coding methods also provide a means of identifying areas of the phylogeny that are strongly supported by the data, regardless of coding (i.e., less sensitive areas) and those that are weakly supported (highly coding-sensitive; Simmons and Geisler 2002). Then coding-sensitive topological regions of the tree can aid in making more efficient decisions for data collection or taxon sampling in future studies aimed at improving the phylogenetic estimate (Simmons and Geisler 2002).

Coding of morphological data, whether 'qualitative' or 'quantitative', is still open for exploration. There is no universal coding method, or "holy grail" of coding, that solves all of these interesting and diverse questions about how to represent data for the most accurate reconstruction of phylogeny (Swiderski et al. 1998, Thiele 1993). But through a combination of simulation studies and empirical studies, measuring sensitivity of data to coding methods, some of these questions can be answered, providing guidelines for future evaluation of various types of data. 


\section{Mantle Height versus Valve Diameter for Four Populations of Aulacoseira}

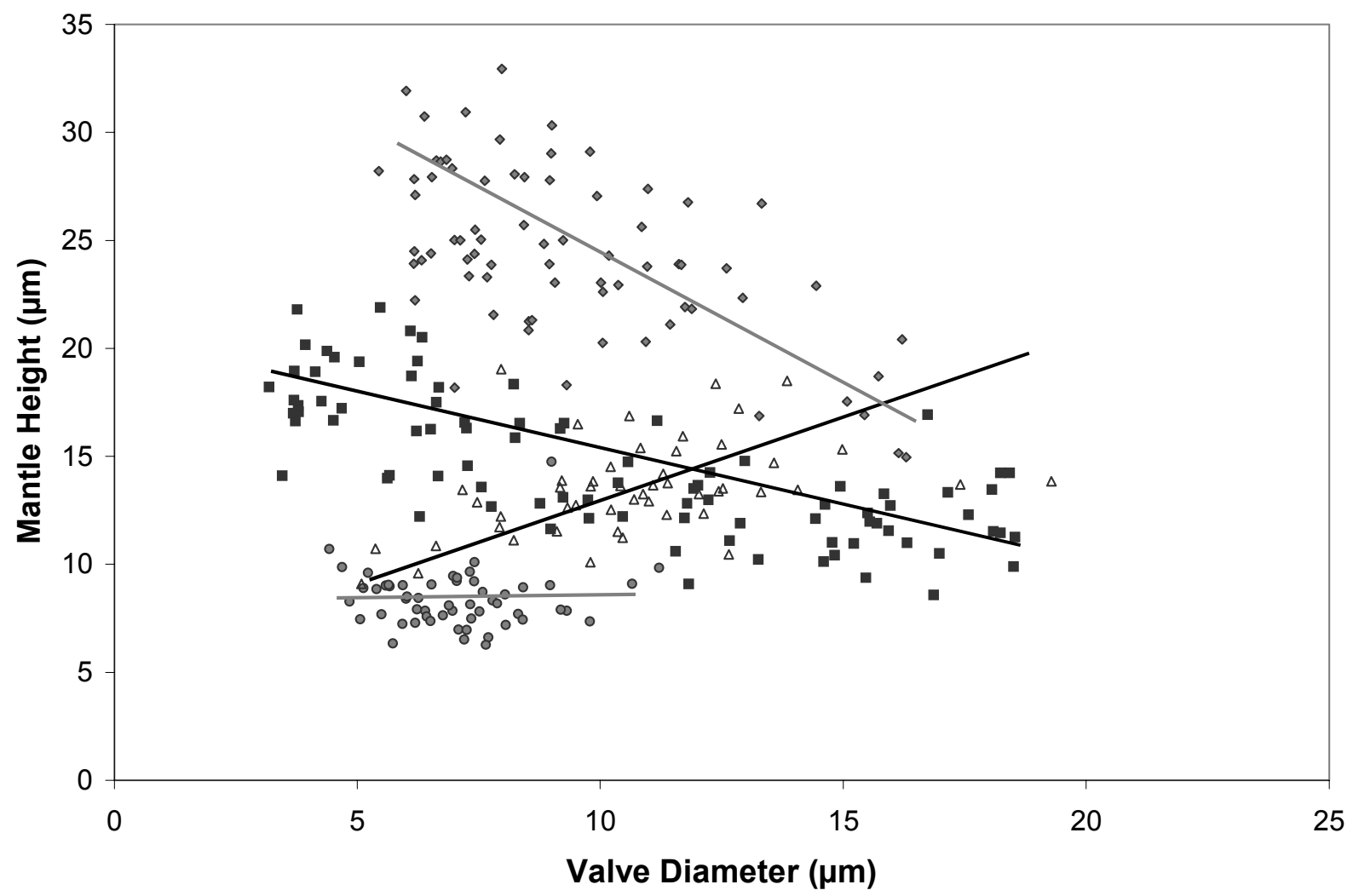

Figure 12. Variation among Aulacoseira species in ontogenetic-allometric trajectories of mantle height is shown. A. granulata fide Van Heurk (diamonds) as well as $A$. granulata fide $\mathrm{H}$. L. Smith (squares) show an increase in mantle height as valve diameter decreases over the life cycle, in $A$. ambigua (circles) there is no change in mantle height as diameter decreases, and in A. valida (triangles) mantle height decreases as valve diameter decreases. Lines represent the principal axis regressions for each of the species shown. 


\section{CHAPTER 2. Phylogeny of Aulacoseira (Bacillariophyta)}

\section{INTRODUCTION}

Aulacoseira Thwaites (previously Melosira Agardh, in part) is globally distributed and seasonally composes a considerable portion of the phytoplankton community in many lakes, ponds, and large rivers (Kilham 1990, Round 1981, Tilzer and Serruya 1990). It can play a significant ecological role as a primary producer in grazer-based aquatic food webs and in lacustrine biogeochemical cycles (Hutchinson 1957, 1967, Tilzer and Serruya 1990). Species of Aulacoseira (along with other diatom species) are used for reconstruction of paleoenvironments and ecological monitoring (Battarbee 1986, Charles et al. 1994, Stoermer and Smol 2001). It has a strong fossil record going back at least 45 million years (Khursevich 1995, Krebs 1994), and is commonplace in Tertiary lacustrine deposits (Haworth and Sabater 1993, Khursevich 1995). For nearly four decades, diatom workers have continued to reiterate that the systematics of this group needs re-evaluation (e.g., Crawford and Likhoshway 2002, Gasse 1980, Krammer 1991a, b, VanLandingham 1967).

Despite a broad distribution in space and time and major ecological importance there is no comprehensive phylogeny of species within Aulacoseira. Two narrowly focused phylogenies have been proposed. Gasse (1980) proposed the divergence of two highly cosmopolitan species of Aulacoseira from a common ancestor (Aulacoseira granulata var. granulata and var. valida, plus A. agassizii var. agassizii and var. 
malayensis) that was strictly based on Plio-Pleistocene fossil diatoms from a few stratigraphic sequences from Ethiopia. Shcherbakova et al. (1998) used 18S rDNA to determine the age and divergence of two highly endemic Aulacoseira species found in Lake Baikal, Russia, and included three other cosmopolitan Aulacoseira species previously sequenced by other research groups in these analyses (A. baicalensis and $A$. skvortzowii from Lake Baikal, and A. subarctica, A. ambigua and A. distans from other lakes). Because these approaches are localized geographically and temporally, and restricted by so few taxa, their contribution is extremely limited.

Accurate reconstruction of the phylogenetic relationships among Aulacoseira species requires a reasonable sampling of taxa. In diatoms, which have a spatially and temporally extensive fossil record, this translates to a need for inclusion of fossil species as well as extant species. The abundance and diversity of extinct and living Aulacoseira species in the fossil record offer an unusual opportunity to test the effects of taxon sampling, the relationship of cladistic branching order to appearance in the fossil record, and to resolve an important problem in biogeographic analysis (relevant extinctions). Not only does this make understanding Aulacoseira systematics of general importance, it emphasizes why an understanding and subsequent cladistic analysis of its morphological characters are vital. Eventually, extinct Aulacoseira species should be understood in the same context as living species. When available, molecular data are a good way to gather information on which to base a phylogenetic estimate. However, morphological data are invaluable in their applicability to fossil taxa, and to extant taxa 
that are not able to be, or have not yet been, cultured. Therefore, the inclusion of both molecular and morphological data will potentially inform a more complete and accurate estimate of phylogeny than either type of data alone.

Diatoms have a diplontic life history, with their most common mode of reproduction being asexual division. As a result, the mean cell size of the descendants decreases (Fritsch 1935). Under appropriate conditions, when individuals are stressed and a cell reaches a certain size threshold for the species, c. $30-40 \%$ of the maximum diameter for centric diatoms, sexual reproduction can occur (Drebes 1977, Edlund and Stoermer 1997). Due to the diminution in cell size over a life cycle, morphological characters are often size-correlated (Edgar and Kociolek MS. submitted 2002, Haworth and Sabater 1993, McBride and Edgar 1998, Theriot 1988). For this reason, sampling characters over the full size range of each species is required for a complete description of the species. Quantitative consideration of variation with respect to size is largely overlooked in diatom systematics.

The first species of Aulacoseira was described by Ehrenberg in 1836 as Gallionella distans. As of 1964, VanLandingham (1971, 1979) recognized 30 described species, 52 varieties and 34 forms, which now fall in Aulacoseira but were included in the more comprehensive genus Melosira at that time. Simonsen (1979) was the first worker to transfer out of Melosira the majority of species and varieties now recognized as Aulacoseira. The majority of varieties fell largely within only a couple of species; for instance, A. distans reportedly had 18 varieties and 8 forms. Aulacoseira distans has 
historically been recognized largely by its low mantle height-to-valve diameter ratio (its boxy shape). Other species that also had low mantle height-to-valve diameter ratios were lumped into $A$. distans as different varieties. Today, many of these varieties have been recognized as sufficiently morphologically different from nominate varieties to be raised to species status (Florin 1981, Krammer 1991b). Recent papers exploring the characters of these species using the SEM have reinforced these taxonomic decisions (Crawford and Likhoshway 2002, Florin 1981, Haworth 1988, Krammer 1991 a, b, Likhoshway and Crawford 2001, Siver and Kling 1997). A review of the literature after 1970 reveals that the number of described species has risen to approximately 60 with 15 common varieties (with approximately $15-20 \%$ of these species reportedly fossil). However, many varieties have yet to be examined in the SEM or morphometrically assessed to see how morphologically different they may be from nominate and other conspecific varieties. The relationships among Aulacoseira species and varieties remain under-explored.

The goal of this study is to estimate the phylogeny of 45 Aulacoseira species using combined morphological and gene sequence data. Data on the species were gathered from: 1) historic collections at the Farlow Herbarium (FH, Harvard University, Cambridge, Massachusetts, USA), or provided by various colleagues (all fossil or preserved materials), 2) personal collections (SME) of living and surface sediment material from which morphological samples were taken and cultures initiated for the collection of molecular sequence data, 3) cultures from the Freshwater Diatom Culture 
Collection (FDCC, Loras College, Dubuque, Iowa, USA) and Culture Collection of Algae and Protozoa (CCAP, Ambleside, Cumbria, UK), 4) sequence data archives of GenBank, and 5) from the literature for the morphological characteristics of species that were not readily accessible for personal examination (Appendices A and B). The types of data used in this analysis included morphometric continuous (or quantitative) data, qualitative morphological data, partial nucleotide sequence data from the $r b c \mathrm{~L}$ region of the chloroplast, as well as nucleotide sequence data from the $18 \mathrm{~S}$ rDNA region of the nuclear genome. These data were analyzed as a combined data set to provide the best global estimate of phylogeny. Phylogenies produced from the independent analyses of these four sets of characters were compared to one another and to the combined estimate in order to evaluate the strength of phylogenetic signal, to assess potential conflicts in this signal among the different types of data, and to identify strongly- and weaklysupported areas in the global estimate of phylogeny. Qualitative and quantitative morphological data were also combined and analyzed in an independent estimate of phylogeny. $18 \mathrm{~S}$ rDNA and $r b c \mathrm{~L}$ sequence data were also combined in a cladistic analysis to assess their contribution alone to the phylogenetic signal.

Problems arising from the mis-identification of Aulacoseira species, the often poor descriptions of their characters in the literature, and the lack of voucher specimens for GenBank sequences and cultures are addressed. Recommendations are made to improve data collection specific to diatoms for future phylogenetic studies.

Morphometric data, including the newly proposed ontogenetic-allometric characters, are 
evaluated for putative homologies, phylogenetic signal, and usefulness in diatom systematics.

Lastly, in addition to presenting novel continuous and ontogenetic allometric characters in Aulacoseira, I also introduce a novel methodological step to the stepmatrix gap weighting method (Wiens 2001) for coding of continuous characters that allows its application to data sets containing more than 32 taxa with unique means, previously the upper limit permitted by this method.

\section{MATERIALS AND METHODS}

Taxa

In this study a total of 45 species of Aulacoseira (39 named, 6 unnamed) and 1 unnamed species of Alveolophora Moiseeva and Nevretdinova (= Miosira Krammer, Lange-Bertalot, and Schiller) were included in the ingroup. Of these, 10 are reportedly fossil taxa. Alveolophora contains 4 described fossil species very similar in morphology to Aulacoseira (Krammer et al. 1997, Moiseeva and Nevretdinova 1990). All 4 of the species now within Alveolophora, including the type for the genus, A. areolata, were in fact originally described as Melosira, and transferred to Aulacoseira in 1971 before being raised to generic status in 1990 (Moisseeva 1971, Moiseeva and Nevretdinova 1990). Its only major morphological differences from other Aulacoseira species lies in the valve shape (ranging from round to elliptical to trilobed) and the presence of alveoli formed by thick silica struts extending from the valve face to the ringleist along the 
inner face of the mantle wall. One Alveolophora sample contained cells with valves that were round to elliptical, facilitating their morphometric comparison with Aulacoseira.

Outgroup taxa chosen for this study were Melosira varians Agardh and Stephanopyxis cf. broschii Grunow. Melosira varians is the only freshwater species remaining in Melosira after the taxonomic revisions of Crawford (1988; Round et al. 1990) and Simonsen (1979), and is considered a sister group to Aulacoseira in some studies (Medlin et al. 1996a, b). However, in other studies the phylogenies produced are ambiguous about whether $S$. cf. broschii (a species from an entirely marine genus whose members were initially classified as Melosira) or Melosira varians is the sister group to Aulacoseira (Kooistra and Medlin 1996). There have been no studies that use more than one representative species for either Melosira or Stephanopyxis in estimating these relationships (and only one or two species of Aulacoseira). Small subunit rDNA sequences were available via GenBank for both $M$. varians and $S$. cf. broschii. In addition, cultures of $M$. varians initiated from my own living collections were used as a source of DNA for sequencing a portion of the chloroplast $r b c \mathrm{~L}$ gene. Both of these taxa were scored for qualitative morphological data as reported in the literature and from personal observations of samples for M. varians (Crawford 1971, 1978, Round et al. 1990). However, only M. varians was available for morphometric measurements. 


\section{Data collection}

Collections of living material were made in North America from July 1998 through September 2002. Living Aulacoseira were isolated using inverted and dissecting microscopes and a mouth-pipetting technique using sterile pasteur pipets drawn to capillary size over a flame. Each filament that was isolated was put through several washes of media before it was placed into a test-tube containing freshwater WC medium (Guillard 1975), with the exception that the amount of silica was doubled as this is typically the limiting nutrient for the growth of diatoms in culture. Each tube was then placed in either a Percival incubator (Percival Scientific, Perry, Iowa) maintained at $14^{\circ} \mathrm{C}$ or $4^{\circ} \mathrm{C}$ with $12: 12$ light:dark regimens, or left at room temperature in a window that received indirect sunlight. Once cultures began to grow, each one was identified initially in the light microscope at 1000x. Further identification occurred in the scanning electron microscope at a later date for each culture used in this study.

The samples of natural collections, or populations, from which cultures were isolated and DNA sequences were derived, were preserved in ethanol or formaldehyde for later use in the collection of qualitative and quantitative morphological data.

Consequently, DNA data and morphological data were derived from exactly the same population where possible. For three cases, in which sequence data were downloaded from GenBank (A. skvortzowii, A. baicalensis, and A. nyassensis), corresponding morphological data were gathered from populations in the same lakes (although at a different time) as the samples used to generate the GenBank sequences. 
Non-morphological characters - small subunit rDNA

To gather enough material for DNA extraction, batch cultures were generally grown in volumes ranging from 250 to $2000 \mathrm{ml}$, condensed into dense pellets of material in microcentrifuge tubes, and cleared of all excess liquids. At this stage a small aliquot of cells was preserved as voucher material in EtOH. Pellets of fresh culture material were frozen at $-80^{\circ} \mathrm{C}$. In preparation for DNA extraction, each pellet was removed from the freezer and immediately crushed vigorously for several minutes in the microcentrifuge tube with a glass or plastic pestle. DNA was then isolated from crushed pellets following the CTAB method of Doyle and Doyle (1987). Amplification via PCR was first done on five species using a reverse primer designed from sequences available via GenBank (ITS-1dr) and the forward primer used for amplification of the 18S rDNA region in diatoms by Medlin et al. (1988; SSU-1 in Table 1). Success of amplification with these primers was limited, so sequences from the few species that amplified were used to construct new primers more specific to Aulacoseira (Table 1). Purification of PCR products was achieved using QIAquick spin columns according to the manufacturer's protocol. Products were quantified with $1 \mathrm{~Kb}$ DNA mass ladder, and approximately $20 \mathrm{ng}$ of each sample was used directly in sequencing reactions using BigDye Terminator and sequenced with an ABI Prism 377A, ABI 3700, or MJ Research BaseStation. Internal primers were designed from the results of the first partial sequences obtained (Table 1). Sequences were obtained in both the forward and reverse 
direction, which were then combined and edited in Sequencher ${ }^{\mathrm{TM}}$, aligned using ClustalX, and finally adjusted by eye, as necessary.

On occasion, when cultures could not be grown into sufficiently large quantities (anything over $150 \mathrm{ml}$ ) a small droplet of the culture would be placed on an alcohol- and flame-sterilized microscope slide under a dissecting microscope in a Labconco ${ }^{\circledR}$ Purifier ${ }^{\mathrm{TM}}$ Clean Bench. While watching through the microscope flame-sterilized forceps were used to apply gentle pressure on a flame-sterilized coverslip resting on top of the sample until the cells were broken open and their cellular contents released. Approximately 10-15 $\mathrm{ml}$ of the cellular mix would be placed directly into PCR tubes. A standard PCR cocktail with Taq was added to the tubes containing the cellular mix (minus 10-15 $\mu$ l of $\mathrm{ddH}_{2} 0$ depending on how much cellular slurry was added), and PCR reactions were run as above.

Taxa sequenced are listed with their 18S rDNA sequences in Appendix C. Sequences directly downloaded from GenBank and used in this study are also noted with their accession numbers in Appendix A. 
Table 1. Primer sequences used in the amplification and sequencing of the $18 \mathrm{~S}$ rDNA region of the nuclear genome in Aulacoseira. Approximate locations of each primer are shown in the cartoon at the bottom of the table.

\begin{tabular}{|c|c|c|c|}
\hline $\begin{array}{l}\text { Forward } \\
\text { primers }\end{array}$ & Primer sequence $\left(5^{\prime}-3^{\prime}\right)$ & $\begin{array}{l}\text { Reverse } \\
\text { primers }\end{array}$ & Primer sequence $\left(5^{\prime}-3^{\prime}\right)$ \\
\hline SSU-1 & AACCTGGTTGATCCTGCCAGT & ITS- $1 \mathrm{dr}$ & CCTTGTTACGACTTCACCTTCC \\
\hline SSU-F & CGAGACTGCGAATGGCTCATT & SSU-R & TTCTCACAGCCACATCCCA \\
\hline Aul & TTCCAGCTCCAATAGCGTAT & Au3 & ATACGCTATTGGAGCTGGAA \\
\hline $\mathrm{Au} 2$ & TCAGCCTTGCGACCATACTC & $\mathrm{Au} 4$ & GAGTATGGTCGCAAGGCTGA \\
\hline \multirow{2}{*}{$5^{\prime}$} & $\mathrm{Au} 1 \rightarrow$ & $\mathrm{Au} 2$ & \\
\hline & - Au3 & & $\longleftarrow_{\text {SSU-R }}^{\text {ITS-1dr }}$ \\
\hline
\end{tabular}

Non-morphological characters $-r b c L$

Whole genomic DNA extractions from the previous sections were also used for amplification of a portion of the chloroplast $r b c \mathrm{~L}$ gene. Primers used for the first round of PCR amplifications and sequencing reactions were the same as (DPrbcL1 and DP $r b c \mathrm{~L} 7$ ) or modified from (NDrbcL6+ and NDrbcL8+) primers used by Daugbjerg and Andersen (1997; Table 2). Success rates on amplifications were initially poor, so new primers were designed from the few Aulacoseira sequences that amplified (Table 2). These new primers were used for all subsequent amplifications, and PCR products purified using QIAquick spin columns according to the manufacturer's protocol.

Products were quantified with $1 \mathrm{~Kb}$ DNA mass ladder, and approximately $20 \mathrm{ng}$ of each sample was used in sequencing reactions using BigDye Terminator and sequenced with an ABI Prism 377A, ABI 3700, or MJ Research BaseStation. Sequences were obtained in both the forward and reverse direction for about a 1,200 bp fragment of the $r b c \mathrm{~L}$ 
gene. Sequences were then combined and edited in Sequencher ${ }^{\mathrm{TM}}$, aligned using ClustalX, and finally adjusted by eye as necessary.

For this $r b c \mathrm{~L}$ data set, the taxa sequenced, sources of the cultures, and GenBank accession numbers are listed in Appendix A, along with information on sequences obtained directly from GenBank for use in this study.

Table 2. Primer sequences used in the amplification and sequencing of a portion of the $r b c \mathrm{~L}$ region of the chloroplast genome in Aulacoseira. Approximate locations of each primer are shown in the cartoon at the bottom of the table. Sites with mixed bases coded as: D (A/T/G), H (A/T/C), S (C/G), W (A/T), Y (C/T), R (A/G).

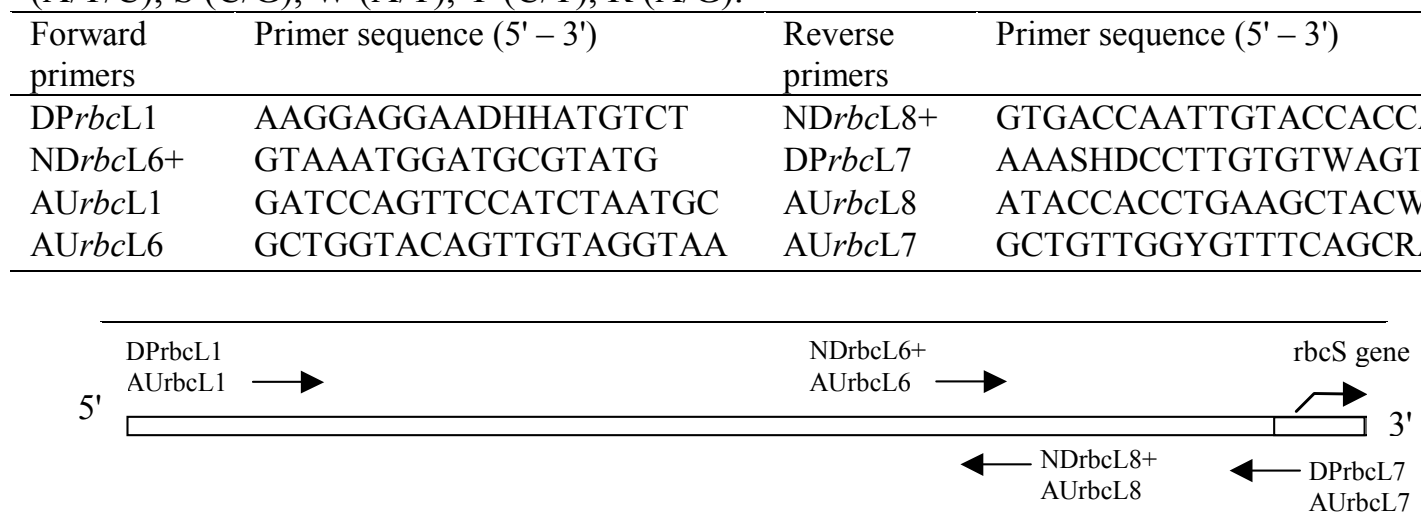

Morphological characters - ultrastructural 'qualitative' characters

Over 1000 samples labeled as containing Aulacoseira in collections from the Farlow Herbarium (Harvard), personal collections of Dr. Edward Theriot and myself, and a few samples from other investigators were screened for this study. Only collections that contained enough valves for measurement $(n \geq 20)$ and had an adequate representation of the size range for the species of interest were used (Appendix A). 
The great majority of samples chosen were available as raw material allowing them to be cleaned and prepared on SEM stubs for the gathering of the more traditional qualitative morphological data. Samples were cleaned with equal amounts of $30 \%$ hydrogen peroxide and distilled water (typically $10 \mathrm{ml}$ each) and three to four crystals of potassium dichromate by gently heating until the color of the sample turned yellow or clear. Samples were then rinsed repeatedly with distilled water before placing aliquots onto coverslips and allowing to dry. An unused coverslip was then placed over half of the specimen-coated coverslip and gentle pressure applied using the eraser of a pencil to fracture the valves exposing their internal structure. Specimens were prepared with the valves broken, as well as intact, to improve chances of viewing all characters regardless of their being internal or external on the valve mantle. The coverslips containing the samples were then attached to SEM stubs via double-sided tape, carbon paint, or carbon tape and grounded with either silver or carbon conductive paint. Samples were sputtercoated with gold-palladium and were generally viewed at 15-30 KV. Images were either captured digitally with a computer attached to a JEOL JSM-330A [Geology Department, UT], or Hitachi FSEM [Texas Materials Institute UT], or by using Polaroid PN/55 film on a JOEL JSM-825 SEM [Biology Department, University of Massachusetts Dartmouth]. All taxa, with the exception of those in two historical collections from the Farlow (HLS 224 and VH 463; Appendix A) and those taxa scored from their literature (Appendix B), had sample material available for SEM and LM examination. 
For preparation of slides for specimens examined under the LM, aliquots of cleaned, well rinsed material were placed on No. 11/2 coverslips and allowed to air dry. Any remaining moisture was driven off by heating the coverslips on a hot plate for 1020 minutes before mounting. Coverslips were typically mounted onto slides using Hyrax ${ }^{\circledR}($ refractive index $=1.71)$, but in some instances, where species possessed more heavily silicified valves, Canada balsam (refractive index $=1.535$ ) was used as a mounting medium to facilitate the imaging of cell wall structure for collection of morphometric data.

Scoring of qualitative morphological characters was largely based on observations for 60 populations representing 36 species of Aulacoseira, one species of Alveolophora, and M. varians in the LM and SEM, except for a few characters and 10 taxa scored from data gathered from the literature (Appendix B). Unfortunately, scanning electron micrographs in the literature often exclusively depict the diatom valve from the outside and focus on the mantle or face of the valve leaving many ultrastructural characters on the inside of valve walls unrevealed, (some exceptions being Crawford and Likhoshway 2002, Likhoshway and Crawford 2001, Siver and Kling 1997), thus leaving some characters unscored for these species.

For the qualitative morphological data gathered via the LM and SEM, each character was evaluated and delineated into character states according to the similarity criterion advocated by Patterson $(1982,1988)$. Multiple populations representing each species were examined where available, and character states assessed and assigned 
independently for each population. These characters did not vary within species as has been previously suggested by Likhoshway and Crawford (2001).

From the variety of samples from herbarium collections (Farlow Herbarium), personal collections of the author, and collections from various colleagues (Appendix A) that were chosen based on the presence of acceptable cell size ranges, 58 populations representing 36 species of Aulacoseira, one species of Alveolophora (unnamed) and $M$. varians were available for collection of morphometric data. Initially, approximately 50 valves were randomly sampled from any one population. If valves were found in filaments or chains, only 1 chosen valve per filament was examined, because all cells contained in that chain are genetically identical. For each valve, images were collected using an Olympus C3040Z digital camera with 2048x1536 pixel resolution attached to an Olympus Photomax microscope using a planachromatic oil immersion objective $(\mathrm{N} . \mathrm{A} \cdot \max =1.25)$. Captured images had about 44 pixels covering $1 \mu \mathrm{m}$ of specimen distance. Two images were collected, generally at $1800 \mathrm{X}$, for each valve examined, one focusing on the cross-section of the valve along the pervalvar axis with brightfield illumination (fig. 13), the other focusing on the areolar patterns on the mantle of the valve generally using Hoffman Modulation contrast optics (fig. 14). Landmarks and pseudo-landmarks were taken for each level of focus using TPSdig morphometric digitizing software (Rohlf 2001). Twenty-six landmarks and pseudo-landmarks were used for the cross-sectional focus on the mantle wall (Fig. 15). On the images focusing on mantle areolae, pseudo-landmarks were made in the center of each areolus within a 
pervalvar stria proceeding from the valve face to the collum for typically five striae, but never fewer than two striae. Generally, the total number of pseudo-landmarks taken on an image of a valve focusing on the areolar patterns in the mantle wall ranged from 15 to 100 , depending on how many areolae occurred within each stria and density of the striae on the valve (Fig. 16). In addition, 20 pseudo-landmarks were taken, 4 for each of 5 areolae, in order to estimate areolar size for each valve (Fig. 17); each pair of these landmarks was used to mark the ends of the major and minor axes of the areolar ellipse. If an areolus was circular, its major axis was taken as parallel to the trend of the pervalvar stria at the areolus. All (pseudo-)landmark x,y coordinates for both levels of focus for each valve were analyzed in Microsoft Excel ${ }^{\circledR}$ using a specifically designed set of Visual Basic macros which: 1) translated and rotated the valves into a standard position and orientation in a Cartesian coordinate system, 2) "unrolled" the cylindrical valve mantle surface which had been digitized to a flat valve surface on which distance measurements would be made in a single flat plane, 3) calculated from the adjusted x,y coordinate data of the distance-based characters of interest for each valve (see below), 4) calculated and plotted summary descriptive univariate or bivariate (with respect to size) statistics for the characters, and 5) calculated the principal axis regression slopes and their $95 \%$ confidence intervals for selected continuous characters versus size.

In addition to random sampling of valves for these specimens, each population measured was examined for under-represented size groups. If any were identified, a stratified random sampling scheme was applied to fill in those size ranges. The total 
number of individuals (represented by one of their two valves) measured for this study was 3575 , ranging from 36 to 144 valves for each species, depending on how many populations were available for measurement.
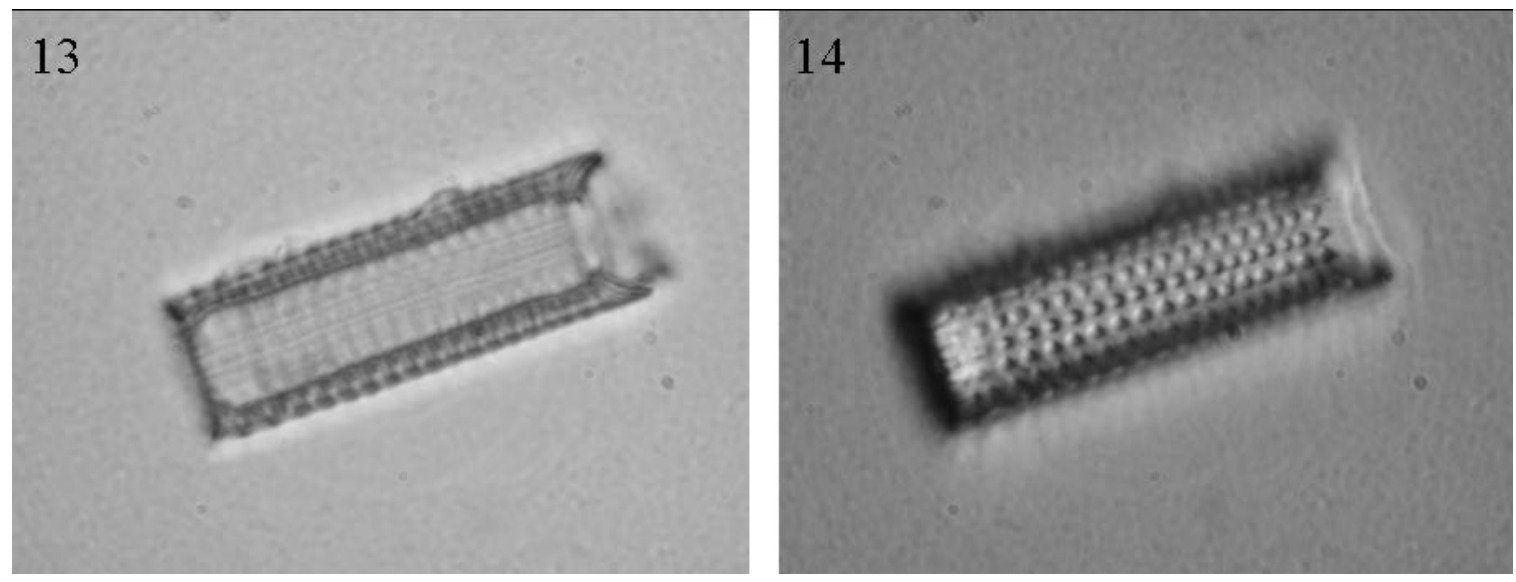

Figures 13 and 14. Images captured in the LM for each valve examined and from which morphometric data were collected. Fig. 13. The first image captured is that of each valve where the level of focus provides a cross sectional view of the mantle wall through the pervalvar axis. Fig. 14. The second image is capture of each valve focusing on the areolar patterns on the mantle wall surface. 


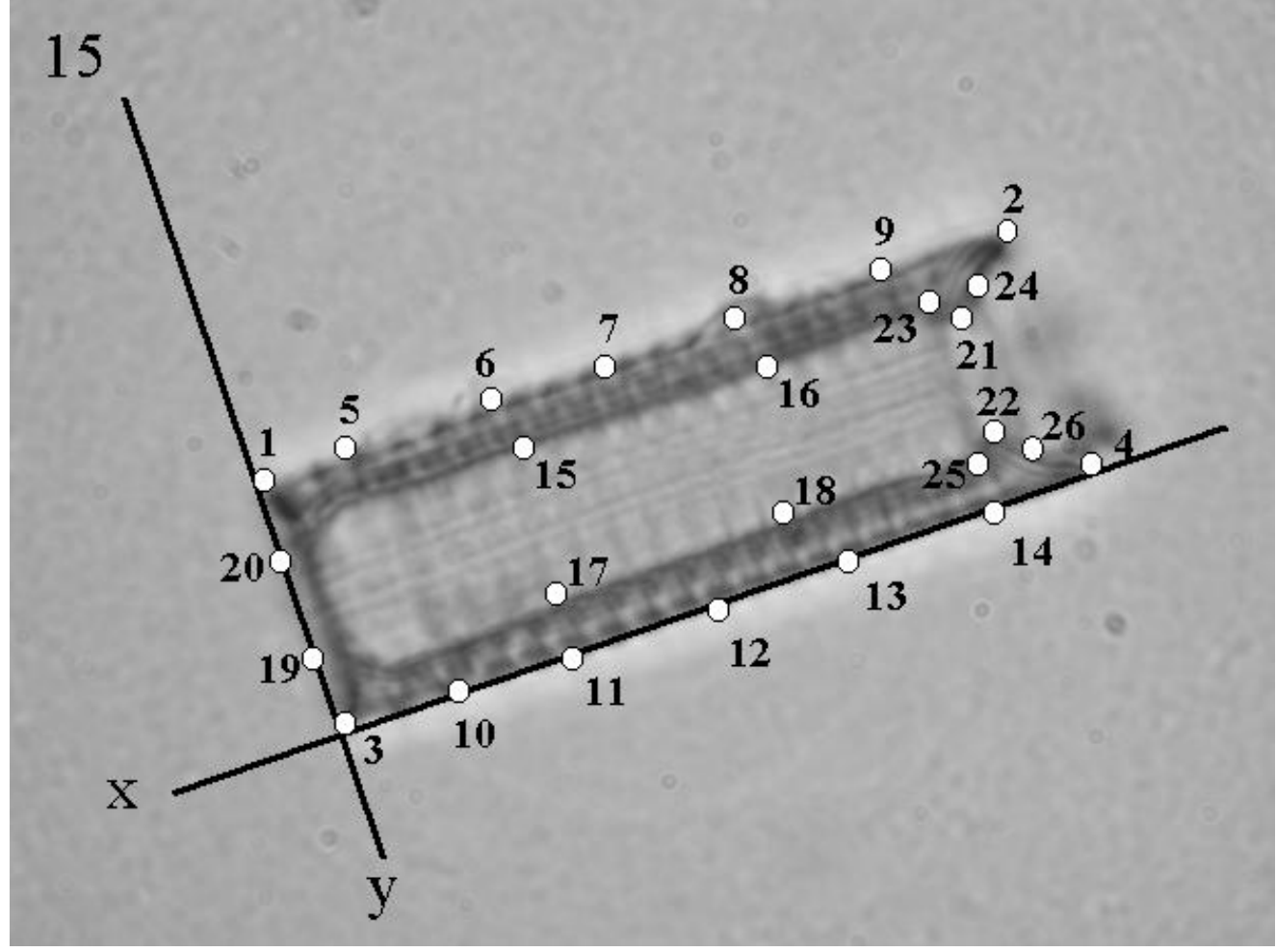

Figure 15. Landmarks and pseudo-landmarks (numbered 1 to 26) are digitized on images focusing on the cross sectional view of the mantle wall. Each valve imaged is rotated into the same orientation where the valve face rests against the $\mathrm{x}$-axis of a Cartesian $\mathrm{x}, \mathrm{y}$-coordinate system and one of the mantle walls rests against the $\mathrm{x}$-axis, and where the rest of the valve is above the $\mathrm{x}$-axis. 


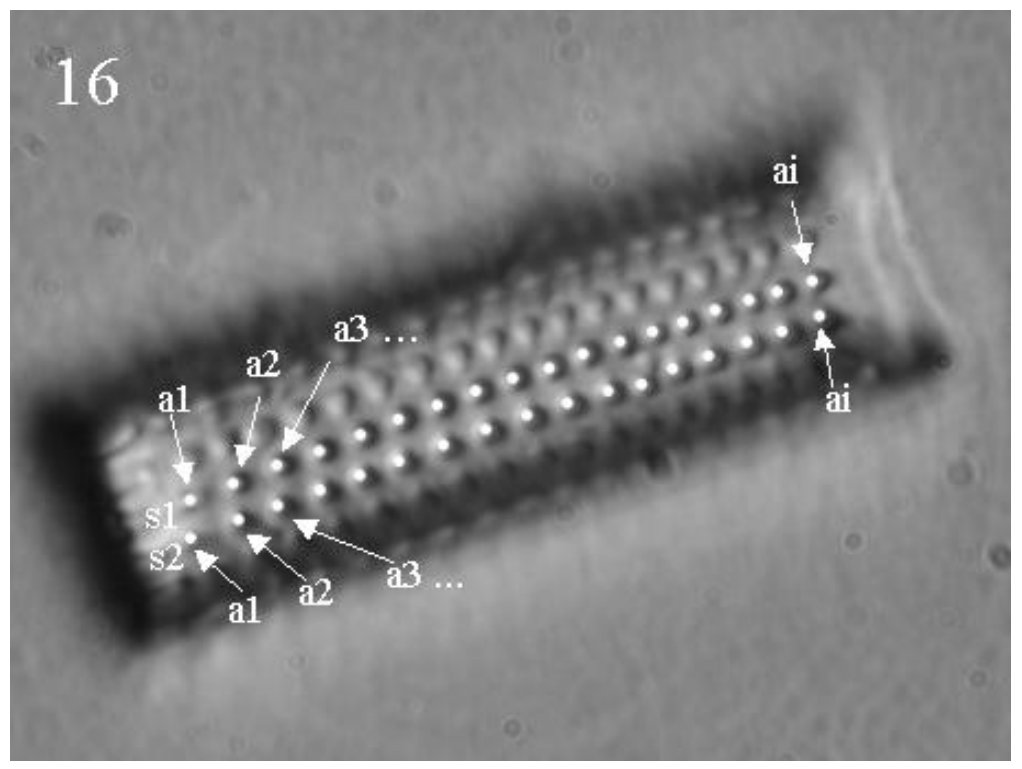

Figure 16. A pseudo-landmark is made at the center of each areolus (a1, a2, ... ai) within a stria (s1 or s2), starting at the valve face, or distal, end of the valve and proceeding to the collum, or proximal, end of the valve for multiple striae per valve.

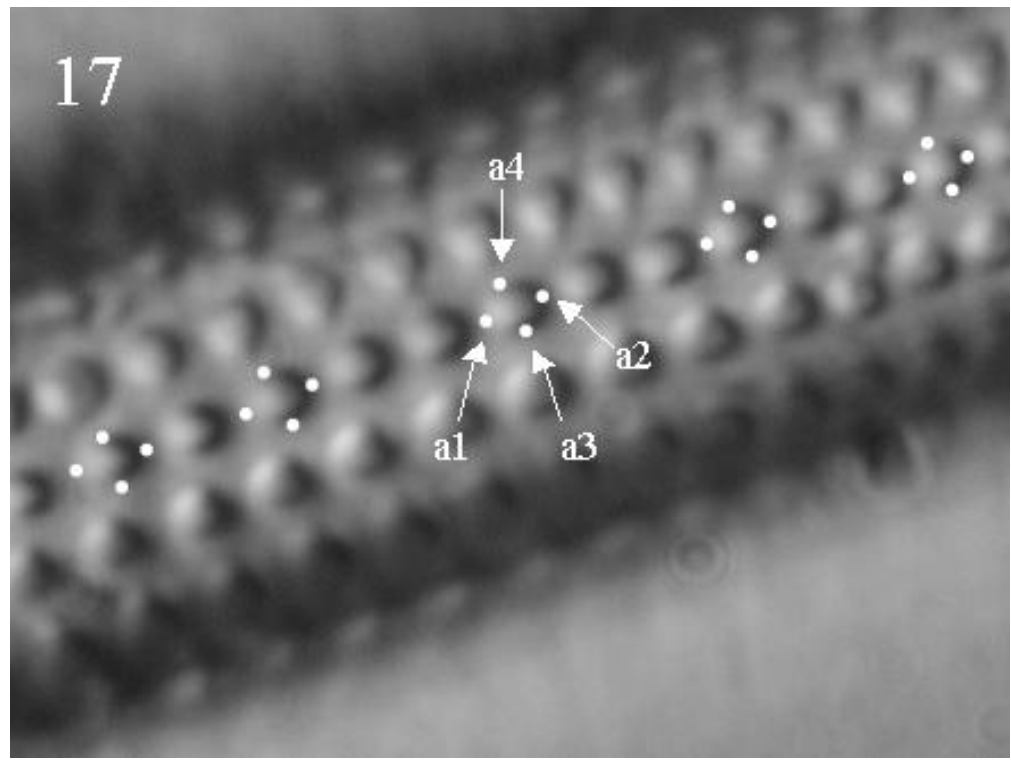

Figure 17. To estimate areolar area, four pseudo-landmarks are made around each of five areolae spread out over the length of the valve mantle. The first two pseudolandmarks for an areolus, a1 and a2, mark the ends of the major axis, while the last two, a3 and a4, mark the minor axis of the areolus. 


\section{Coding of morphological data}

Morphometric data were gathered for 36 species of Aulacoseira, 1 species of Alveolophora and M. varians. To improve taxon sampling, characters for 9 additional Aulacoseira species (where no materials were available for examination) were extracted from the literature for many of the morphometric as well as qualitative characters (Appendix B). However, due to the lack of either original data or bivariate correlations of characters with size reported in the literature for these 9 species, all of the 9 ontogenetic-allometric morphological characters (as measured by principal axis regressions) were left unscored for these taxa, with the exception of mantle height versus diameter reported in Haworth and Sabater (1993). However, more than half of the full set of morphological characters was gathered, and the taxa were included because of the comparative advantage of more thorough taxon sampling despite the increase in missing data (Wiens 1998b, Wiens and Reeder 1995). Quantitative data and qualitative observations extracted from the literature and their sources are listed in Appendix B, the final scores for the data are presented in Appendix E, and the stepmatrices used to weight changes among character states in the phylogenetic analysis for these characters are provided in Appendix F.

All morphometric characters (means and principal axis regression slopes) were coded according to the following method: The taxa are first ranked by their means from lowest to highest. The second step is a modification (addition) to Wiens' (2001) step-matrix gap weighting method to deal with having a number of taxa with unique 
means that exceeds the number of character states allowable by the systematic software being employed. In this case the limitation of 32 character states is imposed by PAUP, so if the number of taxa with unique means is greater than 32 , the means are successively rounded into bins using the smallest amount of rounding (as deemed appropriate depending on the scale of measurement) and increasing bin size bit-by-bit, as necessary, until the 32-character threshold is reached. By successive bouts of rounding by small increments, the maximum number of character states for that character given the data at hand that fits maximally within the constraints of the character state limit imposed has been maintained. This effectively combines taxa with very similar means into the same bin, or code, before the next step of rescaling or range standardization is applied to determine weights for the step-matrix for the character being coded. The steps following this initial rounding are the same as those followed by Wiens (2001). The following equation is used to range standardize and rescale the means so gaps between character states can be assigned a weight via a step-matrix that will be applied to the ordered character:

$$
\mathrm{w}^{\prime}=\frac{(\mathrm{x}-\mathrm{min})}{(\mathrm{max}-\mathrm{min})} * 0.999
$$

where $\mathrm{w}^{\prime}$ is the weight to be applied in the step-matrix to the character state, min is the minimum mean found in the series, $x$ is the mean of the character state being assigned 
the weight, and max is the maximum mean in the series. This first factor is then multiplied by the maximum weight to be assigned in the step-matrix. In this case, decimal weights are being used, and the range for weighting maximized to 0.999 to cover essentially the full range allowable by PAUP and therefore maintain the highest level of resolution in the weighting as is technologically possible. Finally, the ranked means are assigned coded states using integers and letters, and the differences among character states weighted accordingly using step-matrices created via the weights calculated for each taxon's mean or ontogenetic-allometric slope by the equation above.

Using this coding method once a data set reaches a certain size or complexity, integer weights can become too encumbering for PAUP to process, consequently decimal weights were chosen here over integer weights.

Characters 1-18 were treated as unordered with a weight of 1; characters 19-39 were coded following a modified version of the step-matrix gap weighting method outlined by Wiens (2001) and given a weight of 4 (equal to the average number of character states in characters 1-18). Final scores of the characters for each taxon are in Appendix E, and step-matrices used for weighting gaps for each morphometric character are in Appendix F. The following list summarizes each morphological character, its character states and, where appropriate, how the character was measured.

1 Mantle Areolae: (0) absent, (1) present on the mantle, but in a more-or-less irregular pattern, (2) present on the mantle in regular pervalvar rows. 
2 Ringleist: (0) absent, (1) solid, (2) hollow.

3 Separation spine length: separation spines are (0) absent, (1) short-pointed and all of equal length within a valve, (2) moderately long (extending $\sim 1 / 3$ to $2 / 3$ of the length of the valve mantle from which they arise) and all of equal length within a valve, (3) long (extending $\sim 3 / 4$ to full length of the valve mantle from which they arise) and all of equal length within a valve, (4) pointed spines greatly varying in length from short to long within a valve.

4 Pseudosulcus: valve face is $(0)$ very convex resulting in a clear and strong pseudosulcus, (1) slightly convex resulting in a weak but apparent pseudosulcus, (2) flat with no detectable pseudosulcus, (3) concave while that of its sibling valve is convex and they fit together so well that there is no detectable pseudosulcus, (4) dimorphic with states 0 and 1 both present, (5) dimorphic with states 1 and 3 both present.

$5 \quad$ Linking spine rooting: linking spines are (0) absent, (1) rooted by a single pervalvar costa, (2) rooted by two coalescing pervalvar costae, (3) rooted by three coalescing pervalvar costae, (4) rooted by four coalescing pervalvar costae, (5) present but the mantle is hyaline. 
6 Linking spines stalked: linking spines are (0) absent, (1) present and their inflations rest directly on the valve face/mantle junction, (2) present and their inflations are raised above the valve face/mantle junction on a stalk.

$7 \quad$ Shape of linking spine inflation: linking spines are (0) absent, (1) present and their inflations are irregular in shape, (2) present but have no inflations and are short and pointed, (3) present and inflations are very small and round, anchor-like, or shaped like an arrowhead, (4) present and inflations are in the middle of the tapering spine, (5) present and inflations are cruciform or antler-like, (6) present and inflations are triangular, round, or Y-shaped, (7) present and inflations are broad and spathulate, (8) present and inflations are broad and rounded like a teardrop, (9) present but have no inflations as the whole spine is long and rectangular.

8 Linking spine ornamentation: linking spines are (0) absent, (1) present and have smooth edges, (2) present and have silica protrusions around their edge.

9 Separation spine rooting: separation spines are (0) absent, (1) present and are rooted by a single pervalvar costa, (2) present and are rooted by the coalescence of two pervalvar costae, (3) present and are rooted by the coalescence of three pervalvar costae, (4) present and are rooted by the coalescence of five pervalvar costae, (5) present but the mantle is hyaline (i.e., costae are absent). 
10 Spacing between separation spines: separation spines are (0) absent, (1) present with roots separated by a single pervalvar stria, (2) present with roots separated by three pervalvar striae, (3) present with roots separated by numerous $(n>3)$ pervalvar striae, (4) present and evenly spaced, but the mantle is hyaline (i.e., no pervalvar striae).

11 Grooves associated with separation spines: separation spines are (0) absent, (1) present with no grooves in the mantle of sibling valves developed in conjunction with separation spines, (2) present with grooves on the mantles of sibling valves associated with separation spines.

12 Valve face areolae: the valve face (0) is entirely hyaline (i.e., no areolae), (1) has a ring of areolae around the periphery of the valve face but the center is hyaline, (2) has areolae evenly dispersed over the entire valve face.

13 Spacing between linking spines: linking spines are (0) absent, (1) present with roots separated by a single pervalvar stria, (2) present with roots separated by two pervalvar striae, (3) present and evenly spaced, but the mantle is hyaline.

14 Internal opening of the rimoportula: the internal opening of the rimoportula is (0) slit-like with parallel inflations surrounding it in lip-like fashion, (1) slit-like or 
pore-like with no accompanying inflations, (2) a rounded pore surrounded by a tube-like structure.

\section{Structure of rimoportula between internal opening and its exit through the}

mantle wall: the internal opening of the rimoportula is positioned (0) directly on the mantle wall with no other structures associated with it, (1) at the end of a raised spiral hyaline tube fused to and running parallel to the mantle wall, (2) at the end of a straight, slightly raised hyaline tube fused to the internal mantle wall and running parallel to the ringleist, (3) at the end of a straight hyaline area of the internal mantle wall running parallel to the ringleist, (4) at the end of a hyaline tube fused to the ringleist where the opening rests near the ringleist aperture and the tube runs from the opening either along the top fused to the ringleist, or through the ringleist itself, to the mantle wall (sometimes termed 'ringleist canals').

16 Internal location of rimoportulae: intracellularly, rimoportula are located (0) on the ringleist, (1) just above (distal to) the ringleist without intervening areolae, (2) distal to the ringleist and separated from it by one to a few areolae, (3) just above the ringleist and on the mantle proximal to the valve face/ mantle junction, (4) just above the mantle edge and in a ring on the valve face, (5) scattered all over the mantle, (6) solely below the valve face/mantle junction, (7) scattered over the mantle as well as the valve face. 
17 External rimoportular opening: external opening of the rimoportula is $(0)$ small and opens into a mantle areola, (1) larger than and distinct from areolae, (2) small and opens between areolae, (3) small and its opening is associated with an areolus, (4) a tubular extension with a pore at the terminal end.

18 Number of rimoportulae: rimoportulae present per valve is (0) 1 to 3 , (1) 5 to $6,(2)$ numerous ( $\mathrm{n}>6)$, typically 8 to 20 .

Characters 19-39 are all derived from morphometric measurements to which the following applies. 1) All valves were measured in a standard orientation of the valves in which the pervalvar axis is parallel to the coordinate system's x-axis, the apical axis is parallel to the coordinate system's y-axis, one exterior mantle wall rests on the $\mathrm{x}$-axis, and the other above it, and the valve face is flush with the y-axis at its most distal point (fig. 15). 2) Notation used in the following character descriptions is as follows:

$$
\begin{aligned}
& \mathrm{n}, \mathrm{m}=\text { landmark or pseudo-landmark number (figs. } 15 \text { to17) } \\
& \mathrm{a}_{(\mathrm{n})}=\text { areolar landmark numbers (in center of areola; figs. } 16 \text { and 17) } \\
& \mathrm{d}_{\mathrm{n} . \mathrm{m}}=\text { distance between landmarks } \mathrm{n} \text { and } \mathrm{m} \\
& \mathrm{L}_{\mathrm{n} . \mathrm{m}}=\text { line segment between landmarks } \mathrm{n} \text { and } \mathrm{m}
\end{aligned}
$$

3) All distances are assessed as absolute distances, except those which are used in assessing striae curvature. 4) All distances between a point and a line segment are 
measured perpendicular to the line segment. 5) Proximal and distal as used here are in reference to the collum end of the valve, which is the center of a complete frustule or cell.

19 Mean mantle height (fig. 15):

$$
=0.5\left(\mathrm{~d}_{1.3}+\mathrm{d}_{2.4}\right) \quad\{\mathrm{x} \text {-dimension only }
$$

20 Ontogenetic-allometric trajectory for mantle height: slope of the principal axis regression of mantle height versus valve diameter.

21 Mean mantle thickness (fig. 15):

$$
=0.25\left(\mathrm{~d}_{\mathrm{y} .15}+\mathrm{d}_{\mathrm{y} \cdot 16}+\mathrm{d}_{\mathrm{y}^{\prime} .17}+\mathrm{d}_{\mathrm{y}^{\prime} \cdot 18}\right) \quad\{\mathrm{y} \text {-dimension only }\}
$$

where $y$ is the mean y-coordinate value of landmarks $1,3,5-9$, and $y^{\prime}$ is the mean $y$ coordinate value of landmarks $2,4,10-14$.

22 Ontogenetic-allometric trajectory for mantle thickness: slope of the principal axis regression of mantle thickness versus valve diameter.

23 Collum height (fig. 16):

$$
=0.5\left(\mathrm{~d}_{1.3}-\mathrm{D}-0.5 \mathrm{~d}_{\text {majax }}\right)+\left(\mathrm{d}_{2.4}-\mathrm{D}-0.5 \mathrm{~d}_{\text {majax }}\right) \quad\{\mathrm{x} \text {-dimension only }
$$


where $\mathrm{D}$ is the distance from the center of the most proximal areola on the mantle to the valve face (measured perpendicularly to the $\mathrm{L}_{1.2}$ ), and where $\mathrm{d}_{\text {majax }}$ is the mean length of the major axis of areolae on the mantle (See character 38.).

24 Ontogenetic-allometric trajectory for collum height: slope of the principal axis regression of collum height versus valve diameter.

25 Mean ringleist height (fig. 15):

$$
=0.5\left(\mathrm{~d}_{(\mathrm{L} 3.4) .21}+\mathrm{d}_{(\mathrm{L} 3.4) .22}\right) \quad\{\mathrm{x} \text {-dimension only }\}
$$

26 Ontogenetic-allometric trajectory for ringleist height: slope of the principal axis regression of ringleist height versus valve diameter.

27 Mean depth of penetration of the ringleist into valve lumen (fig. 15):

$$
=0.5\left(\mathrm{~d}_{21 .(\mathrm{L} 23.24)}+\mathrm{d}_{22 .(\mathrm{L} 25.26)}\right) \quad\{\mathrm{y} \text {-dimension only }\}
$$

28 Ontogenetic-allometric trajectory for depth of ringleist penetration: slope of the principal axis regression of ringleist depth of penetration versus valve diameter.

29 Mean ringleist thickness (fig. 15):

$$
=0.5\left(\mathrm{~d}_{23.24}+\mathrm{d}_{25.26}\right) \quad\{\mathrm{x} \text {-dimension only }
$$


30 Ontogenetic-allometric trajectory for ringleist thickness: slope of the principal axis regression of ringleist thickness versus valve diameter.

\section{Mean interareolar distance (fig. 16):}

for each areola the interareolar distance is $=\mathrm{d}_{\mathrm{a}(\mathrm{i}) \mathrm{a}(\mathrm{i}+1)}$, where (i) is one areolus and (i+1) is the next most proximal areolus in the same stria. The mean is based on all digitized areolae except the most proximal in each stria. \{Both $\mathrm{x}$ and $\mathrm{y}$ dimensions are considered.\}

32 Ontogenetic-allometric trajectory for mean interareolar distance: slope of the principal axis regression of distance between areolae within pervalvar striae versus valve diameter.

\section{Mean interstriae distance (fig. 16):}

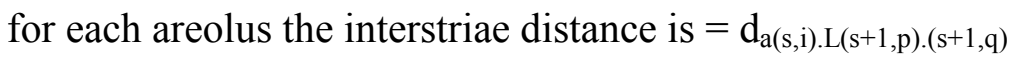

where $\mathrm{a}(\mathrm{s}, \mathrm{i})$ is the $\mathrm{i}^{\text {th }}$ areola in the $\mathrm{s}^{\text {th }}$ stria and $\mathrm{L}$ is a line segment between two adjacent areolae ( $\mathrm{p}$ and $\mathrm{q})$ in the adjacent (clockwise as seen from valve face) stria $(\mathrm{s}+1)$, one distal ( $\mathrm{p}$; in the $\mathrm{x}$ dimension) to $\mathrm{a}(\mathrm{s}, \mathrm{i})$ and one proximal ( $\mathrm{q}$; in $\mathrm{x}$ dimension) to $\mathrm{a}(\mathrm{s}, \mathrm{i})$. The mean interstriae distance is based on this distance for all areolae in a striae for which adjacent striae areolae meeting the requirements of the measurement. 
Occasionally the first areola in a stria did not meet these requirements, and usually the last areola in a stria did not. All areolae in the last stria were necessarily excluded. \{Both $\mathrm{x}$ and $\mathrm{y}$ dimensions are considered.\}

34 Ontogenetic-allometric trajectory for mean interstriae distance: slope of the principal axis regression of distance between pervalvar striae versus valve diameter.

35 Mean curvature of pervalvar striae in linking valves (fig. 16):

For each areolus the striae curvature was defined as the smaller of the two angles (in degrees) formed by $\mathrm{L}_{\mathrm{a}(\mathrm{i}) . \mathrm{a}(\mathrm{i}+1)}$ within the same stria and the pervalvar axis. The mean curvature of pervalvar striae on a valve was based on all areolae except the most proximal in each stria. \{Both $\mathrm{x}$ and $\mathrm{y}$ dimensions are considered.\}

36 Mean curvature in pervalvar striae in separation valves: same as Character 34 but applied to separation valves.

37 Mean valve diameter (fig. 15):

$$
=\mathrm{d}_{\mathrm{y} \cdot \mathrm{y}^{\prime}} \quad\{\mathrm{y} \text {-dimension only }\}
$$

where $y$ is the mean y-coordinate value of landmarks $1,3,5-9$, and $y^{\prime}$ is the mean $y$ coordinate value of landmarks $2,4,10-14$. 
38 Mean areolar area (fig. 17):

For each areolus, areolar area was calculated as $=0.5 \pi\left(\mathrm{d}_{\mathrm{a} 1 . \mathrm{a} 2}\right)\left(\mathrm{d}_{\mathrm{a} 3 . \mathrm{a} 4}\right)$, where a1 and a2 mark ends of the major axis of the areolar ellipse, or, if circular, the axis parallel to the striae slope at that areolus, and a3 and a4 mark the ends of the minor axis of the areolar ellipse, a line segment across the center of the areola perpendicular to the major axis $\left(\mathrm{L}_{\mathrm{a} 1 . \mathrm{a} 2}\right)$. The mean areolar area for each valve is based on 5 areolae distributed along the length of the mantle.

39 Ontogenetic-allometric trajectory for areolar area: slope of the principal axis regression of areolar area versus valve diameter.

Analyses

All matrices and step-matrices were constructed in PAUP v4b10 (Swofford 2000). Maximum parsimony analyses were run in PAUP v4b10 except where noted. All quantitative overlapping data were treated as ordered, whereas all other data (qualitative morphological characters, gene sequence data) were treated as unordered. Quantitative characters were weighted so that their whole range of variation was on average equal to the average number of character states (4) found in the qualitative data. Gaps were treated as missing data. All most parsimonious trees were identified using 1000 replicates of heuristic searches employing the tree bisection and reconnection (TBR) branch swapping algorithm with random sequence addition. In the independent 
analyses for qualitative data and $18 \mathrm{~S}$ rDNA data, a time limit of 90 seconds was introduced for the heuristic search. Non-parametric bootstrap analyses were performed for 250 to 500 pseudo-replicates of 10 TBR addition sequence replicates each for the combined analysis, as well as for the independent data set analyses. Bootstrap estimates for the independent analyses of qualitative data, $18 \mathrm{~S}$ rDNA data, morphometric data, as well as for the combined DNA ( $r b c \mathrm{~L}$ and $18 \mathrm{~S}$ rDNA) analysis, a time limit of 90 seconds for the addition sequence replicates was imposed. Nodes with bootstrap values $\geq 70$, are considered to be well supported. Bremer support (Bremer 1994) was estimated for the total evidence tree using 125 addition sequence replicates and the TBR branch swapping algorithm for each node. The command file to perform these searches based on constraint trees for each node of the most parsimonious tree from the original 1000 rep heuristic search was produced using TreeRot.v2 (Sorenson 1999).

\section{RESULTS}

The combined analysis of all data (total evidence; quantitative and qualitative morphological characters, $18 \mathrm{~S} \mathrm{rDNA}, r b c \mathrm{~L}$ ) produced a single most parsimonious tree (MPT; Fig. 18; length (L) =1280.392, consistency index excluding uninformative characters $(\mathrm{ci})=0.41$, retention index $(\mathrm{ri})=0.65)$. There are five major clades that can be identified in this global or combined phylogenetic estimate (Fig. 18): 1) a most basal clade containing Aulacoseira crenulata, A. italica, three hyaline species and a couple of unnamed species, 2) a clade containing $A$. subarctica, $A$. herzogii, and a mix of species 
typically described as possessing low mantle-height-to-valve-diameter ratios, including A. distans, 3) a clade containing the $A$. granulata complex, 4) a clade containing $A$. ambigua, A. nyassensis, and A. valida, and 5) a clade containing A. islandica, A. skvortzowii, A. baicalensis, Alveolophora and many extinct Aulacoseira taxa. All seven species represented by more than one population (sample) were found to be monophyletic.

Both the $18 \mathrm{~S}$ rDNA and the $r b c \mathrm{~L}$ data sets were missing for two-thirds of the taxa. The taxa that are represented by these data fall evenly within each of the five major clades in the combined tree. The strict consensus of the 822,225 most parsimonious trees (MPTs) produced by the 18S rDNA data alone (fig. 19; component tree statistics: $\mathrm{L}=512, \mathrm{ci}=0.65, \mathrm{ri}=0.74$ ), is somewhat less resolved, but agrees entirely with the strict consensus of the $150 \mathrm{MPTs}$ from the analysis of the $r b c \mathrm{~L}$ data alone (fig. 20; component tree statistics: $\mathrm{L}=197, \mathrm{ci}=0.63$, $\mathrm{ri}=0.78$ ). When the $18 \mathrm{~S}$ rDNA and $r b c \mathrm{~L}$ gene sequence data were analyzed together, the basal relationships were also less resolved than in the independent analysis of $r b c \mathrm{~L}$, and the basal position of the A. subarctica clade was unresolved (fig. 21 ; component tree statistics: $\mathrm{L}=705$, ci $=$ $0.65, \mathrm{ri}=0.76)$.

The strict consensus tree of 16,965 MPTs from the phylogenetic estimate based on qualitative morphological data (fig. 22; component tree statistics: $\mathrm{L}=143$, ci $=0.46$, $\mathrm{ri}=0.80)$ indicates that this type of data provides a very limited and specific signal. For species represented by multiple populations (samples), they group populations together 
into clades for each species, with the exception of $A$. canadensis. However, these qualitative data alone are unable to resolve relationships among populations within a species, as well as relationships among species.

In contrast to the qualitative character results, the phylogenetic estimate (fig. 23; 2 MPTs; component tree statistics: $\mathrm{L}=86.731, \mathrm{ci}=0.23, \mathrm{ri}=0.57$ ) based solely on morphometric quantitative data is highly resolved at all levels of the tree, but species containing multiple representatives (populations/samples) are only found to be monophyletic for A. ambigua, A. crenulata and A. sp. 3 .

The combination of the qualitative and quantitative morphological characters in a maximum parsimony analysis results in a single MPT. Each of the seven species with more than one representative population is found to be monophyletic (fig. 24; $\mathrm{L}=$ $553.432, \mathrm{ci}=0.26, \mathrm{ri}=0.59)$. The combination of both 'types' of morphological data also show major changes in which taxa fall together into clades and in the basal relationships among these clades. Although the qualitative data alone do not reveal any basal relationships in the strict consensus of MPTs, when they are combined with the morphometric data, five major clades (fig. 24) that are very different from those presented by the morphometric data alone (fig.23) are resolved. These five clades found with all morphological data combined closely match in population composition the five major clades found in the DNA combined phylogeny for those populations included in both data sets (fig. 21). The basal relationships hypothesized by each of these analyses, however, are in slight disagreement. 
When the MPT from the combined morphological data (fig. 24) is compared with the MPT from the total evidence data set (fig. 18), the five major clades largely remain the same in population composition, but there are some slight shifts in terminal relationships as well as some taxa $(n=4)$ positioned in different clades or displaced from the clades altogether $(n=5)$. Also, the basal relationships among these five major clades change in the combined morphology tree (fig. 24) compared to the total evidence tree (fig. 18). The total evidence MPT (fig. 18) agrees with the combined DNA tree (fig. 21) that clade 1 is the most basal of the five clades. But, then clade 2 is the next to branch off in the total evidence tree (which is the most basal clade in the combined morphology tree) instead of clade 5, which is the next clade to branch off in the combined DNA tree. Clades 3 and 4 are always sister groups in the combined morphology (fig. 24), combined DNA (fig. 21), and total evidence trees (fig. 18), and they are always the most terminal or derived clades as well. Because of the small number of species contained in each clade for the combined DNA data (fig. 21), evaluation of agreement in terminal relationships among taxa within clades compared to other data sets is precluded. Overall, the branching patterns of the total evidence tree is well supported, or not strongly contradicted, by the phylogenetic analyses of independent data sets. 


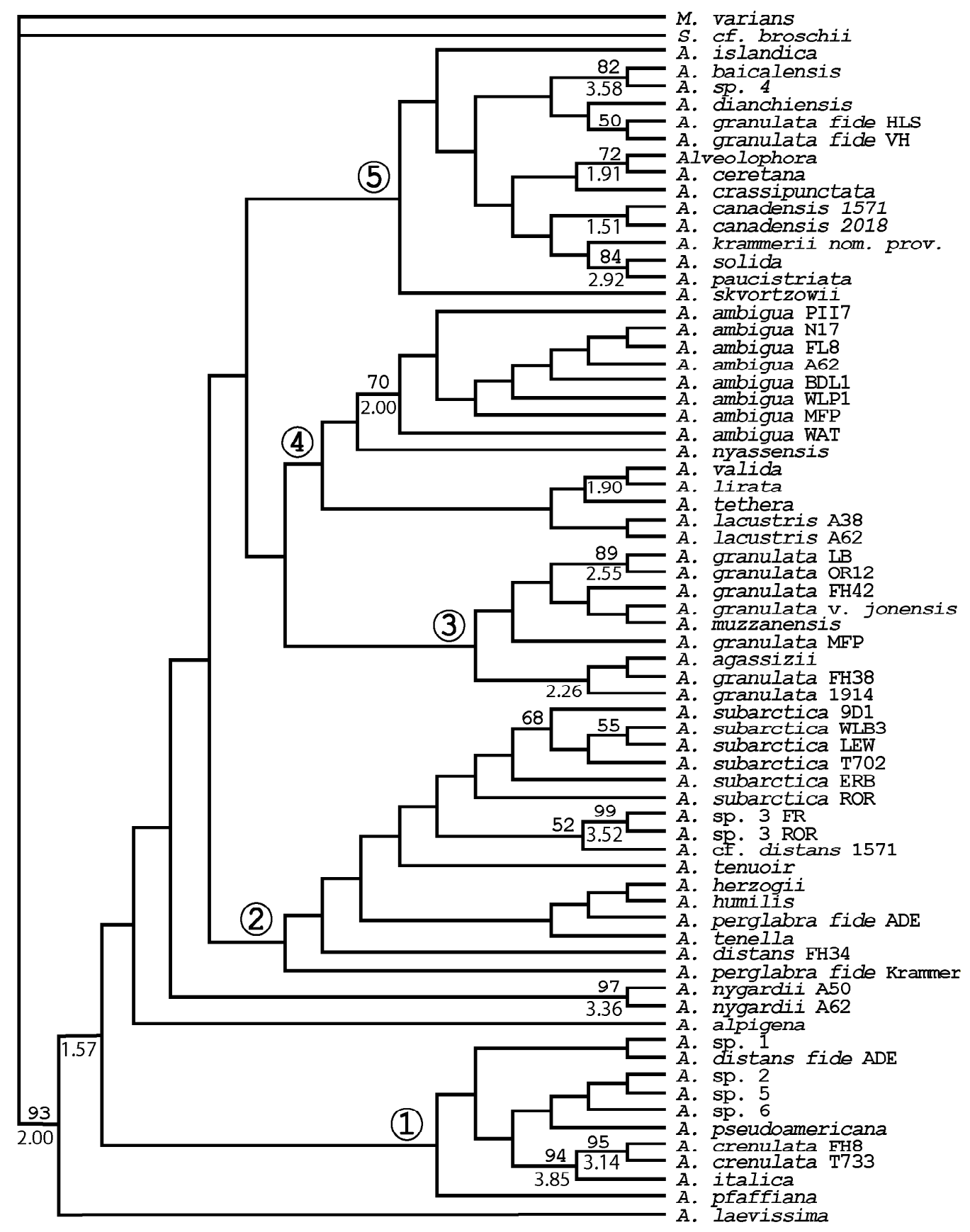

Figure 18. Total evidence phylogeny of Aulacoseira (1 MPT). Bootstrap values are shown above branches are based on 500 pseudo-replicates of 10 addition sequence replicates employing the TBR branch swapping algorithm. Bremer supports are shown below selected branches and are based on 125 addition sequence replicates per node. Length $(\mathrm{L})=1280.392$, consistency index excluding uninformative characters $(\mathrm{ci})=0.41$, retention index $(\mathrm{ri})=0.65$. Five major clades are labeled at their nodes $($ circled numbers). 


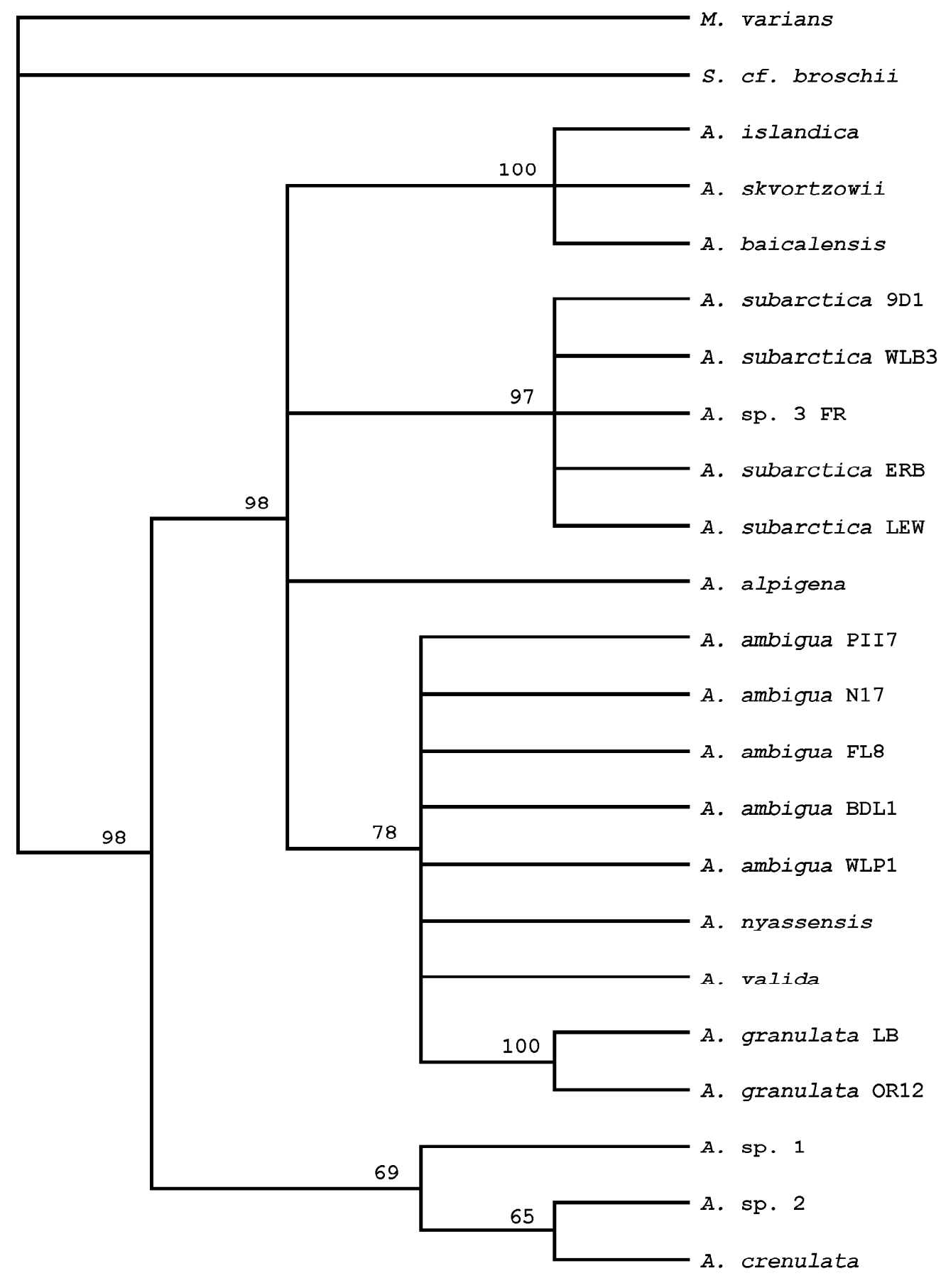

Figure 19. Strict consensus of 822,255 MPTs found in an independent analysis of $18 \mathrm{~S}$ rDNA. Bootstrap values above branches are based on 338 pseudo-replicates of 10 addition sequence replicates employing TBR branch swapping. Statistics for component trees from which this strict consensus tree was derived: $\mathrm{L}=512, \mathrm{ci}=0.65, \mathrm{ri}=0.74$. 


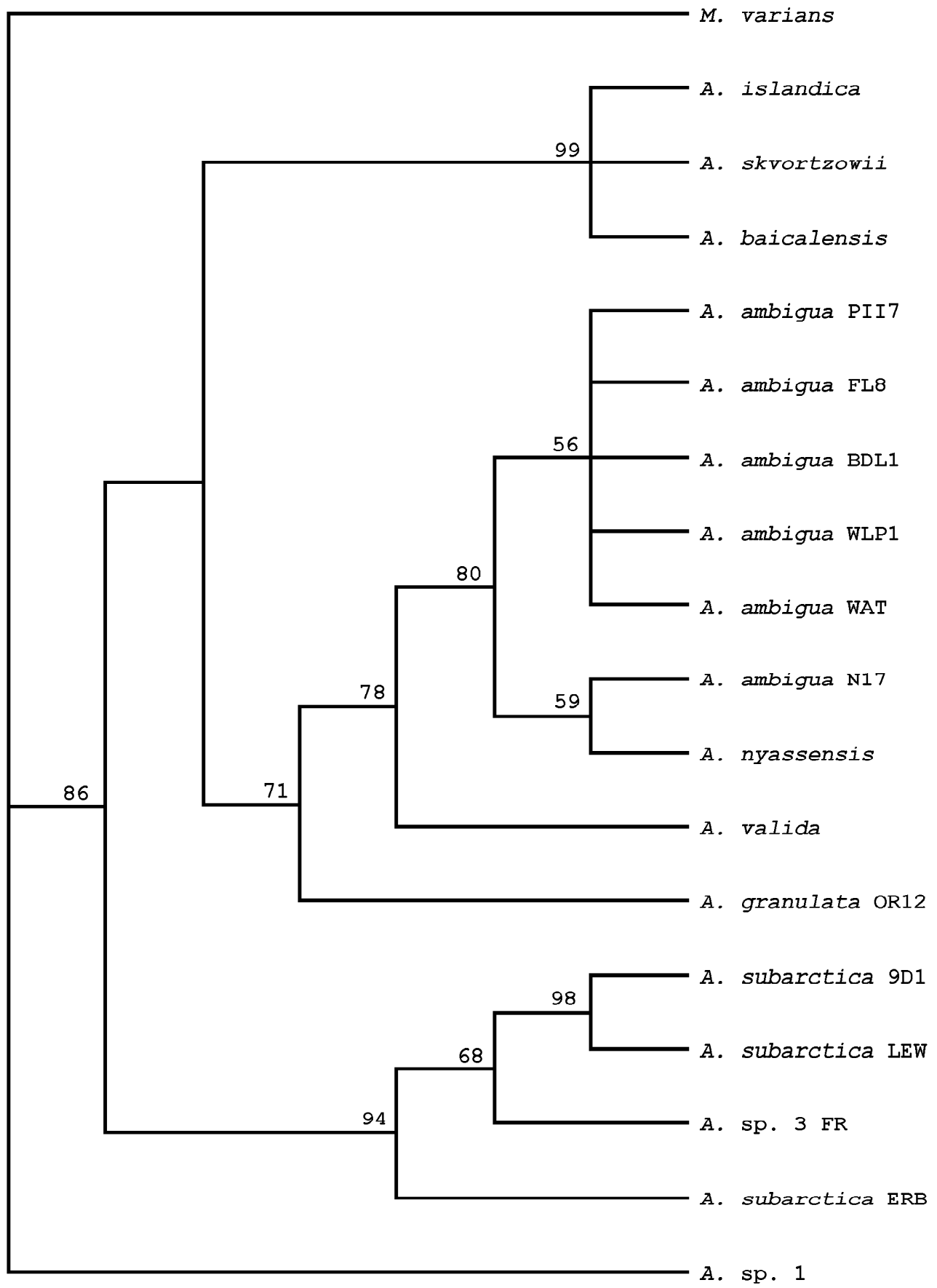

Figure 20. Strict consensus of $150 \mathrm{MPTs}$ found in an independent analysis of $r b c \mathrm{~L}$ data. Bootstrap values above branches are based on 500 pseudo-replicates of 10 addition sequence replicates employing TBR branch swapping. Statistics for component trees from which this strict consensus tree was derived: $\mathrm{L}=197, \mathrm{ci}=0.63, \mathrm{ri}=0.78$. 


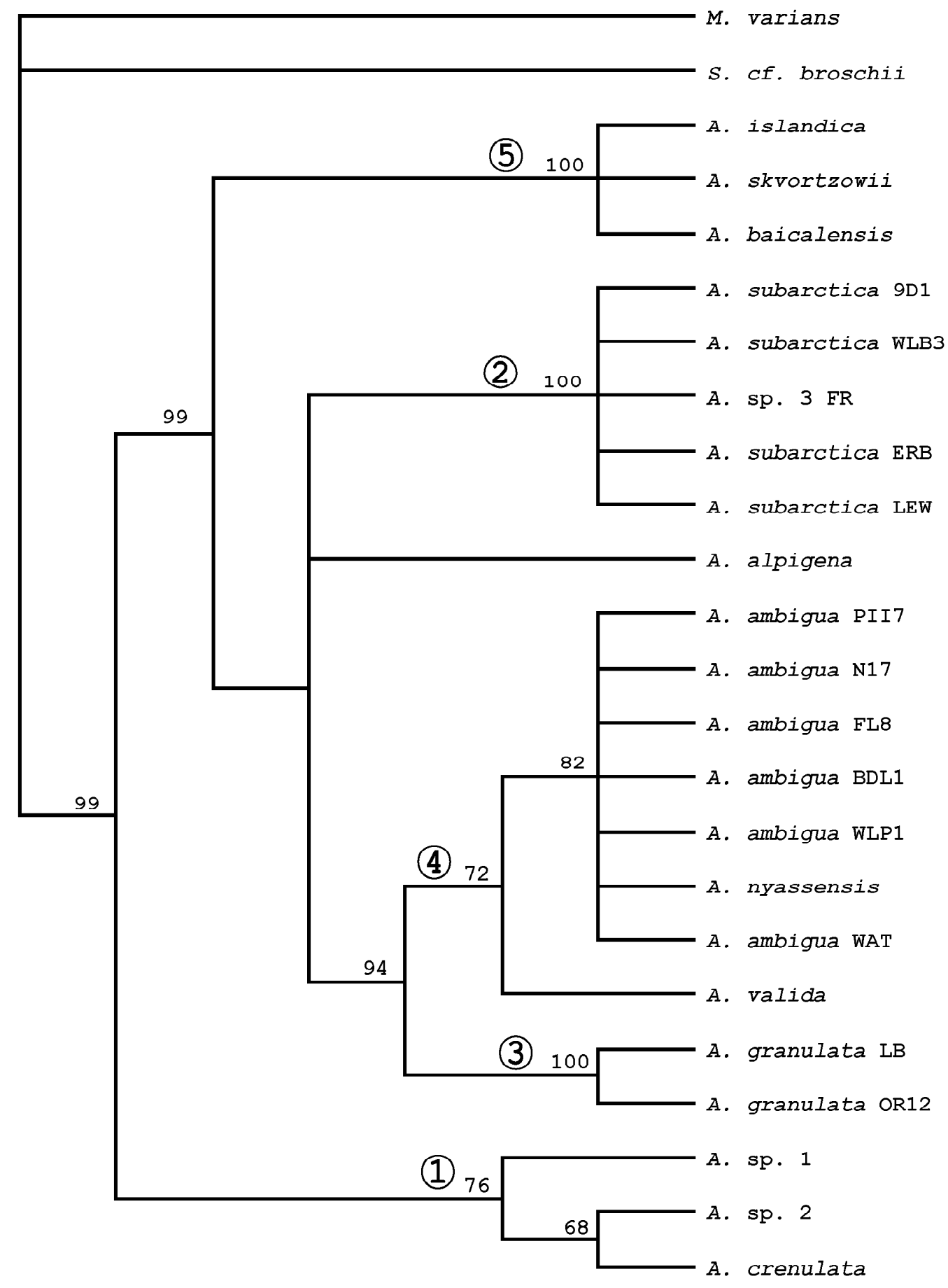

Figure 21. Strict consensus of 3,948 MPTs for DNA data combined (18S plus $r b c \mathrm{~L}$ ). Bootstrap values above branches are based on 500 pseudo-replicates of 10 addition sequence replicates employing TBR branch swapping. Statistics for component trees from which this strict consensus tree was derived: $\mathrm{L}=705, \mathrm{ci}=0.65, \mathrm{ri}=0.76$. Five major clades are labeled at their nodes (circled numbers). 


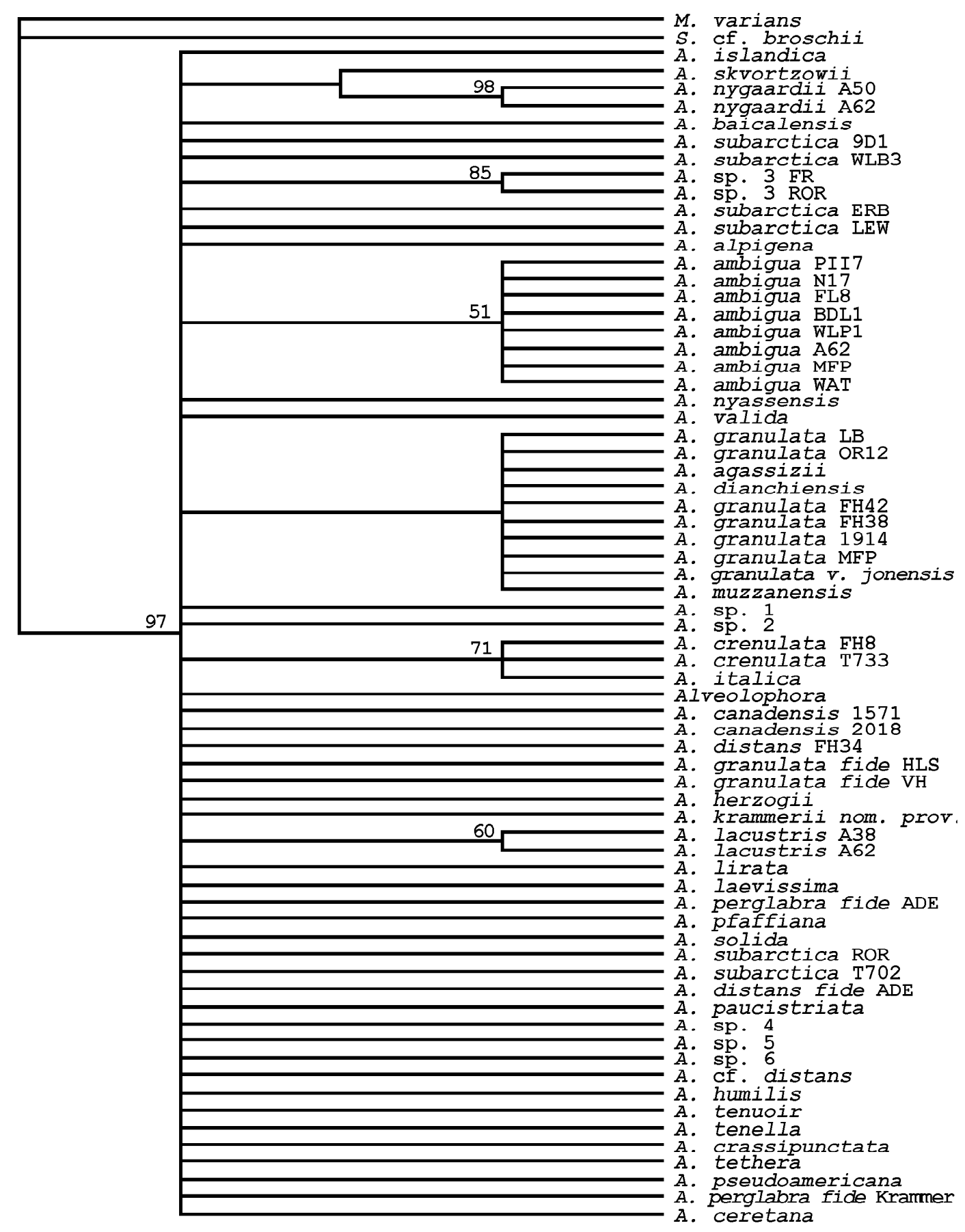

Figure 22. Strict consensus of 16,965 MPTs from independent analysis of qualitative morphological characters. Bootstrap values above branches are based on 370 pseudo-replicates of 10 addition sequence replicates employing TBR branch swapping. Statistics for component trees from which this strict consensus tree was derived: $\mathrm{L}=143, \mathrm{ci}=0.46, \mathrm{ri}=0.80$. 


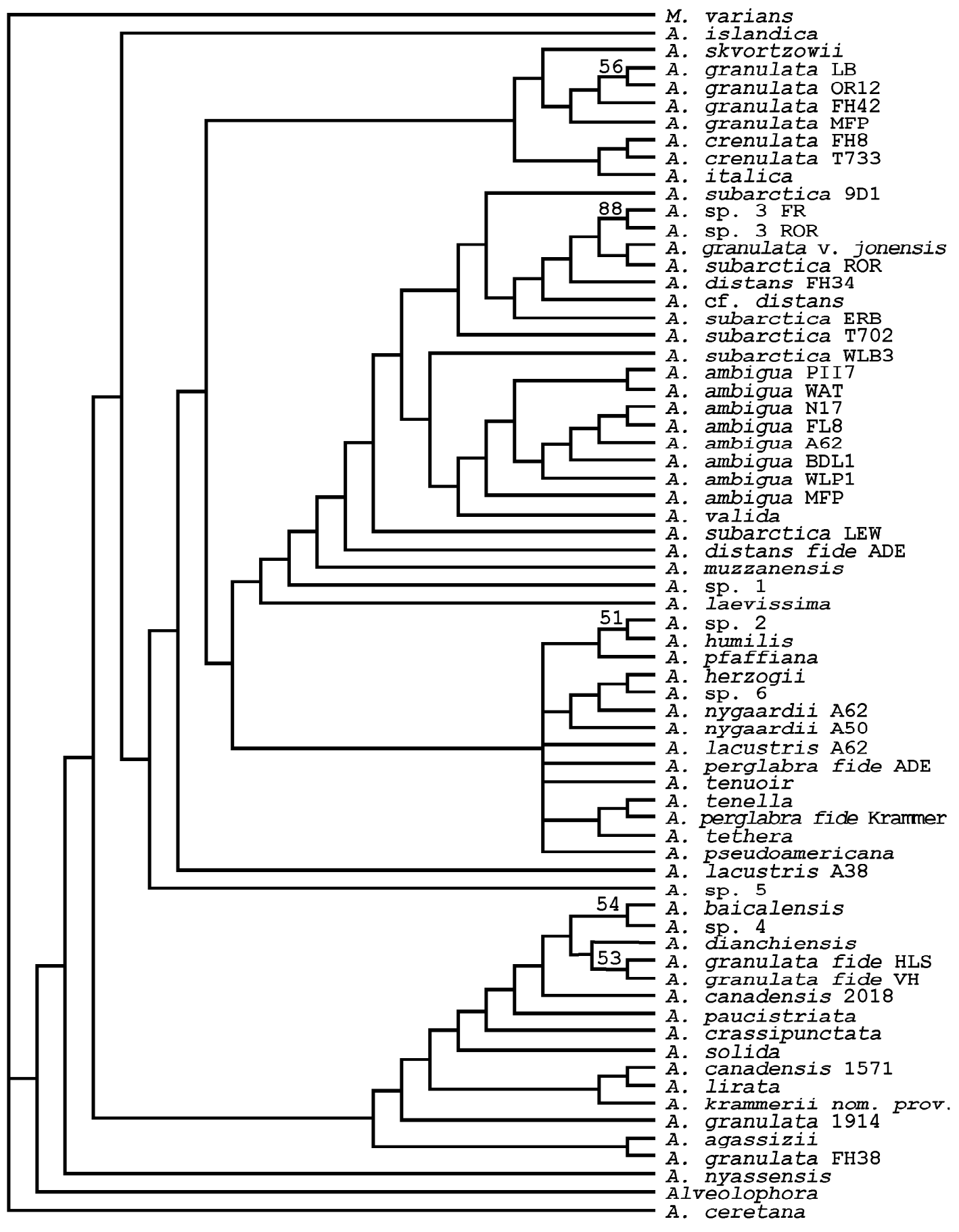

Figure 23. Strict consensus of 2 MPTs found in an independent analysis of morphometric data. Bootstrap values above branches are based on 500 pseudo-replicates of 10 addition sequence replicates employing TBR branch swapping. Statistics for component trees from which this strict consensus tree was derived: L $=86.731, \mathrm{ci}=0.23, \mathrm{ri}=0.57$. 


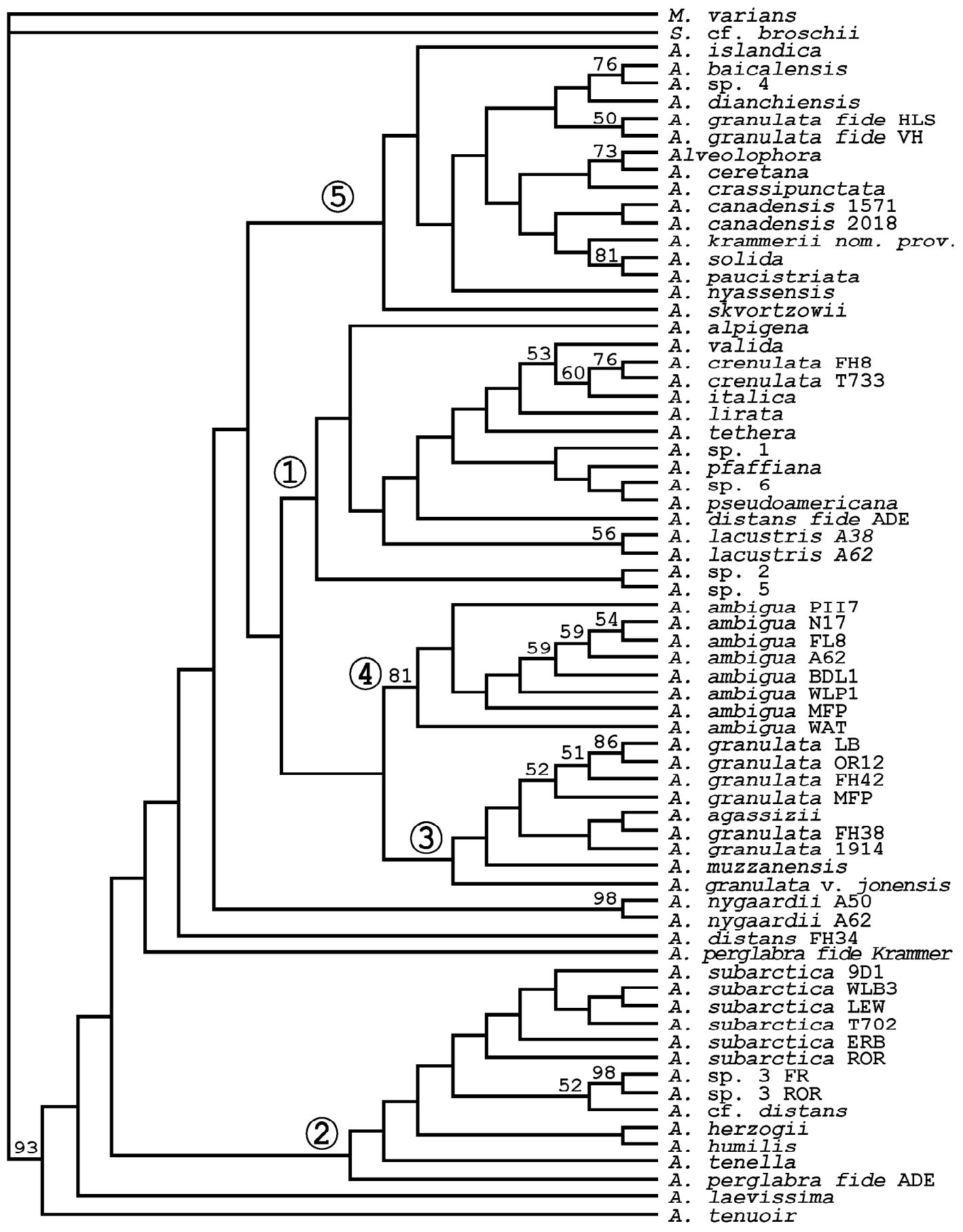

Figure 24. Most parsimonious tree found in an analysis of the combined morphological data (qualitative plus morphometric). Bootstrap values above branches are based on 300 pseudo-replicates of 10 addition sequence replicates employing TBR branch swapping. $\mathrm{L}=553.432, \mathrm{ci}=0.26, \mathrm{ri}=0.59$. Five major clades are labeled at their nodes (circled numbers). 


\section{DISCUSSION}

There have been four basic approaches to estimating phylogenies when multiple sources or 'types' of data are employed: 1) the taxonomic congruence approach, in which each data 'type' or partition is analyzed separately and then compared to one another via consensus among data types (Mickevich 1978, Miyamoto and Fitch 1995, Nelson 1979), 2) the total evidence approach, in which data types are combined for analyses to come up with a phylogenetic estimate that is able to most parsimoniously explain all of the data (Chippindale and Wiens 1994, Kluge 1989, Kluge and Farris 1969, Omland 1994), 3) the prior agreement approach, in which data are combined if they are found to be congruent with one another via a statistical test, such as the homogeneity partition test implemented in PAUP, otherwise the data are analyzed separately (Bull et al. 1993, de Queiroz 1993), and 4) the approach proposed by Wiens (1998a) that advocates combining the data for a best global estimate of phylogeny, but also examining data partitions independently to identify areas in the global phylogeny that may be weak due to localized differences in phylogenetic histories among data sets (Cannatella et al. 1998, Poe 1996). This study follows the fourth approach.

Unfortunately, in the data set gathered for this study, several taxa were unable to be scored for the DNA data because: 1) they were fossil taxa, 2) they were unable to be found in culture collections, 3 ) they were unable to be found in bloom (living and in large enough numbers to detect) in live field samples, or 4) they were found living, but would not grow up when isolated for cultures. This left the number of taxa with 
complete data for DNA and morphology at 14, compared to 44 Aulacoseira species scored for both qualitative and quantitative characters.

Although complete data for all taxa in this study would have been preferable, Wiens (1998b) and Wiens and Reeder (1995) have shown via simulation studies that inclusion of taxa with large, non-random chunks of missing data (e.g., missing data partitions for some taxa), can still lead to relatively accurate phylogenetic estimates. And the argument follows that it is better to have pretty good estimates of phylogeny where all taxa of interest are included than to have really good estimates of phylogeny limited to just a few taxa (Wiens and Reeder 1995).

By evaluating topology of the various data partitions separately and in combination, it becomes possible to detect differences in phylogenetic signal from these different data sources and to assess their approximate contribution to the combined estimate of phylogeny. For instance, evaluation of qualitative morphological data alone revealed an overall weak phylogenetic signal, with the exception that 6 out of 7 species represented by multiple populations were found to be monophyletic. This is apparently due to the conservativeness of the qualitative data within species (such as rimoportula characteristics; Likhoshway and Crawford 2001). Morphometric continuous data, on the other hand, apparently reflect character variation or transformations within species, thereby providing some hypothesis of relationships among populations within species, as well as among species. However, these characters seem to vary too greatly to mark uniquely populations belonging to the same species (via clades) on their own. Only 
when both of these 'types' of morphological data are combined does the analysis result in a fully resolved tree with all populations as monophyletic for each species, as well as a monophyletic $A$. granulata complex. Also, with the combination of both sets of morphological data (qualitative and quantitative) the five major clades are revealed in the hypothesis of phylogeny (fig. 24). It is notable that the morphometric data alone yielded little semblance of these five major clades (fig. 23), in that members from these clades are found spread about the tree. Neither do the qualitative data alone (fig. 22), where a large polytomy exists and no hypothesis of clade relations is apparent. However, the five clades obtained by the combination of these two morphological data sets (fig. 24) matches the five clades found by the DNA data when analyzed alone (fig. 21), despite the DNA data sets suffering from poor taxon sampling. Based on both the monophyly of species represented by multiple populations and the possession of the same five major clades in independent analyses based on all morphology alone and combined DNA data alone, the combination of these two 'types' of morphological data clearly provides a better estimate of phylogeny than either one does alone.

In the tree based on combined morphological data (fig. 24), there are only 3 taxa (A. sp. 2, A. valida, and A. nyassensis) that disagree with the estimate based on combined DNA data as to which of the five major clades they belong. But in the total evidence tree (fig. 18), the agreement in the placement of these three taxa is with the DNA data. 
Results based on the molecular data indicate that there may be some disagreement, at least in basal relationships among the five major clades. But, further collection of this type of data for the other species in the study (that are not fossil) is needed before this can be said to be a true disagreement and not simply the results of poor taxon sampling within the DNA data set. As it stands now, the total evidence phylogenetic estimate is the best working hypothesis of phylogeny given the data available.

\section{Implications for Aulacoseira Taxonomy}

Species delineation has been commonly described as problematic within Aulacoseira (Crawford and Likhoshway 2002, Siver and Kling 1997). Poor availability of type specimens, type specimens often being available already mounted on slides that are too thick for adequate examination in the LM at high resolution, and species descriptions that largely rely on light microscopy or sketches with a very limited and relatively primitive characterization of obviously quantitative characters, or of quantitative characters masquerading as qualitative ones (Stevens 1991, 2000) add to the problem of species recognition and comparison. Given this, one might expect that species identifications in the current literature are inconsistent among studies, ranging from identifying the same species as different, to even more commonly, clumping many different species under the same species epithet. 
Krammer $(1991 \mathrm{a}, \mathrm{b})$ is one of the few workers who examined type materials in taxonomic studies, generally providing both LM and SEM images to display important characteristics of cell walls. Unfortunately, Krammer (1991 a, b) and Krammer and Lange-Bertalot (1991) generally do not provide SEM micrographs displaying important characteristics of the internal cell wall structure. Likhoshway and Crawford (2001) have recently provided many SEM micrographs of internal cell wall structure with recommendations for crushing of samples in order for inside mantle pictures to be obtained.

Due to the difficulty in gathering type specimen data in diatoms, it is particularly important to present as much descriptive data as possible. This includes providing appropriate LM and SEM micrographs of all pertinent structures in taxonomic studies to eliminate any confusion about what species or entity is receiving the name. But more importantly, specimens (samples) should be deposited in an herbarium. Light and Scanning Electron Micrographs used in this study can be found in Appendix N, or if data were collected from the literature, references to the sources of data and figures examined are given in Appendix B.

Taxonomic studies on Aulacoseira have only recently begun to provide more SEM micrographs revealing many of the ultrastructural silica cell wall characteristics that are proving to be useful in phylogenetic reconstruction (Crawford and Likhoshway 1999, 2002, Edgar and Kociolek MS. submitted 2002, Haworth 1988, Krammer 1991a, b, Likhoshway and Crawford 2001, Siver and Kling 1997). With the exceptions of 
Crawford and Likhoshway's (1999) work describing the original material of $A$. distans and Edgar and Kociolek's (MS. submitted 2002) description and diagnosis of $A$. krammeri nom. prov., taxonomic studies have generally focused on a few diagnostic characters while overlooking a complete description of the taxa. And only a handful of studies (Edgar and Kociolek MS. submitted 2002, Haworth and Sabater 1993) have gathered any kind of quantitative data in species descriptions that retain information on bivariate correlations of quantitative characters with valve size (diameter).

Difficulties and inconsistencies in species determinations are shown in the examination of the total evidence estimate of phylogeny (fig. 18). Isotype material for A. distans was measured and included in this study (A. distans FH34). It is apparent that what has been identified as $A$. distans fide Americanarum Diatomarum Exsiccata (ADE; Hamilton et al. 1992) in clade 1 is very different from A. distans FH34 in clade 2. Differences in morphometric characteristics are found between these two taxa. For instance, A. distans FH34 is smaller in diameter (3.6-7.3 $\mu \mathrm{m})$ than $A$. distans fide ADE (6.2-13 $\mu \mathrm{m})$, despite sharing similar ranges for mantle heights (2.7-7.1 $\mu \mathrm{m}$, and 2.9-8.4 $\mu \mathrm{m}$, respectively). And their ontogenetic-allometric trajectories for mantle height are also different, where $A$. distans fide $\mathrm{ADE}$ has a has a significantly positive principal axis regression slope $(b=0.599 ; p \leq 0.05)$, or there is an increase in mantle height with increasing valve diameter, and $A$. distans $\mathrm{FH} 34$ has a significantly negative principal axis regression slope $(b=-0.783 ; p \leq 0.05)$, where mantle height is reduced as valve 
diameter increases. Also, $A$. distans $\mathrm{FH} 34$ possesses noticeably smaller areolae $($ mean $=$ $0.10 \mu \mathrm{m}^{2}$ ) than $A$. distans fide $\operatorname{ADE}\left(\right.$ mean $=0.17 \mu \mathrm{m}^{2} ;$ t-test, $\left.\mathrm{p}<0.01\right)$.

Siver and Kling (1997) pointed out difficulty in distinguishing between $A$. alpigena and A. lirata, believing them to be the same species, and proposing that differences in morphology reflect size-related differences found at opposite ends of the size range. This would not be the first time that workers have named the two ends of the asexual size range of a single species as different species or varieties. A study by Kilham and Kilham (1975) used cultures grown over the size range of $A$. granulata to demonstrate that the cells at the smaller end of the size range matched the description of A. granulata var. angustissima, and effectively revealing these differences to be false. As pointed out in Chapter 1, with diatoms and great differences in size over a life cycle, understanding of ontogenetic-allometric trajectories of various characters allows us to see many previously described 'polymorphisms' in Aulacoseira to be simply a result of the limitations of the human eye to detect the gradual changes among these two opposite ends of the spectrum. These perceived polymorphisms may also be an artifact of the distribution of sizes in a sample or a set of samples examined. It is unclear yet whether A. alpigena and $A$. lirata are in fact the same species. The phylogenetic estimate suggests that they are not. But $A$. alpigena was represented by DNA data and some qualitative data from the literature while $A$. lirata was represented by qualitative data and morphometric data leaving very little overlap in shared scored characters. Because of the lack of morphometric data for A. alpigena (only a culture was available to 
examine), it was not placed in any of the five major clades. Perhaps with collection of additional data for both species, $A$. alpigena will be pulled into the same clade as $A$. lirata and their relationship to one another better revealed.

Aulacoseira perglabra fide Krammer (1991a), the description of which is based on original material, and A. perglabra fide ADE are found to belong to the same clade (clade 2), although not as sister taxa. There are some obvious differences between the two taxa, the most obvious one being the possession of mantle areolae in A. perglabra fide Krammer and the apparent hyaline mantle of $A$. perglabra fide ADE. The 'hyaline' mantle of $A$. perglabra fide ADE does, sometimes, possess areolae between the bases of the spines at the mantle valve face junction that extend down onto the mantle to a degree, and SEM micrographs of the internal cell wall structure (Siver and Kling 1997) reveal that areolae were formed on the mantle at one point and then subsequently filled with silica. This is evident from the cribra that remain internally as part of the wall structure, despite the apparent filling of the external portion of the areolae giving it its hyaline appearance when viewed from the outside or in the LM. This is different from the other hyaline species included in this phylogeny where no evidence exists of areolae ever having been formed (A. sp. 5, A. sp. 6, A. pseudoamericana). These 'truly' hyaline species all fall together as sister taxa in clade 1, separate from $A$. perglabra fide ADE (areolae filled in to appear hyaline) in clade 2.

Other interesting findings based on the total evidence phylogeny involve clade 5: A. baicalensis and A. skvortzowii are living species endemic to Lake Baikal, Siberia, 
which is one of the oldest lakes in the world, estimated at 25 million years by Edlund et al. (1996), and they are found in clade 5 with mostly fossil taxa. It can be hypothesized from this clade that $A$. skvortzowii may have given rise to $A$. islandica, a cosmopolitan species with very similar morphology to $A$. skvortzowii (and, in fact, historically identified as that species). Another interesting hypothesis is that $A$. baicalensis and the extinct taxa in clade 5 were both derived from a common ancestor after the split between $A$. islandica and A. skvortzowii, making them comparatively younger than these extant taxa. Shcherbakova et al. (1998), based on their phylogeny of five Aulacoseira species, dated the split between A. skvortzowii and A. baicalensis to have occurred within the existence of Lake Baikal ( $\leq 25 \mathrm{Mya})$. From reports of first appearances in the fossil record, the extinct taxa in clade 5 do not seem to exist in the fossil record prior to the beginning of the Miocene (22.5 mya or 25 mya; Haworth and Sabater 1993, Kaczmarska 1985, Krammer et al. 1997, Shcherbakova et al. 1998, VanLandingham 1964, 1967). The few reports of their occurrence before the Miocene are accompanied by question marks, indicating the authors' recognition of the uncertainty in their dating. Although this seemingly supports the hypothesis that the extinct taxa and A. baicalensis are a recently derived subclade compared to the other extant taxa in clade 5 , it is not overwhelming support because the dates for the taxa are at best uncertain and identification of the taxa is uncertain. However, it is interesting to note that the majority of extinct taxa are not randomly distributed across the tree. With the exception of $A$. distans and $A$. cf. distans in clade 2, all of the extinct taxa are clumped together in clade 
5 , raising questions as to whether this very non-random distribution represents an artifact of the fossil record, a result of missing data, or biologically or environmentally interesting phenomena.

Also, among the fossil taxa there are three very similar species that are grouped together: A. krammeri nom. prov., A. paucistriata, and A. solida. Only in collections of A. krammeri were linking valves found, despite their being fragile and often fragmented. They also show a wide spectrum of variation in their shape with diminution of valve diameter, which was reduced by $84 \%$ from the largest valves to the smallest over the life cycle. Substantial differences in spine morphology changing from separation spines to linking spines over a small size range $(6-8 \mu \mathrm{m}$ in the full range of $2.7-17 \mu \mathrm{m})$ can also be observed with the change in cell diameter (Edgar and Kociolek MS. submitted 2002). Despite the mean values for the morphometric data being lowered when the smaller end of the spectrum is present in the sample, the phylogenetic analysis still recognizes the similarity between $A$. krammeri and its sister taxa, $A$ solida and $A$. paucistriata. It might be expected that all of these species possess linking valves at the smallest end of the size range, but they simply are as yet uncovered in fossil samples due to the light silicification of these gracile forms.

The Aulacoseira granulata complex and the historically evolving concept of $A$. granulata are also of interest. In some cases, the assignment of the specific epithet was applied based on a concept that has evolved or historically changed. In samples which H. L. Smith (HLS) and H. Van Heurck (VH) labeled as A. granulata, where the 
lanceolate separation spines of unequal length used to recognize the species today, no such separation spines are present. Instead the species in their samples have small, pointed separation spines of equal length. Historically, it seems that $A$. granulata was recognized for its possession of large mantle areolae giving it a coarse "granular" look, which is reflected in its name. Therefore, when A. granulata fide HLS and A. granulata fide $\mathrm{VH}$ are in another clade, away from the more contemporary A. granulata-like species, it is no great surprise.

Within the Aulacoseira granulata complex which falls under the current concept for this species, there is a notably large amount of variation in characters among populations. Of the species represented by more than one population, A. granulata is the only one that possesses variation in qualitative characteristics as well as morphometric characters, strongly suggesting that it is a species complex and not a single species. This is also supported by the non-monophyly of populations identified as $A$. granulata within the $A$. granulata complex itself.

Finally, monophyly of Aulacoseira can only be achieved if Alveolophora, which contains four described fossil species very similar in morphology to Aulacoseira, is no longer given generic status. All four species within Alveolophora were originally described as Melosira species, and subsequently transferred to Aulacoseira, their only major morphological differences being valve shape and the presence of alveoli formed by thick silica struts extending from the valve face to the ringleist along the inner face of 
the mantle wall. And it may be that Alveolophora species are simply highly derived Aulacoseira.

This study offers the first comprehensive estimate of phylogeny for Aulacoseira, the first comprehensive estimate of phylogeny to be made at the species level within diatoms, and the first estimate of phylogenetic relationships among diatoms that evaluates morphological and molecular characters, especially quantitative morphometric characters. However, there are other data sources (e.g., cytological, such as chloroplast number, shape, and position in the cell) that have been used in estimates of phylogeny (Cox and Williams 2000) that have not yet been examined here. Phylogenies are hypotheses, which should be challenged by the full and diverse repertoire of characters descriptive of the species (or other taxa) constituting the terminal taxa. 


\section{Chapter 3: Phylogenetic Sensitivity to Coding of Overlapping Continuous Morphometric Characters: an example from Aulacoseira (Bacillariophyta)}

\section{INTRODUCTION}

In the past 30 years there has been growing discussion about the use of continuous characters with overlapping ranges in the cladistic estimation of phylogenies. Generally there are two basic schools of thought: 1) they are inappropriate and should not be used (Cranston and Humphries 1988, Pimentel and Riggins 1987) and 2) they are appropriate and should be evaluated for inclusion (Rae 1998, Stevens 1991, Strait et al. 1996, Swiderski et al. 1998, Thiele 1993, Wiens 2001). For those in this second school the challenge lies in finding a means of coding these data that is objective, unbiased, repeatable, and can be applied to different types of quantitative characters to present the data most accurately in the integer form required by phylogenetic software programs and in a form that allows potential phylogenetic signal to be detected.

There are two basic arguments made by proponents of the "exclusion" school for not including continuous overlapping data in cladistic analyses. The first is a claim that this 'type' of data is simply not cladistic in nature (Pimentel and Riggins 1987). Rae (1998) clearly shows that arguments that fall into this class do not proffer up reasons to support their claims, but instead tried to gloss over them with rhetorical statements. With no justification or evidence that quantitative overlapping data are 
inherently different from qualitative morphological data, it is difficult to give any weight to these types of argument (Rae 1998, Stevens 1991, 2000, Thiele 1993).

A more substantial argument against the use of continuous overlapping data in cladistics has focused on the lack of non-arbitrary methods to code continuous overlapping variables. If there is some arbitrariness in a method used to identify gaps in overlapping data, how can we be sure that the postulates of homologous character states are in any way meaningful? There have been major advances over the years to ameliorate this complaint as more principled, objective, and repeatable methods have been developed to handle the transformation of raw overlapping variables into integer form for coding in current phylogenetic software packages (Archie 1985, Simon 1983, Thiele 1993, Wiens 2001).

Some compelling arguments for the inclusion of quantitative overlapping data in phylogenetic analysis have been put forth throughout this debate, the strongest of which are: 1) 'Quantitative' data are not inherently different from 'qualitative' data and thus $a$ priori exclusion is unwarranted as well as difficult to attain if, for instance, any of the qualitative characters being included are in fact just word descriptors of quantitative variables, such as shape or color, which is frequently the case (Stevens 1991, 2000). 2) Many morphological variables are continuous, heritable, and useful in diagnosing taxa (Stearns 1992, Stevens 1991, 2000, Strait et al. 1996, Thiele 1993). 3) Continuous data are precise and replicable (Reid and Sidwell 2002). 4) Continuous data "fulfill the sole criterion for inclusion in phylogenetic analysis, the presence of homologous character states, and thus cannot be excluded as a class of data" (Rae 1998). 
Many methods for coding continuous or meristic overlapping morphological characters have been proposed, but there is a lack of agreement as to which one is the best in terms of providing sound postulates of homology for character state identification. The coding methods proposed basically fall within two categories: gap identification (Almeida and Bisby 1984, Archie 1985, Chappill 1989, Gift and Stevens 1997, Michevich and Johnson 1976, Simon 1983, Stevens 1991, 2000, Swiderski et al. 1998, Thorpe 1984) and gap weighting (Thiele 1993, Wiens 2001). Gap identification methods are focused on breaking up the overlap in the data into character states by using some measure of dis-similarity or threshold for how different central tendencies have to be before they are considered different states. Gap weighting methods focus more on representing the overlapping scale of variation in the coding while trying to maintain the data in as raw a form as possible.

The gap identification methods can be broken down further into three distinct classes: 1) non-statistical gap identification methods, where data (or summaries of data) are displayed graphically and broken into character states by visually examining the graphs for apparent breaks in the continuous data (Almeida and Bisby 1984, Gift and Stevens 1997, Swiderski et al. 1998), 2) quasi-statistical or threshold methods, where some arbitrary unit is chosen as a critical gap size between means, usually based on a descriptive statistic (such as the mean pooled standard deviation of a character), and is used to identify differences between means large enough to justify grouping taxa into different character states (Archie 1985, Chappill 1989, Michevich and Johnson 1976, Thorpe 1984), and 3) statistical methods applied usually to raw data (or log-transformed 
raw data) to assess the probability that the means of the observed distributions for each taxon were sampled from populations with the same character state (Archie 1985, Rae 1998, Simon 1983, Strait et al. 1996).

Class 1 of the gap identification methods can be disregarded as a sound method for coding continuous variables based on Gift and Stevens (1997), which clearly show them to be biased and nonreplicable on many levels. Not only is coding choice, or gap identification greatly affected by how the data are graphically displayed (i.e., with or without standard deviations or confidence intervals, arithmetic or logarithmic scale), but also on the preferences of the investigator doing the splitting (Gift and Stevens 1997).

Class 2, or quasi-statistical methods, showed up in the literature in an effort to provide more stringent guidelines that would make the method repeatable and remove the need for visual recognition in order to group taxa into character states. Unfortunately, the thresholds chosen in these various methods are arbitrary and can create nonsensical gaps in character data that are not based on any underlying biological principles (homology) and would not necessarily reflect any phylogenetic signal (Archie 1985, Rae 1998). The problems with these arbitrary thresholds and a need for a well-grounded postulate of homology when breaking characters into character states have been the main stimuli for both class 3 statistical methods of gap identification as well as of gap weighting.

Statistical methods proposed for identifying gaps in continuous overlapping data greatly reduce the unprincipled or arbitrary nature of gap identification by relying on basic biometric and evolutionary principles to assess the probability that the 
observations made for each taxon were sampled from populations with the same, or homologous, character states as the other taxa. In other words, they determine the probability that the taxa possess homologous character states (Rae 1998). Generally these methods rely on good variance estimates in the test as opposed to just the mean or central tendency, and because of this sample sizes become important factors in the tests. However, if sufficient sample sizes are available, tests such as a posteriori multiple comparisons, as in homogeneous subset coding applied by Archie (1985) and Simon (1983), are objective and repeatable methods for assigning character states to taxa based on solidly grounded probability models (Rae 1998).

The biological basis for statistical gap identification methods is clear and compelling as pointed out by Rae (1998):

These procedures, which are both objective and repeatable, determine the probability that two taxa possess an homologous character state; that is, if they have inherited a particular central tendency and distribution of individual variates unchanged from a common ancestor. Thus, the application of statistical tests to quantitative data empirically detects the presence of evolutionary change, the raw material of phylogenetic reconstruction.

There have been arguments against the use of homogeneous subset coding as implemented by Archie (1985) and Simon (1983), because the statistical test used to identify homogeneous subsets of taxa seems violated by the second step of designation of character states (Farris 1990, Swiderski et al. 1998). The first step in the method of homogeneous subset coding is to employ an a posteriori test to identify groups of taxa as having central tendencies that are similar to one other (statistically indistinguishable), 
and as being statistically different from exactly the same set of taxa. With quantitative overlapping data, it is rarely the case that these homogeneous subsets of taxa do not overlap with other homogeneous subsets of taxa. These overlaps complicate the designation of character states in comparison to distribution patterns in which there is no overlap among the subsets, and identity that is used to postulate homology is simply the subset itself. Archie (1985; Simon 1983) had proposed a method of designating character states for taxa using additive binary coding from the overlapping homogeneous subsets that was able to consistently represent the shared overlaps. There have been complaints that this type of coding method can assign different character states to taxa with means that cannot be statistically distinguished from one another (Farris 1990, Swiderski et al. 1998). This would happen in the areas of overlap in subsets where the central tendency of taxon $y$ is not significantly different from the taxon before it $(\operatorname{taxon} x)$, which in ranked order has a slightly smaller mean, and is also indistinguishable from central tendency of the taxon $(z)$, after it in rank order, but when $\operatorname{taxon} x$ and taxon $z$ are statistically distinguishable in central tendency. This, however, is not really inconsistent with the statistical test (Rae 1998). In fact, it picks up a finer scale of differences in means than is represented by the homogeneous subsets when considered independently of one another. As Rae (1998) points out, the overlap in subsets can potentially represent change that has occurred from the mean and distribution of that character for taxon $x$ and taxon $y$ that would justify the two character states being assigned two adjacent codes. If no change had occurred in the central tendency, the expectation would be that the two taxa would be found to have the same 
character state and different from the exact same subset of taxa. When taxa belong to exactly the same homogeneous subset(s) as one another, they should (and do) get assigned the same character state. The use of multiple comparisons combined with the information in the overlap of homogeneous subsets found can then inform us on a finer scale about the levels of similarity among taxa means from which we can base postulates of homology via character state assignments.

Gap weighting methods also avoid the arbitrary nature of most gap identification models, because they require no identification of gaps to code the data. The objective of gap weighting (Thiele 1993) or step-matrix gap weighting (Wiens 2001) is to represent the continuous data, via the central tendencies or means, in as raw a form as possible within the constraints of coding with integers, which is required by the phylogenetic software being employed (e.g., PAUP). It is similarly based on the biological principles stated above by Rae (1998), but in addition to detecting evolutionary change, it also quantifies the amount of evolutionary change detected among means possessed by taxa for a character in the coding to allow all of the potential phylogenetic signal contained in the data to be expressed. In Thiele's (1993) method, the means of the characters are ranked from lowest to highest, then scaled to the maximum number of character states allowable by whatever computer program is being employed. The scaled means would then be rounded to the nearest integer, which is assigned as the character state, and the character is analyzed as ordered. As long as there are fewer character states than the maximum number of character states allowable by the program, the empty character states stand to weight the differences between 
means among the taxa. A serious restriction of Thiele's (1993) method, largely due to the restricted number of character states allowable by phylogenetic programs, is the more taxa included, the less accurate the weighting of the differences among the means will be, and the less resolved the potential phylogenetic signal from the data. Character states are at a premium under these conditions, so it is wasteful to use them for weighting if that can be accomplished by step-matrices.

Wiens (2001) recognized this limitation of Thiele's (1993) method, but he also recognized the appeal of the logical and biological underpinnings of this method for coding of continuous data. In order to reduce the influence of the character state limitation and improve the gap weighting method, Wiens (2001) utilized step-matrices to weight the differences in the means thereby allowing all character states to be filled! This effectively allows more taxa to be coded without reducing the accuracy or resolution of the weighting of the differences among means.

In Chapter 2, a phylogeny of Aulacoseira was estimated using a combined data set containing morphological characteristics of the siliceous cell wall and a limited amount of gene sequence data from the chloroplast $(r b c \mathrm{~L})$ and nuclear (18S rDNA) genomes. Because of the need to understand extinct taxa in the same context as living taxa, as well as limited availability of cultures for DNA data for these taxa, it is imperative to include morphological data in our phylogenetic estimates. About half (21 of 39) of the morphological characters collected for Aulacoseira are continuous and overlapping. This, in combination with the ability to generate large sample sizes 
$(\mathrm{n} \geq 50)$ for measurement of these characters makes this a good data set for application of statistical gap-identification and gap-weighting methods.

The purpose of this study is to evaluate sensitivity of the phylogeny of Aulacoseira derived from combined data to four different methods of coding: 1) a new variation of a quasi-statistical (class 2) model using Student's t-tests to identify gaps instead of a fixed, critical gap value, 2) a statistical gap identification method utilizing Tukey-Kramer multiple comparisons to identify homogeneous subsets, as was applied in Archie (1985) and is argued for by Rae (1998), 3) the gap weighting method of Thiele (1993), and 4) a modified version of the step-matrix gap weighting method of Wiens (2001). In addition, within the gap weighting regimes (methods 3 and 4 above), evaluation is made of the effects of different levels of resolution determined by character state limitations of current software packages available for creation, editing and/or analysis of phylogenetic data sets (Winclada: 10 character states, Nixon 1999; MacClade: 26 character states, Maddison and Maddison 1992; and PAUP: 32 character states, Swofford 2000).

\section{MATERIALS AND METHODS}

The data set used here was gathered in a previous study to estimate the phylogeny of Aulacoseira (Chapter 2). This data set includes 45 species of Aulacoseira Thwaites, 1 species of Alveolophora Moiseeva and Nevretdinova, and 2 outgroup genera represented by 1 species each (Melosira varians Agardh and Stephanopyxis cf. 
broschii Grunow). Of these, 8 species have more than 1 population (sample) measured for morphological character and/or gene sequence data giving a total of 70 populations representing the 48 species in the analysis. Twenty-three populations representing 15 species have sequence data for the nuclear 18S rDNA gene (Appendix C), including both outgroups. Nineteen populations representing 11 species have partial sequence data for the chloroplast $r b c \mathrm{~L}$ gene (Appendix D), including one of the two outgroups (M. varians). All 70 populations in this study representing 48 species have some or all of the 18 'qualitative' characteristics of the silica cell wall scored, whereas 68 populations representing 46 species have had the morphometric characters measured and scored for them via the light microscope (Appendix E) or in some few cases, where samples of taxa have been unavailable, via information from the literature (Appendix B). The morphometric methods used, explanation of the characters, and sample sizes are provided in Chapter 2 and Appendix A.

Seven methods of coding quantitative overlapping data are employed in this study, including a new quasi-statistical model following Michevich and Johnson (1976), and a modification of the step-matrix gap weighting method of Wiens (2001) are as follows:

1) Quasi-statistical gap-recognition method using Student's t-test

Taxa were ranked from lowest to highest according to their means for each quantitative character. Pairwise comparisons were limited to all adjacent taxa, using a one-tailed Student's t-test ( $\alpha=0.05$; Sokal and Rohlf 1995). Wherever the t-test identified a statistical difference between means, a gap was introduced in the ranked 
series of taxa and the character state assignments increased by one for the taxon with the higher of the two means. Taxa that fell between any two gaps in the series were assigned the same character state. All 12 quantitative characters with distributions of raw data around means were coded in this way and treated as ordered characters (Appendix L).

Ontogenetic-allometric trajectory characters $(n=8)$ did not contain data permitting the application of Student's t-test because there was a single value per population, and consequently no variance, and therefore were coded using the $95 \%$ confidence intervals of their principal axis regressions. If the $95 \%$ confidence intervals for the slope of the principal axis regression excluded zero and fell above it, that taxon was coded as having a positive relationship among variables for that character. If the $95 \%$ confidence intervals of the slope included zero, that taxon was coded as having no relationship among the variables for that character (or a zero slope). Finally, if the $95 \%$ confidence intervals for the slope excluded zero and fell below it for the principal axis regression, that taxon was coded as having a negative relationship between variables for that ontogenetic-allometric character. One ontogenetic-allometric character (mean mantle thickness vs. valve diameter) was variable in magnitude, but it was found in all taxa to have a significantly $(\mathrm{p} \leq 0.05)$ positive slope and was therefore uninformative when coded by this method and thus excluded from the data set (Appendix L). 2) Tukey-Kramer multiple comparisons or homogeneous subset coding Tukey-Kramer multiple comparisons $(\alpha=0.01)$ were used to identify homogeneous subsets of means to group taxa for 12 descriptive characters with full raw 
data available for most taxa. Because measurements of ontogenetic-allometric characters within a taxon (sample) are not replicated, there is no variance and thus multiple comparisons are precluded. Again, the $95 \%$ confidence intervals for the principal axis regression slopes were used to determine character states for these eight characters.

For the application of the multiple comparisons, taxa were first ranked in order by their means. The Tukey-Kramer method for multiple comparisons among pairs of means was chosen, because it is best among those available for handling unequal sample sizes, but also performs quite well when sample sizes are nearly equal (Archie 1985, Sokal and Rohlf 1995). The raw data were entered into Microsoft EXCEL ${ }^{\circledR}$ and log-transformed because of the wide range of variance among taxa for most characters. A taxon-pair specific minimum significant difference (MSD) for each character was determined based on its standard error and the critical value from the studentized range table (Sokal and Rohlf 1995). These MSDs were then used as a threshold by which each respective pairwise comparison of means was judged, where differences in means larger than the MSD for that character indicated that those means belonged to taxa in different homogeneous subsets. Codes were assigned according to the recommendations of Archie (1985) and Simon (1983). The taxon with the smallest mean for character $x$ was always scored with a state of 0 . Then each ranked taxon after the first was examined in order and was given an additional 0.5 to the cumulative score if it began a new homogeneous subset, and 0.5 if it ended a prior homogeneous subset. If the taxon shared exactly the same homogeneous subset with the taxon before it, 
nothing was added to the score. When scored this way, characters almost always had more than 32 character states, precluding ordered multistate character coding (Appendix M). Therefore, these scores were translated into additive binary characters for coding (Archie 1985).

For some characters, certain taxa for which data were gathered from the literature, had too few observations ( $\mathrm{n} \leq 10$; Appendix $\mathrm{B})$ to include them in the multiple comparisons, but were assigned the same score a posteriori as the taxon with the most similar mean that was included in the multiple comparisons. This was done to preserve information in the coding as opposed to simply a designation as missing data (?).

3) Gap weighting - with a 10 character state limitation

To apply the gap weighting method of Thiele (1993), taxa were first ranked according to their means for each character to be coded. The mean values of all taxa were then scaled so that the whole range of data would fit into 10 character states (maximum number of allowable character states in Hennig86 and Winclada; Farris 1989, Nixon 1999) according to the following equation:

$$
\mathrm{x}^{\prime}=\frac{(\mathrm{x}-\min )}{(\mathrm{max}-\min )}(\mathrm{N}-1)
$$


where $\mathrm{x}$ ' is the "new" rescaled, range-standardized mean, $\mathrm{x}$ is the original mean, min is the minimum mean in the set, and max is the maximum mean in the set. This is then rescaled to a range of $\mathrm{N}-1$, where $\mathrm{N}$ is the number of character states allowable by the phylogenetic software package being employed. In this case $\mathrm{N}=10$, the maximum number of character states allowable in Hennig86 and Winclada (Farris 1989, Nixon 1999). The first factor in the equation (x-min/max-min) standardizes the mean (i.e., scales it to 1), and the second factor $(\mathrm{N}-1)$ rescales the mean to the maximum number of character states allowable by the phylogenetic program.

The rescaled range-standardized means (x') for each taxon for the character of interest are then rounded to the nearest integer, coded as that integer (or symbols representing the integers if greater than 10 character states are being used), and treated as an ordered character in the cladistic analysis. Ontogenetic-allometric slope characters (9) as well as central tendency characters (12) were coded according to this method for a total of 21 morphometric gap weighted characters (Appendix K). 4) Gap weighting - with a 26 character state limitation

This method is the same as the previous method (method3), but with the character state restriction raised to 26 (i.e., rescaled range-standardized to 25), that maximally allowable by MacClade (Maddison and Maddison 1992; Appendix J). 5) Gap weighting - with a 32 character state limitation

Again, the method remains the same as above, but with rescaling range limited to the maximal number of character states allowable by PAUP (32; Appendix I). 
6) Step-matrix gap weighting - with a 26 character state limitation

This method, introduced by Wiens (2001) is very similar to the gap weighting method used in methods 3 to 5. The taxa were first ranked in order of their means. Wiens' (2001) method was modified in the following step to deal with having a number of taxa with unique means that exceeds the number of character states allowable by the systematic software being employed. In this case the limitation of 26 character states is imposed by MacClade, so if the number of taxa with unique means was greater than 26 , then the means were successively rounded using larger rounding bins until 26 character states or fewer were reached. By successively rounding by small increments, the maximum number of character states for that character, given the data at hand, that fit maximally within the constraints of the character state limit imposed are maintained. For example, the smallest amount of rounding (typically to 0.001) for each character was applied first. If the number of unique means were still greater than the character state restriction, another level of rounding would be applied (in this example, to 0.002), and so forth until the maximum number of unique means was retained that fit the limitation of character states imposed by the phylogenetic software. This effectively combined taxa with very similar means into the same bin, or code, before the next step of range-standardization was applied to determine weights for the step-matrix for the character being coded. The steps following this initial rounding are the same as that followed by Wiens (2001). The following equation is used to range-standardize the means so gaps between character states can be assigned a weight via a step-matrix that will be applied to the ordered character: 


$$
\text { w' }=\frac{(x-\min )}{(\max -\min )}
$$

where $\mathrm{w}^{\prime}$ is the weight to be applied in the step-matrix to the character state $\mathrm{x}$, $\min$ is the smallest mean in the set for the character being coded, and max is the largest mean found in the set. This is then multiplied by the maximum weight to be assigned in the step-matrix. In this case, decimal weights are being used, and the maximum allowable by MacClade is 0.999 (or 999 if integer weights are used; Maddison and Maddison 1992). Finally, the ranked means are assigned coded states using integers and symbols (Appendix G), and the differences among all pairs of character states are weighted accordingly, using step-matrices created via the range-standardized weights (Appendix $\mathrm{H})$.

Using the maximum range allowable by the software for weighting maximizes the resolution in coding allowable by the program. Once a data set using this coding method reaches a certain size or complexity, integer weights can become too encumbering for PAUP to handle. Decimal weights were chosen here over integer weights for this reason.

7) Step-matrix gap weighting - with a 32 character state limitation

This follows the procedures states above in method 6, except that when more than 32 unique means among taxa are found, rounding of means only proceeds until the number of unique means attained does not exceed 32 (Appendices $\mathrm{E}$ and F). 
By limiting the number of character states in the gap weighting methods, differences in signal that might result from different technological restrictions can be examined. MacClade and PAUP are the two major software packages that allow creation and editing of nexus files for phylogenetic analysis that can also incorporate step-matrix weighting (Maddison and Maddison 1992, Swofford 2000). Therefore, the limitations of character states by these two programs were chosen for analyses 5 and 6 .

Analyses

All matrices and step matrices were constructed in, and maximum-parsimony analyses implemented in, PAUP v4b10 (Swofford 2000). All quantitative overlapping data were treated as ordered, whereas all other data (qualitative morphological characters, gene sequence data) were treated as unordered. In all cases of the coding described above, quantitative characters were scaled so that their whole range of variation was on average equal to the average number of character states found in the qualitative data. Gaps were treated as missing data. All most parsimonious trees were identified using 1000 replicates of heuristic searches employing TBR branch swapping with random sequence addition. Non-parametric bootstrap analyses were performed for 250 to 500 pseudo-replicates of $10 \mathrm{TBR}$ addition sequence replicates each for each of the 7 coding analyses. Nodes with bootstrap values $\geq 70$, are considered to be well supported. In order to test whether the phylogenetic signals for the differently coded morphometric data (means as well as slopes) were significantly different from one that might be expected due to chance alone, permutation tail probability tests (PTP) of 
Archie (1989), Faith (1991) and Faith and Cranston (1991) were run in PAUP using the permute command with 100 replicates for each of the seven analyses.

The sensitivity of the phylogenetic estimate to coding methods for continuous overlapping variables was evaluated by comparison of tree topology and clade composition in each analysis as well as measures of bootstrap support. There were seven species included which contained more than one representative population (Aulacoseira crenulata, A. canadensis, A. lacustris, A. nygaardii, A. subarctica, A. ambigua, and $A$. sp. 3), as well as the $A$. granulata complex with multiple populations, which were used to judge methods of coding by their ability to resolve monophyletic relationships for each species or species complex. Also, the resolution of the coding methods was judged by the number of most parsimonious trees (MPTs) found by the heuristic searches, where the fewer MPTs reflected more resolution or phylogenetic signal held in the coding.

\section{RESULTS}

Five major clades can be identified in the great majority of trees (figs. 25-31): 1) a most basal clade containing Aulacoseira crenulata, A. italica, three hyaline species and a couple of unnamed species, 2) a clade containing $A$. subarctica, $A$. herzogii, and a mix of species commonly described as having low mantle-height-to-width ratios, including $A$. distans, 3) a clade containing the A. granulata complex, 4) a clade containing $A$. ambigua, A. nyassensis, and $A$. valida, and 5) a clade containing $A$. islandica, A. skvortzowii, A. baicalensis, Alveolophora and many extinct Aulacoseira 
taxa. Only the homogeneous subset coding method (analysis 2; fig. 26) failed to recover clade 3, the A. granulata clade, although in the Student's t-test method (analysis 1; fig. 25) this clade was greatly reduced in number of species in it. Overall, the clades differed in some taxa from one analysis to the next, but the majority of taxa included in each clade remained the same among analyses.

When the results from the quasi-statistical gap identification method (analysis 1) were compared to the statistical homogeneous subset coding method (analysis 2), there was a reduction in number of most parsimonious trees (MPTs) from 176 to 6 . But despite this increase in resolution, the more statistically based homogeneous subset coding had lost clade 3 and had equal difficulty in maintaining species' clades. Out of the seven cases in which two or more populations represent the same species, both analyses 1 and 2 had difficulty resolving monophyletic relationships for $A$. canadensis, A. subarctica, $A$. lacustris, and the $A$. granulata complex. In addition to these, analysis 1 (quasi-statistical gap identification) could not resolve A. ambigua as monophyletic. Non-monophyly for the $A$. granulata clade was somewhat expected, because although new species names have not yet been published for various subsets of this taxon, it has been suspected that this nominal species is much more of a complex of different species that have been assigned the same name due to possession of relatively few synapomorphic characters, but commonly misunderstood as autapomorphies for the species. And as was pointed out in Chapter 2, in some cases (A. granulata fide Van Heurck and fide H. L. Smith), the assignment of the specific epithet was applied based on a historical concept of the species that has since evolved, again explaining the 
position of those historical samples being in a separate clade from the $A$. granulata complex. However, the complete undoing of this contemporary A. granulata complex/clade as the result of the homogeneous subset coding is unexpected.

Besides the difference in the absence of the $A$. granulata clade, there also are major shifts in positions of 15 OTUs from analysis 1 to analysis 2, and most of the terminal relationships within clades differ (with the big exception being the structure of clade 1). However, the basal relationships among the four clades left in analysis 2 (homogeneous subset coding) are more in agreement with the estimated phylogenies of analyses 4-7 (figs. 28 - 31) than with analyses 1 or 3 (figs. 25 and 27, respectively). Apparently this is due to slightly higher resolution of phylogenetic signal when incorporating the information on shifts in taxon means gleaned from overlap patterns in the coding method applied to the homogeneous subsets. However, the terminal relationships among taxa within each of the four major clades contained by this analysis vary greatly from those found by all of the gap weighting analyses (3 to 7). Also, there are eight taxa which shift positions among the major clades compared to analysis 3 (fig. 27; most restrictive gap weighting) and two more shifts in taxa for a total of 10 by the time the highest resolution of gap weighting is reached (fig. 31; step-matrix gap weighting of analysis 7).

A comparison of analysis 1 (quasi-statistical gap identification method) to analysis 3 (gap weighting method restricted to 10 character states, the limit in Winclada; Nixon 1999) reveals no major change in relationships among the five major clades. The contemporary Aulacoseira granulata complex is monophyletic as in analysis 3 (fig. 27), 
and of those species containing multiple representatives, only A. ambigua is nonmonophyletic, having $A$. nyassensis, which is quite similar in morphology to $A$. ambigua, nested in the middle of the clade. Again, 15 taxa shift positions among the five major clades when comparing analysis 1 to analysis 3, but, in general, both analyses agree on which taxa are contained by each clade. However, the relationships among taxa within clades vary widely.

When there are as many OTUs as are in this study (70), the large restriction of character states in analysis 3 due to a character state limitation of 10, causes the coding of the characters to be ranked and grouped into more or less adjacent character states (depending on the character), thereby effectively removing any of the actual weighting of the gaps that is intended by the method. This may in part explain why the basal relationships among the five major clades do not change from the quasi-statistical gap identification method (analysis 1; fig. 25) to the most limited of gap weighting methods (analysis 3; fig. 27). This may also explain why analysis 3 had 60 MPTs, the second largest number of MPTs found of all the analyses, the largest being 176 MPTs found for the quasi-statistical gap identification method.

However, once the character state limit is raised to 26 (maximum allowable by MacClade) in analysis 4 (fig. 28), the weighting of the gaps results in a shift of basal relationships among the five major clades. The taxa that are included within each of these major clades remains intact from analysis 3 to analysis 4 with the exception of the position of four OTUs (Aulacoseira nyassensis, A. distans fide ADE, and the two populations of $A$. lacustris). In the previous two analyses, $A$. nyassensis was placed in 
the middle of the A. ambigua populations, thereby breaking up A. ambigua, where the lack of resolution in the coding apparently caused the inability to differentiate between these two similar taxa. However, with the increase in coding resolution in analysis $4, A$. nyassensis has been moved out of, and positioned as a sister taxon to, A. ambigua. There is also a slight increase in the resolution of the strict consensus tree for analysis 4 (6 MPTs) compared to analyses 1 and 3 (176 and 60 MPTs, respectively).

In analysis 5 (fig. 29), when gap weighting is still implemented but with the limit of character states increased to 32 (maximum allowable in PAUP), there is no change from that of analysis 4 in terminal relationships among OTUs or the relationships among the five major clades. In fact, the only change between the two analyses is one of resolution in the tree at a single node. Analysis 5 (fig. 29) found only three MPTs compared to the six found in analysis 4 (fig. 28).

In analysis 6 (fig. 30), the number of character states was reduced to 26 , but the gaps weighted via step-matrices allowing all character states to be used making the resolution of this coding method greater than that of analysis 5 (but following the character state limits in MacClade which can handle step-matrix weighting). When the strict consensus tree from analysis 5 (three MPTs) is compared to the strict consensus of three MPTs in analysis 6, there is little change. The positions of four OTUs are shifted (A. distans fide $\mathrm{ADE}$, and two populations of A. lacustris), and the relationships among the A. ambigua populations change.

When the step-matrix gap weighting method in analysis 7 (fig. 31) raises the character state limit to 32 , the same three OTUs which shifted from analysis 5 to 6 shift 
back into the position they held in analysis 5 . The relationships among populations of A. ambigua, however, stay the same as in analysis 6 with the exception of one population (WAT). Also, we find only a single most parsimonious tree.

Across all analyses there were multiple MPTs found with the exception of analysis 7. In analyses 3-6, the placement of $A$. tenella consistently caused disagreement among MPTs. In analyses 3 and 4 there is also disagreement as to the placement of A. dianchiensis among MPTs. PAUP, of all phylogenetic software to date, now provides the maximum allowable character states within a single character, or column in the data matrix, in combination with the ability to use step-matrices for highest accuracy of weighting of differences in central tendencies of these characters, thereby setting the technical limitation to this coding method. However, as this limitation is reached, even with over twice as many OTUs (70) as number of allowable character states (32), a fully resolved estimate of relationships among the OTUs is found. This perhaps indicates that a large amount of phylogenetic signal from the raw data is being expressed regardless of the limitation in character states at this level.

Permutation tail probability tests were run on the morphometric (including slopes) data for each of the analyses above. All were found to have significant phylogenetic signal $(\mathrm{p} \leq 0.01)$. Non-parametric bootstrap estimates were very similar for all analyses, largely supporting 10 nodes in phylogenetic estimates regardless of the coding method used.

Over the course of increase in resolution via changes in coding methods and relaxation of software-imposed character state restrictions, there was an increase in 
resolution of phylogenetic signal and a decrease in the number of most parsimonious trees found indicating that the signal has reached its peak in analysis 7 with its most parsimonious tree being the best estimate of phylogeny for this group given the data at hand. 


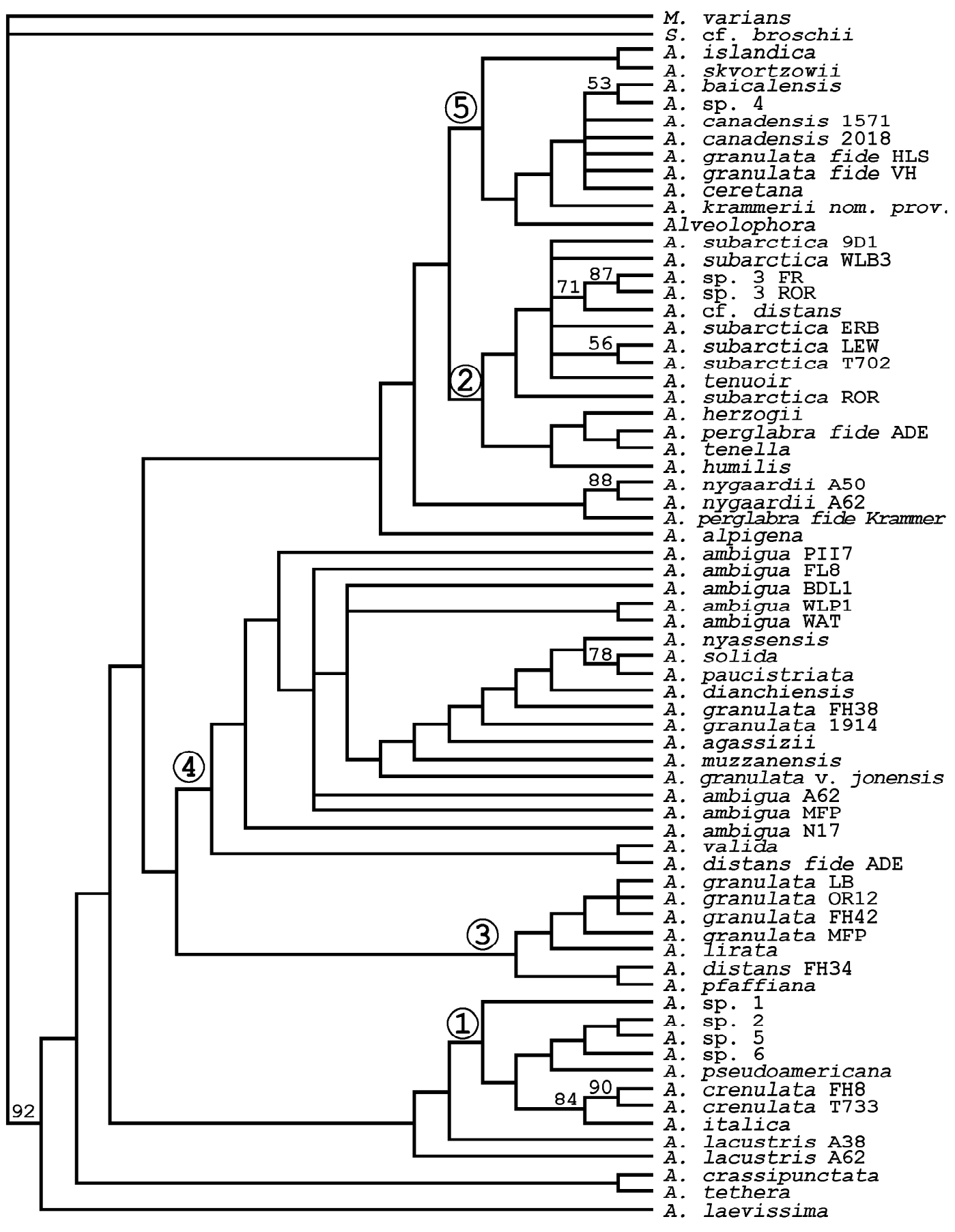

Figure 25. Analysis 1. Quasi-statistical gap-identification coding method (Student's t-test). Strict consensus of 176 MPTs. Bootstrap values above branches are based on 466 pseudo-replicates of 10 addition sequence replicates employing TBR branch swapping. Length $(\mathrm{L})=2,562$, consistency index excluding characters that are not parsimony informative (ci) $=0.40$, retention index (ri) $=0.65$. Five major clades are labeled at their nodes (circled numbers). 


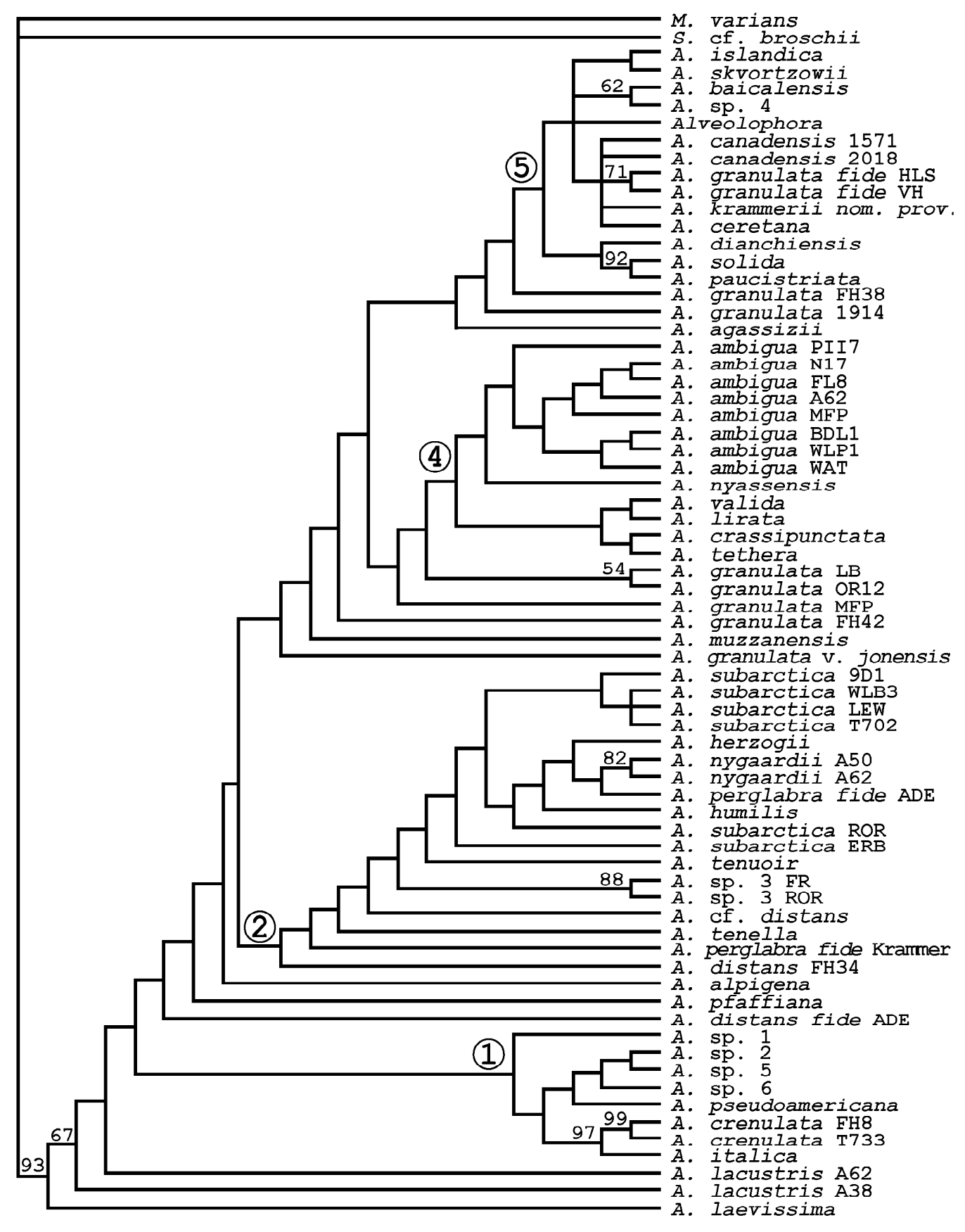

Figure 26. Analysis 2. Statistical gap-identification coding method - Homogeneous subset coding using Tukey-Kramer multiple comparisons. Strict consensus of 6 MPTs. Bootstrap values above branches are based on 500 pseudo-replicates of 10 addition sequence replicates employing TBR branch swapping. $\mathrm{L}=$ $11,562, \mathrm{ci}=0.38, \mathrm{ri}=0.65$. Four of the five major clades are labeled at their nodes (circled numbers), clade 3 was not recovered by this coding method. 


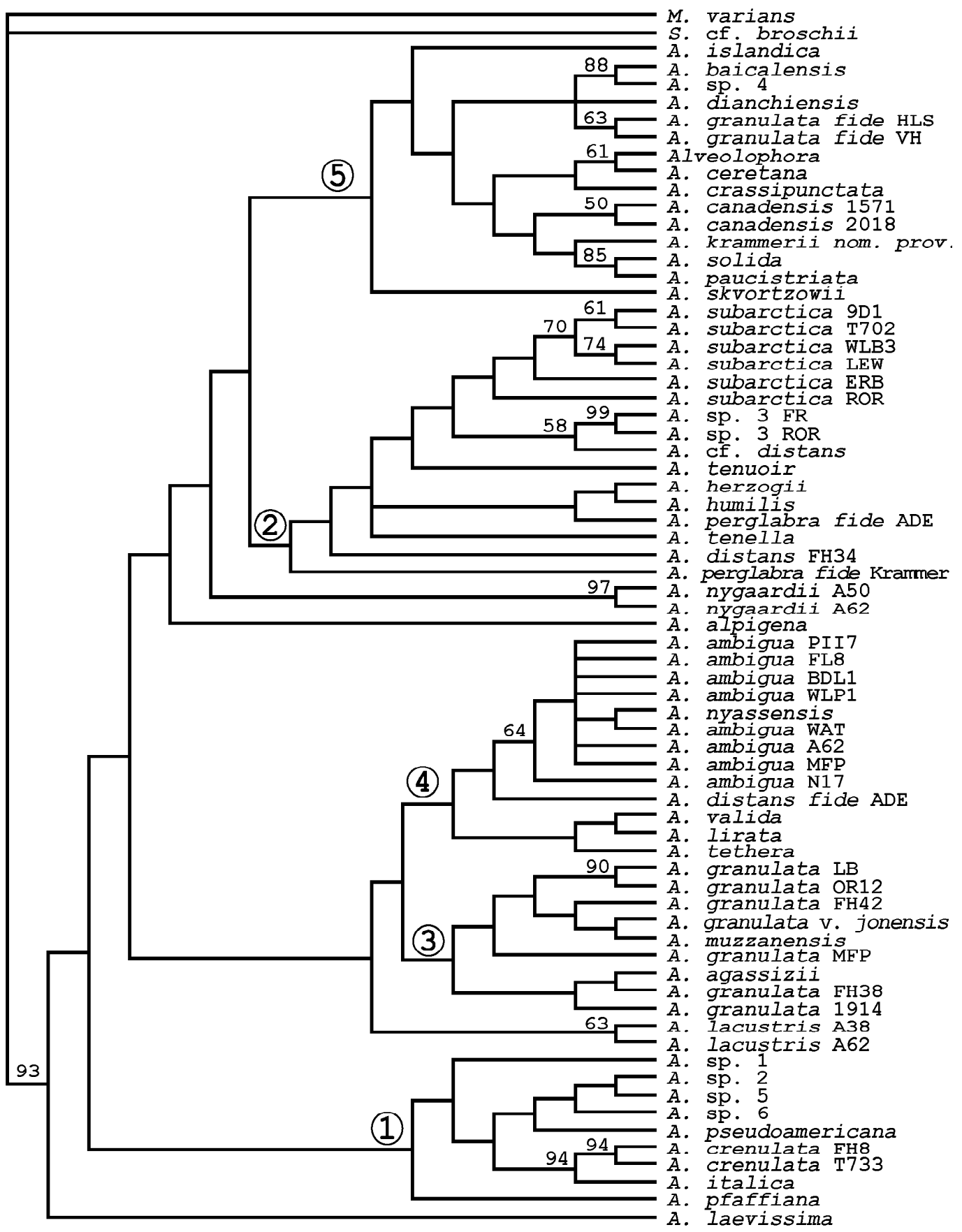

Figure 27. Analysis 3. Gap-weighting coding method of Thiele (1993) limited to 10 character states. Bootstrap values above branches are based on 500 pseudo-replicates of 10 addition sequence replicates employing TBR branch swapping. Strict consensus of 60 MPTs. $\mathrm{L}=3,543, \mathrm{ci}=0.44, \mathrm{ri}=0.67$. Five major clades are labeled at their nodes (circled numbers). 


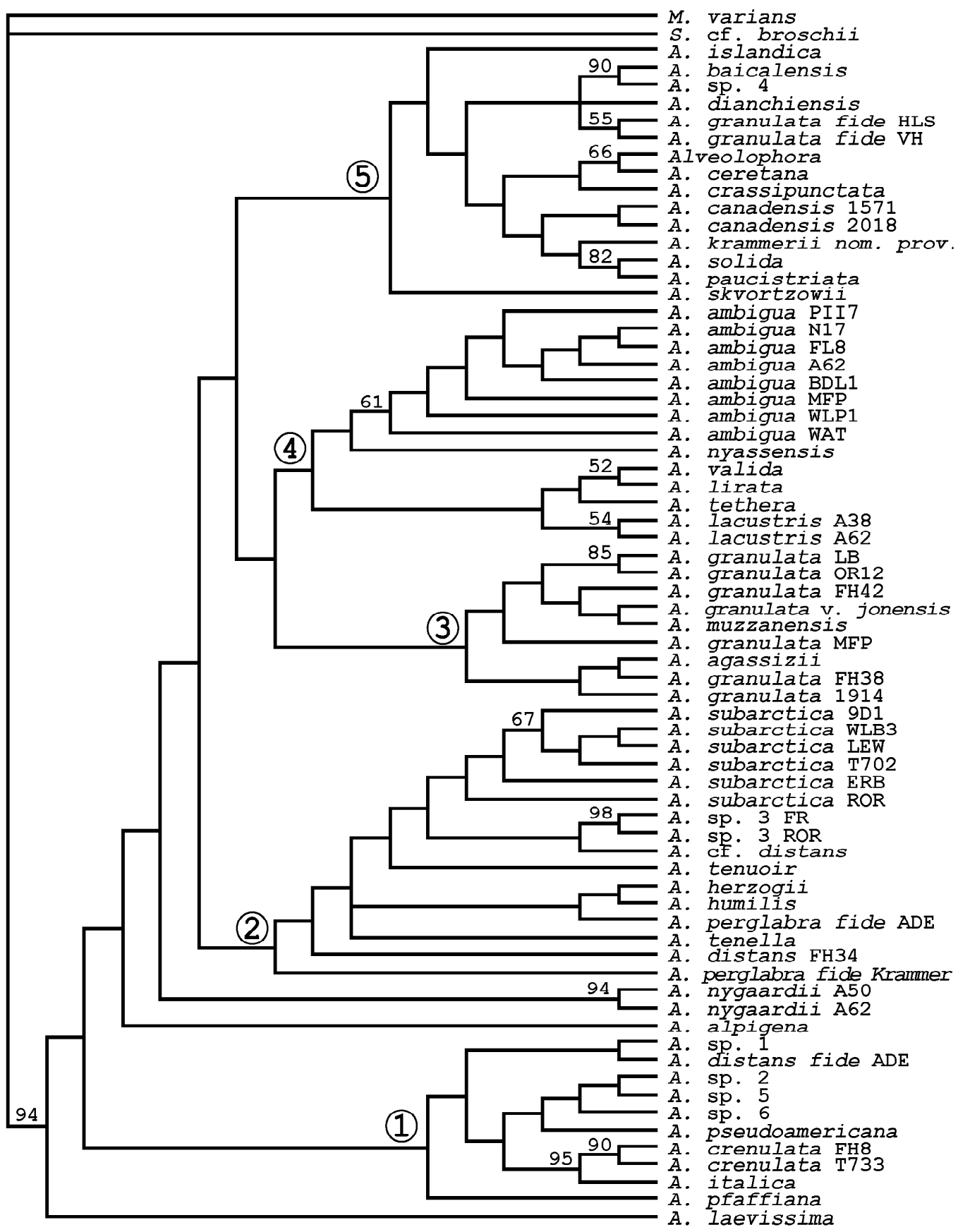

Figure 28. Analysis 4. Gap-weighting coding method of Thiele (1993) limited to 26 character states. Strict consensus of 6 MPTs. Bootstrap values above branches are based on 302 pseudo-replicates of 10 addition sequence replicates employing TBR branch swapping. $\mathrm{L}=10,465, \mathrm{ci}=0.44, \mathrm{ri}=0.67$. Five major clades are labeled at their nodes (circled numbers). 


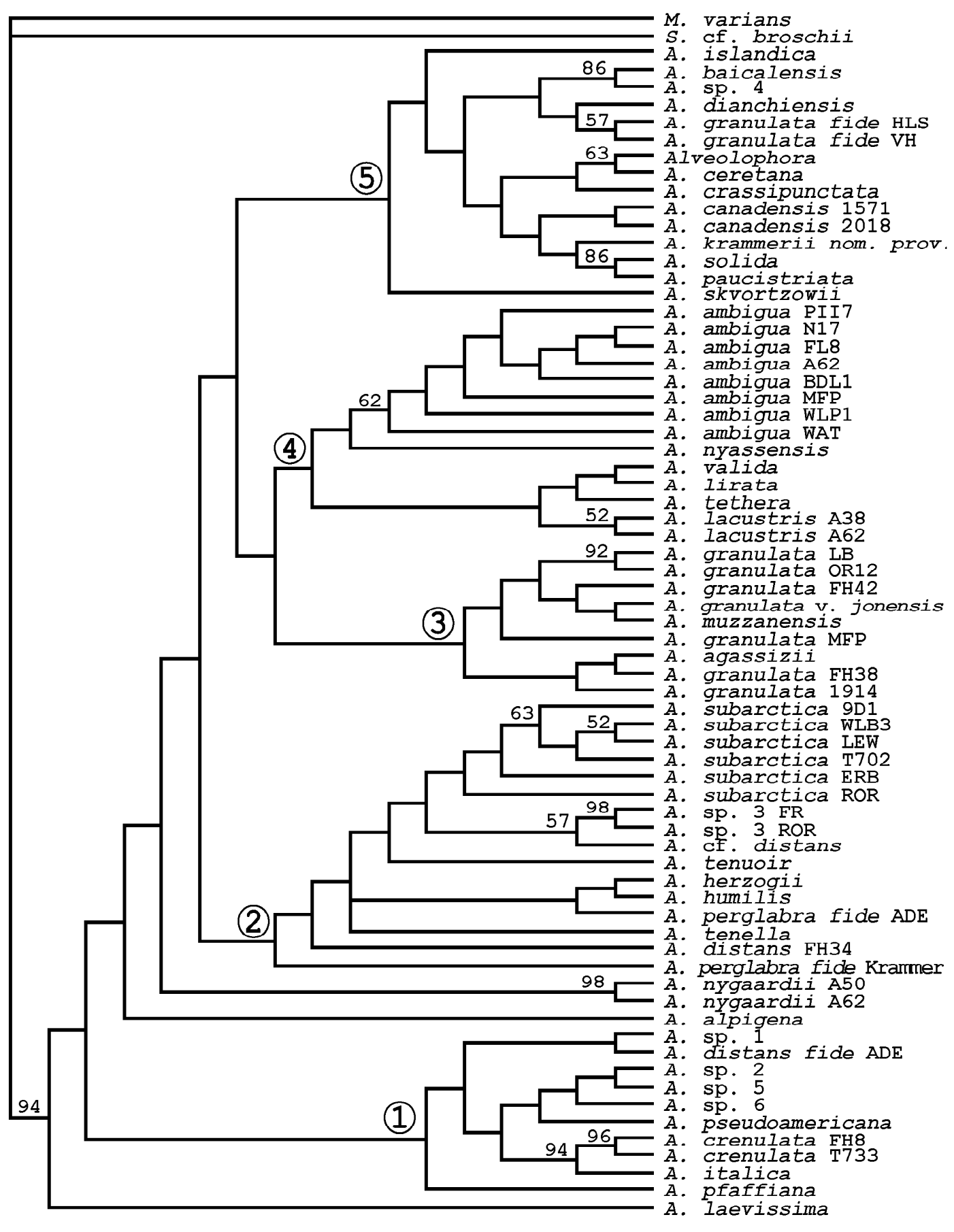

Figure 29. Analysis 5. Gap-weighting coding method of Thiele (1993) with 32 character state limitation. Strict consensus of 3 MPTs. Bootstrap values above branches are based on 265 pseudo-replicates of 10 addition sequence replicates employing TBR branch swapping. $\mathrm{L}=12,794, \mathrm{ci}=0.44, \mathrm{ri}=0.67$. Five major clades are labeled at their nodes (circled numbers). 


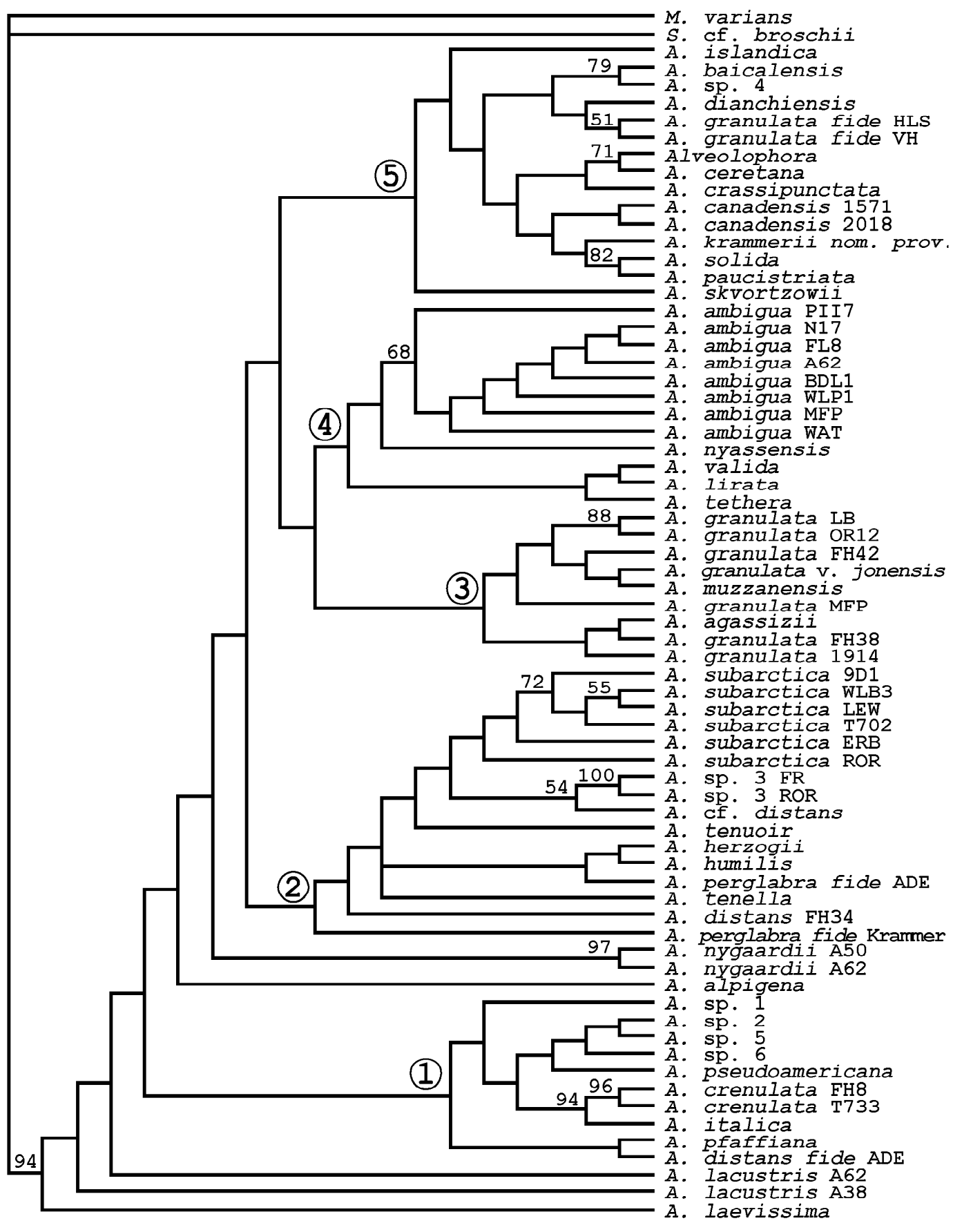

Figure 30. Analysis 6. Step-matrix gap weighting coding method modified from Wiens (2001) with a character state restriction of 26. Strict consensus of 3 MPTs. Bootstrap values above branches are based on 317 pseudo-replicates of 10 addition sequence replicates employing TBR branch swapping. $\mathrm{L}=$ $1,265.380, \mathrm{ci}=0.41, \mathrm{ri}=0.65$. Five major clades are labeled at their nodes (circled numbers). 


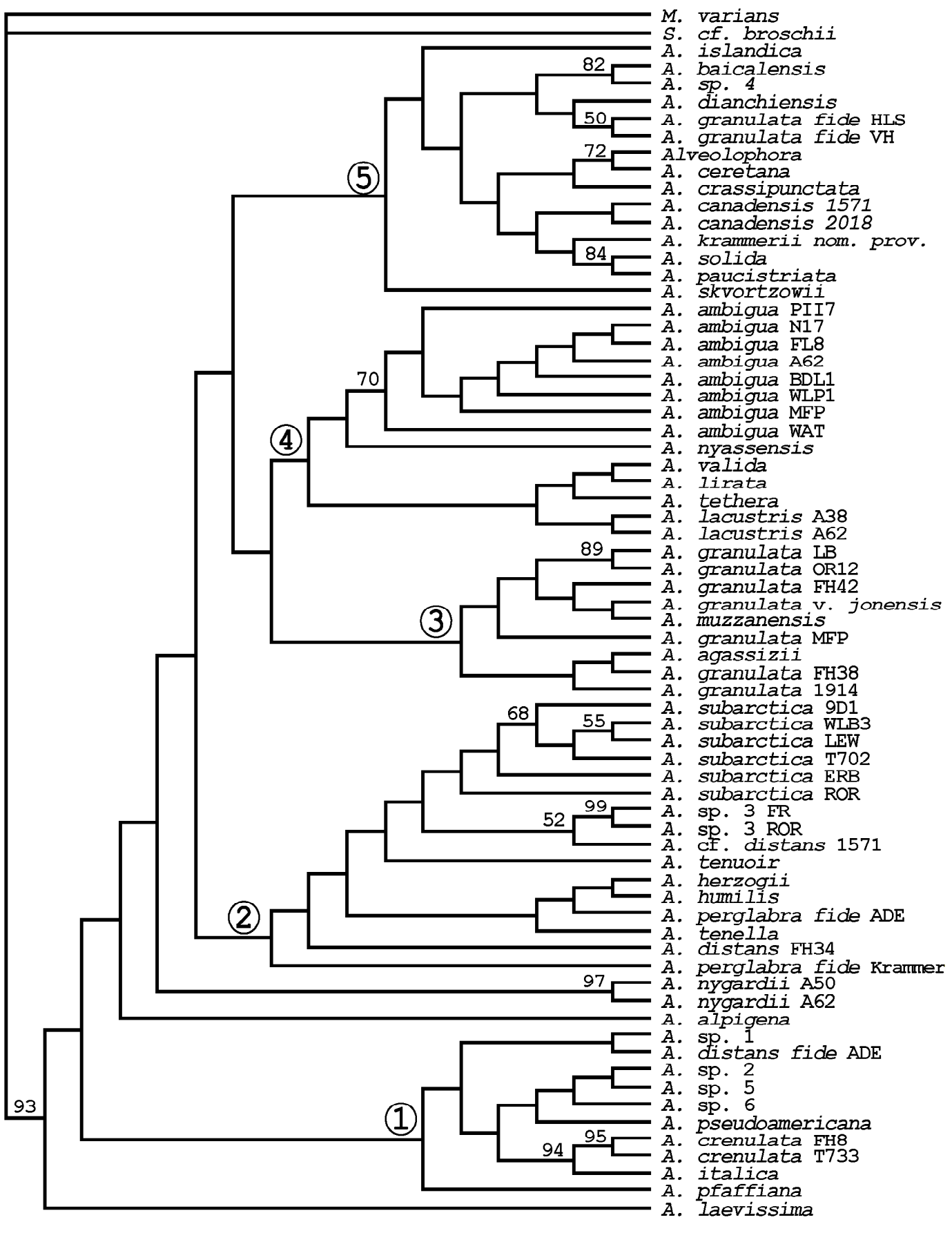

Figure 31. Analysis 7. Step-matrix gap weighting coding method modified from Wiens (2001) with a character state restriction of 32 (single MPT). Bootstrap values above branches are based on 500 pseudoreplicates of 10 addition sequence replicates employing TBR branch swapping. $\mathrm{L}=1280.392, \mathrm{ci}=0.41$, $\mathrm{ri}=0.65$. Five major clades are labeled at their nodes (circled numbers). 


\section{DISCUSSION}

Among all analyses run, five major clades are identified based on their high similarity in population composition (figs. 25 - 31). These five clades generally occur regardless of coding method (with the exception of losing clade 3 in analysis 2; fig. 26) or character state restrictions, with the exception of only a handful of taxa. This in combination with the PTP test results, indicating that the morphometric data were in fact contributing a statistically significant phylogenetic signal regardless of coding method, lends strong support to the hypothesized homologies (internested synapomorphies) in the morphometric continuous overlapping characters and their use in phylogenetic estimates. One might worry that since a phylogeny based on combined data is being tested for sensitivity here that the underlying similarity in signal is due to the non-morphometric characters which prevail and is shown despite different manipulations or coding of the morphometric data. There are three points of evidence against this being the case: 1) Taxa represented by DNA data are too few, and too evenly distributed amongst the five clades, to contribute to hypotheses of relationships among taxa within clades. 2) The PTP tests do indicate significant phylogenetic signal in the morphometric data in all permutations of coding. 3) The qualitative morphological data alone produce a highly unresolved estimate of phylogeny (strict consensus tree 16,965 MPTs; fig. 22). This indicates that the great majority of the signal is in fact coming from the morphometric data. The quantitative morphometric data will produce a highly resolved phylogenetic estimate without the presence of any other data type. The qualitative morphological data do seem to contribute some signal 
in combination with the morphometric data, because when combined it does pull multiple populations of species together, in some cases (fig. 24). The qualitative morphological data are generally describing ultrastructural characteristics, such as rimoportula position on the valve mantle, that do not generally vary among populations of the same species, whereas the morphometric characters do vary among populations to varying degrees potentially picking up more recent transformations of these types of characters.

As was shown in Thiele (1993), Wiens (2001), and herein, morphometric data can be a robust type of data given some standardized method of coding, such as stepmatrix gap weighting (Wiens 2001) and should by no means be excluded a priori. Instead, they should be gathered and explored empirically within the context of each analysis to evaluate their utility, as has been suggested by proponents such as Humphries (2002), Stevens (2000), and Thiele (1993).

The empirical evidence presented here leads to a recommendation of coding continuous overlapping characters with the highest amount of resolution available, i.e., using the highest number of character states allowable by a program that also allows weighting of gaps via step-matrices (at present PAUP alone holds this place with 32 allowable character states).

In addition to the empirical evidence presented here, there are also compelling logical arguments for this choice of coding method for continuous overlapping variables when using them in an estimate of phylogeny. First, this method, of all methods, has the least amount of manipulation of the raw data. Yet, it has very specific rules for its 
application, containing no ambiguous threshold, and it is highly repeatable. The biological and theoretical justification for recognizing similarities in this type of data, which can then be proposed as internested synapomorphies or homologies, are clearly laid out by Rae (1998). The basic argument falls back on our understanding of evolutionary processes. There are now a plethora of examples in scientific journals, books and general texts that have come out in support of and development of our understanding of these processes since the famous publication of Darwin in 1859. Whether changes in characters are moved by natural selection, genetic drift, or any combination of evolutionary forces, we generally see the same trend - the central tendencies of quantitative characters are gradually shifted as groups differentiate (cladogenesis), or adapt (anagenesis; Cronin and Schneider 1990, Falconer 1981, Freeman and Herron 2001, Ridley 1993, Theriot 1992).

When examining what can be considered to be homologous characters and the initial step of detecting them via the criterion or test of similarity (Patterson 1982, 1988) Cracraft (1981) states "similarity is the factor that compels us to postulate homology." Similarities in central tendencies of characters generally reflect a shared history (i.e., common descent) leading us to postulate that these characters may well be homologous and that the actual differences in the central tendencies among taxa for this characteristic generally will reflect their phylogenetic history. Using internested sets of similarity that we observe in continuous overlapping data then would compel us to postulate ordered transformation series, the same as we would for qualitative morphological characters (Lipscomb 1992). As Lipscomb points out for qualitative 
morphological data, "states are modifications of either the original attribute or other states so that a character is an internested set of synapomorphies" (emphasis added). This description fits anagenesis of continuous characters as well. And following Patterson $(1982,1988)$, any proposed homologous character, whether it be qualitative or quantitative, overlapping or discrete, can be subjected to a test of congruence with other proposed homologous characters allowing us an a posteriori test of our postulates (Lipscomb 1992, Thiele 1993, Wiens 2001).

Previous attempts to judge whether or not continuous overlapping data are appropriate for phylogenetic estimates have unfortunately and unnecessarily focused on the more primitive means of coding this type of data (Gift and Stevens 1997, Reid and Sidwell 2002). Furthermore, Reid and Sidwell (2002) used an arguably insufficient amount of data for the type of criteria they attempted to evaluate, leaving their conclusions less than compelling.

Despite many papers proposing statistical means of coding continuous overlapping characters with a major goal of removing investigator bias from coding techniques in the literature from 1976 to 1997 (Archie 1985, Colless 1980, Mickevich and Johnson 1976, Thiele 1993, Thorpe 1984), Gift and Stevens (1997) chose to focus on the most primitive means of gap identification method - graphical representation of the data and the eye of the investigator to perceive gaps. Gift and Stevens (1997) were aware of Thiele's (1993) method that did not require gap identification for coding and therefore eliminating many of the problems raised regarding gap identification methods of coding. Unfortunately, they considered Thiele's (1993) method to be beyond the 
scope of their paper, resulting in their work being an incomplete test of continuous character utility.

Reid and Sidwell (2002) only tested methods employing gap identification (Archie 1985, Mickevich and Johnson 1976, Thorpe 1984) despite citing a more recent and arguably less ambiguous gap weighting method (Thiele 1993) and without any discussion as to why it was not chosen. The diatom data set they used contained only 13 characters, 4 of which were considered quantitative and continuous. However, the quantitative characters chosen included two ratios and were not considered relative to any understanding of how characteristics change with size (i.e., life cycle).

There are still some concerns when applying step-matrix gap weighting that are currently being investigated, such as use of ratios in range standardization, limitation of character states (as a technological imposition only), and methods used to scale data coded this way being comparable to other data types that may be employed in a phylogeny estimated from a combined analysis of multiple types of data (Wiens, personal communication). Despite having over twice as many OTUs as character states in this study, a fully resolved estimate of phylogeny was obtained that can be argued to be the most reasonable working hypothesis for phylogeny for this group given the data at hand. This suggests that the technical limitation of maximum number of character states, although still a concern for larger data sets, may not be quite as restrictive as once thought. One would hope, however, that technical limitations such as these will disappear in the near future as phylogenetic software and processing hardware become 
more powerful, allowing larger data sets to employ this type of data in phylogenetic estimates.

Every study that includes this type of data will want to explore the data and its potential signal as best it can, and this is where sensitivity analyses can lend a hand. As suggested by Simmons and Geisler (2002), sensitivity analyses are a useful tool for independent evaluation of a phylogeny more in tune to a particular dataset than some other estimates of support, such as bootstrap values. Sensitivity analyses are a very good means of testing the ambiguity or robustness of clades given the data at hand to changes in coding choice and do not necessarily speak to whether they are a best estimate of phylogeny overall. But as Simmons and Geisler (2002) point out, it is best to know and understand the strengths and limitations of a data set in order to plan most efficiently future research. Sensitivity analyses permit pinpointing ambiguities in phylogenetic signal and helps in planning more efficient sampling for future estimates, by focusing on ambiguous or sensitive areas of the phylogeny via additional data collection or addition of taxa (Simmons and Geisler 2002).

Every type of data has its difficulties when seriously considering how to recognize characters, define character states, and how to treat these states (ordered, unordered, multistate, binary, concerns of redundancy in coding, treating gaps as fifth character states versus missing data, weighting transitions and transversions, etc.) for matrix construction for phylogenetic estimation (Forey and Kitching 2000, Hawkins 2000, Lipscomb 1992, Simmons and Ochoterena 2000, Strong and Lipscomb 1999). There have been technological and methodological difficulties with including multistate 
data or more complex data, such as overlapping continuous data that may have caused workers to historically avoid this type of data but such difficulties no longer exist. Even in a study that found major bias from one investigator to another in coding the same sets of data (Gift and Stevens 1997), the ultimate recommendation was to explore methods that might remove this investigator bias and allow coding of overlapping continuous data in an unbiased and repeatable manner. Coding of all types of data is still open for exploration. As of yet, nobody has discovered the universal coding method that solves all of these interesting and diverse questions about how to represent data for the most accurate reconstruction of phylogeny (Swiderski et al. 1998). But perhaps through a combination of additional empirical studies exhibiting sensitivity of data to coding methods and simulation studies, we will be able to answer some of these coding questions as they relate to our data at hand and provide guidelines for future examination and evaluation of various types of data. 
APPENDIX A. Specimens Examined. 


\begin{tabular}{|c|c|c|c|c|c|c|c|}
\hline Species & Identifier & Collection. & Coll. No. & Locality Info. & $\begin{array}{l}\text { Date of } \\
\text { Coll. }\end{array}$ & $\begin{array}{c}\text { Culture } \\
\text { ID/*GenBank } \\
\text { Accession } \\
\text { No. }\end{array}$ & $\begin{array}{l}\text { Culture } \\
\text { Collection }\end{array}$ \\
\hline Melosira varians Agardh & $\begin{array}{l}\text { FFP } \\
\text { CHMP-7 }\end{array}$ & $\begin{array}{l}\text { SME } \\
\text { SME }\end{array}$ & $\begin{array}{l}\text { SME-123 } \\
\text { SME-20 }\end{array}$ & $\begin{array}{l}\text { Franklin Falls Pond, NY } \\
\text { Lake Champlain, VT }\end{array}$ & $\begin{array}{l}28 X 2001 \\
12 \mathrm{i} 2000\end{array}$ & $\begin{array}{l}\text { N/A } \\
\text { CHMP-7 }\end{array}$ & $\begin{array}{l}\text { N/A } \\
\text { SME }\end{array}$ \\
\hline Stephanopyxis cf. broschii & GB-18S & & & & & *M87330 & \\
\hline $\begin{array}{l}\text { Alveolophora Moiseeva and } \\
\text { Nevretdinova }\end{array}$ & 2019 & Theriot - personal & ECT2019 & Mann Creek, ID & 24vi1988 & $\mathrm{N} / \mathrm{A}$ & $\mathrm{N} / \mathrm{A}$ \\
\hline $\begin{array}{l}\text { Aulacoseira islandica (O. } \\
\text { Müller) Simonsen }\end{array}$ & $\begin{array}{l}\text { MICH } \\
\text { CHMP-1 }\end{array}$ & $\begin{array}{l}\text { SME } \\
\text { SME }\end{array}$ & $\begin{array}{l}\text { SME-56 } \\
\text { SME-20 }\end{array}$ & $\begin{array}{l}\text { Lake Michigan } \\
\text { Lake Champlain, VT }\end{array}$ & $\begin{array}{l}10 \times 2000 \\
12 \mathrm{i} 2000\end{array}$ & $\begin{array}{l}\text { N/A } \\
\text { CHMP-1 }\end{array}$ & $\begin{array}{l}\text { N/A } \\
\text { SME }\end{array}$ \\
\hline $\begin{array}{l}\text { A. skvortzowii Edlund, Stoermer } \\
\text { \& Taylor }\end{array}$ & $\begin{array}{l}\text { LkBkl } \\
\text { GB-18S } \\
\text { GB- } r b c \mathrm{~L}\end{array}$ & Edlund - personal & 319-v9 & $\begin{array}{l}\text { Lake Baikal, Siberia } \\
\text { Lake Baikal, Siberia } \\
\text { Lake Baikal, Siberia }\end{array}$ & 1991 & $\begin{array}{l}\text { N/A } \\
\text { *AY121822 } \\
\text { *AF522279 } \\
\end{array}$ & $\mathrm{N} / \mathrm{A}$ \\
\hline $\begin{array}{l}\text { A. baicalensis (K. Meyer) } \\
\text { Simonsen }\end{array}$ & $\begin{array}{l}\text { LkBkl } \\
\text { GB-18S } \\
\text { GB- } r b c \mathrm{~L}\end{array}$ & Edlund - personal & 319-v9 & $\begin{array}{l}\text { Lake Baikal, Siberia } \\
\text { Lake Baikal, Siberia } \\
\text { Lake Baikal, Siberia }\end{array}$ & 1991 & $\begin{array}{l}\text { N/A } \\
\text { *AY121821 } \\
\text { *AF522281 }\end{array}$ & $\mathrm{N} / \mathrm{A}$ \\
\hline $\begin{array}{l}\text { A. subarctica (O. Müller) } \\
\text { Haworth }\end{array}$ & $\begin{array}{l}\text { 9D1 } \\
\text { WLB } \\
\text { ERB } \\
\text { LEW } \\
\text { ROR } \\
\text { T702 }\end{array}$ & $\begin{array}{l}\text { SME } \\
\text { SME } \\
\text { SME } \\
\text { SME } \\
\text { SME } \\
\text { Terry/FH }\end{array}$ & $\begin{array}{l}\text { SME-19 } \\
\text { SME-135 } \\
702\end{array}$ & $\begin{array}{l}\text { Yellowstone Lake, WY } \\
\text { Lake Willoughby, VT } \\
\text { East Rosebud Lake, MT } \\
\text { Lewis Lake, WY } \\
\text { Raccourci Old River, LA } \\
\text { Oregon }\end{array}$ & $\begin{array}{l}11 \mathrm{i} 2000 \\
8 \mathrm{iii} 2002\end{array}$ & $\begin{array}{l}\text { 9D1 } \\
\text { WLB-3 } \\
\text { ERB-68A } \\
\text { L55B } \\
\text { N/A } \\
\text { N/A }\end{array}$ & $\begin{array}{l}\text { SME } \\
\text { SME } \\
\text { SME } \\
\text { SME } \\
\text { N/A } \\
\text { N/A }\end{array}$ \\
\hline A. sp. 1 & ODR & SME & SME-140 & Old Duke Road Pond, VT & ix2002 & ODR-3 & SME \\
\hline A. sp. 2 & LPNH & SME & SME-65 & Long Pond, NH & 10vii2001 & LPNH-1 & SME \\
\hline A. sp. 3 & $\begin{array}{l}\text { FR } \\
\text { ROR }\end{array}$ & $\begin{array}{l}\text { SME } \\
\text { SME }\end{array}$ & $\begin{array}{l}\text { SME-136 } \\
\text { SME-135 }\end{array}$ & $\begin{array}{l}\text { False River, LA } \\
\text { Raccourci Old River, LA }\end{array}$ & $\begin{array}{l}\text { 9iii2002 } \\
\text { 8iii2002 }\end{array}$ & $\begin{array}{l}\text { FR-9 } \\
\text { N/A }\end{array}$ & $\begin{array}{l}\text { SME } \\
\text { N/A }\end{array}$ \\
\hline A. sp. 4 & T733 & Terry/FH & 733 & Wellington, New Zealand & & $\mathrm{N} / \mathrm{A}$ & N/A \\
\hline A. sp. 5 & HDSM & RKE & 2377 & & & $\mathrm{~N} / \mathrm{A}$ & $\mathrm{N} / \mathrm{A}$ \\
\hline A. sp. 6 & HDSM & RKE & 2377 & & & N/A & N/A \\
\hline A. alpigena (Grunow) Krammer & CCAP & $\begin{array}{l}\text { Culture Collection of } \\
\text { Algae and Protozoa }\end{array}$ & CCAP-6 & Cumbria, UK & & CCAP-6 & CCAP \\
\hline $\begin{array}{l}\text { A. nyassensis (O. Müller) } \\
\text { Simonsen }\end{array}$ & $\begin{array}{l}\text { H.K. } \\
\text { GB-18S } \\
\text { GB-rbcL }\end{array}$ & Kling - personal & 886 & Lake Malawi, Kenya & $13 \mathrm{v} 1997$ & $\begin{array}{l}\text { N/A } \\
\text { *AY121819 } \\
\text { *AF522282 }\end{array}$ & $\mathrm{N} / \mathrm{A}$ \\
\hline
\end{tabular}




\begin{tabular}{|c|c|c|c|c|c|c|c|}
\hline Species & Identifier & Collection. & Coll. No. & Locality Info. & $\begin{array}{l}\text { Date of } \\
\text { Coll. }\end{array}$ & $\begin{array}{c}\text { Culture } \\
\text { ID/*GenBank } \\
\text { Accession } \\
\text { No. }\end{array}$ & $\begin{array}{l}\text { Culture } \\
\text { Collection }\end{array}$ \\
\hline A. ambigua (Grunow) Simonsen & $\begin{array}{l}\text { PII7 } \\
\text { N17 } \\
\text { FL8 } \\
\text { BDL1 } \\
\text { WLP1 } \\
\text { A62 } \\
\text { MFP } \\
\text { WAT }\end{array}$ & $\begin{array}{l}\text { SME } \\
\text { SME } \\
\text { SME } \\
\text { SME } \\
\text { SME } \\
\text { ADE/FH } \\
\text { SME } \\
\text { SME }\end{array}$ & $\begin{array}{l}\text { SME-69 } \\
\text { SME-85 } \\
\text { SME-118 } \\
\text { SME-16 } \\
\text { SME-15 } \\
\text { sl. no. } 62 \\
\text { SME-43 } \\
\text { SME-14 }\end{array}$ & $\begin{array}{l}\text { Perch Pond II, NH } \\
\text { Newport Pond, NY } \\
\text { Lake Flower, NY } \\
\text { Budd Lake, NJ } \\
\text { Lake Wallenpaupack, PN } \\
\text { Big Moose Lake, NY } \\
\text { Molly's Falls Pond, VT } \\
\text { South Watuppa Pond, MA }\end{array}$ & $\begin{array}{l}\text { 10vii2001 } \\
\text { 26vii2001 } \\
28 X 2001 \\
9 \mathrm{i} 2000 \\
7 \mathrm{i} 2000 \\
1794 \\
\text { 30vii2000 } \\
4 \mathrm{i} 2000\end{array}$ & $\begin{array}{l}\text { PII7 } \\
\text { N17 } \\
\text { FL8 } \\
\text { BDL1 } \\
\text { WLP1 } \\
\text { N/A } \\
\text { N/A } \\
\text { WAT-1 }\end{array}$ & $\begin{array}{l}\text { SME } \\
\text { SME } \\
\text { SME } \\
\text { SME } \\
\text { SME } \\
\text { N/A } \\
\text { N/A } \\
\text { SME }\end{array}$ \\
\hline $\begin{array}{l}\text { A. granulata (Ehrenburg) } \\
\text { Simonsen }\end{array}$ & $\begin{array}{l}\text { LB } \\
\text { OR12 } \\
\text { FH42 } \\
\text { FH38 } \\
\\
1914 \\
\text { MFP } \\
\end{array}$ & $\begin{array}{l}\text { SME } \\
\text { SME } \\
\text { Tilden Amer. Alg./FH } \\
\text { Rabenhorst Alg. Eur./FH } \\
\text { Theriot - personal } \\
\text { SME }\end{array}$ & $\begin{array}{l}\text { SME-134 } \\
\text { SME-137 } \\
367 \\
2212 \\
\\
\text { ECT1914 } \\
\text { SME-43 } \\
\end{array}$ & $\begin{array}{l}\text { Lake Buchanan, TX } \\
\text { Raccourci Old River, LA } \\
\text { Strout's Claim Pit, ID } \\
\text { Molly's Falls Pond, VT }\end{array}$ & $\begin{array}{l}\text { 11xii2001 } \\
\text { 4iv2000 } \\
\text { 30vii2000 } \\
\end{array}$ & $\begin{array}{l}\text { LB-Aul } \\
\text { OR-12 } \\
\text { N/A } \\
\text { N/A } \\
\text { N/A } \\
\text { N/A } \\
\end{array}$ & $\begin{array}{l}\text { SME } \\
\text { SME } \\
\text { N/A } \\
\text { N/A } \\
\text { N/A } \\
\text { N/A } \\
\end{array}$ \\
\hline $\begin{array}{l}\text { A. crenulata (Ehrenburg) } \\
\text { Thwaites }\end{array}$ & $\begin{array}{l}\text { FH8 } \\
\text { T733 } \\
\text { L639 }\end{array}$ & $\begin{array}{l}\text { FH- herbarium sheets } \\
\text { Terry/FH } \\
\text { Freshwater Diatom } \\
\text { Culture Collection }\end{array}$ & $\begin{array}{l}4058 \\
733 \\
\text { L639 }\end{array}$ & $\begin{array}{l}\text { Wellington, New Zealand } \\
\text { Iowa }\end{array}$ & $10 X 1940$ & $\begin{array}{l}\text { N/A } \\
\text { N/A } \\
\text { L639 }\end{array}$ & $\begin{array}{l}\text { N/A } \\
\text { N/A } \\
\text { FDCC }\end{array}$ \\
\hline $\begin{array}{l}\text { A. canadensis (Hustedt) } \\
\text { Simonsen }\end{array}$ & $\begin{array}{l}1571 \\
2018 \\
\end{array}$ & $\begin{array}{l}\text { Theriot - personal } \\
\text { Theriot - personal }\end{array}$ & $\begin{array}{l}\text { ECT1571 } \\
\text { ECT2018 } \\
\end{array}$ & $\begin{array}{l}\text { Nevada } \\
\text { Mann Creek, ID }\end{array}$ & $\begin{array}{l}8 \text { ix1987 } \\
\text { 24vii1988 } \\
\end{array}$ & $\begin{array}{l}\text { N/A } \\
\text { N/A }\end{array}$ & $\begin{array}{l}\text { N/A } \\
\text { N/A }\end{array}$ \\
\hline $\begin{array}{l}\text { A. dianchiensis Yang, Stoermer } \\
\text { \& Kociolek }\end{array}$ & & Whitmore -personal & & $\begin{array}{l}\text { Er Hai,Yunnan Provence, } \\
\text { China }\end{array}$ & 1998 & $\mathrm{~N} / \mathrm{A}$ & $\mathrm{N} / \mathrm{A}$ \\
\hline A. distans (Ehrenburg) Simonsen & FH34 & Rabenhorst/FH & 2202 & Bilin & & N/A & N/A \\
\hline $\begin{array}{l}\text { A. granulata var. jonensis } \\
\text { (Grunow) Simonsen }\end{array}$ & 3016 & R. Edgar - personal & RKE3016 & & & N/A & N/A \\
\hline A. granulata fide Van Heurck & $\mathrm{VH}$ & $\mathrm{FH}$ & 463 & & & $\mathrm{~N} / \mathrm{A}$ & N/A \\
\hline A. granulata fide H. L. Smith & HLS & FH & 224 & & & $\mathrm{~N} / \mathrm{A}$ & N/A \\
\hline $\begin{array}{l}\text { A. herzogii (Lemmermann) } \\
\text { Simonsen }\end{array}$ & HDSM & R. Edgar - personal & RKE2377 & & & $\mathrm{N} / \mathrm{A}$ & N/A \\
\hline
\end{tabular}




\begin{tabular}{|c|c|c|c|c|c|c|c|}
\hline Species & Identifier & Collection. & Coll. No. & Locality Info. & $\begin{array}{l}\text { Date of } \\
\text { Coll. }\end{array}$ & $\begin{array}{c}\text { Culture } \\
\text { ID/*GenBank } \\
\text { Accession } \\
\text { No. }\end{array}$ & $\begin{array}{l}\text { Culture } \\
\text { Collection }\end{array}$ \\
\hline A. pfaffiana (Reinsch) Krammer & FH37 & Rhabenhorst/FH & 1912 & & & $\mathrm{~N} / \mathrm{A}$ & $\mathrm{N} / \mathrm{A}$ \\
\hline A. solida (Eulenstein) Krammer & FH23 & Bartholomew/FH & 64 & Pitt River, CA & & $\mathrm{N} / \mathrm{A}$ & $\mathrm{N} / \mathrm{A}$ \\
\hline A. distans fide ADE & HRT & SME & SME-70 & Heart Lake, NY & 26vii2001 & N/A & N/A \\
\hline $\begin{array}{l}\text { A. krammeri nom. prov. Edgar \& } \\
\text { Kociolek }\end{array}$ & CAS & $\begin{array}{l}\text { California Academy of } \\
\text { Sciences }\end{array}$ & $\begin{array}{l}\text { CAS } \\
600215 \\
\end{array}$ & & & N/A & $\mathrm{N} / \mathrm{A}$ \\
\hline A. lirata (Ehrenburg) Simonsen & A37 & $\mathrm{ADE} / \mathrm{FH}$ & sl. no. 37 & West Pond, NY & 1981 & $\mathrm{~N} / \mathrm{A}$ & $\mathrm{N} / \mathrm{A}$ \\
\hline $\begin{array}{l}\text { A. laevissima (Grunow) } \\
\text { Krammer }\end{array}$ & HDSM & R. Edgar - personal & RKE2377 & & & $\mathrm{N} / \mathrm{A}$ & $\mathrm{N} / \mathrm{A}$ \\
\hline A. nygaardii (Camburn) & $\begin{array}{l}\text { A50 } \\
\text { A62 }\end{array}$ & $\begin{array}{l}\mathrm{ADE} / \mathrm{FH} \\
\mathrm{ADE} / \mathrm{FH}\end{array}$ & $\begin{array}{l}\text { sl. no. } 50 \\
\text { sl. no. } 62 \\
\end{array}$ & $\begin{array}{l}\text { Big Moose Lake, NY } \\
\text { Big Moose Lake, NY }\end{array}$ & $\begin{array}{l}1963 \\
1794 \\
\end{array}$ & $\begin{array}{l}\text { N/A } \\
\text { N/A }\end{array}$ & $\begin{array}{l}\text { N/A } \\
\text { N/A }\end{array}$ \\
\hline A. perglabra fide ADE & $\mathrm{ADE}$ & $\mathrm{ADE} / \mathrm{FH}$ & sl. no. 38 & West Pond, NY & 1970 & N/A & $\mathrm{N} / \mathrm{A}$ \\
\hline A. paucistriata Bradbury & 2035 & Theriot - personal & ECT2035 & Thorn Lake, OR & 25vi1988 & N/A & N/A \\
\hline A. cf. distans & 1571 & Theriot - personal & ECT1571 & Nevada & $8 \mathrm{ix} 1987$ & $\mathrm{~N} / \mathrm{A}$ & $\mathrm{N} / \mathrm{A}$ \\
\hline A. valida (Grunow) Krammer & N2 & SME & SME-85 & Newport Pond, NH & 26vii2001 & NWPT-2 & SME \\
\hline A. italica (Ehrenburg) Simonsen & FH15 & Bartholomew/FH & 718 & Santa Fiora, Italy & & N/A & $\mathrm{N} / \mathrm{A}$ \\
\hline $\begin{array}{l}\text { A. agassizii (Ostenfeld) } \\
\text { Simonsen }\end{array}$ & $\mathrm{FH} 21$ & Bartholomew/FH & & Paso Fiores, Argentina & & N/A & $\mathrm{N} / \mathrm{A}$ \\
\hline
\end{tabular}


APPENDIX B. Species data gathered from literature. 


\begin{tabular}{|c|c|c|c|}
\hline Species & Reference & $\begin{array}{c}\text { Character Examined or } \\
\text { Measured }\end{array}$ & Figures or Tables \\
\hline $\begin{array}{l}\text { Aulacoseira ceretana } \\
\text { Haworth \& Sabater }\end{array}$ & $\begin{array}{l}\text { Haworth and Sabater } \\
1993\end{array}$ & $\begin{array}{l}\text { Interareolar Distance } \\
\text { Interstriae Distance } \\
\text { Mantle Thickness } \\
\text { Collum Height } \\
\text { Ringleist Height } \\
\text { Ringleist penetration } \\
\text { into Lumen } \\
\text { Ringleist Thickness } \\
\text { Areolar Area } \\
\text { Striae Curvature - } \\
\text { Linking Valves } \\
\text { Striae Curvature- } \\
\text { Separation Valves }\end{array}$ & $\begin{array}{l}\text { figs. 10-13 } \\
\text { figs. } 10-13 \\
\text { fig. } 14 \\
\text { figs. } 4-6,10-13,16 \\
\text { fig. } 14 \\
\text { fig. } 14 \\
\text { fig. } 14 \\
\text { figs. } 10-13 \\
\text { figs. } 10-13 \\
\text { figs. } 12,16\end{array}$ \\
\hline $\begin{array}{l}\text { A. tenella (Nygaard) } \\
\text { Simonsen }\end{array}$ & Siver and Kling 1997 & $\begin{array}{l}\text { Interareolar Distance } \\
\text { Interstriae Distance } \\
\text { Collum Height } \\
\text { Areolar Area } \\
\text { Striae Curvature - } \\
\text { Separation Valves } \\
\end{array}$ & $\begin{array}{l}\text { figs. } 87-90 \\
\text { figs. } 87-90 \\
\text { figs. } 87-90,92 \\
\text { figs. } 87-90 \\
\text { figs. } 87-90\end{array}$ \\
\hline A. tethera Haworth & Haworth 1988 & $\begin{array}{l}\text { Interareolar Distance } \\
\text { Interstriae Distance } \\
\text { Collum Height } \\
\text { Areolar Area } \\
\text { Striae Curvature - } \\
\text { Linking Valves }\end{array}$ & $\begin{array}{l}\text { figs. } 28,30 \\
\text { figs. } 28,30 \\
\text { figs. } 28,30,31 \\
\text { figs. } 28,30 \\
\text { figs. } 28,30\end{array}$ \\
\hline $\begin{array}{l}\text { A. tenuior (Grunow) } \\
\text { Krammer }\end{array}$ & Krammer 1991a & $\begin{array}{l}\text { Interareolar Distance } \\
\text { Interstriae Distance } \\
\text { Collum Height } \\
\text { Areolar Area } \\
\text { Striae Curvature - } \\
\text { Separation Valves } \\
\end{array}$ & $\begin{array}{l}\text { fig. } 88 \\
\text { fig. } 88 \\
\text { figs. } 83,88 \\
\text { fig. } 88 \\
\text { fig. } 88\end{array}$ \\
\hline
\end{tabular}




\begin{tabular}{|c|c|c|c|}
\hline Species & Reference & $\begin{array}{c}\text { Character Examined or } \\
\text { Measured }\end{array}$ & Figures or Tables \\
\hline $\begin{array}{l}\text { A. muzzanensis } \\
\text { (Meister) Krammer }\end{array}$ & Krammer 1991b & $\begin{array}{l}\text { Interareolar Distance } \\
\text { Interstriae Distance } \\
\text { Mantle Thickness } \\
\text { Collum Height } \\
\text { Ringleist Height } \\
\text { Ringleist penetration } \\
\text { into Lumen } \\
\text { Ringleist Thickness } \\
\text { Areolar Area } \\
\text { Striae Curvature - } \\
\text { Linking Valves } \\
\text { Striae Curvature - } \\
\text { Separation Valves }\end{array}$ & $\begin{array}{l}\text { figs. } 3,10,10 \mathrm{a}, 17,18, \\
20,21 \mathrm{a} \\
\text { figs. } 3,10,10 \mathrm{a}, 17,18, \\
20,21 \mathrm{a} \\
\text { figs. } 5,9 \\
\text { figs. } 4,6-8,10-19,21,22 \\
\text { fig. } 9 \\
\text { fig. } 9 \\
\text { fig. } 9 \\
\text { figs. } 3,10,10 \mathrm{a}, 17,18 \text {, } \\
20,21 \mathrm{a} \\
\text { figs. } 3,4,8,15,16 \\
\text { figs. } 10-15,17,19,22\end{array}$ \\
\hline $\begin{array}{l}\text { A. crassipunctata } \\
\text { Krammer }\end{array}$ & Krammer 1991b & $\begin{array}{l}\text { Interareolar Distance } \\
\text { Interstriae Distance } \\
\text { Collum Height } \\
\text { Ringleist penetration } \\
\text { into Lumen } \\
\text { Areolar Area } \\
\text { Striae Curvature - } \\
\text { Linking Valves }\end{array}$ & $\begin{array}{l}\text { figs. } 75-78 \\
\text { figs. } 75-78 \\
\text { figs. } 71,72,75,77,78 \\
\text { figs. } 73,74 \\
\text { figs. } 76,77,78,79 \\
\text { figs. } 76,77,78,79\end{array}$ \\
\hline $\begin{array}{l}\text { A. perglabra (Østrup) } \\
\text { Haworth }\end{array}$ & Krammer 1991a & $\begin{array}{l}\text { Interareolar Distance } \\
\text { Interstriae Distance } \\
\text { Collum Height } \\
\text { Ringleist penetration } \\
\text { into Lumen } \\
\text { Areolar Area } \\
\text { Striae Curvature - } \\
\text { Linking Valves } \\
\text { Striae Curvature - } \\
\text { Separation Valves }\end{array}$ & $\begin{array}{l}\text { figs. } 62-64 \\
\text { figs. } 62-64 \\
\text { figs. } 55,62,63,64 \\
\text { figs. } 62,64,66-68,71 \\
\text { figs. } 62-64 \\
\text { figs. } 62-64,72 \\
\text { figs. } 68,71\end{array}$ \\
\hline $\begin{array}{l}\text { A. pseudoamericana } \\
\text { Camburn }\end{array}$ & $\begin{array}{l}\text { Camburn and Kingston } \\
1986\end{array}$ & $\begin{array}{l}\text { Mantle Height } \\
\text { Valve Diameter }\end{array}$ & $\begin{array}{l}\text { plate III: } 55-58 \text {, } \\
\text { plate V: } 82\end{array}$ \\
\hline
\end{tabular}


APPENDIX C. Molecular characters: 18S rDNA. 
[

M._varians_CHMP7_FFP

S._Cf. broschii GB

A._islāndica_CHM̄P1_MICH

A._skvortzowi

A._baicalensis

A. sub_9D1

A._sub_WLB3

A. $-\mathrm{sp} \cdot{ }^{3} \mathrm{FR}$

A. sub LEW

A._alpigena

A._amb_PII 7

A. $a m b$ N17

A._amb_FL8

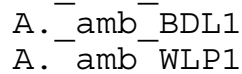

A._nyassensis_GB

A._valida_N2

A. -gran LB

A. gran OR12

A. $\mathrm{sp} \cdot \overline{1}$

A. $\operatorname{sp} .2$

A._cren_FH8_L639

10

20

30

40

50

]

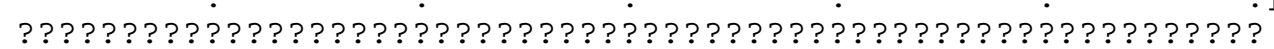
? ? ? ? ? ? ? ? ? ? ? ? ? ? ? ? ? ? ? ? ? ? ? ? ? ? ? ? ? ? ? ? ? ? ? ? ? ? ? ? ? ? ? ? ? ? ? ? ? ? ? ? ? ? ? ? ? ? ? ? ? ? ? ? ? ? ? ? ? ? ? ? ? ? ? ? ? ? ? ? ? ? ? ? ? ?

AACCTGGTTGATCCTGCCAGTAGTGATACGCTGTCATTAAGGACTAAGCCATGC-ATGTG AACCTGGTTGATCCTGCCAGTAGTGATACGCTGTCATTAAGGACTAAGCCATGCCATGTG

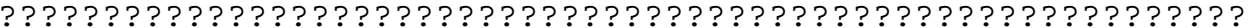
ใ? ? ? ? ? ? ? ? ? ? ? ? ? ? ? ? ? ? ? ? ? ? ? ? ? ? ? ? ? ? ? ? ? ? ? ? ?

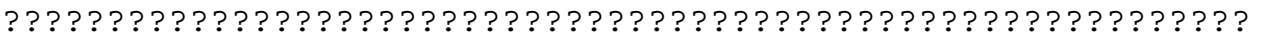
? ? ? ? ? ? ? ? ? ? ? ? ? ? ? ? ? ? ? ? ? ? ? ? ? ? ? ? ? ? ? ? ? ? ? ? ? ? ? ? ? ? ? ? ? ? ? ? ? ? ? ? ? ? ? ? ? ? ? ? ? ? ? ? ? ? ? ? ? ? ? ? ? ? ? ? ? ? ? ? ว? ? ? ? ? ? ? ? ? ? ? ? ? ? ? ? ? ? ? ? ? ? ? ? ? ? ? ? ? ? ? ? ? ? ? ? ? ? ? ? ? ? ? ? ? ? ? ? ? ? ? ? ? ? ? ? ? ? ? ? ? ? ? ? ? ? ? ? ? ? ? ? ? ? ? ? ? ? ? ? ? ? ? ? ? ? ? ?

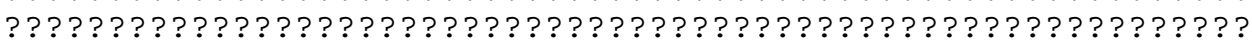

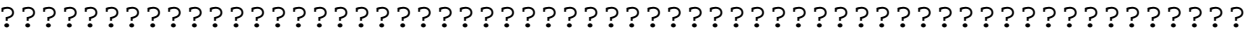
? ? ? ? ? ? ? ? ? ? ? ? ? ? ? ? ? ? ? ? ? ? ? ? ? ? ? ? ? ? ? ? ? ? ? ? ? ? ? ?

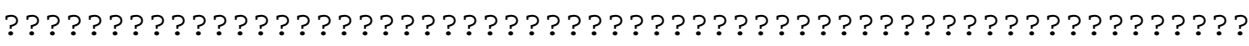
? ? ? ? ? ? ? ? ? ? ? ? ? ? ? ? ? ? ? ? ? ? ? ? ? ? ? ? ? ? ? ? ? ? ? ? ? ? ? ? ? ? ? ? ?

AACCTGGTTGATTGTGTGAGTAGTGATATGGTGTGATTAAAGAGTAAGGGATGC-ATGTG ? ? ? ? ? ? ? ? ? ? ? ? ? ? ? ? ? ? ? ? ? ? ? ? ? ? ? ? ? ? ? ? ? ? ? ? ? ? ? ? ? ? ? ? ?

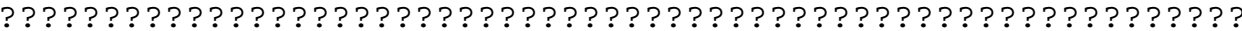

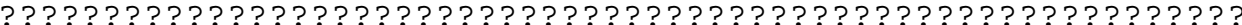

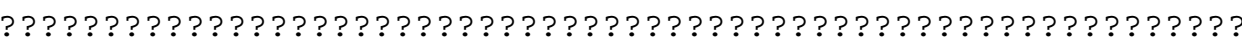
?? ? ? ? ? ? ? ? ? ? ? ? ? ? ? ? ? ? ? ? ? ? ? ? ? ? ? ? ? ? ? ? ? ? ? ? ? ? ? ? ? ? ? ?? ?? ? ? ? ? ? ? ? ? ? ? ? ? ? ? ? ? ? ? ? ? ? ? ? ? ? ? ? ? ? ? ? ? ? ? ? ? ? ? 
[

M._varians_CHMP7_FFP

S._Cf._bros̄chii_ $\overline{G B}$

A._islāndica_CHM̄P1_MICH

A._skvortzowi

A._baicalensis

A. sub_9D1

A._sub_WLB3

A. $-s p \cdot{ }^{3}$ FR

A._sub_E $\mathrm{R} B$

A. Sub_LEW

A._alpigena

A._amb_PII 7

A. $a m b$ N17

A._amb_FL8

A. ${ }^{a m b}$ BDL1

A. $a m b$ WLP1

A._nyassensis_GB

A._valida_N2

A. -gran LB

A. gran OR12

A. $\mathrm{sp} \cdot \overline{1}$

A. $\operatorname{sp} .2$

A._cren_FH8_L639

? ? ? ? ? ? ? ? ? ? ? ? ? ? ? ? ? ? ? ? ? ? ? ? ? ? ? ? ? ? ? ? ? ? ? ? ? ? ? ? ? ? ? ?

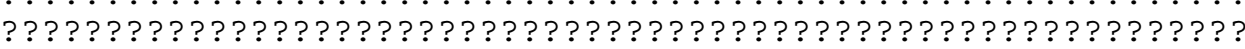
? ? ? ? ? ? ? ? ? ? ? ? ? ? ? ? ? ? ? ? ? ? ? ? ? ? ? ? ? ? ? ? ? ? ? ? ? ? ? ? ? ? ? ?

TAAGTATAAGAACT - TTCGAGACTGCGAATGGCTCATTATATCAGTTATAGTTTATTTGA TAAGTATAAGAACT - TTCGAGACTGCGAATGGCTCATTATATCAGTTATAGTTTATTTGA

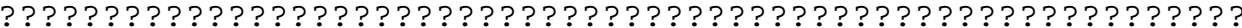

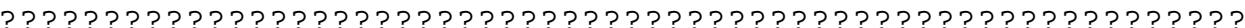

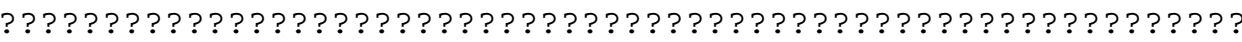

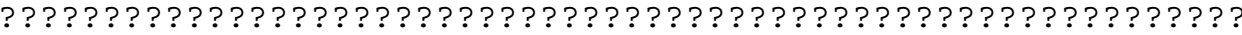

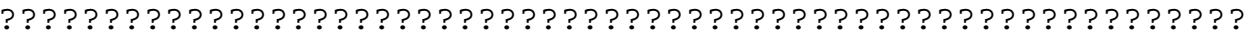
? ? ? ? ? ? ? ? ? ? ? ? ? ? ? ? ? ? ? ? ? ? ? ? ? ? ? ? ? ? ? ? ? ? ? ? ? ? ? ? ? ? ? ? ? ? ?

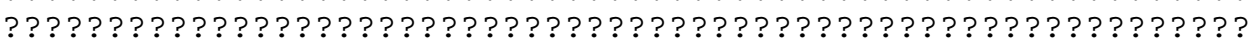
?? ?? ? ? ? ? ? ? ? ? ? ? ? ? ? ? ? ? ? ? ? ? ? ? ? ? ? ? ? ? ? ? ? ? ? ? ? ? ? ? ? ? ? ว? ? ? ? ? ? ? ? ? ? ? ? ? ? ? ? ? ? ? ? ? ? ? ? ? ? ? ? ? ? ? ? ? ? ? ? ? ? ?

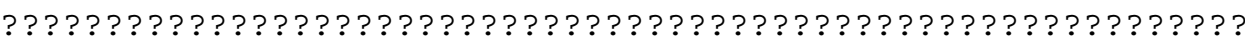
?? ? ? ? ? ? ? ? ? ? ? ? ? ? ? ? ? ? ? ? ? ? ? ? ? ? ? ? ? ? ? ? ? ? ? ? ? ? ? ? ? ? ?

TAAGTATAAGAAGTACTCGAGACTGTGAATGGCTCATTATATCAGTTATAGTTTATTTGA

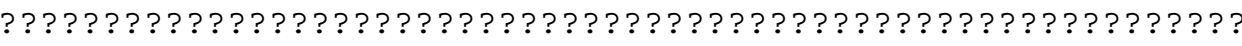

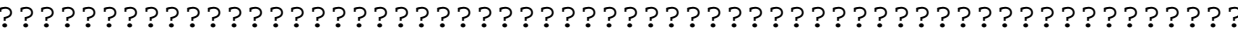
? ? ? ? ? ? ? ? ? ? ? ? ? ? ? ? ? ? ? ? ? ? ? ? ? ? ? ? ? ? ? ? ? ? ? ? ? ? ? ? ? ? ?

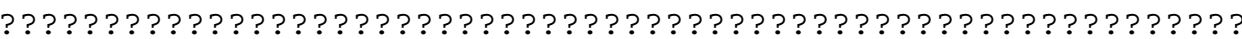

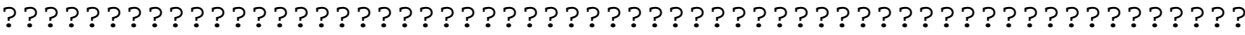
?? ?? ? ? ? ? ? ? ? ? ? ? ? ? ? ? ? ? ? ? ? ? ? ? ? ? ? ? ? ? ? ? ? ? ? ? ? ? ? ? 
[

M._varians_CHMP7_FFP

S._Cf. broschii GB

A._islāndica_CHM̄P1_MICH

A._skvortzowi

A._baicalensis

A._sub_9D1

A._sub_WLB3

A. $-s p \cdot{ }^{3}$ FR

A._sub_E $\mathrm{R} B$

A. sub_LEW

A._alpigena

A._amb_PII 7

A. $a m b$ N17

A._amb_FL8

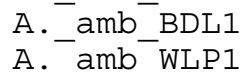

A._nyassensis_GB

A._valida_N2

A. -gran LB

A. gran OR12

A. $\mathrm{sp} \cdot \overline{1}$

A. sp. 2

A._cren_FH8_L639
130

140

150

160

170

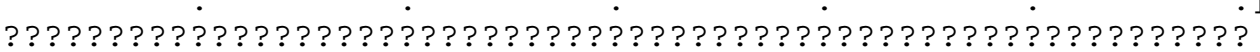

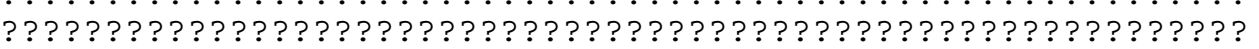
? ? ? ? ? ? ? ? ? ? ? ? ? ? ? ? ? ? ? ? ? ? ? ? ? ? ? ? ? ? ? ? ? ? ? ? ? ? ? ? ? ? ? ? ? ? ? ?

TAGTCCCTTACTACTTGGATAACCGTAGTAATTCTAGAGCTAATACATGCGTCAAGATCC TAGTCCCTTACTACTTGGATTACCGTAGTAATTCTAGAGCTAATACATGCGTCAAGATCC

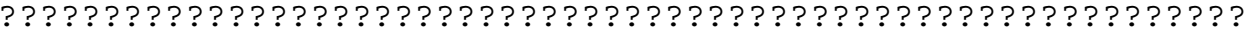

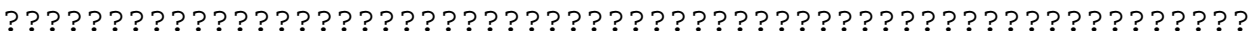

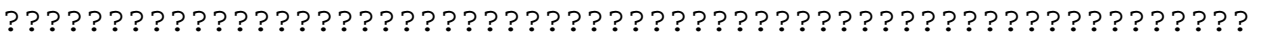

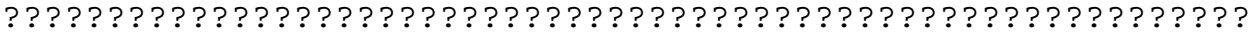
ใ? ? ? ? ? ? ? ? ? ? ? ? ? ? ? ? ? ? ? ? ? ? ? ? ? ? ? ? ? ? ? ? ? ? ? ? ? ? ? ? ? ? ? ? ? ? ? ? ? ? ? ? ? ? ? ? ? ? ? ? ? ? ? ? ? ? ? ? ? ? ? ? ? ? ? ? ? ? ? ? ? ? ? ? ? ? ? ?

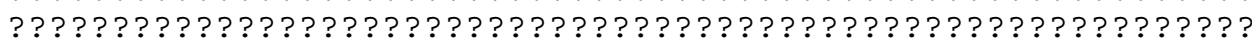
?? ?? ?? ? ? ? ? ? ? ? ? ? ? ? ? ? ? ? ? ? ? ? ? ? ? ? ? ? ? ? ? ? ? ? ? ? ? ? ? ? ? ว? ? ? ? ? ? ? ? ? ? ? ? ? ? ? ? ? ? ? ? ? ? ? ? ? ? ? ? ? ? ? ? ? ? ? ? ? ? ?

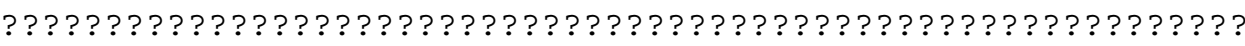
? ? ? ? ? ? ? ? ? ? ? ? ? ? ? ? ? ? ? ? ? ? ? ? ? ? ? ? ? ? ? ? ? ? ? ? ? ? ? ? ? ? ? ?

TAGTCCCTTACTACTTGGATAACCGTAGTAATTCTAGAGCTAATACATGCGTCAAGATCC

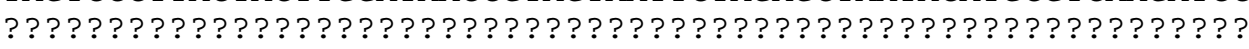

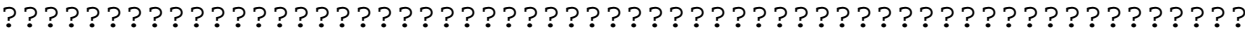

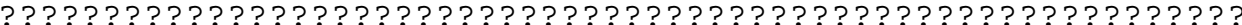

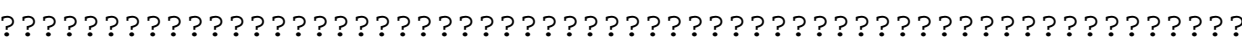

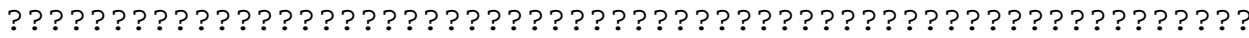
? ? ? ? ? ? ? ? ? ? ? ? ? ? ? ? ? ? ? ? ? ? ? ? ? ? ? ? ? ? ? ? ? ? ? ? ? ? ? ? 
[

M._varians_CHMP7_FFP

S._Cf. broschii GB

A._islāndica_CHM̄P1_MICH

A._skvortzowi

A._baicalensis

A._sub_9D1

A._sub_WLB3

A. $-s p \cdot{ }^{3}$ FR

A._sub_E $\mathrm{R} B$

A. sub_LEW

A._alpigena

A._amb_PII 7

A. $a m b$ N17

A._amb_FL8

A. $a m b$ BDL1

A. $a m b$ WLP1

A._nyassensis_GB

A._valida_N2

A. -gran LB

A. gran OR12

A. $\mathrm{sp} \cdot \overline{1}$

A. sp. 2

A._cren_FH8_L639
190

200

210

220

230

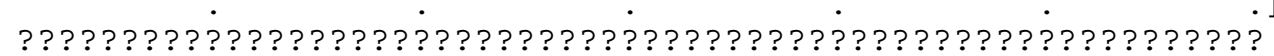

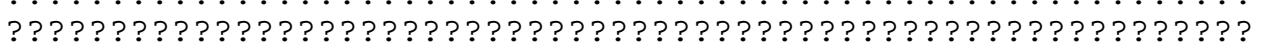
? ? ? ? ? ? ? ? ? ? ? ? ? ? ? ? ? ? ? ? ? ? ? ? ? ? ? ? ? ? ? ? ? ? ? ? ? ? ? ? ? ? ? ? ? ? ? ?

GACTTCGGGAGGATCGTATTTATTAGGTTACAAAACCAACCCTCTTTCGGGAGGTTCTCT GACTTCGGGAGGATCGTATTTATTAGGTTACAAAACCAACCCTCTTTCGGGAGGTTCTCT

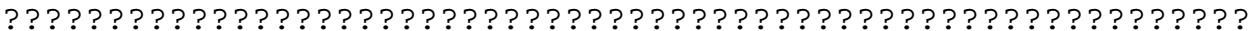

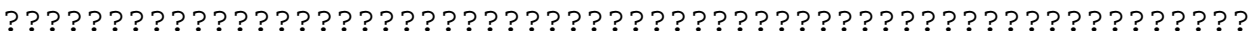

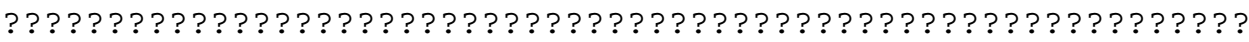

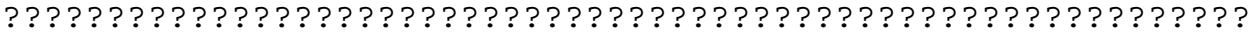

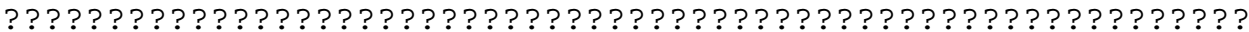
? ? ? ? ? ? ? ? ? ? ? ? ? ? ? ? ? ? ? ? ? ? ? ? ? ? ? ? ? ? ? ? ? ? ? ? ? ? ? ? ? ? ? ? ? ? ? ? ? ? ? ? ? ? ? ? ? ? ? ? ? ? ? ? ? ? ? ? ? ? ? ? ? ? ? ? ? ? ? ? ? ? ? ? ? ? ? ?? ?? ?? ? ? ? ? ? ? ? ? ? ? ? ? ? ? ? ? ? ? ? ? ? ? ? ? ? ? ? ? ? ? ? ? ? ? ? ? ? ? ว? ? ? ? ? ? ? ? ? ? ? ? ? ? ? ? ? ? ? ? ? ? ? ? ? ? ? ? ? ? ? ? ? ? ? ? ? ? ?

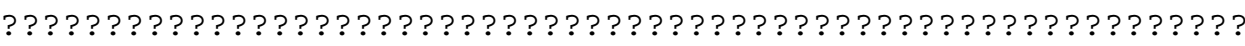
? ? ? ? ? ? ? ? ? ? ? ? ? ? ? ? ? ? ? ? ? ? ? ? ? ? ? ? ? ? ? ? ? ? ? ? ? ? ? ? ? ? ? ?

GACTTCGGGAGGATTGTATTTATTAGGTTACAAAGCCAACCCTCTCTCGGGAGGTAGTTT

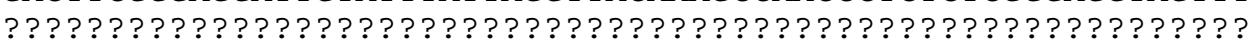

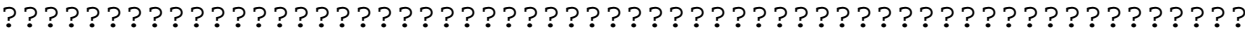

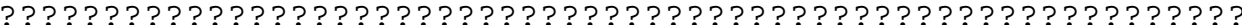

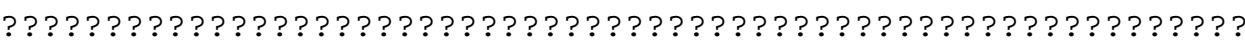

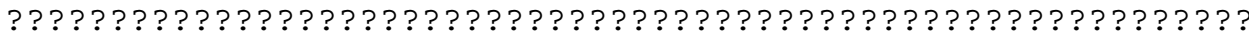
? ? ? ? ? ? ? ? ? ? ? ? ? ? ? ? ? ? ? ? ? ? ? ? ? ? ? ? ? ? ? ? ? ? ? ? ? ? ? ? ? 
[

M._varians_CHMP7_FFP

S._Cf. broschii GB

A._islāndica_CHM̄P1_MICH

A._skvortzowi

A._baicalensis

A. sub_9D1

A._sub_WLB3

A. $-s p \cdot{ }^{3}$ FR

A._sub_E $\mathrm{R} B$

A. sub_LEW

A._alpigena

A._amb_PII 7

A. $a m b$ N17

A._amb_FL8

A. $a m b$ BDL1

A. $a m b$ WLP1

A._nyas̄sensis_GB

A._valida_N2

A. -gran LB

A. gran OR12

A. $\mathrm{sp} \cdot \overline{1}$

A. sp. 2

A._cren_FH8_L639

250

260

270

280

290

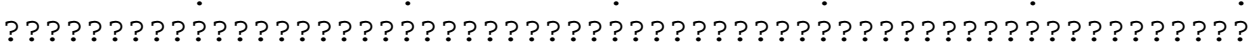

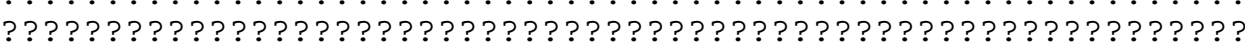
? ? ? ? ? ? ? ? ? ? ? ? ? ? ? ? ? ? ? ? ? ? ? ? ? ? ? ? ? ? ? ? ? ? ? ? ? ? ? ? ? ? ? ?

GGGTGAGTCATGATAAGCATGCGGATCGCACGGCTTTATGGCCGGCGACGGATCATGTAA GGGTGAGTCATGATAAGCATGCGCATCGCACGGCTTTATGGC- - GCATCGGATCAGGTAA ?? ?? ? ? ? ? ? ? ? ? ? ? ? ? ? ? ? ? ? ? ? ? ? ? ? ? ? ? ? ? ? ? ? ? ? ? ? ? ? ? ? ?

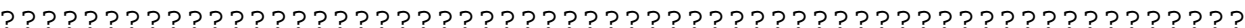

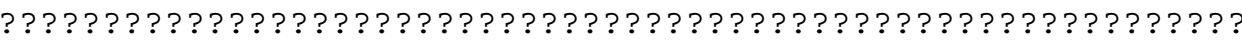

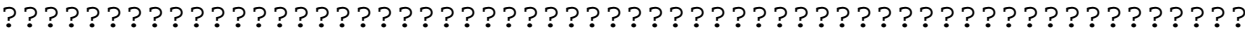

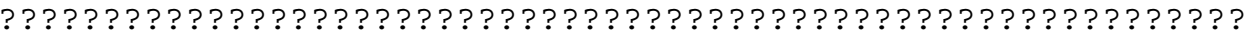
? ? ? ? ? ? ? ? ? ? ? ? ? ? ? ? ? ? ? ? ? ? ? ? ? ? ? ? ? ? ? ? ? ? ? ? ? ? ? ? ? ? ? ? ? ? ?

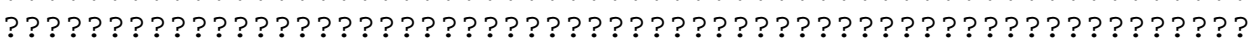

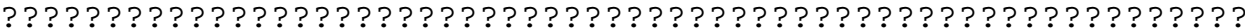
ว? ? ? ? ? ? ? ? ? ? ? ? ? ? ? ? ? ? ? ? ? ? ? ? ? ? ? ? ? ? ? ? ? ? ? ? ? ? ?

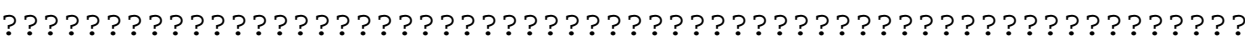
?? ? ? ? ? ? ? ? ? ? ? ? ? ? ? ? ? ? ? ? ? ? ? ? ? ? ? ? ? ? ? ? ? ? ? ? ? ? ? ? ? ? ? ?

GGGTGAGTCATGATAAGCATGCGGATCGCAGGGCTTTACGGCCGGCGACGGATCATGTAA

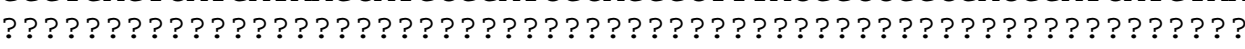

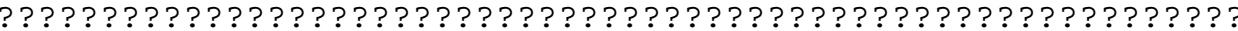
ใ? ? ? ? ? ? ? ? ? ? ? ? ? ? ? ? ? ? ? ? ? ? ? ? ? ? ? ? ? ? ? ? ? ? ? ? ? ? ? ? ? ?

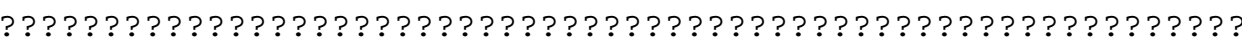
?? ? ? ? ? ? ? ? ? ? ? ? ? ? ? ? ? ? ? ? ? ? ? ? ? ? ? ? ? ? ? ? ? ? ? ? ? ? ? ? ? ? ? ? ? ? ? ? ? ? ? ? ? ? ? ? ? ? ? ? ? ? ? ? ? ? ? ? ? ? ? ? ? ? ? ? ? ? ? ? ? ? ? 
[

M._varians_CHMP7_FFP

S._Cf. broschii GB

A._islāndica_CHM̄P1_MICH

A._skvortzowi

A._baicalensis

A._sub_9D1

A._sub_WLB3

A. $-s p \cdot{ }^{3}$ FR

A._sub_E $\mathrm{R} B$

A. Sub_LEW

A._alpigena

A._amb_PII 7

A. $a m b$ N17

A._amb_FL8

A. $a m b$ BDL1

A. $a m b$ WLP1

A._nyas̄sensis_GB

A._valida_N2

A. -gran LB

A. gran OR12

A. $\mathrm{sp} \cdot \overline{1}$

A. sp. 2

A._cren_FH8_L639

310

320

330

340

350

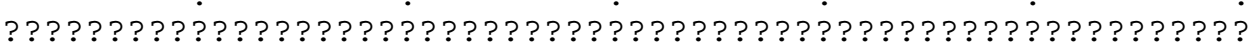

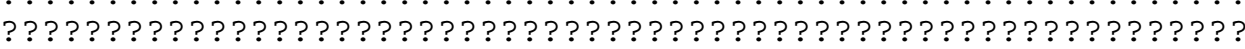
? ? ? ? ? ? ? ? ? ? ? ? ? ? ? ? ? ? ? ? ? ? ? ? ? ? ? ? ? ? ? ? ? ? ? ? ? ? ? ? ? ? ? ?

GTTCCTGCCCCATCAGCTTCGGATGGTAGAGTATTGGCCTACCATGGCGTTAACGGGTAA GTTCCTGCCCTATCAGCTTCGGATGGTAGGGTATTGGCCTACCATGGCGTTAACGGGTAA ?? ?? ? ? ? ? ? ? ? ? ? ? ? ? ? ? ? ? ? ? ? ? ? ? ? ? ? ? ? ? ? ? ? ? ? ? ? ? ? ? ? ?

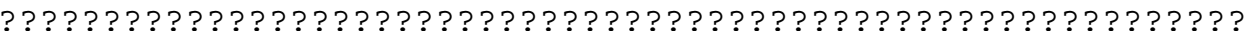

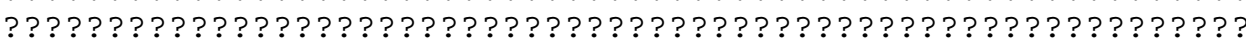

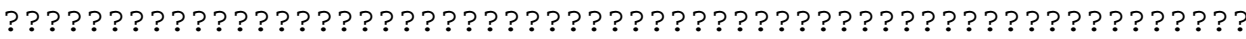

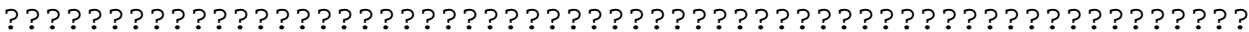
? ? ? ? ? ? ? ? ? ? ? ? ? ? ? ? ? ? ? ? ? ? ? ? ? ? ? ? ? ? ? ? ? ? ? ? ? ? ? ? ? ? ? ? ? ? ?

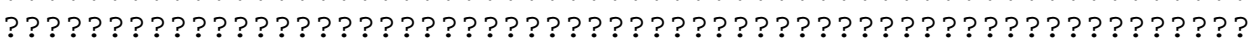

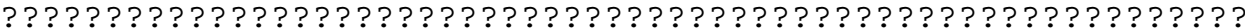
ว? ? ? ? ? ? ? ? ? ? ? ? ? ? ? ? ? ? ? ? ? ? ? ? ? ? ? ? ? ? ? ? ? ? ? ? ? ? ?

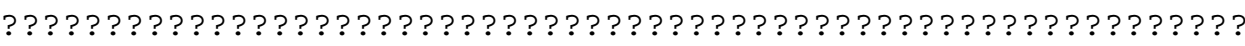
?? ? ? ? ? ? ? ? ? ? ? ? ? ? ? ? ? ? ? ? ? ? ? ? ? ? ? ? ? ? ? ? ? ? ? ? ? ? ? ? ? ? ? ?

GCTCCTGCCCTATCAGCTTCGGATGGTAGGGTATTGGCCTACCATGGCATTAACGGGTAA

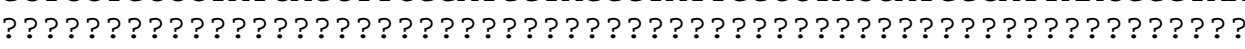

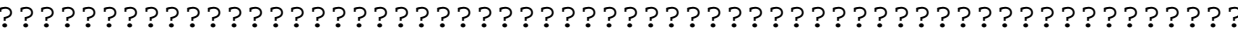

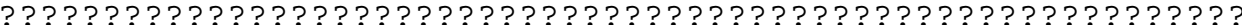

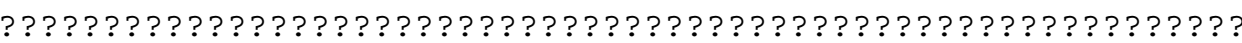

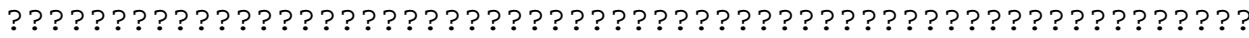
? ? ? ? ? ? ? ? ? ? ? ? ? ? ? ? ? ? ? ? ? ? ? ? ? ? ? ? ? ? ? ? ? ? ? ? ? ? ? ? 
[

M._varians_CHMP7_FFP

S._Cf. broschii GB

A._islāndica_CHM̄P1_MICH

A._skvortzowi

A._baicalensis

A._sub_9D1

A._sub_WLB3

A. $-s p \cdot{ }^{3}$ FR

A._sub_E $\mathrm{R} B$

A. Sub_LEW

A._alpigena

A._amb_PII 7

A. $a m b$ N17

A._amb_FL8

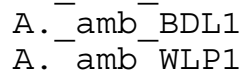

A._nyas̄sensis_GB

A._valida_N2

A. -gran LB

A. gran OR12

A. $\mathrm{sp} \cdot \overline{1}$

A. sp. 2

A._cren_FH8_L639
370

380

390

400

410

? ? ? ? ? ? ? ? ? ? ? ? ? ? ? ? ? ? ? ? ? ? ? ? ? ? ? ? ? ? ? ? ? ? ? ? ? ? ? ? ? ? ? ? ?

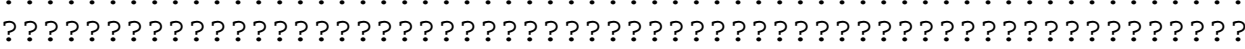
? ? ? ? ? ? ? ? ? ? ? ? ? ? ? ? ? ? ? ? ? ? ? ? ? ? ? ? ? ? ? ? ? ? ? ? ? ? ? ? ? ? ? ?

CGGGAGATCAGGGTTTGACTCCGGAGAGGGAGCCTGAGAGACGGCTACCACATCCAAGGA CGGGAGATCAGGGTTTGACTCCGGAGAGGGAGCCTGAGAGACGGCTACCACATCCAAGGA ? ? ? ? ? ? ? ? ? ? ? ? ? ? ? ? ? ? ? ? ? ? ? ? ? ? ? ? ? ? ? ? ? ? ? ? ? ? ? ? ? ? ? ว?ว? ? ? ? ? ? ? ? ? ? ? ? ? ? ? ? ? ? ? ? ? ? ? ? ? ? ? ? ? ? ? ? ? ? ? ? ?

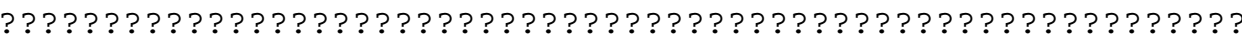

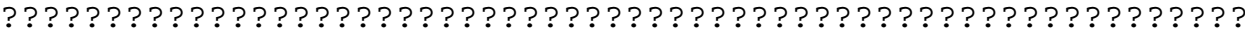

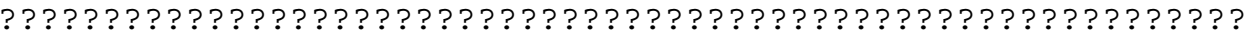
? ? ? ? ? ? ? ? ? ? ? ? ? ? ? ? ? ? ? ? ? ? ? ? ? ? ? ? ? ? ? ? ? ? ? ? ? ? ? ? ? ? ? ? ? ? ?

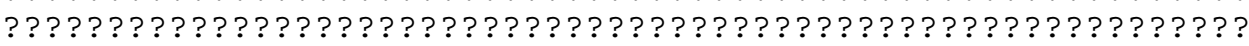

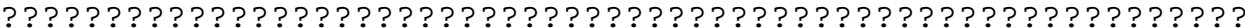
ว? ? ? ? ? ? ? ? ? ? ? ? ? ? ? ? ? ? ? ? ? ? ? ? ? ? ? ? ? ? ? ? ? ? ? ? ? ? ?

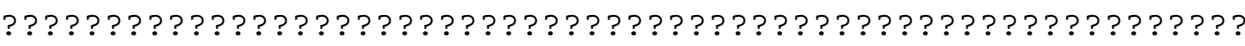
?? ? ? ? ? ? ? ? ? ? ? ? ? ? ? ? ? ? ? ? ? ? ? ? ? ? ? ? ? ? ? ? ? ? ? ? ? ? ? ? ? ? ? ?

CGGGAGATCAGGGTTTGACTCCGGAGAGGGAGCCTGAGAGACGGCTACCACATCCAAGGA ? ? ? ? ? ? ? ? ? ? ? ? ? ? ? ? ? ? ? ? ? ? ? ? ? ? ? ? ? ? ? ? ? ? ? ? ? ? ? ? ? ?

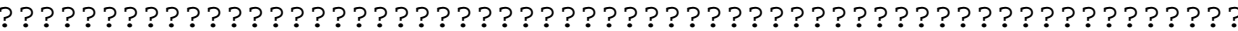

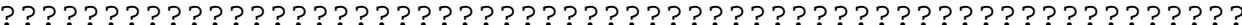

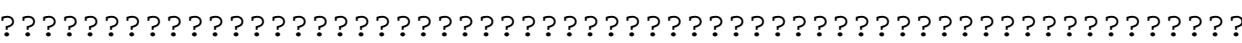

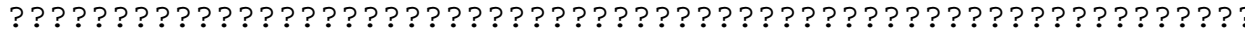
?? ?? ? ? ? ? ? ? ? ? ? ? ? ? ? ? ? ? ? ? ? ? ? ? ? ? ? ? ? ? ? ? ? ? ? ? ? ? ? ? 
[

M._varians_CHMP7_FFP

S._Cf._bros̄chii_GB

A. islāndica CHM̄P1 MICH

A._skvortzowi

A._baicalensis

A. sub_9D1

A._sub_WLB3

A. $-s p \cdot{ }^{3}$ FR

A._sub_E $\mathrm{R} B$

A. Sub LEW

A._alpigena

A._amb_PII 7

A. $a m b$ N17

A._amb_FL8

A. $a m b$ BDL1

A. $a m b$ WLP1

A._nyassensis_GB

A._valida_N2

A. -gran_LB

A. gran OR12

A. $\mathrm{sp} \cdot \overline{1}$

A. sp. 2

A._cren_FH8_L639
430

440

450

460

470

]

?? ? ? ? ? ? ? ? ? ? ? ? ? ? ? ? ? ? ? ? ? ? ? ? ? ? ? ? ? ? ? ? ? ? ? ? ? ? ? ? ? ? ?AATAACA

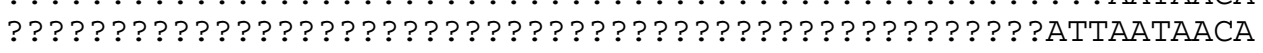
? ? ? ? ? ? ? ? ? ? ? ? ? ? ? ? ? ? ? ? ? ? ? ? ? ? ? ? ? ? ? ? ? ? ? ? ? ? ? ? ? ? ? ? ? ? ? ?

AGGCAGCAGGCGCGTAAATTACCCAATCCTGATACAGGGAGGTAGTGACGATAAATAACA AGGCAGCAGGCGCGTAAATTACCCAATCCTGATACAGGGAGGTAGTGACGATAAATAACA

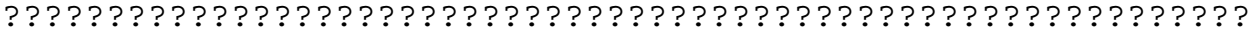

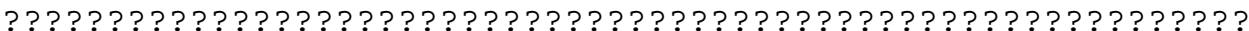
? ? ? ? ? ? ? ? ? ? ? ? ? ? ? ? ? ? ? ? ? ? ? ? ? ? ? ? ? ? ? ? ? ? ? ? ? ? ? ? ? ? ?

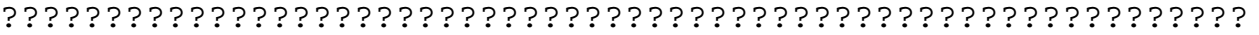

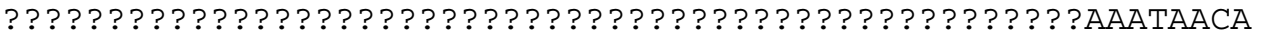

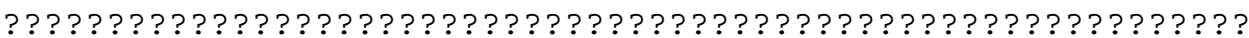
?? ?? ?? ? ? ? ? ? ? ? ? ? ? ? ? ? ? ? ? ? ? ? ? ? ? ? ? ? ? ? ? ? ? ? ? ? ? ? ? ?? ?? ?? ? ? ? ? ? ? ? ? ? ? ? ? ? ? ? ? ? ? ? ? ? ? ? ? ? ? ? ? ? ? ? ? ? ? ? ? ? ? ว? ? ? ? ? ? ? ? ? ? ? ? ? ? ? ? ? ? ? ? ? ? ? ? ? ? ? ? ? ? ? ? ? ? ? ? ? ? ?

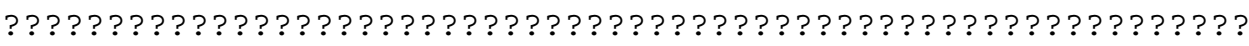
? ? ? ? ? ? ? ? ? ? ? ? ? ? ? ? ? ? ? ? ? ? ? ? ? ? ? ? ? ? ? ? ? ? ? ? ? ? ? ? ? ? ? ?

AGCAGGCAGGCGCGTAAATTACCCAATCCTGACACAGGGAGGTAGTGACGATAAATAACA

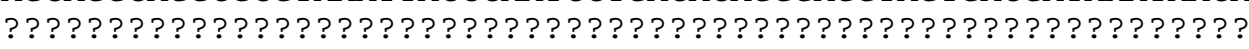

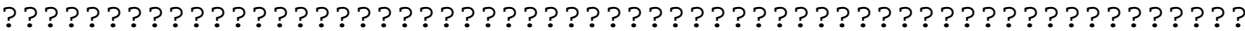

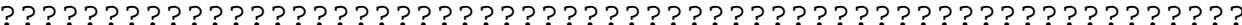

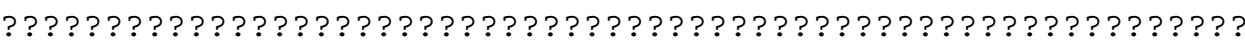

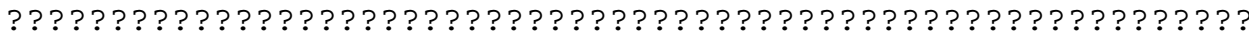
?? ?? ?? ? ? ? ? ? ? ? ? ? ? ? ? ? ? ? ? ? ? ? ? ? ? ? ? ? ? ? ? ? ? ? ? ? ? ? 
[

M._varians_CHMP7_FFP

S._Cf._bros̄chii GB

A. islāndica $\mathrm{CHM} P 1 \mathrm{MICH}$

A._skvortzowi

A._baicalensis

A._sub_9D1

A._sub_WLB3

A. $-\mathrm{sp} \cdot{ }^{3} \mathrm{FR}$

A. sub LEW

A._alpigena

A._amb_PII 7

A. $a m b$ N17

A._amb_FL8

A. $a m b$ BDL1

A. $a m b$ WLP1

A._nyas̄sensis_GB

A._valida_N2

A. -gran LB

A. gran OR12

A. $-\mathrm{sp} \cdot \overline{\overline{1}}$

A. $\operatorname{sp} .2$

A._cren_FH8_L639
490

500

510

520

530

]

ATGTCGGGCCTTTTCAGG - - - - TCTGACÄATGGAATGÄGAACGATGTÄAACATCCTAT ATGCCGGGCCTTTTCAGG- - - - TCTGGCAATTGGAATGAGAACGATGTAAACACCCTAT ? ? ? ? ? ? ? ? ? ? ? ? ? ? ? ? ? ? ? ? ? ? ? ? ? ? ? ? ? ? ? ? ? ? ? ? ? ? ? ? ? ? ? ? ? ?

ATGCCGGGCCTTTTTAGG - - - - TCTGGCAATTGGAATGAGAACGATGCAAATACCCTAT ATGCCGGGCCTTTTTAGG - - - - TCTGGCAATTGGAATGAGAACGATGCAAATACCCTAT

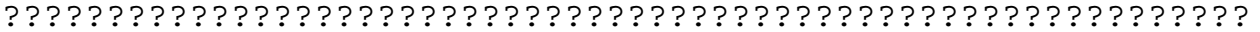
ว? ? ? ? ? ? ? ? ? ? ? ? ? ? ? ? ? ? ? ? ? ? ? ? ? ? ? ? ? ? ? ? ? ? ? ? ? ? ? ? ? ? ? ? ? ? ? ? ? ? ? ? ? ? ? ? ? ? ? ? ? ? ? ? ? ? ? ? ? ? ? ? ? ? ? ? ? ? ? ? ? ? ? ? ? ? ? ? ATGCCGGGCCTTTCTAGG - - - - TCTGGCAATTGGAATGAGAACGATGCAAACACCCTAT ? ? ? ? ? ? ? ? ? ? ? ? ? ? ? ? ? ? ? ? ? ? ? ? ? ? ? ? ? ? ? ? ? ? ? ? ? ? ? ? ? ? ? ? ?? ?? ? ? ? ? ? ? ? ? ? ? ? ? ? ? ? ? ? ? ? ? ? ? ? ? ? ? ? ? ? ? ? ? ? ? ? ? ? ?

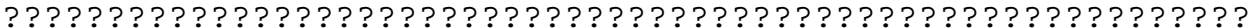
ว? ? ? ? ? ? ? ? ? ? ? ? ? ? ? ? ? ? ? ? ? ? ? ? ? ? ? ? ? ? ? ? ? ? ? ? ? ? ?

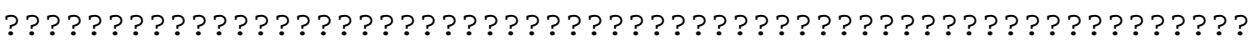
? ? ? ? ? ? ? ? ? ? ? ? ? ? ? ? ? ? ? ? ? ? ? ? ? ? ? ? ? ? ? ? ? ? ? ? ? ? ? ? ? ? ? ? ATGCAGGGACTTTGTAGGCTCTGGCAATTGGAATGCAGCAACGATGCAAACACCCTACTC ? ? ? ? ? ? ? ? ? ? ? ? ? ? ? ? ? ? ? ? ? ? ? ? ? ? ? ? ? ? ? ? ? ? ? ? ? ? ? ? ? ?

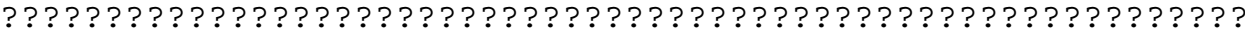

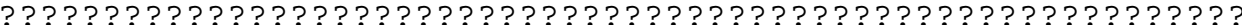

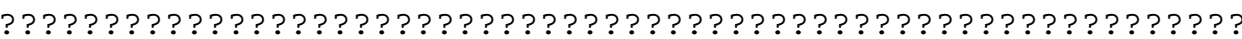

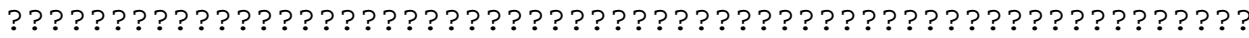
? ?? ? ? ? ? ? ? ? ? ? ? ? ? ? ? ? ? ? ? ? ? ? ? ? ? ? ? ? ? ? ? ? ? ? ? ? ? ? ? ? 
CAAGGATCAATTGGAGGGCÄAGTCTGGTGCCAGCAGCCGC-GGTAATTCCAGCTCCAATA CGAGGACCAATTGGAGGGCAAGTCTGGTGCCAGCAGCCGC-GGTAATTCCAGCTCCAATA ? ? ? ? ? ? ? ? ? ? ? ? ? ? ? ? ? ? ? ? ? ? ? ? ? ? CAGCCGC-GGTAATTCCAGCTCCAATA CGAGGATCAATTGGAGGGCAAGTCTGGTGCCAGCAGCCGC-GGTAATTCCAGCTCCAATA CGAGGATCAATTGGAGGGCAAGTCTGGTGCCAGCAGCCGC-GGTAATTCCAGCTCCAATA

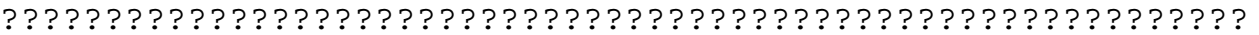
? ? ? ? ? ? ? ? ? ? ? ? ? ? ? ? ?AAGTCTGGTGCCAGCAACCGC-GGTAATTCCAGCTCCAATA ? ? ? ? ? ? ? ? ? ? ? ? ? GGGCAAGTCTGGTGCCAGCAGCCGC-GGTAATTCCAGCTCCAATA ? ? ? ? ? ? ? ? ? ? ? ? AGGGCAAGTCTGGTGCCAGCAGCCGC-GGTAATTCCAGCTCCAATA CGAGGATCAATTGGAGGGCAAGTCTGGTGCCAGCAGCCGC-GGTAATTCCAGCTCCAATA ? ? ? ? ? ? ? ? ? ? ? ? ? ? ? ? ? ? ? ? GTGCCAGCAAGCCGCGGTAATTCCAGCTCCAATA ? ? ? ? ? ? ? ? ? ? ? GGGCAAGTCTGGTGCCAGCAGCCGC-GGTAATTCCAGCTCCAATA ? ? ? ? ? ? ? ? ? ? ? ? GGGCAAGTCTGGTGCCAGCAGCCGC-GGTAATTCCAGCTCCAATA ? ? ? ? ? ? ? ? ? TTGGAGGGCAAGTCTGGTGCCAGCAGCCGC-GGTAATTCCAGCTCCAATA ? ? ? ? ? ? ? ? ? ? ? ? ? ? ? ? ? ? TCTGGTGCCAGCAGCCGC-GGTAATTCCAGCTCCAATA

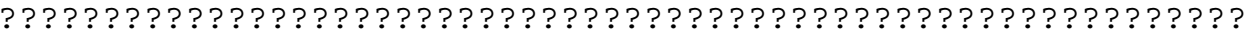
GAGGCATCAATTTGAGGGCAAGTCTAGTGCCAGCAGCCGC-GGTAATTCCAACTCCAATA ? ? ? ? ? ? ?ATTGGAGGGCAAGTCTGGTGCCAGCAGCCGC-GGTAATTCCAGCTCCAATA ? ? ? ? ? ? ? ? ? ? ? ? ? ? ? ? ? ? ? ? ? ? ? TGCCAGCAGCCGC-GGTAATTCAGCCTCCAATA ? ? ? ? ? ? ? ? ? ? ? ? ?GGGCAAGTCTGGTGCCAGCAGCCGC-GGTAATTCCAGCTCCAATA ? ? ? ? ? ? ? ? ? ? ? ? ? ? ? CAAGTCTGGTGCCAGCAGCCGC-GGTAATTCCAGCTCCAATA ? ? ? ? ? ? CAATTGGAGGGCAAGTCTGGTGCCAGCAGCCAC-AGTAATTCCAGCTCCAATA ? ? ? ? ? ? ? ? ? ? ? ? ? ? ? ? ? ? ? ? ? ? GCCAGCAGCCGC-GGTAATTCCAGCTCCAATA 
A._sub_WLB3

A. $s p .{ }^{3}$ FR

A. S sub_ERB

A. sub_LEW

A._alpigena

A. amb_PII 7

A. amb N17

A._amb_FL8

A._amb_BDL1

A. $a m b$ WLP1

A._nyas̄sensis_GB

A._valida_N2

A. $\operatorname{gran} L \bar{B}$

A._gran_oR12

A. $-\mathrm{sp} \cdot \overline{\overline{1}}$

A._creñ_FH8_L639

GC-GTATATTAAA-GTTGTTGCAGTT- - AAAAAAGCTCGTAGTTGAATTTTAGGTTGTGT GC-GTATATTAAA-GTTGTTGCAGTT- -AAAAAGCTCGTAGTTGAATTTCTGGCGGGAG GC-GTATATTAAA-GTTGTTGCAGTT - -AAAAAGCTCGTAGTTGAATTTCTGGTGGAGT SC-GTATATTAAA-GTTGTTGCAGTT - -AAAAAGCTCGTAGTTGAATTTCTGGTGGAGT GC-GTATATTAAA-GTTGTTGCAGTT- -AAAAAGCTCGTAGTTGAATTTCTGGTGGAGT ? ? ? ? ? ? ? ? ? ? ? ? ? ? ? ? ? ? ? ? ? ? ? ? ? ? ? ? ? ? ? ? ? ? ? ? ? ? ? ? ? ? ? ? ? GC-GTATATTAAA - GTTGTNGCAGTT - -AAAAAGCTCGTAGTTGAATTTCTGGTGGAGT GC-GTATATTAAA-GTTGTTGCAGTT - -AAAAAGCTCGTAGTTGAATTTCTGGTGGAGT GC-GTATATTAAA-GTTGTTGCAGTT- -AAAAAGCTCGTAGTTGAATTTCTGGTGGAGT GCCGTATATTAAA - GTTGTTGCAGTT - -AAAAAGCTCGTAGTTGAATTTCTGGTGGAGT GGCGTATATTAAAGGTTGTTGCAAGTTAAAAAAGCTCGTAGTTGAATTTCTGGTGGAGT GC-GTATATTAAA - GTTGTTGCAGTT - -AAAAAGCTCGTAGTTGAATTTCTGGTGGAGT GC - GTATATTAAA - GTTGTTGCAGTT - -AAAAAGCTCGTAGTTGAATTTCTGGTGGAGT GC-GTATATTAAA - GTTGTTGCAGTT - -AAAAAGCTCGTAGTTGAATTTCTGGTGGAGT GC-GTATATTAAA-GTTGTTGCAGTT- -AAAAAGCTCGTAGTTGAATTTCTGGTGGAGT ? ? ? ? ? ? ? ? ? ? A-GTTGTTGCAGTT - -AAAAAGCTCGTAGTTGAATTTCTGGTGGAGT GC-GTATATTAAA-GTTGCTGCAGTT-AGAAAAGCTCGTAGTTGGATTTCTGGTGGAGT GC-GTATATTAAA-GTTGTTGCAGTT - -AAAAAGCTCGTAGTTGAATTTCTGGTAGAGT GC-GTATATTAAA - GTTGTTGCAGTTT-AAAAAGCTCGTAGTTGAATTTCTGGTGGAGT GC-GTATATTAAA - GTTGTTGCAGTT - - AAAAAGCTCGTAGTTGAATTTCTGGTGGAGT GC-GTATATTAAA - GTTGTTGCAGTT - -AAAAAGCTCGTAGTTGAATTTCTGGCGGAGT GC-GTATATTAAA-GTTGTTGCAGTT- - AAAAAGCTCATGGTTGAATTTCTGGCAGAGT GC-GTATATTAAA-GTTGTTGCAGTT- -AAAAAGCTCGTAGTTGAATTTTTGGTGGAGC 
- GGCTTATTCT-CACATTGTGAGAAAGAGTCGTTTAGCCTTTAATGATTAATGATAAAACAACCGGTCCTTCACTTGGTGTTGGTACTTGTGT-TGTCTCTGGCCATCCTTGGGGAGAG CGGTCTGTCCTTCACTTGGTGTTGGTTCGTGACTGTATCTCTGCCC-TCCTTGGTTGGAG CGGTCTGTCCTTCACTTGGTGTTGGTTCGTGACTGTATCTCTGCCC-TCCTTGGTTGGAG CGGTCTGTCCTTCACTTGGTGTTGGTTCGTGACTGTATCTCTGCCC-TCCTTGGTTGGAG ? ? ? ? ? ? ? ? ? ? ? ? ? ? ? ? ? ? ? ? ? ? ? ? ? ? ? ? ? ? ? ? ? ? ? ? ? ? ? ? ? ? ?

A._sub_9D1

A. sub WLB3

A. $-\mathrm{sp} \cdot{ }^{3}$ FR

A. $-\mathrm{sub}-\mathrm{ER} B$

A. sub LEW

A._alpigena

A._amb_PII 7

A. $a m b-\mathrm{N} 17$

A._amb_FL8

A._amb_BDL1

A. $a m b$ WLP1

A._nyas̄sensis_GB

A._valida_N2

A. gran_LB

A. gran_OR12

A. _sp. $\overline{1}$

A. sp. 2

A. creñ FH8 L639 GGGTCTGTCCTGCACTTGGTGTAGGTTCGTGACT? TGTCTCTGCCC-TCCTTGGCCGGAG GGGTCTGTCCTGCACTTGGTGTAGGTTCGTGACT? TGTCTCTGCCC-TCCTTGGCCGGAG GGGTCTGTCCTGCACTTGGTGTAGGTTCGTGACT? TGTCTCTGCCC - TCCCTGGCCGGAG CGGTCTGTCCTTCACTTGGTGTTGGTTCGTGACTGTATCTCTGCCC-TCCTTGGTTGGAG CGGTCTGTCCTTCACTTGGTGTTGGTACGTGACTGTATCTCTGCCC-TCCTTGGTTGGAT CGGTCTGTCCTTCACTTGGTGTTGGTACGTGACTGTATCTCTGCCC-TCCTTGGTTGGAT CGGTCTGTCCTTCACTTGGTGTTGGTACGTGACTGTATCTCTGCCC-TCCTTGGTTGGAT CGGTCTGTCCTTCACTTGGTGTTGGTACGTGACTGTATCTCTGCCC-TCCTTGGTTGGAT CGGTCTGTCCTTCACTTGGTGTTGGTACGTGACTGTATCTCTGCCC-TCCTTGGTTGGAT CGGTCTGTCCTTCACTTGGTGTTGGTACGTGACTGTATCTCTGCCC-TCCTTGGTTGGAT CGGTCTGTCCTTCACTTGGTGTTGGCACGTGACTGTGTCTCTACCC-TCCTTGGTTGGAC TAGTCTGTCCTTCACTTGGTGTTGGTACGTGACTGTATCTCTGCCC-TCCTTGGTTGGAT TAGTCTGTCCTTCACTTGGTGTTGGTACGTGACTGTATCTCTGCCC-TCCTTGGTTGGAT CGGCCTGTCCTTCACTTGGTGTTGGCACGTGGTTGGTTCTCCGCCC-TCCTTGGTTGGAG TGGCCTGTCCTTCACTTGGTGTTGGCACTTGGTTGGATCTCTGCCC-TCCTTTGCTGGAG CATCCTGTCCTTCACTTGGTGTTGGCACTTGGTTGGTTCTCTGCCC-TCCTTGGCTGGAG 
A. sub_WLB3

A. $s p .{ }^{3}$ FR

A. Sub_ERB

A. sub LEW

A._alpigena

A. amb_PII 7

A. amb N17

A._amb_FL8

A._amb_BDL1

A. $a m b$ WLP1

A._nyas̄sensis_GB

A._valida_N2

A. gran_L $\bar{B}$

A._gran_oR12

A. $\operatorname{sp} \cdot \overline{1}$

A. sp. 2

A._creñ_FH8_L639

- - - - - TAGCATTAATTTGTTGTTTTGTTAGGAATCATCTTTTACTGTGAAGAAATTAG CCTGTG-TGGCATTAAGTTGTCGGGCGGGGGATCCCCATCGTTTACTGTGAAGAAATTAG CCAGTGTTGGCATTCAGTTGTTGGGCTGGGGACAATCATCGTTTACTGTGAAGAAATTAG CCAGTGTTGGCATTCAGTTGTTGGGCTGGGGACAATCATCGTTTACTGTGAAGAAATTAG CCAGTGTTGGCATTCAGTTGTTGGGCTGGGGACAATCATCGTTTACTGTGAAGAAATTAG ? ? ? ? ? ? ? ? ? ? ? ? ? ? ? ? ? ? ? ? ? ? ? ? ? ? ? ? ? ? ? CGTTTACTGTGAAGAAATTAG CCAGTGTTGGCATTAAGTTGTCGGGCTGGGGACGGTCATCGTTTACTGTGAAGAAATTAG CCAGTGTTGGCATTAAGTTGTCGGGCTGGGGACGGTCATCGTTTACTGTGAAGAAATTAG CCAGTGTTGGCATTAAGTTGTCGGGCTGGGGACGGTCATCGTTTACTGTGAAGAAATTAG CCAGTGTTGGCATTAAGTTGTCGGGCTGGGGACGGTCATCGTTTACTGTGAAGAAATTAG CCAGCGTTGGCATTTAGTTGTCGGGCTGGGGACGATCATCGTTTACTGTGAAGAAATTAG CCAGTGCTGGCATTTGGTTGTCGGTCTGGGGACGGCCATCGTTTACTGTGAAGAAATTAG CCAGTGCTGGCATTTGGTTGTCGGTCTGGGGACGGCCATCGTTTACTGTGAAGAAATTAG CCAGTGCTGGCATTTGGTTGTCGGTCTGGGGACGGCCATCGTTTACTGTGAAGAAATTAG CCAGTGCTGGCATTTGGTTGTCGGTCTGGGGACGGCCATCGTTTACTGTGAAGAAATTAG CCAGTGCTGGCATTTGGTTGTCGGTCTGGGGACGGCCATCGTTTACTGTGAAGAAATTAG CTAGTGCTGGCATTTGGTTGTCGGTCTGGGGACGGCCATCGTTTACTGTGAAGAAATTAG CCGGTGCTGGCATTTGGTTGTCGGCCTGGGCACGGCCATCGTTTACTGTGAAGAAATTAG CTGGTGCTGGCATTTGGTTGTCGGCCTGGGGACGGCCATCATTTACTGTGAAGAAATTAG CTGGTGCTGGCATTTGGTTGTCGGCCTGGGGACGGCCATCGTTTACTGTGAAGAAATTAG CCAGCG - TGGCATTTGGTTGTCGGGCTGGGGACGACCATCGTTTACTGTGAAGAAATTAG CCAGTG-TGGCATTTGGGTGTCATGCTGGGGATGGTCATTGTTTACTGTGAAGAAATTAG CTAGCA - TGGCATTTGGTTGTCGTGTTGGGGACGGTCATCTTTTACTGTGAAGAAATTAG 
AGTGTTCAAÄGCAGGCATTGCTG - TGAATACATTAGCATGGAATAATGÄAATAGGACTC AGTGTTCAAAGCAGGCTTATGCCGCTGAATACATTAGCATGGAATAATAAGATAGGACCT AGTGTTCAAAGCAGGCTTAGGCC - TTGAATACATTAGCATGGAATAATGAAATAGGGTTT AGTGTTCAAAGCAGGCTTAGGCC-TTGAATACATTAGCATGGAATAATGAAATAGGGTTT AGTGTTCAAAGCAGGCTTAGGCC - TTGAATACATTAGCATGGAATAATGAAATAGGGTTT AGTGTTCAAAGCAGGCTTAGGCC - TTGAATACATTAGCATGGAATAATGAAATAGGGTTT AGTGTTCAAAGCAGGCTTAGGCC - TTGAATACATTAGCATGGAATAATGAAATAGGGTTT AGTGTTCAAAGCAGGCTTAGGCC-TTGAATACATTAGCATGGAATAATGAAATAGGGTTT AGTGTTCAAAGCAGGCTTAGGCC-TTGAATACATTAGCATGGAATAATGAAATAGGGTTT AGTGTTCAAAGCAGGCTTAGGCC - TTGAATACATTAGCATGGAATAATGAAATAGGGTTT AGTGTTCAAAGCAGGCTTAGGCC - TTGAATACATTAGCATGGAATAATGAAATAGGGTTT AGTGTTCAAAGCAGGCTTAGGCC - TTGAATACATTAGCATGGAATAATGAAATAGGGTTT AGTGTTCAAAGCAGGCTTAGGCC - TTGAATACATTAGCATGGAATAATGAAATAGGGTTT AGTGTTCAAAGCAGGCTTAGGCC - TTGAATACATTAGCATGGAATAATGAAATAGGGTTT AGTGTTCAAAGCAGGCTTAGGCC - TTGAATACATTAGCATGGAATAATGAAATAGGGTTT AGTGTTCAAAGCAGGCTTAGGCC - TTGAATACATTAGCATGGAATAATGAAATAGGGTTT AGTGTTCAAAGCAGGCTTAGGCC - TTGAATAGATTAGCATGGAATAATGAAATAGGGTTT AGTGTTCAAAGCAGGCTTAGGCC - TTGAATACATTAGCATGGAATAATGAAATAGGGTTT AGTGTTCAAAGCAGGCTTAGGCC - TTGAATACATTAGCATGGAATAATGAGATAGGGTTT AGTGTTCAAAGCAGGCTTAGGCC - TTGAATACATTAGCATGGAATAATGAGATAGGGTTT AGTGTTCAAAGCAGGCTTAGGCC - TTGAATACATTAGCATGGAATAATGAAATAGGGTTT GGTGTTCAAAGCAGGCTTAGGCC-TTGAATACATTAGCATGGAATAATGAAATAGGGTTT AGTGTTCAAAGCAGGCTTAGGCC - TTGAATACATTAGCATGGAATAATGAAATAGGGTTT 
A._sub_WLB3

A. $s p .{ }^{3}$ FR

A. S sub_ERB

A. sub LEW

A._alpigena

A. amb_PII 7

A. amb N17

A._amb_FL8

A._amb_BDL1

A. $a m b$ WLP1

A._nyas̄sensis_GB

A._valida_N2

A. -gran LB

A._gran_oR12

A. $-\mathrm{sp} \cdot \overline{-}-\overline{2}$

A._sp._2

A._cren_FH8_L639

A-GGTTCTAтTTTGTTGGTTTAAGACCCAंTAATAATGATTAACAGGAACAG-TTGGGGG T-GGTACTATTTTGTTGGTTTGCGCATCA-AGGTAATGATTAATAGGGACAG-TTGGGGG T-GGCACTATTTTGTTGGTTTGCGCGCCA-AAATAATGATTAACAGGGACAGTTTGGGGA T-GGCACTATTTTGTTGGTTTGCGCGCCA-AAATAATGATTAACAGGGACAGTTTGGGGA T-GGCACTATTTTGTTGGTTTGCGCGCCA-AAATAATGATTAACAGGGACAGTTTGGGGA T-GGCACTATTTTGTTGGTTTGCGCGCCG-AAACAATGATTAATAGGGACAGTTTGGGGA T-GGCACTATTTTGTTGGTTTGCGCGCCG-AAACAATGATTAATAGGGACAGTTTGGGGA T-GGCACTATTTTGTTGGTTTGCGCGCCG-AAACAATGATTAATAGGGACAGTTTGGGGA T-GGCACTATTTTGTTGGTTTGCGCGCCG-AAACAATGATTAATAGGGACAGTTTGGGGA T-GGCACTATTTTGTTGGTTTGCGCGCCG-AAACAATGATTAATAGGGACAGTTTGGGGA T-GGCACTATTTTGTTGGTTTGCGCGCCG-AAACAATGATTAATAGGGACAGTTTGGGGA T-GGCACTATTTCGTTGGTTTGCGCGCCG-AAACAATGATTAATAGGGACAGTTTGGGGA T-GGCACTATTTCGTTGGTTTGCGCGCCG-AAACAATGATTAATAGGGACAGTTTGGGGA T-GGCACTATTTCGTTGGTTTGCGCGCCG-AAACAATGATTAATAGGGACAGTTTGGGGA T-GGCACTATTTCGTTGGTTTGCGCGCCG-AAACAATGATTAATAGGGACAGTTTGGGGA T-GGCACTATTTCGTTGGTTTGCGCGCCG-AAACAATGATTAATAGGGACAGTTTGGGGA T-GGCACTATTTCGTTGGTTTGCGCGCCG-AAACAATGACTAATAGGGACAGTTTGGGGA T-GGCACTATTTCGTTGGTTTGCGCGCCG-AAACAATGATTAATAGGGACAGTTTGGGGA T-GGCACTATTTCGTTGGTTTGCGCGCCG-AAACAATGATTAATAGGGACAGTTTGGGGA T-GGCACTATTTCGTTGGTTTGCGCGCCG-AAACAATGATTAATAGGGACAGTTTGGGGA C-GGCACTATTTTGTTGGTTTGCGCGCCG-AAATAATGATTAATAGGGACAG- TTGGGGG CAGGCACTATTTTGTTGGTTTGCACACCA-AAACAATAATTAATAGGGACAAGTTGGGGG T-GGCACTATTTTGTTGGTTTGCGCGCCG-AAACAATGATTAATAGGGACAG-TTGGGGG 
A._sub_WLB3

A. $s p .{ }^{3}$ FR

A._sub_ERB

A. sub_LEW

A._alpigena

A. amb_PII 7

A. amb N17

A._amb_FL8

A._amb_BDL1

A. $a m b$ WLP1

A._nyas̄sensis_GB

A._valida_N2

A. -gran LB

A._gran_oR12

A. $-\mathrm{sp} \cdot \overline{\overline{1}}$

A. sp. 2

A._creñ_FH8_L639

TATTTGTATTCCAGTGTCAGAGGTGAAATTCTTGGATTTCंTGGAAGACAÄACTACTGCGA TATTCGTATTCAATTGTCAGAGGTGAAATTCTTGGATTTCTGGAAGACGAACTACTGCGA TATTCGTATTCAATTGTCAGAGGTGAAATTCTTGGATTTCTGGAAGACGAACGACTGCGA TATTCGTATTCAATTGTCAGAGGTGAAATTCTTGGATTTCTGGAAGACGAACGACTGCGA TATTCGTATTCAATTGTCAGAGGTGAAATTCTTGGATTTCTGGAAGACGAACGACTGCGA TATTCGTATTCAATTGTCAGAGGTGAAATTCTTGGATTTCTGGAAGACGAACGACTGCGA TATTCGTATTCAATTGTCAGAGGTGAAATTCTTGGATTTCTGGAAGACGAACGACTGCGA TATTCGTATTCAATTGTCAGAGGTGAAATTCTTGGATTTCTGGAAGACGAACGACTGCGA TATTCGTATTCAATTGTCAGAGGTGAAATTCTTGGATTTCTGGAAGACGAACGACTGCGA TATTCGTATTCAATTGTCAGAGGTGAAATTCTTGGATTTCTGGAAGACGAACGACTGCGA TATTCGTATTCAATTGTCAGAGGTGAAATTCTTGGATTTCTGGAAGACGAACGACTGCGA TATTCGTATTCAATTGTCAGAGGTGAAATTCTTGGATTTCTGGAAGACGAACGACTGCGA TATTCGTATTCAATTGTCAGAGGTGAAATTCTTGGATTTCTGGAAGACGAACGACTGCGA TATTCGTATTCAATTGTCAGAGGTGAAATTCTTGGATTTCTGGAAGACGAACGACTGCGA TATTCGTATTCAATTGTCAGAGGTGAAATTCTTGGATTTCTGGAAGACGAACGACTGCGA TATTCGTATTCAATTGTCAGAGGTGAAATTCTTGGATTTCTGGAAGACGAACGACTGCGA TATTCGTATTCAATTGTCAGAGGTGAAATTCTTGGATTTCTGGAAGACGAACGACTGCGA TATTCGTATTCAATTGTCAGAGGTGAAATTCTTGGATTTCTGGAAGACGAACGACTGCGA TATTCGTATTCCATTGTCAGAGGTGAAATTCTTGGATTTCTGGAAGACGAACGACTGCGA TATTCGTATTCCATTGTCAGAGGTGAAATTCTTGGATTTCTGGAAGACGAACGACTGCGA TATTCGTATTCAATTGTCAGAGGTGAAATTCTTGGATTTCTGGAAGACGAACGACTGCGA TATTTGTATTCAATTGTCAGAGGTGAAATTCTTGGATTTCTGGAAGACGAATGACTGTGA TATTCGTATTCAATTGTCAGAGGTGAAATTCTTGGATTTCTGGAAGACGAACAACTGCGA 
AAGCATTTACCAAA-GGATGTTTTCACTAATCAAGAACGAÄAGTTAGGGGÄTCGAAGATGA AAGCATTTACCAA-GGATGTTTTCATTAATCAAGAACGAAAGTTGGGGGATCGAAGATGA AAGCATTTATCAA- GGATGTTTTCATTAATCAAGAACGAAAGTTAGGGGATCGAAGATGA A._skvortzowi

A._baicalensis

A._sub_9D1

A. Sub_WLB3

A. $-\mathrm{sp} \cdot{ }^{3}$ FR

A._sub_ERB

A. sub LEW

A._alpigena

A. amb_PII 7

A. amb N17

A._amb_FL8

A._amb_BDL1

A. $a m b$ WLP1

A._nyas̄sensis_GB

A._valida_N2

A. -gran_LB

A._gran_oR12

A. $-\mathrm{sp} \cdot \overline{\overline{1}}$

A.. sp. 2

A._creñ_FH8_L639

AAGCATTTATCAA-GGATGTTTTCATTAATCAAGAACGAAAGTTAGGGGATCGAAGATGA AACGATTTATCAA-GGATGTTTTCATTAATCAAGAACGAAAGTTAGGGGATCGAAGATGA AAGCATTTATCAA - GGATGTTTTCATTAATCAAGAACGAAAGTTAGGGGATCGAAGATGA AAGCATTTATCAA - GGATGTTTTCATTAATCAAGAACGAAAGTTAGGGGATCGAAGATGA AAGCATTTATCAA- GGATGTTTTCATTAATCAAGAACGAAAGTTAGGGGATCGAAGATGA AAGCATTTATCAA- GGATGTTTTCATTAATCAAGAACGAAAGTTAGGGGATCGAAGATGA AAGCATTTATCAA- GGATGTTTTCATTAATCAAGAACGAAAGTTAGGGGATCGAAGATGA AAGCATTTATCAAAGGATGTTTTCATTAATCAAGAACGAAAGTTAGGGGATCGAAGATGA AAGCATTTATCAA - GGATGTTTTCATTAATCAAGAACGAAAGTTAGGGGATCGAAGATGA AAGCATTTATCAA - GGATGTTTTCATTAATCAAGAACGAAAGTTAGGGGATCGAAGATGA AAGCATTTATCAA - GGATGTTTTCATTAATCAAGAACGAAAGTTAGGGGATCGAAGATGA AAGCATTTATCAA - GGATGTTTTCATTAATCAAGAACGAAAGTTAGGGGATCGAAGATGA AAGCATTTATCAA - GGATGTTTTCATTAATCAAGAACGAAAGTTAGGGGATCGAAGATGA AAGCATTTATCAA - GGATGTTTTCATTAATCAAGAACGAAAGTTAGGGGATCGAAGATGA AAGCATTTATCAA - GGATGTTTTCATTAATCAAGAACGAAAGTTAGGGGATCGAAGATGA AAGCATTTATCAA - GGATGTTTTCATTAATCAAGAACGAAAGTTAGGGGATCGAAGATGA AAGCATTTATCAA-GGATGTTTTCATTAATCAAGAACGAAAGTTAGGGGATCGAAGATGA AAGCATTTACCAA - GGATGTTTTCATTAATCAAGAACGAAAGTTAGGGGATCGAAGATGA AAGCATTTACCAA - GGATGTTTTCATTAATCAAGAATGAAAGTTAGGGGATCAGAGATGA AAGCATTTACCAA - GGATGTTTTCATTAATCAAGAACGAAAGTTAGGGGATCGAAGATGA 
A._sub_WLB3

A. $s p .{ }^{3}$ FR

A. S sub_ERB

A. sub_LEW

A._alpigena

A. amb_PII 7

A. amb N17

A._amb_FL8

A._amb_BDL1

A. $a m b$ WLP1

A._nyas̄sensis_GB

A._valida_N2

A. $-g r a n \_L \bar{B}$

A._gran_oR12

A. $-\mathrm{sp} \cdot \overline{\overline{1}}$

A. sp. 2

A._cren_FH8_L639

$\begin{array}{llllll}1030 & 1040 & 1050 & 1060 & 1070 & .] \\ . & . & . & . & . & ]\end{array}$

TTAGATACCÄTCGTAGTCTTAACCATAAACTATGCCAACTAGGGATCATCAGTTGTTATT TTAGATACCATCGTAGTCTCAACCATAAACTATGCCAACTAGGGATTAGCGGTCGTTTAT TTAGATACCATCGTAGTCTTAACCATAAACTATGCCGACTAGGGATTTGCGGTTGTTCTT TTAGATACCATCGTAGTCTTAACCATAAACTATGCCGACTAGGGATTTGCGGTTGTTCTT TTAGATACCATCGTAGTCTTAACCATAAACTATGCCGACTAGGGATTTGCGGTTGTTCTT TTAGATACCATCGTAGTCTTAACCATAAACCATGCCGACTAGGGATTTGCGGTTGTTCTT TTAGATACCATCGTAGTCTTAACCATAAACCATGCCGACTAGGGATTTGCGGTTGTTCTT TTAGATACCATCGTAGTCTTAACCATAAACCATGCCGACTAGGGATTTGCGGTTGTTCTT TTAGATACCATCGTAGTCTTAACCATAAACCATGCCGACTAGGGATTTGCGGTTGTTCTT TTAGATACCATCGTAGTCTTAACCATAAACCATGCCGACTAGGGATTTGCGGTTGTTCTT TTAGATACCATCGTAGTCTTAACCATAAACCATGCCGACTAGGGATTTGCGGTTGTTCTT TTAGATACCATCGTAGTCTTAACCATAAACCATGCCGACTAGGGATTTGCGGTTGTTCTT TTAGATACCATCGTAGTCTTAACCATAAACCATGCCGACTAGGGATTTGCGGTTGTTCTT TTAGATACCATCGTAGTCTTAACCATAAACCATGCCGACTAGGGATTTGCGGTTGTTCTT TTAGATACCATCGTAGTCTTAACCATAAACCATGCCGACTAGGGATTTGCGGTTGTTCTT TTAGATACCATCGTAGTCTTAACCATAAACCATGCCGACTAGGGATTTGCGGTTGTTCTT TTAGATACCATCGTAGTCTTAACCATAAACCATGCCGACTAGGGATTTGCGGTTGTTCTT TTAGATACCATCGTAGTCTTAACCATAAACCATGCCGACTAGGGATTTGCGGTTGTTCTT TTAGATACCATCGTAGTCTTAACCATAAACCATGCCGACTAGGGATTTGCGGTTGTTCTT TTAGATACCATCGTAGTCTTAACCATAAACCATGCCGACTAGGGATTTGCGGTTGTTCTT TTAGATACCATCGTAGTCTTAACCATAAACTATGCCGACTAGGGATTGGCGGTTGTTCTA TTAGATACCATCATAGTCTTAACCATAAACTATGCTGACTAGGGATTGGTGGTTGTTCTG TTAGATACCATCGTAGTCTTAACCATAAACTATGCCGACTAGGGATTGGCGGTTGTTTCT 
A._sub_WLB3

A. $s p .{ }^{3}$ FR

A. S sub_ERB

A. sub LEW

A._alpigena

A. amb_PII 7

A. amb N17

A._amb_FL8

A._amb_BDL1

A. $a m b$ WLP1

A._nyas̄sensis_GB

A._valida_N2

A. gran LB

A._gran_oR12

A. $-\mathrm{sp} \cdot \overline{-}-\overline{2}$

A._sp._2

A._cren_FH8_L639

TA - TGACACTGG - - TGGCACCTTATGAGAÄATCAAAG - TCंTTTGGGTTCTGGGGGGAGTA TAGTGACTCCGT - TAGCACCTTATGAGAAATCAAAG - TCTTTGGGTTCCGGGGGGAGTA GT - AGACTCCGT - AAGCACCTTATGAGAAATCAAAG - TGTTTGGGTTCCGGGGGGAGTA GT-AGACTCCGT - -AAGCACCTTATGAGAAATCAAAG - TGTTTGGGTTCCGGGGGGAGTA GT-AGACTCCGTGTAAGCACCTTATGAGAAATCAAAG - TGTTTGGGTTCCGGGGGGAGTA GT -AGACTCCGT - -AAGCACCTTATGAGAAATCAAAG - TGTTTGGGTTCCGGGGGGAGTA GT - AGACTCCGT - -AAGCACCTTATGAGAAATCAAAG - TGTTTGGGTTCCGGGGGGAGTA GTCAGACTCCGA - -AAGCACCTTATGAGAAATCAAAGGTTGTCCGGTTCCGGGGGGAGTA GT -AGACTCCGT - -AAGCACCTTATGAGAAATCAAAG - TGTTTGGGTTCCGGGGGGAGTA GT - AGACTCCGT - - AAGCACCTTATGAGAAATCAAAG - TGTTTGGGTTCCGGGGGGAGTA GT-AGACTCCGT - -AAGCACCTTATGAGAAATCAAAG - TGTTTGGGTTCCGGGGGGAGTA GT -AGACTCCGT - -AAGCACCTTATGAGAAATCAAAG - TGTTTGGGTTCCGGGGGGAGTA GT - AGACTCCGT - - AAGCACCTTATGAGAAATCAAAG - TGTTTGGGTTCCGGGGGGAGTA GT - AGACTCCGT - -AAGCACCTTATGAGAAATCAAAG - TGTTTGGGTTCCGGGGGGAGTA GT-AGACTCCGT - AAGCACCTTATGAGAAATCAAAG - TGTTTGGGTTCCGGGGGGAGTA GT -AGACTCCGT - -AAGCACCTTATGAGAAATCAAAG - TGTTTGGGTTCCGGGGGGAGTA GT - AGACTCCGT - - AAGCACCTTATGAGAAATCAAAG-TGTTTGGGTTCCGGGGGGAGTA GT-AGACTCCGT - -AAGCACCTTATGAGAAATCAAAG-TGTTTGGGTTCCGGGGGGAGTA GT-AGACTCCGT - -AAGCACCTTATGAGAAATCAAAG-TGTTTGGGTTCCGGGGGGAGTA GT - AGACTCCGT - -AAGCACCTTATGAGAAATCAAAG - TGTTTGGGTTCCGGGGGGAGTA TTT-GGCTCCGT - - CAGCACCTTATGAGAAATCAAAG - TTTTTGGGTTCCGGGGGGAGTA TTTTGACTCCAT - CAGCACCTTCTGAGAAATCAAAG - TGTTTGGGTTCCAGGGGGAGTA TTTTGACTCCGT - - CAGCACCTTATGAGAAATCAAAG - TGTTTGGGTTCCGGGGGGAGTA 
A. sub_9D1

A._sub_WLB3

A., $\mathrm{sp} \cdot{ }^{3}$ FR

A. S sub_ERB

A. sub_LEW

A._alpigena

A. amb_PII 7

A. amb N17

A._amb_FL8

A._amb_BDL1

A. $a m b$ WLP1

A._nyas̄sensis_GB

A._valida_N2

A. gran_LB

A._gran_oR12

A. $-\mathrm{sp} \cdot \overline{\overline{1}}$

A. sp. 2

A._creñ_FH8_L639

TGG-TCGCÄ̈GGCTGAAA- CTTAAAGAAA-TTGACGGAAGGGCACCACCÄGGAGTGGAGC TGG-TCGCAAGGCTGAAA - CTTAAAGAAA - TTGACGGAAGGGCACCACCAGGAGTG? AGC TGG - TCGCAAGGCTGAAA - CTTAAAGAAA - TTGACGGAAGGGCACCACCAGGAGTGGAGC TGG - TCGCAAGGCTGAAA - CTTAAAGAAA - TTGACGGAAGGGCACCACCAGGAGTGGAGC TGGTTGCCAAGGCTGAAA - CTTAAAGAAA - TTGACGGAAGGGCACCACCAGGAGTGGAGC TGG - TCGCAAGGCTGAAA - CTTAAAGAAA - TTGACGGAAGGGCACCACCAGGAGTGGAGC TGG-TCGCAAGGCTGAAA - CTTAAAGAAA - TTGACGGAAGGGCACCACCAGGAGTGGAGC TGGGTCGCAAGGCTAAAA - CTTAAAGAAAATTGACGGAAGGGCACCACCAGGAGTGGAGC TGG - TCGCAAGGCTGAAA - CTTAAAGAAA - TTGACGGAAGGGCACCACCAGGAGTGGAGC TGG - TCGCAAGGCTGAAAACTTAAAGAAA - TTGACGGAAGGGCACCACCAGGAGTGGAGC TGG-TCGCAAGGCTGAAA - CTTAAAGAAA - TTGACGGAAGGGCACCACCAGGAGTGGAGC TGG-TCGCAAGGCTGAAA- CTTAAAGAAA - TTGACGGAAGGGCACCACCAGGAGTGGAGC TGG - TCGCAAGGCTGAAA - CTTAAAGAAA - TTGACGGAAGGGCACCACCAGGAGTGGAGC TGG-TCGCAAGGCTGAAA - CTTAAAGAAA - TTGACGGAAGGGCACCACCAGGAGTGGAGC TGG - TCGCAAGGCTGAAA - CTTAAAGAAA - TTGACGGAAGGGCACCACCAGGAGTGGAGC TGG - TCGCAAGGCTGAAA - CTTAAAGAAA - TTGACGGAAGGGCACCACCAGGAGTGGAGC TGG-TCGCAAGGCTGAAA - CTTAAAGAAA - TTGACGGAAGGGCACCACCAGGAGTGGAGC TGG-TCGCAAGGCTGAAA - CTTAAAGAAA - TTGACGGAAGGGCACCACCAGGAGTGGAGC TGG-TCGCAAGGCTGAAA - CTTAAAGAAA - TTGACGGAAGGGCACCACCAGGAGTGGAGC TGG-TCGCAAGGCTGAAA - CTTAAAGAAA - TTGACGGAAGGGCACCACCAGGAGTGGAGC TGG-TCGCAAGGCTGAAA - CTTAAAGAAA - TTGACGGAAGGGCACCACCAGGAGTGGAGC TGG - TCACAAGGCTGAAA - CTTAAAGAAA - TTGATGGAAGGGTACCACCAGGAGTGGAGC TGG-TCGCAAGGCTGAAA - CTTAAAGAAA - TTGACGGAAGGGCACCACCAGGAGTGGAGC 
A._sub_9D1

A._sub_WLB3

A. $s p .{ }^{3}$ FR

A. S sub_ERB

A. sub LEW

A._alpigena

A. amb_PII 7

A. amb N17

A._amb_FL8

A._amb_BDL1

A. $a m b$ WLP1

A._nyas̄sensis_GB

A._valida_N2

A. gran LB

A._gran_oR12

A. $-\mathrm{sp} \cdot \overline{\overline{1}}$

A. sp. 2

A._cren_FH8_L639

CTGCGGCTTAATTTGACTCAACACGGGAAAACTTACCAGGTCCAGACATAGTAAGGATTG CTGCGGCTTAATTTGACTCAACACGGGAAAACTTACCAGGTCCAGACATAGTGAGGATTG CTGCGGCTTAATTTGACTCAACACGGGAAAACTTACCAGGTCCAGACATAGTGAGGATTG CTGCGGCTTAATTTGACTCAACACGGGAAAACTTACCAGGTCCAGACATAGTGAGGATTG CTGCGGCTTAATTTGACTCAACACGGGAAAACTTACCAGGTCCAGACATAGTGAGGATTG CTGCGGCTTAATTTGACTCAACACGGGAAAACTTACCAGGTCCAGACATAGTGAGGATTG CTGCGGCTTAATTTGACTCAACACGGGAAAACTTACCAGGTCCAGACATAGTGAGGATTG CTGCGGCTTAATTTGACTCAACACGGGAAAACTTACCAGGTCCAGACATAGTGAGGATTG CTGCGGCTTAATTTGACTCAACACGGGAAAACTTACCAGGTCCAGACATAGTGAGGATTG CTGCGGCTTAATTTGACTCAACACGGGAAAACTTACCAGGTCCAGACATAGTGAGGATTG CTGCGGCTTAATTTGACTCAACACGGGAAAACTTACCAGGTCCAGACATAGTGAGGATTG CTGCGGCTTAATTTGACTCAACACGGGAAAACTTACCAGGTCCAGACATAGTGAGGATTG CTGCGGCTTAATTTGACTCAACACGGGAAAACTTACCAGGTCCAGACATAGTGAGGATTG CTGCGGCTTAATTTGACTCAACACGGGAAAACTTACCAGGTCCAGACATAGTGAGGATTG CTGCGGCTTAATTTGACTCAACACGGGAAAACTTACCAGGTCCAGACATAGTGAGGATTG CTGCGGCTTAATTTGACTCAACACGGGAAAACTTACCAGGTCCAGACATAGTGAGGATTG CTGCGGCTTAATTTGACTCAACACGGGAAAACTTACCAGGTCCAGACATAGTGAGGATTG CTGCGGCTTAATTTGACTCAACACGGGAAAACTTACCAGGTCCAGACATAGTGAGGATTG CTGCGGCTTAATTTGACTCAACACGGGAAAACTTACCAGGTCCAGACATAGTGAGGATTG CTGCGGCTTAATTTGACTCAACACGGGAAAACTTACCAGGTCCAGACATAGTGAGGATTG CTGCGGCTTAATTTGACTCAACACGGGAAAACTTACCAGGTCCAGACATAGTGAGGATTG CTGTGGCTTAATTTGACTCAACATGGGAAAACTTACCAGGTCCAGACATAGTGAGGATTG CTGCGGCTTAATTTGACTCAACACGGGAAAACTTACCAGGTCCAGACATAGTGAGGATTG 
A. sub_WLB3

A. $-\mathrm{sp} \cdot{ }^{3}$ FR

A._sub_ERB

A. sub LEW

A._alpigena

A. amb_PII 7

A. amb N17

A._amb_FL8

A._amb_BDL1

A. $a m b$ WLP1

A._nyas̄sensis_GB

A._valida_N2

A. gran_LB

A._gran-OR12

A. $\operatorname{sp} \cdot \overline{1}$

A. sp. 2

A._cren_FH8_L639

$\left.\begin{array}{ccccc}1270 & 1280 & 1290 & 1300 & 1310 \\ \cdot & \cdot & \cdot & \cdot & \cdot\end{array}\right]$ ACAGATTGAGAGCTCTTTCTTGATTCTATGGGTGGTGGTGCATGGCCGTTCTTAGTTGGT
ACAGATTGAGAGCTCTTTCTTGATTCTATGGGTGGTGGTGCATGGCCGTTCTTAGTTGGT ACAGATTGAGAGCTCTTTCTTGATTCTATGGGTGGTGGTGCATGGCCGTTCTTAGTTGGT ACAGATTGAGAGCTCTTTCTTGATTCTATGGGTGGTGGTGCATGGCCGTTCTTAGTTGGT ACAGATTGAGAGCTCTTTCTTGATTCTATGGGTGGTGGTGCATGGCCGTTCTTAGTTGGT ACAGATTGAGAGCTCTTTCTTGATTCTATGGGTGGTGGTGCATGGCCGTTCTTAGTTGGT ACAGATTGAGAGCTCTTTCTTGATTCTATGGGTGGTGGTGCATGGCCGTTCTTAGTTGGT ACAGATTGAGAGCTCTTTCTTGATTCTATGGGTGGTGGTGCATGGCCGTTCTTAGTTGGT ACAGATTGAGAGCTCTTTCTTGATTCTATGGGTGGTGGTGCATGGCCGTTCTTAGTTGGT ACAGATTGAGAGCTCTTTCTTGATTCTATGGGTGGTGGTGCATGGCCGTTCTTAGTTGGT ACAGATTGAGAGCTCTTTCTTGATTCTATGGGTGGTGGTGCATGGCCGTTCTTAGTTGGT ACAGATTGAGAGCTCTTTCTTGATTCTATGGGTGGTGGTGCATGGCCGTTCTTAGTTGGT ACAGATTGAGAGCTCTTTCTTGATTCTATGGGTGGTGGTGCATGGCCGTTCTTAGTTGGT ACAGATTGAGAGCTCTTTCTTGATTCTATGGGTGGTGGTGCATGGCCGTTCTTAGTTGGT ACAGATTGAGAGCTCTTTCTTGATTCTATGGGTGGTGGTGCATGGCCGTTCTTAGTTGGT ACAGATTGAGAGCTCTTTCTTGATTCTATGGGTGGTGGTGCATGGCCGTTCTTAGTTGGT ACAGATTGAGAGCTCTTTCTTGATTCTATGGGTGGTGGTGCATGGC-GT-CT-AGGTG-T ACAGATTGAGAGCTCTTTCTTGATTCTATGGGTGGTGGTGCATGGCCGTTCTTAGTTGGT ACAGATTGAGAGCTCTTTCTTGATTCTATGGGTGGTGGTGCATGGCCGTTCTTAGTTGGT ACAGATTGAGAGCTCTTTCTTGATTCTATGGGTGGTGGTGCATGGCCGTTCTTAGTTGGT ACAGATTGAGAGCTCTTTCTTGATTCTATGGGTGGTGGTGCATGGCCGTTCTTAGTTGGT ACAGATTGAGAGCTCTTTCTTGATTCTATGGGTGGTGGTGCATGGCCGTTCTTAGTTGGT ACAGATTGAGAGCTCTTTCTTGATTCTATGGGTGGTGGTGCATGGCCGTTCTTAGTTGGT 
A. sub_WLB3

A. $s p .{ }^{3}$ FR

A. S sub_ERB

A. sub LEW

A._alpigena

A. amb_PII 7

A. amb N17

A._amb_FL8

A. amb_BDL1

A. $a m b$ WLP1

A._nyas̄sensis_GB

A._valida_N2

A. gran LB

A._gran_oR12

A. $\operatorname{sp} \cdot \overline{1}$

A. sp. 2

A._cren_FH8_L639

GGAGTGATTTGTCTGGTTAÄTTCCGTTAACGAACGAGACCCCTGCCTGCTAAATAGTTAC GGAGTGATTTGTCTGGTTAATTCCGTTAACGAACGAGACCCCCGCCTGCTAAATAGTCCT GGAGTGATTTGTCTGGTTAATTCCGTTAACGAACGAGACCCCCGCCTGCTAAATAGTCCG GGAGTGATTTGTCTGGTTAATTCCGTTAACGAACGAGACCCCCGCCTGCTAAATAGTCCG GGAGTGATTTGTCTGGTTAATTCCGTTAACGAACGAGACCCCCGCCTGCTAAATAGTCCG GGAGTGATTTGTCTGGTTAATTCCGTTAACGAACGAGACCCCCGCCTGCTAAATAGACTG GGAGTGATTTGTCTGGTTAATTCCGTTAACGAACGAGACCCCCGCCTGCTAAATAGACTG GGAGTGATTTGTCTGGTTAATTCCGTTAACGAACGAGACCCCCGCCTGCTAAATAGACTG GGAGTGATTTGTCTGGTTAATTCCGTTAACGAACGAGACCCCCGCCTGCTAAATAGACTG GGAGTGATTTGTCTGGTTAATTCCGTTAACGAACGAGACCCCCGCCTGCTAAATAGACTG GGAGTGATTTGTCTGGTTAATTCCGTTAACGAACGAGACCCCCGCCTGCTAAATAGTCCG GGAGTGATTTGTCTGGTTAATTCCGTTAACGAACGAGACCCCCGCCTGCTAAATAGTCCG GGAGTGATTTGTCTGGTTAATTCCGTTAACGAACGAGACCCCCGCCTGCTAAATAGTCCG GGAGTGATTTGTCTGGTTAATTCCGTTAACGAACGAGACCCCCGCCTGCTAAATAGTCCG GGAGTGATTTGTCTGGTTAATTCCGTTAACGAACGAGACCCCCGCCTGCTAAATAGTCCG GGAGTGATTTGTCTGGTTAATTCCGTTAACGAACGAGACCCCCGCCTGCTAAATAGTCCG - GAGTGATTTGTCTGGTTAATTCCGTTAACGAACGAGACCCCCGCCTGCTAAATAGTCCG GGAGTGATTTGTCTGGTTAATTCCGTTAACGAACGAGACCCCCGCCTGCTAAATAGTCCG GGAGTGATTTGTCTGGTTAATTCCGTTAACGAACGAGACCCCCGCCTGCTAAATAGTCCG GGAGTGATTTGTCTGGTTAATTCCGTTAACGAACGAGACCCCCGCCTGCTAAATAGTCCG GGAGTGATTTGTCTGGTTAATTCCGTTAACGAACGAGACCCCCGCCTGCTAAATAGTCCG GGAGTGATTTGTCTGGTTAATTCCTTTAATGAACAAGACCCCCGCCTGCTAAATAGTCTG GGAGTGATTTGTCTGGTTAATTCCGTTAACGAACGAGACCCCCGCCTGCTAAATAGTCTG 
A._sub_WLB3

A. $s p .{ }^{3}$ FR

A. S sub_ERB

A. sub LEW

A._alpigena

A. amb_PII 7

A. amb N17

A._amb_FL8

A._amb_BDL1

A. $a m b$ WLP1

A._nyas̄sensis_GB

A._valida_N2

A. gran LB

A._gran_oR12

A. $-\mathrm{sp} \cdot \overline{\overline{1}}$

A. sp. 2

A._cren_FH8_L639

ACTAATG - - TTTT-ATTTGTGTTAACTTCTTAGAGGGACTTTCAGTGACTAACTGAAGGA GTGAATGAATTTTCATTGACAAGGTCTTCTTAGAGGGACTTTCGGTGACTAACCGAAGGA GTGAATGAGTTTTCATTGACCTGGTCTTCTTAGAGGGACTTTCGGTGACTAACCGAAGGA GTGAATGAGTTTTCATTGACCTGGTCTTCTTAGAGGGACTTTCGGTGACTAACCGAAGGA GTGAATGAGTTTTCATTGACCTGGTCTTCTTAGAGGGACTTTCGGTGRCTAACCGAAGGA GTGAATGAGTTTTCATTGACTTGGTCTTCTTAGAGGGACTTTCGGTGACTAACCGAAGGA GTGAATGAGTTTTCATTGACTTGGTCTTCTTAGAGGGACTTTCGGTGACTAACCGAAGGA GTGAATGAGTTTTCATTGACTTGGTCTTCTTAGAGGGACTTTCGGTGACTAACCGAAGGA GTGAATGAGTTTTCATTGACTTGGTCTTCTTAGAGGGACTTTCGGTGACTAACCGAAGGA GTGAATGAGTTTTCATTGACTTGGTCTTCTTAGGGGGACTTTCGGTGACTAACCGAAGGA GTGAATGAGTTTTCATTGACCTGGTCTTCTTAGAGGGACTTTCGGTGACTAACCGAAGGA GTGAATGAGTTTTCATTGACCTGGTCTTCTTAGAGGGACTTTCGGTGACTAACCGAAGGA GTGAATGAGTTTTCATTGACCTGGTCTTCTTAGAGGGACTTTCGGTGACTAACCGAAGGA GTGAATGAGTTTTCATTGACCTGGTCTTCTTAGAGGGACTTTCGGTGACTAACCGAAGGA GTGAATGAGTTTTCATTGACCTGGTCTTCTTAGAGGGACTTTCGGTGACTAACCGAAGGA GTGAATGAGTTTTCATTGACCTGGTCTTCTTAGAGGGACTTTCGGTGACTAACCGAAGGA GTGAATGAGTTTTCATTGACCTGGTCTTCTTAGAGGGACTTTCGGTGACTAACCGAAG?A GTGAATGAGTTTTCATTGACCTGGTCTTCTTAGAGGGACTTTCGGTGACTAACCGAAGGA GTGAATGAGTCTTCATTGACCTGGTCTTCTTAGAGGGACTTTTGGTGACTAACCGAAGGA GTGAATGAGTCTTCATTGACCTGGTCTTCTTAGAGGGACTTTTGGTGACTAACCGAAGGA GTGAATGAGTGTTCATTGACCTGGTCTTCTTAGAGGGACTTTCGGTGACTAACCGAAGGA GTGAATGAGTGTTCATTGACCTGGTCTTCTTAGAGGGACTTTTGGTGACTAACCGAAGGA GTGAATGAGTATTCATTGACTTGGACTTCTTAGAGGGACTTTCGGTGACTAACTGAAGGA 
A._sub_9D1

A._sub_WLB3

A. $s p .{ }^{3}$ FR

A._sub_ERB

A. sub_LEW

A._alpigena

A. amb_PII 7

A. amb N17

A._amb_FL8

A. amb_BDL1

A. $a m b$ WLP1

A._nyas̄sensis_GB

A._valida_N2

A. gran_L $\bar{B}$

A._gran_oR12

A. $\operatorname{sp} \cdot \overline{1}$

A. sp. 2

A._creñ_FH8_L639

AGATGGGGGCAATAACAGGTCTGTGATGCCCTTAGATGTCCTGGGCCGCÄCGCGCGCTAC AGTTGGGGGCAATAACAGGTCTGTGATGCCCTTAGATGTCCTGGGCGGCACGCGCGCTAC AGTTGGGGGCAATAACAGGTCTGTGATGCCCTTAGATGATCTGGGCCGCACGCGCGCTAC AGTTGGGGGCAATAACAGGTCTGTGATGCCCTTAGATGATCTGGGCCGCACGCGCGCTAC AGTTGGGGGCAATAACAGGTCTGTGATGCCCTTAGATGATCTGGGCCGCACGCGCGCTAC AGTTGGGGGCAATAACAGGTCTGTGATGCCCTTAGATGATCTGGGCCGCACGCGCGCTAC AGTTGGGGGCAATAACAGGTCTGTGATGCCCTTAGATGATCTGGGCCGCACGCGCGCTAC AGTTGGGGGCAATAACAGGTCTGTGATGCCCTTAGATGATCTGGGCCGCACGCGCGCTAC AGTTGGGGGCAATAACAGGTCTGTGATGCCCTTAGATGATCTGGGCCGCACGCGCGCTAC AGTTGGGGGCAATAACAGGTCTGTGATGCCCTTAGATGATCTGGGCCGCACGCGCGCTAC AGTTGGGGGCAATAACAGGTCTGTGATGCCCTTAGATGATCTGGGCCGCACGCGCGCTAC AGTTGGGGGCAATAACAGGTCTGTGATGCCCTTAGATGATCTGGGCCGCACGCGCGCTAC AGTTGGGGGCAATAACAGGTCTGTGATGCCCTTAGATGATCTGGGCCGCACGCGCGCTAC AGTTGGGGGCAATAACAGGTCTGTGATGCCCTTAGATGATCTGGGCCGCACGCGCGCTAC AGTTGGGGGCAATAACAGGTCTGTGATGCCCTTAGATGATCTGGGCCGCACGCGCGCTAC AGTTGGGGGCAATAACAGGTCTGTGATGCCCTTAGATGATCTGGGCCGCACGCGCGCTAC AGTTGGGGGCAATAACAGGTCTGTGATGCCCTTAGATGATCTGGGCCGCACGCGCGCTAC AGTTGGGGGCAATAACAGGTCTGTGATGCCCTTAGATGATCTGGGCCGCACGCGCGCTAC AGTTGGGGGCAATAACAGGTCTGTGATGCCCTTAGATGATCTGGGCCGCACGCGCGCTAC AGTTGGGGGCAATAACAGGTCTGTGATGCCCTTAGATGATCTGGGCCGCACGCGCGCTAC AGTTGGGGGCAATAACAGGTCTGTGATGCCCTTAGATGTCCTGGGCCGCACGCGCGCTAC AGTTGGGGGCAATAACAGGTCTGTGATGCCCTTAGATGTCCTGGGCTGAATGCGTGCTAC AGTTGGGGGCAATAACAGGTCTGTGATGCCCTTAGATGTTCTGGGCCGCACGCGCGCTAC 
A._sub_WLB3

A. $s p .{ }^{3}$ FR

A. S sub_ERB

A. sub LEW

A._alpigena

A. amb_PII 7

A. amb N17

A._amb_FL8

A._amb_BDL1

A. $a m b$ WLP1

A._nyas̄sensis_GB

A._valida_N2

A. gran LB

A._gran_oR12

A. $-\mathrm{sp} \cdot \overline{\overline{1}}$

A. sp. 2

A._creñ_FH8_L639

ACTGATGCATTCAATAAGTCTTT- - CCTTGGCCGAGAGGCTTAGGTAAACTTTT-AATAT ACTGATGCATTCAACAAGCATATAACCTTGGCCGAGAGGCCTGGGTAATCTTTGAAACGT ACTGATGCATTCAACGAGTCTAGAACCTCGGCCGTGAGGCCTGGGTAATCTTATGAACGT ACTGATGCATTCAACGAGTCTAGAACCTCGGCCGTGAGGCCTGGGTAATCTTATGAACGT ACTGATGCATTCAACGAGTCTAGAACCTNCRCCGTGAGGCCTGGGTAATCTTATGAACGT ACTGATGCATTCAACGAGTCTAGAACCTTGGCCGAGAGGCCTGGGTAATCTTGGGAACGT ACTGATGCATTCAACGAGTCTAGAACCTTGGCCGAGAGGCCTGGGTAATCTTGGGAACGT ACTGATGCATTCAACGAGTCTAGAACCTTGGCCGAGAGGCCTGGGTAATCTTAGGAACGT ACTGATGCATTCAACGAGTCTAGAACCTTGGCCGAGAGGCCTGGGTAATCTTGGGAACGT ACTGATGCATTCAACGAGTCTAGAACCATGGCCGAGAGGCCTGGGTAATCTTGGGAACGT ACTGATGCATTCAACGAGTCTAGAACCTTGGCCGAGAGGCCTGGGTAATCTTGGGAACGT ACTGATGCATTCAACGAGTCTAGAACCTTGGCCGAGAGGCCTGGGTAATCTTGTGAACGT ACTGATGCATTCAACGAGTCTAGAACCTTGGCCGAGAGGCCTGGGTAATCTTGTGAACGT ACTGATGCATTCAACGAGTGTAGAACCTTGGCCGAGAGGCCTGGGTAATCTTGTGAACGT ACTGATGCATTCAACGAGTCTAGAACCTTGGCCGAGAGGCCTGGGTAATCTTGTGAACGT ACTGATGCATTCAACGAGTGTAGAACCTTGGCCGAGAGGCCTGGGTAATCTTGTGAACGT ACTGATGCATTCAACGAGTGTAGAACCTTGGCCGAGAGGC - TGGGTAATCTTGTGAACGT ACTGATGCATTCAACGAGTCTAGAACCTTGGCCGAGAGGCCTGGGTAATCTTGTGAACGT ACTGATGCATTCAACGAGTGTAGAACCTTGGCCGAGAGGCCTGGGTAATCTTGTGAACGT ACTGATGCATTCAACGAGTGTAGAACCTTGGCCGAGAGGCCTGGGTAATCTTGTGAACGT ACTGATGCATTCAACGAGTGTAGAACCTTGGCCGAGAGGCCTGGGTAATCTTGTGAACGT ACTGATGCATTCAATGAGTGTAGAACCTTGGCTGAGAGGCCTGGGTAATCTTGGGAATGT ACTGATGCATTCAACGAGTGTAGAACCTTGGCCGAGAGGCTTGGGTAATCTTTTGAACTT 
A. sub_WLB3

A. $s p .{ }^{3}$ FR

A._sub_ERB

A. sub LEW

A._alpigena

A. amb_PII 7

A. amb N17

A._amb_FL8

A._amb_BDL1

A. $a m b$ WLP1

A._nyas̄sensis_GB

A._valida_N2

A. gran_LB

A._gran_oR12

A. $-\mathrm{sp} \cdot \overline{1}$

A. sp. 2

A._creñ_FH8_L639

$\begin{array}{llllll}1570 & 1580 & 1590 & 1600 & 1610 & .] \\ . & . & . & . & . & .]\end{array}$

GTTTCGTGATAGGGATTGAATCTTGTAATTATTATTCATGAACGAGGAATTCCTAGTAAC GCATCGTGATAGGGATAGATTATTGCAATTATTAATCTTGAACGAGGAATTCCTAGTAAA GCATCGTGCTAGGGATTGAATATTGCAATTATTATTCATGAACGAGGAATTCCTAGTAAA GCATCGTGCTAGGGATTGAATATTGCAATTATTATTCATGAACGAGGAATTCCTAGTAAA GCATCGTGCTAGGGATTGAATATTGCAATTATTATTCATGAACGAGGAATTCCTAGTAAA GCATCGTGATAGGGATTGAATATTGCAATTATTATTCATGAACGAGGAATTCCTAGTAAA GCATCGTGATAGGGATTGAATATTGCAATTATTATTCATGAACGAGGAATTCCTAGTAAA GCATCGTGATAGGGATTGAATATTGCAATTATTATTCATGAACGAGGAATTCCTAGTAAA GCATCGTGATAGGGATTGAATATTGCAATTATTATTCATGAACGAGGAATTCCTAGTAAA GCATCGTGATAGGGATTGAATATTGCAATTATTATTCATGAACGAGGAATTCCTAGTAAA GCATCGTGATAGGGATTGAATATTGCAATTATTATTCATGAACGAGGAATTCCTAGTAAA GCATCGTGATAGGGATTGAATATTGCAATTATTATTCATGAACGAGGAATTCCTAGTAAA GCATCGTGATAGGGATTGAATATTGCAATTATTATTCATGAACGAGGAATTCCTAGTAAA GCATCGTGATAGGGATTGAATATTGCAATTATTATTCATGAACGAGGAATTCCTAGTAAA GCATCGTGATAGGGATTGAATATTGCAATTATTATTCATGAACGAGGAATTCCTAGTAAA GCATCGTGATAGGGATTGAATATTGCAATTATTATTCATGAACGAGGAATTCCTAGTAAA GCATCGTGATAGGGATTGAATATTGCAATTATTATTCATGAACGAGGAATTCCTAGTAAA GCATCGTGATAGGGATTGAATATTGCAATTATTATTCATGAACGAGGAATTCCTAGTAAA GCATCGTGATAGGGATTGAATATTGCAATTATTATTCATGAACGAGGAATTCCTAGTAAA GCATCGTGATAGGGATTGAATATTGCAATTATTATTCATGAACGAGGAATTCCTAGTAAA GCATCGTGATAGGGATTGAATATTGCAATTATTATTCATGAACGAGGAATTCCTAGTAAA GCATCATGATAGGGATTGAATATTGCAATTATTATTCATGAATGAGGAATTCCTAGTAAA GCATCGTGATAGGGATTGAATATTGCAATTATTATTCATGAACGAGGAATTCCTAGTAAA 
A. sub_9D1

A._sub_WLB3

A. $s p .{ }^{3}$ FR

A. S sub_ERB

A. sub_LEW

A._alpigena

A. amb_PII 7

A. amb N17

A._amb_FL8

A._amb_BDL1

A. $a m b$ WLP1

A._nyas̄sensis_GB

A._valida_N2

A. gran_L $\bar{B}$

A._gran_oR12

A. $\operatorname{sp} \cdot \overline{1}$

A. sp. 2

A._cren_FH8_L639

CGCAGATCATTAATCTGCAGTGATTACGTCCCTGCCCTTTGTACACACCGCCCGTCGCA. CGCAGATCATCAATCTGCATTGATTACGTCCCTGCCCTTTGTACACACCGCCCGTCGCAC CGCAGATCATCAATCTGCATTGATTACGTCCCTGCCCTTTGTACACACCGCCCGTCGCAC CGCAGATCATCAATCTGCATTGATTACGGCCCTGCCCTTTGTACACACCGCCCGTCGCAC CGCAGATCATCAATCTGCWTTGATTACGTCCCTGCCCTTTGTACACACCGCCCGTCGCAC CGCAGATCATCAATCTGCATTGATTACGTCCCTGCCCTTTGTACACACCGCCCGTCGCAC CGCAGATCATCAATCTGCATTGATTACGTCCCTGCCCTTTGTACACACCGCCCGTCGCAC CGCAGATCATCAATCTGCATTGATTACGTCCCTGCCCTTTGTACACACCGCCCGTCGCAC CGCAGATCATCAATCTGCATTGATTACGTCCCTGCCCTTTGTACACACCGCCCGTCGCAC CGCAGATCATCAATCTGCATTGATTACGTCCCTGCCCTTTGTACACACCGCCCGTCGCAC CGCAGATCATCAATCTGCATTGATTACGTCCCTGCCCTTTGTACACACCGCCCGTCGCAC CGCAGATCATCAATCTGCATTGATTACGTCCCTGCCCTTTGTACACACCGCCCGTCGCAC CGCAGATCATCAATCTGCATTGATTACGTCCCTGCCCTTTGTACACACCGCCCGTCGCAC CGCAGATCATCAATCTGCATTGATTACGTCCCTGCCCTTTGTACACACCGCCCGTCGCAC CGCAGATCATCAATCTGCATTGATTACGTCCCTGCCCTTTGTACACACCGCCCGTCGCAC CGCAGATCATCAATCTGCATTGATTACGTCCCTGCCCTTTGTACACACCGCCCGTCGCAC CGCAGATCATCAATCTGCATTGATTACGTCCCTGCCCTTTGTACACACCGCCCGTCGCAC CGCAGATCATCAATCTGCATTGATTACGTCCCTGCCCTTTGTACACACCGCCCGTCGCAC CGCAGATCATCAATCTGCATTGATTACGTCCCTGCCCTTTGTACACACCGCCCGTCGCAC CGCAGATCATCAATCTGCATTGATTACGTCCCTGCCCTTTGTACACACCGCCCGTCGCAC CGCAGATCATCAATCTGCATTGATTACGTCCCTGCCCTTTGTACACACCGCCCGTCGCAC TGCAGATCATCAATCTGCATTGATTATGTCCCTGCCCTTTGTACACACCACCCATTGCAC CGCGGATCATCAATCCGCATTGATTACGTCCCTGCCCTTTGTACACACCGCCCGTCGCAC 
A. sub_WLB3

A. $s p .{ }^{3}$ FR

A. S sub_ERB

A. sub LEW

A._alpigena

A. amb_PII 7

A. amb N17

A._amb_FL8

A. $a m b$ BDL1

A. $a m b$ WLP1

A._nyas̄sensis_GB

A._valida_N2

A. $-g r a n \_L \bar{B}$

A. -gran-oR12

A. $-\mathrm{sp} \cdot \overline{1}$

A. sp. 2

A._cren_fH8_L639

$\begin{array}{cccccc}1690 & 1700 & 1710 & 1720 & 1730 & .] \\ \cdot & \cdot & \cdot & \cdot & \cdot & \text { ] }\end{array}$ CTACCGATTGAATGATCCGGTGAGGACTGGAGATTAAGGCAATCTTTCTTCACTGGAAGT
CTACCGATTGAATGTTCCGGTGAGGACTCGGGATTGATGTTAGCTTCCTTTGTTGGGACT CTACCGATTGAATGGTCCGGTGAAGCCTCGGGATTGTGGTC-CGTTCCTTCAGTGGGATG CTACCGATTGAATGGTCCGGTGAAGCCTCGGGATTGTGGTC- CGTTCCTTCAGTGGGATG CTACC?ATTGAATGGTCCGGTGAAGCCTCGGGATTGTGGTC-CGTTCCTTCAGTGGGATG CTACCGATTGAATGGTCCGGTGAAGCCTCGGGATTGTGGTC-CGTTCCTTT? ? ? ? ? ? ? CTACCGATTGAATGGTCCGGTGAAGCCTCGGGATTGTGGTC-CGTTCCTTTAGTGGGATG CTACCGATTGAATGGTCCGGTGAAGCCTCGGGATTGTGGTC-CGTTCCTTTA ? ? ? ? ? ? CTACCGATTGAATGGTCCGGTGAAGCCTCGGGATTGTGGTC-CGTTCCTTTAGTG? ? ? ? CTACCGATTGAATGGTCCGGTGAAGCCTCGGGATTGTGGTC-CGTTCCTTTAGTGGGATG CTACCGATTGAATGGTCCGGTGAAGCCTCGGGATTGTGGTC-CGTTCCTTTACTGGGATG CTACCGATTGAATGGTCCGGTGAAGCCTCGGGATTGCGGTC-CGTTCCTTTACTG? ? ? ? CTACCGATTGAATGGTCCGGTGAAGCCTCGGGATTGCGGTC-CGTTCCTTTACTG? ? ? ? CTACCGATTGAATGGTCCGGTGAAGCCTCGGGATTGCGGTC-CGTTCCTTTACTG? ? ? ? CTACCGATTGAATGGTCCGGTGAAGCCTCGGGATTGCGGTC-CGTTCCTTTACTGGGATG CTACCGATTGAATGGTCCGGTGAAGCCTCGGGATTGCGGTC-CGTTCCTTTACTGGGATG CTACCGATTGAATGGTCCGGTGAAGCCTCGGGATTGCGGTC-CGTTCCTTTACTGGGATG CTACCGATTGAATGGTCCGGTGAAGCCTCGGGATTGCGGCC-CGTTCCT? ? ? ? ? ? ? ? ? CTACCGATTGAATCATCAGGTGAAGCCTCGGGATTGCGGTC-CGTTCCTTCAGTGGGATG CTACCGATTGAATGGTCCGGTGAAGCCTCGGGATTGCGGTC-CGTTCCTTCAGTG? ? ? ? ? CTACCGATTGAATGGTCCGGTGAAGCCTCGGGATCGCGGTCAGGTTCCTTCACTGGGAT? CTACTGATTGAATGGTCCGGTGCAGCCTCAGGACTGCGGTC-GGTTCCTTTACTG? ? ? ? CTACCGATTGAATGGTCC? ? ? ? ? ? ? ? ? ? ? ? ? ? ? ? ? ? ? ? ? ? ? ? ? ? ? ? ? ? 


\author{
[ \\ M._varians_CHMP7_FFP \\ S._Cf._broschii_GB \\ A._islandica_CHM̄PI_MICH \\ A._skvortzowi \\ A._baicalensis \\ A. sub_9D1 \\ A._sub_WLB3 \\ A. $-s p \cdot{ }^{3}$ FR \\ A._sub_ERB \\ A. sub LEW \\ A._alpigena \\ A._amb_PII 7 \\ A. amb N17 \\ A._amb_FL8 \\ A. amb_BDL1 \\ A. $a m b$ WLPI \\ A._nyassensis_GB \\ A._valida_N2 \\ A. gran $L \bar{B}$ \\ A. gran OR12 \\ A. $\mathrm{sp} \cdot \overline{1}$ \\ A. sp. 2 \\ A. creñ FH8 L639
}
1750
1760
1770
1780
1790
]

GCGCCATGAGAACTTGTCCAAATCTTATCATTT? ? ? ? ? ? ? ? ? ? ? ? ? ? ? ? ? ? ? ? ? ? ? TGATAATGCGAACTCGTCCAAACCTTATCATTTAGAGGAAGGTGAAGTCGTAACAAGGTT $\mathrm{TG}$ ? ? ? ?? ? ? ? ? ? ? ? ? ? ? ? ? ? ? ? ? ? ? ? ? ? ? ? ? ? ? ? ? ? ? ? ? ? ? ? ?

TGGCTGTAAGAACTTGTCTAAACCTTATCATTTAGAGGAAGGTGAAGTCGTAACAAGGTT TGGCTGTAAGAACTTGTCTAAACCTTATCATTTAGAGGAAGGTGAAGTCGTAACAAGGTT

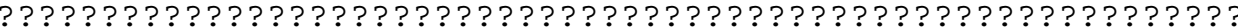

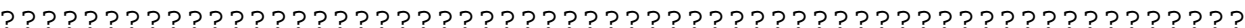

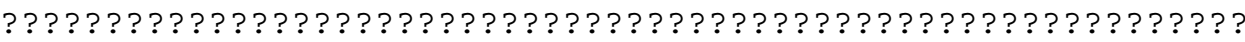
? ? ? ? ? ? ? ? ? ? ? ? ? ? ? ? ? ? ? ? ? ? ? ? ? ? ? ? ? ? ? ? ? ? ? ? ? ? ? ? ? ? ? ? TGGCTGTGAGAACTTGTCTAAACCTTATCATTTAGA ? ? ? ? ? ? ? ? ? ? ? ? ? ? ? ? ? ? ? ? ? ? ? ? ? ? ? ? ? ? ? ? ? ? ? ? ? ? ? ? ? ? ? ? ? ? ? ? ? ? ? ? ? ? ? ? ? ? ? ? ? ? ?? ?? ?? ? ? ? ? ? ? ? ? ? ? ? ? ? ? ? ? ? ? ? ? ? ? ? ? ? ? ? ? ? ? ? ? ? ? ? ?

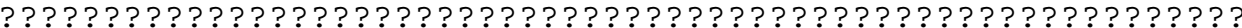
? ? ? ? ? ? ? ? ? ? ? ? ? ? ? ? ? ? ? ? ? ? ? ? ? ? ? ? ? ? ? ? ? ? ? ? ? ? ? ?

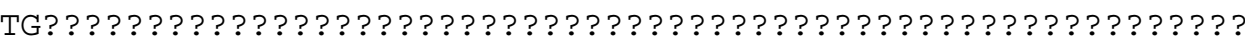
? ? ? ? ? ? ? ? ? ? ? ? ? ? ? ? ? ? ? ? ? ? ? ? ? ? ? ? ? ? ? ? ? ? ? ? ? ? ? ? ? ? ? ?

TGGCTGTGAGAACTTGTCTAAACCTTATCATTTAGAGGAAGGTGAAGTCGTAACAAGGTT ? ? ? ? ? ? ? ? ? ? ? ? ? ? ? ? ? ? ? ? ? ? ? ? ? ? ? ? ? ? ? ? ? ? ? ? ? ? ? ? TGGGCT? ? ? ? ? ? ? ? ? ? ? ? ? ? ? ? ? ? ? ? ? ? ? ? ? ? ? ? ? ? ? ? ? ? ? ? ? ? ? ? ? ? ? ? ? ? ? ? ? ? ? ? ? ? ? ? ? ? ? ? ? ? ? ? ? ? ? ? ? ? ? ? ? ? ? ? ? ? ? ? ? ? ? ? ? ? ? ? ? ? ? ? ? ? ? ? ? ? ? ? ? ? ? ? ? ? ? ? ? ? ? ? ? ? ? ? ? ? ? ? ? ? ? ? ? ? ? ? ? ? ? ? ? ? ? ? ? ? ? ? ? ? ? ? ? ? ? ? ? ? ? ? ? ? ? ? ? ? ? ? ? ? ? ? ? ? ? ? ? ? ? ? ? ? ? ? ?? ? ? ? ? ? ? ? ? ? ? ? ? ? ? ? ? ? ? ? ? ? ? ? ? ? ? ? ? ? ? ? ? ? ? ? ? ? ? ? 


\author{
[ \\ M._varians_CHMP7_FFP \\ S. Cf. bros̄chii $\overline{G B}$ \\ A. islandica_CHM̄P1_MICH \\ A._skvortzowi \\ A._baicalensis \\ A. sub_9D1 \\ A._sub_WLB3 \\ A. $-\mathrm{sp} \cdot{ }^{3} \mathrm{FR}$ \\ A. sub LEW \\ A._alpigena \\ A._amb_PII 7 \\ A. amb N17 \\ A._amb_FL8 \\ A._amb_BDL1 \\ A. amb $\mathrm{WLP} 1$ \\ A._nyas̄sensis_GB \\ A._valida_N2 \\ A. gran_L $\bar{B}$ \\ A. gran OR12 \\ A. $-\mathrm{sp} \cdot \overline{1}$ \\ A. sp. 2 \\ A. creñ FH8 L639
}

$\begin{array}{lll}1810 & 1820 & 1826]\end{array}$

END SSU ]

? ? ? ? ? ? ? ? ? ? ? ? ? ? ? ? ? ? ? ? $\mathrm{TCC}$ ? ? ? ? ? ? ? ? ? ? ? ? ? ? ? ? ?? ? ? ? ? ? ? ? ? ? ? ? ? ? ? ? ? ? ? ?

TCCGTAGGTGAACCTGCAGAAGGATCA TCCGTAGGTGAACCTRCAGAAGGATCA ?? ? ? ? ? ? ? ? ? ? ? ? ? ? ? ? ? ? ? ?

ใ? ? ? ? ? ? ? ? ? ? ? ? ? ? ? ? ? ? ? ? ? ? ? ? ? ? ? ? ? ? ? ? ? ? ? ? ? ? ? ? ? ? ? ? ? ? ? ? ? ? ? ? ? ? ? ? ? ? ? ใ? ? ? ? ? ? ? ? ? ? ? ? ? ? ? ? ? ? ? ? ? ? ? ? ? ? ? ? ? ? ? ? ? ? ? ? ? ?? ? ? ?? ? ? ? ?? ? ? ? ? ? ? ? ? ? ? ? ? ? ? ? ? ? ? ? ? ? ? ? ? ? ? ? ? ?

ว? ? ? ? ? ? ? ? ? ? ? ? ? ? ? ? ? ? ? ? ? ? ? ? ? ? ? ? ? ? ? ? ? ? ? ? ? ? ?? ? ? ? ? ? ? ? ? ? ? ? ? ? ? ? ? ? ? ? TCCGTAGGTGAACCTGCAGAAGGATCA ? ? ? ? ? ? ? ? ? ? ? ? ? ? ? ? ? ? ? ? ? ? ? ? ? ? ? ? ? ? ? ? ? ? ? ? ? ? ? ? ว?ว? ? ? ? ? ? ? ? ? ? ? ? ? ? ? ? ? ? ? ? ? ? ? ? ? ? ? ? ? ? ? ? ? ? ? ? ? ? ? ? ? ? ? ? ? ? ? ? ? ? ? ? ? ? ? ? ? ? ? ? ? ? ? ? ? ? ? ? ? ? ? ? ? ? ? ? 
APPENDIX D. Molecular characters: $r b c \mathrm{~L}$. 
M._varians_CHMP7_FFP A. islandi $\bar{C}$ _ CHMP 1 MICH A. - skvortzowīi

A._baicalensis

A._sub_9D1

A. - sp. 3 - FR

A. sub $E \bar{R} B$

A._sub_LEW

A._amb_PII 7

A. amb N17

A._amb_FL8

A. - amb_BDL1

A. amb WLP1

A._nyas̄sensis_GB

A. valida N2

A. gran_oR 12

A. sp. 1

A._amb_WAT

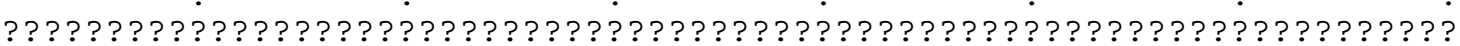

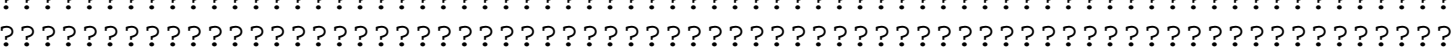

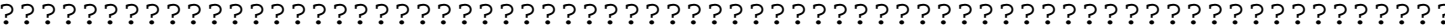
? ? ? ? ? ? ? ? ? ? ? ? ? ? ? ? ? ? ? ? ? ? ? ? ? ? ? ? ? ? ? ? ? ? ? ? ? ? ? ? ? ? ? ? ? ? ? ? ? ? ? ? ?

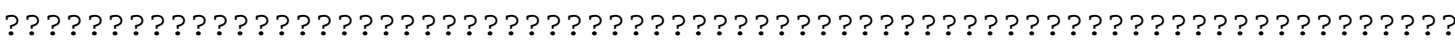
? ? ? ? ? ? ? ? ? ? ? ? ? ? ? ? ? ? ? ? ? ? ? ? ? ? ? ? ? ? ? ? ? ? ? ? ? ? ? ? ? ? ? ? ? ? ? ? ? ? ? ? ? ? ? ? ? ? ? ? ? ? ? ? ? ? ? ? ? ? ? ? ? ? ? ? ? ? ? ? ? ? ? ? ? ? ? ? ? ? ? ? ? ? ? ? ? ? ? ? ? ? ? ? ? ? ? ? ? ? ? ? ? ? ? ? ? ? ? ? ? ? ? ? ? ? ? ? ? ? ? ? ? ? ? ? ? ? ?

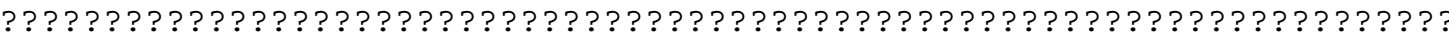
? ? ? ? ? ? ? ? ? ? ? ? ? ? ? ? ? ? ? ? ? ? ? ? ? ? ? ? ? ? ? ? ? ? ? ? ? ? ? ? ? ? ? ? ? ? ? ? ? ? ? ? ? ? ? ? ? ? ? ? ? ? ? ? ? ? ? ? ? ? ? ? ? ? ? ? ? ? ? ? ? ? ? ? ? ? ? ? ? ? ? ? ? ? ? ? ?

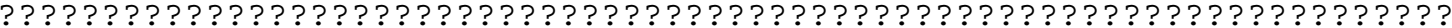

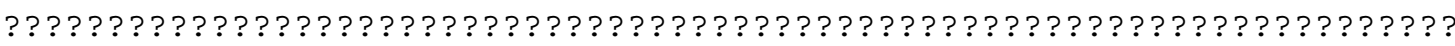

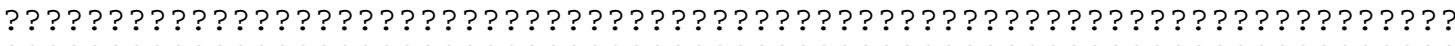
? ? ? ? ? ? ? ? ? ? ? ? ? ? ? ? ? ? ? ? ? ? ? ? ? ? ? ? ? ? ? ? ? ? ? ? ? ? ? ? ? ? ? ? ? ? ? ? ? ? ? ? ? ? ? ? ? ? ? ? ? ? ? ? ? ? ? ? ? ? ? ? ? ? ? ? ? ? ? ? ? ? ? ? ? ?? ? ? ? ? ? ? ? ? ? ? ? ? ? ? ? ? ? ? ? ? ? ? ? ? ? ? ? ? ? ? ? ? ? ? ? ? ? ? ? ? ? ? ? ? ? ? ? ? ? ? ? ? ? ? ? ? ? ? ? ? ? ? ? ? ? ? ? ? ? ? ? ? ? ? ? ? ? ? ? ? ? ? ? ? ? ? ? ? ? ? ? ? ? ? ? ? ? ? ? ? ? ? TGTAATTTATGAAGGATTAAAAGGTGGTTTANATTTCTTAAAAGATGATGAGAATATTAACTCACAACCA

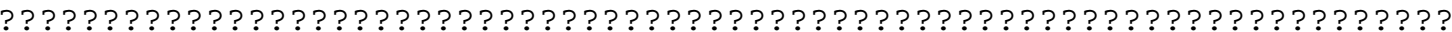
? ? ? ? ? ? ? ? ? ? ? ? ? ? ? ? ? ? ? ? ? ? ? ? ? ? ? ? ? ? ? ? ? ? ? ? ? ? ? ? ? ? ? ? ? ? ? ? ? ? 
M. varians_CHMP7 FFP A. islandi $\bar{C} a$ CHMP 1 MICH A. - skvortzowīi

A._baicalensis

A._sub_9D1

A. - sp. 3 - FR

A. sub $E \bar{R} B$

A._sub_LEW

A. - amb PII 7

A. $a m b \mathrm{~N} 17$

A._amb_FL8

A. - amb_BDL1

A. amb_WLP1

A._nyas̄sensis_GB

A. - valida N2

A._gran_o $\bar{R} 12$

A. sp. 1

A._amb_WAT

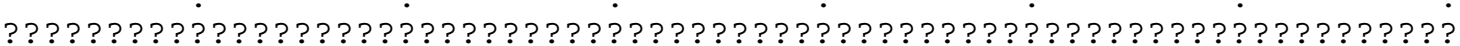

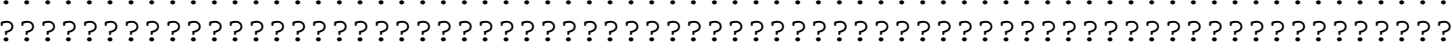

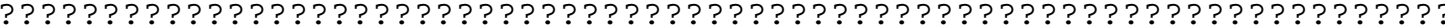
? ? ? ? ? ? ? ? ? ? ? ? ? ? ? ? ? ? ? ? ? ? ? ? ? ? ? ? ? ? ? ? ? ? ? ? ? ? ? ? ? ? ? ? ? ? ? ? ? ? ?

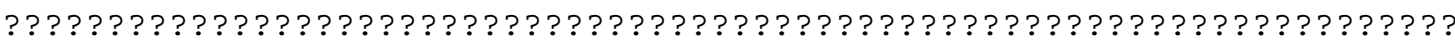
? ? ? ? ? ? ? ? ? ? ? ? ? ? ? ? ? ? ? ? ? ? ? ? ? ? ? ? ? ? ? ? ? ? ? ? ? ? ? ? ? ? ? ? ? ? ? ? ? ? ? ? ? ? ? ? ? ? ? ? ? ? ? ? ? ? ? ? ? ? ? ? ? ? ? ? ? ? ? ? ? ? ? ? ? ? ? ? ? ? ? ? ? ? ? ?

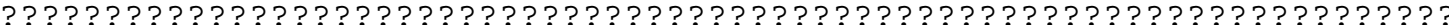

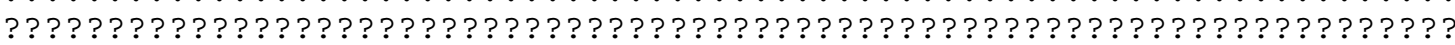
? ? ? ? ? ? ? ? ? ? ? ? ? ? ? ? ? ? ? ? ? ? ? ? ? ? ? ? ? ? ? ? ? ? ? ? ? ? ? ? ? ? ? ? ? ? ? ? ? ? ? ? ? ? ? ? ? ? ? ? ? ? ? ? ? ? ? ? ? ? ? ? ? ? ? ? ? ? ? ? ? ? ? ? ? ? ? ? ? ? ? ? ? ? ? ? ?

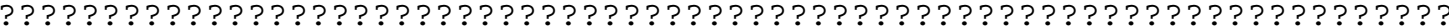

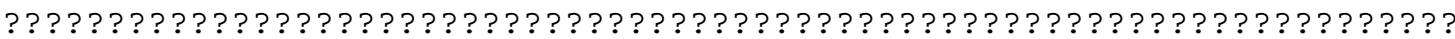

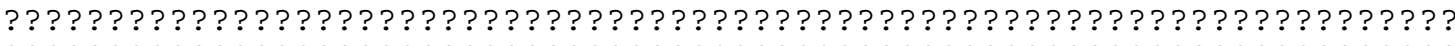
?? ? ? ? ? ? ? ? ? ? ? ? ? ? ? ? ? ? ? ? ? ? ? ? ? ? ? ? ? ? ? ? ? ? ? ? ? ? ? ? ? ? ? ? ? ? ? ? ? ? ? ? ? ? ? ? ? ? ? ? ? ? ? ? ? ? ? ? ? ? ? ? ? ? ? ? ? ? ? ? ? ? ? ? ? ? ?? ? ? ? ? ? ? ? ? ? ? ? ? ? ? ? ? ? ? ? ? ? ? ? ? ? ? ? ? ? ? ? ? ? ? ? ? ? ? ? ? ? ? ? ? ? ? ? ? ? ? ? ? ? ? ? ? ? ? ? ? ? ? ? ? ? ? ? ? ? ? ? ? ? ? ? ? ? ? ? ? ? ? ? ? ? ? ? ? ? ? ? ? ? ? ? ? ? ? ? ? ? ? TTTATGCGTTGGAGAGAACGTTTCTTAAATTGTATGGAAGGTATTAACCGTGCTTCTGCTGCAACAGGTG

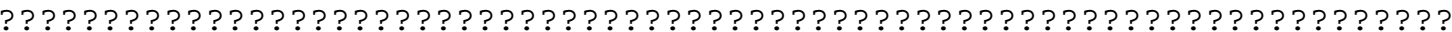
? ? ? ? ? ? ? ? ? ? ? ? ? ? ? ? ? ? ? ? ? ? ? ? ? ? ? ? ? ? ? ? ? ? ? ? ? ? ? ? ? ? ? ? ? ? ? ? ? ? ? 
M._varians_CHMP7_FFP A. islandi $\bar{c}$ _CHMP 1 MICH A. skvortzowi

A._baicalensis

A._sub_9D1

A. - sp. 3 - FR

A. sub_ERB

A._sub_LEW

A._amb_PII 7

A. amb N17

A._amb_FL8

A. - amb_BDL1

A. amb WLP1

A._nyas̄sensis_GB

A. valida N2

A. gran_oR 12

A. sp. 1

A._amb_WAT

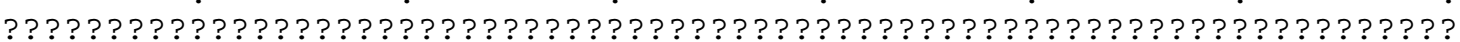
? ? ? ? ? ? ? ? ? ? ? ? ? ? ? ? ? ? ? ? ? ? ? ? ? ? ? ? ? ? ? ? ? ? ? ? ? ? ? ? ? ? ? ? ? ? ? ? ?

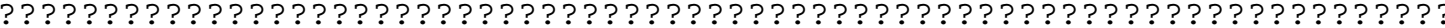
? ? ? ? ? ? ? ? ? ? ? ? ? ? ? ? ? ? ? ? ? ? ? ? ? ? ? ? ? ? ? ? ? ? ? ? ? ? ? ? ? ? ? ? ? ? ? ? ? ? ? ?

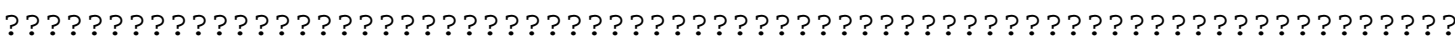
? ? ? ? ? ? ? ? ? ? ? ? ? ? ? ? ? ? ? ? ? ? ? ? ? ? ? ? ? ? ? ? ? ? ? ? ? ? ? ? ? ? ? ? ? ? ? ? ? ? ? ? ? ? ? ? ? ? ? ? ? ? ? ? ? ? ? ? ? ? ? ? ? ? ? ? ? ? ? ? ? ? ? ? ? ? ? ? ? ? ? ? ?

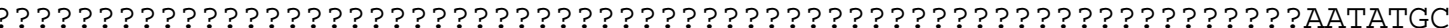

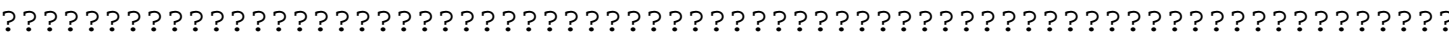

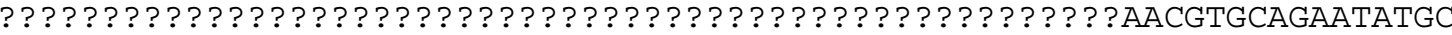

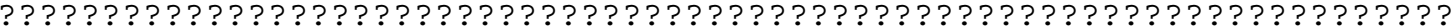

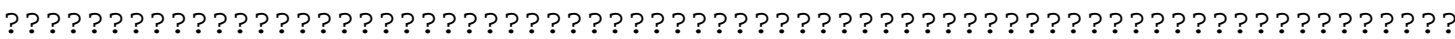
? ? ? ? ? ? ? ? ? ? ? ? ? ? ? ? ? ? ? ? ? ? ? ? ? ? ? ? ? ? ? ? ? ? ? ? ? ? ? ? ? ? ? ? ? ? ? ? ? ? ? ? ? ?AATATGC ? ? ? ? ? ? ? ? ? ? ? ? ? ? ? ? ? ? ? ? ? ? ? ? ? ? ? ? ? ? ? ? ? ? ? ? ? ? ? ?GATGAAGTTATTAAACGTGCAGAATATGC

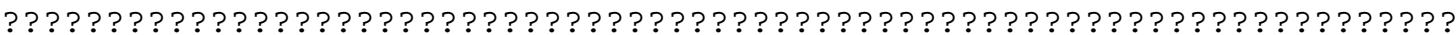
? ? ? ? ? ? ? ? ? ? ? ? ? ? ? ? ? ? ? ? ? ? ? ? ? ? ? ? ? ? ? ? ? ? ? ? ? ? ? ? AACGTGCAGAATATGC AAGTTAAAGGTTCATATTTAAACATTACAGCTGCAACAATGGAAGAAGTTATTAAACGTGCAGAATATGC

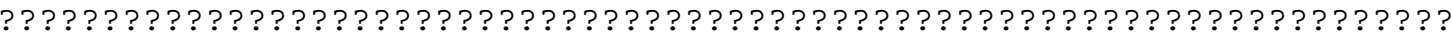
? ? ? ? ? ? ? ? ? ? ? ? ? ? ? ? ? ? ? ? ? ? ? ? ? ? ? ? ? TGAAGTTATTAAACGTGCAGAATATGC 
M._varians_CHMP7_FFP

A. - islandi $\bar{C} a$ CHM $\bar{P} 1$ MICH

A. skvortzowi

A. baicalensis

A._sub_9D1

A. $s p \cdot{ }^{3}$ FR

A._sub_ERB

A._sub_LEW

A._amb_PII 7

A. $a m b$ N17

A._amb_FL8

A. amb_BDL1

A. amb WLP1

A._nyas̄sensis_GB

A. valida N2

A. gran OR 12

A. sp. 1

A._amb_WAT

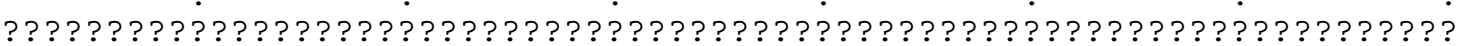
? ? ? ? ? ? ? ? ? ? ? ? ? ? ? ? ? ? ? ? ? ? ? ? ? ? ? ? ? ? ? ? ? ? ? ? ? ? ? ? ? ? ? ? ? ? ? ? ?

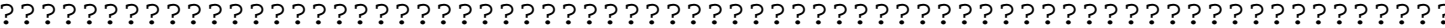
? ? ? ? ? ? ? ? ? ? ? ? ? ? ? ? ? ? ? ? ? ? ? ? ? ? ? ? ? ? ? ? ? ? ? ? ? ? ? ? ? ? ? ? ? ? ? ? ? ? ? ? ? ? ? ? ? GAAGTAGGTTCTGTAATTATCATGATTGACTTAGTTATGGGTTACACAGCAATTCAATCAATTGCT ?? ? ? ? ? ? ? ? ? ? ? ? ? ? ? ? ? ? ? ? ? ? ? ? ? ? ? ? ? ? ? ? ? ? ? ? ? ? ? ? ? ? ? ? ? ? ? ? ? ? TAAAGAAGTAGGTTCTATAATTGTAATGATCGACTTAGTTATGGGGTATACAGCAATTCAAACAGCTGCT ? ? ? ? ? ? ? ? ? ? ? ? ? ? GTAATTATCATGATTGACTTAGTTATGGGTTACACAGCAATTCAATCAATTGCT TAAAGAAGTAGGTTCTGTAATTGTAATGATCGATTTAGTTATGGGTTACACAGCAATTCAAACCGCTGCT ? ? ? ? ? ? ? ? ? ? ? ? ? ? ? ? ? ? ? ? ? ? ? ? ? ? ? ? ? TTATGGGTTACACAGCAATTCAAACAGCTGCT ? ? ? ? ? ? GTAGGTTCTGTAATTGTAATGATCGATTTAGTTATGGGTTACACAGCAATTCAAACCGCTGCT TAAAGAAGTAGGTTCTGTAATTGTAATGATCGATTTAGTTATGGGTTACACAGCAATTCAAACCGCTGCT TAAAGAAGTAGGTTCTGTAATTGTAATGATCGATTTAGTTATGGGTTACACAGCAATTCAAACCGCTGCT ?? ? ? ? ? ? ? ? ? ? ? ? ? ? ? ? ? ? ? ? ? ? ? ? ? ? ? ? ? ? ? ? ? ? ? ? ? ? ? ? ? ? ? ? ? ? ? ? ? ? TAAAGAAGTAGGTTCTATAATTGTAATGATCGACTTAGTTATGGGCTATACAGCAATTCAAACAGCTGCT TAAANAAGTTGGTTCTGTAATTGTAATGATCGACTTAGTTATGGGTTACACAGCAATTCAATCAGCTGCT ? ? ? ? ? ? ? ? ? ? ? ? ? ? ? ? ? TAATGATCGATTTAGTTATGGGTTACACAGCAATTCAAACCGCTGCT TAAAGAAGTAGGTTCTGTAATTGTAATGATCGATTTAGTTATGGGTTACACAGCAATTCAAACCGCTGCT 
M._varians_CHMP7_FFP

A. - islandica_CHMP 1 MICH

A. skvortzowi

A. baicalensis

A._sub_9D1

A. $\mathrm{sp} .3 \mathrm{FR}$

A._sub_ERB

A._sub_LEW

A._amb_PII 7

A. $a m b$ N17

A._amb_FL8

A._amb_BDL1

A. amb WLP1

A._nyas̄sensis_GB

A. valida N2

A. gran OR 12

A. sp. 1

A._amb_WAT

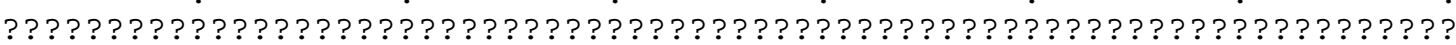

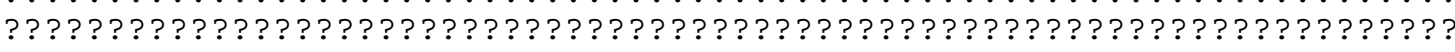

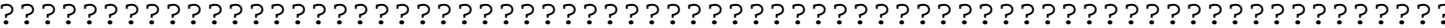
? ? ? ? ? ? ? ? ? ? ? ? ? ? ? ? ? ? ? ? ? ? ? ? ? ? ? ? ? ? ? ? ? ? ? ? ? ? ? ? ? ? ? ? ? ? ? ? ? ? ? ? ? ? ? CTTTGGTCTCGTGATAATGATATGATTTTACATTTACACCGTGCAGGTAATTCAACTTATGCTCGTCAAA

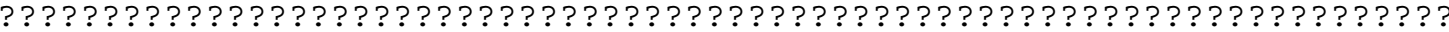
ATTTGGGCTCGTGAAAACGATATGCTTTTACATTTACACCGTGCAGGTAACTCAACATATGCTCGTCAAA CTTTGGTCTCGTGATAATGATATGATTTTACATTTACACCGTGCAGGTAATTCAACTTATGCTCGTCAAA ATTTGGTCTCGTGAAAACGATATGCTTTTACATTTACACCGTGCAGGTAACTCTACATATGCTCGCCAAA ATTTGGGCTCGTGAAAACGATATGCTTTTACATTTACACCGTGCAGGTAACTCTACATATGCTCGCCAAA ATTTGGTCTCGTGAAAACGATATGCTTTTACATTTACACCGTGCAGGTAACTCTACATATGCTCGCCAAA ATTTGGTCTCGTGAAAACGATATGCTTTTACATTTACACCGTGCAGGTAACTCTACATATGCTCGCCAAA ATTTGGTCTCGTGAAAACGATATGCTTTTACATTTACACCGTGCAGGTAACTCTACATATGCTCGCCAAA

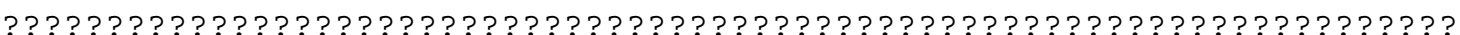
ATTTGGGCTCGTGAAAACGATATGCTTTTACATTTACACCGTGCAGGTAACTCAACATATGCTCGTCAAA ATTTGGGCGCGTGAAAATGATATGATTTTACATTTACACCGTGCGGGTAATTCTACTTATGCTCGTCAAA ATTTGGTCTCGTGAAAACGATATGCTTTTACATTTACACCGTGCAGGTAACTCTACATATGCTCGCCAAA ATTTGGTCTCGTGAAAACGATATGCTTTTACATTTACACCGTGCAGGTAACTCTACATATGCTCGCCAAA 
M._varians_CHMP7_FFP

A._islandi $\bar{C} a \_C H M \bar{P} 1$ MICH

A. skvortzowi

A. baicalensis

A._sub_9D1

A. $\mathrm{sp} .3 \mathrm{FR}$

A._sub_ERB

A._sub_LEW

A._amb_PII 7

A. amb N17

A._amb_FL8

A._amb_BDL1

A. amb WLP1

A._nyas̄sensis_GB

A. valida N2

A. gran OR 12

A. sp. 1

A._amb_WAT

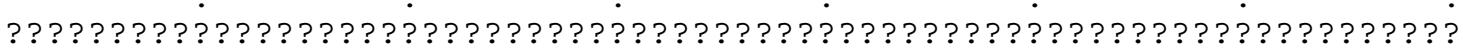
? ? ? ? ? ? ? ? ? ? ? ? ? ? ? ? ? ? ? ? ? ? ? ? ? ? ? ? ? ? ? ? ? ? ? ? ? ? ? ? ? ? ? ? ? ? ? ? ?

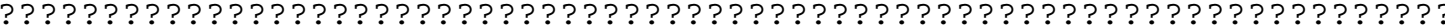
? ? ? ? ? ? ? ? ? ? ? ? ? ? ? ? ? ? ? ? ? ? ? ? ? ? ? ? ? ? ? ? ? ? ? ? ? ? ? ? ? ? ? ? ? ? ? ? ? ? ? ? ? ? ? AAAATCATGGTATTAACTTCCGTGTAATTGTAAATGGATGCGTATGTCTGG-TGTAGATCATATCCACG

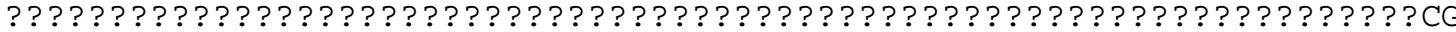
AAAATCATGGTATTAACTTCCGTGTAATCTGTAAATGGATGCGTATGTCTGGGTGTAGATCATATTCATG AAAATCATGGTATTAACTTCCGTGTAATTTGTAAATGGATGCGTATGTCTGG-TGTAGATCATATCCACG AAAATCATGGTATTAACTTCCGTGTAATCTGTAAATGGATGCGTATGTCTGG-TGTAGATCATATTCATG AAAATCATGGTATTAACTTCCGTGTAATCTGTAAATGGATGCGTATGTCTGG-TGTAGATCATATTCATG AAAATCATGGTATTAACTTCCGTGTAATCTGTAAATGGATGCGTATGTCTGG-TGTAGATCATATTCATG AAAATCATGGTATTAACTTCCGTGTAATCTGTAAATGGATGCGTATGTCTGG-TGTAGATCATATTCATG AAAATCATGGTATTAACTTCCGTGTAATCTGTAAATGGATGCGTATGTCTGG - TGTAGATCATATTCATG ? ? ? ?? ?? ? ? ? ? ? ? ? ? ? ? ? ? ? ? ? ? ? ? ? ? ? ? ? ? ? ? ? ? ? ? ? ? ? ? ? ? ? ? ? ? ? ? AAAATCATGGGATTAACTTCCGTGTAATCTGTAAATGGATGCGTATGTCTGG-TGTAGATCATATTCATG AAAATCATGGTATCAACTTCCGTGTAATCTGTAAATGGATGCGTATGTCTGG - TGTAGACCATATTCATG AAAATCATGGTATTAACTTCCGTGTAATCTGTAAATGGATGCGTATGTCTGG-TGTAGATCATATTCATG AAAATCATGGTATTAACTTCCGTGTAATCTGTAAATGGATGCGTATGTCTGG-TGTAGATCATATTCATG 
M._varians_CHMP7_FFP

A. islandi $\bar{C} a$ CHM $\bar{P} 1$ MICH

A. skvortzowi

A. baicalensis

A._sub_9D1

A. sp. 3 FR

A. sub $E \overline{R B}$

A._sub_LEW

A. amb PII7

A. $a m b$ N17

A._amb_FL8

A. amb BDLI

A. amb WLP1

A._nyas̄sensis_GB

A. valida N2

A. gran OR12

A. sp. 1

A._amb_WAT

? ? ? ? ? ? ? ? ? ? ? ? ? ? ? ? ? ? ? ? ? ? ? ? ? ? ? ? ? ? ? ? TGATTAAAGGTTTCTACACTACTTTATTAGC ? ? ? ? ? ? ? ? ? ? ? ? ? ? ? ? ? ? ? ? ? ? ? ? ? ? ? ? ? ? ? ?AAGGTTTTTACATTACTTTATTAGC

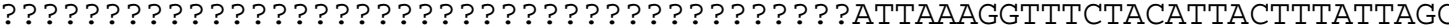
? ? ? ? ? ? ? ? ? ? ? ? ? ? ? ? ? ? ? ? ? ? ? ? ? ? ? ? ? ? ? ? ?ATTAAAGGTTCCTACATTACTTTATTAGC CTGGTACAGTTGTAGGTAAATTAGAAGGTGATCCTTTAATGATTAGAGGTTTCTATATTACCTTATTAGC CTGGTACAGTTGTAGGTAAATTAGAAGGTGATCCTTTAATGATTAAAGGTTTCTATACTACTTTATTAGC CTGGTACAGTTGTAGGTAAATTAGAAGGTGATCCTTTAATGATCAAAGGTTTCTATACTACTTTATTAGC CTGGTACAGTTGTAGGTAAATTAGAAGGTGATCCTTTAATGATTAGAGGTTTCTATATTACCTTATTAGC CTGGTACAGTTGTAGGTAAATTAGAAGGTGATCCTTTAATGATCAAAGGTTTCTATACTACTTTATTAGC CTGGTACAGTTGTAGGTAAATTAGAAGGTGATCCTTTAATGATCAAAGGTTTCTATACTACTTTATTAGC CTGGTACAGTTGTAGGTAAATTAGAAGGTGATCCTTTAATGATCAAAGGTTTCTATACTACTTTATTAGC CTGGTACAGTTGTAGGTAAATTAGAAGGTGATCCTTTAATGATCAAAGGTTTCTATACTACTTTATTAGC CTGGTACAGTTGTAGGTAAATTAGAAGGTGATCCTTTAATGATCAAAGGTTTCTATACTACTTTATTAGC ? ? ? ? ? ? ? ? ? ? ? ? ? ? ? ? ? ? ? ? ? ? ? ? ? ? ? ? ? ? ?ATCAAAGGTTTCTATACTACTTTATTAGC CTGGTACAGTTGTAGGTAAATTAGAAGGTGATCCTTTAATGATCAAAGGTTTCTATACTACTTTATTAGC CTGGTACAGTTGTAGGTAAATTAGAAGGTGATCCTTTAATGATCAAAGGTTTCTATACTACGTTATTAGC CTGGTACAAGTGTAGGTAAATTAGAAGGTGATCCTTTAATGATTAAAGGTTTCTACACTACTTTATTAGC CTGGTACAGTTGTAGGTAAATTAGAAGGTGATCCTTTAATGATCAAAGGTTTCTATACTACTTTATTAGC 
._varians_CHMP7_FFP

A. islandi $\bar{C} a$ CHM $\bar{P} 1$ MICH

A. - skvortzowīi

A. baicalensis

A._sub_9D1

A. sp. 3 FR

A. sub $E \overline{R B}$

A._sub_LEW

A. amb PII7

A. amb N17

A._amb_FL8

A. amb BDL1

A. amb WLP1

A._nyas̄sensis_GB

A. valida N2

A. gran OR12

A. $s p .1$

A._amb_WAT
AACAGAGTTAAAAGTTAACTTACCATATGGTATCTTCTTCGATATGGATTGGGCTAGTTTACGTAGATGT TACTGAATTAAAAGTTAATTTAGCTTACGGTATTTTCTTCGATATGGACTGGGCTAGTTTACGTAAATGT TACTGAATTAAAAGTTAATTTAGCTTACGGTATTTTCTTCGATATGGATTGGGCTAGTTTACGTAAATGT TACTGAATTAAAAGTTAATTTAGCTTACGGTATTTTCTTCGATATGGATTGGGCTAGTTTACGTAAATGT TACTGAATTAAAAGTTAATTTAGCATACGGTATTTTCTTTGATATGGATTGGGCTAGTTTACGTAAATGT TACTGAATTAAAAGTTAATTTAGCATACGGTATTTTCTTTGATATGGATTGGGCTAGTTTACGTAAATGT TACTGAATTAAAAGTTAATTTAGCATACGGTATTTTCTTTGATATGGATTGGGCTAGTTTACGTAAATGT TACTGAATTAAAAGTTAATTTAGCATACGGTATTTTCTTTGATATGGATTGGGCTAGTTTACGTAAATGT TACAGAATTAAAAGTTAATTTAGCTTACGGTATTTTCTTTGATATGGATTGGGCTAGTTTACGTAAATGT TACAGAATTAAAAGTTAATTTAGCTTACGGTATTTTCTTTGACATGGATTGGGCTAGTTTACGTAAATGT TACAGAATTAAAAGTTAATTTAGCTTACGGTATTTTCTTTGATATGGATTGGGCTAGTTTACGTAAATGT TACAGAATTAAAAGTTAATTTAGCTTACGGTATTTTCTTTGATATGGATTGGGCTAGTTTACGTAAATGT TACAGAATTAAAAGTTAATTTAGCTTACGGTATTTTCTTTGATATGGATTGGGCTAGTTTACGTAAATGT TACAGAATTAAAAGTTAATTTAGCTTACGGTATTTTCTTTGACATGGATTGGGCTAGTTTACGTAAATGT TACTGAATTAAAAGTTAATTTAGCTTACGGTATTTTCTTTGATATGGATTGGGCTAGTTTACGTAAATGT AACTGAATTAAAAGTTAATTTAGCATATGGTATTTTCTTTGATATGGACTGGGCTAGTTTACGTAAATGT TACTGAATTAAAAGTTAACTTACCATACGGTATCTTTTTCGATATGGATTGGGCTAGTTTACGTAAATGT TACAGAATTAAAAGTTAATTTAGCTTACGGTATTTTCTTTGATATGGATTGGGCTAGTTTACGTAAATGT 
._varians_CHMP7_FFP

A._islandi $\bar{C} a$ CHMP 1 _MICH

A. - skvortzowīi

A. baicalensis

A._sub_9D1

A. sp. 3 FR

A._sub_ERB

A._sub_LEW

A. amb_PII7

A. $a m b$ N17

A._amb_FL8

A. amb BDL1

A. amb WLP1

A._nyas̄sensis_GB

A. valida N2

A. gran OR12

A. $s p .1$

A._amb_WAT
ATGCCGGTAGCATCTGGTGGTATCCACTGTGGTCAAATGCACCAATTATTAATTTATTTAGGTGATGACG ATGCCAGTAGCTTCTGGTGGTATTCATTGTGGTCAAATGCACCAATTACTTACATATTTAGGTGATGATG ATGCCAGTAGCTTCTGGTGGTATTCATTGTGGTCAAATGCACCAATTACTTACATATTTAGGTGATGATG ATGCCAGTAGCTTCTGGTGGTATTCATTGTGGTCAAATGCACCAATTACTTACATATTTAGGTGATGATG TTACCTGTAGCTTCTGGCGGTATCCATTGTGGTCAAATGCACCAATTAATTACATACTTAGGTGATGACG TTACCTGTAGCTTCTGGCGGTATCCATTGTGGTCAAATGCACCAATTAATTACATACTTAGGTGACGACG TTACCTGTAGCTTCTGGCGGTATCCATTGTGGTCAAATGCACCAATTAATTACATACTTAGGTGATGACG TTACCTGTAGCTTCTGGCGGTATCCATTGTGGTCAAATGCACCAATTAATTACATACTTAGGTGATGACG ATGCCTGTAGCTTCAGGTGGTATCCACTGTGGTCAAATGCACCAATTAATTACATATTTAGGTGATGATG ATGCCTGTAGCTTCAGGTGGTATCCACTGTGGTCAAATGCACCAATTAATTACATATTTAGGTGATGATG ATGCCTGTAGCTTCAGGTGGTATCCACTGTGGTCAAATGCACCAATTAATTACATATTTAGGTGATGATG ATGCCTGTAGCTTCAGGTGGTATCCACTGTGGTCAAATGCACCAATTAATTACATATTTAGGTGATGATG ATGCCTGTAGCTTCAGGTGGTATCCACTGTGGTCAAATGCACCAATTAATTACATATTTAGGTGATGATG ATGCCTGTAGCTTCAGGTGGTATCCACTGTGGTCAAATGCACCAATTAATTACATATTTAGGTGATGATG ATGCCTGTAGCTTCAGGTGGTATTCACTGTGGTCAAATGCATCAATTAATTACATATTTAGGTGATGATG ATGCCAGTAGCTTCAGGTGGTATTCACTGTGGTCAAATGCATCAATTAATCACATATTTAGGTGATGATG TTACCAGTAGCTTCTGGTGGTATTCACTGTGGTCAAATGCACCAATTAATCACTTATTTAGGTGATGATG ATGCCTGTAGCTTCAGGTGGTATCCACTGTGGTCAAATGCACCAATTAATTACATATTTAGGTGATGATG 
A._islandi $\bar{C} a$ CHMP 1 MICH

A. skvortzowi

A. baicalensis

A._sub_9D1

A. $\mathrm{sp} .3 \mathrm{FR}$

A. $s u b-E \bar{R} B$

A._sub_LEW

A._amb_PII 7

A. $a m b$ N17

A._amb_FL8

A. $a m b$ BDL1

A. amb WLP1

A._nyas̄sensis_GB

A. valida N2

A. gran OR 12

A. sp. 1

A._amb_WAT
TAGTATTACAATTTGGTGGTGGTACTATTGGTCACCCTGATGGTATTCAAGCTGGTGCTACAGCTAACCG TAATTTTACAATTTGGTGGTGGTACAATTGGTCACCCTGATGGTATTCAAGCTGGTGCAACAGCAAACCG TAATTTTACAATTTGGTGGTGGTACAATTGGTCACCCTGATGGTATTCAAGCGGGTGCAACAGCAAACCG TAATTTTACAATTTGGTGGTGGTACAATTGGTCACCCTGATGGTATTCAAGCGGGTGCAACAGCAAACCG TAGTATTACAATTGGTGGTGGTACAATTGGTCACCCTGATGGTATTCAAGCGGGTGCAACAGCTAACCG TAGTATTACAATTTGGTGGTGGTACAATTGGTCACCCTGATGGTATTCAAGCGGGTGCAACAGCTAACCG TAGTATTACAATTTGGTGGTGGTACAATTGGTCACCCTGATGGTATTCAAGCGGGTGCAACAGCTAACCG TAGTATTACAATTGGTGGTGGTACAATTGGTCACCCTGATGGTATTCAAGCGGGTGCAACAGCTAACCG TAGTATTACAATTTGGTGGTGGTACAATTGGTCACCCTGATGGTATTCAAGCAGGTGCAACAGCAAACCG TAGTATTACAATTTGGTGGTGGTACAATTGGTCACCCTGATGGTATTCAAGCAGGTGCAACAGCAAACCG TAGTATTACAATTTGGTGGTGGTACAATTGGTCACCCTGATGGTATTCAAGCAGGTGCAACAGCAAACCG TAGTATTACAATTTGGTGGTGGTACAATTGGTCACCCTGATGGTATTCAAGCAGGTGCAACAGCAAACCG TAGTATTACAATTTGGTGGTGGTACAATTGGTCACCCTGATGGTATTCAAGCAGGTGCAACAGCAAACCG TAGTATTACAATTTGGTGGTGGTACAATTGGTCACCCTGATGGTATTCAAGCAGGTGCAACAGCAAACCG TAGTATTACAATTTGGTGGTGGTACAATTGGTCACCCTGATGGTATTCAAGCAGGTGCAACAGCAAACCG TTGTGTTACAATTTGGTGGTGGTACAATTGGTCACCCTGATGGTATTCAAGCAGGTGCAACAGCAAACCG TAGTATTACAATTTGGTGGTGGTACAATTGGTCACCCTGATGGTATTCAAGCAGGTGCAACAGCTAACCG TAGTATTACAATTGGTGGTGGTACAATTGGTCACCCTGATGGTATTCAAGCAGGTGCAACAGCAAACCG 
M._varians_CHMP7_FFP

A._islandi $\bar{C} a \_C H M \bar{P} 1$ MICH

A. skvortzowii

A. baicalensis

A._sub_9D1

A. $\mathrm{sp} \cdot 3$ FR

A. sub_ERB

A._sub_LEW

A._amb_PII 7

A. $a m b$ N17

A._amb_FL8

A. $a m b$ BDL1

A. amb WLP1

A._nyas̄sensis_GB

A. -valida_N2

A. gran OR 12

A. sp. 1

A._amb_WAT
TGTAGCTTTAGAAGCTATGGTATTAGCTCGTAACGAAGGTTTAGACATTTTCAGTAATGAAGTTGGTCCT TGTAGCTATGGAATCTATGGTATTAGCACGTAACGAAGGTGCGGATATCTTCAGTAATGAAGTTGGTCCA TGTAGCTATGGAATCTATGGTATTAGCACGTAACGAAGGTGCGGATGTCTTCAGTAATGAAGTTGGTCCA TGTAGCTATGGAATCTATGGTATTAGCACGTAACGAAGGCGCGGATGTCTTCAGTAATGAAGTTGGGCCA TGTAGCGATGGAATCTATGGTATTAGCTCGTAATGAAGGTCTAGATGTTTTCAGTAACGAAGTTGGTCCA TGTAGCGATGGAATCTATGGTATTAGCTCGTAATGAAGGCTTAGATGTTTTCAGTAACGAAGTTGGTCCA TGTAGCGATGGAATCTATGGTATTAGCTCGTAATGAAGGTCTAGATGTTTTCAGTAACGAAGTTGGTCCA TGTAGCGATGGAATCTATGGTATTAGCTCGTAATGAAGGTCTAGATGTTTTCAGTAACGAAGTTGGTCCA TGTAGCTATGGAATCTATGGTACTTGCACGTAATGAAGGTGCAGATGTCTTCAGTAACGAAGTGGGTCCA TGTAGCTATGGAATCTATGGTACTTGCACGTAATGAAGGTGCAGATATCTTCAGTAACGAAGTGGGTCCA TGTAGCTATGGAATCTATGGTACTTGCACGTAATGAAGGTGCAGATGTCTTCAGTAACGAAGTGGGTCCA TGTAGCTATGGAATCTATGGTACTTGCACGTAATGAAGGTGCAGATGTCTTCAGTAACGAAGTGGGTCCA TGTAGCTATGGAATCTATGGTACTTGCACGTAATGAAGGTGCAGATGTCTTCAGTAACGAAGTGGGTCCA TGTAGCTATGGAATCTATGGTACTTGCACGTAATGAAGGTGCAGATGTCTTCAGTAATGAAGTGGGTCCA TGTAGCTATGGAATCTATGGTACTAGCACGTAATGAAGGCTTAGATATCTTCAGTAACGAAGTGGGTCCA TGTAGCAATGGAGTCTATGGTATTAGCACGTAATGAAGGTGCAGATGTTTTCAGTAATGAAGTAGGTCCA TGTTGCTTTAGAAGCTATGGTATTAGCTCGTAACGAAGGTCAAGATGTCTTCAGTAACGAAGTTGGTCCG TGTAGCTATGGAATCTATGGTACTTGCACGTAATGAAGGTGCAGATGTCTTCAGTAACGAAGTGGGTCCA 
M._varians_CHMP7_FFP

A._islandi $\bar{C} a$ CHM $\bar{P} 1$ MICH

A. skvortzowii

A. baicalensis

A._sub_9D1

A. $\mathrm{sp} \cdot 3$ FR

A. sub_ERB

A._sub_LEW

A._amb_PII 7

A. amb N17

A._amb_FL8

A. $a m b$ BDL1

A. amb WLP1

A._nyas̄sensis_GB

A. valida N2

A. gran OR 12

A. $s p .1$

A._amb_WAT
CGTATTTTACGCGACGCTGCTAAAACATGTGGTCCTTTACAAACAGCTTTAGATTTATGGAAAGATATTA AAAATTTTACGTGACGCAGCTAAAACATGTGGTCCTTTACAAACAGCTTTAGATTTATGGAAAGATATTA AAAATTTTACGTGACGCAGCTAAAACATGTGGTCCTTTACAAACAGCTTTAGATTTATGGAAAGATATTA AAAATTTTACGTGACGCAGCTAAAACATGTGGTCCTTTACAAACAGCTTTAGATTTATGGAAAGATATTA AAAATTTTACGTGACGCAGCTAAAACATGTGGTCCTTTACAA ? ? ? ? ? ? ? ? ? ? ? ? ? ? ? ? ? ? ? ? ? AAAATTTTACGTGATGCAGCTAAAACATGTGGTCCTCTACAAACAGCTTTAGATTTATGGAAAGATATTA AAAATTTTACGTGACGCAGCTAAAACATGTGGTCCTTTAC? ? ? ? ? ? ? ? ? ? ? ? ? ? ? ? ? ? ? ? ? ? AAAATTTTACGTGACGCAGCTAAAACATGTGGTCCTTTACAAACAGCTTTAGATT? ? ? ? ? ? ? ? ? ? ? ? AGAATTTTACGTGATGCAGC? ? ? ? ? ? ? ? ? ? ? ? ? ? ? ? ? ? ? ? ? ? ? ? ? ? ? ? ? ? ? ? ? ? ? ? ? ? AGAATTTTACGTGATGCAGCTAAAACATGTGGTCCTCTACAAACAGCTTTAGACTTATGGAAAGATATTA AGAATTTTACGTGATGCAGCTAAAACATGTGGTCCTCTACAAACAGCTTTAGACTTATGGAAAGATATTA AGAATTTTACGTGATGCAGCTAAAACATGTGGTCCTCTACAAACAGCTTTAGACTTATGGAAAGATATTA AGAATTTTACG? ? ? ? ? ? ? ? ? ? ? ? ? ? ? ? ? ? ? ? ? ? ? ? ? ? ? ? ? ? ? ? ? ? ? ? ? ? ? ? ? AGAATTTTACGTGATGCAGCTAAAACATGTGGTCCTCTACAAACAGCTTTAGACTTATGGAAAGATATTA AGAATTTTACGTGATGCAGCTAAAACATGTGGTCCTCTACAAACAGCTTTAGACTTATGGAAAGATATTA AAAATTTTACGTGATGCAGCTAAAACATGTGGTCCTCTACAAACAGCTTTAGATTTATGGAAAGATATTA AAAATTTTACGTGATGCAGCTAAAACTTGCGGTCCGTTACAAACAGCTTTAGATTTATGGAAAGATATTA AGAATTTTACGTGATGCAGCTAAAA? ? ? ? ? ? ? ? ? ? ? ? ? ? ? ? ? ? ? ? ? ? ? ? ? ? ? ? ? ? ? ? 
varians CHMP7 FFP

A. islandi $\bar{c}$. CHMP 1 MICH

A. skvortzowi

A. baicalensis

A._sub_9D1

A. $s p \cdot{ }^{3}$ FR

A. $s u b-E \bar{R} B$

A._sub_LEW

A._amb_PII 7

A. amb N17

A._amb_FL8

A._amb_BDL1

A. amb WLP1

A._nyas̄sensis_GB

A. valida N2

A. gran OR 12

A. sp. 1

A._amb_WAT

GTTTTAACTATA ? ? ? ? ? ? ? ? ? ? ? ? ? ? ? ? ? ? ? ? ? ? ? ? ? ? ? ? ? ? ? ? ? ? ? ? ? ? ? $\mathrm{GTTT}$ ? ? ? ? ? ? ? ? ? ? ? ? ? ? ? ? ? ? ? ? ? ? ? ? ? ? ? ? ? ? ? ? ? ? ? ? ? ? ? ? ? ? ? ? ? ? ? GTTTTAACTATACTTCTACAGATACTGCTGATTTCGCTGAAACACCAACAGCTAACTTATAATT- - - - T GTTTTAACTATACTTCTACAGATACTGCTGATTTCGCTGAAACACCAACAGCTAACTTATAATT- - - - - T ? ? ? ? ? ? ? ? ? ? ? ? ? ? ? ? ? ? ? ? ? ? ? ? ? ? ? ? ? ? ? ? ? ? ? ? ? ? ? ? ? ? ? ? ? ? ? ? ? ? ? GTTTTAACTATACTTCTACAGATACTGCTGATTTCGCTGAAACGCCAACAGCTAACGTATAACTA- - - T ?? ?? ? ? ? ? ? ? ? ? ? ? ? ? ? ? ? ? ? ? ? ? ? ? ? ? ? ? ? ? ? ? ? ? ? ? ? ? ? ? ? ? ? ? ? ?

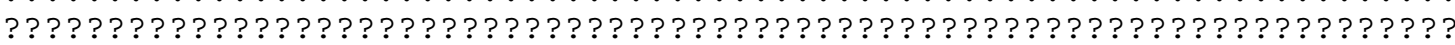

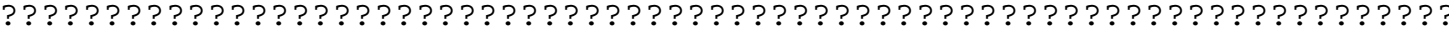

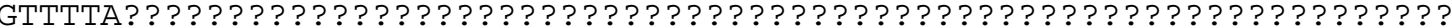
GTTTTAACTATACTTCTACAGA ? ? ? ? ? ? ? ? ? ? ? ? ? ? ? ? ? ? ? ? ? ? ? ? ? ? ? ? ? ? ? ? ? ? ? ? ? ? GTTTTAACTATACTTCTACAGATACTGCTGACTTTGCTGAAACACCAACAGCTAACT? ? ? ? ? ? ? ? ?

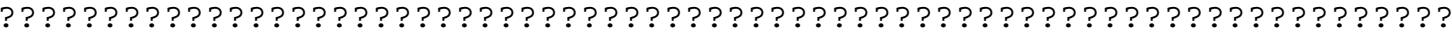
GTTTTAACTATACTTCTACAGATACTGCTGACTTTGCTGAAACACCAACAGCTAACTTATAATT- - - - A G? ? ? ? ? ? ? ? ? ? ? ? ? ? ? ? ? ? ? ? ? ? ? ? ? ? ? ? ? ? ? ? ? ? ? ? ? ? ? ? ? ? ? ? ? ? ? GTTTCAACTATACTTCTACAGATACTGCTGATTTTGCTGAAACACCAACAGCTAACTTATAATTACAATA GTTTCAACTATAGTTCTACAGACACTGCTGACTTC? ? ? ? ? ? ? ? ? ? ? ? ? ? ? ? ? ? ? ? ? ? ? ? ? ? ? ?? ? ? ? ? ? ? ? ? ? ? ? ? ? ? ? ? ? ? ? ? ? ? ? ? ? ? ? ? ? ? ? ? ? ? ? ? ? ? ? ? ? ? ? ? 
. varians_CHMP7_FFP A. islandi $\bar{c}$ _ CHMP 1 MICH

A. skvortzowi

A._baicalensis

A._sub_9D1

A. - sp. 3 - FR

A. sub_ERB

A._sub_LEW

A._amb_PII 7

A. amb N17

A._amb_FL8

A. - amb_BDL1

A. amb_WLP1

A._nyas̄sensis_GB

A. valida N2

A. gran_oR 12

A. sp. 1

A._amb_WAT

?? ?? ? ? ? ? ? ? ? ? ? ? ? ? ? ? ? ? ? ? ? ? ? ? ? ? ? ? ? ? ? ? ? ? ? ? ? ? ? ? ? ? ? ? ? ? ? ? ? ? ? ? ? ? ? ? ? ? ? ? ? ? ? ? ? ? ? ? ? ? ? ? ? ? ? ? ? ? ? ? ? ? ? ? ? ? ? ? ? ? ? ? ? ? ? ? ? ? TAATAACACTCAAAGGAGTATTTGAATAGTGAGACTTACACAAGGTTGCTTCTCTTTTTTACCTGATTTA TAATAACACTCAAAGGAGTATTTGAATAGTGAGACTTACACAAGGTTGCTTCTCTTTTTTACCTGATTTA ? ? ? ? ? ? ? ? ? ? ? ? ? ? ? ? ? ? ? ? ? ? ? ? ? ? ? ? ? ? ? ? ? ? ? ? ? ? ? ? ? ? ? ? ? ? ? ? ? ? ? TAATTATAATAAAAACTAAA? ? ? ? ? ? ? ? ? ? ? ? ? ? ? ? ? ? ? ? ? ? ? ? ? ? ? ? ? ? ? ? ? ? ? ? ? ? ? ? ? ? ? ? ? ? ? ? ? ? ? ? ? ? ? ? ? ? ? ? ? ? ? ? ? ? ? ? ? ? ? ? ? ? ? ? ? ? ? ? ? ? ? ? ? ? ? ? ? ? ?

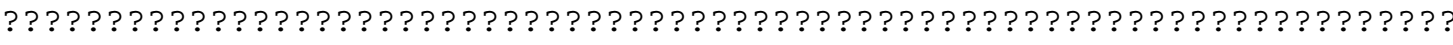

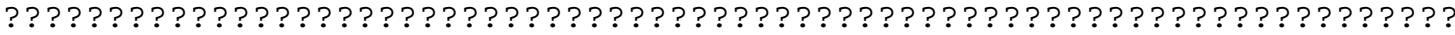
?? ? ? ? ? ? ? ? ? ? ? ? ? ? ? ? ? ? ? ? ? ? ? ? ? ? ? ? ? ? ? ? ? ? ? ? ? ? ? ? ? ? ? ? ? ? ? ? ?

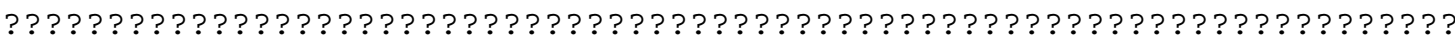

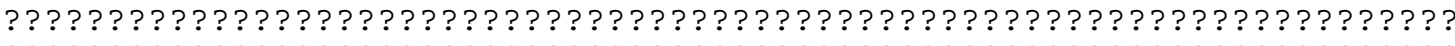
?? ? ? ? ? ? ? ? ? ? ? ? ? ? ? ? ? ? ? ? ? ? ? ? ? ? ? ? ? ? ? ? ? ? ? ? ? ? ? ? ? ? ? ? ? ? ? ? ? ? ? ? ?

TAATAAAAATTAAAGGAGTATTTGAATAGTGAGACTTACACAAGGTTGCTTCTCTTTTTTACCTGATTTA ? ? ? ? ? ? ? ? ? ? ? ? ? ? ? ? ? ? ? ? ? ? ? ? ? ? ? ? ? ? ? ? ? ? ? ? ? ? ? ? ? ? ? ? ? ? ? ?

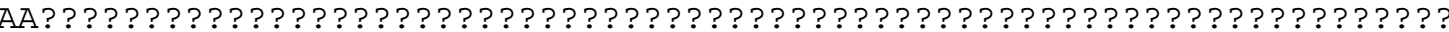
? ? ? ? ? ? ? ? ? ? ? ? ? ? ? ? ? ? ? ? ? ? ? ? ? ? ? ? ? ? ? ? ? ? ? ? ? ? ? ? ? ? ? ? ? ? ? ? ?? ? ? ? ? ? ? ? ? ? ? ? ? ? ? ? ? ? ? ? ? ? ? ? ? ? ? ? ? ? ? ? ? ? ? ? ? ? ? ? ? ? ? ? ? 


\begin{tabular}{|c|c|}
\hline [ & END] \\
\hline[ & . END $r b c L]$ \\
\hline M. varians_CHMP7_FFP & ?? ? ?? ? ? ? ? ? ? ? ? ? ? \\
\hline A. islandica_CHMP 1 MICH & ????????????????? \\
\hline A. skvortzowii & ACTGATTCACAAATCG \\
\hline A. baicalensis & ACTGATTCACAAATCG \\
\hline A. sub_9D1 & ???????????????? \\
\hline A._sp._3_FR & ????????????????? \\
\hline A._sub_ERB & ????????????????? \\
\hline A._sub_LEW & ????????????????? \\
\hline A. ${ }^{-}$amb_PII7 & ????????????????? \\
\hline A._amb_N17 & ????????????????? \\
\hline A._amb_FL8 & ????????????????? \\
\hline A. ${ }^{-} \mathrm{amb} \mathrm{BDL}^{-} \mathrm{C}$ & ????????????????? \\
\hline A. ${ }^{-} b^{-}$WLP 1 & ????????????????? \\
\hline A. nyassensis GB & ACAGATGCTCAAATCG \\
\hline A. valida N2 & ???????????????? \\
\hline A. gran oR 12 & ????????????????? \\
\hline A. $\operatorname{sp.} \overline{1}$ & ???????????????? \\
\hline amb WAT & ? ????? ? ? ? ? ? ? ? ? ? \\
\hline
\end{tabular}


Appendix E. Morphological characters: Morphometric Character Data Coded via a Modified Step-Matrix Gap Weighting Method (32 Character State Limit). 
[

M._varians_CHMP7_FFP

S. Cf. broschii GB

A._islāndica_CHM̄P1_MICH

A._skvortzowi $i$

A. baicalensis

A._sub_9D1

A._sub_WLB3

A. $\mathrm{sp} \cdot 3 \mathrm{FR}$

A. Sub_ERB

A. sub_LEW

A. alpigena

A. amb PII7

A._amb_N17

A. amb FL8

A. $a m b$ BDL1

A._amb_WLP1

A._nyas̄sensis_GB

A. valida N2

A. gran_L $\bar{B}$

A._gran_OR12

A. $-\mathrm{sp} \cdot \overline{1}$

A. $\mathrm{sp} \cdot 2$

A._cren_FH8_L639

A. cren T73 $3 \overline{3}$ L639

Alv̄eolophora

A._agassizii_FH21

A. amb A62

A._amb_MFP

A. amb WAT

A._canad_1571

A. canad 2018

A. dianchiensis

A._distans_FH34

A. $\operatorname{gran} \mathrm{FH}_{\overline{4}} 2$

A. gran FH38

A._gran_1914

A. gran MFP

A._gran_v. jonensis

A._gran_f_HLS

A. gran f $-\mathrm{VH}$

A. herzogii HDSM

A._italica_F $\bar{F} 15$

A._krammerii_nom._prov.

A. lac A38

A. 1 - 1 ac_A62

A. lirāta_A37

A. laevissima_HDSM

A._nyg_A50
Qualitative Chars

1001000000020000713

100000000002020443 ??????????????????

211111611112100503 PAGDC7HA72E98678JGEA2

211511611112100513 NB6DC7HQ77DD587CPL893

21111141111 ? 100523 UMLHQPVUD9NSLHFJMJJT6

211100002120000011 G4BJ6977BEA8685H?D598

211100002120000011 C4EH5291CCDG6255?5B70

$211100002123000102 \quad 37022$ F2B1706171C? 1126

211100002120000011 B28J4E43CD4B494J?9678

211100002120000011 8AJF5A88JEAG6C5C?CA89

2111126111001 ????? ??????????????????

221111611110100011 GEC79FKM85HM694A66B84

221111611110100011 CA7G5KAH78CT5E4E3256B

221111611110100011 DK7A4CBH68EH5D4D43478

221111611110100011 G348K0G9389U6B4A10375

221111611110100011 K69C9DKB76FD694A21696

2211116111121001 ?3 PCJ5DRL883G8H3A9JGLK4

210022720001100011 MDKLCCQBFCKJ8D540?CA9

2141116121201 ?? ?? ? Q0AB8BAL467EDMBUDK6LR

214111612120100231 KL647MCS276JBH9WCJ2GP

210512620001100202 9GBMBNAR559QAL80N?9AH

2111126111101 ????? AN3FEQLV3 0A5546KNK17K

2100327100012032 ? 1 MCGHMCUPA6LPBJ4JSQDAE

2100327100012032 ? 1 LCTENGTDM5RKAA5ARPPC7

2112116111121000 ?3 8BS5B9H8W1D4B7G4QMQJ3

2141116121221001 ? 1 F9QKE7J5P5G7A8A3KKGE6

221111611110100011 E?683DBJ7AER6B5B5558A

221111611110100011 K7A99AJB87EJ5B4877877

221111611110100011 HAAA68F585GB664G88894

211211611110114033 B9DJA3B8DJABFCGTLH5HM

211211611110114033 C8PNC8E6LKHNK4MMQM9SQ

21311161212 ? 111601 TJN6J7R0B9J7MOLLPLAUG

210111211102100123 571C5C539C154C3GNK14 7

214111612120101201 LAA8E9MB2 48BCFCQEK9FH

214111612120101201 KASMF6Q9G6JAF5EBHMFL6

2141???? 21201003 ? 2 K1MPCCKFKLFCB9A1?LAD7

2141116121201001 ? 1 P9CCBDJC56BLCBBEDK7JF

2241116121201012 ? 1 B952176802464A58AJ653

2111 ? ? ? 11121 ? ? ? ? P841F5M2NF22K5HNMJCQF

2111????11121????? S3C5L1S0EG91J8KPNKAPJ

213100005422000311 LH7A887F448A4102NK742

21004271000?2122? 1 QFEEGFNG53FF8H2ETRC89

212411411121114003 HOHCC3C2HPF2B3 JVPL7E7

2101111100002 ????? 9CCDC8GF93D96B77PLE98

2101111100002 ?? ?? ? 9CA999DJ86AA596ALHB77

21011251000?1????? E9FECDGNGMJGDGBSFECGL

213100003312000103 7CDE97BG75CC9E8JPLFA9

2115129213122 ? ? ?? BC8AABEE84DE2A2DMJC3 7 
A. nyg A62

A._pergla_f_A38

A._pfaffiāna_FH37

A. solida $\mathrm{FH}_{\overline{2}} 3$

A. sub ROR

A. sub_T702

A. $\mathrm{sp} .{ }^{3} \mathrm{ROR}$

A._distañs_f ADE

A._paucistriāta_2035

A._sp. 4 T733

A. $\mathrm{sp} \cdot{ }^{5} \mathrm{~h}$

A._sp. ${ }^{-}{ }^{-}$hwg

A._cf._distans_1571

A. humilis

A. tenuoir

A. tenella

A. crassipunctata

A. tethera

A._pseudoamericana

A. muzzanensis

A._pergla_f_KI

A._ceretaña

\begin{tabular}{|c|c|}
\hline & $C$ \\
\hline$\cdot$ & \\
\hline$\perp \perp 3 \perp Z 92 \perp 3 \perp 2$ & AD7A9DDG63FG1526NKAN1 \\
\hline $121003285110003 ? 1$ & 2C7C??3E1339????? ?C? \\
\hline 10112310002100102 & 69399J9835336K7FGF67B \\
\hline 12000002120014003 & CCNLECH4RNE9B3GG?MFF4 \\
\hline 11100002120000011 & 948045595657595 C?A672 \\
\hline 211100002120000011 & E7BG5484BHAG6G5L?A578 \\
\hline 211100002123000102 & $3523252825102 \mathrm{~A} 1 \mathrm{E} ? 4138$ \\
\hline 2101126100001 ?? ??? & 6D9F7J8K87AR5C5BBB98A \\
\hline 213000001120014003 & GCRAHHP 6 TKPDE 7NH? MKMC \\
\hline 2113111111101????? & RDTFPERJG6QDNCKLMJMRN \\
\hline 0111526185203 ????? & 5BCF? ? $\mathrm{CB} 22 \mathrm{E} 6$ ? ? ? ? ?D? \\
\hline 0101523111113 ? ? ?? & 4B59 ? ? 8N2 4BL? ? ? ? ? 6 ? ? \\
\hline 111000021220 ?? ? ? & 1B4M231E9B1C3J2R9A03D \\
\hline 2130000011220 ????? & 4A4G6L7T2532 77AA?N38D \\
\hline 2?11000021120????? & A?? ? 6? ?? S? ? 4 ? 4 ?Q? HE? \\
\hline $2 ? 12000011120001 ? 1$ & 0 ? ? ? 0 ? ? ? ? ? ? 3 ? 2 ? ?M60 ? \\
\hline $2 ? 03111100021 ? 40$ ? 3 & M? ? ? J? ? ?U? ? ? G? K? Q ? 78 ? \\
\hline 2?01115100011000?1 & 2???1?????? ?0? 8?Q? C1? \\
\hline $0 ? 01529100013006 ? 1$ & 3 ? ? ? ? ? ? ? ? ? ? ? ? ? ? ?D? ? \\
\hline 2?41116121211????? & 7?A? 5 ? 0 ? 8 ?B ? 8 ?D? BMLB? \\
\hline 2?11216111121????? & 1 ? ? ? 2 ? ? ?Q? ? ? 1 ? 1 ?QMG1 ? \\
\hline 211211611112104033 & P9D? 9 ?H?V?M?D?H?QMPN? \\
\hline
\end{tabular}

Characters in columns from left to right are as listed 1-39 in the Material and Methods 
Appendix F: Step-Matrices Used to Weight Morphometric Characters Coded via a Modified Step-Matrix Gap Weighting Method (32 Character State Limit). 
USERTYPE meanmantleheight STEPMATRIX $=29$

$\begin{array}{llllll}0 & 1 & 2 & 3 & 4 & 5\end{array}$

.0580 .078

0970.117

[0] $\begin{array}{llllllllllllllllllllllllll}0.078 & 0.02 & 0 & 0.019 & 0.039 & 0.059 & 0.078 & 0.098 & 0.117 & 0.137 & 0.157 & 0.176 & 0.196 & 0.215 & 0.235\end{array}$ $\begin{array}{lllllllllllllllllllllll}0.097 & 0.039 & 0.019 & 0.0 .04 & 0.04 & 0.04 & 0.059 & 0.079 & 0.098 & 0.118 & 0.138 & 0.157 & 0.177 & 0.196 & 0.216\end{array}$

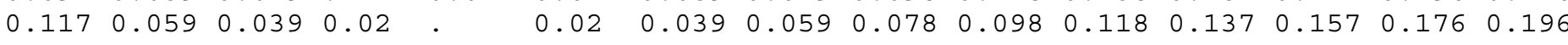

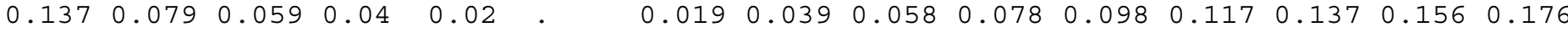
$\begin{array}{llllllllllllllllllllllllll}0.156 & 0.098 & 0.078 & 0.059 & 0.039 & 0.019 & 0.02 & 0.039 & 0.059 & 0.079 & 0.098 & 0.118 & 0.137 & 0.157\end{array}$ $\begin{array}{llllllllllllllllll}0.176 & 0.118 & 0.098 & 0.079 & 0.059 & 0.039 & 0.02 & 0.019 & 0.039 & 0.059 & 0.078 & 0.098 & 0.117 & 0.137\end{array}$ $\begin{array}{lllllllllllllllll}0.195 & 0.137 & 0.117 & 0.098 & 0.078 & 0.058 & 0.039 & 0.019 & 0.02 & 0.04 & 0.059 & 0.079 & 0.098 & 0.118\end{array}$ $\begin{array}{lllllllllllllllll}0.215 & 0.157 & 0.137 & 0.118 & 0.098 & 0.078 & 0.059 & 0.039 & 0.02 & 0.03 & 0.02 & 0.039 & 0.059 & 0.078 & 0.098\end{array}$

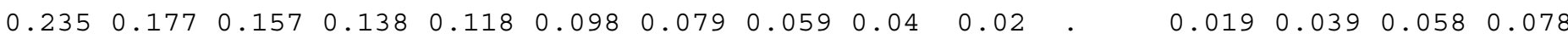
$\begin{array}{llllllllllllllll}0.254 & 0.196 & 0.176 & 0.157 & 0.137 & 0.117 & 0.098 & 0.078 & 0.059 & 0.039 & 0.019 & . & 0.02 & 0.039 & 0.059\end{array}$ $\begin{array}{llllllllllllllllll}0.274 & 0.216 & 0.196 & 0.177 & 0.157 & 0.137 & 0.118 & 0.098 & 0.079 & 0.059 & 0.039 & 0.02 & . & 0.019 & 0.039\end{array}$ $\begin{array}{lllllllllllllllllll}0.293 & 0.235 & 0.215 & 0.196 & 0.176 & 0.156 & 0.137 & 0.117 & 0.098 & 0.078 & 0.058 & 0.039 & 0.019 & 0.03 & 0.02\end{array}$ $\begin{array}{llllllllllllllllll}0.313 & 0.255 & 0.235 & 0.216 & 0.196 & 0.176 & 0.157 & 0.137 & 0.118 & 0.098 & 0.078 & 0.059 & 0.039 & 0.02 & .\end{array}$ $\begin{array}{llllllllllllllllll}0.333 & 0.275 & 0.255 & 0.236 & 0.216 & 0.196 & 0.177 & 0.157 & 0.138 & 0.118 & 0.098 & 0.079 & 0.059 & 0.04 & 0.02\end{array}$ $\begin{array}{lllllllllllllll}0.352 & 0.294 & 0.274 & 0.255 & 0.235 & 0.215 & 0.196 & 0.176 & 0.157 & 0.137 & 0.117 & 0.098 & 0.078 & 0.059 & 0.039\end{array}$ $\begin{array}{lllllllllllllllll}0.372 & 0.314 & 0.294 & 0.275 & 0.255 & 0.235 & 0.216 & 0.196 & 0.177 & 0.157 & 0.137 & 0.118 & 0.098 & 0.079 & 0.059\end{array}$ $\begin{array}{llllllllllllllllll}0.391 & 0.333 & 0.313 & 0.294 & 0.274 & 0.254 & 0.235 & 0.215 & 0.196 & 0.176 & 0.156 & 0.137 & 0.117 & 0.098 & 0.078\end{array}$ $\begin{array}{llllllllllllllll}0.43 & 0.372 & 0.352 & 0.333 & 0.313 & 0.293 & 0.274 & 0.254 & 0.235 & 0.215 & 0.195 & 0.176 & 0.156 & 0.137 & 0.117\end{array}$ $\begin{array}{lllllllllllllll}0.45 & 0.392 & 0.372 & 0.353 & 0.333 & 0.313 & 0.294 & 0.274 & 0.255 & 0.235 & 0.215 & 0.196 & 0.176 & 0.157 & 0.137\end{array}$ $\begin{array}{llllllllllllllll}0.47 & 0.412 & 0.392 & 0.373 & 0.353 & 0.333 & 0.314 & 0.294 & 0.275 & 0.255 & 0.235 & 0.216 & 0.196 & 0.177 & 0.157\end{array}$ $\begin{array}{llllllllllllllll}0.489 & 0.431 & 0.411 & 0.392 & 0.372 & 0.352 & 0.333 & 0.313 & 0.294 & 0.274 & 0.254 & 0.235 & 0.215 & 0.196 & 0.176\end{array}$ $\begin{array}{lllllllllllllll}0.509 & 0.451 & 0.431 & 0.412 & 0.392 & 0.372 & 0.353 & 0.333 & 0.314 & 0.294 & 0.274 & 0.255 & 0.235 & 0.216 & 0.196\end{array}$ $\begin{array}{lllllllllllllll}0.528 & 0.47 & 0.45 & 0.431 & 0.411 & 0.391 & 0.372 & 0.352 & 0.333 & 0.313 & 0.293 & 0.274 & 0.254 & 0.235 & 0.215\end{array}$ $\begin{array}{llllllllllllllllll}0.587 & 0.529 & 0.509 & 0.49 & 0.47 & 0.45 & 0.431 & 0.411 & 0.392 & 0.372 & 0.352 & 0.333 & 0.313 & 0.294 & 0.274\end{array}$ $\begin{array}{lllllllllllllllll}0.901 & 0.843 & 0.823 & 0.804 & 0.784 & 0.764 & 0.745 & 0.725 & 0.706 & 0.686 & 0.666 & 0.647 & 0.627 & 0.608 & 0.588\end{array}$ $\begin{array}{lllllllllllllllllll}0.959 & 0.901 & 0.881 & 0.862 & 0.842 & 0.822 & 0.803 & 0.783 & 0.764 & 0.744 & 0.724 & 0.705 & 0.685 & 0.666 & 0.646\end{array}$ $\begin{array}{llllllllllllllllll}0.999 & 0.941 & 0.921 & 0.902 & 0.882 & 0.862 & 0.843 & 0.823 & 0.804 & 0.784 & 0.764 & 0.745 & 0.725 & 0.706 & 0.686\end{array}$ 
[0] $\quad \begin{array}{lllllllllllllllll}0.333 & 0.352 & 0.372 & 0.391 & 0.43 & 0.45 & 0.47 & 0.489 & 0.509 & 0.528 & 0.587 & 0.901 & 0.959 & 0.999\end{array}$

[1] $\quad \begin{array}{llllllllllllllll}0.275 & 0.294 & 0.314 & 0.333 & 0.372 & 0.392 & 0.412 & 0.431 & 0.451 & 0.47 & 0.529 & 0.843 & 0.901 & 0.941\end{array}$

[2] $\quad \begin{array}{llllllllllllllllll}0.255 & 0.274 & 0.294 & 0.313 & 0.352 & 0.372 & 0.392 & 0.411 & 0.431 & 0.45 & 0.509 & 0.823 & 0.881 & 0.921\end{array}$

[3] $\quad \begin{array}{lllllllllllllll}0.236 & 0.255 & 0.275 & 0.294 & 0.333 & 0.353 & 0.373 & 0.392 & 0.412 & 0.431 & 0.49 & 0.804 & 0.862 & 0.902\end{array}$

[4] $\quad \begin{array}{llllllllllllllll}0.216 & 0.235 & 0.255 & 0.274 & 0.313 & 0.333 & 0.353 & 0.372 & 0.392 & 0.411 & 0.47 & 0.784 & 0.842 & 0.882\end{array}$

[5] $\quad \begin{array}{llllllllllllllllll}0.196 & 0.215 & 0.235 & 0.254 & 0.293 & 0.313 & 0.333 & 0.352 & 0.372 & 0.391 & 0.45 & 0.764 & 0.822 & 0.862\end{array}$

[6] $\quad \begin{array}{llllllllllllllll}0.177 & 0.196 & 0.216 & 0.235 & 0.274 & 0.294 & 0.314 & 0.333 & 0.353 & 0.372 & 0.431 & 0.745 & 0.803 & 0.843\end{array}$

[7] $\quad \begin{array}{lllllllllllllllll}0.157 & 0.176 & 0.196 & 0.215 & 0.254 & 0.274 & 0.294 & 0.313 & 0.333 & 0.352 & 0.411 & 0.725 & 0.783 & 0.823\end{array}$

[8] $\quad \begin{array}{llllllllllllllllll}0.138 & 0.157 & 0.177 & 0.196 & 0.235 & 0.255 & 0.275 & 0.294 & 0.314 & 0.333 & 0.392 & 0.706 & 0.764 & 0.804\end{array}$

[9] $\quad \begin{array}{llllllllllllllllll}0.118 & 0.137 & 0.157 & 0.176 & 0.215 & 0.235 & 0.255 & 0.274 & 0.294 & 0.313 & 0.372 & 0.686 & 0.744 & 0.784\end{array}$

[A] $\quad \begin{array}{llllllllllllllll}0.098 & 0.117 & 0.137 & 0.156 & 0.195 & 0.215 & 0.235 & 0.254 & 0.274 & 0.293 & 0.352 & 0.666 & 0.724 & 0.764\end{array}$

[B] $\quad \begin{array}{lllllllllllllllll}0.079 & 0.098 & 0.118 & 0.137 & 0.176 & 0.196 & 0.216 & 0.235 & 0.255 & 0.274 & 0.333 & 0.647 & 0.705 & 0.745\end{array}$

[C] $\quad \begin{array}{llllllllllllllllll}0.059 & 0.078 & 0.098 & 0.117 & 0.156 & 0.176 & 0.196 & 0.215 & 0.235 & 0.254 & 0.313 & 0.627 & 0.685 & 0.725\end{array}$

[D] $\quad \begin{array}{lllllllllllllllll}0.04 & 0.059 & 0.079 & 0.098 & 0.137 & 0.157 & 0.177 & 0.196 & 0.216 & 0.235 & 0.294 & 0.608 & 0.666 & 0.706\end{array}$

[E] $\quad \begin{array}{lllllllllllllllll}0.02 & 0.039 & 0.059 & 0.078 & 0.117 & 0.137 & 0.157 & 0.176 & 0.196 & 0.215 & 0.274 & 0.588 & 0.646 & 0.686\end{array}$

$[\mathrm{F}] \quad \begin{array}{lllllllllllllllll} & 0.019 & 0.039 & 0.058 & 0.097 & 0.117 & 0.137 & 0.156 & 0.176 & 0.195 & 0.254 & 0.568 & 0.626 & 0.666\end{array}$

[G] $\quad \begin{array}{llllllllllllllllll}0.019 & 0 & 0.02 & 0.039 & 0.078 & 0.098 & 0.118 & 0.137 & 0.157 & 0.176 & 0.235 & 0.549 & 0.607 & 0.647\end{array}$

$[\mathrm{H}] \quad \begin{array}{llllllllllllllllll}0.039 & 0.02 & . & & 0.019 & 0.058 & 0.078 & 0.098 & 0.117 & 0.137 & 0.156 & 0.215 & 0.529 & 0.587 & 0.627\end{array}$

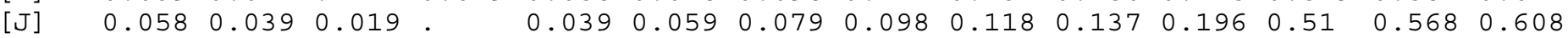

$[\mathrm{K}] \quad \begin{array}{llllllllllllllllll}0.097 & 0.078 & 0.058 & 0.039 & 0.0 .04 & 0.02 & 0.04 & 0.059 & 0.079 & 0.098 & 0.157 & 0.471 & 0.529 & 0.569\end{array}$

$[\mathrm{L}] \quad \begin{array}{lllllllllllllllll}0.117 & 0.098 & 0.078 & 0.059 & 0.02 & . & 0.02 & 0.039 & 0.059 & 0.078 & 0.137 & 0.451 & 0.509 & 0.549\end{array}$

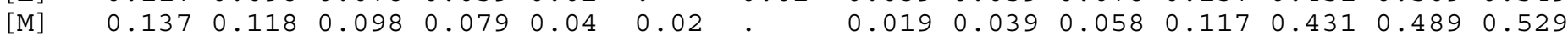

[N] $\quad \begin{array}{llllllllllllllllllllll}0.156 & 0.137 & 0.117 & 0.098 & 0.059 & 0.039 & 0.019 & 0.02 & 0.039 & 0.098 & 0.412 & 0.47 & 0.51\end{array}$

[P] $\quad \begin{array}{lllllllllllllllllll}0.176 & 0.157 & 0.137 & 0.118 & 0.079 & 0.059 & 0.039 & 0.02 & . & 0.019 & 0.078 & 0.392 & 0.45 & 0.49\end{array}$

[Q] $\quad \begin{array}{llllllllllllllll}0.195 & 0.176 & 0.156 & 0.137 & 0.098 & 0.078 & 0.058 & 0.039 & 0.019 & 0.059 & 0.059 & 0.373 & 0.431 & 0.471\end{array}$

[R] $\quad \begin{array}{lllllllllllllllll}0.254 & 0.235 & 0.215 & 0.196 & 0.157 & 0.137 & 0.117 & 0.098 & 0.078 & 0.059 & . & 0.314 & 0.372 & 0.412\end{array}$

[S] $\quad \begin{array}{lllllllllllllllll}0.568 & 0.549 & 0.529 & 0.51 & 0.471 & 0.451 & 0.431 & 0.412 & 0.392 & 0.373 & 0.314 & . & 0.058 & 0.098\end{array}$

[T] $\quad \begin{array}{lllllllllllllllll}0.626 & 0.607 & 0.587 & 0.568 & 0.529 & 0.509 & 0.489 & 0.47 & 0.45 & 0.431 & 0.372 & 0.058 & . & 0.04\end{array}$

[U] $\quad \begin{array}{llllllllllllllllllll}0.666 & 0.647 & 0.627 & 0.608 & 0.569 & 0.549 & 0.529 & 0.51 & 0.49 & 0.471 & 0.412 & 0.098 & 0.04 & .\end{array}$ 
USERTYPE pamantleheight STEPMATRIX $=23$

\begin{tabular}{|c|c|c|c|c|c|c|c|c|c|c|c|c|c|c|c|}
\hline & 0 & 1 & 2 & 3 & 4 & 5 & 6 & 7 & 8 & 9 & A & B & $\mathrm{C}$ & $\mathrm{D}$ & $\mathrm{E}$ \\
\hline ] & & 0 & 62 & 72 & 3 & 93 & 4 & -4 & 24 & 35 & 145 & 56 & 166 & 187 & 0.208 \\
\hline & 0.01 & & 052 & 0.062 & 073 & 083 & 0.094 & 104 & 0.114 & 0.125 & 0.135 & 0.146 & 0.156 & 0.177 & \\
\hline$[2$ & 0.062 & 0.052 & & 0.01 & 0.021 & 0.031 & 0.042 & 0.052 & 0.062 & 0.073 & 0.083 & 0.094 & 0.104 & 0.125 & 0.146 \\
\hline & 072 & 062 & 0.01 & & 011 & .021 & 0.032 & .042 & 0.052 & 0.063 & 0.073 & 0.084 & 0.094 & 0.115 & 136 \\
\hline & .083 & 0.073 & 0.021 & 0.011 & & 0.01 & 0.021 & 0.031 & 0.041 & 0.052 & 0.062 & 0.073 & 0.083 & 0.104 & 0.125 \\
\hline & 093 & .083 & 031 & 0.021 & $U_{\perp}$ & & 0.011 & 0.021 & 0.031 & 0.042 & 0.052 & 0.063 & & 0.094 & 115 \\
\hline & 104 & 094 & .042 & 0.032 & 0.021 & 0.011 & & 0.01 & 0.02 & 0.031 & 0.041 & 0.052 & 62 & 0.083 & 104 \\
\hline & .114 & .104 & .052 & 0.042 & .031 & 0.021 & 0.01 & & 0.01 & 0.021 & 0.031 & 0.042 & & .073 & 0.094 \\
\hline & .124 & 114 & .062 & 0.052 & .041 & 0.031 & 0.02 & 0.01 & & 0.011 & 0.021 & 0.032 & & 63 & 084 \\
\hline & .135 & .125 & .073 & 0.063 & .052 & 0.042 & 0.031 & 0.021 & 0.011 & & 0.01 & 0.021 & 0 . & 52 & 0.073 \\
\hline & .145 & 0.135 & .083 & 0.073 & 0.062 & 0.052 & 0.041 & 0.031 & 0.021 & 0.01 & & 0.011 & 0.021 & 0.042 & 0.063 \\
\hline & .156 & 0.146 & .094 & 84 & 3 & 0.063 & 0.052 & 0.042 & 032 & 0.021 & 0.011 & & 0.01 & 31 & 52 \\
\hline & .166 & 0.156 & .104 & 0.094 & 0.083 & 0.073 & 0.062 & 0.052 & 0.042 & 0.031 & 0.021 & 0.01 & & .021 & 0.042 \\
\hline & .187 & .177 & 125 & 0.115 & & 0.094 & & 0.073 & & & 42 & 0.031 & 0. & & 0.021 \\
\hline & .208 & .198 & 6 & 36 & & 5 & 04 & 4 & 84 & 73 & 63 & 52 & 42 & 0.021 & \\
\hline & .218 & 0.208 & 6 & 0.146 & 5 & 0.125 & 0.114 & 0. & 94 & 0. & 73 & 62 & & 31 & 1 \\
\hline & .249 & 0.239 & 7 & & & 0.156 & 0.145 & 35 & 0.125 & 14 & 04 & 93 & 83 & 62 & 41 \\
\hline & .27 & 0.26 & 0.208 & 0.198 & .187 & 0.177 & 0.166 & 0.156 & 0.146 & 0.135 & 0.125 & 0 . & & 0.083 & 0.062 \\
\hline & 28 & 027 & & & & 0.187 & & 0.166 & 0.156 & & 0.135 & 0.124 & & 0.093 & $\begin{array}{lll}0 & 072\end{array}$ \\
\hline & 0.312 & 0.302 & 0.25 & 0.24 & 0.229 & 0.219 & 0.208 & 0.198 & 0.188 & 0.177 & 0.167 & 0.156 & 0.146 & 0.125 & 0.104 \\
\hline & .395 & 0.385 & 0.333 & & & & & & & 026 & & & & 0.208 & \\
\hline$[\mathrm{M}$ & 0.707 & 0.697 & 0.645 & 0.635 & 0.624 & 0.614 & 0.603 & 0.593 & 0.583 & 0.572 & 0.562 & 0.551 & 0.541 & 0.52 & 0.499 \\
\hline & .999 & 0.989 & 0.937 & 0.927 & 0.916 & 0.906 & 0.895 & 0.885 & 0.875 & 0.864 & 0.854 & 0.843 & 0.833 & 0.812 & 0.791 \\
\hline
\end{tabular}


[0] $\quad \begin{array}{lllllllll}0.218 & 0.249 & 0.27 & 0.28 & 0.312 & 0.395 & 0.707 & 0.999\end{array}$

[1] $\quad \begin{array}{lllllllll}0.208 & 0.239 & 0.26 & 0.27 & 0.302 & 0.385 & 0.697 & 0.989\end{array}$

[2] $\quad \begin{array}{lllllllll}0.156 & 0.187 & 0.208 & 0.218 & 0.25 & 0.333 & 0.645 & 0.937\end{array}$

[3] $\quad \begin{array}{lllllllll}0.146 & 0.177 & 0.198 & 0.208 & 0.24 & 0.323 & 0.635 & 0.927\end{array}$

[4] $\quad \begin{array}{llllllllll}0.135 & 0.166 & 0.187 & 0.197 & 0.229 & 0.312 & 0.624 & 0.916\end{array}$

[5] $\quad \begin{array}{llllllllll}0.125 & 0.156 & 0.177 & 0.187 & 0.219 & 0.302 & 0.614 & 0.906\end{array}$

[6] $\quad \begin{array}{llllllllll}0.114 & 0.145 & 0.166 & 0.176 & 0.208 & 0.291 & 0.603 & 0.895\end{array}$

[7] $\quad \begin{array}{llllllllll}0.104 & 0.135 & 0.156 & 0.166 & 0.198 & 0.281 & 0.593 & 0.885\end{array}$

[8] $\quad \begin{array}{lllllllllll}0.094 & 0.125 & 0.146 & 0.156 & 0.188 & 0.271 & 0.583 & 0.875\end{array}$

[9] $\quad \begin{array}{llllllllll}0.083 & 0.114 & 0.135 & 0.145 & 0.177 & 0.26 & 0.572 & 0.864\end{array}$

[A] $\quad \begin{array}{llllllllll}0.073 & 0.104 & 0.125 & 0.135 & 0.167 & 0.25 & 0.562 & 0.854\end{array}$

[B] $\quad \begin{array}{llllllllll}0.062 & 0.093 & 0.114 & 0.124 & 0.156 & 0.239 & 0.551 & 0.843\end{array}$

[C] $\quad \begin{array}{llllllllll}0.052 & 0.083 & 0.104 & 0.114 & 0.146 & 0.229 & 0.541 & 0.833\end{array}$

[D] $\quad \begin{array}{llllllllll}0.031 & 0.062 & 0.083 & 0.093 & 0.125 & 0.208 & 0.52 & 0.812\end{array}$

[E] $\quad \begin{array}{llllllllll}0.01 & 0.041 & 0.062 & 0.072 & 0.104 & 0.187 & 0.499 & 0.791\end{array}$

[F] $\quad \begin{array}{lllllllll} & 0.031 & 0.052 & 0.062 & 0.094 & 0.177 & 0.489 & 0.781\end{array}$

[G] $\quad \begin{array}{lllllllll}0.031 & 0.031 & 0.021 & 0.031 & 0.063 & 0.146 & 0.458 & 0.75\end{array}$

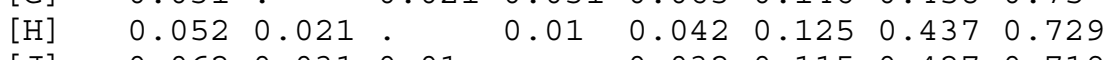

[J] $\quad \begin{array}{lllllllll}0.062 & 0.031 & 0.01 & 0.032 & 0.115 & 0.427 & 0.719\end{array}$

$[\mathrm{K}] \quad \begin{array}{lllllllll}0.094 & 0.063 & 0.042 & 0.032 & 0.0 .039 & 0.083 & 0.395 & 0.687\end{array}$

[L] $\quad \begin{array}{llllllllll} & 0.177 & 0.146 & 0.125 & 0.115 & 0.083 & . & 0.312 & 0.604\end{array}$

[M] $\quad \begin{array}{lllllllll}0.489 & 0.458 & 0.437 & 0.427 & 0.395 & 0.312 & . & 0.292\end{array}$

[N] $\quad \begin{array}{lllllllll}0.781 & 0.75 & 0.729 & 0.719 & 0.687 & 0.604 & 0.292\end{array}$. 
USERTYPE meanmantlethickness STEPMATRIX $=28$

\begin{tabular}{|c|c|c|c|c|c|c|c|c|c|c|c|c|c|c|c|}
\hline & 0 & 1 & 2 & 3 & 4 & 5 & 6 & 7 & 8 & 9 & A & B & $\mathrm{C}$ & $\mathrm{D}$ & $\mathrm{E}$ \\
\hline$[0]$ & . & 0.03 & 06 & 09 & 121 & 0.151 & 0.211 & 242 & 272 & 0.302 & 0.333 & 0.363 & 0.393 & 0.423 & .454 \\
\hline [1] & 0.03 & . & 0.03 & 0.06 & 0.091 & 0.121 & 0.181 & 0.212 & 0.242 & 0.272 & 0.303 & 0.333 & 0.363 & 0.393 & 0.424 \\
\hline [2] & .06 & 0.03 & . & 0.03 & 0.061 & 0.091 & 0.151 & 0.182 & 0.212 & 0.242 & 0.273 & 0.303 & 0.333 & 0.363 & 0.394 \\
\hline [3] & 0.09 & 0.06 & 0.03 & . & 0.031 & 0.061 & 0.121 & 0.152 & 0.182 & 0.212 & 0.243 & 0.273 & 0.303 & 0.333 & 0.364 \\
\hline$[4$ & 0.121 & 0.091 & 0.061 & 0.031 & . & 0.03 & 0.09 & 0.121 & 0.151 & 0.181 & 0.212 & 0.242 & 0.272 & 0.302 & 0.333 \\
\hline$[5$ & 0.151 & 0.121 & 0.091 & 0.061 & 0.03 & 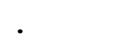 & 0.06 & 0.091 & 0.121 & 0.151 & 0.182 & 0.212 & 0.242 & 0.272 & 0.303 \\
\hline 10 & 0.211 & .181 & 0.151 & .121 & 0.09 & 0.06 & . & 0.031 & 0.061 & 0.091 & 0.122 & 0.152 & 0.182 & 0.212 & 0.243 \\
\hline$[7$ & 0.242 & 0.212 & 0.182 & 0.152 & 0.121 & 0.091 & 0.031 & . & 0.03 & 0.06 & 0.091 & 0.121 & 0.151 & 0.181 & 0.212 \\
\hline [8] & 0.272 & 0.242 & 0.212 & 0.182 & 0.151 & 0.121 & 0.061 & 0.03 & . & 0.03 & 0.061 & 0.091 & 0.121 & 0.151 & 0.182 \\
\hline [9] & 0.302 & 0.272 & 0.242 & 0.212 & 0.181 & 0.151 & 0.091 & 0.06 & 0.03 & 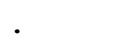 & 0.031 & 0.061 & 0.091 & 0.121 & 0.152 \\
\hline$[\mathrm{A}]$ & 0.333 & 0.303 & 0.273 & 0.243 & 0.212 & 0.182 & 0.122 & 0.091 & 0.061 & 0.031 & & 0.03 & 0.06 & 0.09 & 0.121 \\
\hline [B. & 0.363 & 0.333 & 0.303 & 0.273 & 0.242 & 0.212 & 0.152 & 0.121 & 0.091 & 0.061 & 0.03 & & 0.03 & 0.06 & 0.091 \\
\hline [C. & 0.393 & 0.363 & 0.333 & 0.303 & 0.272 & 0.242 & 0.182 & 0.151 & 0.121 & 0.091 & 0.06 & 0.03 & 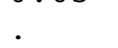 & 0.03 & 0.061 \\
\hline - & 0.423 & .393 & 0.363 & 0.333 & 0.302 & 0.272 & 0.212 & 0.181 & 0.151 & 0.121 & 0.09 & 0.06 & 0.03 & . & 0.031 \\
\hline$[E]$ & 0.454 & 0.424 & 0.394 & 0.364 & 0.333 & 0.303 & 0.243 & 0.212 & 0.182 & 0.152 & 0.121 & 0.091 & 0.061 & 0.031 & \\
\hline$[\mathrm{F}]$ & 0.484 & 0.454 & 0.424 & 0.394 & 0.363 & 0.333 & 0.273 & 0.242 & 0.212 & 0.182 & 0.151 & 0.121 & 0.091 & 0.061 & 0.03 \\
\hline [G] & 0.544 & 0.514 & 0.484 & 0.454 & 0.423 & 0.393 & 0.333 & 0.302 & 0.272 & 0.242 & 0.211 & 0.181 & 0.151 & 0.121 & 0.09 \\
\hline$[\mathrm{H}]$ & 0.575 & 0.545 & 0.515 & 0.485 & 0.454 & 0.424 & 0.364 & 0.333 & 0.303 & 0.273 & 0.242 & 0.212 & 0.182 & 0.152 & 0.121 \\
\hline$[\mathrm{J}$. & 0.605 & 0.575 & 0.545 & 0.515 & 0.484 & 0.454 & 0.394 & 0.363 & 0.333 & 0.303 & 0.272 & 0.242 & 0.212 & 0.182 & 0.151 \\
\hline$K K$ & 0.635 & 0.605 & 0.575 & 0.545 & 0.514 & 0.484 & 0.424 & 0.393 & 0.363 & 0.333 & 0.302 & 0.272 & 0.242 & 0.212 & 0.181 \\
\hline$[\mathrm{L}$ & 0.666 & 0.636 & 0.606 & 0. & 0.545 & 0.515 & 0.455 & 0.424 & 0.394 & 0.364 & 0.333 & 0.303 & 0.273 & 0.243 & 0.212 \\
\hline$[\mathrm{M}]$ & 0.696 & 0.666 & 0.636 & 0.606 & 0.575 & 0.545 & 0.485 & 0.454 & 0.424 & 0.394 & 0.363 & 0.333 & 0.303 & 0.273 & 0.242 \\
\hline$[\mathrm{N}]$ & 0.726 & 0.696 & 0.666 & 0.636 & 0.605 & 0.575 & 0.515 & 0.484 & 0.454 & 0.424 & 0.393 & 0.363 & 0.333 & 0.303 & 0.272 \\
\hline$[\mathrm{P}]$ & 0.756 & 0.726 & 0.696 & 0.666 & 0.635 & 0.605 & 0.545 & 0.514 & 0.484 & 0.454 & 0.423 & 0.393 & 0.363 & 0.333 & 0.302 \\
\hline$[Q$ & 0.787 & 0.757 & 0.727 & 0.697 & 0.666 & 0.636 & 0.576 & 0.545 & 0.515 & 0.485 & 0.454 & 0.424 & 0.394 & 0.364 & 0.333 \\
\hline$[\mathrm{R}]$ & 0.847 & 0.817 & 0.787 & 0.757 & 0.726 & 0.696 & 0.636 & 0.605 & 0.575 & 0.545 & 0.514 & 0.484 & 0.454 & 0.424 & 0.393 \\
\hline$[S$ & 0.877 & 0.847 & 0.817 & 0.787 & 0.756 & 0.726 & 0.666 & 0.635 & 0.605 & 0.575 & 0.544 & 0.514 & 0.484 & 0.454 & 0.423 \\
\hline$[\mathrm{T}]$ & 0.999 & 0.969 & 0.939 & 0.909 & 0.878 & 0.848 & 0.788 & 0.757 & 0.727 & 0.697 & 0.666 & 0.636 & 0.606 & 0.576 & 0.545 \\
\hline
\end{tabular}


[0] $\quad \begin{array}{llllllllllllllllll}0.484 & 0.544 & 0.575 & 0.605 & 0.635 & 0.666 & 0.696 & 0.726 & 0.756 & 0.787 & 0.847 & 0.877 & 0.999\end{array}$

$[1] \quad \begin{array}{llllllllllllllll}0.454 & 0.514 & 0.545 & 0.575 & 0.605 & 0.636 & 0.666 & 0.696 & 0.726 & 0.757 & 0.817 & 0.847 & 0.969\end{array}$

[2] $\quad \begin{array}{llllllllllllllll}0.424 & 0.484 & 0.515 & 0.545 & 0.575 & 0.606 & 0.636 & 0.666 & 0.696 & 0.727 & 0.787 & 0.817 & 0.939\end{array}$

[3] $\quad \begin{array}{lllllllllllllllll}0.394 & 0.454 & 0.485 & 0.515 & 0.545 & 0.576 & 0.606 & 0.636 & 0.666 & 0.697 & 0.757 & 0.787 & 0.909\end{array}$

[4] $\quad \begin{array}{llllllllllllllllll}0.363 & 0.423 & 0.454 & 0.484 & 0.514 & 0.545 & 0.575 & 0.605 & 0.635 & 0.666 & 0.726 & 0.756 & 0.878\end{array}$

[5] $\quad \begin{array}{lllllllllllllllll}0.333 & 0.393 & 0.424 & 0.454 & 0.484 & 0.515 & 0.545 & 0.575 & 0.605 & 0.636 & 0.696 & 0.726 & 0.848\end{array}$

[6] $\quad \begin{array}{llllllllllllllll}0.273 & 0.333 & 0.364 & 0.394 & 0.424 & 0.455 & 0.485 & 0.515 & 0.545 & 0.576 & 0.636 & 0.666 & 0.788\end{array}$

[7] $\quad \begin{array}{lllllllllllllllll}0.242 & 0.302 & 0.333 & 0.363 & 0.393 & 0.424 & 0.454 & 0.484 & 0.514 & 0.545 & 0.605 & 0.635 & 0.757\end{array}$

[8] $\quad \begin{array}{lllllllllllllllll}0.212 & 0.272 & 0.303 & 0.333 & 0.363 & 0.394 & 0.424 & 0.454 & 0.484 & 0.515 & 0.575 & 0.605 & 0.727\end{array}$

[9] $\quad \begin{array}{llllllllllllllll}0.182 & 0.242 & 0.273 & 0.303 & 0.333 & 0.364 & 0.394 & 0.424 & 0.454 & 0.485 & 0.545 & 0.575 & 0.697\end{array}$

[A] $\quad \begin{array}{llllllllllllllll}0.151 & 0.211 & 0.242 & 0.272 & 0.302 & 0.333 & 0.363 & 0.393 & 0.423 & 0.454 & 0.514 & 0.544 & 0.666\end{array}$

[B] $\quad \begin{array}{llllllllllllllll}0.121 & 0.181 & 0.212 & 0.242 & 0.272 & 0.303 & 0.333 & 0.363 & 0.393 & 0.424 & 0.484 & 0.514 & 0.636\end{array}$

[C] $\quad \begin{array}{llllllllllllllll}0.091 & 0.151 & 0.182 & 0.212 & 0.242 & 0.273 & 0.303 & 0.333 & 0.363 & 0.394 & 0.454 & 0.484 & 0.606\end{array}$

[D] $\quad \begin{array}{lllllllllllllll}0.061 & 0.121 & 0.152 & 0.182 & 0.212 & 0.243 & 0.273 & 0.303 & 0.333 & 0.364 & 0.424 & 0.454 & 0.576\end{array}$

$\left[\begin{array}{llllllllllllllll} & 0.03 & 0.09 & 0.121 & 0.151 & 0.181 & 0.212 & 0.242 & 0.272 & 0.302 & 0.333 & 0.393 & 0.423 & 0.545\end{array}\right.$

[F] $\quad \begin{array}{lllllllllllllllll} & & 0.06 & 0.091 & 0.121 & 0.151 & 0.182 & 0.212 & 0.242 & 0.272 & 0.303 & 0.363 & 0.393 & 0.515\end{array}$

[G] $\quad \begin{array}{llllllllllllllll}0.06 & 0.0 & 0.031 & 0.061 & 0.091 & 0.122 & 0.152 & 0.182 & 0.212 & 0.243 & 0.303 & 0.333 & 0.455\end{array}$

$[\mathrm{H}] \quad \begin{array}{llllllllllllllllll} & 0.091 & 0.031 & . & 0.03 & 0.06 & 0.091 & 0.121 & 0.151 & 0.181 & 0.212 & 0.272 & 0.302 & 0.424\end{array}$

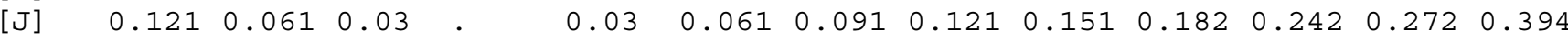

$[\mathrm{K}] \quad \begin{array}{llllllllllllllllllll}0.151 & 0.091 & 0.06 & 0.03 & 0.031 & 0.061 & 0.091 & 0.121 & 0.152 & 0.212 & 0.242 & 0.364\end{array}$

[L] $\quad \begin{array}{lllllllllllllll} & 0.182 & 0.122 & 0.091 & 0.061 & 0.031 & 0.0 .03 & 0.03 & 0.06 & 0.09 & 0.121 & 0.181 & 0.211 & 0.333\end{array}$

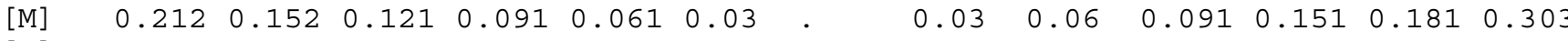

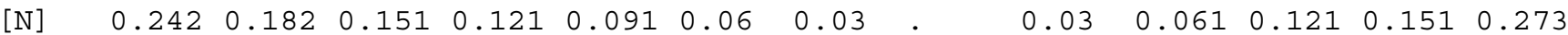

[P] $\quad \begin{array}{llllllllllllllll} & 0.272 & 0.212 & 0.181 & 0.151 & 0.121 & 0.09 & 0.06 & 0.03 & . & 0.031 & 0.091 & 0.121 & 0.243\end{array}$

[Q] $\quad \begin{array}{llllllllllllllll}0.303 & 0.243 & 0.212 & 0.182 & 0.152 & 0.121 & 0.091 & 0.061 & 0.031 & . & 0.06 & 0.09 & 0.212\end{array}$

[R] $\quad \begin{array}{llllllllllllllll}0.363 & 0.303 & 0.272 & 0.242 & 0.212 & 0.181 & 0.151 & 0.121 & 0.091 & 0.06 & . & 0.03 & 0.152\end{array}$

[S] $\quad \begin{array}{llllllllllllllll}0.393 & 0.333 & 0.302 & 0.272 & 0.242 & 0.211 & 0.181 & 0.151 & 0.121 & 0.09 & 0.03 & . & 0.122\end{array}$

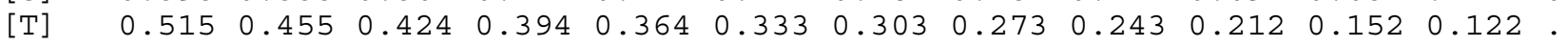


USERTYPE pamantlethickness STEPMATRIX $=24$

\begin{tabular}{|c|c|c|c|c|c|c|c|c|c|c|c|c|c|c|c|}
\hline & 0 & 1 & 2 & 3 & 4 & 5 & 6 & 7 & 8 & 9 & A & B & $\mathrm{C}$ & D & $\mathrm{E}$ \\
\hline [0] & . & 0.066 & 0.099 & 0.133 & 0.199 & 0.233 & 0.299 & 0.333 & 0.399 & 0.432 & 0.466 & 0.499 & 0.532 & 0.566 & 0.599 \\
\hline [1] & 0.066 & . & 0.033 & 0.067 & 0.133 & 0.167 & 0.233 & 0.267 & 0.333 & 0.366 & 0.4 & 0.433 & 0.466 & 0.5 & 0.533 \\
\hline [2] & 0.099 & 0.033 & . & 0.034 & 0.1 & 0.134 & 0.2 & 0.234 & 0.3 & 0.333 & 0.367 & 0.4 & 0.433 & 0.467 & 0.5 \\
\hline [3] & 0.133 & 0.067 & 0.034 & . & 0.066 & 0.1 & 0.166 & 0.2 & 0.266 & 0.299 & 0.333 & 0.366 & 0.399 & 0.433 & 0.466 \\
\hline [4] & 0.199 & 0.133 & 0.1 & 0.066 & . & 0.034 & 0.1 & 0.134 & 0.2 & 0.233 & 0.267 & 0.3 & 0.333 & 0.367 & 0.4 \\
\hline [5] & 0.233 & 0.167 & 0.134 & 0.1 & 0.034 & & 0.066 & 0.1 & 0.166 & 0.199 & 0.233 & 0.266 & 0.299 & 0.333 & 0.366 \\
\hline [6] & 0.299 & 0.233 & 0.2 & 0.166 & 0.1 & 0.066 & . & 0.034 & 0.1 & 0.133 & 0.167 & 0.2 & 0.233 & 0.267 & 0.3 \\
\hline [7] & 0.333 & 0.267 & 0.234 & 0.2 & 0.134 & 0.1 & 0.034 & . & 0.066 & 0.099 & 0.133 & 0.166 & 0.199 & 0.233 & 0.266 \\
\hline [8] & 0.399 & 0.333 & 0.3 & 0.266 & 0.2 & 0.166 & 0.1 & 0.066 & . & 0.033 & 0.067 & 0.1 & 0.133 & 0.167 & 0.20 \\
\hline [9] & 0.432 & 0.366 & 0.333 & 0.299 & 0.233 & 0.199 & 0.133 & 0.099 & 0.033 & . & 0.034 & 0.067 & 0.1 & 0.134 & 0.167 \\
\hline [A] & 0.466 & 0.4 & 0.367 & 0.333 & 0.267 & 0.233 & 0.167 & 0.133 & 0.067 & 0.034 & . & 0.033 & 0.066 & 0.1 & 0.133 \\
\hline [B] & 0.499 & 0.433 & 0.4 & 0.366 & 0.3 & 0.266 & 0.2 & 0.166 & 0.1 & 0.067 & 0.033 & & 0.033 & 0.067 & 0.1 \\
\hline$[\mathrm{C}]$ & 0.532 & 0.466 & 0.433 & 0.399 & 0.333 & 0.299 & 0.233 & 0.199 & 0.133 & 0.1 & 0.066 & 0.033 & . & 0.034 & 0.067 \\
\hline [D] & 0.566 & 0.5 & 0.467 & 0.433 & 0.367 & 0.333 & 0.267 & 0.233 & 0.167 & 0.134 & 0.1 & 0.067 & 0.034 & . & 0.033 \\
\hline$[E]$ & 0.599 & 0.533 & 0.5 & 0.466 & 0.4 & 0.366 & 0.3 & 0.266 & 0.2 & 0.167 & 0.133 & 0.1 & 0.067 & 0.033 & \\
\hline$[F]$ & 0.632 & 0.566 & 0.533 & 0.499 & 0.433 & 0.399 & 0.333 & 0.299 & 0.233 & 0.2 & 0.166 & 0.133 & 0.1 & 0.066 & 0.033 \\
\hline [G] & 0.666 & 0.6 & 0.567 & 0.533 & 0.467 & 0.433 & 0.367 & 0.333 & 0.267 & 0.234 & 0.2 & 0.167 & 0.134 & 0.1 & 0.067 \\
\hline$[\mathrm{H}]$ & 0.699 & 0.633 & 0.6 & 0.566 & 0.5 & 0.466 & 0.4 & 0.366 & 0.3 & 0.267 & 0.233 & 0.2 & 0.167 & 0.133 & 0.1 \\
\hline$[\mathrm{J}]$ & 0.732 & 0.666 & 0.633 & 0.599 & 0.533 & 0.499 & 0.433 & 0.399 & 0.333 & 0.3 & 0.266 & 0.233 & 0.2 & 0.166 & 0.133 \\
\hline$[\mathrm{K}]$ & 0.799 & 0.733 & 0.7 & 0.666 & 0.6 & 0.566 & 0.5 & 0.466 & 0.4 & 0.367 & 0.333 & 0.3 & 0.267 & 0.233 & 0.2 \\
\hline [L] & 0.832 & 0.766 & 0.733 & 0.699 & 0.633 & 0.599 & 0.533 & 0.499 & 0.433 & 0.4 & 0.366 & 0.333 & 0.3 & 0.266 & 0.233 \\
\hline$[\mathrm{M}]$ & 0.865 & 0.799 & 0.766 & 0.732 & 0.666 & 0.632 & 0.566 & 0.532 & 0.466 & 0.433 & 0.399 & 0.366 & 0.333 & 0.299 & 0.266 \\
\hline$[\mathrm{N}]$ & 0.899 & 0.833 & 0.8 & 0.766 & 0.7 & 0.666 & 0.6 & 0.566 & 0.5 & 0.467 & 0.433 & 0.4 & 0.367 & 0.333 & 0.3 \\
\hline$[\mathrm{P}]$ & 0.999 & 0.933 & 0.9 & 0.866 & 0.8 & 0.766 & 0.7 & 0.666 & 0.6 & 0.567 & 0.533 & 0.5 & 0.467 & 0.433 & 0.4 \\
\hline
\end{tabular}


[0] $\quad \begin{array}{lllllllllll}0.632 & 0.666 & 0.699 & 0.732 & 0.799 & 0.832 & 0.865 & 0.899 & 0.999\end{array}$

[1] $\begin{array}{llllllllll}0.566 & 0.6 & 0.633 & 0.666 & 0.733 & 0.766 & 0.799 & 0.833 & 0.933\end{array}$

$[2] \quad \begin{array}{llllllllll}0.533 & 0.567 & 0.6 & 0.633 & 0.7 & 0.733 & 0.766 & 0.8 & 0.9\end{array}$

[3] $\quad \begin{array}{lllllllllll}0.499 & 0.533 & 0.566 & 0.599 & 0.666 & 0.699 & 0.732 & 0.766 & 0.866\end{array}$

[4] $\quad \begin{array}{llllllllll}0.433 & 0.467 & 0.5 & 0.533 & 0.6 & 0.633 & 0.666 & 0.7 & 0.8\end{array}$

[5] $\quad \begin{array}{lllllllllll}0.399 & 0.433 & 0.466 & 0.499 & 0.566 & 0.599 & 0.632 & 0.666 & 0.766\end{array}$

$\begin{array}{llllllllll}\text { [6] } & 0.333 & 0.367 & 0.4 & 0.433 & 0.5 & 0.533 & 0.566 & 0.6 & 0.7\end{array}$

[7] $\quad \begin{array}{lllllllllll}0.299 & 0.333 & 0.366 & 0.399 & 0.466 & 0.499 & 0.532 & 0.566 & 0.666\end{array}$

[8] $\quad \begin{array}{llllllllll}0.233 & 0.267 & 0.3 & 0.333 & 0.4 & 0.433 & 0.466 & 0.5 & 0.6\end{array}$

[9] $\begin{array}{lllllllll}0.2 & 0.234 & 0.267 & 0.3 & 0.367 & 0.4 & 0.433 & 0.467 & 0.567\end{array}$

[A] $\begin{array}{lllllllllll}0.166 & 0.2 & 0.233 & 0.266 & 0.333 & 0.366 & 0.399 & 0.433 & 0.533\end{array}$

[B] $\quad \begin{array}{llllllllll}0.133 & 0.167 & 0.2 & 0.233 & 0.3 & 0.333 & 0.366 & 0.4 & 0.5\end{array}$

[C] $\begin{array}{llllllllll}0.1 & 0.134 & 0.167 & 0.2 & 0.267 & 0.3 & 0.333 & 0.367 & 0.467\end{array}$

[D] $\begin{array}{lllllllllll}0.066 & 0.1 & 0.133 & 0.166 & 0.233 & 0.266 & 0.299 & 0.333 & 0.433\end{array}$

[E] $\quad \begin{array}{llllllllll}0.033 & 0.067 & 0.1 & 0.133 & 0.2 & 0.233 & 0.266 & 0.3 & 0.4\end{array}$

$[F] \quad \begin{array}{lllllllll}0 & 0.034 & 0.067 & 0.1 & 0.167 & 0.2 & 0.233 & 0.267 & 0.367\end{array}$

[G] $\quad \begin{array}{lllllllllllll}0.034 & 0.0 .133 & 0.033 & 0.066 & 0.133 & 0.166 & 0.199 & 0.233 & 0.333\end{array}$

[H] $\quad \begin{array}{llllllllll}0.067 & 0.033 & 0 . & 0.033 & 0.1 & 0.133 & 0.166 & 0.2 & 0.3\end{array}$

[J] $\quad \begin{array}{llllllllllll}0.1 & 0.066 & 0.033 & . & 0.067 & 0.1 & 0.133 & 0.167 & 0.267\end{array}$

$\begin{array}{lllllllllll}\mathrm{K}] & 0.167 & 0.133 & 0.1 & 0.067 & 0.033 & 0.066 & 0.1 & 0.2\end{array}$

$\begin{array}{llllllllll}\text { [L] } & 0.2 & 0.166 & 0.133 & 0.1 & 0.033 & . & 0.033 & 0.067 & 0.167\end{array}$

[M] $\quad \begin{array}{llllllllllll}0.233 & 0.199 & 0.166 & 0.133 & 0.066 & 0.033 & . & 0.034 & 0.134\end{array}$

[N] $\quad \begin{array}{lllllllllll}0.267 & 0.233 & 0.2 & 0.167 & 0.1 & 0.067 & 0.034 & . & 0.1\end{array}$

[P] $\quad \begin{array}{lllllllllll}0.367 & 0.333 & 0.3 & 0.267 & 0.2 & 0.167 & 0.134 & 0.1\end{array}$ 
USERTYPE meancollumheight STEPMATRIX $=25$

\begin{tabular}{|c|c|c|c|c|c|c|c|c|c|c|c|c|c|c|c|}
\hline & & 1 & & 3 & 4 & 5 & 6 & & 8 & & $\mathrm{~A}$ & B & C & D & E \\
\hline 0] & & 0 & .09 & 36 & + & 4 & 27 & 9 & 72 & 95 & 0. & 0.34 & 85 & 0.408 & 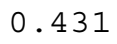 \\
\hline & 068 & & 022 & 068 & 173 & 136 & 0.159 & .181 & 0.204 & 0.227 & 0.249 & & 317 & & \\
\hline & .09 & 0.022 & & 46 & & & & & & & & 0 & & & \\
\hline & 136 & 0.068 & 046 & & & & 0.091 & & & & & & & & \\
\hline & 181 & 113 & .091 & 0.045 & & .023 & & & 91 & & & & & & \\
\hline & 204 & 136 & 114 & 0.068 & 3 & & 23 & 5 & & & & & & & \\
\hline & 27 & & & & & .023 & & & & & & & & & \\
\hline & 249 & 181 & 159 & 0.113 & & 0.045 & 0.022 & & 23 & & & & & & \\
\hline & 272 & & & & & & & .023 & & & & & & & \\
\hline & 95 & 227 & 5 & 59 & & & & & 0.023 & & & & & & \\
\hline & 17 & 249 & 27 & & & & & & & & & & & & \\
\hline & 3 & 272 & 25 & 0.204 & 59 & 36 & 13 & 91 & 68 & 0.045 & 023 & & 0.045 & & \\
\hline & & & & & & & & & & & & & & 23 & \\
\hline & .408 & 34 & 318 & 0.272 & 227 & .204 & 0.181 & 59 & 136 & 13 & 91 & 0.068 & 0.023 & & 023 \\
\hline & 31 & & & & & & & & & & & & & & \\
\hline & 454 & 386 & 364 & 0.318 & 3 & 0.2 & 27 & 5 & 82 & 0.159 & & 14 & & 046 & \\
\hline & & & & & & & & & & & & & & & \\
\hline & 99 & 1 & 9 & & & 95 & & & 27 & 4 & 82 & 59 & & 91 & 68 \\
\hline & & & & & & & & & & & & & & & \\
\hline & 635 & 7 & 5 & & & & & & & & & 95 & 0 & 27 & 04 \\
\hline & 681 & & & & & & & & & & & & & & \\
\hline & 703 & & 3 & & & & & & & & & & & & 72 \\
\hline & 771 & 3 & 681 & & & & 0. & & & & & & & & \\
\hline & & & & & & & & & & & & & & & \\
\hline & 999 & .931 & .909 & 0.863 & .818 & 0.795 & 0.772 & 0.15 & 0.727 & 0.704 & 0.682 & 0.659 & 0.614 & 0.591 & 0.568 \\
\hline
\end{tabular}


[0] $\quad \begin{array}{llllllllllll}0.454 & 0.476 & 0.499 & 0.544 & 0.635 & 0.681 & 0.703 & 0.771 & 0.862 & 0.999\end{array}$

[1] $\quad \begin{array}{llllllllllll}0.386 & 0.408 & 0.431 & 0.476 & 0.567 & 0.613 & 0.635 & 0.703 & 0.794 & 0.931\end{array}$

[2] $\quad \begin{array}{lllllllllllll}0.364 & 0.386 & 0.409 & 0.454 & 0.545 & 0.591 & 0.613 & 0.681 & 0.772 & 0.909\end{array}$

[3] $\quad \begin{array}{llllllllllll}0.318 & 0.34 & 0.363 & 0.408 & 0.499 & 0.545 & 0.567 & 0.635 & 0.726 & 0.863\end{array}$

[4] $\quad \begin{array}{llllllllll}0.273 & 0.295 & 0.318 & 0.363 & 0.454 & 0.5 & 0.522 & 0.59 & 0.681 & 0.818\end{array}$

[5] $\quad \begin{array}{llllllllllll}0.25 & 0.272 & 0.295 & 0.34 & 0.431 & 0.477 & 0.499 & 0.567 & 0.658 & 0.795\end{array}$

[6] $\quad \begin{array}{llllllllllll}0.227 & 0.249 & 0.272 & 0.317 & 0.408 & 0.454 & 0.476 & 0.544 & 0.635 & 0.772\end{array}$

[7] $\quad \begin{array}{llllllllllll}0.205 & 0.227 & 0.25 & 0.295 & 0.386 & 0.432 & 0.454 & 0.522 & 0.613 & 0.75\end{array}$

[8] $\quad \begin{array}{llllllllllll}0.182 & 0.204 & 0.227 & 0.272 & 0.363 & 0.409 & 0.431 & 0.499 & 0.59 & 0.727\end{array}$

[9] $\quad \begin{array}{llllllllllll}0.159 & 0.181 & 0.204 & 0.249 & 0.34 & 0.386 & 0.408 & 0.476 & 0.567 & 0.704\end{array}$

[A] $\quad \begin{array}{llllllllllll}0.137 & 0.159 & 0.182 & 0.227 & 0.318 & 0.364 & 0.386 & 0.454 & 0.545 & 0.682\end{array}$

[B] $\quad \begin{array}{llllllllllll}0.114 & 0.136 & 0.159 & 0.204 & 0.295 & 0.341 & 0.363 & 0.431 & 0.522 & 0.659\end{array}$

[C] $\quad \begin{array}{lllllllllllll}0.069 & 0.091 & 0.114 & 0.159 & 0.25 & 0.296 & 0.318 & 0.386 & 0.477 & 0.614\end{array}$

[D] $\quad \begin{array}{llllllllllll}0.046 & 0.068 & 0.091 & 0.136 & 0.227 & 0.273 & 0.295 & 0.363 & 0.454 & 0.591\end{array}$

[E] $\quad \begin{array}{llllllllllll}0.023 & 0.045 & 0.068 & 0.113 & 0.204 & 0.25 & 0.272 & 0.34 & 0.431 & 0.568\end{array}$

[F] $\quad \begin{array}{llllllllllll}0.022 & 0.045 & 0.09 & 0.181 & 0.227 & 0.249 & 0.317 & 0.408 & 0.545\end{array}$

[G] $\quad \begin{array}{llllllllllll}0.022 & 0 & 0.023 & 0.068 & 0.159 & 0.205 & 0.227 & 0.295 & 0.386 & 0.523\end{array}$

[H] $\quad \begin{array}{lllllllllllll}0.045 & 0.023 & . & & 0.045 & 0.136 & 0.182 & 0.204 & 0.272 & 0.363 & 0.5\end{array}$

[J] $\quad \begin{array}{llllllllllllll}0.09 & 0.068 & 0.045 & . & 0.091 & 0.137 & 0.159 & 0.227 & 0.318 & 0.455\end{array}$

$[\mathrm{K}] \quad \begin{array}{lllllllllllll}0.181 & 0.159 & 0.136 & 0.091 & & 0.0 .046 & 0.046 & 0.068 & 0.136 & 0.227 & 0.364\end{array}$

[L] $\quad \begin{array}{llllllllllll} & 0.227 & 0.205 & 0.182 & 0.137 & 0.046 & 0.0 .04 & 0.022 & 0.09 & 0.181 & 0.318\end{array}$

[M] $\quad \begin{array}{lllllllllll}0.249 & 0.227 & 0.204 & 0.159 & 0.068 & 0.022 & 0.068 & 0.159 & 0.296\end{array}$

[N] $\quad \begin{array}{lllllllllll}0.317 & 0.295 & 0.272 & 0.227 & 0.136 & 0.09 & 0.068 & 0.091 & 0.228\end{array}$

[P] $\quad \begin{array}{lllllllllllll}0.408 & 0.386 & 0.363 & 0.318 & 0.227 & 0.181 & 0.159 & 0.091 & . & & 0.137\end{array}$

[Q] $\quad \begin{array}{llllllllllll}0.545 & 0.523 & 0.5 & 0.455 & 0.364 & 0.318 & 0.296 & 0.228 & 0.137\end{array}$ 
USERTYPE pacollumheight STEPMATRIX $=26$

\begin{tabular}{|c|c|c|c|c|c|c|c|c|c|c|c|c|c|c|c|}
\hline & & 1 & 2 & 3 & 4 & 5 & 6 & & 8 & 9 & A & B & C & $D$ & $\mathrm{E}$ \\
\hline & & 5 & 7 & 7 & 1 & 6 & 8 & 0.18 & 2 & 4 & 6 & 8 & 92 & 96 & \\
\hline & 155 & & & 012 & & & & & & & & & & & \\
\hline & .157 & .002 & & .01 & 014 & 019 & 0.021 & .023 & 0.025 & .027 & .029 & 0.031 & 0.035 & 0.039 & .043 \\
\hline & 167 & 012 & 01 & & & & & & & & & & & & \\
\hline & 171 & 016 & .014 & 0.004 & & 0.005 & .007 & .009 & 11 & 13 & 15 & 0.017 & 21 & 25 & 029 \\
\hline & 176 & 021 & & & .005 & & & & & & & & & & \\
\hline & & 023 & & & 007 & 0.002 & & & & & & & & & \\
\hline & 18 & 025 & & & & & 0 & & & & & & & & \\
\hline & & 027 & & & & & & 0.002 & & & & & & & \\
\hline & 4 & & & & & & & & & & & & & & \\
\hline & 186 & 031 & & & & & 08 & & 04 & 2 & & & & & \\
\hline & 188 & & & & & & & & & & & & & & \\
\hline & 192 & 037 & & & & & & & & & & & & & \\
\hline & & & & & & & & & & & & & & & \\
\hline & & & & & & & & & & & & & & & \\
\hline & & & & & & & & & & & & & & & \\
\hline & & & & & & & & & & & & & & & \\
\hline & 206 & 1 & & & & & & & & & & & & & \\
\hline & & & & & & & & & & & & & & & 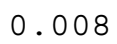 \\
\hline & .214 & .059 & 7 & 0.047 & & 38 & 36 & 4 & 32 & & 28 & 26 & & 18 & 0.014 \\
\hline & .223 & & & & & & & & & & & & & & \\
\hline & .227 & 0.072 & & 0. & & .051 & 49 & 7 & 45 & 3 & 41 & 39 & & 0.031 & 0.027 \\
\hline & .241 & 086 & & & & & & & & & & & & & \\
\hline & 5 & & & & & & & & & & & & & & 55 \\
\hline & & & & & & & & & & & & & & & \\
\hline & & & & & & & & & & & & & & & .79 \\
\hline
\end{tabular}


[0]

[1]

[2]

[3]

$[4]$

[5]

[6]

[7]

$[8]$

[9]

[A]

[B]

$[\mathrm{C}]$

[D]
$[E]$

[E]

$[\mathrm{G}]$
$[\mathrm{H}]$

J]

[J]

[L]

[M]

[N]

[P]

[Q]

$[\mathrm{R}]$

$\begin{array}{lllllllllll}0.202 & 0.204 & 0.206 & 0.208 & 0.214 & 0.223 & 0.227 & 0.241 & 0.255 & 0.436 & 0.999\end{array}$ $\begin{array}{llllllllllll}0.047 & 0.049 & 0.051 & 0.053 & 0.059 & 0.068 & 0.072 & 0.086 & 0.1 & 0.281 & 0.844\end{array}$ $\begin{array}{llllllllllll}0.045 & 0.047 & 0.049 & 0.051 & 0.057 & 0.066 & 0.07 & 0.084 & 0.098 & 0.279 & 0.842\end{array}$ $\begin{array}{llllllllllll}0.035 & 0.037 & 0.039 & 0.041 & 0.047 & 0.056 & 0.06 & 0.074 & 0.088 & 0.269 & 0.832\end{array}$ $\begin{array}{llllllllllll}0.031 & 0.033 & 0.035 & 0.037 & 0.043 & 0.052 & 0.056 & 0.07 & 0.084 & 0.265 & 0.828\end{array}$ $\begin{array}{llllllllllll}0.026 & 0.028 & 0.03 & 0.032 & 0.038 & 0.047 & 0.051 & 0.065 & 0.079 & 0.26 & 0.823\end{array}$ $\begin{array}{llllllllllll}0.024 & 0.026 & 0.028 & 0.03 & 0.036 & 0.045 & 0.049 & 0.063 & 0.077 & 0.258 & 0.821\end{array}$ $\begin{array}{llllllllllll}0.022 & 0.024 & 0.026 & 0.028 & 0.034 & 0.043 & 0.047 & 0.061 & 0.075 & 0.256 & 0.819\end{array}$ $\begin{array}{lllllllllllll}0.02 & 0.022 & 0.024 & 0.026 & 0.032 & 0.041 & 0.045 & 0.059 & 0.073 & 0.254 & 0.817\end{array}$ $\begin{array}{lllllllllllll}0.018 & 0.02 & 0.022 & 0.024 & 0.03 & 0.039 & 0.043 & 0.057 & 0.071 & 0.252 & 0.815\end{array}$ $\begin{array}{llllllllllll}0.016 & 0.018 & 0.02 & 0.022 & 0.028 & 0.037 & 0.041 & 0.055 & 0.069 & 0.25 & 0.813\end{array}$ $\begin{array}{llllllllllll}0.014 & 0.016 & 0.018 & 0.02 & 0.026 & 0.035 & 0.039 & 0.053 & 0.067 & 0.248 & 0.811\end{array}$ $\begin{array}{lllllllllllll}0.01 & 0.012 & 0.014 & 0.016 & 0.022 & 0.031 & 0.035 & 0.049 & 0.063 & 0.244 & 0.807\end{array}$ $\begin{array}{lllllllllllll}0.006 & 0.008 & 0.01 & 0.012 & 0.018 & 0.027 & 0.031 & 0.045 & 0.059 & 0.24 & 0.803\end{array}$ $\begin{array}{llllllllllll}0.002 & 0.004 & 0.006 & 0.008 & 0.014 & 0.023 & 0.027 & 0.041 & 0.055 & 0.236 & 0.799\end{array}$ $\begin{array}{llllllllllll}0.002 & 0.004 & 0.006 & 0.012 & 0.021 & 0.025 & 0.039 & 0.053 & 0.234 & 0.797\end{array}$ $\begin{array}{llllllllllll}0.002 & 0 & 0.002 & 0.004 & 0.01 & 0.019 & 0.023 & 0.037 & 0.051 & 0.232 & 0.795\end{array}$ $\begin{array}{llllllllllllll}0.004 & 0.002 & . & 0.002 & 0.008 & 0.017 & 0.021 & 0.035 & 0.049 & 0.23 & 0.793\end{array}$ $\begin{array}{llllllllllllll}0.006 & 0.004 & 0.002 & 0.006 & 0.015 & 0.019 & 0.033 & 0.047 & 0.228 & 0.791\end{array}$ $\begin{array}{llllllllllllll}0.012 & 0.01 & 0.008 & 0.006 & 0.009 & 0.013 & 0.027 & 0.041 & 0.222 & 0.785\end{array}$ $\begin{array}{lllllllllllll}0.021 & 0.019 & 0.017 & 0.015 & 0.009 & 0.004 & 0.018 & 0.032 & 0.213 & 0.776\end{array}$ $\begin{array}{llllllllllllll}0.025 & 0.023 & 0.021 & 0.019 & 0.013 & 0.004 & 0.014 & 0.028 & 0.209 & 0.772\end{array}$ $\begin{array}{llllllllllll}0.039 & 0.037 & 0.035 & 0.033 & 0.027 & 0.018 & 0.014 & 0.014 & 0.195 & 0.758\end{array}$ $\begin{array}{llllllllllll}0.053 & 0.051 & 0.049 & 0.047 & 0.041 & 0.032 & 0.028 & 0.014 & 0.181 & 0.744\end{array}$ $\begin{array}{lllllllllllll}0.234 & 0.232 & 0.23 & 0.228 & 0.222 & 0.213 & 0.209 & 0.195 & 0.181 & 0.563\end{array}$ $\begin{array}{lllllllllllll}0.797 & 0.795 & 0.793 & 0.791 & 0.785 & 0.776 & 0.772 & 0.758 & 0.744 & 0.563\end{array}$. 
USERTYPE meanringleistheight STEPMATRIX $=30$

\begin{tabular}{|c|c|c|c|c|c|c|c|c|c|c|c|c|c|c|c|}
\hline & & 1 & & 3 & 4 & 5 & 6 & & 8 & & A & B & C & D & $\mathrm{E}$ \\
\hline ] & & 8 & $\$ 3$ & 5 & .23 & 15 & 1 & 7 & 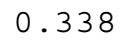 & 53 & 0. & 0. & 99 & 14 & 0.43 \\
\hline & 138 & & 015 & 077 & 092 & 107 & 0.123 & 169 & 02 & & 0.23 & & & & \\
\hline & 153 & 015 & & 62 & & & & & 185 & 0 & & & & & \\
\hline & 215 & 077 & 062 & & & & 0.046 & & & & & & & & \\
\hline & 23 & 092 & .077 & 0.015 & & 15 & & & & & & & & & \\
\hline & 245 & 107 & 092 & 0.03 & 5 & & & 62 & & & & & & & \\
\hline & & & & & & 0.016 & & & & & & & & & \\
\hline & 307 & 169 & 54 & 092 & & 0.062 & 0 & & & & & & & & \\
\hline & & & & & & & & 1 & & & & & & & \\
\hline & 53 & 15 & 2 & & & & & & & & & & & & \\
\hline & 8 & & & & & & & & & & & & & & \\
\hline & .384 & 246 & 1 & 59 & & 39 & 23 & & & 31 & .016 & & 15 & & \\
\hline & & & & & & & & & & & & & & & \\
\hline & 14 & 276 & 1 & 99 & 84 & 59 & 53 & 07 & 76 & 61 & & 0 & 0.015 & & 016 \\
\hline & & & & & & & & & & & & & & & \\
\hline & 445 & 07 & & 0 & & & 84 & 38 & & & & & & & 15 \\
\hline & & & & & & & & & & & & & & & \\
\hline & 476 & 338 & & & & 31 & & 59 & 38 & 3 & & & & 62 & \\
\hline & & & & & & & & & & & & & & & \\
\hline & 507 & 69 & & & & & & & & & & & & 93 & \\
\hline & & & & & & & & & & & & & & & \\
\hline & 537 & & 84 & & & & & & & 84 & & & & & \\
\hline & & & & & & & & & & & & & & & \\
\hline & 84 & & & & & & & & & & & & & & \\
\hline & 599 & 61 & & & & & & & & & & & & & \\
\hline & & & & & & & & & & & & & & & \\
\hline & & & & & & & & & & & & & & & \\
\hline & & & & & & & & & & & & & & & \\
\hline & 010 & 8 & & & & & & & & & & & & & \\
\hline & 999 & .861 & & & & & 0.738 & & 61 & & & & & 0.585 & 0.569 \\
\hline
\end{tabular}


[0] $\quad \begin{array}{llllllllllllllllllll}0.445 & 0.461 & 0.476 & 0.491 & 0.507 & 0.522 & 0.537 & 0.553 & 0.584 & 0.599 & 0.63 & 0.768 & 0.814 & 0.876 & 0.999\end{array}$

[1] $\quad \begin{array}{llllllllllllllllll}0.307 & 0.323 & 0.338 & 0.353 & 0.369 & 0.384 & 0.399 & 0.415 & 0.446 & 0.461 & 0.492 & 0.63 & 0.676 & 0.738 & 0.861\end{array}$

[2] $\quad \begin{array}{lllllllllllllllllllllll}0.292 & 0.308 & 0.323 & 0.338 & 0.354 & 0.369 & 0.384 & 0.4 & 0.431 & 0.446 & 0.477 & 0.615 & 0.661 & 0.723 & 0.846\end{array}$

[3] $\quad \begin{array}{lllllllllllllll}0.23 & 0.246 & 0.261 & 0.276 & 0.292 & 0.307 & 0.322 & 0.338 & 0.369 & 0.384 & 0.415 & 0.553 & 0.599 & 0.661 & 0.784\end{array}$

[4] $\quad \begin{array}{lllllllllllllllll}0.215 & 0.231 & 0.246 & 0.261 & 0.277 & 0.292 & 0.307 & 0.323 & 0.354 & 0.369 & 0.4 & 0.538 & 0.584 & 0.646 & 0.769\end{array}$

[5] $\quad \begin{array}{lllllllllllllllll}0.2 & 0.216 & 0.231 & 0.246 & 0.262 & 0.277 & 0.292 & 0.308 & 0.339 & 0.354 & 0.385 & 0.523 & 0.569 & 0.631 & 0.754\end{array}$

[6] $\quad \begin{array}{lllllllllllllllll}0.184 & 0.2 & 0.215 & 0.23 & 0.246 & 0.261 & 0.276 & 0.292 & 0.323 & 0.338 & 0.369 & 0.507 & 0.553 & 0.615 & 0.738\end{array}$

[7] $\quad \begin{array}{llllllllllllllll}0.138 & 0.154 & 0.169 & 0.184 & 0.2 & 0.215 & 0.23 & 0.246 & 0.277 & 0.292 & 0.323 & 0.461 & 0.507 & 0.569 & 0.692\end{array}$

[8] $\quad \begin{array}{llllllllllllllllll}0.107 & 0.123 & 0.138 & 0.153 & 0.169 & 0.184 & 0.199 & 0.215 & 0.246 & 0.261 & 0.292 & 0.43 & 0.476 & 0.538 & 0.661\end{array}$

[9] $\quad \begin{array}{llllllllllllllll}0.092 & 0.108 & 0.123 & 0.138 & 0.154 & 0.169 & 0.184 & 0.2 & 0.231 & 0.246 & 0.277 & 0.415 & 0.461 & 0.523 & 0.646\end{array}$

[A] $\quad \begin{array}{lllllllllllllll}0.077 & 0.093 & 0.108 & 0.123 & 0.139 & 0.154 & 0.169 & 0.185 & 0.216 & 0.231 & 0.262 & 0.4 & 0.446 & 0.508 & 0.631\end{array}$

[B] $\quad \begin{array}{llllllllllllllll}0.061 & 0.077 & 0.092 & 0.107 & 0.123 & 0.138 & 0.153 & 0.169 & 0.2 & 0.215 & 0.246 & 0.384 & 0.43 & 0.492 & 0.615\end{array}$

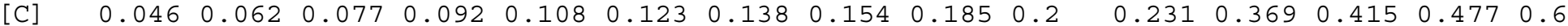

[D] $\quad \begin{array}{llllllllllllllll}0.031 & 0.047 & 0.062 & 0.077 & 0.093 & 0.108 & 0.123 & 0.139 & 0.17 & 0.185 & 0.216 & 0.354 & 0.4 & 0.462 & 0.585\end{array}$

[E] $\quad \begin{array}{lllllllllllllllll}0.015 & 0.031 & 0.046 & 0.061 & 0.077 & 0.092 & 0.107 & 0.123 & 0.154 & 0.169 & 0.2 & 0.338 & 0.384 & 0.446 & 0.569\end{array}$

[F] $\quad \begin{array}{lllllllllllllllllll}0.016 & 0.031 & 0.046 & 0.062 & 0.077 & 0.092 & 0.108 & 0.139 & 0.154 & 0.185 & 0.323 & 0.369 & 0.431 & 0.554\end{array}$

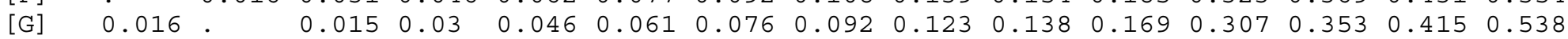

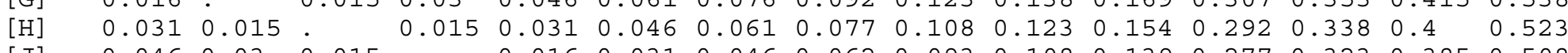

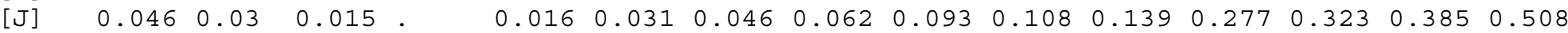

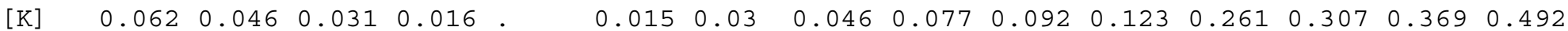

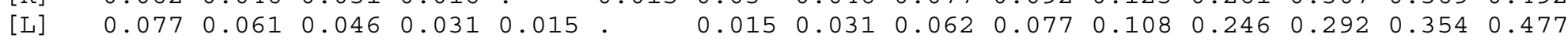

[M] $\quad \begin{array}{lllllllllllllllllllllllll}0.092 & 0.076 & 0.061 & 0.046 & 0.03 & 0.015 & 0.016 & 0.016 & 0.047 & 0.062 & 0.093 & 0.231 & 0.277 & 0.339 & 0.462\end{array}$

[N] $\quad \begin{array}{llllllllllllllllllllllllll}0.108 & 0.092 & 0.077 & 0.062 & 0.046 & 0.031 & 0.016 & 0.031 & 0.046 & 0.077 & 0.215 & 0.261 & 0.323 & 0.446\end{array}$

[P] $\quad \begin{array}{lllllllllllllllllll} & 0.139 & 0.123 & 0.108 & 0.093 & 0.077 & 0.062 & 0.047 & 0.031 & 0.015 & 0.046 & 0.184 & 0.23 & 0.292 & 0.415\end{array}$

[Q] $\quad \begin{array}{llllllllllllllllll} & 0.154 & 0.138 & 0.123 & 0.108 & 0.092 & 0.077 & 0.062 & 0.046 & 0.015 & . & 0.031 & 0.169 & 0.215 & 0.277 & 0.4\end{array}$

[R] $\quad \begin{array}{llllllllllllllllll}0.185 & 0.169 & 0.154 & 0.139 & 0.123 & 0.108 & 0.093 & 0.077 & 0.046 & 0.031 & . & 0.138 & 0.184 & 0.246 & 0.369\end{array}$

[S] $\quad \begin{array}{lllllllllllllllll}0.323 & 0.307 & 0.292 & 0.277 & 0.261 & 0.246 & 0.231 & 0.215 & 0.184 & 0.169 & 0.138 & . & 0.046 & 0.108 & 0.231\end{array}$

[T] $\quad \begin{array}{llllllllllllllllll}0.369 & 0.353 & 0.338 & 0.323 & 0.307 & 0.292 & 0.277 & 0.261 & 0.23 & 0.215 & 0.184 & 0.046 & . & 0.062 & 0.185\end{array}$

[U] $\quad \begin{array}{lllllllllllllllll}0.431 & 0.415 & 0.4 & 0.385 & 0.369 & 0.354 & 0.339 & 0.323 & 0.292 & 0.277 & 0.246 & 0.108 & 0.062 . & 0.123\end{array}$

[V] $\quad \begin{array}{llllllllllllll}0.554 & 0.538 & 0.523 & 0.508 & 0.492 & 0.477 & 0.462 & 0.446 & 0.415 & 0.4 & 0.369 & 0.231 & 0.1850 .123 & .\end{array}$ 
USERTYPE paringleistheight STEPMATRIX $=30$

\begin{tabular}{|c|c|c|c|c|c|c|c|c|c|c|c|c|c|c|c|}
\hline & & 1 & 2 & 3 & 4 & 5 & 6 & & 8 & 9 & 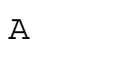 & B & C & D & $\mathrm{E}$ \\
\hline 0] & & 1 & 3 & 4 & 8 & 9 & 1 & 4 & 6 & 27 & 51 & 62 & 74 & 35 & 197 \\
\hline & 011 & & & 023 & & 058 & 07 & & & & & & & & \\
\hline & .023 & 012 & & 011 & 035 & .046 & 0.058 & .081 & .093 & 0.104 & 0.128 & 0.139 & 0.151 & 0.162 & .174 \\
\hline & 034 & 023 & 11 & & & & & & & & & & & & \\
\hline & 058 & 047 & .035 & 0.024 & & .011 & 023 & .046 & 058 & 69 & 93 & 0.104 & 16 & 27 & 139 \\
\hline & 069 & 058 & 046 & 0.035 & .011 & & & & & & & & & & \\
\hline & & 07 & & & 023 & 0.012 & & 23 & & & & & & & \\
\hline & 4 & 093 & & & & 0.035 & 23 & & & & & & & & \\
\hline & & & & & & & & .012 & & & & & & & \\
\hline & 7 & & & & & & & & & & & & & & \\
\hline & & & & & & & & & & 4 & & & & & \\
\hline & 62 & & & & & & & & & & & & & & \\
\hline & & 63 & & & & & & & & & & & & & \\
\hline & 35 & 174 & & & & & & & & & & & & & \\
\hline & & & & & & & & & & & & & & & \\
\hline & & & & & & & & & & & & & & & .012 \\
\hline & & & & & & & & & & & & & & & \\
\hline & .243 & 32 & & & & & & & & & & & & & \\
\hline & & & & & & & & & & & & & & & \\
\hline & 267 & 0.256 & 14 & 0.233 & & 98 & & & 51 & 4 & & & & 82 & 07 \\
\hline & & & & & & & & & & & & & & & \\
\hline & 302 & 291 & & & & 33 & 21 & & 86 & 75 & 51 & & & 17 & 05 \\
\hline & & & & & & & & & & & & & & & \\
\hline & 3 & & & & & & & & & & & & & & 86 \\
\hline & & & & & & & & & & & & & & & \\
\hline & & & & & & & & & & & & & & 21 & 9 \\
\hline & & & & & & & & & & & & & & & \\
\hline & & & & & & & & & & & & & & & \\
\hline & & 0.778 & & & & & & & & & & & & & \\
\hline & & & & & & & & & & & & & 25 & 0 & \\
\hline
\end{tabular}


[0] $\quad \begin{array}{lllllllllllllllll}0.209 & 0.232 & 0.243 & 0.255 & 0.267 & 0.29 & 0.302 & 0.325 & 0.383 & 0.394 & 0.406 & 0.429 & 0.499 & 0.789 & 0.999\end{array}$

[1] $\quad \begin{array}{llllllllllllllllll}0.198 & 0.221 & 0.232 & 0.244 & 0.256 & 0.279 & 0.291 & 0.314 & 0.372 & 0.383 & 0.395 & 0.418 & 0.488 & 0.778 & 0.988\end{array}$

[2] $\quad \begin{array}{lllllllllllllllll}0.186 & 0.209 & 0.22 & 0.232 & 0.244 & 0.267 & 0.279 & 0.302 & 0.36 & 0.371 & 0.383 & 0.406 & 0.476 & 0.766 & 0.976\end{array}$

[3] $\quad \begin{array}{llllllllllllllll}0.175 & 0.198 & 0.209 & 0.221 & 0.233 & 0.256 & 0.268 & 0.291 & 0.349 & 0.36 & 0.372 & 0.395 & 0.465 & 0.755 & 0.965\end{array}$

[4] $\quad \begin{array}{llllllllllllllllllll}0.151 & 0.174 & 0.185 & 0.197 & 0.209 & 0.232 & 0.244 & 0.267 & 0.325 & 0.336 & 0.348 & 0.371 & 0.441 & 0.731 & 0.941\end{array}$

[5] $\quad \begin{array}{llllllllllllllll}0.14 & 0.163 & 0.174 & 0.186 & 0.198 & 0.221 & 0.233 & 0.256 & 0.314 & 0.325 & 0.337 & 0.36 & 0.43 & 0.72 & 0.93\end{array}$

[6] $\quad \begin{array}{llllllllllllllll}0.128 & 0.151 & 0.162 & 0.174 & 0.186 & 0.209 & 0.221 & 0.244 & 0.302 & 0.313 & 0.325 & 0.348 & 0.418 & 0.708 & 0.918\end{array}$

[7] $\quad \begin{array}{lllllllllllllllll}0.105 & 0.128 & 0.139 & 0.151 & 0.163 & 0.186 & 0.198 & 0.221 & 0.279 & 0.29 & 0.302 & 0.325 & 0.395 & 0.685 & 0.895\end{array}$

[8] $\quad \begin{array}{lllllllllllllllllll}0.093 & 0.116 & 0.127 & 0.139 & 0.151 & 0.174 & 0.186 & 0.209 & 0.267 & 0.278 & 0.29 & 0.313 & 0.383 & 0.673 & 0.883\end{array}$

[9] $\quad \begin{array}{lllllllllllllllll}0.082 & 0.105 & 0.116 & 0.128 & 0.14 & 0.163 & 0.175 & 0.198 & 0.256 & 0.267 & 0.279 & 0.302 & 0.372 & 0.662 & 0.872\end{array}$

[A] $\quad \begin{array}{llllllllllllllllll}0.058 & 0.081 & 0.092 & 0.104 & 0.116 & 0.139 & 0.151 & 0.174 & 0.232 & 0.243 & 0.255 & 0.278 & 0.348 & 0.638 & 0.848\end{array}$

[B] $\quad \begin{array}{llllllllllllllllll}0.047 & 0.07 & 0.081 & 0.093 & 0.105 & 0.128 & 0.14 & 0.163 & 0.221 & 0.232 & 0.244 & 0.267 & 0.337 & 0.627 & 0.837\end{array}$

[C] $\quad \begin{array}{llllllllllllllllll}0.035 & 0.058 & 0.069 & 0.081 & 0.093 & 0.116 & 0.128 & 0.151 & 0.209 & 0.22 & 0.232 & 0.255 & 0.325 & 0.615 & 0.825\end{array}$

[D] $\quad \begin{array}{lllllllllllllllll}0.024 & 0.047 & 0.058 & 0.07 & 0.082 & 0.105 & 0.117 & 0.14 & 0.198 & 0.209 & 0.221 & 0.244 & 0.314 & 0.604 & 0.814\end{array}$

$[\mathrm{E}] \quad \begin{array}{llllllllllllllllll}0.012 & 0.035 & 0.046 & 0.058 & 0.07 & 0.093 & 0.105 & 0.128 & 0.186 & 0.197 & 0.209 & 0.232 & 0.302 & 0.592 & 0.802\end{array}$

[F] $\quad \begin{array}{llllllllllllllll}0 & 0.023 & 0.034 & 0.046 & 0.058 & 0.081 & 0.093 & 0.116 & 0.174 & 0.185 & 0.197 & 0.22 & 0.29 & 0.58 & 0.79\end{array}$

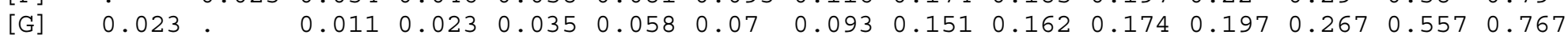

$[\mathrm{H}] \quad \begin{array}{llllllllllllllllllllllll} & 0.034 & 0.011 & . & & 0.012 & 0.024 & 0.047 & 0.059 & 0.082 & 0.14 & 0.151 & 0.163 & 0.186 & 0.256 & 0.546 & 0.756\end{array}$

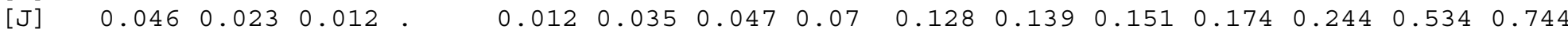

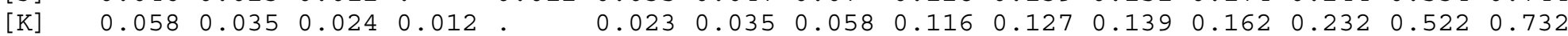

[L] $\quad \begin{array}{lllllllllllllllllllllllll} & 0.081 & 0.058 & 0.047 & 0.035 & 0.023 & . & 0.012 & 0.035 & 0.093 & 0.104 & 0.116 & 0.139 & 0.209 & 0.499 & 0.709\end{array}$

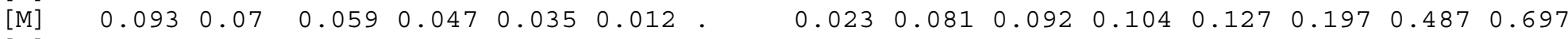

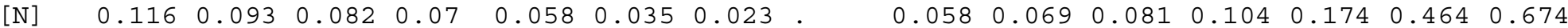

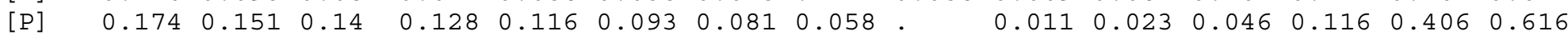

[Q] $\quad \begin{array}{lllllllllllllllllll}0.185 & 0.162 & 0.151 & 0.139 & 0.127 & 0.104 & 0.092 & 0.069 & 0.011 & 0.012 & 0.035 & 0.105 & 0.395 & 0.605\end{array}$

[R] $\quad \begin{array}{llllllllllllllllllll}0.197 & 0.174 & 0.163 & 0.151 & 0.139 & 0.116 & 0.104 & 0.081 & 0.023 & 0.012 & . & 0.023 & 0.093 & 0.383 & 0.593\end{array}$

[S] $\quad \begin{array}{lllllllllllllllll}0.22 & 0.197 & 0.186 & 0.174 & 0.162 & 0.139 & 0.127 & 0.104 & 0.046 & 0.035 & 0.023 & . & 0.07 & 0.36 & 0.57\end{array}$

[T] $\quad \begin{array}{llllllllllllllllll} & 0.29 & 0.267 & 0.256 & 0.244 & 0.232 & 0.209 & 0.197 & 0.174 & 0.116 & 0.105 & 0.093 & 0.07 & . & 0.29 & 0.5\end{array}$

[U] $\quad \begin{array}{llllllllllllllllll}0.58 & 0.557 & 0.546 & 0.534 & 0.522 & 0.499 & 0.487 & 0.464 & 0.406 & 0.395 & 0.383 & 0.36 & 0.29 & . & 0.21\end{array}$

[V] $\quad \begin{array}{llllllllllllllll}0.79 & 0.767 & 0.756 & 0.744 & 0.732 & 0.709 & 0.697 & 0.674 & 0.616 & 0.605 & 0.593 & 0.57 & 0.5 & 0.21 & .\end{array}$ 
USERTYPE meanringleistdpi STEPMATRIX = 31

\begin{tabular}{|c|c|c|c|c|c|c|c|c|c|c|c|c|c|c|c|}
\hline & 0 & 1 & 2 & 3 & 4 & 5 & 6 & 7 & 8 & 9 & A & B & $\mathrm{C}$ & D & $\mathrm{E}$ \\
\hline [0] & . & 0.013 & 0.027 & .041 & 0.054 & 0.068 & 0.082 & 0.095 & 0.109 & 0.123 & 0.136 & 0.164 & 0.177 & 0.205 & 0.218 \\
\hline [1] & 0.013 & 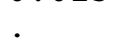 & 0.014 & 0.028 & 0.041 & 0.055 & 0.069 & 0.082 & 0.096 & 0.11 & 0.123 & 0.151 & 0.164 & 0.192 & 0.205 \\
\hline [2] & 0.027 & 0.014 & . & 0.014 & 0.027 & 0.041 & 0.055 & 0.068 & 0.082 & 0.096 & 0.109 & 0.137 & 0.15 & 0.178 & 0.191 \\
\hline [3] & 0.041 & 0.028 & 0.014 & 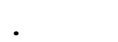 & 0.013 & 0.027 & 0.041 & 0.054 & 0.068 & 0.082 & 0.095 & 0.123 & 0.136 & 0.164 & 0.177 \\
\hline [4] & 0.054 & 0.041 & 0.027 & 0.013 & . & 0.014 & 0.028 & 0.041 & 0.055 & 0.069 & 0.082 & 0.11 & 0.123 & 0.151 & 0.164 \\
\hline [5] & 0.068 & 0.055 & 0.041 & 0.027 & 0.014 & • & 0.014 & 0.027 & 0.041 & 0.055 & 0.068 & 0.096 & 0.109 & 0.137 & 0.15 \\
\hline [6] & 0.082 & 0.069 & 0.055 & 0.041 & 0.028 & 0.014 & & 0.013 & 0.027 & 0.041 & 0.054 & 0.082 & 0.095 & 0.123 & 0.136 \\
\hline [7] & 0.095 & 0.082 & 0.068 & 0.054 & 0.041 & 0.027 & 0.013 & & 0.014 & 0.028 & 0.041 & 0.069 & 0.082 & 0.11 & 0.123 \\
\hline [8] & 0.109 & 0.096 & 0.082 & 0.068 & 0.055 & 0.041 & 0.027 & 0.014 & $\theta^{\circ}$ & 0.014 & 0.027 & 0.055 & 0.068 & 0.096 & 0.109 \\
\hline [9] & 0.123 & 0.11 & 0.096 & 0.082 & 0.069 & 0.055 & 0.041 & 0.028 & 0.014 & . & 0.013 & 0.041 & 0.054 & 0.082 & 0.095 \\
\hline [A] & 0.136 & 0.123 & 0.109 & 0.095 & 0.082 & 0.068 & 0.054 & 0.041 & 0.027 & 0.013 & & 0.028 & 0.041 & 0.069 & 0.082 \\
\hline [B] & 0.164 & 0.151 & 0.137 & 0.123 & 0.11 & 0.096 & 0.082 & 0.069 & 0.055 & 0.041 & 0.028 & & 0.013 & 0.041 & 0.054 \\
\hline [C] & 0.177 & 0.164 & 0.15 & 0.136 & 0.123 & 0.109 & 0.095 & 0.082 & 0.068 & 0.054 & 0.041 & 0.013 & . & 0.028 & 0.041 \\
\hline [D] & 0.205 & 0.192 & 0.178 & 0.164 & 0.151 & 0.137 & 0.123 & 0.11 & 0.096 & 0.082 & 0.069 & 0.041 & 0.028 & 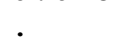 & 0.013 \\
\hline$[E]$ & 0.218 & 0.205 & 0.191 & 0.177 & 0.164 & 0.15 & 0.136 & 0.123 & 0.109 & 0.095 & 0.082 & 0.054 & 0.041 & & \\
\hline$[\mathrm{F}]$ & 0.232 & 0.219 & 0.205 & 0.191 & 0.178 & 0.164 & 0.15 & 0.137 & 0.123 & 0.109 & 0.096 & 0.068 & 0.055 & 0.027 & 0.014 \\
\hline [G] & 0.246 & 0.233 & 0.219 & 0.205 & 0.192 & 0.178 & 0.164 & 0.151 & 0.137 & 0.123 & 0.11 & 0.082 & 0.069 & 0.041 & 0.028 \\
\hline [H] & 0.287 & 0.274 & 0.26 & 0.246 & 0.233 & 0.219 & 0.205 & 0.192 & 0.178 & 0.164 & 0.151 & 0.123 & 0.11 & 0.082 & 0.069 \\
\hline$[\mathrm{J}]$ & 0.301 & 0.288 & 0.274 & 0.26 & 0.247 & 0.233 & 0.219 & 0.206 & 0.192 & 0.178 & 0.165 & 0.137 & 0.124 & 0.096 & 0.083 \\
\hline$[\mathrm{K}]$ & 0.314 & 0.301 & 0.287 & 0.273 & 0.26 & 0.246 & 0.232 & 0.219 & 0.205 & 0.191 & 0.178 & 0.15 & 0.137 & 0.109 & 0.096 \\
\hline [L] & 0.328 & 0.315 & 0.301 & 0.287 & 0.274 & 0.26 & 0.246 & 0.233 & 0.219 & 0.205 & 0.192 & 0.164 & 0.151 & 0.123 & 0.11 \\
\hline [M] & 0.342 & 0.329 & 0.315 & 0.301 & 0.288 & 0.274 & 0.26 & 0.247 & 0.233 & 0.219 & 0.206 & 0.178 & 0.165 & 0.137 & 0.124 \\
\hline$[\mathrm{N}]$ & 0.355 & 0.342 & 0.328 & 0.314 & 0.301 & 0.287 & 0.273 & 0.26 & 0.246 & 0.232 & 0.219 & 0.191 & 0.178 & 0.15 & 0.137 \\
\hline$[\mathrm{P}]$ & 0.383 & 0.37 & 0.356 & 0.342 & 0.329 & 0.315 & 0.301 & 0.288 & 0.274 & 0.26 & 0.247 & 0.219 & 0.206 & 0.178 & 0.165 \\
\hline [Q] & 0.396 & 0.383 & 0.369 & 0.355 & 0.342 & 0.328 & 0.314 & 0.301 & 0.287 & 0.273 & 0.26 & 0.232 & 0.219 & 0.191 & 0.178 \\
\hline$[\mathrm{R}]$ & 0.629 & 0.616 & 0.602 & 0.588 & 0.575 & 0.561 & 0.547 & 0.534 & 0.52 & 0.506 & 0.493 & 0.465 & 0.452 & 0.424 & 0.411 \\
\hline$[S]$ & 0.643 & 0.63 & 0.616 & 0.602 & 0.589 & 0.575 & 0.561 & 0.548 & 0.534 & 0.52 & 0.507 & 0.479 & 0.466 & 0.438 & 0.425 \\
\hline$[\mathrm{T}]$ & 0.711 & 0.698 & 0.684 & 0.67 & 0.657 & 0.643 & 0.629 & 0.616 & 0.602 & 0.588 & 0.575 & 0.547 & 0.534 & 0.506 & 0.493 \\
\hline [U] & 0.848 & 0.835 & 0.821 & 0.807 & 0.794 & 0.78 & 0.766 & 0.753 & 0.739 & 0.725 & 0.712 & 0.684 & 0.671 & 0.643 & 0.63 \\
\hline [V] & 0.957 & 0.944 & 0.93 & 0.916 & 0.903 & 0.889 & 0.875 & 0.862 & 0.848 & 0.834 & 0.821 & 0.793 & 0.78 & 0.752 & 0.739 \\
\hline [W] & 0.999 & 0.986 & 0.972 & 0.958 & 0.945 & 0.931 & 0.917 & 0.904 & 0.89 & 0.876 & 0.863 & 0.835 & 0.822 & 0.794 & 0.781 \\
\hline
\end{tabular}


[0] $\quad \begin{array}{llllllllllllllllllll}0.232 & 0.246 & 0.287 & 0.301 & 0.314 & 0.328 & 0.342 & 0.355 & 0.383 & 0.396 & 0.629 & 0.643 & 0.711 & 0.848 & 0.957\end{array}$

[1] $\quad \begin{array}{llllllllllllllllll}0.219 & 0.233 & 0.274 & 0.288 & 0.301 & 0.315 & 0.329 & 0.342 & 0.37 & 0.383 & 0.616 & 0.63 & 0.698 & 0.835 & 0.944\end{array}$

[2] $\quad \begin{array}{llllllllllllllllllll}0.205 & 0.219 & 0.26 & 0.274 & 0.287 & 0.301 & 0.315 & 0.328 & 0.356 & 0.369 & 0.602 & 0.616 & 0.684 & 0.821 & 0.93\end{array}$

[3] $\quad \begin{array}{llllllllllllllllll}0.191 & 0.205 & 0.246 & 0.26 & 0.273 & 0.287 & 0.301 & 0.314 & 0.342 & 0.355 & 0.588 & 0.602 & 0.67 & 0.807 & 0.916\end{array}$

[4] $\quad \begin{array}{llllllllllllllllll}0.178 & 0.192 & 0.233 & 0.247 & 0.26 & 0.274 & 0.288 & 0.301 & 0.329 & 0.342 & 0.575 & 0.589 & 0.657 & 0.794 & 0.903\end{array}$

[5] $\quad \begin{array}{llllllllllllllllll}0.164 & 0.178 & 0.219 & 0.233 & 0.246 & 0.26 & 0.274 & 0.287 & 0.315 & 0.328 & 0.561 & 0.575 & 0.643 & 0.78 & 0.889\end{array}$

[6] $\quad \begin{array}{llllllllllllllll}0.15 & 0.164 & 0.205 & 0.219 & 0.232 & 0.246 & 0.26 & 0.273 & 0.301 & 0.314 & 0.547 & 0.561 & 0.629 & 0.766 & 0.875\end{array}$

[7] $\quad \begin{array}{llllllllllllllll}0.137 & 0.151 & 0.192 & 0.206 & 0.219 & 0.233 & 0.247 & 0.26 & 0.288 & 0.301 & 0.534 & 0.548 & 0.616 & 0.753 & 0.862\end{array}$

[8] $\quad \begin{array}{lllllllllllllllllll}0.123 & 0.137 & 0.178 & 0.192 & 0.205 & 0.219 & 0.233 & 0.246 & 0.274 & 0.287 & 0.52 & 0.534 & 0.602 & 0.739 & 0.848\end{array}$

[9] $\quad \begin{array}{llllllllllllllll}0.109 & 0.123 & 0.164 & 0.178 & 0.191 & 0.205 & 0.219 & 0.232 & 0.26 & 0.273 & 0.506 & 0.52 & 0.588 & 0.725 & 0.834\end{array}$

[A] $\quad \begin{array}{llllllllllllllllll}0.096 & 0.11 & 0.151 & 0.165 & 0.178 & 0.192 & 0.206 & 0.219 & 0.247 & 0.26 & 0.493 & 0.507 & 0.575 & 0.712 & 0.821\end{array}$

[B] $\quad \begin{array}{llllllllllllllllll}0.068 & 0.082 & 0.123 & 0.137 & 0.15 & 0.164 & 0.178 & 0.191 & 0.219 & 0.232 & 0.465 & 0.479 & 0.547 & 0.684 & 0.793\end{array}$

[C] $\quad \begin{array}{llllllllllllllllllll}0.055 & 0.069 & 0.11 & 0.124 & 0.137 & 0.151 & 0.165 & 0.178 & 0.206 & 0.219 & 0.452 & 0.466 & 0.534 & 0.671 & 0.78\end{array}$

[D] $\quad \begin{array}{lllllllllllllllll}0.027 & 0.041 & 0.082 & 0.096 & 0.109 & 0.123 & 0.137 & 0.15 & 0.178 & 0.191 & 0.424 & 0.438 & 0.506 & 0.643 & 0.752\end{array}$

[E] $\quad \begin{array}{lllllllllllllllll}0.014 & 0.028 & 0.069 & 0.083 & 0.096 & 0.11 & 0.124 & 0.137 & 0.165 & 0.178 & 0.411 & 0.425 & 0.493 & 0.63 & 0.739\end{array}$

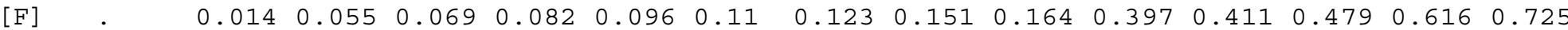

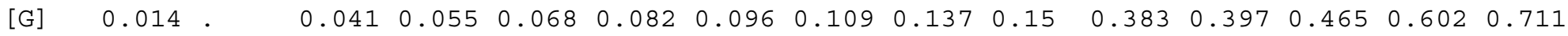

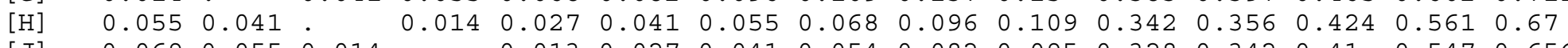

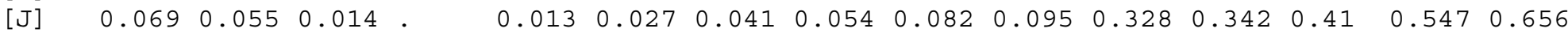

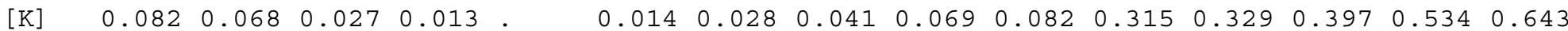

[L] $\quad \begin{array}{lllllllllllllllllllllllll}0.096 & 0.082 & 0.041 & 0.027 & 0.014 & . & 0.014 & 0.027 & 0.055 & 0.068 & 0.301 & 0.315 & 0.383 & 0.52 & 0.629\end{array}$

[M] $\quad \begin{array}{llllllllllllllllllllllllll}0.11 & 0.096 & 0.055 & 0.041 & 0.028 & 0.014 & 0.013 & 0.041 & 0.054 & 0.287 & 0.301 & 0.369 & 0.506 & 0.615\end{array}$

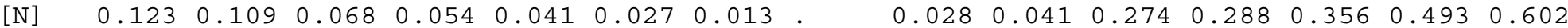

[P] $\quad \begin{array}{lllllllllllllllll}0.151 & 0.137 & 0.096 & 0.082 & 0.069 & 0.055 & 0.041 & 0.028 & . & 0.013 & 0.246 & 0.26 & 0.328 & 0.465 & 0.574\end{array}$

[Q] $\quad \begin{array}{llllllllllllllllll}0.164 & 0.15 & 0.109 & 0.095 & 0.082 & 0.068 & 0.054 & 0.041 & 0.013 & . & 0.233 & 0.247 & 0.315 & 0.452 & 0.561\end{array}$

[R] $\quad \begin{array}{llllllllllllllllllll}0.397 & 0.383 & 0.342 & 0.328 & 0.315 & 0.301 & 0.287 & 0.274 & 0.246 & 0.233 & . & 0.014 & 0.082 & 0.219 & 0.328\end{array}$

[S] $\quad \begin{array}{lllllllllllllllll}0.411 & 0.397 & 0.356 & 0.342 & 0.329 & 0.315 & 0.301 & 0.288 & 0.26 & 0.247 & 0.014 & . & 0.068 & 0.205 & 0.314\end{array}$

[T] $\quad \begin{array}{llllllllllllllllll}0.479 & 0.465 & 0.424 & 0.41 & 0.397 & 0.383 & 0.369 & 0.356 & 0.328 & 0.315 & 0.082 & 0.068 & 0.0 .137 & 0.246\end{array}$

[U] $\quad \begin{array}{llllllllllllllllllll}0.616 & 0.602 & 0.561 & 0.547 & 0.534 & 0.52 & 0.506 & 0.493 & 0.465 & 0.452 & 0.219 & 0.205 & 0.137 & . & 0.109\end{array}$

[V] $\quad \begin{array}{llllllllllllllllll}0.725 & 0.711 & 0.67 & 0.656 & 0.643 & 0.629 & 0.615 & 0.602 & 0.574 & 0.561 & 0.328 & 0.314 & 0.246 & 0.109 & .\end{array}$

[W] $\quad \begin{array}{lllllllllllllllll}0.767 & 0.753 & 0.712 & 0.698 & 0.685 & 0.671 & 0.657 & 0.644 & 0.616 & 0.603 & 0.37 & 0.356 & 0.288 & 0.151 & 0.042\end{array}$ 


$\begin{array}{ll}\text { [O] } & 0.999 \\ {[1]} & 0.986 \\ {[2]} & 0.972 \\ {[3]} & 0.958 \\ {[4]} & 0.945 \\ {[5]} & 0.931 \\ {[6]} & 0.917 \\ {[7]} & 0.904 \\ {[8]} & 0.89 \\ {[9]} & 0.876 \\ {[\mathrm{~A}]} & 0.863 \\ {[\mathrm{~B}]} & 0.835 \\ {[\mathrm{C}]} & 0.822 \\ {[\mathrm{D}]} & 0.794 \\ {[\mathrm{E}]} & 0.781 \\ {[\mathrm{~F}]} & 0.767 \\ {[\mathrm{G}]} & 0.753 \\ {[\mathrm{H}]} & 0.712 \\ {[\mathrm{~J}]} & 0.698 \\ {[\mathrm{~K}]} & 0.685 \\ {[\mathrm{~L}]} & 0.671 \\ {[\mathrm{M}]} & 0.657 \\ {[\mathrm{~N}]} & 0.644 \\ {[\mathrm{P}]} & 0.616 \\ {[\mathrm{Q}]} & 0.603 \\ {[\mathrm{R}]} & 0.37 \\ {[\mathrm{~S}]} & 0.356 \\ {[\mathrm{~T}]} & 0.288 \\ {[\mathrm{U}]} & 0.151 \\ {[\mathrm{~V}]} & 0.042 \\ {[\mathrm{~W}]} & . \\ \end{array}$


USERTYPE paringleistdpi STEPMATRIX $=24$

\begin{tabular}{|c|c|c|c|c|c|c|c|c|c|c|c|c|c|c|c|}
\hline & & 1 & & 3 & 4 & 5 & 6 & 1 & 8 & 9 & A & B & C & D & $\mathrm{E}$ \\
\hline 0] & & 0 & 5 & 53 & & 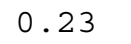 & 58 & 7 & 5 & & 0. & 0. & 99 & 537 & \\
\hline & 076 & & 039 & 077 & & 154 & & & & & & & & & \\
\hline & .115 & .039 & & 38 & & & & & & & & & & & \\
\hline & 53 & 077 & 038 & & & & & & & & & & & & \\
\hline & 192 & 116 & .077 & 0.039 & & 38 & & & & & & & & & \\
\hline & .23 & 154 & 15 & 0.077 & 8 & & & & & & & & & & \\
\hline & & & & & & 0.038 & & & & & & & & & \\
\hline & 307 & 231 & 92 & & & & & & & & & & & & \\
\hline & 5 & & & & & & & & & & & & & & \\
\hline & & 8 & & & & & & & & & & & & & \\
\hline & 22 & & & & & & & & & & & & & & \\
\hline & 51 & 35 & 6 & & & 31 & & 54 & & & 39 & & & & 15 \\
\hline & & & & & & & & & & & & & & & \\
\hline & .537 & 461 & 22 & 84 & 45 & 07 & 59 & 3 & & 53 & & & 8 & & 39 \\
\hline & & & & & & & & & & & & & & & \\
\hline & .614 & 538 & 99 & 0.461 & 22 & 384 & 46 & .307 & & 0.23 & & & & & 38 \\
\hline & & & & & & & & & & 9 & & & & & 077 \\
\hline & 91 & & & & & & & & & 7 & & & & .54 & 115 \\
\hline & & & & & & & & & & & & & & & \\
\hline & 58 & & & & & & & & & & & & & 31 & 92 \\
\hline & & & & & & & & & & & & & & & \\
\hline & & & & & & & & & & & & & & & \\
\hline & & & & & & & & & & & & & & & \\
\hline & & 23 & & 6 & 07 & 69 & 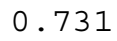 & .692 & 54 & 0.615 & 577 & 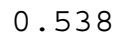 & & 0.462 & 0.42 \\
\hline
\end{tabular}


[0] $\quad \begin{array}{llllllllll}0.614 & 0.653 & 0.691 & 0.73 & 0.768 & 0.806 & 0.845 & 0.922 & 0.999\end{array}$

[1] $\quad \begin{array}{llllllllll}0.538 & 0.577 & 0.615 & 0.654 & 0.692 & 0.73 & 0.769 & 0.846 & 0.923\end{array}$

[2] $\quad \begin{array}{lllllllllll}0.499 & 0.538 & 0.576 & 0.615 & 0.653 & 0.691 & 0.73 & 0.807 & 0.884\end{array}$

[3] $\quad \begin{array}{lllllllllll}0.461 & 0.5 & 0.538 & 0.577 & 0.615 & 0.653 & 0.692 & 0.769 & 0.846\end{array}$

[4] $\quad \begin{array}{llllllllll}0.422 & 0.461 & 0.499 & 0.538 & 0.576 & 0.614 & 0.653 & 0.73 & 0.807\end{array}$

[5] $\quad \begin{array}{lllllllllll}0.384 & 0.423 & 0.461 & 0.5 & 0.538 & 0.576 & 0.615 & 0.692 & 0.769\end{array}$

[6] $\quad \begin{array}{llllllllll}0.346 & 0.385 & 0.423 & 0.462 & 0.5 & 0.538 & 0.577 & 0.654 & 0.731\end{array}$

[7] $\quad \begin{array}{lllllllllll}0.307 & 0.346 & 0.384 & 0.423 & 0.461 & 0.499 & 0.538 & 0.615 & 0.692\end{array}$

[8] $\quad \begin{array}{lllllllllll}0.269 & 0.308 & 0.346 & 0.385 & 0.423 & 0.461 & 0.5 & 0.577 & 0.654\end{array}$

[9] $\quad \begin{array}{lllllllllll}0.23 & 0.269 & 0.307 & 0.346 & 0.384 & 0.422 & 0.461 & 0.538 & 0.615\end{array}$

[A] $\quad \begin{array}{llllllllll}0.192 & 0.231 & 0.269 & 0.308 & 0.346 & 0.384 & 0.423 & 0.5 & 0.577\end{array}$

[B] $\quad \begin{array}{llllllllllll}0.153 & 0.192 & 0.23 & 0.269 & 0.307 & 0.345 & 0.384 & 0.461 & 0.538\end{array}$

[C] $\quad \begin{array}{llllllllllll}0.115 & 0.154 & 0.192 & 0.231 & 0.269 & 0.307 & 0.346 & 0.423 & 0.5\end{array}$

[D] $\quad \begin{array}{lllllllllll}0.077 & 0.116 & 0.154 & 0.193 & 0.231 & 0.269 & 0.308 & 0.385 & 0.462\end{array}$

[E] $\quad \begin{array}{lllllllllll}0.038 & 0.077 & 0.115 & 0.154 & 0.192 & 0.23 & 0.269 & 0.346 & 0.423\end{array}$

[F] $\quad \begin{array}{lllllllllll} & 0.039 & 0.077 & 0.116 & 0.154 & 0.192 & 0.231 & 0.308 & 0.385\end{array}$

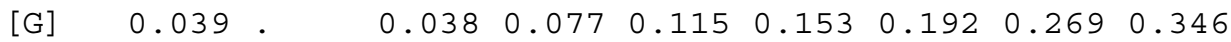

[H] $\quad \begin{array}{lllllllllllll} & 0.077 & 0.038 & . & 0.039 & 0.077 & 0.115 & 0.154 & 0.231 & 0.308\end{array}$

[J] $\quad \begin{array}{llllllllllll}0.116 & 0.077 & 0.039 & 0.038 & 0.076 & 0.115 & 0.192 & 0.269\end{array}$

[K] $\quad \begin{array}{lllllllllll}0.154 & 0.115 & 0.077 & 0.038 & . & 0.038 & 0.077 & 0.154 & 0.231\end{array}$

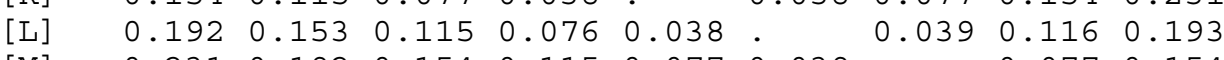

[M] $\quad \begin{array}{llllllllllll}0.231 & 0.192 & 0.154 & 0.115 & 0.077 & 0.039 & . & 0.077 & 0.154\end{array}$

$[\mathrm{N}] \quad \begin{array}{lllllllll}0.308 & 0.269 & 0.231 & 0.192 & 0.154 & 0.116 & 0.077 & 0.077\end{array}$

$\begin{array}{lllllllllll}{[P]} & 0.385 & 0.346 & 0.308 & 0.269 & 0.231 & 0.193 & 0.154 & 0.077\end{array}$. 
USERTYPE meanringleistthickness STEPMATRIX = 26

\begin{tabular}{|c|c|c|c|c|c|c|c|c|c|c|c|c|c|c|c|}
\hline & 0 & 1 & 2 & 3 & 4 & 5 & 6 & 7 & 8 & 9 & A & B & $\mathrm{C}$ & $\mathrm{D}$ & $\mathrm{E}$ \\
\hline$[0]$ & . & 0.041 & 0.083 & 0.104 & 0.124 & 0.166 & 0.187 & 0.228 & 0.249 & 0.27 & 0.291 & 0.291 & 0.312 & 0.333 & 0.353 \\
\hline [1] & 0.041 & . & 0.042 & 0.063 & 0.083 & 0.125 & 0.146 & 0.187 & 0.208 & 0.229 & 0.25 & 0.25 & 0.271 & 0.292 & 0.312 \\
\hline [2] & 0.083 & 0.042 & 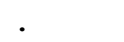 & 0.021 & 0.041 & 0.083 & 0.104 & 0.145 & 0.166 & 0.187 & 0.208 & 0.208 & 0.229 & 0.25 & 0.27 \\
\hline [3] & 0.104 & 0.063 & 0.021 & . & 0.02 & 0.062 & 0.083 & 0.124 & 0.145 & 0.166 & 0.187 & 0.187 & 0.208 & 0.229 & 0.249 \\
\hline$[4$ & 0.124 & 0.083 & 0.041 & 0.02 & . & 0.042 & 0.063 & 0.104 & 0.125 & 0.146 & 0.167 & 0.167 & 0.188 & 0.209 & 0.229 \\
\hline$[0$ & 0.166 & 0.125 & 0.083 & 0.062 & 0.042 & & 0.021 & 0.062 & 0.083 & 0.104 & 0.125 & 0.125 & 0.146 & 0.167 & 0.187 \\
\hline$[6]$ & 0.187 & 0.146 & 0.104 & 0.083 & 0.063 & 0.021 & . & 0.041 & 0.062 & 0.083 & 0.104 & 0.104 & 0.125 & 0.146 & 0.166 \\
\hline$[7]$ & 0.228 & 0.187 & 0.145 & 0.124 & 0.104 & 0.062 & 0.041 & . & 0.021 & 0.042 & 0.063 & 0.063 & 0.084 & 0.105 & 0.125 \\
\hline$[8$ & 0.249 & 0.208 & 0.166 & 0.145 & 0.125 & 0.083 & 0.062 & 0.021 & & 0.021 & 0.042 & 0.042 & 0.063 & 0.084 & 0.104 \\
\hline 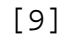 & 0.27 & 0.229 & 0.187 & 0.166 & 0.146 & 0.104 & 0.083 & 0.042 & 0.021 & & 0.021 & 0.021 & 0.042 & 0.063 & 0.083 \\
\hline$[\mathrm{A}$ & 0.291 & 0.25 & 0.208 & 0.187 & 0.167 & 0.125 & 0.104 & 0.063 & 0.042 & 0.021 & . & 0 & 0.021 & 0.042 & 0.062 \\
\hline [B & 0.291 & 0.25 & 0.208 & 0.187 & 0.167 & 0.125 & 0.104 & 0.063 & 0.042 & 0.021 & 0 & . & 0.021 & 0.042 & 0.062 \\
\hline$[\mathrm{C}$ & 0.312 & 0.271 & 0.229 & 0.208 & 0.188 & 0.146 & 0.125 & 0.084 & 0.063 & 0.042 & 0.021 & 0.021 & & 0.021 & 0.041 \\
\hline$[\mathrm{D}$ & 0.333 & 0.292 & 0.25 & 0.229 & 0.209 & 0.167 & 0.146 & 0.105 & 0.084 & 0.063 & 0.042 & 0.042 & 0.021 & & 0.02 \\
\hline$[E]$ & 0.353 & 0.312 & 0.27 & 0.249 & 0.229 & 0.187 & 0.166 & 0.125 & 0.104 & 0.083 & 0.062 & 0.062 & 0.041 & 0.02 & \\
\hline$[F]$ & 0.374 & 0.333 & 0.291 & 0.27 & 0.25 & 0.208 & 0.187 & 0.146 & 0.125 & 0.104 & 0.083 & 0.083 & 0.062 & 0.041 & 0.021 \\
\hline$[\mathrm{G}]$ & 0.437 & 0.396 & 0.354 & 0.333 & 0.313 & 0.271 & 0.25 & 0.209 & 0.188 & 0.167 & 0.146 & 0.146 & 0.125 & 0.104 & 0.084 \\
\hline$[\mathrm{H}$ & 0.457 & 0.416 & 0.374 & 0.353 & 0.333 & 0.291 & 0.27 & 0.229 & 0.208 & 0.187 & 0.166 & 0.166 & 0.145 & 0.124 & 0.104 \\
\hline$[\mathrm{J}$ & 0.478 & 0.437 & 0.395 & 0.374 & 0.354 & 0.312 & 0.291 & 0.25 & 0.229 & 0.208 & 0.187 & 0.187 & 0.166 & 0.145 & 0.125 \\
\hline 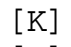 & 0.52 & 0.479 & 0.437 & 0.416 & 0.396 & 0.354 & 0.333 & 0.292 & 0.271 & 0.25 & 0.229 & 0.229 & 0.208 & 0.187 & 0.167 \\
\hline [L & 0.541 & 0.5 & 0.458 & 0.437 & 0.417 & 0.375 & 0.354 & 0.313 & 0.292 & 0.271 & 0.25 & 0.25 & 0.229 & 0.208 & 0.188 \\
\hline$[\mathrm{M}$ & 0.666 & 0.625 & 0.583 & 0.562 & 0.542 & 0.5 & 0.479 & 0.438 & 0.417 & 0.396 & 0.375 & 0.375 & 0.354 & 0.333 & 0.313 \\
\hline$[\mathrm{N}]$ & 0.707 & 0.666 & 0.624 & 0.603 & 0.583 & 0.541 & 0.52 & 0.479 & 0.458 & 0.437 & 0.416 & 0.416 & 0.395 & 0.374 & 0.354 \\
\hline$[\mathrm{P}]$ & 0.77 & 0.729 & 0.687 & 0.666 & 0.646 & 0.604 & 0.583 & 0.542 & 0.521 & 0.5 & 0.479 & 0.479 & 0.458 & 0.437 & 0.417 \\
\hline$[\mathrm{Q}$ & 0.936 & 0.895 & 0.853 & 0.832 & 0.812 & 0.77 & 0.749 & 0.708 & 0.687 & 0.666 & 0.645 & 0.645 & 0.624 & 0.603 & 0.583 \\
\hline D & 0.999 & 0.958 & 0.916 & 0.895 & 0.875 & 0.833 & 0.812 & 0.771 & 0.75 & 0.729 & 0.708 & 0.708 & 0.687 & 0.666 & 0.646 \\
\hline
\end{tabular}


$[0]$

$[1]$

$\begin{array}{lllllllllll}0.374 & 0.437 & 0.457 & 0.478 & 0.52 & 0.541 & 0.666 & 0.707 & 0.77 & 0.936 & 0.999\end{array}$ $\begin{array}{lllllllllll}0.333 & 0.396 & 0.416 & 0.437 & 0.479 & 0.5 & 0.625 & 0.666 & 0.729 & 0.895 & 0.958\end{array}$ $\begin{array}{llllllllllll}0.291 & 0.354 & 0.374 & 0.395 & 0.437 & 0.458 & 0.583 & 0.624 & 0.687 & 0.853 & 0.916\end{array}$ $\begin{array}{lllllllllll}0.27 & 0.333 & 0.353 & 0.374 & 0.416 & 0.437 & 0.562 & 0.603 & 0.666 & 0.832 & 0.895\end{array}$ $\begin{array}{llllllllllll}0.25 & 0.313 & 0.333 & 0.354 & 0.396 & 0.417 & 0.542 & 0.583 & 0.646 & 0.812 & 0.875\end{array}$ $\begin{array}{lllllllllll}0.208 & 0.271 & 0.291 & 0.312 & 0.354 & 0.375 & 0.5 & 0.541 & 0.604 & 0.77 & 0.833\end{array}$ $\begin{array}{lllllllllll}0.187 & 0.25 & 0.27 & 0.291 & 0.333 & 0.354 & 0.479 & 0.52 & 0.583 & 0.749 & 0.812\end{array}$ $\begin{array}{llllllllllll}0.146 & 0.209 & 0.229 & 0.25 & 0.292 & 0.313 & 0.438 & 0.479 & 0.542 & 0.708 & 0.771\end{array}$ $\begin{array}{llllllllllll}0.125 & 0.188 & 0.208 & 0.229 & 0.271 & 0.292 & 0.417 & 0.458 & 0.521 & 0.687 & 0.75\end{array}$ $\begin{array}{lllllllllll}0.104 & 0.167 & 0.187 & 0.208 & 0.25 & 0.271 & 0.396 & 0.437 & 0.5 & 0.666 & 0.729\end{array}$ $\begin{array}{lllllllllll}0.083 & 0.146 & 0.166 & 0.187 & 0.229 & 0.25 & 0.375 & 0.416 & 0.479 & 0.645 & 0.708\end{array}$ $\begin{array}{lllllllllll}0.083 & 0.146 & 0.166 & 0.187 & 0.229 & 0.25 & 0.375 & 0.416 & 0.479 & 0.645 & 0.708\end{array}$ $\begin{array}{llllllllllll}0.062 & 0.125 & 0.145 & 0.166 & 0.208 & 0.229 & 0.354 & 0.395 & 0.458 & 0.624 & 0.687\end{array}$ $\begin{array}{lllllllllllll}0.041 & 0.104 & 0.124 & 0.145 & 0.187 & 0.208 & 0.333 & 0.374 & 0.437 & 0.603 & 0.666\end{array}$ $\begin{array}{llllllllllll}0.021 & 0.084 & 0.104 & 0.125 & 0.167 & 0.188 & 0.313 & 0.354 & 0.417 & 0.583 & 0.646\end{array}$ $\begin{array}{lllllllllllll}. & 0.063 & 0.083 & 0.104 & 0.146 & 0.167 & 0.292 & 0.333 & 0.396 & 0.562 & 0.625\end{array}$ $\begin{array}{llllllllllll}0.063 & 0.0 .04 & 0.041 & 0.083 & 0.104 & 0.229 & 0.27 & 0.333 & 0.499 & 0.562\end{array}$ $\begin{array}{llllllllllll}0.083 & 0.02 & . & 0.021 & 0.063 & 0.084 & 0.209 & 0.25 & 0.313 & 0.479 & 0.542\end{array}$ $\begin{array}{llllllllllllll}0.104 & 0.041 & 0.021 & 0.0 .042 & 0.042 & 0.063 & 0.188 & 0.229 & 0.292 & 0.458 & 0.521\end{array}$ $\begin{array}{llllllllllll}0.146 & 0.083 & 0.063 & 0.042 & 0.0 .021 & 0.146 & 0.187 & 0.25 & 0.416 & 0.479\end{array}$ $\begin{array}{lllllllllllllll}0.167 & 0.104 & 0.084 & 0.063 & 0.021 & & 0.125 & 0.166 & 0.229 & 0.395 & 0.458\end{array}$ $\begin{array}{lllllllllllll}0.292 & 0.229 & 0.209 & 0.188 & 0.146 & 0.125 & 0.041 & 0.104 & 0.27 & 0.333\end{array}$ $\begin{array}{lllllllllll}0.333 & 0.27 & 0.25 & 0.229 & 0.187 & 0.166 & 0.041 & 0.063 & 0.229 & 0.292\end{array}$ $\begin{array}{lllllllllll}0.396 & 0.333 & 0.313 & 0.292 & 0.25 & 0.229 & 0.104 & 0.063 & 0.166 & 0.229\end{array}$ $\begin{array}{llllllllll}0.562 & 0.499 & 0.479 & 0.458 & 0.416 & 0.395 & 0.27 & 0.229 & 0.166\end{array}$. $\begin{array}{lllllllllll}0.625 & 0.562 & 0.542 & 0.521 & 0.479 & 0.458 & 0.333 & 0.292 & 0.229 & 0.063\end{array}$. 
USERTYPE paringleistthickness STEPMATRIX $=29$

\begin{tabular}{|c|c|c|c|c|c|c|c|c|c|c|c|c|c|c|c|}
\hline & & & 2 & 3 & & 5 & 6 & 7 & 8 & 7 & $\mathrm{~A}$ & B & C & $\mathrm{D}$ & $E$ \\
\hline 1 & & 5 & 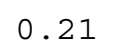 & 6 & 2 & 9 & 5 & 1 & 58 & 0 & 73 & 99 & 525 & 52 & 0.578 \\
\hline & .105 & & & & & & & & & & 368 & & & & \\
\hline & .21 & .105 & & .026 & 052 & .079 & .105 & .131 & 0.158 & 21 & .263 & 0.289 & 0.315 & .342 & .368 \\
\hline & & 131 & 026 & & & & & & & & & & & & \\
\hline & 262 & 157 & .052 & .026 & & 0.027 & 053 & .079 & 06 & & 0.211 & 37 & .263 & 0.29 & 316 \\
\hline & 289 & 184 & & .053 & 7 & & & & & & & & & & \\
\hline & & 21 & & & 053 & 0.026 & & 26 & & & & & & & \\
\hline & & 36 & & & & 0.052 & 5 & & & & & & & & \\
\hline & & & & & & & & 0.027 & & & & & & & \\
\hline & & 5 & & & & & & & & & & & & & \\
\hline & & & & & & & & & & 53 & & & & & \\
\hline & & & & & & & & & & & & & & & \\
\hline & & 2 & & & & & & & & & & & & & \\
\hline & & & & & & & & & & & & & & & \\
\hline & & & & & & & & & & & & & & & \\
\hline & & & & & & & & & & & & & & & \\
\hline & & & & & & & & & & & & & & & \\
\hline & & 2 & & & & & & & & & & & & & \\
\hline & & & & & & & & & & & & & & & \\
\hline & 09 & 604 & 9 & 73 & 47 & & & & \pm 1 & & 36 & & 84 & & 131 \\
\hline & & 631 & & & & & & & & & & & & & \\
\hline & 2 & 657 & 2 & 0.526 & & 73 & 47 & 0.421 & 94 & & 0.289 & 63 & 0.237 & 1 & .184 \\
\hline & & & & & & & & & & & & & & & \\
\hline & & & & & & & & & & & & & & & \\
\hline & & & & & & & & & & & & & & & \\
\hline & & & & & & & & & & & & & & & \\
\hline & & & & & & & & & & & & & & & \\
\hline$T$ & & & & & & & & & & & & & & & \\
\hline & בתק. & .094 & .809 & .103 & 8131 & 0.11 & U.684 & 0.658 & 0.631 & צונ. & $.5<6$ & & 0.474 & 0.447 & $0.4 \angle 1$ \\
\hline
\end{tabular}


[0] $\quad \begin{array}{lllllllllllllllllll}0.604 & 0.63 & 0.657 & 0.683 & 0.709 & 0.736 & 0.762 & 0.788 & 0.814 & 0.841 & 0.867 & 0.893 & 0.92 & 0.999\end{array}$

[1] $\quad \begin{array}{llllllllllllllllll}0.499 & 0.525 & 0.552 & 0.578 & 0.604 & 0.631 & 0.657 & 0.683 & 0.709 & 0.736 & 0.762 & 0.788 & 0.815 & 0.894\end{array}$ [2] $\quad \begin{array}{llllllllllllllllll}0.394 & 0.42 & 0.447 & 0.473 & 0.499 & 0.526 & 0.552 & 0.578 & 0.604 & 0.631 & 0.657 & 0.683 & 0.71 & 0.789\end{array}$ [3] $\quad \begin{array}{lllllllllllllllll}0.368 & 0.394 & 0.421 & 0.447 & 0.473 & 0.5 & 0.526 & 0.552 & 0.578 & 0.605 & 0.631 & 0.657 & 0.684 & 0.763\end{array}$ [4] $\quad \begin{array}{llllllllllllllll}0.342 & 0.368 & 0.395 & 0.421 & 0.447 & 0.474 & 0.5 & 0.526 & 0.552 & 0.579 & 0.605 & 0.631 & 0.658 & 0.737\end{array}$ [5] $\quad \begin{array}{lllllllllllllllllll}0.315 & 0.341 & 0.368 & 0.394 & 0.42 & 0.447 & 0.473 & 0.499 & 0.525 & 0.552 & 0.578 & 0.604 & 0.631 & 0.71\end{array}$

[6] $\quad \begin{array}{llllllllllllllll}0.289 & 0.315 & 0.342 & 0.368 & 0.394 & 0.421 & 0.447 & 0.473 & 0.499 & 0.526 & 0.552 & 0.578 & 0.605 & 0.684\end{array}$

[7] $\quad \begin{array}{llllllllllllllll}0.263 & 0.289 & 0.316 & 0.342 & 0.368 & 0.395 & 0.421 & 0.447 & 0.473 & 0.5 & 0.526 & 0.552 & 0.579 & 0.658\end{array}$ [8] $\quad \begin{array}{llllllllllllllll}0.236 & 0.262 & 0.289 & 0.315 & 0.341 & 0.368 & 0.394 & 0.42 & 0.446 & 0.473 & 0.499 & 0.525 & 0.552 & 0.631\end{array}$ [9] $\quad \begin{array}{llllllllllllllll}0.184 & 0.21 & 0.237 & 0.263 & 0.289 & 0.316 & 0.342 & 0.368 & 0.394 & 0.421 & 0.447 & 0.473 & 0.5 & 0.579\end{array}$ [A] $\quad \begin{array}{lllllllllllllllll}0.131 & 0.157 & 0.184 & 0.21 & 0.236 & 0.263 & 0.289 & 0.315 & 0.341 & 0.368 & 0.394 & 0.42 & 0.447 & 0.526\end{array}$ [B] $\quad \begin{array}{lllllllllllllllll}0.105 & 0.131 & 0.158 & 0.184 & 0.21 & 0.237 & 0.263 & 0.289 & 0.315 & 0.342 & 0.368 & 0.394 & 0.421 & 0.5\end{array}$

[C] $\quad \begin{array}{llllllllllllllll}0.079 & 0.105 & 0.132 & 0.158 & 0.184 & 0.211 & 0.237 & 0.263 & 0.289 & 0.316 & 0.342 & 0.368 & 0.395 & 0.474\end{array}$

[D] $\quad \begin{array}{lllllllllllllllll}0.052 & 0.078 & 0.105 & 0.131 & 0.157 & 0.184 & 0.21 & 0.236 & 0.262 & 0.289 & 0.315 & 0.341 & 0.368 & 0.447\end{array}$

[E] $\quad \begin{array}{llllllllllllllll}0.026 & 0.052 & 0.079 & 0.105 & 0.131 & 0.158 & 0.184 & 0.21 & 0.236 & 0.263 & 0.289 & 0.315 & 0.342 & 0.421\end{array}$

[F] $\quad \begin{array}{lllllllllllllll} & 0.026 & 0.053 & 0.079 & 0.105 & 0.132 & 0.158 & 0.184 & 0.21 & 0.237 & 0.263 & 0.289 & 0.316 & 0.395\end{array}$

[G] $\quad \begin{array}{llllllllllllllllll}0.026 & 0 & 0.027 & 0.053 & 0.079 & 0.106 & 0.132 & 0.158 & 0.184 & 0.211 & 0.237 & 0.263 & 0.29 & 0.369\end{array}$

$[\mathrm{H}] \quad \begin{array}{lllllllllllllllll}0.053 & 0.027 & . & & 0.026 & 0.052 & 0.079 & 0.105 & 0.131 & 0.157 & 0.184 & 0.21 & 0.236 & 0.263 & 0.342\end{array}$

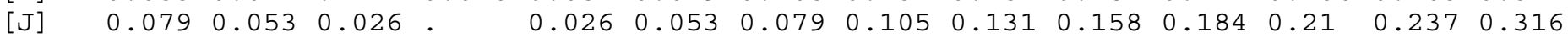

$[\mathrm{K}] \quad \begin{array}{lllllllllllllllll}0.105 & 0.079 & 0.052 & 0.026 & 0.027 & 0.053 & 0.079 & 0.105 & 0.132 & 0.158 & 0.184 & 0.211 & 0.29\end{array}$

[L] $\quad \begin{array}{llllllllllllllllllllll} & 0.132 & 0.106 & 0.079 & 0.053 & 0.027 & . & 0.026 & 0.052 & 0.078 & 0.105 & 0.131 & 0.157 & 0.184 & 0.263\end{array}$

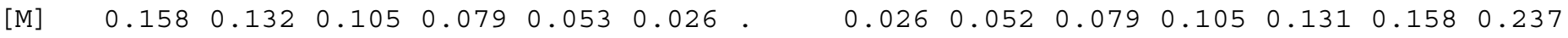

[N] $\quad \begin{array}{llllllllllllllllllllllllll}0.184 & 0.158 & 0.131 & 0.105 & 0.079 & 0.052 & 0.026 & 0.026 & 0.053 & 0.079 & 0.105 & 0.132 & 0.211\end{array}$

$\left[\begin{array}{lllllllllllllll}\mathrm{P}] & 0.21 & 0.184 & 0.157 & 0.131 & 0.105 & 0.078 & 0.052 & 0.026 & . & 0.027 & 0.053 & 0.079 & 0.106 & 0.185\end{array}\right.$

[Q] $\quad \begin{array}{lllllllllllllllll}0.237 & 0.211 & 0.184 & 0.158 & 0.132 & 0.105 & 0.079 & 0.053 & 0.027 & 0.026 & 0.026 & 0.052 & 0.079 & 0.158\end{array}$

[R] $\quad \begin{array}{llllllllllllllll}0.263 & 0.237 & 0.21 & 0.184 & 0.158 & 0.131 & 0.105 & 0.079 & 0.053 & 0.026 & .026 & 0.026 & 0.053 & 0.132\end{array}$

[S] $\quad \begin{array}{lllllllllllllllll}0.289 & 0.263 & 0.236 & 0.21 & 0.184 & 0.157 & 0.131 & 0.105 & 0.079 & 0.052 & 0.026 & . & 0.027 & 0.106\end{array}$

$[\mathrm{T}] \quad \begin{array}{lllllllllllllllll}0.316 & 0.29 & 0.263 & 0.237 & 0.211 & 0.184 & 0.158 & 0.132 & 0.106 & 0.079 & 0.053 & 0.027 & .0 .079\end{array}$

[U] $\quad \begin{array}{lllllllllllllllll}0.395 & 0.369 & 0.342 & 0.316 & 0.29 & 0.263 & 0.237 & 0.211 & 0.185 & 0.158 & 0.132 & 0.106 & 0.079\end{array}$ 
USERTYPE meanard1 STEPMATRIX $=23$

\begin{tabular}{|c|c|c|c|c|c|c|c|c|c|c|c|c|c|c|c|}
\hline & 0 & 1 & 2 & 3 & 4 & 5 & 6 & 7 & 8 & 9 & A & B & $\mathrm{C}$ & $\mathrm{D}$ & $\mathrm{E}$ \\
\hline$[0]$ & . & 0.024 & 0.049 & 0.074 & 0.099 & 0.124 & 0.149 & 0.174 & 0.199 & 0.249 & 0.274 & 0.299 & 0.349 & 0.399 & 0.424 \\
\hline [1] & 0.024 & 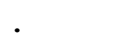 & 0.025 & 0.05 & 0.075 & 0.1 & 0.125 & 0.15 & 0.175 & 0.225 & 0.25 & 0.275 & 0.325 & 0.375 & 0.4 \\
\hline [2] & 0.049 & 0.025 & . & 0.025 & 0.05 & 0.075 & 0.1 & 0.125 & 0.15 & 0.2 & 0.225 & 0.25 & 0.3 & 0.35 & 0.375 \\
\hline [3] & 0.074 & 0.05 & 0.025 & . & 0.025 & 0.05 & 0.075 & 0.1 & 0.125 & 0.175 & 0.2 & 0.225 & 0.275 & 0.325 & 0.35 \\
\hline$[4]$ & 0.099 & 0.075 & 0.05 & 0.025 & . & 0.025 & 0.05 & 0.075 & 0.1 & 0.15 & 0.175 & 0.2 & 0.25 & 0.3 & 0.325 \\
\hline [5] & 0.124 & 0.1 & 0.075 & 0.05 & 0.025 & $\cdot$ & 0.025 & 0.05 & 0.075 & 0.125 & 0.15 & 0.175 & 0.225 & 0.275 & 0.3 \\
\hline [6] & 0.149 & 0.125 & 0.1 & 0.075 & 0.05 & 0.025 & . & 0.025 & 0.05 & 0.1 & 0.125 & 0.15 & 0.2 & 0.25 & 0.275 \\
\hline [7] & 0.174 & 0.15 & 0.125 & 0.1 & 0.075 & 0.05 & 0.025 & . & 0.025 & 0.075 & 0.1 & 0.125 & 0.175 & 0.225 & 0.25 \\
\hline [8] & 0.199 & 0.175 & 0.15 & 0.125 & 0.1 & 0.075 & 0.05 & 0.025 & - & 0.05 & 0.075 & 0.1 & 0.15 & 0.2 & 0.225 \\
\hline [9] & 0.249 & 0.225 & 0.2 & 0.175 & 0.15 & 0.125 & 0.1 & 0.075 & 0.05 & . & 0.025 & 0.05 & 0.1 & 0.15 & 0.175 \\
\hline$[\mathrm{A}]$ & 0.274 & 0.25 & 0.225 & 0.2 & 0.175 & 0.15 & 0.125 & 0.1 & 0.075 & 0.025 & & 0.025 & 0.075 & 0.125 & 0.15 \\
\hline [B] & 0.299 & 0.275 & 0.25 & 0.225 & 0.2 & 0.175 & 0.15 & 0.125 & 0.1 & 0.05 & 0.025 & . & 0.05 & 0.1 & 0.125 \\
\hline [C] & 0.349 & 0.325 & 0.3 & 0.275 & 0.25 & 0.225 & 0.2 & 0.175 & 0.15 & 0.1 & 0.075 & 0.05 & . & 0.05 & 0.075 \\
\hline [D] & 0.399 & 0.375 & 0.35 & 0.325 & 0.3 & 0.275 & 0.25 & 0.225 & 0.2 & 0.15 & 0.125 & 0.1 & 0.05 & . & 0.025 \\
\hline$[\mathrm{E}]$ & 0.424 & 0.4 & 0.375 & 0.35 & 0.325 & 0.3 & 0.275 & 0.25 & 0.225 & 0.175 & 0.15 & 0.125 & 0.075 & 0.025 & - \\
\hline$[\mathrm{F}]$ & 0.449 & 0.425 & 0.4 & 0.375 & 0.35 & 0.325 & 0.3 & 0.275 & 0.25 & 0.2 & 0.175 & 0.15 & 0.1 & 0.05 & 0.025 \\
\hline [G] & 0.524 & 0.5 & 0.475 & 0.45 & 0.425 & 0.4 & 0.375 & 0.35 & 0.325 & 0.275 & 0.25 & 0.225 & 0.175 & 0.125 & 0.1 \\
\hline$[\mathrm{H}]$ & 0.549 & 0.525 & 0.5 & 0.475 & 0.45 & 0.425 & 0.4 & 0.375 & 0.35 & 0.3 & 0.275 & 0.25 & 0.2 & 0.15 & 0.125 \\
\hline$[\mathrm{J}]$ & 0.574 & 0.55 & 0.525 & 0.5 & 0.475 & 0.45 & 0.425 & 0.4 & 0.375 & 0.325 & 0.3 & 0.275 & 0.225 & 0.175 & 0.15 \\
\hline [K] & 0.624 & 0.6 & 0.575 & 0.55 & 0.525 & 0.5 & 0.475 & 0.45 & 0.425 & 0.375 & 0.35 & 0.325 & 0.275 & 0.225 & 0.2 \\
\hline$[\mathrm{L}]$ & 0.674 & 0.65 & 0.625 & 0.6 & 0.575 & 0.55 & 0.525 & 0.5 & 0.475 & 0.425 & 0.4 & 0.375 & 0.325 & 0.275 & 0.25 \\
\hline$[\mathrm{M}]$ & 0.899 & 0.875 & 0.85 & 0.825 & 0.8 & 0.775 & 0.75 & 0.725 & 0.7 & 0.65 & 0.625 & 0.6 & 0.55 & 0.5 & 0.475 \\
\hline$[\mathrm{N}]$ & 0.999 & 0.975 & 0.95 & 0.925 & 0.9 & 0.875 & 0.85 & 0.825 & 0.8 & 0.75 & 0.725 & 0.7 & 0.65 & 0.6 & 0.575 \\
\hline
\end{tabular}


[0] $\quad \begin{array}{llllllllll}0.449 & 0.524 & 0.549 & 0.574 & 0.624 & 0.674 & 0.899 & 0.999\end{array}$

[1] $\begin{array}{lllllllll}0.425 & 0.5 & 0.525 & 0.55 & 0.6 & 0.65 & 0.875 & 0.975\end{array}$

$\left[\begin{array}{lllllllll}{[2]} & 0.4 & 0.475 & 0.5 & 0.525 & 0.575 & 0.625 & 0.85 & 0.95\end{array}\right.$

[3] $\quad \begin{array}{lllllllll}0.375 & 0.45 & 0.475 & 0.5 & 0.55 & 0.6 & 0.825 & 0.925\end{array}$

[4] $\quad \begin{array}{lllllllll}0.35 & 0.425 & 0.45 & 0.475 & 0.525 & 0.575 & 0.8 & 0.9\end{array}$

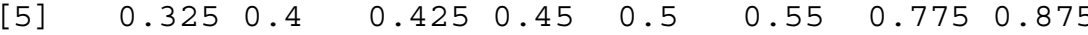

$\begin{array}{lllllllll}{[6]} & 0.3 & 0.375 & 0.4 & 0.425 & 0.475 & 0.525 & 0.75 & 0.85\end{array}$

[7] $\quad \begin{array}{llllllll}0.275 & 0.35 & 0.375 & 0.4 & 0.45 & 0.5 & 0.725 & 0.825\end{array}$

[8] $\quad \begin{array}{lllllllll}0.25 & 0.325 & 0.35 & 0.375 & 0.425 & 0.475 & 0.7 & 0.8\end{array}$

$\begin{array}{lllllllll}9] & 0.2 & 0.275 & 0.3 & 0.325 & 0.375 & 0.425 & 0.65 & 0.75\end{array}$

[A] $\quad \begin{array}{llllllll}0.175 & 0.25 & 0.275 & 0.3 & 0.35 & 0.4 & 0.625 & 0.725\end{array}$

[B] $\quad \begin{array}{lllllllll}0.15 & 0.225 & 0.25 & 0.275 & 0.325 & 0.375 & 0.6 & 0.7\end{array}$

[C] $\begin{array}{lllllllll}0.1 & 0.175 & 0.2 & 0.225 & 0.275 & 0.325 & 0.55 & 0.65\end{array}$

[D] $\quad \begin{array}{llllllll}0.05 & 0.125 & 0.15 & 0.175 & 0.225 & 0.275 & 0.5 & 0.6\end{array}$

[E] $\quad \begin{array}{llllllll}0.025 & 0.1 & 0.125 & 0.15 & 0.2 & 0.25 & 0.475 & 0.575\end{array}$

[F] $\quad \begin{array}{lllllllll} & 0.075 & 0.1 & 0.125 & 0.175 & 0.225 & 0.45 & 0.55\end{array}$

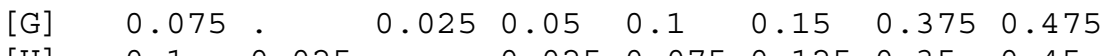

$\begin{array}{llllllllll}{[\mathrm{H}]} & 0.1 & 0.025 & . & 0.025 & 0.075 & 0.125 & 0.35 & 0.45\end{array}$

[J] $\quad \begin{array}{lllllllll}0.125 & 0.05 & 0.025 & 0.04 & 0.05 & 0.1 & 0.325 & 0.425\end{array}$

$\begin{array}{llllllll}{[\mathrm{K}]} & 0.175 & 0.1 & 0.075 & 0.05 & 0.05 & 0.275 & 0.375\end{array}$

[L] $\quad \begin{array}{lllllllll}0.225 & 0.15 & 0.125 & 0.1 & 0.05 & . & 0.225 & 0.325\end{array}$

[M] $\quad \begin{array}{lllllllll}0.45 & 0.375 & 0.35 & 0.325 & 0.275 & 0.225 & . & 0.1\end{array}$

[N] $\quad \begin{array}{llllllll}0.55 & 0.475 & 0.45 & 0.425 & 0.375 & 0.325 & 0.1\end{array}$. 
USERTYPE paardI STEPMATRIX $=22$

\begin{tabular}{|c|c|c|c|c|c|c|c|c|c|c|c|c|c|c|c|}
\hline & & 1 & 2 & 3 & 4 & 5 & 6 & & 8 & 9 & A & B & C & $\mathrm{D}$ & $E$ \\
\hline & & 3 & 6 & 8 & 3 & 5 & 0.37 & 2 & 7 & 19 & 1 & 56 & 68 & 81 & 0.493 \\
\hline & 283 & & & & & & & & & & & & & & \\
\hline & .296 & 013 & & .012 & 037 & .049 & 0.074 & .086 & .111 & 0.123 & 0.135 & 0.16 & 0.172 & 0.185 & .197 \\
\hline & 308 & 025 & 2 & & & & & & & & & & & & \\
\hline & 333 & 05 & .037 & 0.025 & & 0.012 & 037 & .049 & & 36 & .098 & 0.123 & & 48 & 16 \\
\hline & 345 & 062 & 049 & 0.037 & 2 & & & & & & & & & & \\
\hline & 37 & 087 & 74 & 062 & .037 & 0.025 & & & & & & & & & \\
\hline & 2 & 099 & & & & 0.037 & & & & & & & & & \\
\hline & & 124 & & & & & 0.037 & 25 & & & & & & & \\
\hline & & & & & & & & & & & & & & & \\
\hline & 431 & 148 & & 23 & & 86 & 0. & 49 & 0.024 & & & & & & \\
\hline & & 173 & & & & & & & & & & & & & \\
\hline & & 5 & & & & & & & 61 & & & & & & 25 \\
\hline & 1 & 98 & & & & & & & & & & & & & \\
\hline & & & & & & & & & & & & & & & \\
\hline & .505 & 0.222 & 9 & 0.197 & & & 5 & & & & & 9 & & & \\
\hline & .518 & 0235 & & & & & & & & & & & & & \\
\hline & .5 & 0.247 & 4 & 22 & 7 & & & & 23 & 11 & & & & 0.049 & .037 \\
\hline & & & & & & & & & & & & & & & \\
\hline & 592 & 9 & & & & 7 & 0 & & & 73 & 61 & & & 11 & .099 \\
\hline & & & & & & & & & & & & & & & \\
\hline & 999 & 16 & & & & & & & 92 & 0 & & 43 & 31 & 18 & 0.50 \\
\hline
\end{tabular}


[0] $\quad \begin{array}{lllllllll}0.505 & 0.518 & 0.53 & 0.555 & 0.592 & 0.875 & 0.999\end{array}$

[1] $\quad \begin{array}{lllllllll}0.222 & 0.235 & 0.247 & 0.272 & 0.309 & 0.592 & 0.716\end{array}$

$\begin{array}{lllllllll}\text { [2] } & 0.209 & 0.222 & 0.234 & 0.259 & 0.296 & 0.579 & 0.703\end{array}$

[3] $\quad \begin{array}{lllllllll}0.197 & 0.21 & 0.222 & 0.247 & 0.284 & 0.567 & 0.691\end{array}$

[4] $\quad \begin{array}{lllllllll}0.172 & 0.185 & 0.197 & 0.222 & 0.259 & 0.542 & 0.666\end{array}$

$\begin{array}{lllllllll}{[5]} & 0.16 & 0.173 & 0.185 & 0.21 & 0.247 & 0.53 & 0.654\end{array}$

[6] $\quad \begin{array}{llllllll}0.135 & 0.148 & 0.16 & 0.185 & 0.222 & 0.505 & 0.629\end{array}$

[7] $\quad \begin{array}{llllllll}0.123 & 0.136 & 0.148 & 0.173 & 0.21 & 0.493 & 0.617\end{array}$

[8] $\quad \begin{array}{lllllllll}0.098 & 0.111 & 0.123 & 0.148 & 0.185 & 0.468 & 0.592\end{array}$

[9] $\quad \begin{array}{llllllllll}0.086 & 0.099 & 0.111 & 0.136 & 0.173 & 0.456 & 0.58\end{array}$

[A] $\quad \begin{array}{llllllllll}0.074 & 0.087 & 0.099 & 0.124 & 0.161 & 0.444 & 0.568\end{array}$

[B] $\quad \begin{array}{lllllllll}0.049 & 0.062 & 0.074 & 0.099 & 0.136 & 0.419 & 0.543\end{array}$

[C] $\quad \begin{array}{lllllllll}0.037 & 0.05 & 0.062 & 0.087 & 0.124 & 0.407 & 0.531\end{array}$

[D] $\quad \begin{array}{llllllllll}0.024 & 0.037 & 0.049 & 0.074 & 0.111 & 0.394 & 0.518\end{array}$

[E] $\quad \begin{array}{lllllllll}0.012 & 0.025 & 0.037 & 0.062 & 0.099 & 0.382 & 0.506\end{array}$

F] $\begin{array}{llllllll}0.013 & 0.025 & 0.05 & 0.087 & 0.37 & 0.494\end{array}$

[G] $\quad \begin{array}{llllllll}0.013 & 0.012 & 0.037 & 0.074 & 0.357 & 0.481\end{array}$

$\begin{array}{llllllllll}\text { [H] } & 0.025 & 0.012 & . & 0.025 & 0.062 & 0.345 & 0.469\end{array}$

[J] $\quad \begin{array}{lllllllll}0.05 & 0.037 & 0.025 & 0.037 & 0.037 & 0.32 & 0.444\end{array}$

$\begin{array}{lllllllll}{[\mathrm{K}]} & 0.087 & 0.074 & 0.062 & 0.037 & 0.283 & 0.407\end{array}$

$\begin{array}{lllllllll}\text { [L] } & 0.37 & 0.357 & 0.345 & 0.32 & 0.283 & . & 0.124\end{array}$

[M] $\quad \begin{array}{llllllllll}0.494 & 0.481 & 0.469 & 0.444 & 0.407 & 0.124\end{array}$ 
USERTYPE meanisd STEPMATRIX $=23$

\begin{tabular}{|c|c|c|c|c|c|c|c|c|c|c|c|c|c|c|c|}
\hline & 0 & 1 & 2 & 3 & 4 & 5 & 6 & 7 & 8 & 9 & A & B & $\mathrm{C}$ & D & $E$ \\
\hline$[0]$ & & 0.034 & 0.068 & 0.103 & 0.137 & 0.172 & 0.206 & 0.241 & 0.275 & 0.31 & 0.344 & 0.378 & 0.413 & 0.447 & 0.516 \\
\hline [1] & 0.034 & . & 0.034 & 0.069 & 0.103 & 0.138 & 0.172 & 0.207 & 0.241 & 0.276 & 0.31 & 0.344 & 0.379 & 0.413 & 0.482 \\
\hline [2] & 0.068 & 0.034 & 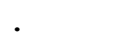 & 0.035 & 0.069 & 0.104 & 0.138 & 0.173 & 0.207 & 0.242 & 0.276 & 0.31 & 0.345 & 0.379 & 0.448 \\
\hline [3] & 0.103 & 0.069 & 0.035 & . & 0.034 & 0.069 & 0.103 & 0.138 & 0.172 & 0.207 & 0.241 & 0.275 & 0.31 & 0.344 & 0.413 \\
\hline [4] & 0.137 & 0.103 & 0.069 & 0.034 & . & 0.035 & 0.069 & 0.104 & 0.138 & 0.173 & 0.207 & 0.241 & 0.276 & 0.31 & 0.379 \\
\hline [5] & 0.172 & 0.138 & 0.104 & 0.069 & 0.035 & . & 0.034 & 0.069 & 0.103 & 0.138 & 0.172 & 0.206 & 0.241 & 0.275 & 0.344 \\
\hline [6] & 0.206 & 0.172 & 0.138 & 0.103 & 0.069 & 0.034 & 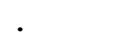 & 0.035 & 0.069 & 0.104 & 0.138 & 0.172 & 0.207 & 0.241 & 0.31 \\
\hline [7] & 0.241 & 0.207 & 0.173 & 0.138 & 0.104 & 0.069 & 0.035 & - & 0.034 & 0.069 & 0.103 & 0.137 & 0.172 & 0.206 & 0.275 \\
\hline [8] & 0.275 & 0.241 & 0.207 & 0.172 & 0.138 & 0.103 & 0.069 & 0.034 & 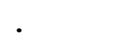 & 0.035 & 0.069 & 0.103 & 0.138 & 0.172 & 0.241 \\
\hline [9] & 0.31 & 0.276 & 0.242 & 0.207 & 0.173 & 0.138 & 0.104 & 0.069 & 0.035 & . & 0.034 & 0.068 & 0.103 & 0.137 & 0.206 \\
\hline$[\mathrm{A}]$ & 0.344 & 0.31 & 0.276 & 0.241 & 0.207 & 0.172 & 0.138 & 0.103 & 0.069 & 0.034 & . & 0.034 & 0.069 & 0.103 & 0.172 \\
\hline [B] & 0.378 & 0.344 & 0.31 & 0.275 & 0.241 & 0.206 & 0.172 & 0.137 & 0.103 & 0.068 & 0.034 & & 0.035 & 0.069 & 0.138 \\
\hline$[\mathrm{C}]$ & 0.413 & 0.379 & 0.345 & 0.31 & 0.276 & 0.241 & 0.207 & 0.172 & 0.138 & 0.103 & 0.069 & 0.035 & . & 0.034 & 0.103 \\
\hline [D] & 0.447 & 0.413 & 0.379 & 0.344 & 0.31 & 0.275 & 0.241 & 0.206 & 0.172 & 0.137 & 0.103 & 0.069 & 0.034 & . & 0.069 \\
\hline$[\mathrm{E}]$ & 0.516 & 0.482 & 0.448 & 0.413 & 0.379 & 0.344 & 0.31 & 0.275 & 0.241 & 0.206 & 0.172 & 0.138 & 0.103 & 0.069 & \\
\hline$[F]$ & 0.551 & 0.517 & 0.483 & 0.448 & 0.414 & 0.379 & 0.345 & 0.31 & 0.276 & 0.241 & 0.207 & 0.173 & 0.138 & 0.104 & 0.035 \\
\hline [G] & 0.585 & 0.551 & 0.517 & 0.482 & 0.448 & 0.413 & 0.379 & 0.344 & 0.31 & 0.275 & 0.241 & 0.207 & 0.172 & 0.138 & 0.069 \\
\hline$[\mathrm{H}]$ & 0.62 & 0.586 & 0.552 & 0.517 & 0.483 & 0.448 & 0.414 & 0.379 & 0.345 & 0.31 & 0.276 & 0.242 & 0.207 & 0.173 & 0.104 \\
\hline$[\mathrm{J}]$ & 0.654 & 0.62 & 0.586 & 0.551 & 0.517 & 0.482 & 0.448 & 0.413 & 0.379 & 0.344 & 0.31 & 0.276 & 0.241 & 0.207 & 0.138 \\
\hline [K] & 0.688 & 0.654 & 0.62 & 0.585 & 0.551 & 0.516 & 0.482 & 0.447 & 0.413 & 0.378 & 0.344 & 0.31 & 0.275 & 0.241 & 0.172 \\
\hline$[\mathrm{L}]$ & 0.792 & 0.758 & 0.724 & 0.689 & 0.655 & 0.62 & 0.586 & 0.551 & 0.517 & 0.482 & 0.448 & 0.414 & 0.379 & 0.345 & 0.276 \\
\hline$[\mathrm{M}]$ & 0.861 & 0.827 & 0.793 & 0.758 & 0.724 & 0.689 & 0.655 & 0.62 & 0.586 & 0.551 & 0.517 & 0.483 & 0.448 & 0.414 & 0.345 \\
\hline$[\mathrm{N}]$ & 0.999 & 0.965 & 0.931 & 0.896 & 0.862 & 0.827 & 0.793 & 0.758 & 0.724 & 0.689 & 0.655 & 0.621 & 0.586 & 0.552 & 0.483 \\
\hline
\end{tabular}


[0] $\quad \begin{array}{lllllllll}0.551 & 0.585 & 0.62 & 0.654 & 0.688 & 0.792 & 0.861 & 0.999\end{array}$

[1] $\quad \begin{array}{llllllllll}0.517 & 0.551 & 0.586 & 0.62 & 0.654 & 0.758 & 0.827 & 0.965\end{array}$

[2] $\quad \begin{array}{llllllllll}0.483 & 0.517 & 0.552 & 0.586 & 0.62 & 0.724 & 0.793 & 0.931\end{array}$

[3] $\quad \begin{array}{lllllllllll}0.448 & 0.482 & 0.517 & 0.551 & 0.585 & 0.689 & 0.758 & 0.896\end{array}$

[4] $\quad \begin{array}{llllllllll}0.414 & 0.448 & 0.483 & 0.517 & 0.551 & 0.655 & 0.724 & 0.862\end{array}$

[5] $\quad \begin{array}{llllllllll}0.379 & 0.413 & 0.448 & 0.482 & 0.516 & 0.62 & 0.689 & 0.827\end{array}$

[6] $\quad \begin{array}{llllllllll}0.345 & 0.379 & 0.414 & 0.448 & 0.482 & 0.586 & 0.655 & 0.793\end{array}$

[7] $\begin{array}{llllllllll}0.31 & 0.344 & 0.379 & 0.413 & 0.447 & 0.551 & 0.62 & 0.758\end{array}$

[8] $\quad \begin{array}{llllllllll}0.276 & 0.31 & 0.345 & 0.379 & 0.413 & 0.517 & 0.586 & 0.724\end{array}$

[9] $\quad \begin{array}{lllllllllll}0.241 & 0.275 & 0.31 & 0.344 & 0.378 & 0.482 & 0.551 & 0.689\end{array}$

[A] $\quad \begin{array}{llllllllll}0.207 & 0.241 & 0.276 & 0.31 & 0.344 & 0.448 & 0.517 & 0.655\end{array}$

[B] $\quad \begin{array}{llllllllll}0.173 & 0.207 & 0.242 & 0.276 & 0.31 & 0.414 & 0.483 & 0.621\end{array}$

[C] $\quad \begin{array}{lllllllllll}0.138 & 0.172 & 0.207 & 0.241 & 0.275 & 0.379 & 0.448 & 0.586\end{array}$

[D] $\quad \begin{array}{lllllllllll}0.104 & 0.138 & 0.173 & 0.207 & 0.241 & 0.345 & 0.414 & 0.552\end{array}$

[E] $\quad \begin{array}{llllllllll}0.035 & 0.069 & 0.104 & 0.138 & 0.172 & 0.276 & 0.345 & 0.483\end{array}$

[F] $\quad \begin{array}{llllllllll}0 & 0.034 & 0.069 & 0.103 & 0.137 & 0.241 & 0.31 & 0.448\end{array}$

[G] $\quad \begin{array}{llllllllll}0.034 & . & 0.035 & 0.069 & 0.103 & 0.207 & 0.276 & 0.414\end{array}$

[H] $\quad \begin{array}{llllllllllll}0.069 & 0.035 & . & 0.034 & 0.068 & 0.172 & 0.241 & 0.379\end{array}$

[J] $\quad \begin{array}{llllllllll}0.103 & 0.069 & 0.034 & 0.034 & 0.034 & 0.138 & 0.207 & 0.345\end{array}$

[K] $\quad \begin{array}{lllllllll}0.137 & 0.103 & 0.068 & 0.034 & 0.104 & 0.173 & 0.311\end{array}$

[L] $\quad \begin{array}{lllllllllll}0.241 & 0.207 & 0.172 & 0.138 & 0.104 & 0.069 & 0.069 & 0.207\end{array}$

[M] $\quad \begin{array}{llllllllll}0.31 & 0.276 & 0.241 & 0.207 & 0.173 & 0.069 & . & 0.138\end{array}$

[N] $\quad \begin{array}{llllllllll}0.448 & 0.414 & 0.379 & 0.345 & 0.311 & 0.207 & 0.138\end{array}$. 
USERTYPE paisd STEPMATRIX $=31$

\begin{tabular}{|c|c|c|c|c|c|c|c|c|c|c|c|c|c|c|c|}
\hline & & 1 & 2 & 3 & 4 & 5 & 6 & 7 & 8 & 9 & A & B & $\mathrm{C}$ & $\mathrm{D}$ & $\mathrm{E}$ \\
\hline [0] & & 1 & 8 & 9 & 3 & 6 & 0.15 & 54 & 7 & 05 & 218 & 32 & 246 & 0 & 0.273 \\
\hline & 041 & & 027 & .068 & 082 & 095 & & 123 & .136 & 0.164 & 0.177 & 0.191 & 0.205 & 0.219 & \\
\hline & .068 & 0.027 & & 0.041 & 055 & 0.068 & 0.082 & .096 & 0.109 & 0.137 & 0.15 & 0.164 & 0.178 & 0.192 & 0.205 \\
\hline & 109 & 068 & 041 & & 074 & 027 & 0.041 & .055 & 0.068 & 0.096 & & & & & 164 \\
\hline & 123 & .082 & .055 & 0.014 & & 0.013 & 0.027 & .041 & 0.054 & 0.082 & 0.095 & 0.109 & 0.123 & 0.137 & 0.15 \\
\hline & 136 & 095 & 068 & 0.027 & 3 & & 0.014 & 028 & 0.041 & 0.069 & 082 & & & & 37 \\
\hline & .15 & 109 & 082 & 0.041 & .027 & 0.014 & & .014 & 027 & 0.055 & 0.068 & .082 & & . & 123 \\
\hline & .164 & 123 & 096 & 0.055 & 041 & 0.028 & 14 & & .013 & 0.041 & 54 & 068 & & & 109 \\
\hline & 177 & 136 & 109 & 0.068 & .054 & & 0.027 & 0.013 & & 0.028 & & & & & \\
\hline & .205 & 164 & 37 & 0.096 & 082 & 0.069 & 0.055 & 0.041 & 0.028 & & 0.013 & .027 & & & 068 \\
\hline & 218 & 177 & & .109 & & 0.082 & 0.068 & & 0.041 & 13 & & & & & \\
\hline & 232 & 0.191 & 4 & 0.123 & & 096 & 0.082 & & 055 & 0.027 & 0.014 & & & & 41 \\
\hline & 246 & 0.205 & 6 & 0.137 & & 7 & 96 & & 69 & & & 4 & & & \\
\hline & 26 & 0.219 & 2 & 0.151 & 37 & 124 & 0.11 & 6 & 883 & 55 & 42 & 0.028 & 0. & & 13 \\
\hline & 273 & 0.232 & & 0.164 & & & & & 96 & & & 0.041 & & & \\
\hline & 287 & 246 & & 78 & & 51 & 0 & & & & 69 & & & & 014 \\
\hline & .301 & 0 & 3 & 92 & & & 0 & & & & 83 & 69 & & & \\
\hline & .314 & 0.273 & 246 & 0.205 & 191 & 0.178 & 0.164 & 5 & 137 & 0.109 & 0.096 & 0.082 & 68 & 54 & 0.041 \\
\hline & .328 & 287 & & .219 & & 92 & 0 & & 51 & 23 & & 96 & & 68 & 055 \\
\hline & .342 & 0.301 & 274 & 0.233 & 219 & 0.206 & 0.192 & .178 & 0.165 & 0.137 & 0.124 & 1 & 96 & 0.082 & .069 \\
\hline & 355 & & & & & & & & & & & & & & \\
\hline & 369 & 0.328 & 301 & 0.26 & 246 & 233 & 0.219 & 205 & 192 & 0.164 & 0.151 & 0.137 & 23 & 109 & 0.096 \\
\hline & & & & & & & & & & & & & & & \\
\hline & 465 & 0.424 & & 56 & 2 & 29 & & & 38 & 0 & 47 & 33 & 19 & 05 & 92 \\
\hline & 478 & & & & & & & & & & & & & & \\
\hline & 52 & 9 & 2 & 11 & 7 & 34 & 0 & 6 & 43 & 0. & 02 & 88 & 74 & 0 & 47 \\
\hline & 588 & 7 & & & & & & & & & & 56 & & & \\
\hline & 602 & 561 & & 0.493 & & & 0.4 & & & 0.3 & & 0.37 & & & 0.329 \\
\hline & 725 & 0.684 & 657 & 0.616 & 2 & 39 & 0.575 & 561 & 48 & 0. & 0.507 & 0.493 & 79 & 0.465 & .452 \\
\hline & & & & & & & & & & & & & & & \\
\hline & .999 & 0.958 & .931 & 0.89 & 0.876 & 0.863 & 0.849 & 0.835 & 0.822 & 0.794 & 0.781 & 0.767 & 0.753 & 0.739 & 0.72 \\
\hline
\end{tabular}


[0] $\quad \begin{array}{lllllllllllllllllll}0.287 & 0.301 & 0.314 & 0.328 & 0.342 & 0.355 & 0.369 & 0.41 & 0.465 & 0.478 & 0.52 & 0.588 & 0.602 & 0.725 & 0.944\end{array}$

[1] $\quad \begin{array}{llllllllllllllllll}0.246 & 0.26 & 0.273 & 0.287 & 0.301 & 0.314 & 0.328 & 0.369 & 0.424 & 0.437 & 0.479 & 0.547 & 0.561 & 0.684 & 0.903\end{array}$ [2] $\quad \begin{array}{lllllllllllllllll}0.219 & 0.233 & 0.246 & 0.26 & 0.274 & 0.287 & 0.301 & 0.342 & 0.397 & 0.41 & 0.452 & 0.52 & 0.534 & 0.657 & 0.876\end{array}$

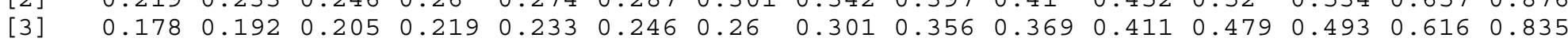

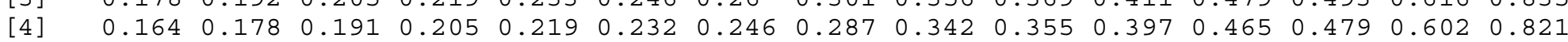
[5] $\quad \begin{array}{llllllllllllllllll}0.151 & 0.165 & 0.178 & 0.192 & 0.206 & 0.219 & 0.233 & 0.274 & 0.329 & 0.342 & 0.384 & 0.452 & 0.466 & 0.589 & 0.808\end{array}$ [6] $\quad \begin{array}{lllllllllllllll}0.137 & 0.151 & 0.164 & 0.178 & 0.192 & 0.205 & 0.219 & 0.26 & 0.315 & 0.328 & 0.37 & 0.438 & 0.452 & 0.575 & 0.794\end{array}$ [7] $\quad \begin{array}{llllllllllllllllll}0.123 & 0.137 & 0.15 & 0.164 & 0.178 & 0.191 & 0.205 & 0.246 & 0.301 & 0.314 & 0.356 & 0.424 & 0.438 & 0.561 & 0.78\end{array}$ [8] $\begin{array}{llllllllllllllllll}0.11 & 0.124 & 0.137 & 0.151 & 0.165 & 0.178 & 0.192 & 0.233 & 0.288 & 0.301 & 0.343 & 0.411 & 0.425 & 0.548 & 0.767\end{array}$ [9] $\quad \begin{array}{llllllllllllllll}0.082 & 0.096 & 0.109 & 0.123 & 0.137 & 0.15 & 0.164 & 0.205 & 0.26 & 0.273 & 0.315 & 0.383 & 0.397 & 0.52 & 0.739\end{array}$ [A] $\quad \begin{array}{llllllllllllllllll}0.069 & 0.083 & 0.096 & 0.11 & 0.124 & 0.137 & 0.151 & 0.192 & 0.247 & 0.26 & 0.302 & 0.37 & 0.384 & 0.507 & 0.726\end{array}$ [B] $\quad \begin{array}{llllllllllllllllll}0.055 & 0.069 & 0.082 & 0.096 & 0.11 & 0.123 & 0.137 & 0.178 & 0.233 & 0.246 & 0.288 & 0.356 & 0.37 & 0.493 & 0.712\end{array}$

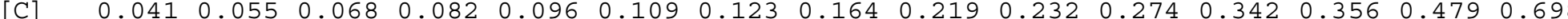
[D] $\quad \begin{array}{lllllllllllllll}0.027 & 0.041 & 0.054 & 0.068 & 0.082 & 0.095 & 0.109 & 0.15 & 0.205 & 0.218 & 0.26 & 0.328 & 0.342 & 0.465 & 0.684\end{array}$ $[\mathrm{E}] \quad \begin{array}{llllllllllllllll}0.014 & 0.028 & 0.041 & 0.055 & 0.069 & 0.082 & 0.096 & 0.137 & 0.192 & 0.205 & 0.247 & 0.315 & 0.329 & 0.452 & 0.671\end{array}$ $\begin{array}{lllllllllllllllllllllllll}0.014 & 0.027 & 0.041 & 0 & 055 & 0.068 & 0.082 & 0.123 & 0.178 & 0.191 & 0.233 & 0.301 & 0.315 & 0.438 & 0.657\end{array}$ $0.0130 .0270 .0410 .0540 .0680 .10990 .1640 .1770 .2190 .287 \quad 0.3010 .4240 .643$ $[\mathrm{H}] \quad \begin{array}{llllllllllllllllllll}0.027 & 0.013 & . & 0.014 & 0.028 & 0.041 & 0.055 & 0.096 & 0.151 & 0.164 & 0.206 & 0.274 & 0.288 & 0.411 & 0.63\end{array}$

[J] $\quad \begin{array}{lllllllllllllllllllllllll}0.041 & 0.027 & 0.014 & . & 0.014 & 0.027 & 0.041 & 0.082 & 0.137 & 0.15 & 0.192 & 0.26 & 0.274 & 0.397 & 0.616\end{array}$

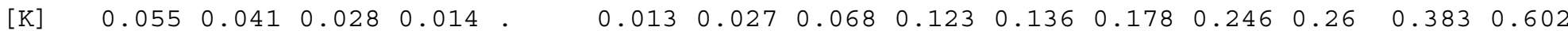
[L] $\quad \begin{array}{llllllllllllllllllll}0.068 & 0.054 & 0.041 & 0.027 & 0.013 & . & 0.014 & 0.055 & 0.11 & 0.123 & 0.165 & 0.233 & 0.247 & 0.37 & 0.589\end{array}$

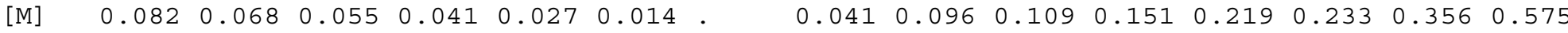

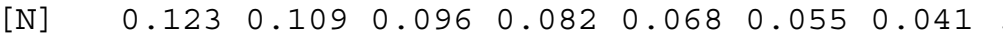

[P] $\quad \begin{array}{llllllllllllllllll} & 0.178 & 0.164 & 0.151 & 0.137 & 0.123 & 0.11 & 0.096 & 0.055 & 0.055 & 0.013 & 0.055 & 0.123 & 0.137 & 0.26 & 0.479\end{array}$ [Q] $\quad \begin{array}{llllllllllllllll}0.191 & 0.177 & 0.164 & 0.15 & 0.136 & 0.123 & 0.109 & 0.068 & 0.013 & 0.0 .042 & 0.11 & 0.124 & 0.247 & 0.466\end{array}$ [R] $\quad \begin{array}{lllllllllllllllll}0.233 & 0.219 & 0.206 & 0.192 & 0.178 & 0.165 & 0.151 & 0.11 & 0.055 & 0.042 & .0\end{array}$ [S] $\quad \begin{array}{lllllllllllllllll} & 0.301 & 0.287 & 0.274 & 0.26 & 0.246 & 0.233 & 0.219 & 0.178 & 0.123 & 0.11 & 0.068 & . & 0.014 & 0.137 & 0.356\end{array}$ $[\mathrm{T}] \quad \begin{array}{llllllllllllllllll} & 0.315 & 0.301 & 0.288 & 0.274 & 0.26 & 0.247 & 0.233 & 0.192 & 0.137 & 0.124 & 0.082 & 0.014 & . & 0.123 & 0.342\end{array}$

[U] $\quad \begin{array}{llllllllllllllllll}0.438 & 0.424 & 0.411 & 0.397 & 0.383 & 0.37 & 0.356 & 0.315 & 0.26 & 0.247 & 0.205 & 0.137 & 0.123 & . & 0.219\end{array}$

[V] $\quad \begin{array}{llllllllllllllllllll}0.657 & 0.643 & 0.63 & 0.616 & 0.602 & 0.589 & 0.575 & 0.534 & 0.479 & 0.466 & 0.424 & 0.356 & 0.342 & 0.219 & .\end{array}$

[W] $\quad \begin{array}{lllllllllllllllll}0.712 & 0.698 & 0.685 & 0.671 & 0.657 & 0.644 & 0.63 & 0.589 & 0.534 & 0.521 & 0.479 & 0.411 & 0.397 & 0.274 & 0.055\end{array}$ 
[0] 0.999

[1] 0.958

0.931

3] 0.89

[4] 0.876

[5] 0.863

[6] $\quad 0.849$

[7] 0.835

[8] 0.822

[9] 0.794

[A] 0.781

[B] 0.767

[C] 0.753

[D] 0.739

[E] 0.726

[F] $\quad 0.712$

[G] $\quad 0.698$

[H] 0.685

[J] $\quad 0.671$

[K] $\quad 0.657$

[L] 0.644

[M] 0.63

[N] $\quad 0.589$

[P] 0.534

[Q] 0.521

[R] 0.479

[S] 0.411

[T] 0.397

[U] 0.274

[V] 0.055

[W] . 
USERTYPE meanthetalnk STEPMATRIX $=28$

\begin{tabular}{|c|c|c|c|c|c|c|c|c|c|c|c|c|c|c|c|}
\hline & 0 & 1 & 2 & 3 & 4 & 5 & 6 & 7 & 8 & 9 & A & B & $\mathrm{C}$ & $\mathrm{D}$ & $\mathrm{E}$ \\
\hline$[0]$ & . & 0.118 & .131 & 17 & 184 & 236 & 0.249 & 276 & 302 & 0.381 & 0.394 & 0.446 & 0.538 & 0.552 & .565 \\
\hline [1] & 0.118 & & 0.013 & 0.052 & 0.066 & 0.118 & 0.131 & 0.158 & 0.184 & 0.263 & 0.276 & 0.328 & 0.42 & 0.434 & 0.447 \\
\hline [2] & 0.131 & 0.013 & & 0.039 & 0.053 & 0.105 & 0.118 & 0.145 & 0.171 & 0.25 & 0.263 & 0.315 & 0.407 & 0.421 & 0.434 \\
\hline [3] & 0.17 & 0.052 & 0.039 & 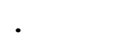 & 0.014 & 0.066 & 0.079 & 0.106 & 0.132 & 0.211 & 0.224 & 0.276 & 0.368 & 0.382 & 0.395 \\
\hline$[4]$ & 0.184 & 0.066 & 0.053 & 0.014 & . & 0.052 & 0.065 & 0.092 & 0.118 & 0.197 & 0.21 & 0.262 & 0.354 & 0.368 & 0.381 \\
\hline$[5$ & 0.236 & 0.118 & 0.105 & 0.066 & 0.052 & & 0.013 & 0.04 & 0.066 & 0.145 & 0.158 & 0.21 & 0.302 & 0.316 & 0.329 \\
\hline 10 & 0.249 & .131 & 0.118 & 0.079 & 0.065 & 0.013 & & 0.027 & 0.053 & 0.132 & 0.145 & 0.197 & 0.289 & 0.303 & 0.316 \\
\hline$[7$ & 0.276 & 0.158 & 0.145 & 0.106 & 0.092 & 0.04 & 0.027 & . & 0.026 & 0.105 & 0.118 & 0.17 & 0.262 & 0.276 & 0.289 \\
\hline [8] & 0.302 & 0.184 & 0.171 & 0.132 & 0.118 & 0.066 & 0.053 & 0.026 & 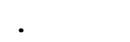 & 0.079 & 0.092 & 0.144 & 0.236 & 0.25 & 0.263 \\
\hline [9] & 0.381 & 0.263 & 0.25 & 0.211 & 0.197 & 0.145 & 0.132 & 0.105 & 0.079 & 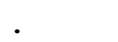 & 0.013 & 0.065 & 0.157 & 0.171 & 0.184 \\
\hline$[\mathrm{A}]$ & 0.394 & 0.276 & 0.263 & 0.224 & 0.21 & 0.158 & 0.145 & 0.118 & 0.092 & 0.013 & & 0.052 & 0.144 & 0.158 & 0.171 \\
\hline [B. & 0.446 & 0.328 & 0.315 & 0.276 & 0.262 & 0.21 & 0.197 & 0.17 & 0.144 & 0.065 & 0.052 & & 0.092 & 0.106 & 0.119 \\
\hline [C. & 0.538 & 0.42 & 0.407 & 0.368 & 0.354 & 0.302 & 0.289 & 0.262 & 0.236 & 0.157 & 0.144 & 0.092 & . & 0.014 & 0.027 \\
\hline$[D]$ & 0.552 & .434 & 0.421 & 0.382 & 0.368 & 0.316 & 0.303 & 0.276 & 0.25 & 0.171 & 0.158 & 0.106 & 0.014 & . & 0.013 \\
\hline$[\mathrm{E}$ & 0.565 & 0.447 & 0.434 & 0.395 & 0.381 & 0.329 & 0.316 & 0.289 & 0.263 & 0.184 & 0.171 & 0.119 & 0.027 & 0.013 & \\
\hline$[\mathrm{F}]$ & 0.617 & 0.499 & 0.486 & 0.447 & 0.433 & 0.381 & 0.368 & 0.341 & 0.315 & 0.236 & 0.223 & 0.171 & 0.079 & 0.065 & 0.052 \\
\hline [G] & 0.644 & 0.526 & 0.513 & 0.474 & 0.46 & 0.408 & 0.395 & 0.368 & 0.342 & 0.263 & 0.25 & 0.198 & 0.106 & 0.092 & 0.079 \\
\hline$[\mathrm{H}]$ & 0.657 & 0.539 & 0.526 & 0.487 & 0.473 & 0.421 & 0.408 & 0.381 & 0.355 & 0.276 & 0.263 & 0.211 & 0.119 & 0.105 & 0.092 \\
\hline$[\mathrm{J}$. & 0.683 & 0.565 & 0.552 & 0.513 & 0.499 & 0.447 & 0.434 & 0.407 & 0.381 & 0.302 & 0.289 & 0.237 & 0.145 & 0.131 & 0.118 \\
\hline$\Gamma K$ & 0.722 & 0.604 & 0.591 & 0.552 & 0.538 & 0.486 & 0.473 & 0.446 & 0.42 & 0.341 & 0.328 & 0.276 & 0.184 & 0.17 & 0.157 \\
\hline$[\mathrm{L}$ & 0.736 & 0.618 & 0. & 0.566 & 0.5 & 0 . & 0.487 & & 0.434 & 0 . & 0.342 & 0.29 & 0 . & 84 & 0.171 \\
\hline$[\mathrm{M}$ & 0.749 & 0.631 & 0.618 & 0.579 & 0.565 & 0.513 & 0.5 & 0.473 & 0.447 & 0.368 & 0.355 & 0.303 & 0.211 & 0.197 & 0.184 \\
\hline$[\mathrm{N}]$ & 0.762 & 0.644 & 0.631 & 0.592 & 0.578 & 0.526 & 0.513 & 0.486 & 0.46 & 0.381 & 0.368 & 0.316 & 0.224 & 0.21 & 0.197 \\
\hline$[\mathrm{P}]$ & 0.775 & 0.657 & 0.644 & 0.605 & 0.591 & 0.539 & 0.526 & 0.499 & 0.473 & 0.394 & 0.381 & 0.329 & 0.237 & 0.223 & 0.21 \\
\hline$[Q]$ & 0.788 & 0.67 & 0.657 & 0.618 & 0.604 & 0.552 & 0.539 & 0.512 & 0.486 & 0.407 & 0.394 & 0.342 & 0.25 & 0.236 & 0.223 \\
\hline$[\mathrm{R}]$ & 0.854 & 0.736 & 0.723 & 0.684 & 0.67 & 0.618 & 0.605 & 0.578 & 0.552 & 0.473 & 0.46 & 0.408 & 0.316 & 0.302 & 0.289 \\
\hline$[S$ & 0.893 & 0.775 & 0.762 & 0.723 & 0.709 & 0.657 & 0.644 & 0.617 & 0.591 & 0.512 & 0.499 & 0.447 & 0.355 & 0.341 & 0.328 \\
\hline$[\mathrm{T}]$ & 0.999 & 0.881 & 0.868 & 0.829 & 0.815 & 0.763 & 0.75 & 0.723 & 0.697 & 0.618 & 0.605 & 0.553 & 0.461 & 0.447 & 0.434 \\
\hline
\end{tabular}


[0] $\quad \begin{array}{lllllllllllllllllll}0.617 & 0.644 & 0.657 & 0.683 & 0.722 & 0.736 & 0.749 & 0.762 & 0.775 & 0.788 & 0.854 & 0.893 & 0.999\end{array}$

[1] $\quad \begin{array}{lllllllllllllllll}0.499 & 0.526 & 0.539 & 0.565 & 0.604 & 0.618 & 0.631 & 0.644 & 0.657 & 0.67 & 0.736 & 0.775 & 0.881\end{array}$

[2] $\quad \begin{array}{llllllllllllllllllll}0.486 & 0.513 & 0.526 & 0.552 & 0.591 & 0.605 & 0.618 & 0.631 & 0.644 & 0.657 & 0.723 & 0.762 & 0.868\end{array}$

[3] $\quad \begin{array}{lllllllllllllllll}0.447 & 0.474 & 0.487 & 0.513 & 0.552 & 0.566 & 0.579 & 0.592 & 0.605 & 0.618 & 0.684 & 0.723 & 0.829\end{array}$

[4] $\quad \begin{array}{llllllllllllllll}0.433 & 0.46 & 0.473 & 0.499 & 0.538 & 0.552 & 0.565 & 0.578 & 0.591 & 0.604 & 0.67 & 0.709 & 0.815\end{array}$

[5] $\quad \begin{array}{llllllllllllllll}0.381 & 0.408 & 0.421 & 0.447 & 0.486 & 0.5 & 0.513 & 0.526 & 0.539 & 0.552 & 0.618 & 0.657 & 0.763\end{array}$

[6] $\quad \begin{array}{lllllllllllllll}0.368 & 0.395 & 0.408 & 0.434 & 0.473 & 0.487 & 0.5 & 0.513 & 0.526 & 0.539 & 0.605 & 0.644 & 0.75\end{array}$

[7] $\quad \begin{array}{llllllllllllllll}0.341 & 0.368 & 0.381 & 0.407 & 0.446 & 0.46 & 0.473 & 0.486 & 0.499 & 0.512 & 0.578 & 0.617 & 0.723\end{array}$

[8] $\quad \begin{array}{llllllllllllllll}0.315 & 0.342 & 0.355 & 0.381 & 0.42 & 0.434 & 0.447 & 0.46 & 0.473 & 0.486 & 0.552 & 0.591 & 0.697\end{array}$

[9] $\quad \begin{array}{llllllllllllllll}0.236 & 0.263 & 0.276 & 0.302 & 0.341 & 0.355 & 0.368 & 0.381 & 0.394 & 0.407 & 0.473 & 0.512 & 0.618\end{array}$

[A] $\quad \begin{array}{lllllllllllllll}0.223 & 0.25 & 0.263 & 0.289 & 0.328 & 0.342 & 0.355 & 0.368 & 0.381 & 0.394 & 0.46 & 0.499 & 0.605\end{array}$

[B] $\quad \begin{array}{lllllllllllllllll}0.171 & 0.198 & 0.211 & 0.237 & 0.276 & 0.29 & 0.303 & 0.316 & 0.329 & 0.342 & 0.408 & 0.447 & 0.553\end{array}$

[C] $\quad \begin{array}{llllllllllllllll}0.079 & 0.106 & 0.119 & 0.145 & 0.184 & 0.198 & 0.211 & 0.224 & 0.237 & 0.25 & 0.316 & 0.355 & 0.461\end{array}$

[D] $\quad \begin{array}{lllllllllllllll}0.065 & 0.092 & 0.105 & 0.131 & 0.17 & 0.184 & 0.197 & 0.21 & 0.223 & 0.236 & 0.302 & 0.341 & 0.447\end{array}$

[E] $\quad \begin{array}{llllllllllllllll}0.052 & 0.079 & 0.092 & 0.118 & 0.157 & 0.171 & 0.184 & 0.197 & 0.21 & 0.223 & 0.289 & 0.328 & 0.434\end{array}$

$[\mathrm{F}] \quad \begin{array}{llllllllllllllll} & & 0.027 & 0.04 & 0.066 & 0.105 & 0.119 & 0.132 & 0.145 & 0.158 & 0.171 & 0.237 & 0.276 & 0.382\end{array}$

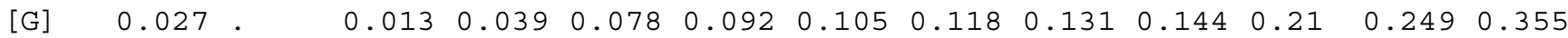

$[\mathrm{H}] \quad \begin{array}{lllllllllllllllllll} & 0.04 & 0.013 & . & & 0.026 & 0.065 & 0.079 & 0.092 & 0.105 & 0.118 & 0.131 & 0.197 & 0.236 & 0.342\end{array}$

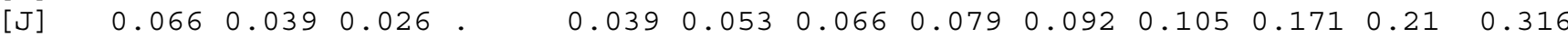

$[\mathrm{K}] \quad \begin{array}{llllllllllllllll}0.105 & 0.078 & 0.065 & 0.039 & 0.014 & 0.014 & 0.027 & 0.04 & 0.053 & 0.066 & 0.132 & 0.171 & 0.277\end{array}$

$[\mathrm{L}] \quad \begin{array}{llllllllllllllllll} & 0.119 & 0.092 & 0.079 & 0.053 & 0.014 & 0 & 0.013 & 0.026 & 0.039 & 0.052 & 0.118 & 0.157 & 0.263\end{array}$

[M] $\quad \begin{array}{llllllllllllllllllllll}0.132 & 0.105 & 0.092 & 0.066 & 0.027 & 0.013 & 0.013 & 0.026 & 0.039 & 0.105 & 0.144 & 0.25\end{array}$

[N] $\quad \begin{array}{llllllllllllllll}0.145 & 0.118 & 0.105 & 0.079 & 0.04 & 0.026 & 0.013 & 0.013 & 0.026 & 0.092 & 0.131 & 0.237\end{array}$

[P] $\quad \begin{array}{llllllllllllllll}0.158 & 0.131 & 0.118 & 0.092 & 0.053 & 0.039 & 0.026 & 0.013 & 0.013 & 0.079 & 0.118 & 0.224\end{array}$

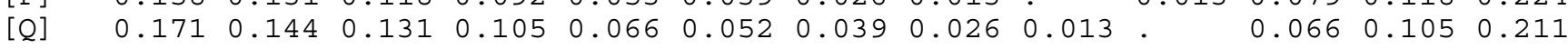

[R] $\quad \begin{array}{llllllllllllllll}0.237 & 0.21 & 0.197 & 0.171 & 0.132 & 0.118 & 0.105 & 0.092 & 0.079 & 0.066 & .0 .039 & 0.145\end{array}$

[S] $\quad \begin{array}{llllllllllllllll}0.276 & 0.249 & 0.236 & 0.21 & 0.171 & 0.157 & 0.144 & 0.131 & 0.118 & 0.105 & 0.039 & . & 0.106\end{array}$

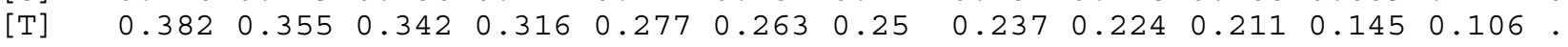


USERTYPE meanthetasep STEPMATRIX $=26$

\begin{tabular}{|c|c|c|c|c|c|c|c|c|c|c|c|c|c|c|c|}
\hline & & & 2 & 3 & & 5 & 6 & 7 & 8 & & $\mathrm{~A}$ & B & C & $\mathrm{D}$ & $\mathrm{E}$ \\
\hline 0] & & 4 & 9 & 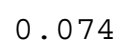 & 9 & 4 & 9 & 8 & 8 & 23 & 98 & 72 & 2 & 32 & 0.566 \\
\hline & .014 & & & & & 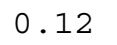 & & & & & & & & & \\
\hline & .059 & .045 & & & 06 & 075 & & .119 & 0.149 & 0.164 & .239 & 0.313 & 0.343 & 0.373 & .507 \\
\hline & & 06 & 015 & & & & & & & & & & & & \\
\hline & 119 & 105 & .06 & .045 & & 0.015 & & .059 & 89 & .04 & .179 & 53 & 83 & 13 & \\
\hline & 134 & 72 & & & 5 & & & .044 & & & & & & & \\
\hline & & & & & & .015 & & .029 & & & & & & & \\
\hline & & 4 & & & & & & & & & & & & & \\
\hline & & & & & & & & 03 & & & & & & & \\
\hline & & & & & & & & & & & & & & & \\
\hline & 298 & 4 & & 4 & & & & 0 & & 75 & & & & & \\
\hline & & & & & & & & & & & & & & & \\
\hline & 402 & 8 & & 8 & & 58 & & 24 & & & 04 & & & & \\
\hline & & & & & & & & & & & & & & & \\
\hline & & & & & & & & & & & & & & & \\
\hline & & & & & & & & & & & & & & & \\
\hline & & & & & & & & & & & & & & & \\
\hline & & 6 & 1 & 6 & & & & & & & & & & & \\
\hline & & & & & & & & & & & & & & & \\
\hline & 0 & .716 & 1 & 656 & 611 & 96 & & 52 & 22 & & 32 & & 0.328 & 98 & 0.164 \\
\hline & & & & & & & & & & & & & & & \\
\hline & 0 & 16 & 0. & .686 & 41 & $0 . \epsilon$ & & 0.582 & 0.552 & & 62 & 88 & 0.358 & 0.328 & .194 \\
\hline & & & & & & & & & & & & & & & \\
\hline & & & & & & & & & & & & & & & \\
\hline & & & & & & & & & & & & & 77 & 47 & \\
\hline & & & & & .88 & .86 & .85 & 0.821 & 0.19 & 0.116 & 0.701 & 0.627 & 0.597 & 0.56 & 0.4 \\
\hline
\end{tabular}


$[0]$

$[1]$

$\begin{array}{lllllllllll}0.611 & 0.641 & 0.7 & 0.715 & 0.73 & 0.745 & 0.76 & 0.775 & 0.834 & 0.879 & 0.999\end{array}$ $\begin{array}{llllllllllll}0.597 & 0.627 & 0.686 & 0.701 & 0.716 & 0.731 & 0.746 & 0.761 & 0.82 & 0.865 & 0.985\end{array}$ $\begin{array}{lllllllllllll}0.552 & 0.582 & 0.641 & 0.656 & 0.671 & 0.686 & 0.701 & 0.716 & 0.775 & 0.82 & 0.94\end{array}$ $\begin{array}{llllllllllll}0.537 & 0.567 & 0.626 & 0.641 & 0.656 & 0.671 & 0.686 & 0.701 & 0.76 & 0.805 & 0.925\end{array}$ $\begin{array}{llllllllllll}0.492 & 0.522 & 0.581 & 0.596 & 0.611 & 0.626 & 0.641 & 0.656 & 0.715 & 0.76 & 0.88\end{array}$ $\begin{array}{lllllllllllll}0.477 & 0.507 & 0.566 & 0.581 & 0.596 & 0.611 & 0.626 & 0.641 & 0.7 & 0.745 & 0.865\end{array}$ $\begin{array}{llllllllllll}0.462 & 0.492 & 0.551 & 0.566 & 0.581 & 0.596 & 0.611 & 0.626 & 0.685 & 0.73 & 0.85\end{array}$ $\begin{array}{llllllllllllll}0.433 & 0.463 & 0.522 & 0.537 & 0.552 & 0.567 & 0.582 & 0.597 & 0.656 & 0.701 & 0.821\end{array}$ $\begin{array}{llllllllllll}0.403 & 0.433 & 0.492 & 0.507 & 0.522 & 0.537 & 0.552 & 0.567 & 0.626 & 0.671 & 0.791\end{array}$ $\begin{array}{lllllllllllll}0.388 & 0.418 & 0.477 & 0.492 & 0.507 & 0.522 & 0.537 & 0.552 & 0.611 & 0.656 & 0.776\end{array}$ $\begin{array}{llllllllllll}0.313 & 0.343 & 0.402 & 0.417 & 0.432 & 0.447 & 0.462 & 0.477 & 0.536 & 0.581 & 0.701\end{array}$ $\begin{array}{lllllllllll}0.239 & 0.269 & 0.328 & 0.343 & 0.358 & 0.373 & 0.388 & 0.403 & 0.462 & 0.507 & 0.627\end{array}$ $\begin{array}{llllllllllll}0.209 & 0.239 & 0.298 & 0.313 & 0.328 & 0.343 & 0.358 & 0.373 & 0.432 & 0.477 & 0.597\end{array}$ $\begin{array}{lllllllllllll}0.179 & 0.209 & 0.268 & 0.283 & 0.298 & 0.313 & 0.328 & 0.343 & 0.402 & 0.447 & 0.567\end{array}$ $\begin{array}{llllllllllll}0.045 & 0.075 & 0.134 & 0.149 & 0.164 & 0.179 & 0.194 & 0.209 & 0.268 & 0.313 & 0.433\end{array}$ $\begin{array}{llllllllllll}. & 0.03 & 0.089 & 0.104 & 0.119 & 0.134 & 0.149 & 0.164 & 0.223 & 0.268 & 0.388\end{array}$ $\begin{array}{llllllllllll}0.03 & 0 & 0.059 & 0.074 & 0.089 & 0.104 & 0.119 & 0.134 & 0.193 & 0.238 & 0.358\end{array}$ $\begin{array}{lllllllllllll}0.089 & 0.059 & 0.015 & 0.03 & 0.045 & 0.06 & 0.075 & 0.134 & 0.179 & 0.299\end{array}$ $\begin{array}{lllllllllllll}0.104 & 0.074 & 0.015 & 0.0 .015 & 0.03 & 0.045 & 0.06 & 0.119 & 0.164 & 0.284\end{array}$ $\begin{array}{lllllllllllll}0.119 & 0.089 & 0.03 & 0.015 & 0.0 .015 & 0.03 & 0.045 & 0.104 & 0.149 & 0.269\end{array}$ $\begin{array}{lllllllllllll}0.134 & 0.104 & 0.045 & 0.03 & 0.015 & 0.015 & 0.015 & 0.03 & 0.089 & 0.134 & 0.254\end{array}$ $\begin{array}{lllllllllllll}0.149 & 0.119 & 0.06 & 0.045 & 0.03 & 0.015 & 0.015 & 0.074 & 0.119 & 0.239\end{array}$ $\begin{array}{llllllllllll}0.164 & 0.134 & 0.075 & 0.06 & 0.045 & 0.03 & 0.015 & & 0.059 & 0.104 & 0.224\end{array}$ $\begin{array}{lllllllllllll}0.223 & 0.193 & 0.134 & 0.119 & 0.104 & 0.089 & 0.074 & 0.059 & 0.045 & 0.165\end{array}$ $\begin{array}{lllllllllll}0.268 & 0.238 & 0.179 & 0.164 & 0.149 & 0.134 & 0.119 & 0.104 & 0.045\end{array}$.

$\begin{array}{llllllllllll}0.388 & 0.358 & 0.299 & 0.284 & 0.269 & 0.254 & 0.239 & 0.224 & 0.165 & 0.12\end{array}$ 
USERTYPE meandiameter STEPMATRIX $=25$

\begin{tabular}{|c|c|c|c|c|c|c|c|c|c|c|c|c|c|c|c|}
\hline & & 1 & & 3 & 4 & 5 & 6 & & 8 & & $\mathrm{~A}$ & B & C & D & E \\
\hline 0] & & 2 & 54 & 6 & 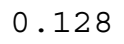 & 51 & 0 & 5 & 57 & 90 & 0. & 0 . & 86 & 18 & 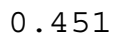 \\
\hline & 032 & & 032 & 064 & 096 & 129 & & 193 & 0.225 & 0.258 & 0.290 & 322 & 0.354 & & \\
\hline & .064 & .032 & & 32 & & & & & 193 & 226 & & 0.290 & & & \\
\hline & 096 & .064 & 032 & & 032 & & & & & & & & & & \\
\hline & 128 & 096 & .064 & 0.032 & & 33 & & & & & & & & & \\
\hline & 161 & 129 & 097 & 0.065 & 3 & & & & & & & & & & \\
\hline & & & & & & .032 & & & & & & & & & \\
\hline & 225 & 193 & .161 & 0.129 & & 64 & 0.032 & & 32 & & & & & & \\
\hline & 57 & & & & & & & & & & & & & & \\
\hline & 0 & 258 & 6 & 94 & & & & & 0.033 & & & & & & \\
\hline & 322 & 290 & & & & & & & & & & & & & \\
\hline & 354 & 322 & 90 & 0.258 & 26 & 93 & 61 & 29 & 97 & 0.064 & 032 & & 0.032 & 64 & 97 \\
\hline & 86 & & & & & & & & & & & & & & \\
\hline & .418 & 386 & 354 & 0.322 & 290 & 0.257 & 25 & 193 & 61 & 0.128 & 96 & 64 & 0.032 & & 033 \\
\hline & 51 & & & & & & & & & & & & & & \\
\hline & 483 & 451 & 419 & 87 & 355 & 22 & & 258 & 26 & 93 & 61 & 29 & & 65 & \\
\hline & & & & & & & & & & & & & & & \\
\hline & 47 & 15 & & & & & & & 90 & 57 & 25 & & 61 & 29 & 96 \\
\hline & & & & & & & & & & & & & & & \\
\hline & 676 & 4 & & & & & & & & & & & & 58 & 25 \\
\hline & & & & & & & & & & & & & & & \\
\hline & 805 & 3 & & & & & & & & & & & & & 54 \\
\hline & 902 & 870 & 3 & & & & 0 & & & & & & & & \\
\hline & & & & & & & & & & & & & & & \\
\hline & 999 & .967 & .935 & 0.903 & .871 & .838 & 0.806 & 0.774 & 0.742 & 0.709 & 0.677 & 0.645 & 0.613 & 0.581 & 0.548 \\
\hline
\end{tabular}


[0] $\quad \begin{array}{llllllllllll}0.483 & 0.515 & 0.547 & 0.580 & 0.676 & 0.773 & 0.805 & 0.902 & 0.966 & 0.999\end{array}$

[1] $\quad \begin{array}{llllllllllll}0.451 & 0.483 & 0.515 & 0.548 & 0.644 & 0.741 & 0.773 & 0.870 & 0.934 & 0.967\end{array}$

[2] $\quad \begin{array}{lllllllllllll}0.419 & 0.451 & 0.483 & 0.516 & 0.612 & 0.709 & 0.741 & 0.838 & 0.902 & 0.935\end{array}$

[3] $\quad \begin{array}{lllllllllllll}0.387 & 0.419 & 0.451 & 0.484 & 0.580 & 0.677 & 0.709 & 0.806 & 0.870 & 0.903\end{array}$

[4] $\quad \begin{array}{llllllllllll}0.355 & 0.387 & 0.419 & 0.452 & 0.548 & 0.645 & 0.677 & 0.774 & 0.838 & 0.871\end{array}$

[5] $\quad \begin{array}{lllllllllllll}0.322 & 0.354 & 0.386 & 0.419 & 0.515 & 0.612 & 0.644 & 0.741 & 0.805 & 0.838\end{array}$

[6] $\quad \begin{array}{llllllllllll}0.290 & 0.322 & 0.354 & 0.387 & 0.483 & 0.580 & 0.612 & 0.709 & 0.773 & 0.806\end{array}$

[7] $\quad \begin{array}{llllllllllll}0.258 & 0.290 & 0.322 & 0.355 & 0.451 & 0.548 & 0.580 & 0.677 & 0.741 & 0.774\end{array}$

[8] $\quad \begin{array}{llllllllllll}0.226 & 0.258 & 0.290 & 0.323 & 0.419 & 0.516 & 0.548 & 0.645 & 0.709 & 0.742\end{array}$

[9] $\quad \begin{array}{lllllllllllll}0.193 & 0.225 & 0.257 & 0.290 & 0.386 & 0.483 & 0.515 & 0.612 & 0.676 & 0.709\end{array}$

[A] $\quad \begin{array}{llllllllllll}0.161 & 0.193 & 0.225 & 0.258 & 0.354 & 0.451 & 0.483 & 0.580 & 0.644 & 0.677\end{array}$

[B] $\quad \begin{array}{llllllllllll}0.129 & 0.161 & 0.193 & 0.226 & 0.322 & 0.419 & 0.451 & 0.548 & 0.612 & 0.645\end{array}$

[C] $\quad \begin{array}{lllllllllllll}0.097 & 0.129 & 0.161 & 0.194 & 0.290 & 0.387 & 0.419 & 0.516 & 0.580 & 0.613\end{array}$

[D] $\quad \begin{array}{llllllllllll}0.065 & 0.097 & 0.129 & 0.162 & 0.258 & 0.355 & 0.387 & 0.484 & 0.548 & 0.581\end{array}$

[E] $\quad \begin{array}{llllllllllll}0.032 & 0.064 & 0.096 & 0.129 & 0.225 & 0.322 & 0.354 & 0.451 & 0.515 & 0.548\end{array}$

[F] $\quad \begin{array}{lllllllllll} & 0.032 & 0.064 & 0.097 & 0.193 & 0.290 & 0.322 & 0.419 & 0.483 & 0.516\end{array}$

[G] $\quad \begin{array}{llllllllllll}0.032 & 0.0 & 0.032 & 0.065 & 0.161 & 0.258 & 0.290 & 0.387 & 0.451 & 0.484\end{array}$

$[\mathrm{H}] \quad \begin{array}{llllllllllll}0.064 & 0.032 & . & & 0.033 & 0.129 & 0.226 & 0.258 & 0.355 & 0.419 & 0.452\end{array}$

[J] $\quad \begin{array}{lllllllllllll}0.097 & 0.065 & 0.033 & 0.0 .036 & 0.096 & 0.193 & 0.225 & 0.322 & 0.386 & 0.419\end{array}$

$[\mathrm{K}] \quad \begin{array}{lllllllllllll}0.193 & 0.161 & 0.129 & 0.096 & & 0.097 & 0.129 & 0.226 & 0.290 & 0.323\end{array}$

[L] $\quad \begin{array}{lllllllllllll}0.290 & 0.258 & 0.226 & 0.193 & 0.097 & . & 0.032 & 0.129 & 0.193 & 0.226\end{array}$

[M] $\quad \begin{array}{llllllllllll}0.322 & 0.290 & 0.258 & 0.225 & 0.129 & 0.032 & . & 0.097 & 0.161 & 0.194\end{array}$

[N] $\quad \begin{array}{lllllllllll}0.419 & 0.387 & 0.355 & 0.322 & 0.226 & 0.129 & 0.097 & 0.064 & 0.097\end{array}$

[P] $\quad \begin{array}{llllllllllllll}0.483 & 0.451 & 0.419 & 0.386 & 0.290 & 0.193 & 0.161 & 0.064 & . & 0.033\end{array}$

[Q] $\quad \begin{array}{llllllllllll}0.516 & 0.484 & 0.452 & 0.419 & 0.323 & 0.226 & 0.194 & 0.097 & 0.033\end{array}$. 
USERTYPE meanareolararea STEPMATRIX = 29

\begin{tabular}{|c|c|c|c|c|c|c|c|c|c|c|c|c|c|c|c|}
\hline & 0 & 1 & 2 & 3 & 4 & 5 & 6 & 7 & 8 & 9 & A & B & $\mathrm{C}$ & $\mathrm{D}$ & $E$ \\
\hline$[0]$ & . & 0.014 & 029 & 043 & 058 & 072 & 087 & 0.101 & 0.116 & 0.130 & .159 & 0.174 & 0.188 & 0.203 & .232 \\
\hline [1] & 0.014 & 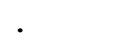 & 0.014 & 0.029 & 0.043 & 0.058 & 0.072 & 0.087 & 0.101 & 0.116 & 0.145 & 0.159 & 0.174 & 0.188 & 0.217 \\
\hline [2] & 0.029 & 0.014 & & 0.014 & 0.029 & 0.043 & 0.058 & 0.072 & 0.087 & 0.101 & 0.130 & 0.145 & 0.159 & 0.174 & 0.203 \\
\hline [3] & 0.043 & 0.029 & 0.014 & - & 0.014 & 0.029 & 0.043 & 0.058 & 0.072 & 0.087 & 0.116 & 0.130 & 0.145 & 0.159 & 0.188 \\
\hline [4] & 0.058 & 0.043 & 0.029 & 0.014 & . & 0.014 & 0.029 & 0.043 & 0.058 & 0.072 & 0.101 & 0.116 & 0.130 & 0.145 & 0.174 \\
\hline [5] & 0.072 & 0.058 & 0.043 & 0.029 & 0.014 & & 0.014 & 0.029 & 0.043 & 0.058 & 0.087 & 0.101 & 0.116 & 0.130 & 0.159 \\
\hline 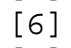 & 0.087 & 0.072 & 0.058 & 0.043 & 0.029 & 0.014 & & 0.014 & 0.029 & 0.043 & 0.072 & 0.087 & 0.101 & 0.116 & 0.145 \\
\hline$[7$ & 0.101 & 0.087 & 0.072 & 0.058 & 0.043 & 0.029 & 0.014 & . & 0.014 & 0.029 & 0.058 & 0.072 & 0.087 & 0.101 & 0.130 \\
\hline [8] & 0.116 & 0.101 & 0.087 & 0.072 & 0.058 & 0.043 & 0.029 & 0.014 & & 0.014 & 0.043 & 0.058 & 0.072 & 0.087 & 0.116 \\
\hline$[9$ & 0.130 & 0.116 & 0.101 & 0.087 & 0.072 & 0.058 & 0.043 & 0.029 & 0.014 & & 0.029 & 0.043 & 0.058 & 0.072 & 0.101 \\
\hline$[\mathrm{A}]$ & 0.159 & 0.145 & 0.130 & 0.116 & 0.101 & 0.087 & 0.072 & 0.058 & 0.043 & 0.029 & & 0.014 & 0.029 & 0.043 & 0.072 \\
\hline [B] & 0.174 & 0.159 & 0.145 & 0.130 & 0.116 & 0.101 & 0.087 & 0.072 & 0.058 & 0.043 & 0.014 & & 0.014 & 0.029 & 0.058 \\
\hline [C] & 0.188 & 0.174 & 0.159 & 0.145 & 0.130 & 0.116 & 0.101 & 0.087 & 0.072 & 0.058 & 0.029 & 0.014 & & 0.014 & 0.043 \\
\hline [D & 0.203 & 0.188 & 0.174 & 0.159 & 0.145 & 0.130 & 0.116 & 0.101 & 0.087 & 0.072 & 0.043 & 29 & 0.014 & & 0.029 \\
\hline$[E$ & 0.232 & 0.217 & 0.203 & 0.188 & 0.174 & 0.159 & 0.145 & 0.130 & 0.116 & 0.101 & 0.072 & 0.058 & 0.043 & 0 & \\
\hline F & 0.246 & 0.232 & 0.217 & 0.203 & 0.188 & 0.174 & 0.159 & 0.145 & 0.130 & 0.116 & 0.087 & 0.072 & 0.058 & 0.043 & 0.014 \\
\hline [G] & 0.261 & 0.246 & 0.232 & 0.217 & 0.203 & 0.188 & 0.174 & 0.159 & 0.145 & 0.130 & 0.101 & 0.087 & 0.072 & 0.058 & 0.029 \\
\hline$[\mathrm{H}]$ & 0.333 & 0.319 & 0.304 & 0.290 & 0.275 & 0.261 & 0.246 & 0.232 & 0.217 & 0.203 & 0.174 & 0.159 & 0.145 & 0.130 & 0.101 \\
\hline$[\mathrm{J}]$ & 0.376 & 0.362 & 0.347 & 0.333 & 0.319 & 0.304 & 0.290 & 0.275 & 0.261 & 0.246 & 0.217 & 0.203 & 0.188 & 0.174 & 0.145 \\
\hline [K & 0.391 & 0.376 & 0.362 & 0.347 & 0.333 & 0.319 & 0.304 & 0.290 & 0.275 & 0.261 & 0.232 & 0.217 & 0.203 & 0.188 & 0.159 \\
\hline$\left[\begin{array}{l}{[-1} \\
]\end{array}\right.$ & 0.405 & 0.391 & 0.376 & 0.362 & 0.347 & 0.333 & 0.319 & 0.304 & 0.290 & 0.275 & 0.246 & 0.232 & 0.217 & 0.203 & 0.174 \\
\hline & .507 & 0.492 & 0.478 & 0.463 & 0.449 & 0.434 & 0.420 & 0.405 & 0.391 & 0.376 & 0.347 & 0.333 & 0.319 & 0.304 & 0.275 \\
\hline$[\mathrm{N}]$ & 0.521 & 0.507 & 0.492 & 0.478 & 0.463 & 0.449 & 0.434 & 0.420 & 0.405 & 0.391 & 0.362 & 0.347 & 0.333 & 0.319 & 0.290 \\
\hline$[\mathrm{P}]$ & 0.579 & 0.565 & 0.550 & 0.536 & 0.521 & 0.507 & 0.492 & 0.478 & 0.463 & 0.449 & 0.420 & 0.405 & 0.391 & 0.376 & 0.347 \\
\hline$[Q]$ & 0.695 & 0.680 & 0.666 & 0.652 & 0.637 & 0.623 & 0.608 & 0.594 & 0.579 & 0.565 & 0.536 & 0.521 & 0.507 & 0.492 & 0.463 \\
\hline I & 0.753 & 0.738 & 0.724 & 0.709 & 0.695 & 0.680 & 0.666 & 0.652 & 0.637 & 0.623 & 0.594 & 0.579 & 0.565 & 0.550 & 0.521 \\
\hline 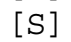 & 0.767 & 0.753 & 0.738 & 0.724 & 0.709 & 0.695 & 0.680 & 0.666 & 0.652 & 0.637 & 0.608 & 0.594 & 0.579 & 0.565 & 0.536 \\
\hline 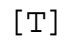 & 0.796 & 0.782 & 0.767 & 0.753 & 0.738 & 0.724 & 0.709 & 0.695 & 0.680 & 0.666 & 0.637 & 0.623 & 0.608 & 0.594 & 0.565 \\
\hline & 0.999 & 0.985 & 0.970 & 0.956 & 0.941 & 0.927 & 0.912 & 0.898 & 0.883 & 0.869 & 0.840 & 0.825 & 0.811 & 0.796 & 0.767 \\
\hline
\end{tabular}


[0] $\quad \begin{array}{lllllllllllllllllllll}0.246 & 0.261 & 0.333 & 0.376 & 0.391 & 0.405 & 0.507 & 0.521 & 0.579 & 0.695 & 0.753 & 0.767 & 0.796 & 0.999\end{array}$

[1] $\quad \begin{array}{llllllllllllllllll}0.232 & 0.246 & 0.319 & 0.362 & 0.376 & 0.391 & 0.492 & 0.507 & 0.565 & 0.680 & 0.738 & 0.753 & 0.782 & 0.985\end{array}$

[2] $\quad \begin{array}{lllllllllllllllllll}0.217 & 0.232 & 0.304 & 0.347 & 0.362 & 0.376 & 0.478 & 0.492 & 0.550 & 0.666 & 0.724 & 0.738 & 0.767 & 0.970\end{array}$

[3] $\quad \begin{array}{lllllllllllllllll}0.203 & 0.217 & 0.290 & 0.333 & 0.347 & 0.362 & 0.463 & 0.478 & 0.536 & 0.652 & 0.709 & 0.724 & 0.753 & 0.956\end{array}$

[4] $\quad \begin{array}{llllllllllllllllll}0.188 & 0.203 & 0.275 & 0.319 & 0.333 & 0.347 & 0.449 & 0.463 & 0.521 & 0.637 & 0.695 & 0.709 & 0.738 & 0.941\end{array}$

[5] $\quad \begin{array}{lllllllllllllllllll}0.174 & 0.188 & 0.261 & 0.304 & 0.319 & 0.333 & 0.434 & 0.449 & 0.507 & 0.623 & 0.680 & 0.695 & 0.724 & 0.927\end{array}$

[6] $\quad \begin{array}{lllllllllllllllll}0.159 & 0.174 & 0.246 & 0.290 & 0.304 & 0.319 & 0.420 & 0.434 & 0.492 & 0.608 & 0.666 & 0.680 & 0.709 & 0.912\end{array}$

[7] $\quad \begin{array}{lllllllllllllllllll}0.145 & 0.159 & 0.232 & 0.275 & 0.290 & 0.304 & 0.405 & 0.420 & 0.478 & 0.594 & 0.652 & 0.666 & 0.695 & 0.898\end{array}$

[8] $\quad \begin{array}{llllllllllllllllllll}0.130 & 0.145 & 0.217 & 0.261 & 0.275 & 0.290 & 0.391 & 0.405 & 0.463 & 0.579 & 0.637 & 0.652 & 0.680 & 0.883\end{array}$

[9] $\quad \begin{array}{llllllllllllllllllllll}0.116 & 0.130 & 0.203 & 0.246 & 0.261 & 0.275 & 0.376 & 0.391 & 0.449 & 0.565 & 0.623 & 0.637 & 0.666 & 0.869\end{array}$

[A] $\quad \begin{array}{lllllllllllllllll}0.087 & 0.101 & 0.174 & 0.217 & 0.232 & 0.246 & 0.347 & 0.362 & 0.420 & 0.536 & 0.594 & 0.608 & 0.637 & 0.840\end{array}$

[B] $\quad \begin{array}{lllllllllllllllll}0.072 & 0.087 & 0.159 & 0.203 & 0.217 & 0.232 & 0.333 & 0.347 & 0.405 & 0.521 & 0.579 & 0.594 & 0.623 & 0.825\end{array}$

[C] $\quad \begin{array}{llllllllllllllllllll}0.058 & 0.072 & 0.145 & 0.188 & 0.203 & 0.217 & 0.319 & 0.333 & 0.391 & 0.507 & 0.565 & 0.579 & 0.608 & 0.811\end{array}$

[D] $\quad \begin{array}{lllllllllllllllll}0.043 & 0.058 & 0.130 & 0.174 & 0.188 & 0.203 & 0.304 & 0.319 & 0.376 & 0.492 & 0.550 & 0.565 & 0.594 & 0.796\end{array}$

[E] $\quad \begin{array}{lllllllllllllllll}0.014 & 0.029 & 0.101 & 0.145 & 0.159 & 0.174 & 0.275 & 0.290 & 0.347 & 0.463 & 0.521 & 0.536 & 0.565 & 0.767\end{array}$

[F] $\quad \begin{array}{lllllllllllllllll} & 0.014 & 0.087 & 0.130 & 0.145 & 0.159 & 0.261 & 0.275 & 0.333 & 0.449 & 0.507 & 0.521 & 0.550 & 0.753\end{array}$

[G] $\quad \begin{array}{lllllllllllllllllllllllll}0.014 & 0 & 0.072 & 0.116 & 0.130 & 0.145 & 0.246 & 0.261 & 0.319 & 0.434 & 0.492 & 0.507 & 0.536 & 0.738\end{array}$

$[\mathrm{H}] \quad \begin{array}{llllllllllllllllllll}0.087 & 0.072 & . & & 0.043 & 0.058 & 0.072 & 0.174 & 0.188 & 0.246 & 0.362 & 0.420 & 0.434 & 0.463 & 0.666\end{array}$

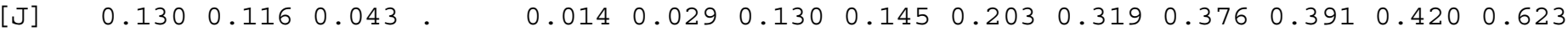

$[\mathrm{K}] \quad \begin{array}{llllllllllllllllll}0.145 & 0.130 & 0.058 & 0.014 & 0.014 & 0.116 & 0.130 & 0.188 & 0.304 & 0.362 & 0.376 & 0.405 & 0.608\end{array}$

[L] $\quad \begin{array}{lllllllllllllllllllllllll} & 0.159 & 0.145 & 0.072 & 0.029 & 0.014 & . & 0.101 & 0.116 & 0.174 & 0.290 & 0.347 & 0.362 & 0.391 & 0.594\end{array}$

[M] $\quad \begin{array}{lllllllllllllllllll}0.261 & 0.246 & 0.174 & 0.130 & 0.116 & 0.101 & 0.014 & 0.072 & 0.188 & 0.246 & 0.261 & 0.290 & 0.492\end{array}$

[N] $\quad \begin{array}{lllllllllllllllllll}0.275 & 0.261 & 0.188 & 0.145 & 0.130 & 0.116 & 0.014 & 0.058 & 0.174 & 0.232 & 0.246 & 0.275 & 0.478\end{array}$

[P] $\quad \begin{array}{lllllllllllllll}0.333 & 0.319 & 0.246 & 0.203 & 0.188 & 0.174 & 0.072 & 0.058 & 0.116 & 0.174 & 0.188 & 0.217 & 0.420\end{array}$

[Q] $\quad \begin{array}{lllllllllllllllll}0.449 & 0.434 & 0.362 & 0.319 & 0.304 & 0.290 & 0.188 & 0.174 & 0.116 & 0.058 & 0.072 & 0.101 & 0.304\end{array}$

[R] $\quad \begin{array}{llllllllllllllllll}0.507 & 0.492 & 0.420 & 0.376 & 0.362 & 0.347 & 0.246 & 0.232 & 0.174 & 0.058 & . & 0.014 & 0.043 & 0.246\end{array}$

[S] $\quad \begin{array}{lllllllllllllllll}0.521 & 0.507 & 0.434 & 0.391 & 0.376 & 0.362 & 0.261 & 0.246 & 0.188 & 0.072 & 0.014 & . & 0.029 & 0.232\end{array}$

[T] $\quad \begin{array}{llllllllllllllllll}0.550 & 0.536 & 0.463 & 0.420 & 0.405 & 0.391 & 0.290 & 0.275 & 0.217 & 0.101 & 0.043 & 0.029 & . & 0.203\end{array}$

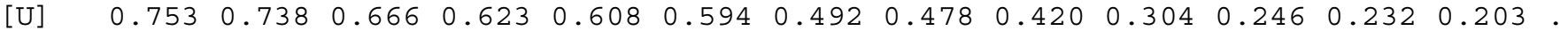


USERTYPE PAareolararea STEPMATRIX $=26$

\begin{tabular}{|c|c|c|c|c|c|c|c|c|c|c|c|c|c|c|c|}
\hline & 0 & 1 & 2 & 3 & 4 & 5 & 6 & 7 & 8 & 9 & A & B & $\mathrm{C}$ & $\mathrm{D}$ & $\mathrm{E}$ \\
\hline$[0]$ & . & 0.039 & 0.052 & 0.065 & 0.078 & 0.091 & 0.104 & 0.117 & 0.130 & 0.143 & 0.156 & 0.169 & 0.195 & 0.208 & 0.221 \\
\hline [1] & 0.039 & . & 0.013 & 0.026 & 0.039 & 0.052 & 0.065 & 0.078 & 0.091 & 0.104 & 0.117 & 0.130 & 0.156 & 0.169 & 0.182 \\
\hline [2] & 0.052 & 0.013 & 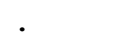 & 0.013 & 0.026 & 0.039 & 0.052 & 0.065 & 0.078 & 0.091 & 0.104 & 0.117 & 0.143 & 0.156 & 0.169 \\
\hline [3] & 0.065 & 0.026 & 0.013 & . & 0.013 & 0.026 & 0.039 & 0.052 & 0.065 & 0.078 & 0.091 & 0.104 & 0.130 & 0.143 & 0.156 \\
\hline$[4$ & 0.078 & 0.039 & 0.026 & 0.013 & & 0.013 & 0.026 & 0.039 & 0.052 & 0.065 & 0.078 & 0.091 & 0.117 & 0.130 & 0.143 \\
\hline$[0$ & 0.091 & 0.052 & 0.039 & 0.026 & 0.013 & & 0.013 & 0.026 & 0.039 & 0.052 & 0.065 & 0.078 & 0.104 & 0.117 & 0.130 \\
\hline [6] & 0.104 & 0.065 & 0.052 & 0.039 & 0.026 & 0.013 & . & 0.013 & 0.026 & 0.039 & 0.052 & 0.065 & 0.091 & 0.104 & 0.117 \\
\hline$[7]$ & 0.117 & 0.078 & 0.065 & 0.052 & 0.039 & 0.026 & 0.013 & . & 0.013 & 0.026 & 0.039 & 0.052 & 0.078 & 0.091 & 0.104 \\
\hline$[8$ & 0.130 & 0.091 & 0.078 & 0.065 & 0.052 & 0.039 & 0.026 & 0.013 & & 0.013 & 0.026 & 0.039 & 0.065 & 0.078 & 0.091 \\
\hline 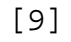 & 0.143 & 0.104 & 0.091 & 0.078 & 0.065 & 0.052 & 0.039 & 0.026 & 0.013 & & 0.013 & 0.026 & 0.052 & 0.065 & 0.078 \\
\hline$[A$ & 0.156 & 0.117 & 0.104 & 0.091 & 0.078 & 0.065 & 0.052 & 0.039 & 0.026 & 0.013 & . & 0.013 & 0.039 & 0.052 & 0.065 \\
\hline [B & 0.169 & 0.130 & 0.117 & 0.104 & 0.091 & 0.078 & 0.065 & 0.052 & 0.039 & 0.026 & 0.013 & & 0.026 & 0.039 & 0.052 \\
\hline$\Gamma \Gamma$ & 0.195 & 0.156 & 0.143 & 0.130 & 0.117 & 0.104 & 0.091 & 0.078 & 0.065 & 0.052 & 0.039 & 0.026 & . & 0.013 & 0.026 \\
\hline [D & 0.208 & 0.169 & 0.156 & 0.143 & 0.130 & 0.117 & 0.104 & 0.091 & 0.078 & 0.065 & 0.052 & 0.039 & 0.013 & & 0.013 \\
\hline$[E]$ & 0.221 & 0.182 & 0.169 & 0.156 & 0.143 & 0.130 & 0.117 & 0.104 & 0.091 & 0.078 & 0.065 & 0.052 & 0.026 & 0.013 & \\
\hline$[F]$ & 0.234 & 0.195 & 0.182 & 0.169 & 0.156 & 0.143 & 0.130 & 0.117 & 0.104 & 0.091 & 0.078 & 0.065 & 0.039 & 0.026 & 0.013 \\
\hline$[\mathrm{G}]$ & 0.259 & 0.221 & 0.208 & 0.195 & 0.182 & 0.169 & 0.156 & 0.143 & 0.130 & 0.117 & 0.104 & 0.091 & 0.065 & 0.052 & 0.039 \\
\hline$[\mathrm{H}$ & 0.272 & 0.234 & 0.221 & 0.208 & 0.195 & 0.182 & 0.169 & 0.156 & 0.143 & 0.130 & 0.117 & 0.104 & 0.078 & 0.065 & 0.052 \\
\hline$[\mathrm{J}$ & 0.285 & 0.247 & 0.234 & 0.221 & 0.208 & 0.195 & 0.182 & 0.169 & 0.156 & 0.143 & 0.130 & 0.117 & 0.091 & 0.078 & 0.065 \\
\hline & 0.337 & 0.298 & 0.285 & 0.272 & 0.259 & 0.247 & 0.234 & 0.221 & 0.208 & 0.195 & 0.182 & 0.169 & 0.143 & 0.130 & 0.117 \\
\hline$[\mathrm{L}$ & 0.350 & 0.311 & 0.298 & 0.285 & 0.272 & 0.259 & 0.247 & 0.234 & 0.221 & 0.208 & 0.195 & 0.182 & 0.156 & 0.143 & 0.130 \\
\hline$[\mathrm{M}$ & 0.389 & 0.350 & 0.337 & 0.324 & 0.311 & 0.298 & 0.285 & 0.272 & 0.259 & 0.247 & 0.234 & 0.221 & 0.195 & 0.182 & 0.169 \\
\hline$[\mathrm{N}]$ & 0.519 & 0.480 & 0.467 & 0.454 & 0.441 & 0.428 & 0.415 & 0.402 & 0.389 & 0.376 & 0.363 & 0.350 & 0.324 & 0.311 & 0.298 \\
\hline$[\mathrm{P}]$ & 0.597 & 0.558 & 0.545 & 0.532 & 0.519 & 0.506 & 0.493 & 0.480 & 0.467 & 0.454 & 0.441 & 0.428 & 0.402 & 0.389 & 0.376 \\
\hline 5 & 0.623 & 0.584 & 0.571 & 0.558 & 0.545 & 0.532 & 0.519 & 0.506 & 0.493 & 0.480 & 0.467 & 0.454 & 0.428 & 0.415 & 0.402 \\
\hline תז & 0.999 & 0.960 & 0.947 & 0.934 & 0.921 & 0.908 & 0.895 & 0.882 & 0.869 & 0.856 & 0.843 & 0.830 & 0.804 & 0.791 & 0.778 \\
\hline
\end{tabular}


[0] $\quad \begin{array}{lllllllllllll}0.234 & 0.259 & 0.272 & 0.285 & 0.337 & 0.350 & 0.389 & 0.519 & 0.597 & 0.623 & 0.999\end{array}$

[1] $\quad \begin{array}{llllllllllllll}0.195 & 0.221 & 0.234 & 0.247 & 0.298 & 0.311 & 0.350 & 0.480 & 0.558 & 0.584 & 0.960\end{array}$

[2] $\quad \begin{array}{llllllllllllll}0.182 & 0.208 & 0.221 & 0.234 & 0.285 & 0.298 & 0.337 & 0.467 & 0.545 & 0.571 & 0.947\end{array}$

[3] $\quad \begin{array}{lllllllllllll}0.169 & 0.195 & 0.208 & 0.221 & 0.272 & 0.285 & 0.324 & 0.454 & 0.532 & 0.558 & 0.934\end{array}$

[4] $\quad \begin{array}{lllllllllllll}0.156 & 0.182 & 0.195 & 0.208 & 0.259 & 0.272 & 0.311 & 0.441 & 0.519 & 0.545 & 0.921\end{array}$

[5] $\quad \begin{array}{llllllllllllll}0.143 & 0.169 & 0.182 & 0.195 & 0.247 & 0.259 & 0.298 & 0.428 & 0.506 & 0.532 & 0.908\end{array}$

[6] $\quad \begin{array}{llllllllllll}0.130 & 0.156 & 0.169 & 0.182 & 0.234 & 0.247 & 0.285 & 0.415 & 0.493 & 0.519 & 0.895\end{array}$

[7] $\quad \begin{array}{lllllllllllll}0.117 & 0.143 & 0.156 & 0.169 & 0.221 & 0.234 & 0.272 & 0.402 & 0.480 & 0.506 & 0.882\end{array}$

[8] $\quad \begin{array}{llllllllllllll}0.104 & 0.130 & 0.143 & 0.156 & 0.208 & 0.221 & 0.259 & 0.389 & 0.467 & 0.493 & 0.869\end{array}$

[9] $\quad \begin{array}{llllllllllllll}0.091 & 0.117 & 0.130 & 0.143 & 0.195 & 0.208 & 0.247 & 0.376 & 0.454 & 0.480 & 0.856\end{array}$

[A] $\quad \begin{array}{llllllllllllll}0.078 & 0.104 & 0.117 & 0.130 & 0.182 & 0.195 & 0.234 & 0.363 & 0.441 & 0.467 & 0.843\end{array}$

[B] $\quad \begin{array}{llllllllllllll}0.065 & 0.091 & 0.104 & 0.117 & 0.169 & 0.182 & 0.221 & 0.350 & 0.428 & 0.454 & 0.830\end{array}$

[C] $\quad \begin{array}{llllllllllllll}0.039 & 0.065 & 0.078 & 0.091 & 0.143 & 0.156 & 0.195 & 0.324 & 0.402 & 0.428 & 0.804\end{array}$

[D] $\quad \begin{array}{llllllllllllll}0.026 & 0.052 & 0.065 & 0.078 & 0.130 & 0.143 & 0.182 & 0.311 & 0.389 & 0.415 & 0.791\end{array}$

[E] $\quad \begin{array}{llllllllllllll}0.013 & 0.039 & 0.052 & 0.065 & 0.117 & 0.130 & 0.169 & 0.298 & 0.376 & 0.402 & 0.778\end{array}$

[F] $\quad \begin{array}{llllllllllllll} & & 0.026 & 0.039 & 0.052 & 0.104 & 0.117 & 0.156 & 0.285 & 0.363 & 0.389 & 0.765\end{array}$

[G] $\quad \begin{array}{llllllllllllll}0.026 & 0 & 0.013 & 0.026 & 0.078 & 0.091 & 0.130 & 0.259 & 0.337 & 0.363 & 0.740\end{array}$

$[\mathrm{H}] \quad \begin{array}{lllllllllllllll}0.039 & 0.013 & . & & 0.013 & 0.065 & 0.078 & 0.117 & 0.247 & 0.324 & 0.350 & 0.727\end{array}$

[J] $\quad \begin{array}{llllllllllllllll} & 0.052 & 0.026 & 0.013 & 0.052 & 0.065 & 0.104 & 0.234 & 0.311 & 0.337 & 0.714\end{array}$

[K] $\quad \begin{array}{llllllllllllll} & 0.104 & 0.078 & 0.065 & 0.052 & 0.013 & 0.013 & 0.052 & 0.182 & 0.259 & 0.285 & 0.662\end{array}$

[L] $\quad \begin{array}{llllllllllllllll} & 0.117 & 0.091 & 0.078 & 0.065 & 0.013 & . & 0.039 & 0.169 & 0.247 & 0.272 & 0.649\end{array}$

[M] $\quad \begin{array}{lllllllllllllll}0.156 & 0.130 & 0.117 & 0.104 & 0.052 & 0.039 & 0.130 & 0.208 & 0.234 & 0.610\end{array}$

[N] $\quad \begin{array}{lllllllllllll}0.285 & 0.259 & 0.247 & 0.234 & 0.182 & 0.169 & 0.130 & 0.078 & 0.104 & 0.480\end{array}$

[P] $\quad \begin{array}{lllllllllllll}0.363 & 0.337 & 0.324 & 0.311 & 0.259 & 0.247 & 0.208 & 0.078 & . & 0.026 & 0.402\end{array}$

[Q] $\quad \begin{array}{llllllllllllll}0.389 & 0.363 & 0.350 & 0.337 & 0.285 & 0.272 & 0.234 & 0.104 & 0.026 & . & 0.376\end{array}$

[R] $\quad \begin{array}{llllllllllllll}0.765 & 0.740 & 0.727 & 0.714 & 0.662 & 0.649 & 0.610 & 0.480 & 0.402 & 0.376\end{array}$. 
Appendix G. Morphological characters: Morphometric Character Data Coded via a Modified Step-Matrix Gap Weighting Method (26 Character State Limit). 
[

M. varians CHMP7 FFP

S._cf._bros̄chii_GB

A._islāndica_CHM̄P1_MICH

A. skvortzowii

A._baicalensis

A._sub_9D1

A. sub_WLB3

A. $s p .{ }^{3}$ FR

A._sub_ERB

A. sub LEW

A._alpigena

A._amb_PII 7

A._amb_N17

A. amb-FL8

A.

A._amb_WLP1

A. nyassensis GB

A._valida_N2

A._gran_LB

A. gran_oR12

A. $-\mathrm{sp} \cdot \overline{1}$

A._sp. 2

A. $\operatorname{cren}$ FH8 L639

A._cren_T733 _L639

Alveolophora

A._agassizii_FH21

A._amb_A62

A._amb_MFP

A. amb_WAT

A. canād 1571

A. canad 2018

A._dianchiensis

A. distans FH34

A._gran_FH $\overline{4} 2$

A._gran_FH38

A. gran_1914

A._gran_MFP

A._gran_V._jonensis

A._gran_f_HLS

A. gran f- $\mathrm{VH}$

A._herzogii_HDSM

A._italica_ $\bar{F} H 15$

A._krammerīi_nom._prov.

A._lac_A38

A. 1 - 1 ac_A62

A. lirāta A37

A._laevissima_HDSM

A._nyg_A50
Qualitative Chars Morphometric Chars

100100000002000713 CB85??????????????N??

100000000002020443 ????????????????????

211111611112100503 FABDC7A642E68676EGE52

211511611112100513 FB4DC79F47D95878JL843

21111141111 ?100523 MMDHQPJK99NFLHFBGJJH6

211100002120000011 A47J69448EA5685B?D548

211100002120000011 94AH52608CDA6254?5B30

$211100002123000102 \quad 37022 \mathrm{~F} 1717041718$ ? 1116

$211100002120000011824 \mathrm{~J} 4 \mathrm{E} 218 \mathrm{D} 47494 \mathrm{~B} ? 9638$

211100002120000011 6ACF5A55CEAA6C58?CA4 9

2111126111001 ???? ???????????????????

221111611110100011 BE879FAC45HC694746B44

221111611110100011 8A4G5K6A48CF5E492253B

221111611110100011 9K4A4C7A48EB5D4833438

221111611110100011 B328K095289G6B4710335

221111611110100011 D65C9DA646F9694711646

2211116111121001 ? 3 HCC5DRB443G5H3A7EGLA4

210022720001100011 FDCLCCD6ACKB8D530?C59

2141116121201 ?? ? ? H07B8B6C2679DMBJBK6BR

214111612120100231 DL447M7H276BBH9LAJ28P

210512620001100202 6G7MBN6G359EAL80H?95H

2111126111101 ?? ?? ? 8N2FEQBL20A4546CHK13K

2100327100012032? 1 FCBHMCHF66LDBJ4BLQD5E

2100327100012032 ? 1 DCJENGG8E5RCAA57KPP67

2112116111121000 ?3 6BH5B995N1D3B7G3JMQA3

2141116121221001 ? 1 A9FKE7A2F5G5A8A3FKG76

221111611110100011 9?383D6B4AEF6B584554A

221111611110100011 D7799AA667EB5B4657837

221111611110100011 BA5A688245G8664A68844

211211611110114033 898JA3649JA8FCGHGH59M

211211611110114033 98ENC883EKHDK4MDJM9HQ

21311161212 ?111601 LJE6J7D079J4M0LCHLAJG

210111211102100123 471C5C316C144C3AHK127

214111612120101201 DA78E9C62487CFCFBK97H

214111612120101201 DAHMF6D5B6J7F5E8DMFA6

2141?? ?? 21201003 ? 2 D1EPCCB8DLF8B9A1?LA6 7

2141116121201001?1 F98CBDA936BCCBB9BK7AF

2241116121201012 ? 1 8922173502444A558J623

2111????11121????? H821F5C0FF22K5HEGJCFF

2111????11121????? K3 75L1F0AG91J8K4HKAEJ

213100005422000311 DH4A884824874102HK722

21004271000 ?2122? 1 HF8EGFC933F98H2 9MRC39

212411411121114003 B0BCC371CPF2B3 JKJL777

2101111100002 ?? ??? 6C7DC89963D66B75JLE4 8

2101111100002 ?? ??? 6C59998B46A75967GHB3 7

21011251000 ?1???? ? 9AECD9EAMJADGBHCEC 7L

213100003312000103 5C8E976A45C89E8BJLF59

2115129213122 ????? 8C5AAB 8 844D92A2 8HJC2 7 
A. nyg A62

A._pergla_f_A38

A._pfaffiāna_FH37

A. solida $\mathrm{FH}_{\overline{2}} 3$

A. sub ROR

A. sub_T702

A. $\mathrm{sp} .{ }^{3} \mathrm{ROR}$

A. distāns_f ADE

A._paucistriāta_2035

A._sp. 4 T733

A. $\mathrm{sp} \cdot{ }^{5} \mathrm{~h}$

A._sp. ${ }^{-}{ }^{-}$hwg

A._cf._distans_1571

A. humilis

A. tenuoir

A. tenella

A. crassipunctata

A. tethera

A._pseudoamericana

A. muzzanensis

A._pergla_f_KI

A._ceretaña

\begin{tabular}{|c|c|}
\hline dalit & $\mathrm{C}$ \\
\hline & \\
\hline 115129213122 ? ? ? ? & 6D4A9D8943 \\
\hline $0121003285110003 ? 1$ & $3 C 4 C ? ? 2813$ \\
\hline 210112310002100102 & 49199J5525 \\
\hline 212000002120014003 & 8CELEC92HNE6B3 GA? MF74 \\
\hline 211100002120000011 & 6440453536555958 ?A632 \\
\hline 211100002120000011 & 977G5452 7HAA6G5C?A538 \\
\hline 211100002123000102 & $3513251415102 \mathrm{~A} 19 ? 4118$ \\
\hline 2101126100001 ?? ?? ? & 5D5F7J5B47AF5C589B93A \\
\hline 213000001120014003 & ACGAHHC3 KKP 8E7NA? MKCC \\
\hline 2113111111101????? & JDJFPEEAA6Q9NCKCHJMGN \\
\hline 0111526185203 ?? ??? & 4B8F? ? $7722 E 4$ ?? ? ? ?D? \\
\hline 0101523111113 ? ? ??? & 4B39? ? 5D14BC? ? ? ? ? 6 ? ? \\
\hline 2111000021220 ????? & 1B2M23086B183J2G7A02D \\
\hline 2130000011220 ????? & 4A2G6L4J253277A ? N34D \\
\hline $2 ? 11000021120$ ?? ??? & 7? ? ? 6 ? ? ? J? ? 4 ? 4 ? J ?H7? \\
\hline $2 ? 12000011120001 ? 1$ & 0 ? ? ? 0 ? ? ? ? ? ? 3 ? 2 ? ? M60 ? \\
\hline $2 ? 03111100021 ? 40 ? 3$ & E? ? ?J? ? ? L? ? ? G? K? J ? 74 ? \\
\hline $2 ? 01115100011000 ? 1$ & 2??? 1? ?? ?? ? ? 0 ? 8 ? ? C1 ? \\
\hline $0 ? 01529100013006 ? 1$ & 3 ? ? ? ? ? ? ? ? ? ? ? ? ? ? ? ? ? \\
\hline 2?41116121211????? & 5 ? 6 ? 5 ? 0 ? 5 ? B ? 8 ? D? 9ML5? \\
\hline 2?11216111121? ? ? ? & 1 ? ? ? 2 ? ? ? G? ? ? 1 ? 1 ? JMG1 ? \\
\hline 1611 & \\
\hline
\end{tabular}

Characters in columns from left to right are as listed 1-39 in the Material and Methods section of Chapter 2, pages 41 to 50 . 
Appendix H. Step-Matrices Used to Weight Morphometric Characters Coded via a Modified Step-Matrix Gap Weighting Method (26 Character State Limit). 
USERTYPE meanmantleheight STEPMATRIX $=22$

$\begin{array}{llllllllllllllllllllllll}0 & 1 & 2 & 3 & 4 & 5 & 6 & 7 & 8 & 9 & \mathrm{~A} & \mathrm{~B} & \mathrm{C} & \mathrm{D} & \mathrm{E}\end{array}$

[0] $\begin{array}{llllllllllllllllllll}0 & 0.058 & 0.078 & 0.097 & 0.137 & 0.176 & 0.215 & 0.235 & 0.254 & 0.293 & 0.333 & 0.372 & 0.411 & 0.450 & 0.470\end{array}$

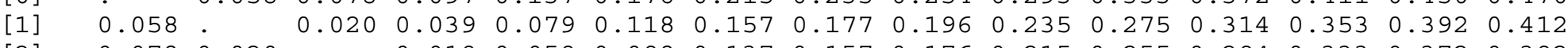

[2] $\quad \begin{array}{lllllllllllllllllllllll}0.078 & 0.020 & 0.019 & 0.019 & 0.059 & 0.098 & 0.137 & 0.157 & 0.176 & 0.215 & 0.255 & 0.294 & 0.333 & 0.372 & 0.392\end{array}$

[3] $\quad \begin{array}{llllllllllllllllll}0.097 & 0.039 & 0.019 & 0.0 .19 & 0.040 & 0.079 & 0.118 & 0.138 & 0.157 & 0.196 & 0.236 & 0.275 & 0.314 & 0.353 & 0.373\end{array}$

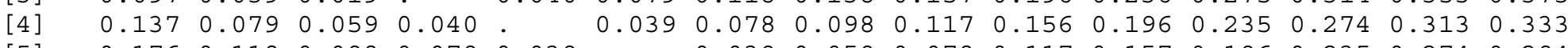

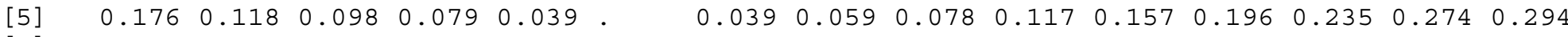

[6] $\quad \begin{array}{lllllllllllllllllllll}0.215 & 0.157 & 0.137 & 0.118 & 0.078 & 0.039 & 0.157 & 0.196 & 0.235 & 0.255\end{array}$

[7] $\quad \begin{array}{lllllllllllllllllllll}0.235 & 0.177 & 0.157 & 0.138 & 0.098 & 0.059 & 0.020 & 0.019 & 0.058 & 0.098 & 0.137 & 0.176 & 0.215 & 0.235\end{array}$

[8] $\quad \begin{array}{lllllllllllllllllll}0.254 & 0.196 & 0.176 & 0.157 & 0.117 & 0.078 & 0.039 & 0.019 & 0.039 & 0.079 & 0.118 & 0.157 & 0.196 & 0.216\end{array}$

[9] $\quad \begin{array}{lllllllllllllllllll}0.293 & 0.235 & 0.215 & 0.196 & 0.156 & 0.117 & 0.078 & 0.058 & 0.039 & . & 0.040 & 0.079 & 0.118 & 0.157 & 0.177\end{array}$

[A] $\quad \begin{array}{lllllllllllllllll}0.333 & 0.275 & 0.255 & 0.236 & 0.196 & 0.157 & 0.118 & 0.098 & 0.079 & 0.040 & . & 0.039 & 0.078 & 0.117 & 0.137\end{array}$

[B] $\quad \begin{array}{llllllllllllllllllll}0.372 & 0.314 & 0.294 & 0.275 & 0.235 & 0.196 & 0.157 & 0.137 & 0.118 & 0.079 & 0.039 & . & 0.039 & 0.078 & 0.098\end{array}$

[C] $\quad \begin{array}{llllllllllllllllllll}0.411 & 0.353 & 0.333 & 0.314 & 0.274 & 0.235 & 0.196 & 0.176 & 0.157 & 0.118 & 0.078 & 0.039 & 0.039 & 0.059\end{array}$

[D] $\quad \begin{array}{lllllllllllllllllll}0.450 & 0.392 & 0.372 & 0.353 & 0.313 & 0.274 & 0.235 & 0.215 & 0.196 & 0.157 & 0.117 & 0.078 & 0.039 & 0.020\end{array}$

$[\mathrm{E}] \quad \begin{array}{lllllllllllllllll}0.470 & 0.412 & 0.392 & 0.373 & 0.333 & 0.294 & 0.255 & 0.235 & 0.216 & 0.177 & 0.137 & 0.098 & 0.059 & 0.020 & \text {. }\end{array}$

[F] $\quad \begin{array}{lllllllllllllllllll}0.489 & 0.431 & 0.411 & 0.392 & 0.352 & 0.313 & 0.274 & 0.254 & 0.235 & 0.196 & 0.156 & 0.117 & 0.078 & 0.039 & 0.019\end{array}$

[G] $\quad \begin{array}{llllllllllllllllll}0.509 & 0.451 & 0.431 & 0.412 & 0.372 & 0.333 & 0.294 & 0.274 & 0.255 & 0.216 & 0.176 & 0.137 & 0.098 & 0.059 & 0.039\end{array}$

[H] $\quad \begin{array}{lllllllllllllllllll}0.528 & 0.470 & 0.450 & 0.431 & 0.391 & 0.352 & 0.313 & 0.293 & 0.274 & 0.235 & 0.195 & 0.156 & 0.117 & 0.078 & 0.058\end{array}$

[J] $\quad \begin{array}{llllllllllllllllll}0.607 & 0.549 & 0.529 & 0.510 & 0.470 & 0.431 & 0.392 & 0.372 & 0.353 & 0.314 & 0.274 & 0.235 & 0.196 & 0.157 & 0.137\end{array}$

$[\mathrm{K}] \quad \begin{array}{llllllllllllllllll}0.881 & 0.823 & 0.803 & 0.784 & 0.744 & 0.705 & 0.666 & 0.646 & 0.627 & 0.588 & 0.548 & 0.509 & 0.470 & 0.431 & 0.411\end{array}$

[L] $\quad \begin{array}{llllllllllllllllll}0.959 & 0.901 & 0.881 & 0.862 & 0.822 & 0.783 & 0.744 & 0.724 & 0.705 & 0.666 & 0.626 & 0.587 & 0.548 & 0.509 & 0.489\end{array}$

[M] $\quad \begin{array}{llllllllllllllllll}0.999 & 0.941 & 0.921 & 0.902 & 0.862 & 0.823 & 0.784 & 0.764 & 0.745 & 0.706 & 0.666 & 0.627 & 0.588 & 0.549 & 0.529\end{array}$ 
[0] $\quad \begin{array}{llllllll}0.489 & 0.509 & 0.528 & 0.607 & 0.881 & 0.959 & 0.999\end{array}$

[1] $\quad \begin{array}{lllllllll}0.431 & 0.451 & 0.470 & 0.549 & 0.823 & 0.901 & 0.941\end{array}$

[2] $\quad \begin{array}{lllllllll}0.411 & 0.431 & 0.450 & 0.529 & 0.803 & 0.881 & 0.921\end{array}$

[3] $\quad \begin{array}{lllllllll}0.392 & 0.412 & 0.431 & 0.510 & 0.784 & 0.862 & 0.902\end{array}$

[4] $\quad \begin{array}{lllllllll}0.352 & 0.372 & 0.391 & 0.470 & 0.744 & 0.822 & 0.862\end{array}$

$\left[\begin{array}{llllllllll}5] & 0.313 & 0.333 & 0.352 & 0.431 & 0.705 & 0.783 & 0.823\end{array}\right.$

[6] $\quad \begin{array}{lllllllll}0.274 & 0.294 & 0.313 & 0.392 & 0.666 & 0.744 & 0.784\end{array}$

[7] $\quad \begin{array}{lllllllll}0.254 & 0.274 & 0.293 & 0.372 & 0.646 & 0.724 & 0.764\end{array}$

[8] $\begin{array}{lllllllll}0.235 & 0.255 & 0.274 & 0.353 & 0.627 & 0.705 & 0.745\end{array}$

[9] $\quad \begin{array}{lllllllll}0.196 & 0.216 & 0.235 & 0.314 & 0.588 & 0.666 & 0.706\end{array}$

[A] $\quad \begin{array}{lllllllll}0.156 & 0.176 & 0.195 & 0.274 & 0.548 & 0.626 & 0.666\end{array}$

[B] $\quad \begin{array}{lllllllll}0.117 & 0.137 & 0.156 & 0.235 & 0.509 & 0.587 & 0.627\end{array}$

[C] $\quad \begin{array}{lllllllll}0.078 & 0.098 & 0.117 & 0.196 & 0.470 & 0.548 & 0.588\end{array}$

[D] $\quad \begin{array}{lllllllll}0.039 & 0.059 & 0.078 & 0.157 & 0.431 & 0.509 & 0.549\end{array}$

[E] $\quad \begin{array}{lllllllll}0.019 & 0.039 & 0.058 & 0.137 & 0.411 & 0.489 & 0.529\end{array}$

[F] $\quad \begin{array}{lllllll}\quad & 0.020 & 0.039 & 0.118 & 0.392 & 0.470 & 0.510\end{array}$

[G] $\quad 0.020 .0 .019 \quad 0.098 \quad 0.372 \quad 0.450 \quad 0.490$

[H] $\quad \begin{array}{llllllllll} & 0.039 & 0.019 & 0 & 0.079 & 0.353 & 0.431 & 0.471\end{array}$

[J] $\quad \begin{array}{lllllllllll}0.118 & 0.098 & 0.079 & 0.374 & 0.274 & 0.352 & 0.392\end{array}$

[K] $\quad \begin{array}{lllllllll}0.392 & 0.372 & 0.353 & 0.274 & . & 0.078 & 0.118\end{array}$

[L] $\quad \begin{array}{llllllll}0.470 & 0.450 & 0.431 & 0.352 & 0.078 & . & 0.040\end{array}$

[M] $\quad \begin{array}{lllllllll}0.510 & 0.490 & 0.471 & 0.392 & 0.118 & 0.040\end{array}$ 
USERTYPE pamantleheight STEPMATRIX $=23$

\begin{tabular}{|c|c|c|c|c|c|c|c|c|c|c|c|c|c|c|c|}
\hline & 0 & 1 & 2 & 3 & 4 & 5 & 6 & 7 & 8 & 9 & A & B & $\mathrm{C}$ & $\mathrm{D}$ & $\mathrm{E}$ \\
\hline ] & & 0 & 62 & 72 & 3 & 93 & 4 & -4 & 24 & 35 & 145 & 56 & 166 & 187 & 0.208 \\
\hline & 0.01 & & 052 & 0.062 & 073 & 083 & 0.094 & 104 & 0.114 & 0.125 & 0.135 & 0.146 & 0.156 & 0.177 & \\
\hline$[2$ & 0.062 & 0.052 & & 0.01 & 0.021 & 0.031 & 0.042 & 0.052 & 0.062 & 0.073 & 0.083 & 0.094 & 0.104 & 0.125 & 0.146 \\
\hline & 072 & 062 & 0.01 & & 011 & .021 & 0.032 & .042 & 0.052 & 0.063 & 0.073 & 0.084 & 0.094 & 0.115 & 136 \\
\hline & .083 & 0.073 & 0.021 & 0.011 & & 0.01 & 0.021 & 0.031 & 0.041 & 0.052 & 0.062 & 0.073 & 0.083 & 0.104 & 0.125 \\
\hline & 093 & .083 & 031 & 0.021 & $U_{\perp}$ & & 0.011 & 0.021 & 0.031 & 0.042 & 0.052 & 0.063 & & 0.094 & 115 \\
\hline & 104 & 094 & .042 & 0.032 & 0.021 & 0.011 & & 0.01 & 0.02 & 0.031 & 0.041 & 0.052 & 62 & 0.083 & 104 \\
\hline & .114 & .104 & .052 & 0.042 & .031 & 0.021 & 0.01 & & 0.01 & 0.021 & 0.031 & 0.042 & & .073 & 0.094 \\
\hline & .124 & 114 & .062 & 0.052 & .041 & 0.031 & 0.02 & 0.01 & & 0.011 & 0.021 & 0.032 & & 63 & 084 \\
\hline & .135 & .125 & .073 & 0.063 & .052 & 0.042 & 0.031 & 0.021 & 0.011 & & 0.01 & 0.021 & 0 . & 52 & 0.073 \\
\hline & .145 & 0.135 & .083 & 0.073 & 0.062 & 0.052 & 0.041 & 0.031 & 0.021 & 0.01 & & 0.011 & 0.021 & 0.042 & 0.063 \\
\hline & .156 & 0.146 & .094 & 84 & 3 & 0.063 & 0.052 & 0.042 & 032 & 0.021 & 0.011 & & 0.01 & 31 & 52 \\
\hline & .166 & 0.156 & .104 & 0.094 & 0.083 & 0.073 & 0.062 & 0.052 & 0.042 & 0.031 & 0.021 & 0.01 & & .021 & 0.042 \\
\hline & .187 & .177 & 125 & 0.115 & & 0.094 & & 0.073 & & & 42 & 0.031 & 0. & & 0.021 \\
\hline & .208 & .198 & 6 & 36 & & 5 & 04 & 4 & 84 & 73 & 63 & 52 & 42 & 0.021 & \\
\hline & .218 & 0.208 & 6 & 0.146 & 5 & 0.125 & 0.114 & 0. & 94 & 0. & 73 & 62 & & 31 & 1 \\
\hline & .249 & 0.239 & 7 & & & 0.156 & 0.145 & 35 & 0.125 & 14 & 04 & 93 & 83 & 62 & 41 \\
\hline & .27 & 0.26 & 0.208 & 0.198 & .187 & 0.177 & 0.166 & 0.156 & 0.146 & 0.135 & 0.125 & 0 . & & 0.083 & 0.062 \\
\hline & 28 & 027 & & & & 0.187 & & 0.166 & 0.156 & & 0.135 & 0.124 & & 0.093 & $\begin{array}{lll}0 & 072\end{array}$ \\
\hline & 0.312 & 0.302 & 0.25 & 0.24 & 0.229 & 0.219 & 0.208 & 0.198 & 0.188 & 0.177 & 0.167 & 0.156 & 0.146 & 0.125 & 0.104 \\
\hline & .395 & 0.385 & 0.333 & & & & & & & 026 & & & & 0.208 & \\
\hline$[\mathrm{M}$ & 0.707 & 0.697 & 0.645 & 0.635 & 0.624 & 0.614 & 0.603 & 0.593 & 0.583 & 0.572 & 0.562 & 0.551 & 0.541 & 0.52 & 0.499 \\
\hline & .999 & 0.989 & 0.937 & 0.927 & 0.916 & 0.906 & 0.895 & 0.885 & 0.875 & 0.864 & 0.854 & 0.843 & 0.833 & 0.812 & 0.791 \\
\hline
\end{tabular}


[0] $\quad \begin{array}{lllllllll}0.218 & 0.249 & 0.27 & 0.28 & 0.312 & 0.395 & 0.707 & 0.999\end{array}$

[1] $\quad \begin{array}{lllllllll}0.208 & 0.239 & 0.26 & 0.27 & 0.302 & 0.385 & 0.697 & 0.989\end{array}$

[2] $\quad \begin{array}{lllllllll}0.156 & 0.187 & 0.208 & 0.218 & 0.25 & 0.333 & 0.645 & 0.937\end{array}$

[3] $\quad \begin{array}{lllllllll}0.146 & 0.177 & 0.198 & 0.208 & 0.24 & 0.323 & 0.635 & 0.927\end{array}$

[4] $\quad \begin{array}{llllllllll}0.135 & 0.166 & 0.187 & 0.197 & 0.229 & 0.312 & 0.624 & 0.916\end{array}$

[5] $\quad \begin{array}{llllllllll}0.125 & 0.156 & 0.177 & 0.187 & 0.219 & 0.302 & 0.614 & 0.906\end{array}$

[6] $\quad \begin{array}{llllllllll}0.114 & 0.145 & 0.166 & 0.176 & 0.208 & 0.291 & 0.603 & 0.895\end{array}$

[7] $\quad \begin{array}{llllllllll}0.104 & 0.135 & 0.156 & 0.166 & 0.198 & 0.281 & 0.593 & 0.885\end{array}$

[8] $\quad \begin{array}{lllllllllll}0.094 & 0.125 & 0.146 & 0.156 & 0.188 & 0.271 & 0.583 & 0.875\end{array}$

[9] $\quad \begin{array}{llllllllll}0.083 & 0.114 & 0.135 & 0.145 & 0.177 & 0.26 & 0.572 & 0.864\end{array}$

[A] $\quad \begin{array}{llllllllll}0.073 & 0.104 & 0.125 & 0.135 & 0.167 & 0.25 & 0.562 & 0.854\end{array}$

[B] $\quad \begin{array}{llllllllll}0.062 & 0.093 & 0.114 & 0.124 & 0.156 & 0.239 & 0.551 & 0.843\end{array}$

[C] $\quad \begin{array}{llllllllll}0.052 & 0.083 & 0.104 & 0.114 & 0.146 & 0.229 & 0.541 & 0.833\end{array}$

[D] $\quad \begin{array}{llllllllll}0.031 & 0.062 & 0.083 & 0.093 & 0.125 & 0.208 & 0.52 & 0.812\end{array}$

[E] $\quad \begin{array}{llllllllll}0.01 & 0.041 & 0.062 & 0.072 & 0.104 & 0.187 & 0.499 & 0.791\end{array}$

[F] $\quad \begin{array}{llllllllll} & 0.031 & 0.052 & 0.062 & 0.094 & 0.177 & 0.489 & 0.781\end{array}$

[G] $\quad \begin{array}{lllllllll}0.031 & 0.031 & 0.021 & 0.031 & 0.063 & 0.146 & 0.458 & 0.75\end{array}$

$[\mathrm{H}] \quad \begin{array}{llllllllll}0.052 & 0.021 & . & 0.01 & 0.042 & 0.125 & 0.437 & 0.729\end{array}$

[J] $\quad \begin{array}{llllllllll}0.062 & 0.031 & 0.01 & 0.032 & 0.115 & 0.427 & 0.719\end{array}$

[K] $\quad \begin{array}{lllllllll}0.094 & 0.063 & 0.042 & 0.032 & 0.083 & 0.083 & 0.395 & 0.687\end{array}$

[L] $\quad \begin{array}{llllllllll} & 0.177 & 0.146 & 0.125 & 0.115 & 0.083 & . & 0.312 & 0.604\end{array}$

[M] $\quad \begin{array}{lllllllll}0.489 & 0.458 & 0.437 & 0.427 & 0.395 & 0.312 & . & 0.292\end{array}$

[N] $\quad \begin{array}{lllllllll}0.781 & 0.75 & 0.729 & 0.719 & 0.687 & 0.604 & 0.292\end{array}$. 
USERTYPE meanmantlethickness STEPMATRIX $=19$
0
12
3
5
6
7
8
9
A
B
C
D
$\mathrm{E}$

4

[0] $\quad \begin{array}{llllllllllllllllll}0 & 0.058 & 0.117 & 0.176 & 0.235 & 0.293 & 0.333 & 0.352 & 0.411 & 0.423 & 0.470 & 0.528 & 0.587 & 0.646 & 0.705\end{array}$

$\left[\begin{array}{llllllllllllllllllllllll} & 0.058 & 0 & 0.059 & 0.118 & 0.177 & 0.235 & 0.275 & 0.294 & 0.353 & 0.365 & 0.412 & 0.470 & 0.529 & 0.588 & 0.647\end{array}\right.$

[2] $\quad \begin{array}{llllllllllllllllllllll}0.117 & 0.059 & 0.059 & 0.118 & 0.176 & 0.216 & 0.235 & 0.294 & 0.306 & 0.353 & 0.411 & 0.470 & 0.529 & 0.588\end{array}$

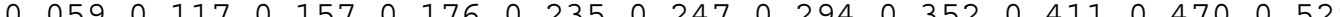

[4] $\quad \begin{array}{lllllllllllllllllllllllllll}0.235 & 0.177 & 0.118 & 0.059 & 0 & 0.058 & 0.098 & 0.117 & 0.176 & 0.188 & 0.235 & 0.293 & 0.352 & 0.411 & 0.470\end{array}$

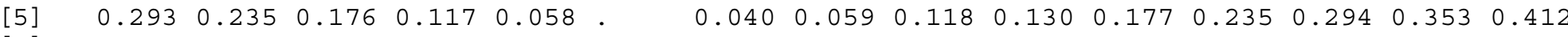

[6] $\quad \begin{array}{llllllll}0.333 & 0.275 & 0.216 & 0.157 & 0.098 & 0.040\end{array}$

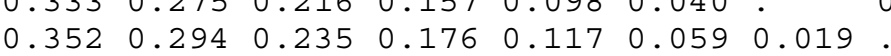

$\begin{array}{lllllllll}.019 & 0.078 & 0.090 & 0.137 & 0.195 & 0.254 & 0.313 & 0.372\end{array}$

$\begin{array}{lllllllllllllllll}0.411 & 0.353 & 0.294 & 0.235 & 0.176 & 0.118 & 0.078 & 0.059 & 0.012 & 0.059 & 0.117 & 0.176 & 0.235 & 0.294\end{array}$

$\begin{array}{lllllllllllllll}0.423 & 0.365 & 0.306 & 0.247 & 0.188 & 0.130 & 0.090 & 0.071 & 0.012 & 0.047 & 0.105 & 0.164 & 0.223 & 0.282\end{array}$

$\begin{array}{lllllllllllllll}0.470 & 0.412 & 0.353 & 0.294 & 0.235 & 0.177 & 0.137 & 0.118 & 0.059 & 0.047 & 0.058 & 0.117 & 0.176 & 0.235\end{array}$

$\begin{array}{lllllllllllllll}0.528 & 0.470 & 0.411 & 0.352 & 0.293 & 0.235 & 0.195 & 0.176 & 0.117 & 0.105 & 0.058 & . & 0.059 & 0.118 & 0.177\end{array}$

$\begin{array}{lllllllllllllllllll}0.587 & 0.529 & 0.470 & 0.411 & 0.352 & 0.294 & 0.254 & 0.235 & 0.176 & 0.164 & 0.117 & 0.059 & 0.059 & 0.118\end{array}$

$\begin{array}{llllllllllllll}0.646 & 0.588 & 0.529 & 0.470 & 0.411 & 0.353 & 0.313 & 0.294 & 0.235 & 0.223 & 0.176 & 0.118 & 0.059\end{array}$

$\begin{array}{llllllllllllllll}0.705 & 0.647 & 0.588 & 0.529 & 0.470 & 0.412 & 0.372 & 0.353 & 0.294 & 0.282 & 0.235 & 0.177 & 0.118 & 0.059 & .0 .13\end{array}$

$\begin{array}{llllllllllllllllll}0.763 & 0.705 & 0.646 & 0.587 & 0.528 & 0.470 & 0.430 & 0.411 & 0.352 & 0.340 & 0.293 & 0.235 & 0.176 & 0.117 & 0.058\end{array}$

$\begin{array}{lllllllllllllllll}0.822 & 0.764 & 0.705 & 0.646 & 0.587 & 0.529 & 0.489 & 0.470 & 0.411 & 0.399 & 0.352 & 0.294 & 0.235 & 0.176 & 0.117\end{array}$

$[\mathrm{H}] \quad \begin{array}{lllllllllllllllll}0.881 & 0.823 & 0.764 & 0.705 & 0.646 & 0.588 & 0.548 & 0.529 & 0.470 & 0.458 & 0.411 & 0.353 & 0.294 & 0.235 & 0.176\end{array}$

[J] $\quad \begin{array}{llllllllllllllllll}0.999 & 0.941 & 0.882 & 0.823 & 0.764 & 0.706 & 0.666 & 0.647 & 0.588 & 0.576 & 0.529 & 0.471 & 0.412 & 0.353 & 0.294\end{array}$ 
F $\quad$ G $\quad \mathrm{H} \quad \mathrm{J}$

[0] $\quad \begin{array}{llllll}0.763 & 0.822 & 0.881 & 0.999\end{array}$

[1] $\quad \begin{array}{llllll}0.705 & 0.764 & 0.823 & 0.941\end{array}$

$\begin{array}{llllll}{[2]} & 0.646 & 0.705 & 0.764 & 0.882\end{array}$

[3] $\quad \begin{array}{llllll}0.587 & 0.646 & 0.705 & 0.823\end{array}$

[4] $\quad \begin{array}{llllll}0.528 & 0.587 & 0.646 & 0.764\end{array}$

$\begin{array}{llllll}{[5]} & 0.470 & 0.529 & 0.588 & 0.706\end{array}$

[6] $\quad \begin{array}{lllll}0.430 & 0.489 & 0.548 & 0.666\end{array}$

[7] $\quad \begin{array}{lllll}0.411 & 0.470 & 0.529 & 0.647\end{array}$

[8] $\quad \begin{array}{llllll}0.352 & 0.411 & 0.470 & 0.588\end{array}$

$\begin{array}{lllllll}\text { [9] } & 0.340 & 0.399 & 0.458 & 0.576\end{array}$

[A] $\quad \begin{array}{llllll}0.293 & 0.352 & 0.411 & 0.529\end{array}$

[B] $\quad \begin{array}{llllll}0.235 & 0.294 & 0.353 & 0.471\end{array}$

[C] $\quad \begin{array}{llllll}0.176 & 0.235 & 0.294 & 0.412\end{array}$

[D] $\quad \begin{array}{llllll}0.117 & 0.176 & 0.235 & 0.353\end{array}$

[E] $\quad \begin{array}{llllll}0.058 & 0.117 & 0.176 & 0.294\end{array}$

$0.059 \quad 0.118 \quad 0.236$

[G] 0.059 . 0.0590 .177

[H] $\quad 0.1180 .059 .0 .118$

[J] $\quad 0.236 \quad 0.177 \quad 0.118$. 
USERTYPE pamantlethickness STEPMATRIX $=24$

\begin{tabular}{|c|c|c|c|c|c|c|c|c|c|c|c|c|c|c|c|}
\hline & 0 & 1 & 2 & 3 & 4 & 5 & 6 & 7 & 8 & 9 & A & B & $\mathrm{C}$ & D & $\mathrm{E}$ \\
\hline [0] & . & 0.066 & 0.099 & 0.133 & 0.199 & 0.233 & 0.299 & 0.333 & 0.399 & 0.432 & 0.466 & 0.499 & 0.532 & 0.566 & 0.599 \\
\hline [1] & 0.066 & . & 0.033 & 0.067 & 0.133 & 0.167 & 0.233 & 0.267 & 0.333 & 0.366 & 0.4 & 0.433 & 0.466 & 0.5 & 0.533 \\
\hline [2] & 0.099 & 0.033 & . & 0.034 & 0.1 & 0.134 & 0.2 & 0.234 & 0.3 & 0.333 & 0.367 & 0.4 & 0.433 & 0.467 & 0.5 \\
\hline [3] & 0.133 & 0.067 & 0.034 & . & 0.066 & 0.1 & 0.166 & 0.2 & 0.266 & 0.299 & 0.333 & 0.366 & 0.399 & 0.433 & 0.466 \\
\hline [4] & 0.199 & 0.133 & 0.1 & 0.066 & . & 0.034 & 0.1 & 0.134 & 0.2 & 0.233 & 0.267 & 0.3 & 0.333 & 0.367 & 0.4 \\
\hline [5] & 0.233 & 0.167 & 0.134 & 0.1 & 0.034 & & 0.066 & 0.1 & 0.166 & 0.199 & 0.233 & 0.266 & 0.299 & 0.333 & 0.366 \\
\hline [6] & 0.299 & 0.233 & 0.2 & 0.166 & 0.1 & 0.066 & . & 0.034 & 0.1 & 0.133 & 0.167 & 0.2 & 0.233 & 0.267 & 0.3 \\
\hline [7] & 0.333 & 0.267 & 0.234 & 0.2 & 0.134 & 0.1 & 0.034 & . & 0.066 & 0.099 & 0.133 & 0.166 & 0.199 & 0.233 & 0.266 \\
\hline [8] & 0.399 & 0.333 & 0.3 & 0.266 & 0.2 & 0.166 & 0.1 & 0.066 & . & 0.033 & 0.067 & 0.1 & 0.133 & 0.167 & 0.20 \\
\hline [9] & 0.432 & 0.366 & 0.333 & 0.299 & 0.233 & 0.199 & 0.133 & 0.099 & 0.033 & . & 0.034 & 0.067 & 0.1 & 0.134 & 0.167 \\
\hline [A] & 0.466 & 0.4 & 0.367 & 0.333 & 0.267 & 0.233 & 0.167 & 0.133 & 0.067 & 0.034 & . & 0.033 & 0.066 & 0.1 & 0.133 \\
\hline [B] & 0.499 & 0.433 & 0.4 & 0.366 & 0.3 & 0.266 & 0.2 & 0.166 & 0.1 & 0.067 & 0.033 & & 0.033 & 0.067 & 0.1 \\
\hline$[\mathrm{C}]$ & 0.532 & 0.466 & 0.433 & 0.399 & 0.333 & 0.299 & 0.233 & 0.199 & 0.133 & 0.1 & 0.066 & 0.033 & . & 0.034 & 0.067 \\
\hline [D] & 0.566 & 0.5 & 0.467 & 0.433 & 0.367 & 0.333 & 0.267 & 0.233 & 0.167 & 0.134 & 0.1 & 0.067 & 0.034 & . & 0.033 \\
\hline$[E]$ & 0.599 & 0.533 & 0.5 & 0.466 & 0.4 & 0.366 & 0.3 & 0.266 & 0.2 & 0.167 & 0.133 & 0.1 & 0.067 & 0.033 & \\
\hline$[F]$ & 0.632 & 0.566 & 0.533 & 0.499 & 0.433 & 0.399 & 0.333 & 0.299 & 0.233 & 0.2 & 0.166 & 0.133 & 0.1 & 0.066 & 0.033 \\
\hline [G] & 0.666 & 0.6 & 0.567 & 0.533 & 0.467 & 0.433 & 0.367 & 0.333 & 0.267 & 0.234 & 0.2 & 0.167 & 0.134 & 0.1 & 0.067 \\
\hline$[\mathrm{H}]$ & 0.699 & 0.633 & 0.6 & 0.566 & 0.5 & 0.466 & 0.4 & 0.366 & 0.3 & 0.267 & 0.233 & 0.2 & 0.167 & 0.133 & 0.1 \\
\hline$[\mathrm{J}]$ & 0.732 & 0.666 & 0.633 & 0.599 & 0.533 & 0.499 & 0.433 & 0.399 & 0.333 & 0.3 & 0.266 & 0.233 & 0.2 & 0.166 & 0.133 \\
\hline$[\mathrm{K}]$ & 0.799 & 0.733 & 0.7 & 0.666 & 0.6 & 0.566 & 0.5 & 0.466 & 0.4 & 0.367 & 0.333 & 0.3 & 0.267 & 0.233 & 0.2 \\
\hline [L] & 0.832 & 0.766 & 0.733 & 0.699 & 0.633 & 0.599 & 0.533 & 0.499 & 0.433 & 0.4 & 0.366 & 0.333 & 0.3 & 0.266 & 0.233 \\
\hline$[\mathrm{M}]$ & 0.865 & 0.799 & 0.766 & 0.732 & 0.666 & 0.632 & 0.566 & 0.532 & 0.466 & 0.433 & 0.399 & 0.366 & 0.333 & 0.299 & 0.266 \\
\hline$[\mathrm{N}]$ & 0.899 & 0.833 & 0.8 & 0.766 & 0.7 & 0.666 & 0.6 & 0.566 & 0.5 & 0.467 & 0.433 & 0.4 & 0.367 & 0.333 & 0.3 \\
\hline$[\mathrm{P}]$ & 0.999 & 0.933 & 0.9 & 0.866 & 0.8 & 0.766 & 0.7 & 0.666 & 0.6 & 0.567 & 0.533 & 0.5 & 0.467 & 0.433 & 0.4 \\
\hline
\end{tabular}


[0] $\quad \begin{array}{lllllllllll}0.632 & 0.666 & 0.699 & 0.732 & 0.799 & 0.832 & 0.865 & 0.899 & 0.999\end{array}$

[1] $\begin{array}{llllllllll}0.566 & 0.6 & 0.633 & 0.666 & 0.733 & 0.766 & 0.799 & 0.833 & 0.933\end{array}$

[2] $\quad \begin{array}{llllllllll}0.533 & 0.567 & 0.6 & 0.633 & 0.7 & 0.733 & 0.766 & 0.8 & 0.9\end{array}$

[3] $\quad \begin{array}{lllllllllll}0.499 & 0.533 & 0.566 & 0.599 & 0.666 & 0.699 & 0.732 & 0.766 & 0.866\end{array}$

[4] $\quad \begin{array}{llllllllll}0.433 & 0.467 & 0.5 & 0.533 & 0.6 & 0.633 & 0.666 & 0.7 & 0.8\end{array}$

[5] $\quad \begin{array}{lllllllllll}0.399 & 0.433 & 0.466 & 0.499 & 0.566 & 0.599 & 0.632 & 0.666 & 0.766\end{array}$

$\begin{array}{llllllllll}\text { [6] } & 0.333 & 0.367 & 0.4 & 0.433 & 0.5 & 0.533 & 0.566 & 0.6 & 0.7\end{array}$

[7] $\quad \begin{array}{lllllllllll}0.299 & 0.333 & 0.366 & 0.399 & 0.466 & 0.499 & 0.532 & 0.566 & 0.666\end{array}$

[8] $\quad \begin{array}{llllllllll}0.233 & 0.267 & 0.3 & 0.333 & 0.4 & 0.433 & 0.466 & 0.5 & 0.6\end{array}$

[9] $\begin{array}{lllllllll}0.2 & 0.234 & 0.267 & 0.3 & 0.367 & 0.4 & 0.433 & 0.467 & 0.567\end{array}$

[A] $\begin{array}{lllllllllll}0.166 & 0.2 & 0.233 & 0.266 & 0.333 & 0.366 & 0.399 & 0.433 & 0.533\end{array}$

[B] $\quad \begin{array}{llllllllll}0.133 & 0.167 & 0.2 & 0.233 & 0.3 & 0.333 & 0.366 & 0.4 & 0.5\end{array}$

[C] $\quad \begin{array}{llllllllll}0.1 & 0.134 & 0.167 & 0.2 & 0.267 & 0.3 & 0.333 & 0.367 & 0.467\end{array}$

[D] $\begin{array}{lllllllllll}0.066 & 0.1 & 0.133 & 0.166 & 0.233 & 0.266 & 0.299 & 0.333 & 0.433\end{array}$

$\left[\begin{array}{llllllllll}{[E]} & 0.033 & 0.067 & 0.1 & 0.133 & 0.2 & 0.233 & 0.266 & 0.3 & 0.4\end{array}\right.$

$[F] \quad \begin{array}{lllllllll}0 & 0.034 & 0.067 & 0.1 & 0.167 & 0.2 & 0.233 & 0.267 & 0.367\end{array}$

[G] $\quad \begin{array}{lllllllllllll}0.034 & 0.0 .133 & 0.033 & 0.066 & 0.133 & 0.166 & 0.199 & 0.233 & 0.333\end{array}$

[H] $\quad \begin{array}{llllllllll}0.067 & 0.033 & 0 . & 0.033 & 0.1 & 0.133 & 0.166 & 0.2 & 0.3\end{array}$

[J] $\quad \begin{array}{lllllllllll}0.1 & 0.066 & 0.033 & . & 0.067 & 0.1 & 0.133 & 0.167 & 0.267\end{array}$

$\left[\begin{array}{llllllllll}\mathrm{K}] & 0.167 & 0.133 & 0.1 & 0.067 & 0.033 & 0.066 & 0.1 & 0.2\end{array}\right.$

$\begin{array}{llllllllll}\text { [L] } & 0.2 & 0.166 & 0.133 & 0.1 & 0.033 & . & 0.033 & 0.067 & 0.167\end{array}$

[M] $\quad \begin{array}{llllllllllll}0.233 & 0.199 & 0.166 & 0.133 & 0.066 & 0.033 & . & 0.034 & 0.134\end{array}$

[N] $\quad \begin{array}{lllllllllll}0.267 & 0.233 & 0.2 & 0.167 & 0.1 & 0.067 & 0.034 & . & 0.1\end{array}$

[P] $\quad \begin{array}{lllllllllll}0.367 & 0.333 & 0.3 & 0.267 & 0.2 & 0.167 & 0.134 & 0.1\end{array}$ 
USERTYPE meancollumheight STEPMATRIX $=25$

\begin{tabular}{|c|c|c|c|c|c|c|c|c|c|c|c|c|c|c|c|}
\hline & & 1 & & 3 & 4 & 5 & 6 & & 8 & & $\mathrm{~A}$ & B & C & D & E \\
\hline 0] & & 0 & .09 & 36 & + & 4 & 27 & 9 & 72 & 95 & 0. & 0.34 & 85 & 0.408 & 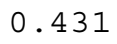 \\
\hline & 068 & & 022 & 068 & 173 & 136 & 0.159 & .181 & 0.204 & 0.227 & 0.249 & & 317 & & \\
\hline & .09 & 0.022 & & 46 & & & & & & & & 0 & & & \\
\hline & 136 & 0.068 & 046 & & & & 0.091 & & & & & & & & \\
\hline & 181 & 113 & .091 & 0.045 & & .023 & & & 91 & & & & & & \\
\hline & 204 & 136 & 114 & 0.068 & 3 & & 23 & 5 & & & & & & & \\
\hline & 27 & & & & & .023 & & & & & & & & & \\
\hline & 249 & 181 & 159 & 0.113 & & 0.045 & 0.022 & & 23 & & & & & & \\
\hline & 272 & & & & & & & .023 & & & & & & & \\
\hline & 95 & 227 & 5 & 59 & & & & & 0.023 & & & & & & \\
\hline & 17 & 249 & 27 & & & & & & & & & & & & \\
\hline & 3 & 272 & 25 & 0.204 & 59 & 36 & 13 & 91 & 68 & 0.045 & 023 & & 0.045 & & \\
\hline & & & & & & & & & & & & & & 23 & \\
\hline & .408 & 34 & 318 & 0.272 & 227 & .204 & 0.181 & 59 & 136 & 13 & 91 & 0.068 & 0.023 & & 023 \\
\hline & 31 & & & & & & & & & & & & & & \\
\hline & 454 & 386 & 364 & 0.318 & 3 & 0.2 & 27 & 5 & 82 & 0.159 & & 14 & & 046 & \\
\hline & & & & & & & & & & & & & & & \\
\hline & 99 & 1 & 9 & & & 95 & & & 27 & 4 & 82 & 59 & & 91 & 68 \\
\hline & & & & & & & & & & & & & & & \\
\hline & 635 & 7 & 5 & & & & & & & & & 95 & 0 & 27 & 04 \\
\hline & 681 & & & & & & & & & & & & & & \\
\hline & 703 & & 3 & & & & & & & & & & & & 72 \\
\hline & 771 & 3 & 681 & & & & 0. & & & & & & & & \\
\hline & & & & & & & & & & & & & & & \\
\hline & 999 & .931 & .909 & 0.863 & .818 & 0.795 & 0.772 & 0.15 & 0.727 & 0.704 & 0.682 & 0.659 & 0.614 & 0.591 & 0.568 \\
\hline
\end{tabular}


[0] $\quad \begin{array}{llllllllllll}0.454 & 0.476 & 0.499 & 0.544 & 0.635 & 0.681 & 0.703 & 0.771 & 0.862 & 0.999\end{array}$

[1] $\quad \begin{array}{llllllllllll}0.386 & 0.408 & 0.431 & 0.476 & 0.567 & 0.613 & 0.635 & 0.703 & 0.794 & 0.931\end{array}$

[2] $\quad \begin{array}{lllllllllllll}0.364 & 0.386 & 0.409 & 0.454 & 0.545 & 0.591 & 0.613 & 0.681 & 0.772 & 0.909\end{array}$

[3] $\quad \begin{array}{llllllllllll}0.318 & 0.34 & 0.363 & 0.408 & 0.499 & 0.545 & 0.567 & 0.635 & 0.726 & 0.863\end{array}$

[4] $\quad \begin{array}{llllllllll}0.273 & 0.295 & 0.318 & 0.363 & 0.454 & 0.5 & 0.522 & 0.59 & 0.681 & 0.818\end{array}$

[5] $\quad \begin{array}{llllllllllll}0.25 & 0.272 & 0.295 & 0.34 & 0.431 & 0.477 & 0.499 & 0.567 & 0.658 & 0.795\end{array}$

[6] $\quad \begin{array}{llllllllllll}0.227 & 0.249 & 0.272 & 0.317 & 0.408 & 0.454 & 0.476 & 0.544 & 0.635 & 0.772\end{array}$

[7] $\quad \begin{array}{llllllllllll}0.205 & 0.227 & 0.25 & 0.295 & 0.386 & 0.432 & 0.454 & 0.522 & 0.613 & 0.75\end{array}$

[8] $\quad \begin{array}{llllllllllll}0.182 & 0.204 & 0.227 & 0.272 & 0.363 & 0.409 & 0.431 & 0.499 & 0.59 & 0.727\end{array}$

[9] $\quad \begin{array}{llllllllllll}0.159 & 0.181 & 0.204 & 0.249 & 0.34 & 0.386 & 0.408 & 0.476 & 0.567 & 0.704\end{array}$

[A] $\quad \begin{array}{llllllllllll}0.137 & 0.159 & 0.182 & 0.227 & 0.318 & 0.364 & 0.386 & 0.454 & 0.545 & 0.682\end{array}$

[B] $\quad \begin{array}{llllllllllll}0.114 & 0.136 & 0.159 & 0.204 & 0.295 & 0.341 & 0.363 & 0.431 & 0.522 & 0.659\end{array}$

[C] $\quad \begin{array}{lllllllllllll}0.069 & 0.091 & 0.114 & 0.159 & 0.25 & 0.296 & 0.318 & 0.386 & 0.477 & 0.614\end{array}$

[D] $\quad \begin{array}{llllllllllll}0.046 & 0.068 & 0.091 & 0.136 & 0.227 & 0.273 & 0.295 & 0.363 & 0.454 & 0.591\end{array}$

[E] $\quad \begin{array}{llllllllllll}0.023 & 0.045 & 0.068 & 0.113 & 0.204 & 0.25 & 0.272 & 0.34 & 0.431 & 0.568\end{array}$

[F] $\quad \begin{array}{llllllllllll}0.022 & 0.045 & 0.09 & 0.181 & 0.227 & 0.249 & 0.317 & 0.408 & 0.545\end{array}$

[G] $\quad \begin{array}{llllllllllll}0.022 & 0 & 0.023 & 0.068 & 0.159 & 0.205 & 0.227 & 0.295 & 0.386 & 0.523\end{array}$

[H] $\quad \begin{array}{lllllllllllll}0.045 & 0.023 & . & & 0.045 & 0.136 & 0.182 & 0.204 & 0.272 & 0.363 & 0.5\end{array}$

[J] $\quad \begin{array}{llllllllllllll}0.09 & 0.068 & 0.045 & . & 0.091 & 0.137 & 0.159 & 0.227 & 0.318 & 0.455\end{array}$

$[\mathrm{K}] \quad \begin{array}{lllllllllllll}0.181 & 0.159 & 0.136 & 0.091 & & 0.0 .046 & 0.046 & 0.068 & 0.136 & 0.227 & 0.364\end{array}$

[L] $\quad \begin{array}{llllllllllll} & 0.227 & 0.205 & 0.182 & 0.137 & 0.046 & 0.0 .04 & 0.022 & 0.09 & 0.181 & 0.318\end{array}$

[M] $\quad \begin{array}{lllllllllll}0.249 & 0.227 & 0.204 & 0.159 & 0.068 & 0.022 & 0.068 & 0.159 & 0.296\end{array}$

[N] $\quad \begin{array}{lllllllllll}0.317 & 0.295 & 0.272 & 0.227 & 0.136 & 0.09 & 0.068 & 0.091 & 0.228\end{array}$

[P] $\quad \begin{array}{lllllllllllll}0.408 & 0.386 & 0.363 & 0.318 & 0.227 & 0.181 & 0.159 & 0.091 & . & & 0.137\end{array}$

[Q] $\quad \begin{array}{llllllllllll}0.545 & 0.523 & 0.5 & 0.455 & 0.364 & 0.318 & 0.296 & 0.228 & 0.137\end{array}$ 
USERTYPE pacollumheight STEPMATRIX $=26$

\begin{tabular}{|c|c|c|c|c|c|c|c|c|c|c|c|c|c|c|c|}
\hline & & 1 & 2 & 3 & 4 & 5 & 6 & & 8 & 9 & A & B & C & $D$ & $\mathrm{E}$ \\
\hline & & 5 & 7 & 7 & 1 & 6 & 8 & 0.18 & 2 & 4 & 6 & 8 & 92 & 96 & \\
\hline & 155 & & & 012 & & & & & & & & & & & \\
\hline & .157 & .002 & & .01 & 014 & 019 & 0.021 & .023 & 0.025 & .027 & .029 & 0.031 & 0.035 & 0.039 & .043 \\
\hline & 167 & 012 & 01 & & & & & & & & & & & & \\
\hline & 171 & 016 & .014 & 0.004 & & 0.005 & .007 & .009 & 11 & 13 & 15 & 0.017 & 21 & 25 & 029 \\
\hline & 176 & 021 & & & .005 & & & & & & & & & & \\
\hline & & 023 & & & 007 & 0.002 & & & & & & & & & \\
\hline & 18 & 025 & & & & & 0 & & & & & & & & \\
\hline & & 027 & & & & & & 0.002 & & & & & & & \\
\hline & 4 & & & & & & & & & & & & & & \\
\hline & 186 & 031 & & & & & 08 & & 04 & 2 & & & & & \\
\hline & 188 & & & & & & & & & & & & & & \\
\hline & 192 & 037 & & & & & & & & & & & & & \\
\hline & & & & & & & & & & & & & & & \\
\hline & & & & & & & & & & & & & & & \\
\hline & & & & & & & & & & & & & & & \\
\hline & & & & & & & & & & & & & & & \\
\hline & 206 & 1 & & & & & & & & & & & & & \\
\hline & & & & & & & & & & & & & & & 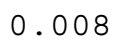 \\
\hline & .214 & .059 & 7 & 0.047 & & 38 & 36 & 4 & 32 & & 28 & 26 & & 18 & 0.014 \\
\hline & .223 & & & & & & & & & & & & & & \\
\hline & .227 & 0.072 & & 0. & & .051 & 49 & 7 & 45 & 3 & 41 & 39 & & 0.031 & 0.027 \\
\hline & .241 & 086 & & & & & & & & & & & & & \\
\hline & 5 & & & & & & & & & & & & & & 55 \\
\hline & & & & & & & & & & & & & & & \\
\hline & & & & & & & & & & & & & & & .79 \\
\hline
\end{tabular}


[0]

[1]

[2]

[3]

$[4]$

[5]

[6]

[7]

$[8]$

[9]

[A]

[B]

$[\mathrm{C}]$

[D]
$[E]$

[E]

$[\mathrm{G}]$
$[\mathrm{H}]$

J]

[J]

[L]

[M]

$[\mathrm{N}]$

[P]

[Q]

$[\mathrm{R}]$

$\begin{array}{lllllllllll}0.202 & 0.204 & 0.206 & 0.208 & 0.214 & 0.223 & 0.227 & 0.241 & 0.255 & 0.436 & 0.999\end{array}$ $\begin{array}{llllllllllll}0.047 & 0.049 & 0.051 & 0.053 & 0.059 & 0.068 & 0.072 & 0.086 & 0.1 & 0.281 & 0.844\end{array}$ $\begin{array}{llllllllllll}0.045 & 0.047 & 0.049 & 0.051 & 0.057 & 0.066 & 0.07 & 0.084 & 0.098 & 0.279 & 0.842\end{array}$ $\begin{array}{llllllllllll}0.035 & 0.037 & 0.039 & 0.041 & 0.047 & 0.056 & 0.06 & 0.074 & 0.088 & 0.269 & 0.832\end{array}$ $\begin{array}{llllllllllll}0.031 & 0.033 & 0.035 & 0.037 & 0.043 & 0.052 & 0.056 & 0.07 & 0.084 & 0.265 & 0.828\end{array}$ $\begin{array}{llllllllllll}0.026 & 0.028 & 0.03 & 0.032 & 0.038 & 0.047 & 0.051 & 0.065 & 0.079 & 0.26 & 0.823\end{array}$ $\begin{array}{llllllllllll}0.024 & 0.026 & 0.028 & 0.03 & 0.036 & 0.045 & 0.049 & 0.063 & 0.077 & 0.258 & 0.821\end{array}$ $\begin{array}{llllllllllll}0.022 & 0.024 & 0.026 & 0.028 & 0.034 & 0.043 & 0.047 & 0.061 & 0.075 & 0.256 & 0.819\end{array}$ $\begin{array}{lllllllllllll}0.02 & 0.022 & 0.024 & 0.026 & 0.032 & 0.041 & 0.045 & 0.059 & 0.073 & 0.254 & 0.817\end{array}$ $\begin{array}{lllllllllllll}0.018 & 0.02 & 0.022 & 0.024 & 0.03 & 0.039 & 0.043 & 0.057 & 0.071 & 0.252 & 0.815\end{array}$ $\begin{array}{llllllllllll}0.016 & 0.018 & 0.02 & 0.022 & 0.028 & 0.037 & 0.041 & 0.055 & 0.069 & 0.25 & 0.813\end{array}$ $\begin{array}{llllllllllll}0.014 & 0.016 & 0.018 & 0.02 & 0.026 & 0.035 & 0.039 & 0.053 & 0.067 & 0.248 & 0.811\end{array}$ $\begin{array}{lllllllllllll}0.01 & 0.012 & 0.014 & 0.016 & 0.022 & 0.031 & 0.035 & 0.049 & 0.063 & 0.244 & 0.807\end{array}$ $\begin{array}{lllllllllllll}0.006 & 0.008 & 0.01 & 0.012 & 0.018 & 0.027 & 0.031 & 0.045 & 0.059 & 0.24 & 0.803\end{array}$ $\begin{array}{llllllllllll}0.002 & 0.004 & 0.006 & 0.008 & 0.014 & 0.023 & 0.027 & 0.041 & 0.055 & 0.236 & 0.799\end{array}$ $\begin{array}{llllllllllll}0.002 & 0.004 & 0.006 & 0.012 & 0.021 & 0.025 & 0.039 & 0.053 & 0.234 & 0.797\end{array}$ $\begin{array}{lllllllllllll}0.002 & 0.002 & 0.004 & 0.01 & 0.019 & 0.023 & 0.037 & 0.051 & 0.232 & 0.795\end{array}$ $\begin{array}{llllllllllllll}0.004 & 0.002 & 0.002 & 0.002 & 0.008 & 0.017 & 0.021 & 0.035 & 0.049 & 0.23 & 0.793\end{array}$ $\begin{array}{llllllllllllll}0.006 & 0.004 & 0.002 & 0.0 .001 & 0.006 & 0.015 & 0.019 & 0.033 & 0.047 & 0.228 & 0.791\end{array}$ $\begin{array}{llllllllllllllll}0.012 & 0.01 & 0.008 & 0.006 & 0.009 & 0.013 & 0.027 & 0.041 & 0.222 & 0.785\end{array}$ $\begin{array}{lllllllllllll}0.021 & 0.019 & 0.017 & 0.015 & 0.009 & 0.0 .004 & 0.004 & 0.018 & 0.032 & 0.213 & 0.776\end{array}$ $\begin{array}{llllllllllllll}0.025 & 0.023 & 0.021 & 0.019 & 0.013 & 0.004 & 0.014 & 0.028 & 0.209 & 0.772\end{array}$ $\begin{array}{llllllllllll}0.039 & 0.037 & 0.035 & 0.033 & 0.027 & 0.018 & 0.014 & 0.014 & 0.195 & 0.758\end{array}$ $\begin{array}{llllllllllll}0.053 & 0.051 & 0.049 & 0.047 & 0.041 & 0.032 & 0.028 & 0.014 & 0.181 & 0.744\end{array}$ $\begin{array}{lllllllllllll}0.234 & 0.232 & 0.23 & 0.228 & 0.222 & 0.213 & 0.209 & 0.195 & 0.181 & 0.563\end{array}$ $\begin{array}{lllllllllllll}0.797 & 0.795 & 0.793 & 0.791 & 0.785 & 0.776 & 0.772 & 0.758 & 0.744 & 0.563\end{array}$. 
USERTYPE meanringleistheight STEPMATRIX = 19

\begin{tabular}{|c|c|c|c|c|c|c|c|c|c|c|c|c|c|c|c|}
\hline & & 1 & 2 & 3 & 4 & & 6 & & 8 & 9 & A & B & E & ) & \\
\hline & & 5 & & & 214 & 249 & 0.285 & 321 & 0.356 & 392 & 0.428 & 0.463 & 499 & 0.57 & .0 \\
\hline & 035 & & & 0.107 & & 4 & & & & & & & & & \\
\hline & & 072 & & 035 & & & & & 249 & & & & & & \\
\hline & 42 & 0.107 & .035 & & & 7 & 0.143 & & & & & & & & \\
\hline & 14 & 179 & & 2 & & & & & & & & & & & \\
\hline & .249 & 0.214 & 142 & 0.107 & .035 & & 0.036 & & & & & & & & \\
\hline & 5 & & & & & & & & & & & & & & \\
\hline & .321 & 286 & 14 & 9 & 07 & 0.072 & 0.036 & & 35 & 71 & 07 & & & & 85 \\
\hline & & & & & & & & & & & & & & & \\
\hline & .392 & 0.357 & 285 & 0. & 8 & 0 . & 0.107 & 71 & 0.036 & & .036 & & & & \\
\hline & & & & & & & & & & & & & & & \\
\hline & 463 & 428 & 356 & 321 & 9 & 0.214 & 0.178 & 42 & 07 & 71 & 0. & & 0.036 & & 43 \\
\hline & & & & & & & & & & & & & & & \\
\hline & 5 & 35 & 53 & 28 & & 0. & 0. & 9 & 14 & 78 & & 07 & 0.071 & & .036 \\
\hline & & & & & & & & & & & & & & & \\
\hline & 9 & 0.714 & & & & & & & & & & & & & \\
\hline & 82 & 0.785 & & & & & & & & & & & & & \\
\hline & & & & & & & & & & & & & & & \\
\hline & .999 & 0.964 & .892 & 0.857 & 0.785 & 0.15 & 0.714 & 0.678 & 0.643 & 0.607 & 0.571 & 0.536 & 0.5 & 0.429 & 0.39 \\
\hline
\end{tabular}


F $\quad$ G $\quad \mathrm{H} \quad \mathrm{J}$

[0] $\quad \begin{array}{llllll}0.749 & 0.82 & 0.856 & 0.999\end{array}$

[1] $\quad \begin{array}{llllll}0.714 & 0.785 & 0.821 & 0.964\end{array}$

$\begin{array}{llllll}{[2]} & 0.642 & 0.713 & 0.749 & 0.892\end{array}$

[3] $\quad \begin{array}{llllll}0.607 & 0.678 & 0.714 & 0.857\end{array}$

[4] $\quad \begin{array}{llllll}0.535 & 0.606 & 0.642 & 0.785\end{array}$

$\begin{array}{llllll}{[5]} & 0.5 & 0.571 & 0.607 & 0.75\end{array}$

[6] $\quad \begin{array}{lllll}0.464 & 0.535 & 0.571 & 0.714\end{array}$

[7] $\quad \begin{array}{lllll}0.428 & 0.499 & 0.535 & 0.678\end{array}$

[8] $\quad \begin{array}{lllll}0.393 & 0.464 & 0.5 & 0.643\end{array}$

$\begin{array}{llllll}\text { [9] } & 0.357 & 0.428 & 0.464 & 0.607\end{array}$

[A] $\quad \begin{array}{llllll}0.321 & 0.392 & 0.428 & 0.571\end{array}$

[B] $\quad \begin{array}{llllll}0.286 & 0.357 & 0.393 & 0.536\end{array}$

[C] $\quad \begin{array}{llllll}0.25 & 0.321 & 0.357 & 0.5\end{array}$

[D] $\quad \begin{array}{llllll}0.179 & 0.25 & 0.286 & 0.429\end{array}$

[E] $\quad \begin{array}{llllll}0.143 & 0.214 & 0.25 & 0.393\end{array}$

$0.0710 .107 \quad 0.25$

[G] $\quad 0.071 .0 .0360 .179$

[H] $\quad 0.107 \quad 0.036 .0 .143$

[J] $\quad 0.25 \quad 0.179 \quad 0.143$. 
USERTYPE paringleistheight STEPMATRIX $=21$

\begin{tabular}{|c|c|c|c|c|c|c|c|c|c|c|c|c|c|c|c|}
\hline & 0 & 1 & 2 & 3 & 4 & & 6 & & 8 & 9 & A & B & C & $\mathrm{D}$ & $\mathrm{E}$ \\
\hline & & 3 & 6 & 9 & 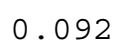 & 6 & 9 & 2 & 5 & 9 & 32 & 55 & 78 & 2 & 325 \\
\hline & 023 & & & & & & & & & & & & & & \\
\hline & .046 & .023 & & .023 & 046 & .070 & 093 & .116 & .139 & 163 & .186 & 209 & .232 & .256 & 79 \\
\hline & & 046 & 3 & & & & & & & & & & & & \\
\hline & 092 & 069 & .046 & 0.023 & & 0.024 & 47 & & 93 & 17 & & & & & \\
\hline & & 093 & & & & & & & & & & & & & \\
\hline & & 116 & & & 7 & 0.023 & & & & & & & & & \\
\hline & & & & & & & & & & & & & & & \\
\hline & & 2 & & & & & & 0 & & & & & & & \\
\hline & & & & & & & & & & & & & & & \\
\hline & 232 & 209 & & 63 & & & 93 & & 0.047 & 0. & & 23 & & & \\
\hline & & & & & & & & & & & & & & & \\
\hline & & 55 & & & & & & & & & & & & 24 & \\
\hline & & 279 & & & & & & & & & & & & & \\
\hline & & & & & & & & & & & & & & & \\
\hline & 371 & 0.348 & & & & & & & & & & & & & \\
\hline & & & & & & & & & & & & & & & \\
\hline & .418 & 0.395 & & 49 & 6 & 22 & & & & & & & & & 093 \\
\hline & & 0.464 & & & & & & & & & & & & & \\
\hline & 789 & 0.766 & 3 & 0.720 & 697 & 0.673 & 0.650 & 27 & 604 & 0.580 & 0.557 & 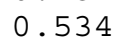 & 0.511 & 0.487 & 0.464 \\
\hline & & 0.976 & & 0.930 & 907 & 0.883 & 0.860 & 0.837 & 0.814 & 0.790 & 0.767 & 0.744 & 0.721 & 0.697 & 0.67 \\
\hline
\end{tabular}


[0] $\quad \begin{array}{llllllll}0.371 & 0.394 & 0.418 & 0.487 & 0.789 & 0.999\end{array}$

[1] $\quad \begin{array}{llllllll}0.348 & 0.371 & 0.395 & 0.464 & 0.766 & 0.976\end{array}$

$\begin{array}{lllllllll}{[2]} & 0.325 & 0.348 & 0.372 & 0.441 & 0.743 & 0.953\end{array}$

[3] $\quad \begin{array}{llllllll}0.302 & 0.325 & 0.349 & 0.418 & 0.720 & 0.930\end{array}$

[4] $\quad \begin{array}{llllllll}0.279 & 0.302 & 0.326 & 0.395 & 0.697 & 0.907\end{array}$

[5] $\quad \begin{array}{llllllll}0.255 & 0.278 & 0.302 & 0.371 & 0.673 & 0.883\end{array}$

[6] $\quad \begin{array}{llllllll}0.232 & 0.255 & 0.279 & 0.348 & 0.650 & 0.860\end{array}$

[7] $\quad \begin{array}{llllllll}0.209 & 0.232 & 0.256 & 0.325 & 0.627 & 0.837\end{array}$

[8] $\quad \begin{array}{llllllll}0.186 & 0.209 & 0.233 & 0.302 & 0.604 & 0.814\end{array}$

[9] $\quad \begin{array}{llllllll}0.162 & 0.185 & 0.209 & 0.278 & 0.580 & 0.790\end{array}$

[A] $\quad \begin{array}{llllllll}0.139 & 0.162 & 0.186 & 0.255 & 0.557 & 0.767\end{array}$

[B] $\quad \begin{array}{llllllll}0.116 & 0.139 & 0.163 & 0.232 & 0.534 & 0.744\end{array}$

[C] $\quad \begin{array}{llllllll}0.093 & 0.116 & 0.140 & 0.209 & 0.511 & 0.721\end{array}$

[D] $\quad \begin{array}{llllllll}0.069 & 0.092 & 0.116 & 0.185 & 0.487 & 0.697\end{array}$

[E] $\quad \begin{array}{llllllll}0.046 & 0.069 & 0.093 & 0.162 & 0.464 & 0.674\end{array}$

[F] $\begin{array}{lllllll}0 & 0.023 & 0.047 & 0.116 & 0.418 & 0.628\end{array}$

[G] $\quad 0.023 .0 .024 \quad 0.093 \quad 0.395 \quad 0.605$

[H] $\quad \begin{array}{lllllllll}0.047 & 0.024 & . & 0.069 & 0.371 & 0.581\end{array}$

[J] $\quad 0.116 \quad 0.0930 .069 .0 .302 \quad 0.512$

[K] $\quad \begin{array}{llllllll}0.418 & 0.395 & 0.371 & 0.302 & . & 0.210\end{array}$

[L] $\quad \begin{array}{lllllll}0.628 & 0.605 & 0.581 & 0.512 & 0.210\end{array}$. 
USERTYPE meanringleistdpi STEPMATRIX $=23$

\begin{tabular}{|c|c|c|c|c|c|c|c|c|c|c|c|c|c|c|c|}
\hline & 0 & 1 & 2 & 3 & 4 & 5 & 6 & 7 & 8 & 9 & A & B & $\mathrm{C}$ & $\mathrm{D}$ & $\mathrm{E}$ \\
\hline ] & & 7 & 4 & 81 & 8 & 9 & 35 & 62 & 89 & 16 & 0.243 & 270 & 297 & 324 & 0.351 \\
\hline & .027 & & 027 & .054 & 081 & 082 & 0.108 & 135 & 0.162 & 0.189 & 0.216 & 0.243 & 0.270 & 0.297 & \\
\hline [2] & 0.054 & 0.027 & & 0.027 & .054 & 0.055 & 0.081 & 0.108 & 0.135 & 0.162 & 0.189 & 0.216 & 0.243 & 0.270 & 0.297 \\
\hline & 081 & 054 & 027 & & 027 & 0.028 & 0.054 & .081 & 0.108 & 0.135 & 0.162 & 0.189 & 0.216 & 0.243 & \\
\hline & .108 & 0.081 & 0.054 & 0.027 & & 0.001 & 0.027 & 0.054 & 0.081 & 0.108 & 0.135 & 0.162 & 0.189 & 0.216 & 0.243 \\
\hline & 109 & 082 & 055 & 0.028 & 1 & & 0.026 & .053 & 0.080 & 0.107 & & & 188 & 215 & \\
\hline & 135 & .108 & 081 & 0.054 & 0.027 & 0.026 & & 0.027 & .054 & 0.081 & 0.108 & 0.135 & 0.162 & 189 & 216 \\
\hline & .162 & .135 & .108 & 0.081 & 0.054 & 0.053 & 0.027 & & 0.027 & 0.054 & 0.081 & .108 & 35 & 62 & 189 \\
\hline & 189 & 162 & 135 & 0.108 & 0.081 & 0.080 & 0.054 & 0.027 & & 0.027 & 54 & 0.081 & 08 & & 62 \\
\hline & .216 & .189 & .162 & 0.135 & .108 & 0.107 & 0.081 & 0.054 & 0.027 & & 0.027 & 0.054 & 81 & 08 & 35 \\
\hline & .243 & 0.216 & .189 & 0.162 & 0.135 & 0.134 & 0.108 & 0.081 & 0.054 & 0.027 & & 0.027 & 0.054 & 81 & 108 \\
\hline & .270 & 0.243 & 216 & 0.189 & 2 & 0.161 & 0.135 & 0.108 & 081 & 0.054 & 0.027 & & 0.027 & 54 & 81 \\
\hline & .297 & 0.270 & .243 & 0.216 & 0.189 & 0.188 & 0.162 & 0.135 & 0.108 & 0.081 & 0.054 & 0.027 & & 0.027 & 0.054 \\
\hline & 324 & 297 & & 0.243 & & 0.215 & & 0.162 & 35 & 0 . & 81 & 0.054 & 27 & & 0.027 \\
\hline & 351 & 324 & 7 & 70 & 3 & 0 . & 16 & 39 & 62 & 0 . & 08 & 0.081 & 54 & 27 & \\
\hline & 378 & 0.351 & 4 & 0.297 & 0 & 0.269 & 0.243 & 0.216 & 89 & 0. & 35 & 0.108 & & & \\
\hline & 396 & 0.369 & 2 & 0.315 & 8 & 0.287 & 61 & 0.234 & 07 & 80 & 0.153 & 0.126 & 99 & 72 & 45 \\
\hline & .621 & 0.594 & .567 & 0.540 & .513 & 0.512 & 0.486 & 0.459 & 0.432 & 0.405 & 0.378 & 0.351 & 0.324 & 97 & 0.270 \\
\hline & 643 & 0.616 & & 0.562 & & 0.534 & & & 0.454 & & 0.400 & 0.373 & & 0.319 & 0292 \\
\hline & 0.729 & 0.702 & 0.675 & 0.648 & 0.621 & 0.620 & 0.594 & 0.567 & 0.540 & 0.513 & 0.486 & 0.459 & 0.432 & 0.405 & 0.378 \\
\hline & .848 & 0.821 & & & & & & & & & 0.605 & & & 0.524 & \\
\hline$[\mathrm{N}$ & 0.957 & 0.930 & 0.903 & 0.876 & 0.849 & 0.848 & 0.822 & 0.795 & 0.768 & 0.741 & 0.714 & 0.687 & 0.660 & 0.633 & 0.606 \\
\hline & .999 & 0.972 & 0.945 & 0.918 & 0.891 & 0.890 & 0.864 & 0.837 & 0.810 & 0.783 & 0.756 & 0.729 & 0.702 & 0.675 & 0.648 \\
\hline
\end{tabular}


[0] $\quad \begin{array}{llllllllll}0.378 & 0.396 & 0.621 & 0.643 & 0.729 & 0.848 & 0.957 & 0.999\end{array}$

[1] $\quad \begin{array}{llllllllll}0.351 & 0.369 & 0.594 & 0.616 & 0.702 & 0.821 & 0.930 & 0.972\end{array}$

[2] $\quad \begin{array}{llllllllll}0.324 & 0.342 & 0.567 & 0.589 & 0.675 & 0.794 & 0.903 & 0.945\end{array}$

$\begin{array}{llllllllll}\text { [3] } & 0.297 & 0.315 & 0.540 & 0.562 & 0.648 & 0.767 & 0.876 & 0.918\end{array}$

[4] $\quad \begin{array}{llllllllll}0.270 & 0.288 & 0.513 & 0.535 & 0.621 & 0.740 & 0.849 & 0.891\end{array}$

[5] $\quad \begin{array}{llllllllll}0.269 & 0.287 & 0.512 & 0.534 & 0.620 & 0.739 & 0.848 & 0.890\end{array}$

[6] $\quad \begin{array}{llllllllll}0.243 & 0.261 & 0.486 & 0.508 & 0.594 & 0.713 & 0.822 & 0.864\end{array}$

[7] $\quad \begin{array}{llllllllll}0.216 & 0.234 & 0.459 & 0.481 & 0.567 & 0.686 & 0.795 & 0.837\end{array}$

[8] $\quad \begin{array}{llllllllll}0.189 & 0.207 & 0.432 & 0.454 & 0.540 & 0.659 & 0.768 & 0.810\end{array}$

[9] $\quad \begin{array}{llllllllll}0.162 & 0.180 & 0.405 & 0.427 & 0.513 & 0.632 & 0.741 & 0.783\end{array}$

[A] $\quad \begin{array}{lllllllllll}0.135 & 0.153 & 0.378 & 0.400 & 0.486 & 0.605 & 0.714 & 0.756\end{array}$

[B] $\quad \begin{array}{lllllllllll}0.108 & 0.126 & 0.351 & 0.373 & 0.459 & 0.578 & 0.687 & 0.729\end{array}$

[C] $\quad \begin{array}{lllllllllll}0.081 & 0.099 & 0.324 & 0.346 & 0.432 & 0.551 & 0.660 & 0.702\end{array}$

[D] $\quad \begin{array}{lllllllllll}0.054 & 0.072 & 0.297 & 0.319 & 0.405 & 0.524 & 0.633 & 0.675\end{array}$

[E] $\quad \begin{array}{llllllllll}0.027 & 0.045 & 0.270 & 0.292 & 0.378 & 0.497 & 0.606 & 0.648\end{array}$

[F] $\quad \begin{array}{llllllllll} & & 0.018 & 0.243 & 0.265 & 0.351 & 0.470 & 0.579 & 0.621\end{array}$

[G] $\quad \begin{array}{lllllllllll}0.018 & 0.225 & 0.247 & 0.333 & 0.452 & 0.561 & 0.603\end{array}$

[H] $\quad \begin{array}{llllllllll} & 0.243 & 0.225 & & 0.022 & 0.108 & 0.227 & 0.336 & 0.378\end{array}$

[J] $\quad \begin{array}{lllllllllll}0.265 & 0.247 & 0.022 & . & 0.086 & 0.205 & 0.314 & 0.356\end{array}$

[K] $\quad 0.351 \quad 0.3330 .108 \quad 0.086 .0 .1190 .2280 .270$

[L] $\quad \begin{array}{lllllllll}0.470 & 0.452 & 0.227 & 0.205 & 0.119 & . & 0.109 & 0.151\end{array}$

[M] $\quad \begin{array}{lllllllllll}0.579 & 0.561 & 0.336 & 0.314 & 0.228 & 0.109 & . & 0.042\end{array}$

[N] $\quad \begin{array}{llllllllll}0.621 & 0.603 & 0.378 & 0.356 & 0.270 & 0.151 & 0.042\end{array}$. 
USERTYPE paringleistdpi STEPMATRIX $=24$

\begin{tabular}{|c|c|c|c|c|c|c|c|c|c|c|c|c|c|c|c|}
\hline & & 1 & & 3 & 4 & 5 & 6 & 1 & 8 & 9 & A & B & C & D & $\mathrm{E}$ \\
\hline 0] & & 0 & 5 & 53 & & 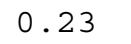 & 58 & 7 & 5 & & 0. & 0. & 99 & 537 & \\
\hline & 076 & & 039 & 077 & & 154 & & & & & & & & & \\
\hline & .115 & .039 & & 38 & & & & & & & & & & & \\
\hline & 53 & 077 & 038 & & & & & & & & & & & & \\
\hline & 192 & 116 & .077 & 0.039 & & 38 & & & & & & & & & \\
\hline & .23 & 154 & 15 & 0.077 & 8 & & & & & & & & & & \\
\hline & & & & & & 0.038 & & & & & & & & & \\
\hline & 307 & 231 & 92 & & & & & & & & & & & & \\
\hline & 5 & & & & & & & & & & & & & & \\
\hline & & 8 & & & & & & & & & & & & & \\
\hline & 22 & & & & & & & & & & & & & & \\
\hline & 51 & 35 & 6 & & & 31 & & 54 & & & 39 & & & & 15 \\
\hline & & & & & & & & & & & & & & & \\
\hline & .537 & 461 & 22 & 84 & 45 & 07 & 59 & 3 & & 53 & & & 8 & & 39 \\
\hline & & & & & & & & & & & & & & & \\
\hline & .614 & 538 & 99 & 0.461 & 22 & 384 & 46 & .307 & & 0.23 & & & & & 38 \\
\hline & & & & & & & & & & 9 & & & & & 077 \\
\hline & 91 & & & & & & & & & 7 & & & & .54 & 115 \\
\hline & & & & & & & & & & & & & & & \\
\hline & 58 & & & & & & & & & & & & & 31 & 92 \\
\hline & & & & & & & & & & & & & & & \\
\hline & & & & & & & & & & & & & & & \\
\hline & & & & & & & & & & & & & & & \\
\hline & & 23 & & 6 & 07 & 69 & 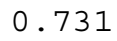 & .692 & 54 & 0.615 & 577 & 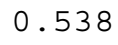 & & 0.462 & 0.42 \\
\hline
\end{tabular}


[0] $\quad \begin{array}{llllllllll}0.614 & 0.653 & 0.691 & 0.73 & 0.768 & 0.806 & 0.845 & 0.922 & 0.999\end{array}$

[1] $\quad \begin{array}{llllllllll}0.538 & 0.577 & 0.615 & 0.654 & 0.692 & 0.73 & 0.769 & 0.846 & 0.923\end{array}$

[2] $\quad \begin{array}{lllllllllll}0.499 & 0.538 & 0.576 & 0.615 & 0.653 & 0.691 & 0.73 & 0.807 & 0.884\end{array}$

[3] $\quad \begin{array}{lllllllllll}0.461 & 0.5 & 0.538 & 0.577 & 0.615 & 0.653 & 0.692 & 0.769 & 0.846\end{array}$

[4] $\quad \begin{array}{llllllllll}0.422 & 0.461 & 0.499 & 0.538 & 0.576 & 0.614 & 0.653 & 0.73 & 0.807\end{array}$

$\left[\begin{array}{lllllllllll}5] & 0.384 & 0.423 & 0.461 & 0.5 & 0.538 & 0.576 & 0.615 & 0.692 & 0.769\end{array}\right.$

[6] $\quad \begin{array}{llllllllll}0.346 & 0.385 & 0.423 & 0.462 & 0.5 & 0.538 & 0.577 & 0.654 & 0.731\end{array}$

[7] $\quad \begin{array}{llllllllllll}0.307 & 0.346 & 0.384 & 0.423 & 0.461 & 0.499 & 0.538 & 0.615 & 0.692\end{array}$

[8] $\quad \begin{array}{lllllllllll}0.269 & 0.308 & 0.346 & 0.385 & 0.423 & 0.461 & 0.5 & 0.577 & 0.654\end{array}$

[9] $\quad \begin{array}{lllllllllll}0.23 & 0.269 & 0.307 & 0.346 & 0.384 & 0.422 & 0.461 & 0.538 & 0.615\end{array}$

[A] $\quad \begin{array}{llllllllll}0.192 & 0.231 & 0.269 & 0.308 & 0.346 & 0.384 & 0.423 & 0.5 & 0.577\end{array}$

[B] $\quad \begin{array}{llllllllllll}0.153 & 0.192 & 0.23 & 0.269 & 0.307 & 0.345 & 0.384 & 0.461 & 0.538\end{array}$

[C] $\quad \begin{array}{llllllllllll}0.115 & 0.154 & 0.192 & 0.231 & 0.269 & 0.307 & 0.346 & 0.423 & 0.5\end{array}$

[D] $\quad \begin{array}{llllllllllll}0.077 & 0.116 & 0.154 & 0.193 & 0.231 & 0.269 & 0.308 & 0.385 & 0.462\end{array}$

[E] $\quad \begin{array}{lllllllllll}0.038 & 0.077 & 0.115 & 0.154 & 0.192 & 0.23 & 0.269 & 0.346 & 0.423\end{array}$

[F] $\quad \begin{array}{lllllllllll} & 0.039 & 0.077 & 0.116 & 0.154 & 0.192 & 0.231 & 0.308 & 0.385\end{array}$

[G] $\quad \begin{array}{llllllllllll}0.039 & 0.038 & 0.077 & 0.115 & 0.153 & 0.192 & 0.269 & 0.346\end{array}$

[H] $\quad \begin{array}{lllllllllllll} & 0.077 & 0.038 & . & 0.039 & 0.077 & 0.115 & 0.154 & 0.231 & 0.308\end{array}$

[J] $\quad \begin{array}{llllllllllll}0.116 & 0.077 & 0.039 & 0.038 & 0.076 & 0.115 & 0.192 & 0.269\end{array}$

[K] $\quad \begin{array}{lllllllllll}0.154 & 0.115 & 0.077 & 0.038 & . & 0.038 & 0.077 & 0.154 & 0.231\end{array}$

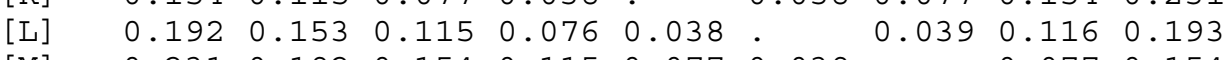

[M] $\quad \begin{array}{llllllllllll}0.231 & 0.192 & 0.154 & 0.115 & 0.077 & 0.039 & . & 0.077 & 0.154\end{array}$

[N] $\quad \begin{array}{lllllllllll}0.308 & 0.269 & 0.231 & 0.192 & 0.154 & 0.116 & 0.077 & 0.077\end{array}$

$\begin{array}{lllllllllll}{[P]} & 0.385 & 0.346 & 0.308 & 0.269 & 0.231 & 0.193 & 0.154 & 0.077\end{array}$. 
USERTYPE meanringleistthickness STEPMATRIX = 26

\begin{tabular}{|c|c|c|c|c|c|c|c|c|c|c|c|c|c|c|c|}
\hline & 0 & 1 & 2 & 3 & 4 & 5 & 6 & 7 & 8 & 9 & A & B & $\mathrm{C}$ & $\mathrm{D}$ & $\mathrm{E}$ \\
\hline$[0]$ & . & 0.041 & 0.083 & 0.104 & 0.124 & 0.166 & 0.187 & 0.228 & 0.249 & 0.27 & 0.291 & 0.291 & 0.312 & 0.333 & 0.353 \\
\hline [1] & 0.041 & . & 0.042 & 0.063 & 0.083 & 0.125 & 0.146 & 0.187 & 0.208 & 0.229 & 0.25 & 0.25 & 0.271 & 0.292 & 0.312 \\
\hline [2] & 0.083 & 0.042 & 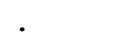 & 0.021 & 0.041 & 0.083 & 0.104 & 0.145 & 0.166 & 0.187 & 0.208 & 0.208 & 0.229 & 0.25 & 0.27 \\
\hline [3] & 0.104 & 0.063 & 0.021 & . & 0.02 & 0.062 & 0.083 & 0.124 & 0.145 & 0.166 & 0.187 & 0.187 & 0.208 & 0.229 & 0.249 \\
\hline$[4$ & 0.124 & 0.083 & 0.041 & 0.02 & . & 0.042 & 0.063 & 0.104 & 0.125 & 0.146 & 0.167 & 0.167 & 0.188 & 0.209 & 0.229 \\
\hline$[0$ & 0.166 & 0.125 & 0.083 & 0.062 & 0.042 & & 0.021 & 0.062 & 0.083 & 0.104 & 0.125 & 0.125 & 0.146 & 0.167 & 0.187 \\
\hline$[6]$ & 0.187 & 0.146 & 0.104 & 0.083 & 0.063 & 0.021 & . & 0.041 & 0.062 & 0.083 & 0.104 & 0.104 & 0.125 & 0.146 & 0.166 \\
\hline$[7]$ & 0.228 & 0.187 & 0.145 & 0.124 & 0.104 & 0.062 & 0.041 & . & 0.021 & 0.042 & 0.063 & 0.063 & 0.084 & 0.105 & 0.125 \\
\hline$[8$ & 0.249 & 0.208 & 0.166 & 0.145 & 0.125 & 0.083 & 0.062 & 0.021 & & 0.021 & 0.042 & 0.042 & 0.063 & 0.084 & 0.104 \\
\hline 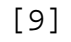 & 0.27 & 0.229 & 0.187 & 0.166 & 0.146 & 0.104 & 0.083 & 0.042 & 0.021 & & 0.021 & 0.021 & 0.042 & 0.063 & 0.083 \\
\hline$[\mathrm{A}$ & 0.291 & 0.25 & 0.208 & 0.187 & 0.167 & 0.125 & 0.104 & 0.063 & 0.042 & 0.021 & . & 0 & 0.021 & 0.042 & 0.062 \\
\hline [B & 0.291 & 0.25 & 0.208 & 0.187 & 0.167 & 0.125 & 0.104 & 0.063 & 0.042 & 0.021 & 0 & . & 0.021 & 0.042 & 0.062 \\
\hline$[\mathrm{C}$ & 0.312 & 0.271 & 0.229 & 0.208 & 0.188 & 0.146 & 0.125 & 0.084 & 0.063 & 0.042 & 0.021 & 0.021 & & 0.021 & 0.041 \\
\hline$[\mathrm{D}$ & 0.333 & 0.292 & 0.25 & 0.229 & 0.209 & 0.167 & 0.146 & 0.105 & 0.084 & 0.063 & 0.042 & 0.042 & 0.021 & & 0.02 \\
\hline$[E]$ & 0.353 & 0.312 & 0.27 & 0.249 & 0.229 & 0.187 & 0.166 & 0.125 & 0.104 & 0.083 & 0.062 & 0.062 & 0.041 & 0.02 & \\
\hline$[F]$ & 0.374 & 0.333 & 0.291 & 0.27 & 0.25 & 0.208 & 0.187 & 0.146 & 0.125 & 0.104 & 0.083 & 0.083 & 0.062 & 0.041 & 0.021 \\
\hline$[\mathrm{G}]$ & 0.437 & 0.396 & 0.354 & 0.333 & 0.313 & 0.271 & 0.25 & 0.209 & 0.188 & 0.167 & 0.146 & 0.146 & 0.125 & 0.104 & 0.084 \\
\hline$[\mathrm{H}$ & 0.457 & 0.416 & 0.374 & 0.353 & 0.333 & 0.291 & 0.27 & 0.229 & 0.208 & 0.187 & 0.166 & 0.166 & 0.145 & 0.124 & 0.104 \\
\hline$[\mathrm{J}$ & 0.478 & 0.437 & 0.395 & 0.374 & 0.354 & 0.312 & 0.291 & 0.25 & 0.229 & 0.208 & 0.187 & 0.187 & 0.166 & 0.145 & 0.125 \\
\hline 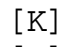 & 0.52 & 0.479 & 0.437 & 0.416 & 0.396 & 0.354 & 0.333 & 0.292 & 0.271 & 0.25 & 0.229 & 0.229 & 0.208 & 0.187 & 0.167 \\
\hline [L & 0.541 & 0.5 & 0.458 & 0.437 & 0.417 & 0.375 & 0.354 & 0.313 & 0.292 & 0.271 & 0.25 & 0.25 & 0.229 & 0.208 & 0.188 \\
\hline$[\mathrm{M}$ & 0.666 & 0.625 & 0.583 & 0.562 & 0.542 & 0.5 & 0.479 & 0.438 & 0.417 & 0.396 & 0.375 & 0.375 & 0.354 & 0.333 & 0.313 \\
\hline$[\mathrm{N}]$ & 0.707 & 0.666 & 0.624 & 0.603 & 0.583 & 0.541 & 0.52 & 0.479 & 0.458 & 0.437 & 0.416 & 0.416 & 0.395 & 0.374 & 0.354 \\
\hline$[\mathrm{P}]$ & 0.77 & 0.729 & 0.687 & 0.666 & 0.646 & 0.604 & 0.583 & 0.542 & 0.521 & 0.5 & 0.479 & 0.479 & 0.458 & 0.437 & 0.417 \\
\hline$[\mathrm{Q}$ & 0.936 & 0.895 & 0.853 & 0.832 & 0.812 & 0.77 & 0.749 & 0.708 & 0.687 & 0.666 & 0.645 & 0.645 & 0.624 & 0.603 & 0.583 \\
\hline D & 0.999 & 0.958 & 0.916 & 0.895 & 0.875 & 0.833 & 0.812 & 0.771 & 0.75 & 0.729 & 0.708 & 0.708 & 0.687 & 0.666 & 0.646 \\
\hline
\end{tabular}


$[0]$

$[1]$

$\begin{array}{lllllllllll}0.374 & 0.437 & 0.457 & 0.478 & 0.52 & 0.541 & 0.666 & 0.707 & 0.77 & 0.936 & 0.999\end{array}$ $\begin{array}{lllllllllll}0.333 & 0.396 & 0.416 & 0.437 & 0.479 & 0.5 & 0.625 & 0.666 & 0.729 & 0.895 & 0.958\end{array}$ $\begin{array}{llllllllllll}0.291 & 0.354 & 0.374 & 0.395 & 0.437 & 0.458 & 0.583 & 0.624 & 0.687 & 0.853 & 0.916\end{array}$ $\begin{array}{lllllllllll}0.27 & 0.333 & 0.353 & 0.374 & 0.416 & 0.437 & 0.562 & 0.603 & 0.666 & 0.832 & 0.895\end{array}$ $\begin{array}{llllllllllll}0.25 & 0.313 & 0.333 & 0.354 & 0.396 & 0.417 & 0.542 & 0.583 & 0.646 & 0.812 & 0.875\end{array}$ $\begin{array}{lllllllllll}0.208 & 0.271 & 0.291 & 0.312 & 0.354 & 0.375 & 0.5 & 0.541 & 0.604 & 0.77 & 0.833\end{array}$ $\begin{array}{lllllllllll}0.187 & 0.25 & 0.27 & 0.291 & 0.333 & 0.354 & 0.479 & 0.52 & 0.583 & 0.749 & 0.812\end{array}$ $\begin{array}{llllllllllll}0.146 & 0.209 & 0.229 & 0.25 & 0.292 & 0.313 & 0.438 & 0.479 & 0.542 & 0.708 & 0.771\end{array}$ $\begin{array}{llllllllllll}0.125 & 0.188 & 0.208 & 0.229 & 0.271 & 0.292 & 0.417 & 0.458 & 0.521 & 0.687 & 0.75\end{array}$ $\begin{array}{lllllllllll}0.104 & 0.167 & 0.187 & 0.208 & 0.25 & 0.271 & 0.396 & 0.437 & 0.5 & 0.666 & 0.729\end{array}$ $\begin{array}{lllllllllll}0.083 & 0.146 & 0.166 & 0.187 & 0.229 & 0.25 & 0.375 & 0.416 & 0.479 & 0.645 & 0.708\end{array}$ $\begin{array}{lllllllllll}0.083 & 0.146 & 0.166 & 0.187 & 0.229 & 0.25 & 0.375 & 0.416 & 0.479 & 0.645 & 0.708\end{array}$ $\begin{array}{llllllllllll}0.062 & 0.125 & 0.145 & 0.166 & 0.208 & 0.229 & 0.354 & 0.395 & 0.458 & 0.624 & 0.687\end{array}$ $\begin{array}{lllllllllllll}0.041 & 0.104 & 0.124 & 0.145 & 0.187 & 0.208 & 0.333 & 0.374 & 0.437 & 0.603 & 0.666\end{array}$ $\begin{array}{llllllllllll}0.021 & 0.084 & 0.104 & 0.125 & 0.167 & 0.188 & 0.313 & 0.354 & 0.417 & 0.583 & 0.646\end{array}$ $\begin{array}{lllllllllllll}. & 0.063 & 0.083 & 0.104 & 0.146 & 0.167 & 0.292 & 0.333 & 0.396 & 0.562 & 0.625\end{array}$ $\begin{array}{llllllllllll}0.063 & 0.0 .04 & 0.041 & 0.083 & 0.104 & 0.229 & 0.27 & 0.333 & 0.499 & 0.562\end{array}$ $\begin{array}{llllllllllll}0.083 & 0.02 & . & 0.021 & 0.063 & 0.084 & 0.209 & 0.25 & 0.313 & 0.479 & 0.542\end{array}$ $\begin{array}{llllllllllllll}0.104 & 0.041 & 0.021 & 0.0 .042 & 0.042 & 0.063 & 0.188 & 0.229 & 0.292 & 0.458 & 0.521\end{array}$ $\begin{array}{llllllllllll}0.146 & 0.083 & 0.063 & 0.042 & 0.0 .021 & 0.146 & 0.187 & 0.25 & 0.416 & 0.479\end{array}$ $\begin{array}{lllllllllllllll}0.167 & 0.104 & 0.084 & 0.063 & 0.021 & & 0.125 & 0.166 & 0.229 & 0.395 & 0.458\end{array}$ $\begin{array}{lllllllllllll}0.292 & 0.229 & 0.209 & 0.188 & 0.146 & 0.125 & 0.041 & 0.104 & 0.27 & 0.333\end{array}$ $\begin{array}{lllllllllll}0.333 & 0.27 & 0.25 & 0.229 & 0.187 & 0.166 & 0.041 & 0.063 & 0.229 & 0.292\end{array}$ $\begin{array}{lllllllllll}0.396 & 0.333 & 0.313 & 0.292 & 0.25 & 0.229 & 0.104 & 0.063 & 0.166 & 0.229\end{array}$ $\begin{array}{llllllllll}0.562 & 0.499 & 0.479 & 0.458 & 0.416 & 0.395 & 0.27 & 0.229 & 0.166\end{array}$. $\begin{array}{lllllllllll}0.625 & 0.562 & 0.542 & 0.521 & 0.479 & 0.458 & 0.333 & 0.292 & 0.229 & 0.063\end{array}$. 
USERTYPE paringleistthickness STEPMATRIX $=21$

\begin{tabular}{|c|c|c|c|c|c|c|c|c|c|c|c|c|c|c|c|}
\hline & & & 2 & 3 & & 5 & 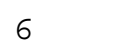 & 7 & 8 & & $\mathrm{~A}$ & B & C & $\mathrm{D}$ & $\mathrm{E}$ \\
\hline & & 3 & 6 & 9 & 92 & & & 2 & & 9 & 2 & 55 & 78 & 02 & \\
\hline & .023 & & & & & & & & & 86 & & & 0.255 & & \\
\hline & 0.046 & .023 & & & .046 & 70 & & 6 & 39 & & & & 0.232 & 56 & \\
\hline & & .046 & 023 & & & $0 \quad 047$ & & & & & & & & & \\
\hline & 0.092 & .069 & 0.046 & 0.023 & & .024 & & & & & & & 86 & & 233 \\
\hline & 6 & & & & 0.024 & & & & & & & & & & \\
\hline & 9 & 6 & & 0 & 0 & 0.023 & & & & & & & & & \\
\hline & 2 & & & & & 0.046 & & & & & & & & & \\
\hline & 5 & 2 & & & 93 & 0. & & 23 & & & & & & & \\
\hline & & & & & & & & & & & & & & & \\
\hline & .232 & 209 & 36 & .163 & 40 & 0.116 & 3 & 70 & 0. & 23 & & 23 & 46 & & 93 \\
\hline & & & & & & & & & & & & & 23 & & \\
\hline & 278 & 2 & & 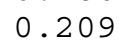 & 86 & 0 . & & & & 69 & 0. & & & .024 & \\
\hline & & & & & & & & & & & & & & & 23 \\
\hline & 5 & 2 & & & & & & & & & & & 47 & & \\
\hline & & & & & & & & & & & & & & & \\
\hline & & & & & & & & & & & & & & & \\
\hline & & 5 & & & & & & & & & & & & & \\
\hline & & & & & & & & & & & & & & & \\
\hline & .789 & .766 & & 0 & & & & & 0 & 80 & 0. & 534 & 0.511 & 0.487 & \\
\hline & .999 & & & & & & .860 & 0.837 & & 790 & 0.767 & 0.744 & 0.721 & 0.697 & .6 \\
\hline
\end{tabular}


[0] $\quad \begin{array}{llllllll}0.371 & 0.394 & 0.418 & 0.487 & 0.789 & 0.999\end{array}$

[1] $\quad \begin{array}{llllllll}0.348 & 0.371 & 0.395 & 0.464 & 0.766 & 0.976\end{array}$

[2] $\quad \begin{array}{llllllll}0.325 & 0.348 & 0.372 & 0.441 & 0.743 & 0.953\end{array}$

[3] $\quad \begin{array}{llllllll}0.302 & 0.325 & 0.349 & 0.418 & 0.720 & 0.930\end{array}$

[4] $\quad \begin{array}{llllllll}0.279 & 0.302 & 0.326 & 0.395 & 0.697 & 0.907\end{array}$

[5] $\quad \begin{array}{llllllll}0.255 & 0.278 & 0.302 & 0.371 & 0.673 & 0.883\end{array}$

[6] $\quad \begin{array}{llllllll}0.232 & 0.255 & 0.279 & 0.348 & 0.650 & 0.860\end{array}$

[7] $\quad \begin{array}{llllllll}0.209 & 0.232 & 0.256 & 0.325 & 0.627 & 0.837\end{array}$

[8] $\quad \begin{array}{llllllll}0.186 & 0.209 & 0.233 & 0.302 & 0.604 & 0.814\end{array}$

[9] $\quad \begin{array}{llllllll}0.162 & 0.185 & 0.209 & 0.278 & 0.580 & 0.790\end{array}$

[A] $\quad \begin{array}{llllllll}0.139 & 0.162 & 0.186 & 0.255 & 0.557 & 0.767\end{array}$

[B] $\quad \begin{array}{llllllll}0.116 & 0.139 & 0.163 & 0.232 & 0.534 & 0.744\end{array}$

[C] $\quad \begin{array}{llllllll}0.093 & 0.116 & 0.140 & 0.209 & 0.511 & 0.721\end{array}$

[D] $\quad \begin{array}{llllllll}0.069 & 0.092 & 0.116 & 0.185 & 0.487 & 0.697\end{array}$

[E] $\quad \begin{array}{llllllll}0.046 & 0.069 & 0.093 & 0.162 & 0.464 & 0.674\end{array}$

[F] $\begin{array}{lllllll}0 & 0.023 & 0.047 & 0.116 & 0.418 & 0.628\end{array}$

[G] $\quad 0.023 .0 .024 \quad 0.093 \quad 0.395 \quad 0.605$

[H] $\quad \begin{array}{lllllllll}0.047 & 0.024 & . & 0.069 & 0.371 & 0.581\end{array}$

[J] $\quad 0.116 \quad 0.0930 .069 .0 .302 \quad 0.512$

[K] $\quad \begin{array}{llllllll}0.418 & 0.395 & 0.371 & 0.302 & . & 0.210\end{array}$

[L] $\quad \begin{array}{lllllll}0.628 & 0.605 & 0.581 & 0.512 & 0.210\end{array}$. 
USERTYPE meanard1 STEPMATRIX $=23$

\begin{tabular}{|c|c|c|c|c|c|c|c|c|c|c|c|c|c|c|c|}
\hline & 0 & 1 & 2 & 3 & 4 & 5 & 6 & 7 & 8 & 9 & A & B & $\mathrm{C}$ & $\mathrm{D}$ & $\mathrm{E}$ \\
\hline$[0]$ & . & 0.024 & 0.049 & 0.074 & 0.099 & 0.124 & 0.149 & 0.174 & 0.199 & 0.249 & 0.274 & 0.299 & 0.349 & 0.399 & 0.424 \\
\hline [1] & 0.024 & 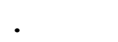 & 0.025 & 0.05 & 0.075 & 0.1 & 0.125 & 0.15 & 0.175 & 0.225 & 0.25 & 0.275 & 0.325 & 0.375 & 0.4 \\
\hline [2] & 0.049 & 0.025 & . & 0.025 & 0.05 & 0.075 & 0.1 & 0.125 & 0.15 & 0.2 & 0.225 & 0.25 & 0.3 & 0.35 & 0.375 \\
\hline [3] & 0.074 & 0.05 & 0.025 & . & 0.025 & 0.05 & 0.075 & 0.1 & 0.125 & 0.175 & 0.2 & 0.225 & 0.275 & 0.325 & 0.35 \\
\hline$[4]$ & 0.099 & 0.075 & 0.05 & 0.025 & . & 0.025 & 0.05 & 0.075 & 0.1 & 0.15 & 0.175 & 0.2 & 0.25 & 0.3 & 0.325 \\
\hline [5] & 0.124 & 0.1 & 0.075 & 0.05 & 0.025 & $\cdot$ & 0.025 & 0.05 & 0.075 & 0.125 & 0.15 & 0.175 & 0.225 & 0.275 & 0.3 \\
\hline [6] & 0.149 & 0.125 & 0.1 & 0.075 & 0.05 & 0.025 & . & 0.025 & 0.05 & 0.1 & 0.125 & 0.15 & 0.2 & 0.25 & 0.275 \\
\hline [7] & 0.174 & 0.15 & 0.125 & 0.1 & 0.075 & 0.05 & 0.025 & . & 0.025 & 0.075 & 0.1 & 0.125 & 0.175 & 0.225 & 0.25 \\
\hline [8] & 0.199 & 0.175 & 0.15 & 0.125 & 0.1 & 0.075 & 0.05 & 0.025 & - & 0.05 & 0.075 & 0.1 & 0.15 & 0.2 & 0.225 \\
\hline [9] & 0.249 & 0.225 & 0.2 & 0.175 & 0.15 & 0.125 & 0.1 & 0.075 & 0.05 & . & 0.025 & 0.05 & 0.1 & 0.15 & 0.175 \\
\hline$[\mathrm{A}]$ & 0.274 & 0.25 & 0.225 & 0.2 & 0.175 & 0.15 & 0.125 & 0.1 & 0.075 & 0.025 & & 0.025 & 0.075 & 0.125 & 0.15 \\
\hline [B] & 0.299 & 0.275 & 0.25 & 0.225 & 0.2 & 0.175 & 0.15 & 0.125 & 0.1 & 0.05 & 0.025 & . & 0.05 & 0.1 & 0.125 \\
\hline [C] & 0.349 & 0.325 & 0.3 & 0.275 & 0.25 & 0.225 & 0.2 & 0.175 & 0.15 & 0.1 & 0.075 & 0.05 & . & 0.05 & 0.075 \\
\hline [D] & 0.399 & 0.375 & 0.35 & 0.325 & 0.3 & 0.275 & 0.25 & 0.225 & 0.2 & 0.15 & 0.125 & 0.1 & 0.05 & . & 0.025 \\
\hline$[\mathrm{E}]$ & 0.424 & 0.4 & 0.375 & 0.35 & 0.325 & 0.3 & 0.275 & 0.25 & 0.225 & 0.175 & 0.15 & 0.125 & 0.075 & 0.025 & - \\
\hline$[\mathrm{F}]$ & 0.449 & 0.425 & 0.4 & 0.375 & 0.35 & 0.325 & 0.3 & 0.275 & 0.25 & 0.2 & 0.175 & 0.15 & 0.1 & 0.05 & 0.025 \\
\hline [G] & 0.524 & 0.5 & 0.475 & 0.45 & 0.425 & 0.4 & 0.375 & 0.35 & 0.325 & 0.275 & 0.25 & 0.225 & 0.175 & 0.125 & 0.1 \\
\hline$[\mathrm{H}]$ & 0.549 & 0.525 & 0.5 & 0.475 & 0.45 & 0.425 & 0.4 & 0.375 & 0.35 & 0.3 & 0.275 & 0.25 & 0.2 & 0.15 & 0.125 \\
\hline$[\mathrm{J}]$ & 0.574 & 0.55 & 0.525 & 0.5 & 0.475 & 0.45 & 0.425 & 0.4 & 0.375 & 0.325 & 0.3 & 0.275 & 0.225 & 0.175 & 0.15 \\
\hline [K] & 0.624 & 0.6 & 0.575 & 0.55 & 0.525 & 0.5 & 0.475 & 0.45 & 0.425 & 0.375 & 0.35 & 0.325 & 0.275 & 0.225 & 0.2 \\
\hline$[\mathrm{L}]$ & 0.674 & 0.65 & 0.625 & 0.6 & 0.575 & 0.55 & 0.525 & 0.5 & 0.475 & 0.425 & 0.4 & 0.375 & 0.325 & 0.275 & 0.25 \\
\hline$[\mathrm{M}]$ & 0.899 & 0.875 & 0.85 & 0.825 & 0.8 & 0.775 & 0.75 & 0.725 & 0.7 & 0.65 & 0.625 & 0.6 & 0.55 & 0.5 & 0.475 \\
\hline$[\mathrm{N}]$ & 0.999 & 0.975 & 0.95 & 0.925 & 0.9 & 0.875 & 0.85 & 0.825 & 0.8 & 0.75 & 0.725 & 0.7 & 0.65 & 0.6 & 0.575 \\
\hline
\end{tabular}


[0] $\quad \begin{array}{llllllllll}0.449 & 0.524 & 0.549 & 0.574 & 0.624 & 0.674 & 0.899 & 0.999\end{array}$

[1] $\begin{array}{lllllllll}0.425 & 0.5 & 0.525 & 0.55 & 0.6 & 0.65 & 0.875 & 0.975\end{array}$

$\left[\begin{array}{lllllllll}{[2]} & 0.4 & 0.475 & 0.5 & 0.525 & 0.575 & 0.625 & 0.85 & 0.95\end{array}\right.$

[3] $\quad \begin{array}{lllllllll}0.375 & 0.45 & 0.475 & 0.5 & 0.55 & 0.6 & 0.825 & 0.925\end{array}$

[4] $\quad \begin{array}{lllllllll}0.35 & 0.425 & 0.45 & 0.475 & 0.525 & 0.575 & 0.8 & 0.9\end{array}$

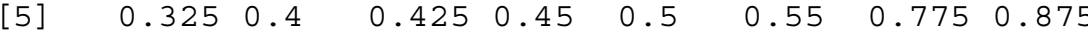

$\begin{array}{lllllllll}{[6]} & 0.3 & 0.375 & 0.4 & 0.425 & 0.475 & 0.525 & 0.75 & 0.85\end{array}$

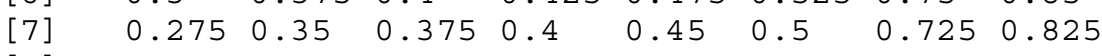

$\begin{array}{lllllllll}\text { [8] } & 0.25 & 0.325 & 0.35 & 0.375 & 0.425 & 0.475 & 0.7 & 0.8\end{array}$

$\begin{array}{lllllllll}9] & 0.2 & 0.275 & 0.3 & 0.325 & 0.375 & 0.425 & 0.65 & 0.75\end{array}$

[A] $\quad \begin{array}{llllllll}0.175 & 0.25 & 0.275 & 0.3 & 0.35 & 0.4 & 0.625 & 0.725\end{array}$

[B] $\quad \begin{array}{lllllllll}0.15 & 0.225 & 0.25 & 0.275 & 0.325 & 0.375 & 0.6 & 0.7\end{array}$

[C] $\begin{array}{lllllllll}0.1 & 0.175 & 0.2 & 0.225 & 0.275 & 0.325 & 0.55 & 0.65\end{array}$

[D] $\quad \begin{array}{llllllll}0.05 & 0.125 & 0.15 & 0.175 & 0.225 & 0.275 & 0.5 & 0.6\end{array}$

[E] $\quad \begin{array}{llllllll}0.025 & 0.1 & 0.125 & 0.15 & 0.2 & 0.25 & 0.475 & 0.575\end{array}$

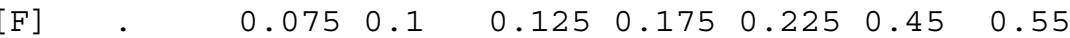

[G] $\quad \begin{array}{lllllllll}0.075 & . & 0.025 & 0.05 & 0.1 & 0.15 & 0.375 & 0.475\end{array}$

$\left[\begin{array}{lllllllll}{[\mathrm{H}]} & 0.1 & 0.025 & . & 0.025 & 0.075 & 0.125 & 0.35 & 0.45\end{array}\right.$

[J] $\quad \begin{array}{lllllllll} & 0.125 & 0.05 & 0.025 & 0.05 & 0.1 & 0.325 & 0.425\end{array}$

$\begin{array}{llllllll}{[\mathrm{K}]} & 0.175 & 0.1 & 0.075 & 0.05 & 0.05 & 0.275 & 0.375\end{array}$

[L] $\quad \begin{array}{lllllllll}0.225 & 0.15 & 0.125 & 0.1 & 0.05 & . & 0.225 & 0.325\end{array}$

[M] $\quad \begin{array}{lllllllll}0.45 & 0.375 & 0.35 & 0.325 & 0.275 & 0.225 & . & 0.1\end{array}$

[N] $\quad \begin{array}{llllllll}0.55 & 0.475 & 0.45 & 0.425 & 0.375 & 0.325 & 0.1\end{array}$. 
USERTYPE paardI STEPMATRIX $=22$

\begin{tabular}{|c|c|c|c|c|c|c|c|c|c|c|c|c|c|c|c|}
\hline & & 1 & 2 & 3 & 4 & 5 & 6 & & 8 & 9 & A & B & C & $\mathrm{D}$ & $E$ \\
\hline & & 3 & 6 & 8 & 3 & 5 & 0.37 & 2 & 7 & 19 & 1 & 56 & 68 & 81 & 0.493 \\
\hline & 283 & & & & & & & & & & & & & & \\
\hline & .296 & 013 & & .012 & 037 & .049 & 0.074 & .086 & .111 & 0.123 & 0.135 & 0.16 & 0.172 & 0.185 & .197 \\
\hline & 308 & 025 & 2 & & & & & & & & & & & & \\
\hline & 333 & 05 & .037 & 0.025 & & 0.012 & 037 & .049 & & 36 & .098 & 0.123 & & 48 & 16 \\
\hline & 345 & 062 & 049 & 0.037 & 2 & & & & & & & & & & \\
\hline & 37 & 087 & 74 & 062 & .037 & 0.025 & & & & & & & & & \\
\hline & 2 & 099 & & & & 0.037 & & & & & & & & & \\
\hline & & 124 & & & & & 0.037 & 25 & & & & & & & \\
\hline & & & & & & & & & & & & & & & \\
\hline & 431 & 148 & & 23 & & 86 & 0. & 49 & 0.024 & & & & & & \\
\hline & & 173 & & & & & & & & & & & & & \\
\hline & & 5 & & & & & & & 61 & & & & & & 25 \\
\hline & 1 & 98 & & & & & & & & & & & & & \\
\hline & & & & & & & & & & & & & & & \\
\hline & .505 & 0.222 & 9 & 0.197 & & & 5 & & & & & 9 & & & \\
\hline & .518 & 0235 & & & & & & & & & & & & & \\
\hline & .5 & 0.247 & 4 & 22 & 7 & & & & 23 & 11 & & & & 0.049 & .037 \\
\hline & & & & & & & & & & & & & & & \\
\hline & 592 & 9 & & & & 7 & 0 & & & 73 & 61 & & & 11 & .099 \\
\hline & & & & & & & & & & & & & & & \\
\hline & 999 & 16 & & & & & & & 92 & 0 & & 43 & 31 & 18 & 0.50 \\
\hline
\end{tabular}


[0] $\quad \begin{array}{lllllllll}0.505 & 0.518 & 0.53 & 0.555 & 0.592 & 0.875 & 0.999\end{array}$

[1] $\quad \begin{array}{lllllllll}0.222 & 0.235 & 0.247 & 0.272 & 0.309 & 0.592 & 0.716\end{array}$

$\begin{array}{lllllllll}\text { [2] } & 0.209 & 0.222 & 0.234 & 0.259 & 0.296 & 0.579 & 0.703\end{array}$

[3] $\quad \begin{array}{lllllllll}0.197 & 0.21 & 0.222 & 0.247 & 0.284 & 0.567 & 0.691\end{array}$

[4] $\quad \begin{array}{lllllllll}0.172 & 0.185 & 0.197 & 0.222 & 0.259 & 0.542 & 0.666\end{array}$

$\begin{array}{lllllllll}{[5]} & 0.16 & 0.173 & 0.185 & 0.21 & 0.247 & 0.53 & 0.654\end{array}$

[6] $\quad \begin{array}{llllllll}0.135 & 0.148 & 0.16 & 0.185 & 0.222 & 0.505 & 0.629\end{array}$

[7] $\quad \begin{array}{llllllll}0.123 & 0.136 & 0.148 & 0.173 & 0.21 & 0.493 & 0.617\end{array}$

[8] $\quad \begin{array}{lllllllll}0.098 & 0.111 & 0.123 & 0.148 & 0.185 & 0.468 & 0.592\end{array}$

[9] $\begin{array}{llllllllll}0.086 & 0.099 & 0.111 & 0.136 & 0.173 & 0.456 & 0.58\end{array}$

[A] $\quad \begin{array}{llllllllll}0.074 & 0.087 & 0.099 & 0.124 & 0.161 & 0.444 & 0.568\end{array}$

[B] $\quad \begin{array}{lllllllll}0.049 & 0.062 & 0.074 & 0.099 & 0.136 & 0.419 & 0.543\end{array}$

[C] $\quad \begin{array}{lllllllll}0.037 & 0.05 & 0.062 & 0.087 & 0.124 & 0.407 & 0.531\end{array}$

[D] $\quad \begin{array}{llllllllll}0.024 & 0.037 & 0.049 & 0.074 & 0.111 & 0.394 & 0.518\end{array}$

[E] $\quad \begin{array}{lllllllll}0.012 & 0.025 & 0.037 & 0.062 & 0.099 & 0.382 & 0.506\end{array}$

F] $\begin{array}{llllllll}0.013 & 0.025 & 0.05 & 0.087 & 0.37 & 0.494\end{array}$

[G] $\quad \begin{array}{llllllll}0.013 & 0.012 & 0.037 & 0.074 & 0.357 & 0.481\end{array}$

$\begin{array}{llllllllll}\text { [H] } & 0.025 & 0.012 & . & 0.025 & 0.062 & 0.345 & 0.469\end{array}$

[J] $\quad \begin{array}{lllllllll}0.05 & 0.037 & 0.025 & 0.037 & 0.037 & 0.32 & 0.444\end{array}$

$\begin{array}{lllllllll}{[\mathrm{K}]} & 0.087 & 0.074 & 0.062 & 0.037 & 0.283 & 0.407\end{array}$

$\begin{array}{lllllllll}\text { [L] } & 0.37 & 0.357 & 0.345 & 0.32 & 0.283 & . & 0.124\end{array}$

[M] $\quad \begin{array}{llllllllll}0.494 & 0.481 & 0.469 & 0.444 & 0.407 & 0.124\end{array}$ 
USERTYPE meanisd STEPMATRIX $=23$

\begin{tabular}{|c|c|c|c|c|c|c|c|c|c|c|c|c|c|c|c|}
\hline & 0 & 1 & 2 & 3 & 4 & 5 & 6 & 7 & 8 & 9 & A & B & $\mathrm{C}$ & D & $E$ \\
\hline$[0]$ & & 0.034 & 0.068 & 0.103 & 0.137 & 0.172 & 0.206 & 0.241 & 0.275 & 0.31 & 0.344 & 0.378 & 0.413 & 0.447 & 0.516 \\
\hline [1] & 0.034 & . & 0.034 & 0.069 & 0.103 & 0.138 & 0.172 & 0.207 & 0.241 & 0.276 & 0.31 & 0.344 & 0.379 & 0.413 & 0.482 \\
\hline [2] & 0.068 & 0.034 & 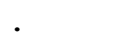 & 0.035 & 0.069 & 0.104 & 0.138 & 0.173 & 0.207 & 0.242 & 0.276 & 0.31 & 0.345 & 0.379 & 0.448 \\
\hline [3] & 0.103 & 0.069 & 0.035 & . & 0.034 & 0.069 & 0.103 & 0.138 & 0.172 & 0.207 & 0.241 & 0.275 & 0.31 & 0.344 & 0.413 \\
\hline [4] & 0.137 & 0.103 & 0.069 & 0.034 & . & 0.035 & 0.069 & 0.104 & 0.138 & 0.173 & 0.207 & 0.241 & 0.276 & 0.31 & 0.379 \\
\hline [5] & 0.172 & 0.138 & 0.104 & 0.069 & 0.035 & . & 0.034 & 0.069 & 0.103 & 0.138 & 0.172 & 0.206 & 0.241 & 0.275 & 0.344 \\
\hline [6] & 0.206 & 0.172 & 0.138 & 0.103 & 0.069 & 0.034 & 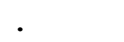 & 0.035 & 0.069 & 0.104 & 0.138 & 0.172 & 0.207 & 0.241 & 0.31 \\
\hline [7] & 0.241 & 0.207 & 0.173 & 0.138 & 0.104 & 0.069 & 0.035 & - & 0.034 & 0.069 & 0.103 & 0.137 & 0.172 & 0.206 & 0.275 \\
\hline [8] & 0.275 & 0.241 & 0.207 & 0.172 & 0.138 & 0.103 & 0.069 & 0.034 & 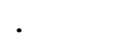 & 0.035 & 0.069 & 0.103 & 0.138 & 0.172 & 0.241 \\
\hline [9] & 0.31 & 0.276 & 0.242 & 0.207 & 0.173 & 0.138 & 0.104 & 0.069 & 0.035 & . & 0.034 & 0.068 & 0.103 & 0.137 & 0.206 \\
\hline$[\mathrm{A}]$ & 0.344 & 0.31 & 0.276 & 0.241 & 0.207 & 0.172 & 0.138 & 0.103 & 0.069 & 0.034 & . & 0.034 & 0.069 & 0.103 & 0.172 \\
\hline [B] & 0.378 & 0.344 & 0.31 & 0.275 & 0.241 & 0.206 & 0.172 & 0.137 & 0.103 & 0.068 & 0.034 & & 0.035 & 0.069 & 0.138 \\
\hline$[\mathrm{C}]$ & 0.413 & 0.379 & 0.345 & 0.31 & 0.276 & 0.241 & 0.207 & 0.172 & 0.138 & 0.103 & 0.069 & 0.035 & . & 0.034 & 0.103 \\
\hline [D] & 0.447 & 0.413 & 0.379 & 0.344 & 0.31 & 0.275 & 0.241 & 0.206 & 0.172 & 0.137 & 0.103 & 0.069 & 0.034 & . & 0.069 \\
\hline$[\mathrm{E}]$ & 0.516 & 0.482 & 0.448 & 0.413 & 0.379 & 0.344 & 0.31 & 0.275 & 0.241 & 0.206 & 0.172 & 0.138 & 0.103 & 0.069 & \\
\hline$[F]$ & 0.551 & 0.517 & 0.483 & 0.448 & 0.414 & 0.379 & 0.345 & 0.31 & 0.276 & 0.241 & 0.207 & 0.173 & 0.138 & 0.104 & 0.035 \\
\hline [G] & 0.585 & 0.551 & 0.517 & 0.482 & 0.448 & 0.413 & 0.379 & 0.344 & 0.31 & 0.275 & 0.241 & 0.207 & 0.172 & 0.138 & 0.069 \\
\hline$[\mathrm{H}]$ & 0.62 & 0.586 & 0.552 & 0.517 & 0.483 & 0.448 & 0.414 & 0.379 & 0.345 & 0.31 & 0.276 & 0.242 & 0.207 & 0.173 & 0.104 \\
\hline$[\mathrm{J}]$ & 0.654 & 0.62 & 0.586 & 0.551 & 0.517 & 0.482 & 0.448 & 0.413 & 0.379 & 0.344 & 0.31 & 0.276 & 0.241 & 0.207 & 0.138 \\
\hline [K] & 0.688 & 0.654 & 0.62 & 0.585 & 0.551 & 0.516 & 0.482 & 0.447 & 0.413 & 0.378 & 0.344 & 0.31 & 0.275 & 0.241 & 0.172 \\
\hline$[\mathrm{L}]$ & 0.792 & 0.758 & 0.724 & 0.689 & 0.655 & 0.62 & 0.586 & 0.551 & 0.517 & 0.482 & 0.448 & 0.414 & 0.379 & 0.345 & 0.276 \\
\hline$[\mathrm{M}]$ & 0.861 & 0.827 & 0.793 & 0.758 & 0.724 & 0.689 & 0.655 & 0.62 & 0.586 & 0.551 & 0.517 & 0.483 & 0.448 & 0.414 & 0.345 \\
\hline$[\mathrm{N}]$ & 0.999 & 0.965 & 0.931 & 0.896 & 0.862 & 0.827 & 0.793 & 0.758 & 0.724 & 0.689 & 0.655 & 0.621 & 0.586 & 0.552 & 0.483 \\
\hline
\end{tabular}


[0] $\quad \begin{array}{lllllllll}0.551 & 0.585 & 0.62 & 0.654 & 0.688 & 0.792 & 0.861 & 0.999\end{array}$

[1] $\quad \begin{array}{llllllllll}0.517 & 0.551 & 0.586 & 0.62 & 0.654 & 0.758 & 0.827 & 0.965\end{array}$

[2] $\quad \begin{array}{llllllllll}0.483 & 0.517 & 0.552 & 0.586 & 0.62 & 0.724 & 0.793 & 0.931\end{array}$

[3] $\quad \begin{array}{lllllllllll}0.448 & 0.482 & 0.517 & 0.551 & 0.585 & 0.689 & 0.758 & 0.896\end{array}$

[4] $\quad \begin{array}{llllllllll}0.414 & 0.448 & 0.483 & 0.517 & 0.551 & 0.655 & 0.724 & 0.862\end{array}$

[5] $\quad \begin{array}{llllllllll}0.379 & 0.413 & 0.448 & 0.482 & 0.516 & 0.62 & 0.689 & 0.827\end{array}$

[6] $\quad \begin{array}{llllllllll}0.345 & 0.379 & 0.414 & 0.448 & 0.482 & 0.586 & 0.655 & 0.793\end{array}$

[7] $\begin{array}{llllllllll}0.31 & 0.344 & 0.379 & 0.413 & 0.447 & 0.551 & 0.62 & 0.758\end{array}$

[8] $\quad \begin{array}{llllllllll}0.276 & 0.31 & 0.345 & 0.379 & 0.413 & 0.517 & 0.586 & 0.724\end{array}$

[9] $\quad \begin{array}{lllllllllll}0.241 & 0.275 & 0.31 & 0.344 & 0.378 & 0.482 & 0.551 & 0.689\end{array}$

[A] $\quad \begin{array}{llllllllll}0.207 & 0.241 & 0.276 & 0.31 & 0.344 & 0.448 & 0.517 & 0.655\end{array}$

[B] $\quad \begin{array}{llllllllll}0.173 & 0.207 & 0.242 & 0.276 & 0.31 & 0.414 & 0.483 & 0.621\end{array}$

[C] $\quad \begin{array}{lllllllllll}0.138 & 0.172 & 0.207 & 0.241 & 0.275 & 0.379 & 0.448 & 0.586\end{array}$

[D] $\quad \begin{array}{lllllllllll}0.104 & 0.138 & 0.173 & 0.207 & 0.241 & 0.345 & 0.414 & 0.552\end{array}$

[E] $\quad \begin{array}{llllllllll}0.035 & 0.069 & 0.104 & 0.138 & 0.172 & 0.276 & 0.345 & 0.483\end{array}$

[F] $\quad \begin{array}{llllllllll}0 & 0.034 & 0.069 & 0.103 & 0.137 & 0.241 & 0.31 & 0.448\end{array}$

[G] $\quad \begin{array}{llllllllll}0.034 & . & 0.035 & 0.069 & 0.103 & 0.207 & 0.276 & 0.414\end{array}$

[H] $\quad \begin{array}{llllllllllll}0.069 & 0.035 & . & 0.034 & 0.068 & 0.172 & 0.241 & 0.379\end{array}$

[J] $\quad \begin{array}{llllllllll}0.103 & 0.069 & 0.034 & 0.034 & 0.034 & 0.138 & 0.207 & 0.345\end{array}$

[K] $\quad \begin{array}{lllllllll}0.137 & 0.103 & 0.068 & 0.034 & 0.104 & 0.173 & 0.311\end{array}$

[L] $\quad \begin{array}{llllllllll}0.241 & 0.207 & 0.172 & 0.138 & 0.104 & . & 0.069 & 0.207\end{array}$

[M] $\quad \begin{array}{llllllllll}0.31 & 0.276 & 0.241 & 0.207 & 0.173 & 0.069 & . & 0.138\end{array}$

[N] $\quad \begin{array}{llllllllll}0.448 & 0.414 & 0.379 & 0.345 & 0.311 & 0.207 & 0.138\end{array}$. 
USERTYPE paisd STEPMATRIX $=21$

\begin{tabular}{|c|c|c|c|c|c|c|c|c|c|c|c|c|c|c|c|}
\hline & & 1 & & 3 & 4 & & 6 & & 8 & & A & B & C & D & $\mathrm{E}$ \\
\hline & & 0 & 33 & 1 & 8 & 66 & & 2 & 49 & 77 & 0. & 0.333 & 60 & 0.388 & ( \\
\hline & 055 & & & & & & & & & & & & & & \\
\hline & .083 & .028 & & 28 & & & & & & & & & & & \\
\hline & 11 & 056 & 028 & & & & & & & & & & & & \\
\hline & 138 & 083 & .055 & 0.027 & & & & & & & & & & & \\
\hline & 56 & & 83 & & & & & & & & & & & & \\
\hline & & & & & & 28 & & & & & & & & & \\
\hline & 22 & 7 & 9 & 1 & & & & & & & & & & & \\
\hline & 249 & & & & & & & & & & & & & & \\
\hline & & & & & & & & & & & & & & & \\
\hline & & & & & & & & & & & & & & & \\
\hline & .333 & 78 & 0 & 2 & & 57 & & 11 & & 56 & 8 & & 27 & & \\
\hline & 360 & & & & & & & & & & & & & 28 & 56 \\
\hline & .388 & 0.333 & 305 & 0.277 & 250 & 0.222 & 94 & .166 & 39 & 0.111 & 83 & 55 & 0.028 & & 0.028 \\
\hline & .416 & & & & & & & & & & & & & & \\
\hline & .471 & 0.416 & 388 & 0.360 & 333 & 0.305 & 77 & 249 & 22 & 0.194 & 66 & 38 & 11 & 083 & 0.055 \\
\hline & 27 & & & & & & & & & & & & & & \\
\hline & 10 & & & & & & & & & & & & & & \\
\hline & & & & & & & & & & & & & & & \\
\hline & 71 & & & & & & & & & & & & & & \\
\hline & .999 & 944 & 10 & .000 & 501 & בכם & 0.805 & . & 0.750 & 0.722 & 0.694 & 0.666 & 0.639 & 0.611 & $.00=$ \\
\hline
\end{tabular}




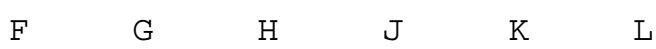

[0] $\quad \begin{array}{llllllll}0.471 & 0.527 & 0.610 & 0.749 & 0.971 & 0.999\end{array}$

[1] $\quad \begin{array}{llllllll}0.416 & 0.472 & 0.555 & 0.694 & 0.916 & 0.944\end{array}$

$\left[\begin{array}{lllllll}{[2]} & 0.388 & 0.444 & 0.527 & 0.666 & 0.888 & 0.916\end{array}\right.$

[3] $\quad \begin{array}{llllllll}0.360 & 0.416 & 0.499 & 0.638 & 0.860 & 0.888\end{array}$

[4] $\quad \begin{array}{llllllll}0.333 & 0.389 & 0.472 & 0.611 & 0.833 & 0.861\end{array}$

[5] $\quad \begin{array}{llllllll}0.305 & 0.361 & 0.444 & 0.583 & 0.805 & 0.833\end{array}$

[6] $\quad \begin{array}{llllllll}0.277 & 0.333 & 0.416 & 0.555 & 0.777 & 0.805\end{array}$

[7] $\quad \begin{array}{llllllll}0.249 & 0.305 & 0.388 & 0.527 & 0.749 & 0.777\end{array}$

[8] $\quad \begin{array}{llllllll}0.222 & 0.278 & 0.361 & 0.500 & 0.722 & 0.750\end{array}$

[9] $\quad \begin{array}{llllllll}0.194 & 0.250 & 0.333 & 0.472 & 0.694 & 0.722\end{array}$

[A] $\quad \begin{array}{llllllll}0.166 & 0.222 & 0.305 & 0.444 & 0.666 & 0.694\end{array}$

[B] $\quad \begin{array}{llllllll}0.138 & 0.194 & 0.277 & 0.416 & 0.638 & 0.666\end{array}$

[C] $\quad \begin{array}{llllllll}0.111 & 0.167 & 0.250 & 0.389 & 0.611 & 0.639\end{array}$

[D] $\quad \begin{array}{llllllll}0.083 & 0.139 & 0.222 & 0.361 & 0.583 & 0.611\end{array}$

[E] $\quad \begin{array}{llllllll}0.055 & 0.111 & 0.194 & 0.333 & 0.555 & 0.583\end{array}$

[F] $\quad \begin{array}{lllllll}. & 0.056 & 0.139 & 0.278 & 0.500 & 0.528\end{array}$

[G] $\quad 0.056 .0 .083 \quad 0.222 \quad 0.444 \quad 0.472$

[H] $\quad \begin{array}{llllllll}0.139 & 0.083 & . & 0.139 & 0.361 & 0.389\end{array}$

[J] $\quad 0.278 \quad 0.2220 .139 \quad 0.2220 .250$

[K] $\quad \begin{array}{lllllllll}0.500 & 0.444 & 0.361 & 0.222 & . & 0.028\end{array}$

[L] $\quad \begin{array}{lllllll}0.528 & 0.472 & 0.389 & 0.250 & 0.028\end{array}$. 
USERTYPE meanthetalnk STEPMATRIX $=22$

\begin{tabular}{|c|c|c|c|c|c|c|c|c|c|c|c|c|c|c|c|}
\hline & & 1 & 2 & 3 & 4 & 5 & 6 & & 8 & 9 & $\mathrm{~A}$ & B & C & $\mathrm{D}$ & $\mathrm{E}$ \\
\hline 0] & & .131 & 7 & 4 & .236 & 2 & 9 & 8 & 4 & 6 & 5 & 52 & 30 & 57 & 0.683 \\
\hline & 131 & & & & & & & & & & & & & & \\
\hline & .157 & 026 & & .027 & 079 & .105 & 0.132 & 211 & 237 & 89 & 0.368 & 0.395 & 0.473 & 0.500 & 526 \\
\hline & 184 & 053 & 7 & & & 078 & & & & & & & & & \\
\hline & 36 & 105 & 079 & 0.052 & & 0.026 & 53 & 32 & 58 & 10 & & 316 & 94 & 21 & \\
\hline & 62 & 131 & & & 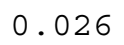 & & & & & & & & & & \\
\hline & 9 & 158 & & & .053 & 0.027 & & & & & & & & & \\
\hline & 8 & 7 & & & & 0.106 & & & & & & & & & \\
\hline & 4 & 263 & & & 8 & 0.132 & 0.105 & 6 & & & & & & & \\
\hline & 6 & & & & & & & & 0.052 & & & & & & \\
\hline & 525 & 394 & & 0.341 & & 0.263 & 0.236 & .157 & 0.131 & & & & & & \\
\hline & & & & & & & & & & & & & & & \\
\hline & 30 & 9 & & & & 58 & & & 36 & & & & & 27 & \\
\hline & 7 & 6 & & & & & & & & & & & & & \\
\hline & & & & & & & & & & & & & & & \\
\hline & .709 & 578 & 2 & 0.5 & & 7 & 0. & 1 & & & & & & & \\
\hline & 736 & 0.605 & & & & & & & & & & & & & \\
\hline & .762 & 0.631 & 5 & 78 & & 0 & & 4 & & 16 & 37 & 10 & & 05 & 079 \\
\hline & & & & & & & & & & & & & & & \\
\hline & 67 & 6 & & & & 5 & & & 73 & & & & 37 & 210 & .184 \\
\hline & & & & & & & & & & & & & & & \\
\hline & 19 & & & & & & & & & & 74 & 47 & 69 & 342 & 0.316 \\
\hline
\end{tabular}


[0] $\quad \begin{array}{lllllllll}0.709 & 0.736 & 0.762 & 0.788 & 0.867 & 0.893 & 0.999\end{array}$

[1] $\quad \begin{array}{lllllllll}0.578 & 0.605 & 0.631 & 0.657 & 0.736 & 0.762 & 0.868\end{array}$

[2] $\quad \begin{array}{lllllllll}0.552 & 0.579 & 0.605 & 0.631 & 0.710 & 0.736 & 0.842\end{array}$

[3] $\quad \begin{array}{lllllllll}0.525 & 0.552 & 0.578 & 0.604 & 0.683 & 0.709 & 0.815\end{array}$

[4] $\quad \begin{array}{lllllllll}0.473 & 0.500 & 0.526 & 0.552 & 0.631 & 0.657 & 0.763\end{array}$

$\begin{array}{llllllllll}{[5]} & 0.447 & 0.474 & 0.500 & 0.526 & 0.605 & 0.631 & 0.737\end{array}$

[6] $\quad \begin{array}{lllllllll}0.420 & 0.447 & 0.473 & 0.499 & 0.578 & 0.604 & 0.710\end{array}$

[7] $\quad \begin{array}{lllllllll}0.341 & 0.368 & 0.394 & 0.420 & 0.499 & 0.525 & 0.631\end{array}$

[8] $\quad \begin{array}{lllllllll}0.315 & 0.342 & 0.368 & 0.394 & 0.473 & 0.499 & 0.605\end{array}$

[9] $\quad \begin{array}{lllllllll}0.263 & 0.290 & 0.316 & 0.342 & 0.421 & 0.447 & 0.553\end{array}$

[A] $\quad \begin{array}{lllllllll}0.184 & 0.211 & 0.237 & 0.263 & 0.342 & 0.368 & 0.474\end{array}$

[B] $\quad \begin{array}{lllllllll}0.157 & 0.184 & 0.210 & 0.236 & 0.315 & 0.341 & 0.447\end{array}$

[C] $\quad \begin{array}{lllllllll}0.079 & 0.106 & 0.132 & 0.158 & 0.237 & 0.263 & 0.369\end{array}$

[D] $\quad \begin{array}{lllllllll}0.052 & 0.079 & 0.105 & 0.131 & 0.210 & 0.236 & 0.342\end{array}$

[E] $\quad \begin{array}{lllllllll}0.026 & 0.053 & 0.079 & 0.105 & 0.184 & 0.210 & 0.316\end{array}$

F] $\begin{array}{llllllll}0.027 & 0.053 & 0.079 & 0.158 & 0.184 & 0.290\end{array}$

[G] $\quad \begin{array}{lllllllll}0.027 & 0.026 & 0.052 & 0.131 & 0.157 & 0.263\end{array}$

[H] $\quad \begin{array}{lllllllll}0.053 & 0.026 & . & 0.026 & 0.105 & 0.131 & 0.237\end{array}$

[J] $\quad \begin{array}{llllllllll}0.079 & 0.052 & 0.026 & 0.079 & 0.105 & 0.211\end{array}$

[K] $\quad \begin{array}{llllllllll}0.158 & 0.131 & 0.105 & 0.079 & 0.026 & 0.132\end{array}$

[L] $\quad \begin{array}{llllllll}0.184 & 0.157 & 0.131 & 0.105 & 0.026 & . & 0.106\end{array}$

[M] $\quad \begin{array}{lllllllll}0.290 & 0.263 & 0.237 & 0.211 & 0.132 & 0.106\end{array}$ 
USERTYPE meanthetasep STEPMATRIX $=26$

\begin{tabular}{|c|c|c|c|c|c|c|c|c|c|c|c|c|c|c|c|}
\hline & & & 2 & 3 & & 5 & 6 & 7 & 8 & & $\mathrm{~A}$ & B & C & $\mathrm{D}$ & $\mathrm{E}$ \\
\hline 0] & & 4 & 9 & 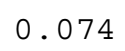 & 9 & 4 & 9 & 8 & 8 & 23 & 98 & 72 & 2 & 32 & 0.566 \\
\hline & .014 & & & & & 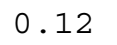 & & & & & & & & & \\
\hline & .059 & .045 & & & 06 & 075 & & .119 & 0.149 & 0.164 & .239 & 0.313 & 0.343 & 0.373 & .507 \\
\hline & & 06 & 015 & & & & & & & & & & & & \\
\hline & 119 & 105 & .06 & .045 & & 0.015 & & .059 & 89 & .04 & .179 & 53 & 83 & 13 & \\
\hline & 134 & 72 & & & 5 & & & .044 & & & & & & & \\
\hline & & & & & & .015 & & .029 & & & & & & & \\
\hline & & 4 & & & & & & & & & & & & & \\
\hline & & & & & & & & 03 & & & & & & & \\
\hline & & & & & & & & & & & & & & & \\
\hline & 298 & 4 & & 4 & & & & 0 & & 75 & & & & & \\
\hline & & & & & & & & & & & & & & & \\
\hline & 402 & 8 & & 8 & & 58 & & 24 & & & 04 & & & & \\
\hline & & & & & & & & & & & & & & & \\
\hline & & & & & & & & & & & & & & & \\
\hline & & & & & & & & & & & & & & & \\
\hline & & & & & & & & & & & & & & & \\
\hline & & 6 & 1 & 6 & & & & & & & & & & & \\
\hline & & & & & & & & & & & & & & & \\
\hline & 0 & .716 & 1 & 656 & 611 & 96 & & 52 & 22 & & 32 & & 0.328 & 98 & 0.164 \\
\hline & & & & & & & & & & & & & & & \\
\hline & 0 & 16 & 0. & .686 & 41 & $0 . \epsilon$ & & 0.582 & 0.552 & & 62 & 88 & 0.358 & 0.328 & .194 \\
\hline & & & & & & & & & & & & & & & \\
\hline & & & & & & & & & & & & & & & \\
\hline & & & & & & & & & & & & & 77 & 47 & \\
\hline & & & & & .88 & .86 & .85 & 0.821 & 0.19 & 0.116 & 0.701 & 0.627 & 0.597 & 0.56 & 0.4 \\
\hline
\end{tabular}


$[0]$

$[1]$

$\begin{array}{lllllllllll}0.611 & 0.641 & 0.7 & 0.715 & 0.73 & 0.745 & 0.76 & 0.775 & 0.834 & 0.879 & 0.999\end{array}$ $\begin{array}{llllllllllll}0.597 & 0.627 & 0.686 & 0.701 & 0.716 & 0.731 & 0.746 & 0.761 & 0.82 & 0.865 & 0.985\end{array}$ $\begin{array}{lllllllllllll}0.552 & 0.582 & 0.641 & 0.656 & 0.671 & 0.686 & 0.701 & 0.716 & 0.775 & 0.82 & 0.94\end{array}$ $\begin{array}{llllllllllll}0.537 & 0.567 & 0.626 & 0.641 & 0.656 & 0.671 & 0.686 & 0.701 & 0.76 & 0.805 & 0.925\end{array}$ $\begin{array}{llllllllllll}0.492 & 0.522 & 0.581 & 0.596 & 0.611 & 0.626 & 0.641 & 0.656 & 0.715 & 0.76 & 0.88\end{array}$ $\begin{array}{lllllllllllll}0.477 & 0.507 & 0.566 & 0.581 & 0.596 & 0.611 & 0.626 & 0.641 & 0.7 & 0.745 & 0.865\end{array}$ $\begin{array}{llllllllllll}0.462 & 0.492 & 0.551 & 0.566 & 0.581 & 0.596 & 0.611 & 0.626 & 0.685 & 0.73 & 0.85\end{array}$ $\begin{array}{llllllllllllll}0.433 & 0.463 & 0.522 & 0.537 & 0.552 & 0.567 & 0.582 & 0.597 & 0.656 & 0.701 & 0.821\end{array}$ $\begin{array}{llllllllllll}0.403 & 0.433 & 0.492 & 0.507 & 0.522 & 0.537 & 0.552 & 0.567 & 0.626 & 0.671 & 0.791\end{array}$ $\begin{array}{lllllllllllll}0.388 & 0.418 & 0.477 & 0.492 & 0.507 & 0.522 & 0.537 & 0.552 & 0.611 & 0.656 & 0.776\end{array}$ $\begin{array}{llllllllllll}0.313 & 0.343 & 0.402 & 0.417 & 0.432 & 0.447 & 0.462 & 0.477 & 0.536 & 0.581 & 0.701\end{array}$ $\begin{array}{lllllllllll}0.239 & 0.269 & 0.328 & 0.343 & 0.358 & 0.373 & 0.388 & 0.403 & 0.462 & 0.507 & 0.627\end{array}$ $\begin{array}{lllllllllllll}0.209 & 0.239 & 0.298 & 0.313 & 0.328 & 0.343 & 0.358 & 0.373 & 0.432 & 0.477 & 0.597\end{array}$ $\begin{array}{lllllllllllll}0.179 & 0.209 & 0.268 & 0.283 & 0.298 & 0.313 & 0.328 & 0.343 & 0.402 & 0.447 & 0.567\end{array}$ $\begin{array}{llllllllllll}0.045 & 0.075 & 0.134 & 0.149 & 0.164 & 0.179 & 0.194 & 0.209 & 0.268 & 0.313 & 0.433\end{array}$ $\begin{array}{llllllllllll}. & 0.03 & 0.089 & 0.104 & 0.119 & 0.134 & 0.149 & 0.164 & 0.223 & 0.268 & 0.388\end{array}$ $\begin{array}{llllllllllll}0.03 & 0 & 0.059 & 0.074 & 0.089 & 0.104 & 0.119 & 0.134 & 0.193 & 0.238 & 0.358\end{array}$ $\begin{array}{lllllllllllll}0.089 & 0.059 & 0.015 & 0.03 & 0.045 & 0.06 & 0.075 & 0.134 & 0.179 & 0.299\end{array}$ $\begin{array}{lllllllllllll}0.104 & 0.074 & 0.015 & 0 & 0.015 & 0.03 & 0.045 & 0.06 & 0.119 & 0.164 & 0.284\end{array}$ $\begin{array}{lllllllllllll}0.119 & 0.089 & 0.03 & 0.015 & 0.0 .015 & 0.03 & 0.045 & 0.104 & 0.149 & 0.269\end{array}$ $\begin{array}{lllllllllllll}0.134 & 0.104 & 0.045 & 0.03 & 0.015 & 0.015 & 0.015 & 0.03 & 0.089 & 0.134 & 0.254\end{array}$ $\begin{array}{lllllllllllll}0.149 & 0.119 & 0.06 & 0.045 & 0.03 & 0.015 & 0.015 & 0.074 & 0.119 & 0.239\end{array}$ $\begin{array}{llllllllllll}0.164 & 0.134 & 0.075 & 0.06 & 0.045 & 0.03 & 0.015 & & 0.059 & 0.104 & 0.224\end{array}$ $\begin{array}{lllllllllllll}0.223 & 0.193 & 0.134 & 0.119 & 0.104 & 0.089 & 0.074 & 0.059 & 0.045 & 0.165\end{array}$ $\begin{array}{lllllllllll}0.268 & 0.238 & 0.179 & 0.164 & 0.149 & 0.134 & 0.119 & 0.104 & 0.045\end{array}$.

$\begin{array}{lllllllllll}0.388 & 0.358 & 0.299 & 0.284 & 0.269 & 0.254 & 0.239 & 0.224 & 0.165 & 0.12\end{array}$ 
USERTYPE meandiameter STEPMATRIX $=25$

\begin{tabular}{|c|c|c|c|c|c|c|c|c|c|c|c|c|c|c|c|}
\hline & & 1 & & 3 & 4 & 5 & 6 & & 8 & & $\mathrm{~A}$ & B & C & D & E \\
\hline 0] & & 2 & 54 & 6 & 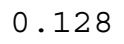 & 51 & 0 & 5 & 57 & 90 & 0. & 0 . & 86 & 18 & 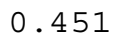 \\
\hline & 032 & & 032 & 064 & 096 & 129 & & 193 & 0.225 & 0.258 & 0.290 & 322 & 0.354 & & \\
\hline & .064 & .032 & & 32 & & & & & 193 & 226 & & 0.290 & & & \\
\hline & 096 & .064 & 032 & & 032 & & & & & & & & & & \\
\hline & 128 & 096 & .064 & 0.032 & & 33 & & & & & & & & & \\
\hline & 161 & 129 & 097 & 0.065 & 3 & & & & & & & & & & \\
\hline & & & & & & .032 & & & & & & & & & \\
\hline & 225 & 193 & .161 & 0.129 & & 64 & 0.032 & & 32 & & & & & & \\
\hline & 57 & & & & & & & & & & & & & & \\
\hline & 0 & 258 & 6 & 94 & & & & & 0.033 & & & & & & \\
\hline & 322 & 290 & & & & & & & & & & & & & \\
\hline & 354 & 322 & 90 & 0.258 & 26 & 93 & 61 & 29 & 97 & 0.064 & 032 & & 0.032 & 64 & 97 \\
\hline & 86 & & & & & & & & & & & & & & \\
\hline & .418 & 386 & 354 & 0.322 & 290 & 0.257 & 25 & 193 & 61 & 0.128 & 96 & 64 & 0.032 & & 033 \\
\hline & 51 & & & & & & & & & & & & & & \\
\hline & 483 & 451 & 419 & 87 & 355 & 22 & & 258 & 26 & 93 & 61 & 29 & & 65 & \\
\hline & & & & & & & & & & & & & & & \\
\hline & 47 & 15 & & & & & & & 90 & 57 & 25 & & 61 & 29 & 96 \\
\hline & & & & & & & & & & & & & & & \\
\hline & 676 & 4 & & & & & & & & & & & & 58 & 25 \\
\hline & & & & & & & & & & & & & & & \\
\hline & 805 & 3 & & & & & & & & & & & & & 54 \\
\hline & 902 & 870 & 3 & & & & 0 & & & & & & & & \\
\hline & & & & & & & & & & & & & & & \\
\hline & 999 & .967 & .935 & 0.903 & .871 & .838 & 0.806 & 0.774 & 0.742 & 0.709 & 0.677 & 0.645 & 0.613 & 0.581 & 0.548 \\
\hline
\end{tabular}


[0] $\quad \begin{array}{llllllllllll}0.483 & 0.515 & 0.547 & 0.580 & 0.676 & 0.773 & 0.805 & 0.902 & 0.966 & 0.999\end{array}$

[1] $\quad \begin{array}{llllllllllll}0.451 & 0.483 & 0.515 & 0.548 & 0.644 & 0.741 & 0.773 & 0.870 & 0.934 & 0.967\end{array}$

[2] $\quad \begin{array}{llllllllllllll}0.419 & 0.451 & 0.483 & 0.516 & 0.612 & 0.709 & 0.741 & 0.838 & 0.902 & 0.935\end{array}$

[3] $\quad \begin{array}{lllllllllllll}0.387 & 0.419 & 0.451 & 0.484 & 0.580 & 0.677 & 0.709 & 0.806 & 0.870 & 0.903\end{array}$

[4] $\quad \begin{array}{llllllllllll}0.355 & 0.387 & 0.419 & 0.452 & 0.548 & 0.645 & 0.677 & 0.774 & 0.838 & 0.871\end{array}$

[5] $\quad \begin{array}{lllllllllllll}0.322 & 0.354 & 0.386 & 0.419 & 0.515 & 0.612 & 0.644 & 0.741 & 0.805 & 0.838\end{array}$

[6] $\quad \begin{array}{llllllllllll}0.290 & 0.322 & 0.354 & 0.387 & 0.483 & 0.580 & 0.612 & 0.709 & 0.773 & 0.806\end{array}$

[7] $\quad \begin{array}{lllllllllllll}0.258 & 0.290 & 0.322 & 0.355 & 0.451 & 0.548 & 0.580 & 0.677 & 0.741 & 0.774\end{array}$

[8] $\quad \begin{array}{llllllllllll}0.226 & 0.258 & 0.290 & 0.323 & 0.419 & 0.516 & 0.548 & 0.645 & 0.709 & 0.742\end{array}$

[9] $\quad \begin{array}{lllllllllllll}0.193 & 0.225 & 0.257 & 0.290 & 0.386 & 0.483 & 0.515 & 0.612 & 0.676 & 0.709\end{array}$

[A] $\quad \begin{array}{llllllllllll}0.161 & 0.193 & 0.225 & 0.258 & 0.354 & 0.451 & 0.483 & 0.580 & 0.644 & 0.677\end{array}$

[B] $\quad \begin{array}{llllllllllll}0.129 & 0.161 & 0.193 & 0.226 & 0.322 & 0.419 & 0.451 & 0.548 & 0.612 & 0.645\end{array}$

[C] $\quad \begin{array}{lllllllllllll}0.097 & 0.129 & 0.161 & 0.194 & 0.290 & 0.387 & 0.419 & 0.516 & 0.580 & 0.613\end{array}$

[D] $\quad \begin{array}{llllllllllll}0.065 & 0.097 & 0.129 & 0.162 & 0.258 & 0.355 & 0.387 & 0.484 & 0.548 & 0.581\end{array}$

[E] $\quad \begin{array}{llllllllllll}0.032 & 0.064 & 0.096 & 0.129 & 0.225 & 0.322 & 0.354 & 0.451 & 0.515 & 0.548\end{array}$

[F] $\quad \begin{array}{lllllllllll} & 0.032 & 0.064 & 0.097 & 0.193 & 0.290 & 0.322 & 0.419 & 0.483 & 0.516\end{array}$

[G] $\quad \begin{array}{llllllllllll}0.032 & 0.032 & 0.032 & 0.065 & 0.161 & 0.258 & 0.290 & 0.387 & 0.451 & 0.484\end{array}$

$[\mathrm{H}] \quad \begin{array}{llllllllllll}0.064 & 0.032 & . & & 0.033 & 0.129 & 0.226 & 0.258 & 0.355 & 0.419 & 0.452\end{array}$

[J] $\quad \begin{array}{lllllllllllll}0.097 & 0.065 & 0.033 & 0.0 .036 & 0.096 & 0.193 & 0.225 & 0.322 & 0.386 & 0.419\end{array}$

$[\mathrm{K}] \quad \begin{array}{lllllllllllll}0.193 & 0.161 & 0.129 & 0.096 & & 0.097 & 0.129 & 0.226 & 0.290 & 0.323\end{array}$

[L] $\quad \begin{array}{lllllllllllll}0.290 & 0.258 & 0.226 & 0.193 & 0.097 & . & 0.032 & 0.129 & 0.193 & 0.226\end{array}$

[M] $\quad \begin{array}{llllllllllll}0.322 & 0.290 & 0.258 & 0.225 & 0.129 & 0.032 & . & 0.097 & 0.161 & 0.194\end{array}$

[N] $\quad \begin{array}{lllllllllll}0.419 & 0.387 & 0.355 & 0.322 & 0.226 & 0.129 & 0.097 & 0.064 & 0.097\end{array}$

[P] $\quad \begin{array}{llllllllllllll}0.483 & 0.451 & 0.419 & 0.386 & 0.290 & 0.193 & 0.161 & 0.064 & . & 0.033\end{array}$

[Q] $\quad \begin{array}{llllllllllll}0.516 & 0.484 & 0.452 & 0.419 & 0.323 & 0.226 & 0.194 & 0.097 & 0.033\end{array}$. 
USERTYPE meanareolararea STEPMATRIX = 19

\begin{tabular}{|c|c|c|c|c|c|c|c|c|c|c|c|c|c|c|c|}
\hline & 0 & 1 & 2 & 3 & 4 & 5 & 6 & 7 & 8 & $y$ & A & B & $\mathrm{C}$ & $\mathrm{D}$ & $E$ \\
\hline [0] & . & 0.036 & .071 & 0.107 & 143 & .178 & 214 & 0.250 & 0.285 & 0.357 & 0.392 & 0.428 & 0.500 & 0.535 & 0.571 \\
\hline [1] & 0.036 & - & 0.036 & 0.071 & 0.107 & 0.143 & 0.178 & 0.214 & 0.250 & 0.321 & 0.357 & 0.392 & 0.464 & 0.500 & 0.535 \\
\hline [2] & 0.071 & 0.036 & 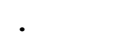 & 0.036 & 0.071 & 0.107 & 0.143 & 0.178 & 0.214 & 0.285 & 0.321 & 0.357 & 0.428 & 0.464 & 0.500 \\
\hline [3] & 0.107 & 0.071 & 0.036 & 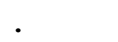 & 0.036 & 0.071 & 0.107 & 0.143 & 0.178 & 0.250 & 0.285 & 0.321 & 0.392 & 0.428 & 0.464 \\
\hline [4] & 0.143 & 0.107 & 0.071 & 0.036 & . & 0.036 & 0.071 & 0.107 & 0.143 & 0.214 & 0.250 & 0.285 & 0.357 & 0.392 & 0.428 \\
\hline$[5$ & 0.178 & 0.143 & 0.107 & 0.071 & 0.036 & & 0.036 & 0.071 & 0.107 & 0.178 & 0.214 & 0.250 & 0.321 & 0.357 & 0.392 \\
\hline & 0.214 & 0.178 & 0.143 & 0.107 & 0.071 & 0.036 & . & 0.036 & 0.071 & 0.143 & 0.178 & 0.214 & 0.285 & 0.321 & 0.357 \\
\hline$L_{1}$ & 0.250 & 0.214 & 0.178 & 0.143 & 0.107 & 0.071 & 0.036 & . & 0.036 & 0.107 & 0.143 & 0.178 & 0.250 & 0.285 & 0.321 \\
\hline$[8$ & 0.285 & 0.250 & 0.214 & 0.178 & 0.143 & 0.107 & 0.071 & 0.036 & & 0.071 & 0.107 & 0.143 & 0.214 & 0.250 & 0.285 \\
\hline[ & 0.357 & 0.321 & 0.285 & 0.250 & 0.214 & 0.178 & 0.143 & 0.107 & 0.071 & & 0.036 & 0.071 & 0.143 & 0.178 & 0.214 \\
\hline A & 0.392 & 0.357 & 0.321 & 0.285 & 0.250 & 0.214 & 0.178 & 0.143 & 0.107 & 0.036 & . & 0.036 & 0.107 & 0.143 & 0.178 \\
\hline [B & 0.428 & 0.392 & 0.357 & 0.321 & 0.285 & 0.250 & 0.214 & 0.178 & 0.143 & 0.071 & 0.036 & . & 0.071 & 0.107 & 0.143 \\
\hline & 0.500 & 0.464 & 0.428 & 0.392 & 0.357 & 0.321 & 0.285 & 0.250 & 0.214 & 0.143 & 0.107 & 0.071 & . & 0.036 & 0.071 \\
\hline$[\mathrm{L}$ & 0.535 & 0.500 & 0.464 & 0.428 & 0.392 & 0.357 & 0.321 & 0.285 & 0.250 & 0.178 & 0.143 & 0.107 & 0.036 & & 0.036 \\
\hline$[\mathrm{E}$ & 0.571 & 0.535 & 0.500 & 0.464 & 0.428 & 0.392 & 0.357 & 0.321 & 0.285 & 0.214 & 0.178 & 0.143 & 0.071 & 0.036 & . \\
\hline$[F]$ & 0.678 & 0.642 & 0.607 & 0.571 & 0.535 & 0.500 & 0.464 & 0.428 & 0.392 & 0.321 & 0.285 & 0.250 & 0.178 & 0.143 & 0.107 \\
\hline 50 & 0.749 & 0.714 & 0.678 & 0.642 & 0.607 & 0.571 & 0.535 & 0.500 & 0.464 & 0.392 & 0.357 & 0.321 & 0.250 & 0.214 & 0.178 \\
\hline$[H$ & 0.785 & 0.749 & 0.714 & 0.678 & 0.642 & 0.607 & 0.571 & 0.535 & 0.500 & 0.428 & 0.392 & 0.357 & 0.285 & 0.250 & 0.214 \\
\hline$[\mathrm{J}$ & 0.999 & 0.963 & 0.928 & 0.892 & 0.856 & 0.821 & 0.785 & 0.749 & 0.714 & 0.642 & 0.607 & 0.571 & 0.500 & 0.464 & 0.428 \\
\hline
\end{tabular}


F $\quad$ G $\quad H \quad$ J

[0] $\quad \begin{array}{lllll}0.678 & 0.749 & 0.785 & 0.999\end{array}$

[1] $\quad \begin{array}{llllll}0.642 & 0.714 & 0.749 & 0.963\end{array}$

$\begin{array}{llllll}{[2]} & 0.607 & 0.678 & 0.714 & 0.928\end{array}$

[3] $\quad \begin{array}{llllll}0.571 & 0.642 & 0.678 & 0.892\end{array}$

[4] $\quad 0.535 \quad 0.607 \quad 0.642 \quad 0.856$

[5] $\quad \begin{array}{lllll}0.500 & 0.571 & 0.607 & 0.821\end{array}$

[6] $\quad \begin{array}{llllll}0.464 & 0.535 & 0.571 & 0.785\end{array}$

[7] $\quad \begin{array}{llllll}0.428 & 0.500 & 0.535 & 0.749\end{array}$

[8] $\quad 0.392 \quad 0.464 \quad 0.500 \quad 0.714$

$\begin{array}{llllll}{[9]} & 0.321 & 0.392 & 0.428 & 0.642\end{array}$

[A] $\quad \begin{array}{llllll}0.285 & 0.357 & 0.392 & 0.607\end{array}$

[B] $\quad \begin{array}{llllll}0.250 & 0.321 & 0.357 & 0.571\end{array}$

[C] $\quad \begin{array}{lllll}0.178 & 0.250 & 0.285 & 0.500\end{array}$

[D] $\quad \begin{array}{llllll}0.143 & 0.214 & 0.250 & 0.464\end{array}$

[E] $\quad \begin{array}{llllll}0.107 & 0.178 & 0.214 & 0.428\end{array}$

$\begin{array}{llll}0.071 & 0.107 & 0.321\end{array}$

[G] $\quad 0.071$. 0.0360 .250

[H] $\quad 0.107 \quad 0.036 .0 .214$

[J] $\quad 0.3210 .250 \quad 0.214$. 
USERTYPE PAareolararea STEPMATRIX $=26$

\begin{tabular}{|c|c|c|c|c|c|c|c|c|c|c|c|c|c|c|c|}
\hline & 0 & 1 & 2 & 3 & 4 & 5 & 6 & 7 & 8 & 9 & A & B & $\mathrm{C}$ & $\mathrm{D}$ & $\mathrm{E}$ \\
\hline$[0]$ & . & 0.039 & 0.052 & 0.065 & 0.078 & 0.091 & 0.104 & 0.117 & 0.130 & 0.143 & 0.156 & 0.169 & 0.195 & 0.208 & 0.221 \\
\hline [1] & 0.039 & . & 0.013 & 0.026 & 0.039 & 0.052 & 0.065 & 0.078 & 0.091 & 0.104 & 0.117 & 0.130 & 0.156 & 0.169 & 0.182 \\
\hline [2] & 0.052 & 0.013 & 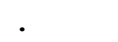 & 0.013 & 0.026 & 0.039 & 0.052 & 0.065 & 0.078 & 0.091 & 0.104 & 0.117 & 0.143 & 0.156 & 0.169 \\
\hline [3] & 0.065 & 0.026 & 0.013 & . & 0.013 & 0.026 & 0.039 & 0.052 & 0.065 & 0.078 & 0.091 & 0.104 & 0.130 & 0.143 & 0.156 \\
\hline$[4$ & 0.078 & 0.039 & 0.026 & 0.013 & & 0.013 & 0.026 & 0.039 & 0.052 & 0.065 & 0.078 & 0.091 & 0.117 & 0.130 & 0.143 \\
\hline$[0$ & 0.091 & 0.052 & 0.039 & 0.026 & 0.013 & & 0.013 & 0.026 & 0.039 & 0.052 & 0.065 & 0.078 & 0.104 & 0.117 & 0.130 \\
\hline [6] & 0.104 & 0.065 & 0.052 & 0.039 & 0.026 & 0.013 & . & 0.013 & 0.026 & 0.039 & 0.052 & 0.065 & 0.091 & 0.104 & 0.117 \\
\hline$[7]$ & 0.117 & 0.078 & 0.065 & 0.052 & 0.039 & 0.026 & 0.013 & . & 0.013 & 0.026 & 0.039 & 0.052 & 0.078 & 0.091 & 0.104 \\
\hline$[8$ & 0.130 & 0.091 & 0.078 & 0.065 & 0.052 & 0.039 & 0.026 & 0.013 & & 0.013 & 0.026 & 0.039 & 0.065 & 0.078 & 0.091 \\
\hline 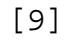 & 0.143 & 0.104 & 0.091 & 0.078 & 0.065 & 0.052 & 0.039 & 0.026 & 0.013 & & 0.013 & 0.026 & 0.052 & 0.065 & 0.078 \\
\hline$[A$ & 0.156 & 0.117 & 0.104 & 0.091 & 0.078 & 0.065 & 0.052 & 0.039 & 0.026 & 0.013 & . & 0.013 & 0.039 & 0.052 & 0.065 \\
\hline [B & 0.169 & 0.130 & 0.117 & 0.104 & 0.091 & 0.078 & 0.065 & 0.052 & 0.039 & 0.026 & 0.013 & & 0.026 & 0.039 & 0.052 \\
\hline$\Gamma \Gamma$ & 0.195 & 0.156 & 0.143 & 0.130 & 0.117 & 0.104 & 0.091 & 0.078 & 0.065 & 0.052 & 0.039 & 0.026 & . & 0.013 & 0.026 \\
\hline [D & 0.208 & 0.169 & 0.156 & 0.143 & 0.130 & 0.117 & 0.104 & 0.091 & 0.078 & 0.065 & 0.052 & 0.039 & 0.013 & & 0.013 \\
\hline$[E]$ & 0.221 & 0.182 & 0.169 & 0.156 & 0.143 & 0.130 & 0.117 & 0.104 & 0.091 & 0.078 & 0.065 & 0.052 & 0.026 & 0.013 & \\
\hline$[F]$ & 0.234 & 0.195 & 0.182 & 0.169 & 0.156 & 0.143 & 0.130 & 0.117 & 0.104 & 0.091 & 0.078 & 0.065 & 0.039 & 0.026 & 0.013 \\
\hline$[\mathrm{G}]$ & 0.259 & 0.221 & 0.208 & 0.195 & 0.182 & 0.169 & 0.156 & 0.143 & 0.130 & 0.117 & 0.104 & 0.091 & 0.065 & 0.052 & 0.039 \\
\hline$[\mathrm{H}$ & 0.272 & 0.234 & 0.221 & 0.208 & 0.195 & 0.182 & 0.169 & 0.156 & 0.143 & 0.130 & 0.117 & 0.104 & 0.078 & 0.065 & 0.052 \\
\hline$[\mathrm{J}$ & 0.285 & 0.247 & 0.234 & 0.221 & 0.208 & 0.195 & 0.182 & 0.169 & 0.156 & 0.143 & 0.130 & 0.117 & 0.091 & 0.078 & 0.065 \\
\hline & 0.337 & 0.298 & 0.285 & 0.272 & 0.259 & 0.247 & 0.234 & 0.221 & 0.208 & 0.195 & 0.182 & 0.169 & 0.143 & 0.130 & 0.117 \\
\hline$[\mathrm{L}$ & 0.350 & 0.311 & 0.298 & 0.285 & 0.272 & 0.259 & 0.247 & 0.234 & 0.221 & 0.208 & 0.195 & 0.182 & 0.156 & 0.143 & 0.130 \\
\hline$[\mathrm{M}$ & 0.389 & 0.350 & 0.337 & 0.324 & 0.311 & 0.298 & 0.285 & 0.272 & 0.259 & 0.247 & 0.234 & 0.221 & 0.195 & 0.182 & 0.169 \\
\hline$[\mathrm{N}]$ & 0.519 & 0.480 & 0.467 & 0.454 & 0.441 & 0.428 & 0.415 & 0.402 & 0.389 & 0.376 & 0.363 & 0.350 & 0.324 & 0.311 & 0.298 \\
\hline$[\mathrm{P}]$ & 0.597 & 0.558 & 0.545 & 0.532 & 0.519 & 0.506 & 0.493 & 0.480 & 0.467 & 0.454 & 0.441 & 0.428 & 0.402 & 0.389 & 0.376 \\
\hline 5 & 0.623 & 0.584 & 0.571 & 0.558 & 0.545 & 0.532 & 0.519 & 0.506 & 0.493 & 0.480 & 0.467 & 0.454 & 0.428 & 0.415 & 0.402 \\
\hline תז & 0.999 & 0.960 & 0.947 & 0.934 & 0.921 & 0.908 & 0.895 & 0.882 & 0.869 & 0.856 & 0.843 & 0.830 & 0.804 & 0.791 & 0.778 \\
\hline
\end{tabular}


[0] $\quad \begin{array}{lllllllllllll}0.234 & 0.259 & 0.272 & 0.285 & 0.337 & 0.350 & 0.389 & 0.519 & 0.597 & 0.623 & 0.999\end{array}$

[1] $\quad \begin{array}{llllllllllllll}0.195 & 0.221 & 0.234 & 0.247 & 0.298 & 0.311 & 0.350 & 0.480 & 0.558 & 0.584 & 0.960\end{array}$

[2] $\quad \begin{array}{llllllllllllll}0.182 & 0.208 & 0.221 & 0.234 & 0.285 & 0.298 & 0.337 & 0.467 & 0.545 & 0.571 & 0.947\end{array}$

[3] $\quad \begin{array}{lllllllllllll}0.169 & 0.195 & 0.208 & 0.221 & 0.272 & 0.285 & 0.324 & 0.454 & 0.532 & 0.558 & 0.934\end{array}$

[4] $\quad \begin{array}{lllllllllllll}0.156 & 0.182 & 0.195 & 0.208 & 0.259 & 0.272 & 0.311 & 0.441 & 0.519 & 0.545 & 0.921\end{array}$

[5] $\quad \begin{array}{llllllllllllll}0.143 & 0.169 & 0.182 & 0.195 & 0.247 & 0.259 & 0.298 & 0.428 & 0.506 & 0.532 & 0.908\end{array}$

[6] $\quad \begin{array}{llllllllllll}0.130 & 0.156 & 0.169 & 0.182 & 0.234 & 0.247 & 0.285 & 0.415 & 0.493 & 0.519 & 0.895\end{array}$

[7] $\quad \begin{array}{lllllllllllll}0.117 & 0.143 & 0.156 & 0.169 & 0.221 & 0.234 & 0.272 & 0.402 & 0.480 & 0.506 & 0.882\end{array}$

[8] $\quad \begin{array}{llllllllllllll}0.104 & 0.130 & 0.143 & 0.156 & 0.208 & 0.221 & 0.259 & 0.389 & 0.467 & 0.493 & 0.869\end{array}$

[9] $\quad \begin{array}{llllllllllllll}0.091 & 0.117 & 0.130 & 0.143 & 0.195 & 0.208 & 0.247 & 0.376 & 0.454 & 0.480 & 0.856\end{array}$

[A] $\quad \begin{array}{llllllllllllll}0.078 & 0.104 & 0.117 & 0.130 & 0.182 & 0.195 & 0.234 & 0.363 & 0.441 & 0.467 & 0.843\end{array}$

[B] $\quad \begin{array}{llllllllllllll}0.065 & 0.091 & 0.104 & 0.117 & 0.169 & 0.182 & 0.221 & 0.350 & 0.428 & 0.454 & 0.830\end{array}$

[C] $\quad \begin{array}{llllllllllllll}0.039 & 0.065 & 0.078 & 0.091 & 0.143 & 0.156 & 0.195 & 0.324 & 0.402 & 0.428 & 0.804\end{array}$

[D] $\quad \begin{array}{llllllllllllll}0.026 & 0.052 & 0.065 & 0.078 & 0.130 & 0.143 & 0.182 & 0.311 & 0.389 & 0.415 & 0.791\end{array}$

[E] $\quad \begin{array}{llllllllllllll}0.013 & 0.039 & 0.052 & 0.065 & 0.117 & 0.130 & 0.169 & 0.298 & 0.376 & 0.402 & 0.778\end{array}$

[F] $\quad \begin{array}{llllllllllllll} & & 0.026 & 0.039 & 0.052 & 0.104 & 0.117 & 0.156 & 0.285 & 0.363 & 0.389 & 0.765\end{array}$

[G] $\quad \begin{array}{lllllllllllll}0.026 & 0 & 0.013 & 0.026 & 0.078 & 0.091 & 0.130 & 0.259 & 0.337 & 0.363 & 0.740\end{array}$

$[\mathrm{H}] \quad \begin{array}{lllllllllllllll}0.039 & 0.013 & . & & 0.013 & 0.065 & 0.078 & 0.117 & 0.247 & 0.324 & 0.350 & 0.727\end{array}$

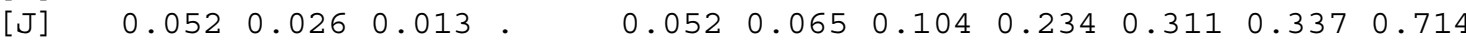

[K] $\quad \begin{array}{llllllllllllll} & 0.104 & 0.078 & 0.065 & 0.052 & 0.013 & 0.013 & 0.052 & 0.182 & 0.259 & 0.285 & 0.662\end{array}$

[L] $\quad \begin{array}{llllllllllllllll} & 0.117 & 0.091 & 0.078 & 0.065 & 0.013 & . & 0.039 & 0.169 & 0.247 & 0.272 & 0.649\end{array}$

[M] $\quad \begin{array}{lllllllllllllll}0.156 & 0.130 & 0.117 & 0.104 & 0.052 & 0.039 & 0.130 & 0.208 & 0.234 & 0.610\end{array}$

[N] $\quad \begin{array}{lllllllllllll}0.285 & 0.259 & 0.247 & 0.234 & 0.182 & 0.169 & 0.130 & 0.078 & 0.104 & 0.480\end{array}$

[P] $\quad \begin{array}{lllllllllllll}0.363 & 0.337 & 0.324 & 0.311 & 0.259 & 0.247 & 0.208 & 0.078 & . & 0.026 & 0.402\end{array}$

[Q] $\quad \begin{array}{llllllllllllll}0.389 & 0.363 & 0.350 & 0.337 & 0.285 & 0.272 & 0.234 & 0.104 & 0.026 & . & 0.376\end{array}$

[R] $\quad \begin{array}{llllllllllllll}0.765 & 0.740 & 0.727 & 0.714 & 0.662 & 0.649 & 0.610 & 0.480 & 0.402 & 0.376\end{array}$. 
Appendix I. Morphological Character States as Scored for Gap Weighting Without Step-Matrices Limited to 32 Character States. 
[

M. varians CHMP7 FFP

S._cf._broschii_GB

A._islāndica_CHM̄P1_MICH

A. skvortzowii

A._baicalensis

A._sub_9D1

A. sub_WLB3

A. $s p .{ }^{3}$ FR

A._sub_ERB

A. sub LEW

A._alpīgena

A._amb_PII 7

A._amb_N17

A. amb-FL8

A.

A._amb_WLP1

A. nyassensis GB

A._valida_N2

A._gran_LB

A._gran_OR12

A. $\mathrm{sp} \cdot \overline{1}$

A._sp. 2

A. cren FH8 L639

A. ${ }^{-}$cren T73 $\overline{3}$ L639

Alveolophora

A._agassizii_FH21

A._amb_A62

A. amb MFP

A. amb_WAT

A. canād 1571

A. canad 2018

A._dianchiensis

A. distans FH34

A._gran_FH4 2

A._gran_FH38

A. gran 1914

A._gran_MFP

A._gran_v. jonensis

A._gran-f_- $\mathrm{HLS}$

A. gran f $-\mathrm{VH}$

A._herzogii_HDSM

A._italica_FH15

A._krammerii_nom. prov.

A._lac_A38

A. Iac_A62

A. lirāta A37

A._laevissima_HDSM

A._nyg_A50
Qualitative Chars Morphometric Chars

100100000002000713 C5E7??????????????U?

100000000002020443 ????????????????????

211111611112100503 G5HJC6F534CD6B76MLE52

211511611112100513 F57JC6FC3ABH4D78QP842

21111141111?100523 XNMNX8XR6CQUMGHAPNJRK

211100002120000011 B3BP76A35J9B5D5A?D544

211100002120000011 93EN65B06GBL5954?4B30

$211100002123000102 \quad 340336550$ A0A1C18?0113

$211100002120000011828 \mathrm{P} 66716 \mathrm{H} 4 \mathrm{G} 3 \mathrm{D} 4 \mathrm{~A}$ ? 7634

211100002120000011 65KL66A49JAL5F58?CA45

2111126111001 ????? ????????????????????

221111611110100011 B6CA96G937FQ5D4785B43

221111611110100011 958M67B83BBV4F4852535

221111611110100011 9A8E66C83BCL4F4862434

221111611110100011 B24CL0E41B9X5E4740333

221111611110100011 D39H96G538EH5D4740643

2211116111121001 ?3 G5K7DXG435EBHAB6MLQC3

210022720001100011 F6LSC6K57GHM6F540?C55

2141116121201????? G0AG86B9287JCXCPHP6DX

214111612120100231 EC7687CD1A6M9GAXHN28J

210512620001100202 78BTB7BD278S9T90Q?958

2111126111101 ?? ? ? 7X3LDEGX10994A6BQP13A

2100327100012032?1 F5HNN6TC48MR9H4AUTD57

2100327100012032?1 E5XKQ6R6B7XN9D57TSW64

$2112116111121000 ? 3$ 65T7B6F4X2C89CJ4QQXC2

2141116121221001? I A4QRD6F2C7EB9DB3NPG73

221111611110100011 A?7C46C83DCT5E5774545

221111611110100011 D4AD96F53AEM4E4696834

221111611110100011 C5AE76E237EG5B4996842

211211611110114033 84DPA5C46P9GEFJKPN5AC

211211611110114033 94PUC6D3AQFQKATBQQ9QK

21311161212 ? 111601 W9P9H6L05CGBU0RBQPAX8

$210111211102100123 \quad 441 \mathrm{H} 66814 \mathrm{G} 193 \mathrm{~F} 39 \mathrm{QP} 124$

214111612120101201 E5ACD6H5168GBGDFJP988

214111612120101201 D5TTE6K488GFEBG7LQFD3

2141????21201003?2 D0NXC6G6AREG9DB1?PA64

2141116121201001?1 G4CHB6F528APBEC8HP7C7

2241116121201012 ? 1 84532684044A3D56CN622

2111????11121????? G442E5H1BK37KBKDPNCN7

2111????11121????? U2C7M5Q07L83JDMEQPAJ9

213100005422000311 E88E86A6268F3902QP722

21004271000 ?2122?1 G7EKF6H725EK6G28XXC45

212411411121114003 COJHC5C19XE79ALVQP774

2101111100002 ?? ? ? 75CJC6E645BD5E75QPE44

2101111100002????? 75AD96D8389F4D67PNB34

21011251000 ?1????? A4FKC6EA8SGLCGCJKJC8B

213100003312000103 55DK96C737AG8F9AQPF55

2115129213122 ????? 858EA6D636BJ2D28PNC14 
A. nyg A62

A._pergla_f_A38

A._pfaffiāna_FH37

A. solida $\mathrm{FH}_{\overline{2}} 3$

A. sub ROR

A. sub_T702

A. $\mathrm{sp} .{ }^{3} \mathrm{ROR}$

A. distañs f ADE

A._paucistriāta_2035

A._sp. 4 - T733

A. $\mathrm{sp} \cdot{ }^{5} \mathrm{~h}$

A._sp. ${ }^{-}{ }^{-}$hwg

A._cf._dístans_1571

A. humilis

A. tenuoir

A. tenella

A._crassipunctata

A. tethera

A._pseudoamericana

A. muzzanensis

A._pergla_f_KI

A._ceretaña
Qualitative Chars Morphometric Chars
2115129213122????? 768E96D735EL1B250PAG1

0121003285110003 ? $158 \mathrm{H}$ ?? 76053D??????C??

210112310002100102 543D96B417375J79LK635

212000002120014003 95PSD6F2LVCD9AJ9?QF83

$21110000212000001173806584285 B 4 D 58$ ? 9632

211100002120000011 A4BM65A25M9L5G5B? 9534

$211100002123000102 \quad 3324355417102 \mathrm{D} 18$ ? 4114

2101126100001 ?? ?? ? 569L86A83A9T4E57EC945

213000001120014003 B5SEG6J3NQQHDCXA?QMG6

2113111111101????? J6XLT6L888VHXFMBPNHPG

0111526185203 ?? ?? 45CL??C514CA? ?? ??B?

0101523111113 ?? ?? 4 55D? ?AA16AP? ? ? ? 6?

2111000021220 ????? 254T35464E1G2H2GC9017

2130000011220 ????? 454M77AG17375CB7?Q347

2 ? 11000021120 ? ? ?? 7? ? 7? ? L? ? 3 ? 4 Q?H7?

2 ? 12000011120001 ? 0 ???0???????2?2??Q60?

2?03111100021?40?3 F???H???S???G?M?Q?74?

2?01115100011000?1 2???2???????0?9?Q?C0?

0 ?01529100013006? 3 ?????????????????D?

2?41116121211????? 5?A?6?0?3 ?A?6?E? EQQ5?

2?11216111121????? 2 ? ? 3 ? ? C? ? 1 ?1 ?QQG0?

211211611112104033 G4D?9?F?W?N?C?K?QQWG?

Characters in columns from left to right are as listed 1-39 in the Material and Methods section of Chapter 2, pages 41 to 50 . 
Appendix J. Morphological Character States as Scored for Gap Weighting Without Step-Matrices Limited to 26 Character States. 
[

M._varians_CHMP7_FFP

S._cf._bros̄chii_̄̄B

A._islāndica_CHM̄P1_MICH

A. skvortzowii

A._baicalensis

A._sub_9D1

A. sub_WLB3

A. $s p .{ }^{3}$ FR

A._sub_ERB

A. sub LEW

A._alpigena

A._amb_PII 7

A._amb_N17

A. amb-FL8

A.

A._amb_WLP1

A. nyassensis GB

A._valida_N2

A._gran_LB

A. gran_oR12

A. $\operatorname{sp} \cdot \overline{1}$

A._sp. 2

A. $\operatorname{cren}$ FH8 L639

A._cren_T733 L639

Alveolophora

A._agassizii_FH21

A. amb_A62

A. amb_MFP

A. amb_WAT

A. canād_1571

A. canad 2018

A._dianchiensis

A. distans FH34

A._gran_FH $\overline{4} 2$

A._gran_FH38

A. gran_1914

A._gran_MFP

A._gran_V._jonensis

A._gran_f_HLS

A. gran f- $\mathrm{VH}$

A._herzogii_HDSM

A._italica_FH15

A._krammerii_nom._prov.

A._lac_A38

A. 1 - 1 - $\mathrm{A} 62$

A. lirāta A37

A._laevissima_HDSM

A._nyg_A50
Qualitative Chars Morphometric Chars

100100000002000713 A4B6? ? ? ? ? ? ? ? ? ? P?

100000000002020443 ???????????????????

211111611112100503 D4EEA5C4239B5964HGB41

211511611112100513 C45EA5CA289E3A66KK632

21111141111 ?100523 RJHJR6RL5AKNHDE8KJFLG

211100002120000011 929J65834E794A48?B433

$21110000212000001172 \mathrm{BJ} 54904 \mathrm{D} 9 \mathrm{G} 4743$ ? 3930

$211100002123000102 \quad 2303254408081 A 16$ ? 0113

211100002120000011 627J55614D3D3A38?6533

211100002120000011 54FG55838E8G4C46?A834

2111126111001 ????? ???????????????????

221111611110100011 95A875D836CK4A3564932

221111611110100011 746H5596299P3C3741424

221111611110100011 786C55A6299G3C3752333

221111611110100011 923AG0C3197R4B3530232

221111611110100011 B38D75D427BE4A3530533

2211116111121001 ?3 D4F6ARD334B9E895HGKA2

210022720001100011 C5GMA5F46DEH5C430?A4 4

2141116121201????? D08D7597176EAR9JEJ5AR

214111612120100231 BA5566AB185H8D8RDJ27F

210512620001100202 569N969A267M7N70K?747

2111126111101 ????? 6R2GBBDR10773859KJ138

2100327100012032 ? 1 C4EJJ5NA37HL8E3 8NNA4 6

2100327100012032 ? 1 B4RFK5L596RJ7B45MKQ53

2112116111121000 ?3 54N695C3R2978AF3LKR92

2141116121221001 ? 1 83LLB5C2A6B97A93JJD63

221111611110100011 8?5A35A62B9N4B4663434

221111611110100011 B38B75C438BH3B3474633

$221111611110100011948 \mathrm{C} 65 \mathrm{~B} 236 \mathrm{BD} 493885632$

211211611110114033 63BJ84A35J7DBCFFJJ48A

211211611110114033 73KPA5B28KCLG8N9LK7KF

21311161212 ?111601 Q7J8E5G04AD9P0L9KK8R7

210111211102100123 331D55613D173C38KJ113

214111612120101201 B48AB5D4157D9DACEJ767

214111612120101201 B4NNB4F367DCB9D6GKCA3

2141????21201003?2 B0HRA5D58LBD8A91?K853

2141116121201001 ? D DAD95C4278J9B97EJ696

2241116121201012 ? 16343257303383 B44AJ522

2111????11121????? D332B4D19F25G9GAKJAH6

2111????11121????? P2A6H4K05G73EAHCKJ8E7

213100005422000311 B76C7585156C3702KJ611

21004271000?2122?1 D5BFC5E624BF5D27RRA34

212411411121114003 90EDA4A17RB588GQKK663

2101111100002 ?? ? ? 54AEA5C5349B4B64KKB33

2101111100002 ?? ??? 548B75A6377C3A55JJ933

21011251000 ?1? ?? ?? 83CFA5C86MDGAD9FFEA79

213100003312000103 44BF75A6268D6C78KKC4 4

2115129213122 ????? 647C85B5359E1B27KJA13 
A. nyg A62

A._pergla_f_A38

A._pfaffiāna_FH37

A. solida $\mathrm{FH}_{\overline{2}} 3$

A. sub ROR

A. sub_T702

A. $\mathrm{sp} .{ }^{3} \mathrm{ROR}$

A._distañs_f_ADE

A._paucistriāta_2035

A. $\mathrm{sp} .4$ T733

A. $\mathrm{sp} \cdot{ }^{5} \mathrm{~h}$

A._sp. ${ }^{-}{ }^{-}$hwg

A._cf._distans_1571

A. humilis

A. tenuoir

A. tenella

A. crassipunctata

A. tethera

A._pseudoamericana

A. muzzanensis

A._pergla_f_KI

A._ceretaña $\begin{array}{cccc}\text { Qualitative Chars Morphometric Chars ] } & \text {. }\end{array}$

2115129213122 ?? ?? $656 \mathrm{C} 75 \mathrm{~A} 624 \mathrm{BG} 1924 \mathrm{KJ} 8 \mathrm{D} 1$

0121003285110003 ? 1 246D? 55043B??????A?

210112310002100102 432B759316364F67GF534

212000002120014003 74JMB5C1GP9B88F8?KC62

$2111000021200000115270546327493 A 46 ? 7531$

$211100002120000011839 H 54814$ H7G4D49?7433

$211100002123000102 \quad 2223244316101 B 17 ? 3113$

2101126100001 ?? ?? 458G6587387N3C46B9734

213000001120014003 94MCD5F2 JKKEBAR8? KHD5

2113111111101????? F5RGN5G667PERCH9KJLKD

0111526185203 ? ? ?? 34AG? ?A41398???? ? A?

0101523111113 ????? 344B? ?8158J????? 5?

2111000021220 ????? 143N24353C1D2E2DA7015

2130000011220 ????? 343H668D16354A95?K235

2 ? 11000021120 ?? ?? 6???6?? ?G???3 ?3 ?L?6E?

2?12000011120001?1 0???0???????2?2??K00?

2?03111100021?40?3 C???E???M???D?H?L?38?

2?01115100011000?1 2???2???????0?7?L?01?

0 ?01529100013006?1 2?????????????????D??

2?41116121211????? 4?8?5?0?3?8?5?B?BKL4?

2? 11216111121 ?? ?? 1???2?? ?A? ? 1 ?1 ?LKG0?

211211611112104033 D1B? 7?C?Q?J?A?G?LKPD?

Characters in columns from left to right are as listed 1-39 in the Material and Methods section of Chapter 2, pages 41 to 50 . 
Appendix K. Morphological Character States as Scored for Gap Weighting Without Step-Matrices Limited to 10 Character States. 
[

M._varians_CHMP7_FFP

S._cf._bros̄chii_̄̄B

A._islāndica_CHM̄P1_MICH

A. skvortzowii

A._baicalensis

A._sub_9D1

A._sub_WLB3

A. $s p .{ }^{3}$ FR

A._sub_ERB

A. sub_LEW

A._alpigena

A._amb_PII 7

A._amb_N17

A. amb-FL8

A.

A._amb_WLP1

A. nyassensis GB

A._valida_N2

A._gran_LB

A._gran_OR12

A. $-\mathrm{sp} \cdot \overline{1}$

A._sp. 2

A. $\operatorname{cren}$ FH8 L639

A._cren_T73 $\overline{3}$ L639

Alveolophora

A._agassizii_FH21

A. amb_A62

A. amb_MFP

A. amb_WAT

A. canād 1571

A. canad 2018

A._dianchiensis

A. distans FH34

A._gran_FH $\overline{4} 2$

A._gran_FH38

A. gran_1914

A._gran_MFP

A._gran_V._jonensis

A._gran_f_HLS

A. gran f- $\mathrm{VH}$

A._herzogii_HDSM

A._italica_FH15

A._krammerii_nom._prov.

A._lac_A38

A. 1 - 1 - $\mathrm{A} 62$

A. lirāta A37

A._laevissima_HDSM

A._nyg_A50
Qualitative Chars Morphometric Chars

1001000000020007134142 ? ? ? ? ? ? ? ? ? ? 8?

100000000002020443 ???????????????????

211111611112100503515532411134232266410

211511611112100513412532441335142277211

$21111141111 ? 100523966692972378655376576$

2111000021200000113137223115331423 ? 4111

2111000021200000112146213025361321 ? 1310

2111000021230001021101121103030302 ?0001

2111000021200000112127222025151413 ?2211

2111000021200000112156223135361422 ? 4311

2111126111001 ? ? ?? ????? ? ? ? ? ?? ?? ? ?

221111611110100011324332531247141221311

221111611110100011212622321338141221111

221111611110100011332422321336141221111

221111611110100011311460410339141210111

221111611110100011413532511245141210211

2211116111121001 ?3 525249511143533266741

21002272000110001142683251255624210 ?311

2141116121201 ????? 503522330225493757249

214111612120100231442222440326353956125

$210512620001100202 \quad 22383234122828207 ? 312$

2111126111101 ????? 291644590033132377013

2100327100012032 ? 1425662831267351388412

2100327100012032 ? 429572723296242288921

2112116111121000 ?3 218232419132335177931

2141116121221001 ? 317742413243243177521

2211116111101000113 ?2412321438142221111

221111611110100011413432411346141222211

221111611110100011313422411245131332211

211211611110114033214732312735445576133

211211611110114033217832413747638377376

21311161212 ?111601 937352601353807377392

210111211102100123110522201503141377011

214111612120101201413442510225354457322

214111612120101201418842512254435267441

2141????21201003?2 $4069325237453430 ? 7321$

2141116121201001 ? 514532421237343257232

2241116121201012 ? 111112210113142246211

2111????11121????? 511142503612636476362

2111????11121????? 814261702621546477353

213100005422000311422422320224130177210

$21004271000 ? 2122$ ? 1524542521145251299311

$212411411121114003 \quad 305532403942336977221$

2101111100002 ?? ?? 224532421134142177411

2101111100002 ????? 223432421234142276311

21011251000 ? ????? 314532432856453565323

213100003312000103224532321235242377411

2115129213122 ????? 222432421235041276301 
A. nyg A62

A._pergla_f_A38

A._pfaffiāna_FH37

A. solida $\mathrm{FH}_{\overline{2}} 3$

A. sub ROR

A. sub_T702

A. $\mathrm{sp} .{ }^{3} \mathrm{ROR}$

A. distāns f $\mathrm{ADE}$

A._paucistriāta_2035

A._sp. 4 - T733

A. $\mathrm{sp} \cdot{ }^{5} \mathrm{~h}$

A._sp. ${ }^{-}{ }^{-}$hwg

A._cf._distans_1571

A. humilis

A. tenuoir

A. tenella

A. crassipunctata

A. tethera

A._pseudoamericana

A. muzzanensis

A. -pergla_f_KI

A._ceretaña
Qualitative Chars Morphometric Chars ]
2115i29213122????? 22243242114603i177350

0121003285110003 ? 1225??220114??????3??

210112310002100102111432310212152366212

$212000002120014003 \quad 2278424168343353$ ? 7421

2111000021200000112120222112231422 ?3210

2111000021200000113136223116361523 ? 3111

2111000021230001021111121102000402 ?1001

2101126100001 ?? ?? 123622321338142243311

$213000001120014003 \quad 3284525167754393$ ? 7652

2113111111101 ????? 529682622285946376775

0111526185203 ????? 1146? ?410133????? 4??

0101523111113 ????? 1114??330237??????2??

2111000021220 ????? 111812121405151533002

2130000011220 ????? 1116223502122332?7112

2?11000021120????? 2???2???6???1?1?7?52?

2?12000011120001?1 0???0???????1?1??720?

2?03111100021?40?3 4???5???8???5?6?7?21?

2?01115100011000?1 1???1???????0?2?7?30?

0 ?01529100013006?1 1?????????????????4??

2?41116121211????? 2?3?2?0?1?3?2?4?4772?

2 ? 11216111121 ????? 1???1???4 ?? 0?0?7750?

211211611112104033 514?3?4?9?6?4?6?7795?

Characters in columns from left to right are as listed 1-39 in the Material and Methods section of Chapter 2, pages 41 to 50. 
Appendix L. Morphological Character States as Scored via Quasi-Statistical Student's T-test Gap Identification Method. 
[

M._varians_CHMP7_FFP

S._cf._bros̄chii_̄̄B

A._islāndica_CHM̄P1_MICH

A. skvortzowii

A._baicalensis

A._sub_9D1

A._sub_WLB3

A. $s p .{ }^{3}$ FR

A._sub_ERB

A. sub_LEW

A._alpigena

A._amb_PII 7

A._amb_N17

A. amb-FL8

A.

A._amb_WLP1

A. nyas̄sensis GB

A._valida_N2

A._gran_LB

A._gran_OR12

A. $-\mathrm{sp} \cdot \overline{1}$

A._sp. 2

A. crēn FH8 L639

A._cren_T73 $\overline{3}$ L639

Alveolophora

A._agassizii_FH21

A. amb_A62

A. amb_MFP

A. amb_WAT

A. canād 1571

A. canad 2018

A._dianchiensis

A. distans FH34

A._gran_FH $\overline{4} 2$

A._gran_FH38

A. gran_1914

A._gran_MFP

A._gran_V._jonensis

A._gran_f_HLS

A. gran f- $\mathrm{VH}$

A._herzogii_HDSM

A._italica_FH15

A._krammerii_nom._prov.

A._lac_A38

A. 1 - 1 - $\mathrm{A} 62$

A. lirāta A37

A._laevissima_HDSM

A._nyg_A50
Qualitative Chars Morphometric Chars

100100000002000713413 ?? ?? ?? ?? ?? ? 5?

100000000002020443 ??????????????????

21111161111210050361321413052414043360

21151161111210051351321423152415154350

$21111141111 ? 10052392342614172 d 171543 F 0$

211100002120000011403214141524131 ?2351

211100002120000011303204141524130 ? 0350

$211100002123000102100111211010110 ? 0130$

211100002120000011303212141223131 ? 0351

211100002120000011313214141524231 ?2351

2111126111001 ?? ?? ?? ?? ????????? ?? ??

22111161111010001142322423162423120350

22111161111010001131322423152423110351

$221111611110100011 \quad 32321423152423110351$

22111161111010001140220412152423110250

22111161111010001150321413162413110350

$2211116111121001 ? 3 \quad 62322423162 \mathrm{~A} 060434 \mathrm{CO}$ $2100227200011000115232242416242300 ? 361$

2141116121201 ????? 613214221529271343C1

$214111612120100231 \quad 523224221427251342 \mathrm{~A} 1$

$210512620001100202 \quad 3232242315272505$ ?361

2111126111101 ????? 31222422051414054151

2100327100012032 ? 15232262317272316 ? 361

2100327100012032 ? 15243262418262315 ? 571

2112116111121000 ?3 $313214250517180545 \mathrm{CO}$

2141116121221001 ? 140321414162626054391

2211116111101000113 ?321423152413020351

22111161111010001150321423162423120351

22111161111010001141321413162413120350

$211211611110114033 \quad 303214141529181543 B 1$

$21121161111011403330321414162 \mathrm{C} 1 \mathrm{~A} 0543 \mathrm{~F} 1$

21311161212 ?111601 $92321414161 \mathrm{E} 090543 \mathrm{G} 0$

2101112111021001232012131311132315 ? 141

$214111612120101201503214121528271343 \mathrm{~A} 1$

$214111612120101201503214141629170443 \mathrm{CO}$

2141 ???? 21201003 ? 2503214141627160 ?4380

$2141116121201001 ? 1 \quad 603224231528271343 \mathrm{Cl}$

2241116121201012 ? 30211310022323034340

2111????11121????? 60221404121C181543E1

2111????11121????? 80320514150B191543D1

$213100005422000311523214221523000 ? 4340$

$21004271000 ? 2122$ ? 16232242316242217 ? 351

21241141112111400340320414161709154391

2101111100002 ????? 3232142315242405?351

2101111100002 ????? 3232142315241415?351

21011251000 ?1????? 3032242416292714?3A1

$213100003312000103 \quad 323214231525251 ? 4361$

2115129213122 ????? 32321423152112154331 
A. nyg A62

A._pergla_f_A38

A._pfaffiāna_FH37

A. solida $\mathrm{FH}_{\overline{2}} 3$

A. sub ROR

A. sub_T702

A. $s p .-3$ ROR

A. distañs f $\mathrm{ADE}$

A._paucistriāta_2035

A._sp. 4 - T733

A. $\mathrm{sp} \cdot{ }^{5} \mathrm{~h}$

A._sp. ${ }^{-}{ }^{-}$hwg

A._cf._distans_1571

A. humilis

A. tenuoir

A. tenella

A. crassipunctata

A. tethera

A. pseudoamericana

A. muzzanensis

A. -pergla_f_KI

A._ceretaña
Qualitative Chars Morphometric Chars ]
2115129213122????? 32321423152011054330

0121003285110003 ? 123 ??221022? ?????3??

$210112310002100102 \quad 30222412121425143351$

$212000002120014003 \quad 323224151527081$ ? 4390

211100002120000011303213231324131 ? 1350

211100002120000011303214141524231 ? 1351

$211100002123000102 \quad 201111121101111$ ? 0131

2101126100001 ?? ?? 32322423152423132351

$213000001120014003 \quad 4232241517291 B 0 ? 44 \mathrm{D} 0$

2113111111101 ????? 71442424182F191544F1

0111526185203 ????? 213??422051??????3??

0101523111113 ?? ?? 212? ?422152? ?? ? ?3 ??

2111000021220 ????? 112111231122221?1031

2130000011220 ????? 212224221214160?4250

2?11000021120????? 3? ?2?? ?5?? 3 ?3 ?5?39?

2?12000011120001? 0 ??0???????2?1?5430?

2 ? 03111100021 ?40?3 5??2???5???A?9?5?35?

2?01115100011000?1 1??1???????E?5?5?31?

0 ?01529100013006? 1 2????????????????3??

2?41116121211????? 3?32?0?3?5?7?7?3447?

2?11216111121????? 1? 1? ? 4 ?? 0?1?5432?

211211611112104033 6032?4?5?7?9?9?545D?

Characters in columns from left to right are as listed 1-39 in the Material and Methods section of Chapter 2, pages 41 to 50, except for the slope of the principal axis regression for mantle thickness versus valve diameter since all taxa had significantly positive slopes. 
Appendix M. Morphological Character States as Scored Using Statistical Homogeneous Subset Coding Method. 
[

M._varians_CHMP7 FFP

S. Cf. broschii GB

A._islāndica_CHM̄P1_MICH

A._skvortzowii

A. baicalensis

A._sub_9D1

A._sub_WLB3

A. $\mathrm{sp} .3 \mathrm{FR}$

A. Sub ERB

A._Sub_LEW

A._alpigena

A. amb PII7

A._amb-N17

A._amb_FL8

A. amb BDL1

A._amb_WLP1

A._nyassensis_GB

A. valida N2

A. gran_LB

A._gran_OR12

A._sp._1

A. $\mathrm{sp} .-2$

A._Creñ_FH8_L639

A. Cren T733 L639

Alv̄eolophora

A._agassizii_FH21

A. amb A62

A. amb MFP

A._amb_WAT

A._canād 1571

A. canad 2018

A._dianch̄iensis

A._distans_FH34

A. gran FH42

A._gran_FH38

A._gran_1914

A._gran_MFP
Qualitative Chars 100100000002000713 100000000002020443

211111611112100503 211511611112100513 21111141111?100523 211100002120000011 211100002120000011 211100002123000102 211100002120000011 211100002120000011 2111126111001 ????? 221111611110100011 221111611110100011 221111611110100011 221111611110100011 221111611110100011 2211116111121001?3 210022720001100011 2141116121201 ????? 214111612120100231 210512620001100202 2111126111101????? 2100327100012032 ? 2100327100012032 ? 1 2112116111121000?3 2141116121221001?1 221111611110100011 221111611110100011 221111611110100011 211211611110114033 211211611110114033 21311161212 ? 111601 210111211102100123 214111612120101201 214111612120101201 2141????21201003?2 2141116121201001?1
SLOPES

1???????

?? ? ? ? ? ?

11102100

11212110

22112110

01112111

00112100

01211100

01112111

11112211

? ? ? ? ? ?

22212210

12212211

21212211

00112210

01112110

22212000

22212201

11212211

22212211

22212201

12201101

22212211

22212211

11201100

01112201

?1212101

01212211

11112110

01112111

01112101

21111000

01111211

01112211

01112100

01112100

02212211 mntl ht ใน? 11111111111110000 11111111111110000 11111111111111111 11111111100000000 11111110000000000 10000000000000000 11111110000000000 11111100000000000 ? ? ? ? ? ? ? ? ? ? ? ? ? ?

11111111000000000 11111110000000000 11111111000000000 11111111100000000 11111111111100000 11111111111110000 11111111111100000 11111111111111100 11111111111110000 11111110000000000 11111110000000000 11111111111100000 11111111111100000 11111100000000000 11111111110000000 11111111000000000 11111111111000000 11111111111000000 11111110000000000 11111111000000000 11111111111111110 11100000000000000 11111111111100000 11111111111110000 11111111111000000 11111111111111000 
[

A. gran_v. jonensis

A._gran_f_HLS

A._gran $f-V H$

A. herzogii HDSM

A. italica FH15

A._krammerīi_nom._prov.

A. lac A38

A. lac A62

A._liráta_A37

A._laevissima_HDSM

A. nyg_A50

A._nyg_A62

A. pergla f A38

A. pfaffiana FH37

A. solida $\mathrm{FH}_{\overline{2}} \overline{3}$

A._sub_ROR

A._sub_T702

A._sp. 3 ROR

A._distañs_f_ADE

A. paucistriata 2035

A. sp. 4 T733

A._sp. -5 - $h$

A. sp. 6 hwg

A. cf. distans 1571

A._humilis

A. tenuoir

A. tenella

A._crassipunctata

A._tethera

A. pseudoamericana

A. muzzanensis

A. -pergla_f_KI

A. ceretaña
Qualitative Chars 2241116121201012?1

2111????11121?????

2111 ??? ?11121????

213100005422000311

$21004271000 ? 2122$ ? 1

212411411121114003

2101111100002 ?????

2101111100002?????

21011251000 ? 1????

213100003312000103

2115129213122 ?????

2115129213122 ?????

0121003285110003 ? 1

210112310002100102

212000002120014003

211100002120000011

211100002120000011

211100002123000102

2101126100001 ????

213000001120014003

2113111111101 ?????

0111526185203 ?? ??

0101523111113 ?????

2111000021220 ?????

2130000011220 ?????

2 ? 11000021120 ????

2 ? 12000011120001 ?

2?03111100021?40?3

2?01115100011000? 1

0 ? 01529100013006 ?

2? 41116121211 ?????

2?11216111121?????

211211611112104033
01011111

00110111

21212000

22212211

00111011

21212201

21212111

02212211

21212211

21212111

21212100

2 ? 202 ?? ?

02111211

22112010

01212110

01112211

01110111

22212211

22112100

12212111

1?201???

1 ?212???

11212211

12211100

? ?? ? ? ?

??????? ?

? ? ? ? ? ?

? ? ? ? ? ?

?? ?? ? ? ?

? ? ? ? ? ?

? ? ? ? ? ?

0 ? ? ? ? ? ? mntl ht

000000000

11111111111110000

11111111111111110

11111111111100000

11111111111110000

11111111110000000

11111110000000000

11111110000000000

11111111000000000

11111100000000000

11111110000000000

11111110000000000

10000000000000000

11110000000000000

11111111000000000

11111110000000000

11111111000000000

11000000000000000

11111000000000000

11111111000000000

11111111111110000

11110000000000000

11100000000000000

10000000000000000

11100000000000000

11111110000000000

00000000000000000

11111111111100000

10000000000000000

11000000000000000

11111100000000000

10000000000000000

11111111111110000 
[ varians CHMP7 FFP S._cf.broschii_GB A._islandica_CHM̄PI_MICH

A. baicalensis

A._sub_9D1

A. sub_WLB3

A. $\mathrm{sp} .3 \mathrm{FR}$

A._sub_ERB

A._sub_LEW

A. alpigena

A._amb_PII 7

A. $a m b$ N17

A. amb FL8

A. amb_BDL1

A._amb_WLP1

A. nyassensis GB

A. valida N2

A._gran_LB

A. gran OR12

A._sp. 1

A. $-\mathrm{sp} \cdot{ }_{-2}$

A. cren FH8 L639

A. cren T73 $\overline{3}$ L639

Alveolophora

A._agassizii FH2I

A. amb A62

A._amb_MFP

A._amb_WAT

A. canad 1571

A. canad 2018

A._dianchiensis

A. distans FH34

A. gran_FH4 2

A._gran_FH38

A. gran 1914

A._gran_MFP mantle thk

1111111111111111111111111110000000000000 ? ? ? ? ? ? ? ? ? ? ? ? ? ? ? ? ? ? ? ? ? ? ? ? ? ? ? ? ? 1111111111111111111111111111111000000000 1111111110000000000000000000000000000000 1111111111111111111111111111111111100000 1111111111111111111110000000000000000000 1111111111111111111111111111000000000000 000000000000000000000000000000000000000 1111111111111000000000000000000000000000 1111111111111111111111111111111110000000 ????????????????????????????????????? ?? 1111111111111111111111111100000000000000 1111111111100000000000000000000000000000 1111111111100000000000000000000000000000 1111100000000000000000000000000000000000 1111111111111111000000000000000000000000 1111111111111111111111111111111110000000 1111111111111111111111111111111111000000 1111111111111111111000000000000000000000 1111111111100000000000000000000000000000 1111111111111111111100000000000000000000 1111000000000000000000000000000000000000 1111111111111111111111111111110000000000 1111111111111111111111111111111111111111 1111111111111111111111111111111111111110 1111111111111111111111111111111111111100 1111111110000000000000000000000000000000 1111111111111111111100000000000000000000 1111111111111111111100000000000000000000 1111111111111111111111111110000000000000 1111111111111111111111111111111111111000 1111111111111111111111111111111111111000 1000000000000000000000000000000000000000 1111111111111111111100000000000000000000 1111111111111111111111111111111111111110 1111111111111111111111111111111111110000 1111111111111111111111111100000000000000 collum height ] ?? ? ? ? ? ? ? ? ? ? ? ? ? ? ? ? ? ? ? ? ? ? ? ? ? ? ? ? ? ? ? ? ? ? ? ? ? 111111111111111100000000000 111111111111111100000000000 111111111111111111111111111 111111100000000000000000000 111111000000000000000000000 110000000000000000000000000 111100000000000000000000000 111111000000000000000000000 ??????? ????????? ???????? ? ? 111111111110000000000000000 111111000000000000000000000 111110000000000000000000000 111111111111111111111110000 111111111110000000000000000 111111111111111110000000000 111111111111111100000000000 111111111000000000000000000 111111110000000000000000000 111111111110000000000000000 111111111111111110000000000 111111111111111111111111000 111111111111111111111111100 111111111110000000000000000 111111111111111111000000000 111000000000000000000000000 111111111110000000000000000 111111100000000000000000000 111111111111110000000000000 111111111111111100000000000 111111111111111111111100000 111111000000000000000000000 111111111111111110000000000 111111111111111111100000000 111111111111111100000000000 111111111111110000000000000 
[ A._gran_v._jonensis A. gran f A. $\operatorname{gran}_{-}^{-} \mathrm{VH}$ A. herzogii HDSM

A. -italica_ $\overline{\mathrm{F}} \mathrm{H} 15$ A._krammerii_nom._prov A._lac_A38

A. lac A62

A._lirata_A37

A._laevissima_HDSM

A. nyg_A50

A._nyg_A62

A._pergla_f_A38

A. pfaffiana FH37

A. solida_ $\mathrm{FH} \overline{2} 3$

A._Sub_ROR

A._sub_T702

A. $\mathrm{sp} .{ }^{3} \mathrm{ROR}$

A._distañs_f_ADE

A. paucistriata_2035

A. sp. 4 T733

A. . sp. ${ }^{5}-\mathrm{h}$

A. sp. 6 hwg

A. Cf. distans 1571

A. humilis

A. tenuoir

A. tenella

A. Crassipunctata

A. tethera

A. pseudoamericana

A. muzzanensis

A._pergla_f_KI

A._ceretaña mantle thk

1111111000000000000000000000000000000000 1111100000000000000000000000000000000000 1111111111111111111111110000000000000000 111111111111000000000000000000000000000 111111111111111111111111110000000000000 .11111111111111111111111111111100000000 11111111111111111111000000000000000000 1111111111111111100000000000000000000000 111111111111111111111111111100000000000 11111111111111111111111100000000000000 1111111111111110000000000000000000000000 1111111111000000000000000000000000000000 1111111111110000000000000000000000000000 1110000000000000000000000000000000000000 1111111111111111111111111111111111111000 111111111111100000000000000000000000000 111111111111111111111100000000000000000 1100000000000000000000000000000000000000 11111111111111000000000000000000000000 111111111111111111111111111111111111110 1111111111111111111111111111111111111111 111111111111111111111111000000000000000 1111111100000000000000000000000000000000 1111100000000000000000000000000000000000 1111110000000000000000000000000000000000

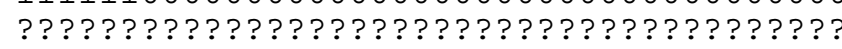

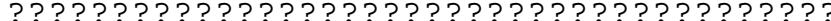

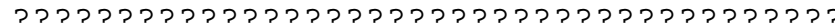

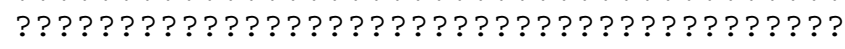

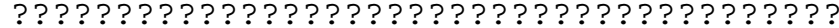
1111111111111111110000000000000000000000

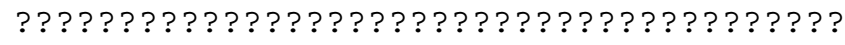
11111111111111111111111110000000000000 collum height ] 00000000000000000000000000 111111111111111111110000000 111111111111111111111111000 111111111000000000000000000 111111111111111111111000000 111111111111111000000000000 111111111111111100000000000 111111111110000000000000000 111111111111111100000000000 111111111100000000000000000 111111111111100000000000000 111111111110000000000000000 ? ? ? ? ? ? ? ? ? ? ? ? ? ? ? ? ? ? ? ? 111111111111000000000000000 111111111111111111000000000 111100000000000000000000000 111100000000000000000000000 110000000000000000000000000 111111100000000000000000000 111111111111111111111000000 111111111111111111111111110 ? ? ? ? ? ? ? ? ? ? ? ? ? ? ? ? ? ? ? ? ? ? ? ? ? ? ? ? ? ? ? ? ? ? ? ? ? ? ? ? 100000000000000000000000000 111111100000000000000000000 111111111100000000000000000 000000000000000000000000000 111111111111111111111100000 000000000000000000000000000 ? ? ? ? ? ? ? ? ? ? ? ? ? ? ? ? ? ? ? ? 111111000000000000000000000 110000000000000000000000000 111111111110000000000000000 
[ varians CHMP7 FFP S._cf.broschii GB A._islandica_CHM̄PI_MICH A. skvortzowii

A._baicalensis

A._sub_9D1

A. sub WLB3

A. $\mathrm{sp} .3 \mathrm{FR}$

A._Sub_ERB

A._Sub_LEW

A. alpigena

A._amb_PII 7

A. $a m b$ N17

A. amb FL8

A. $a m b-B D L 1$

A._amb_WLP1

A. nyassensis GB

A. valida N2

A._gran_L $\overline{\mathrm{B}}$

A. gran OR12

A._sp. 1

A._sp._2

A. cren_FH8 L639

A. cren T733 L639

Al v̄eolophora

A._agassizii_FH2I

A. amb_A62

A._amb_MFP

A._amb_WAT

A. canad 1571

A. ${ }^{-}$canad 2018

A._dianchiensis

A. distans_FH34

A._gran_FH42

A._gran_FH38

A. gran 1914

A._gran_MFP rnglst ht

?????????????????????????????? ???????????????????????? ??? ?? ? 1111111111111111111100000000000 111111111111111110000000000000 1111111111111111111111111111111 1111110000000000000000000000000 1111111110000000000000000000000 0000000000000000000000000000000 1100000000000000000000000000000 1111111100000000000000000000000 ??????????????????????????????? 1111111111111111111111100000000 1111111110000000000000000000000 111111111100000000000000000000 1111111111111111110000000000000 1111111111111111111111100000000 11111111111111111111100000000 1111111111111111111111111100000 1111111110000000000000000000000 1111111111111000000000000000000 1111111110000000000000000000000 1111111111111111111100000000 1111111111111111111111111111111 111111111111111111111111111111 111111111111111111100000000000 1111111111111111111111000000000 1111111111000000000000000000000 1111111111111111111110000000000 1111111111111111000000000000000 1111111111000000000000000000000 1111111111111111000000000000000 1111111111111111111111111111000 1110000000000000000000000000000 1111111111111111111111110000000 11111111111111111111111110000 1111111111111111111111100000000 111111111111111111111000000000 rnglst dpi

??? ? ? ? ? ? ? ? ? ? ? ? ? ? ? ? ? ? ? ? ? ? ? ? ? ? ? ? ? ? ? ? 111111111100000000000000 111111111111000000000000 111111111111111111000000 111111111111111110000000 111111111111111110000000 100000000000000000000000 111111111111111110000000 111111111111111111111000 ???????????????????????? 111111111111000000000000 111111111110000000000000 111111111100000000000000 111111000000000000000000 111111111110000000000000 111111111111000000000000 111111111111111111100000 111111100000000000000000 111100000000000000000000 111111111100000000000000 111110000000000000000000 111111111111111100000000 111111111111111111111000 111111111111111111111111 111111111111111111111000 111111111110000000000000 111111111111100000000000 111111111111000000000000 111111111111111111000000 111111111111111111111000 111111111111111110000000 111111111111110000000000 111000000000000000000000 111111111111111111110000 111111111111111111111000 111111111000000000000000 
[

gran v. jonensis

A._gran_f_HLS

A._gran $f-V H$

A. herzogii HDSM

A. italica FH15

A._krammerii_nom._prov.

A. ${ }^{\prime a c}$ A38

A. lac A62

A._liráta_A37

A._laevissima_HDSM

A. nyg A50

A._nyg_A62

A._pergla f A38

A. pfaffiana FH37

A. solida_FH 23

A._Sub_ROR

A._sub_T702

A. $\mathrm{sp} .{ }^{3} \mathrm{ROR}$

A._distañs_f_ADE

A. paucistriata 2035

A. sp. 4 T733

A. $-\mathrm{sp} \cdot{ }^{-}{ }^{-} \mathrm{h}$

A. sp. 6 hwg

A. Cf. distans 1571

A._humilis

A. tenuoir

A. tenella

A._crassipunctata

A. tethera

A. pseudoamericana

A. muzzanensis

A. -pergla_f_KI

A. ceretaña rnglst ht

1111000000000000000000000000000 1111111111111111111111111000000 1111111111111111111111111111110 1111110000000000000000000000000 1111111111111111111111110000000 111111111110000000000000000000 111111111111111110000000000000 1111111111111100000000000000000 1111111111111111100000000000000 1111111111000000000000000000000 1111111111111110000000000000000 1111111111111100000000000000000 1000000000000000000000000000000 1111111100000000000000000000000 1111111111111111111000000000000 1110000000000000000000000000000 1111111100000000000000000000000 0000000000000000000000000000000 1111111000000000000000000000000 111111111111111111111111100000 1111111111111111111111111111100 1111111111100000000000000000000 1111111000000000000000000000000 0000000000000000000000000000000 1111100000000000000000000000000

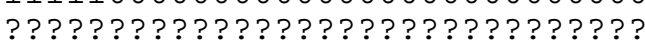
द?

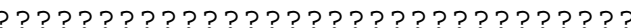

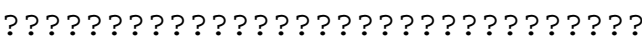
???????????????????????????????? 0000000000000000000000000000000 ? ? ? ? ? ? ? ? ? ? ? ? ? ? ? ? ? ? ? ? ? ? ? ? 111111111111111111100000000000 rnglst dpi ]

000000000000000000000000 11111111111111111111000 111111111111111111000000 111111000000000000000000 111111110000000000000000 11111111111111111100000 11111111111100000000000 111111111111000000000000 111111111111111110000000 111111111111000000000000 111111111111000000000000 111111111100000000000000 110000000000000000000000 111110000000000000000000 111111111111111111111100 111111111000000000000000 111111111111111110000000 111100000000000000000000 111111111111000000000000 11111111111111111111110 111111111111111111100000 111110000000000000000000 111100000000000000000000 111111111111111000000000 111100000000000000000000 111111111111111111111100 ???????????????????????? 111111111111111111111110

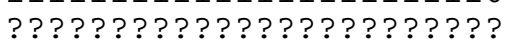
? ? ? ? ? ? ? ? ? ? ? ? ? ? ? ? ? ? ? ? ? 111111111111000000000000 11111111111111111111000 11111111111111111111111 
[ varians CHMP7 FFP S. Cf. bros̄chii $\overline{G B}$ A._islāndica_CHM̄P1_MICH A. skvortzowi

A. baicalensis

A._sub_9D1

A. sub_WLB3

A. $\mathrm{sp} .3 \mathrm{FR}$

A._sub_ERB

A._sub_LEW

A. alpigena

A._amb_PII 7

A. $a m b$ N17

A. amb FL8

A. amb_BDL1

A._amb_WLP1

A. nyassensis GB

A. valida N2

A._gran_LB

A. gran OR12

A._sp. 1

A._sp. 2

A. cren FH8 L639

A. cren T73 $\overline{3}$ L639

Alveolophora

A._agassizii FH2I

A. amb A62

A._amb_MFP

A._amb_WAT

A. canad 1571

A. canad 2018

A._dianchiensis

A. distans FH34

A. gran_FH4 2

A._gran_FH38

A. gran 1914

A._gran_MFP rnglst thk

?? ?? ?? ? ? ? ? ? ? ? ? ? ? ? ? ? ? ? ? ? ? ?? ?? ? ? ? ? ? ? ? ? ? ? ? ? ? ? ? ? ? ? 11111111111111111110000000000000 11111111111111111000000000000000 11111111111111111111111111111100 11111111111110000000000000000000 11111111111111111000000000000000 00000000000000000000000000000000 11111100000000000000000000000000 11111111111111100000000000000000 ??????????????????????????????? 11111111111111111111111111000000 11111111111111111100000000000000 11111111111111111110000000000000 11111111111110000000000000000000 11111111111111111111111100000000 11111111111111111111111100000000 11111111111111111111111111110000 11111111100000000000000000000000 11111111000000000000000000000000 11111111110000000000000000000000 11111111111111000000000000000000 11111111111111111111111111111000 11111111111111111111111111111111 11111111111111111110000000000000 11111111111111111111111110000000 11111111111111111111000000000000 11111111111111111111110000000000 11111111111111111111111100000000 11111111111111000000000000000000 11111111111111111111111110000000 11111111111111111111111111100000 11100000000000000000000000000000 11111111111100000000000000000000 11111111111111111111111111110000 11111111111111111111111000000000 11111111111111110000000000000000 $\operatorname{ard1}$

ใ? ? ? ? ? ? ? ? ? ? ? ? ? ? ? ? ? ? ? ? ? ? ? ? ? ? ? ? ? ? ? ? ? ? ? ? ? ? ? ? ? ? ? ? ? ? 11111111111111100000000000000000 11111111110000000000000000000000 11111111111111111111111111111110 11111111111100000000000000000000 11111111111000000000000000000000 00000000000000000000000000000000 11111100000000000000000000000000 11111111111111000000000000000000 ?? ?? ?? ? ? ? ? ? ? ? ? ? ? ? ? ? ? ? ? ? ? 11111111111000000000000000000000 11111111000000000000000000000000 11111111110000000000000000000000 11111111111000000000000000000000 11111111111111000000000000000000 11111111111111111111111111111000 11111111111111110000000000000000 11111111111111111111111110000000 11111111111111111110000000000000 11111111111111111100000000000000 11111111100000000000000000000000 11111111111111111110000000000000 11111111111111111100000000000000 11111111111111111111000000000000 11111111111111111100000000000000 11111111111110000000000000000000 11111111110000000000000000000000 11111111111110000000000000000000 11111111111111111111111111110000 11111111111111111111111111111100 11111111111111111111111111111111 11110000000000000000000000000000 11111111111111111111110000000000 11111111111111111111111111100000 11111111111111111111100000000000 11111111111111111111111000000000 
[

A. gran_v._jonensis

A. gran f

A._gran $\mathrm{f}_{-\mathrm{VH}}$

A. herzogii HDSM

A. italica_ $\overline{\mathrm{F}} \mathrm{H} 15$

A._krammerii_nom._prov.

A._lac_A38

A. ${ }^{-1 a c}$ A62

A._lirata_A37

A._laeviss̄ima_HDSM

A. nyg_A50

A._nYg_A62

A._pergla_f_A38

A. pfaffiana FH37

A. Solida_FH $\overline{2} 3$

A._sub_ROR

A._sub_T702

A. $\mathrm{sp} .{ }^{3} \mathrm{ROR}$

A._distañs_f_ADE

A. paucistriata 2035

A. sp. 4 T733

A. $-\mathrm{sp} \cdot{ }^{5}-\mathrm{h}$

A. sp. 6 hwg

A. Cf. distans 1571

A. humilis

A. tenuoir

A. tenella

A._crassipunctata

A. tethera

A. pseudoamericana

A._muzzanensis

A. -pergla_f_KI

A._ceretaña rnglst thk

11111100000000000000000000000000 11111000000000000000000000000000 11111111111100000000000000000000 1111111110000000000000000000000 11111111111111111111110000000000 111111111111111111111000000000 1111111111111111100000000000000 11111111111111000000000000000000 1111111111111111111111111100000 111111111111111000000000000000 11111111111111111000000000000000 11111111110000000000000000000000 11111100000000000000000000000000 11111100000000000000000000000000 11111111111111111111100000000000 11111110000000000000000000000000 11111111111111000000000000000000 10000000000000000000000000000000 11111111111110000000000000000000 11111111111111111111111111111100 11111111111111111111111111111110 11111111111111111110000000000000 11111111111111000000000000000000 11000000000000000000000000000000 11110000000000000000000000000000 ? ? ? ? ? ? ? ? ? ? ? ? ? ? ? ? ? ? ? ? ? ? ? ? ? ? ? ? ? ? ? ? ? ? ? ? ? ? ? ? ? ? ? ? ? ? ? ว ? ? ? ? ? ? ? ? ? ? ? ? ? ? ? ? ? ? ? ? ? ? ? ? ? ? ? ? ? ? ? ? ? ? ? ? ? ? ? ? ? ? ? ? ? ? ? ? ? ? ? ? ? ? ? ? ? ? ? ? ? ? ? ? ? ? ? ? 11111111111000000000000000000000 ? ? ? ? ? ? ? ? ? ? ? ? ? ? ? ? ? ? ? ? ? ? ? 11111111111111111111111111111000 $\operatorname{ardl}$

11111000000000000000000000000000 11111111111111111111111111111110 11111111111111111111111111111100 11111110000000000000000000000000 11111111111111110000000000000000 11111111111111111110000000000000 11111111111000000000000000000000 11111111110000000000000000000000 11111111111111111111111100000000 11111111111111111000000000000000 10000000000000000000000000000000 00000000000000000000000000000000 ? ? ? ? ? ? ? ? ? ? ? ? ? ? ? ? ? ? ? ? ? ? ? 11111111111100000000000000000000 11111111111111111110000000000000 11111111111000000000000000000000 11111111111000000000000000000000 10000000000000000000000000000000 11111111110000000000000000000000 11111111111111111111111111000000 11111111111111111111111111111111

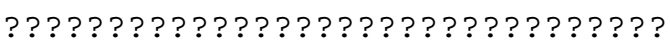
? ? ? ? ? ? ? ? ? ? ? ? ? ? ? ? ? ? ? ? ? ? ? ? ? 11000000000000000000000000000000 11111111111111000000000000000000 11111100000000000000000000000000 11100000000000000000000000000000 11111111111111111111111111111000 11111111111111111111111111111111 ??????????????????????????????? 11111111111111111100000000000000 00000000000000000000000000000000 11111111111111111111111111000000 
[ varians CHMP7 FFP S._cf.broschii_GB A._islāndica_CHM̄P1_MICH A. skvortzowi

A. baicalensis

A._sub_9D1

A. sub WLB3

A. $\mathrm{sp} .3 \mathrm{FR}$

A._sub_ERB

A._sub_LEW

A. alpigena

A._amb_PII 7

A. amb N17

A. amb FL8

A. amb_BDL1

A._amb_WLP1

A. nyassensis GB

A. valida N2

A._gran_L $\bar{B}$

A._gran_OR12

A._sp. 1

A._sp. 2

A._cren_FH8 L639

A. cren T73 $\overline{3}$ L639

Alveolophora

A._agassizii FH2I

A. amb A62

A._amb_MFP

A._amb_WAT

A. canad 1571

A. canad 2018

A._dianchiensis

A. distans_FH34

A. gran_FH4 2

A._gran_FH38

A. gran 1914

A._gran_MFP isd

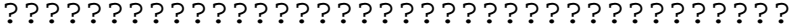
? ? ? ? ? ? ? ? ? ? ? ? ? ? ? ? ? ? ? ? ? ? ? ? ? ? ? ? 11111111111111111110000000000000000000 11111111111111111111000000000000000000 11111111111111111111111111111110000000 11111111111111000000000000000000000000 11111111111000000000000000000000000000 11000000000000000000000000000000000000 11111111110000000000000000000000000000 11111111111110000000000000000000000000

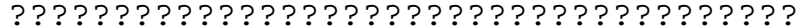
11111111110000000000000000000000000000 11111111000000000000000000000000000000 11111110000000000000000000000000000000 11111111000000000000000000000000000000 11111111100000000000000000000000000000 11111111111111111111111110000000000000 11111111111111100000000000000000000000 11111111111111111111111111110000000000 11111111111111111111111000000000000000 11111111111111111111100000000000000000 11111111111111111100000000000000000000 11111111110000000000000000000000000000 11111111111111000000000000000000000000 11111111111111111111111111111111000000 11111111111111111111111111000000000000 11111111111000000000000000000000000000 11111111100000000000000000000000000000 11111111110000000000000000000000000000 11111111111111111111111111111111000000 11111111111111111111111111111111111110 11111111111111111111111111111111111100 11111100000000000000000000000000000000 11111111111111111111111111111000000000 11111111111111111111111111111100000000 11111111111111111111111111100000000000 11111111111111111111111111110000000000 theta LNK ]

????????????????????? ? ? ? ? ? ? ? ? ? ? ? ? ? ? ? 1111111111111100000000 1111111111111111110000 1111111111111111000000 ? ? ? ? ? ? ? ? ? ? ? ? ? ? ? ? ? ? ? ? ? ? ? ? ? ? ? ? ? ? ? ? ?

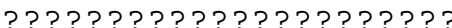
? ? ? ? ? ? ? ? ? ? ? ? ? ? ? ? ? ? ? ? ? ? ? ? ? ? ? ? ? ? ? ? ? ? ? ? ? ? ? ? ? ? ? ? ? ? ? ? ? ? ? ? 1110000000000000000000 1100000000000000000000 1100000000000000000000 1000000000000000000000 1000000000000000000000 1111111111111000000000 0000000000000000000000 1111111100000000000000 1111111000000000000000 1111111111111111100000 1111111111111111000000 1111111111111111111110 1111111111111111111100 1111111111111111111000 1111111111111110000000 1110000000000000000000 1111000000000000000000 1111000000000000000000 1111111111111110000000 1111111111111111110000 1111111111111111100000 1111111111111111100000 1111111110000000000000 1111111111110000000000 ? ?? ? ? ? ? ? ? ? ? ? ? ? ? ? 1111111100000000000000 
A.

A gran v. jonensis

A._gran_f_HLS

A._gran $f$ VH

A. herzogii HDSM

A. italica FH15

A._krammerii_nom._prov

A. $\operatorname{lac}$ A38

A. lac A62

A._lirata_A37

A._laevissima_HDSM

A. nyg A50

A._nyg_A62

A._pergla_f A38

A. pfaffiana FH37

A. solida_FH $\overline{2} 3$

A._Sub_ROR

A. sub T702

A. $\mathrm{sp} .{ }^{3} \mathrm{ROR}$

A._distañs_f_ADE

A. paucistriata 2035

A. sp. 4 T733

A. _sp. ${ }^{5}{ }^{-} \mathrm{h}$

A. sp. 6 hwg

A. Cf. distans 1571

A._humilis

A. tenuoir

A. tenella

A._crassipunctata

A._tethera

A. pseudoamericana

A. muzzanensis

A. -pergla_f_KI

A._ceretaña isd

11111111111111110000000000000000000000 11111111111111111111111111111111100000 11111111111111111111111111111111111000 00000000000000000000000000000000000000 11111000000000000000000000000000000000 11111111111111111111111111111111100000 111111111111111110000000000000000000 11111111111111111000000000000000000000 1111111111111111111111111110000000000 11111111111111111111110000000000000000 11111000000000000000000000000000000000 11100000000000000000000000000000000000 ? ? ? ? ? ? ? ? ? ? ? ? ? ? ? ? ? ? ? ? ? ? ? ? ? ? ? ? ? 1111111111111111111000000000000000000 11111111111111111111111111111111000000 11111111111100000000000000000000000000 11111111111110000000000000000000000000 10000000000000000000000000000000000000 1111111111111000000000000000000000000 1111111111111111111111111111111111111 11111111111111111111111111111111110000 ????????????????????????????????????? ? ? ? ? ? ? ? ? ? ? ? ? ? ? ? ? ? ? ? ? ? ? ? ? ? ? ? ? ? ? ? ? ? ? 11110000000000000000000000000000000000 11111111111111111111111100000000000000 11111111110000000000000000000000000000 11100000000000000000000000000000000000 11111111111111111111111111111111111000 1111111111111111111110000000000000000 ???????????????????????? ? ? ? ? ? ? ? ? ? ? 11111111111111111111111111111100000000 11100000000000000000000000000000000000 11111111111111111111111111111111110000 theta LNK ]

1111100000000000000000 1111111111111110000000 1111111111111111100000 ?????????????????????? 1111111111111111111111 1111111111111111110000 1111111111111111100000 1111111111111100000000 1111111111000000000000 ?????????? ? ? ? ? ? ? ? ? 1111111111111111000000 1111111111111111000000 ? ? ? ? ? ? ? ? ? ? ? ? ? ? ? ? ? 1111111111100000000000 ? ? ?? ? ? ? ? ? ? ? ? ? ? ? ? ? ? ? ? ? ? ? ? ? ? ? ? ? ? ? ? ? ? ? ? ? ? ? ? ? ? ? ? ? ? ? ?? ?? ?? ?? ?? ?? ?? ???? ?? 1111110000000000000000 ?? ? ? ? ? ? ? ? ? ? ? ? ? ? ? ? 1111111111111111000000 ? ? ? ? ? ? ? ? ? ? ? ? ? ? ? ?????? ? ? ? ? ? ? ? ? ? ? ? ?? ?? ? ? ? ? ? ? ? ? ? ? ? ? ? ? ? ? ? ? ? ? ? ? ? ? ? ? ? ? 1111111111111111111100 1111111111111111111100 1111111111111111111100 1111111111111111111100 ?? ?? ?? ? ? ? ? ? ?? ? ? ? ? ? 1111110000000000000000 1111111111111111111100 1111111111111111111100 
[ varians CHMP7 FFP S._cf.broschii_GB A._islāndica_CHM̄P1_MICH A. skvortzowīi

A. baicalensis

A._sub_9D1

A._sub_WLB3

A. sp. 3 FR

A._sub_ERB

A._sub_LEW

A. alpigena

A._amb_PII 7

A. $-a m b-N 17$

A. amb FL8

A. amb_BDL1

A._amb_WLP1

A. nyassensis GB

A. valida N2

A._gran_LB

A. gran OR12

A._sp. 1

A._sp. 2

A._cren_FH8 L639

A. cren T733 L639

Alveolophora

A._agassizii_FH2I

A. amb_A62

A._amb_MFP

A._amb_WAT

A. canad 1571

A. canad 2018

A._dianchiensis

A. distans FH34

A. gran_FH4 2

A._gran_FH38

A. gran 1914

A. gran MFP theta SEP

? ?? ?? ?? ? ? ? ? ? ? ? ? ? ? ? ? ? ? ? ? 111111111111000 111111111111110 111111111111000 111111111000000 111000000000000 000000000000000 111111000000000 111111111000000 ??????????????? 111000000000000 100000000000000 100000000000000 000000000000000 000000000000000 111111111110000 ??????????????? 111111111111000 111111111111000 ??? ?? ????? ? ? ? 111111111111000 ??????? ?? ? ? ? ? ??????????????? 111111111111110 111111111111000 111000000000000 111100000000000 111110000000000 111111111111000 111111111111110 111111111111100 ?? ? ? ?? ?? ??? ? ? 111111111111000 111111111111000 111111111111110 111111111111000 diameter

111111111111111111111111110 ? ? ? ? ? ? ? ? ? ? ? ? ? ? ? ? ? ? ? ? 111111111111111111111110000 111111111111100000000000000 111111111111111111111111000 111111100000000000000000000 111111111111111111000000000 110000000000000000000000000 111111111000000000000000000 111111111111111100000000000 ??????????????????????????? 111111111111111111000000000 111111100000000000000000000 111110000000000000000000000 111100000000000000000000000 111111111000000000000000000 111111111111111111111111110 111111111111111111110000000 111111111100000000000000000 111000000000000000000000000 111111111111111100000000000 100000000000000000000000000 111111111111111111111000000 111111111111111111111111111 111111111111111111111111111 111111111111111111111110000 111111110000000000000000000 111111111111110000000000000 111111111111110000000000000 111111000000000000000000000 111111111111111100000000000 111111111111111110000000000 100000000000000000000000000 111111111111111000000000000 111111111111111111111110000 111111111111111100000000000 111111111110000000000000000 areolar area ว?????? ? ? ? ? ? ? ? ? ? ? ? ? ?? ? ? ? ? ? ? ? ? ? 11111111111000000000 11111111000000000000 11111111111111111110 11111111100000000000 11111100000000000000 11000000000000000000 11110000000000000000 11111110000000000000 ???????????????????? 11111110000000000000 11110000000000000000 11111100000000000000 11111000000000000000 11111111100000000000 11111111111111111000 11111111111000000000 11111111111111111000 11111111111111110000 11111111111000000000 11111000000000000000 11111111111000000000 11111111111100000000 11111111111111111000 11111111111111100000 11111110000000000000 11111100000000000000 11111111100000000000 11111111111111111000 11111111111111111110 11111111111111111111 11100000000000000000 11111111111111100000 11111111111111111000 11111111111111000000 11111111111111111000 
[

A. gran v. jonensis

A._gran_f_HLS

A._gran $f-V H$

A. herzogii HDSM

A. italica FH15

A._krammeríi_nom._prov.

A._lac_A38

A. 1 ac A62

A._liráta_A37

A.laevissima_HDSM

A. nyg A50

A._nyg_A62

A._pergla_f A38

A. pfaffiana FH37

A. solida_FH $\overline{2} 3$

A._Sub_ROR

A. sub_T702

A. $\mathrm{sp} .{ }^{3} \mathrm{ROR}$

A._dist̄añs_f_ADE

A. paucistriata_2035

A. sp. 4 T733

A. $-\mathrm{sp} \cdot{ }^{5}-\mathrm{h}$

A. sp. 6 hwg

A. cf. distans 1571

A._humilis

A. tenuoir

A. tenella

A._crassipunctata

A. tethera

A. pseudoamericana

A. muzzanensis

A. -pergla_f_KI

A. ceretaña theta SEP 111111111111000 111111111111000 111111111111110 111111111111000 ?????????????? 111111111111110 ? ? ? ? ? ? ? ? ? ? ? ? ? ? ? ? ? ? ? ? ? ? ? ? ? ? ? ? ? ? ? 111111111111110 111111111111000 111111111111000 ? ? ? ? ? ? ? ? ? ? 111111111100000

111111111111111 111111110000000 111111110000000 110000000000000 ?? ? ? ? ? ? ? ? ? ? 111111111111110 111111111111000 ? ? ? ? ? ? ? ? ? ? ?????????????? 111111100000000 111111111111110 ??? ??? ?? ?? ? ? 111111111111110 ?????? ????????? ?? ? ? ? ? ? ? ? ? ? ? ?? ?? ?? ?? ? ? ? 111111111111110 111111111111110 111111111111110 diameter

111111111100000000000000000 111111111111111100000000000 111111111111111100000000000 111111111111000000000000000 11111111111111111000000000 111111111100000000000000000 111111111111111111111100000 111111111111111110000000000 111111111111111111100000000 111111111111111111111110000 111111111111111111000000000 111111111111111100000000000 111111111111111111100000000 111111111100000000000000000 111111111111111111111110000 111111111000000000000000000 111111000000000000000000000 100000000000000000000000000 111111111111111100000000000 111111111111111111111111100 111111111111111111111111110 111111111111111111111100000 111111100000000000000000000 000000000000000000000000000 111100000000000000000000000 111111111111111111111111000 111111111100000000000000000 111111111100000000000000000 111111111111111111000000000 111111111111111111111000000 111111111111111111111111110 111111111111111111111110000 111111111111111111111111111 areolar area 11100000000000000000 11111111111111111110 11111111111111111100 11100000000000000000 11111100000000000000 11111111111111100000 11111111100000000000 11111100000000000000 11111111111111100000 11111111110000000000 11000000000000000000 11000000000000000000 ?? ? ? ? ? ? ? ? ? ? ? ? ? ? 11110000000000000000 11111111111111100000 11111000000000000000 11111100000000000000 11000000000000000000 11111100000000000000 11111111111111111100 11111111111111111110 ? ? ? ? ? ? ? ? ? ? ? ? ? ? ? ? ? ? ? ? ? ? ? ? ? ? ? ? ? ? 11000000000000000000 11111100000000000000 11111111111110000000 00000000000000000000 11110000000000000000 00000000000000000000 ???????????????????? 11111111111100000000 10000000000000000000 11111111111111111100 
Appendix N. Images of Species Examined. 

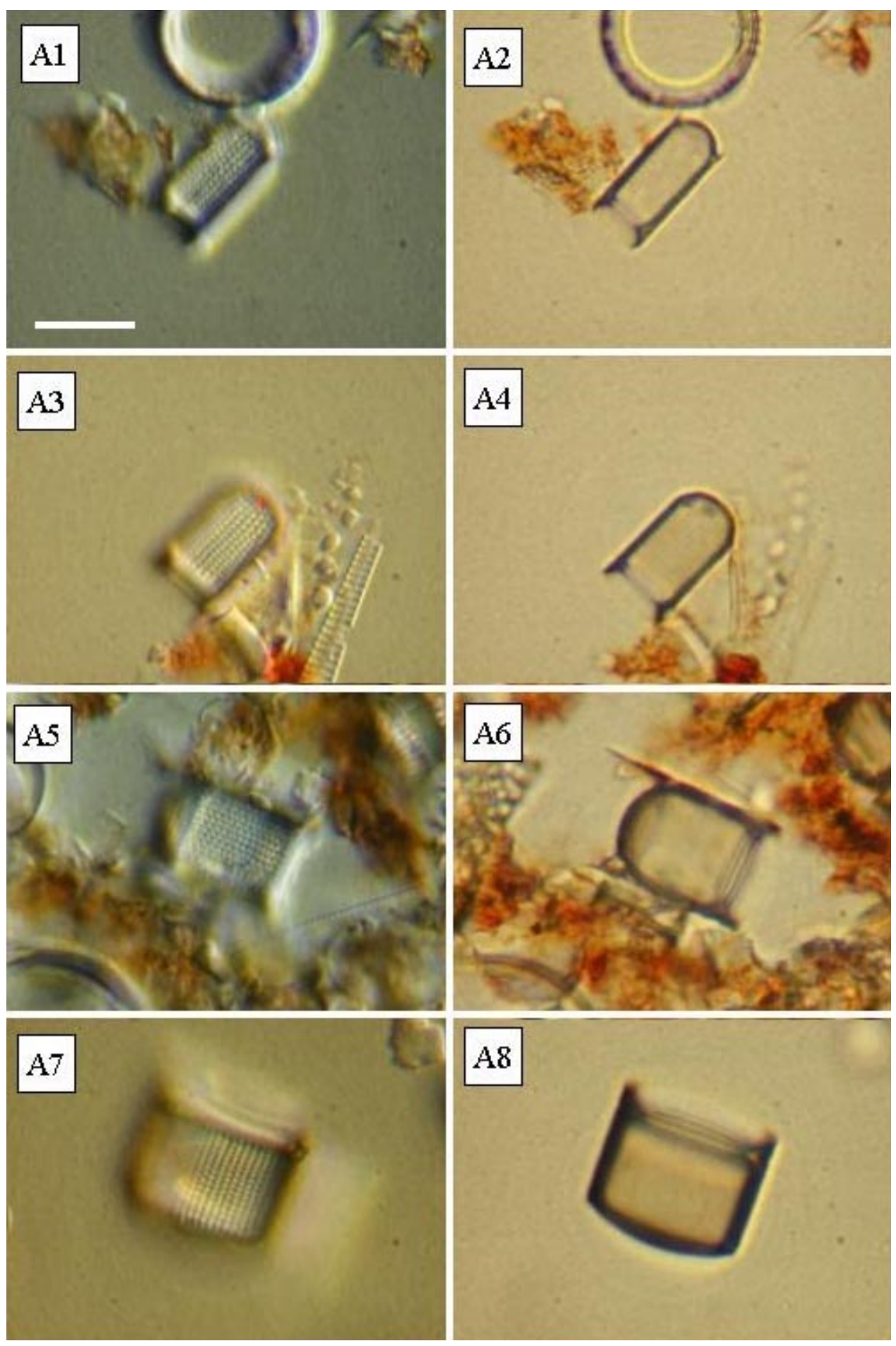

Figures A1-A8. Representative valves of Aulacoseira skvortzowii over the size range for the species (scale bar $=10 \mu \mathrm{m}$ ). 

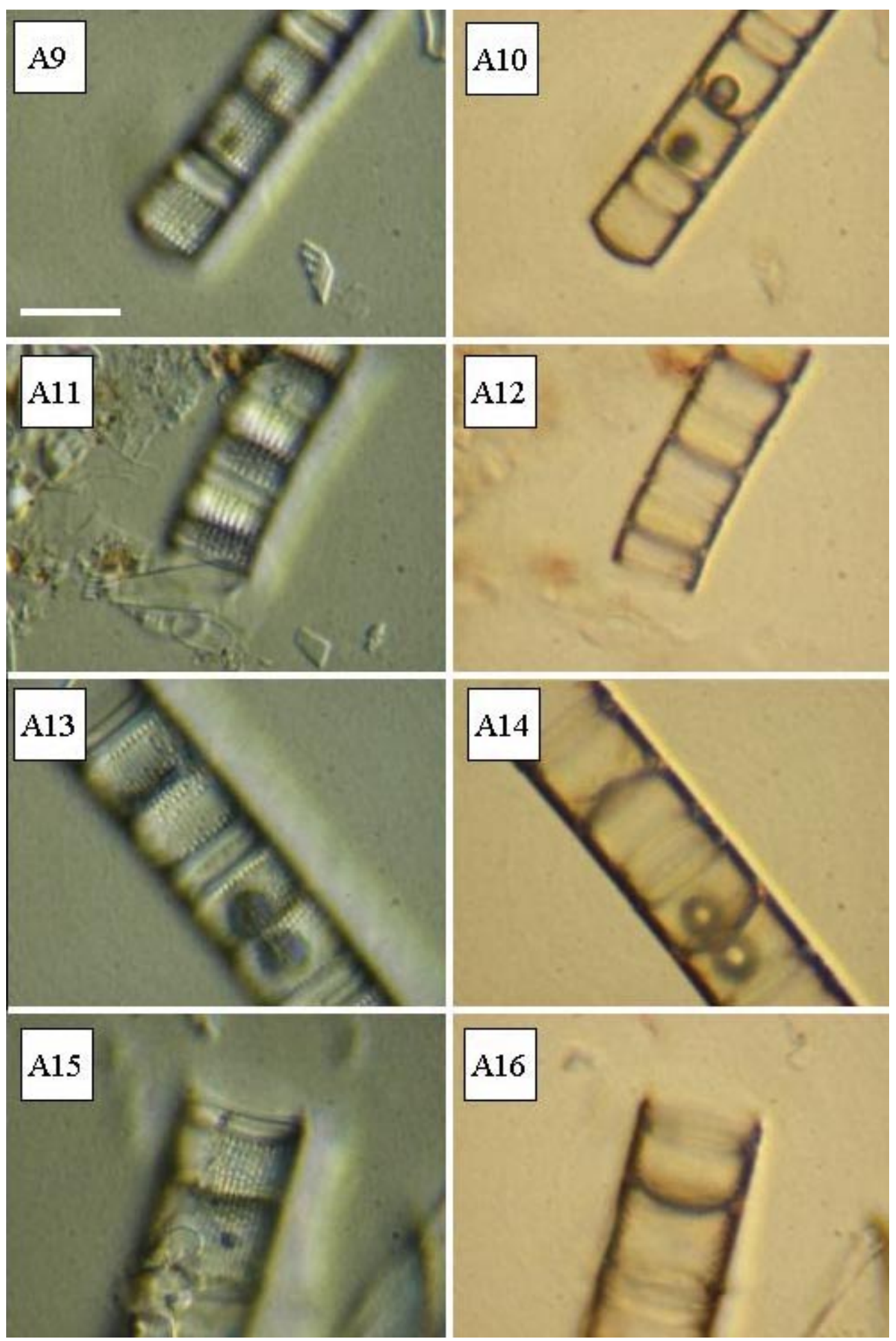

Figures A9-A16. Representative valves of an unidentified Aulacoseira species (A. sp. 1) showing the size range for the species (scale bar $=10 \mu \mathrm{m}$ ). 

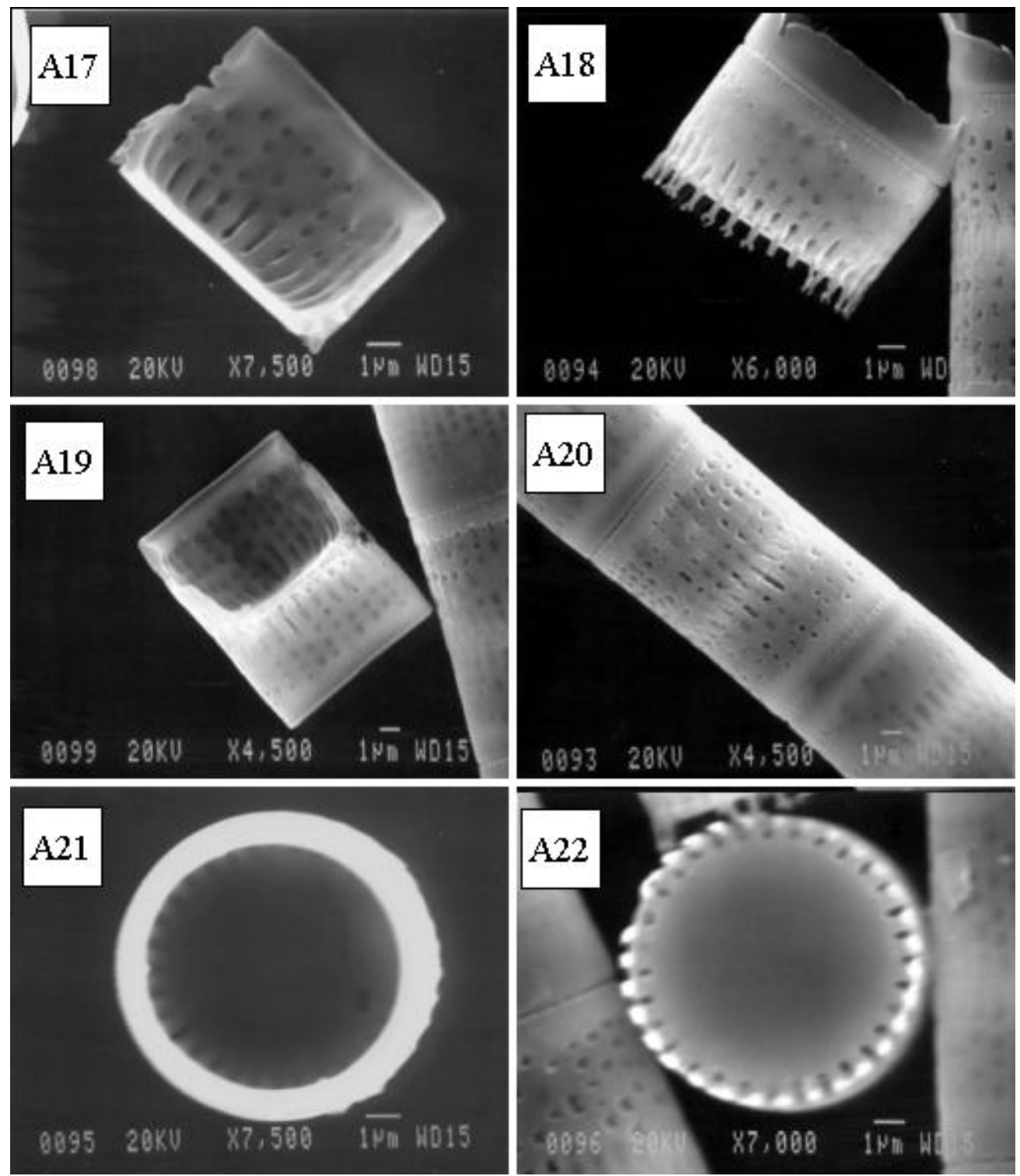

Figures A17-A22. Scanning electron micrographs of valves of an unidentified Aulacoseira species (A. sp. 1). 

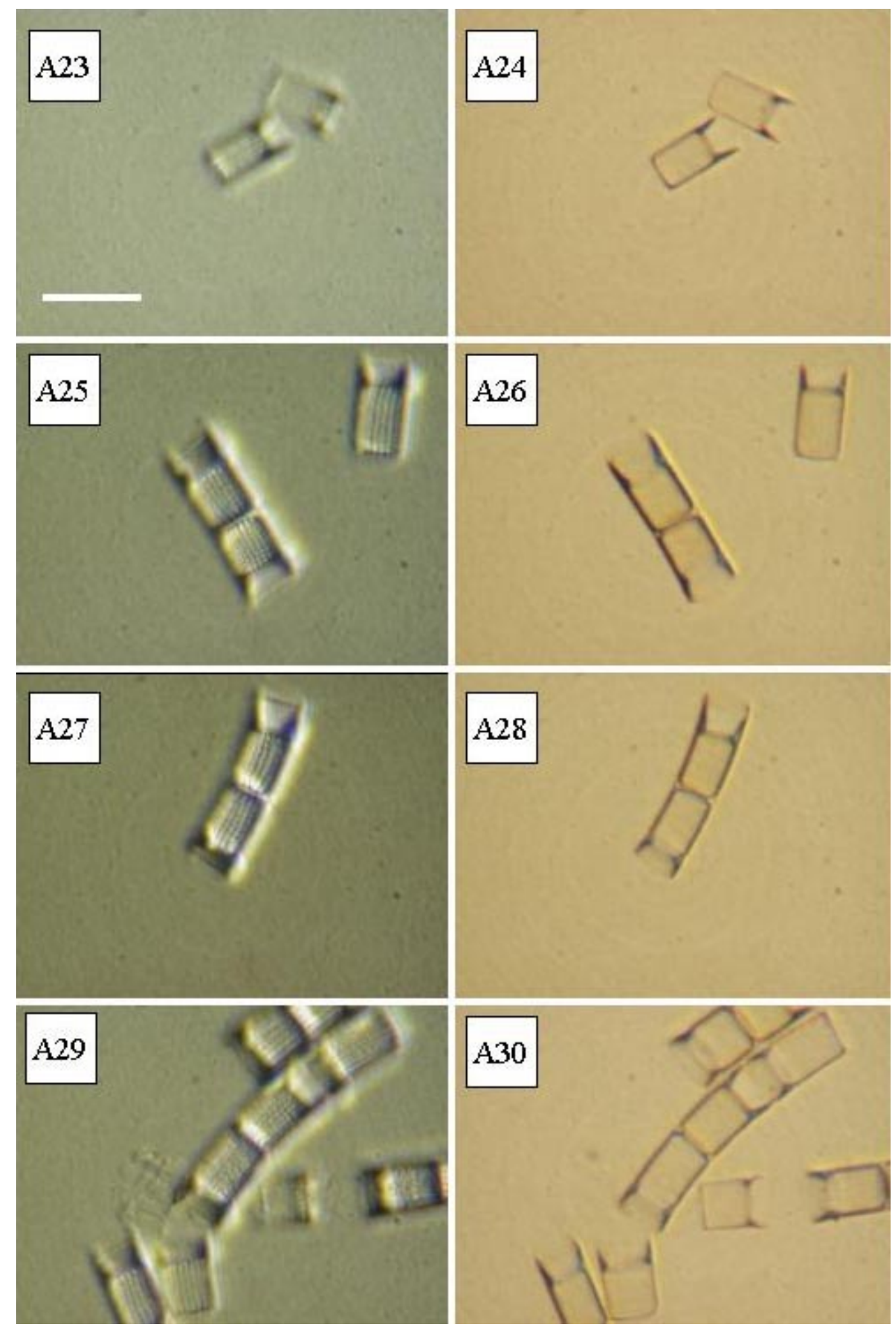

Figures A23-A30. Representative valves of an unidentified Aulacoseira species (A. sp. 2) showing a portion of the size range for the species (scale bar $=10 \mu \mathrm{m}$ ). 

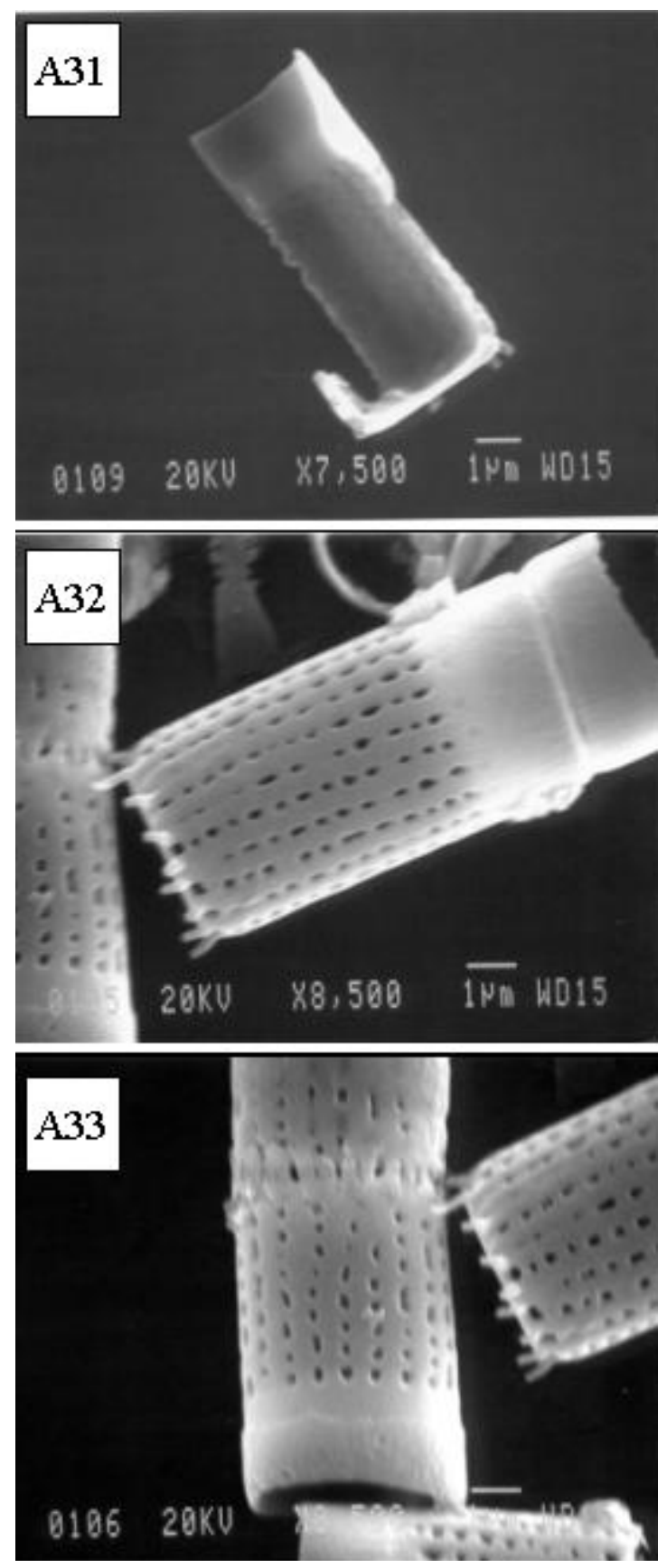

Figures A31-A33. Scanning electron micrographs of valves of an unidentified Aulacoseira species (A. sp. 2). 

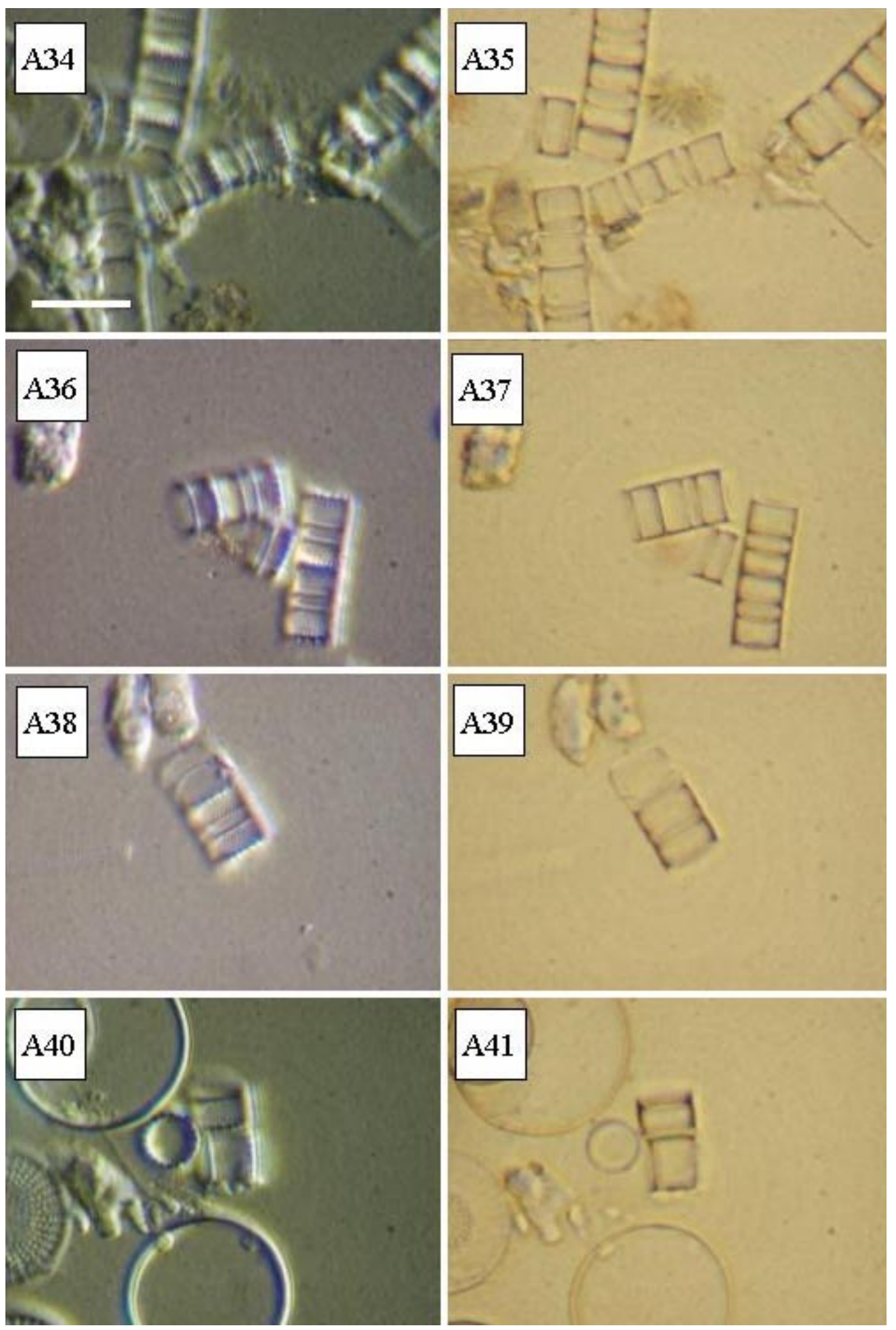

Figures A34-A41. Representative valves of an unidentified Aulacoseira species (A. sp. 3) showing a portion of the size range for the species (scale bar $=10 \mu \mathrm{m}$ ). 

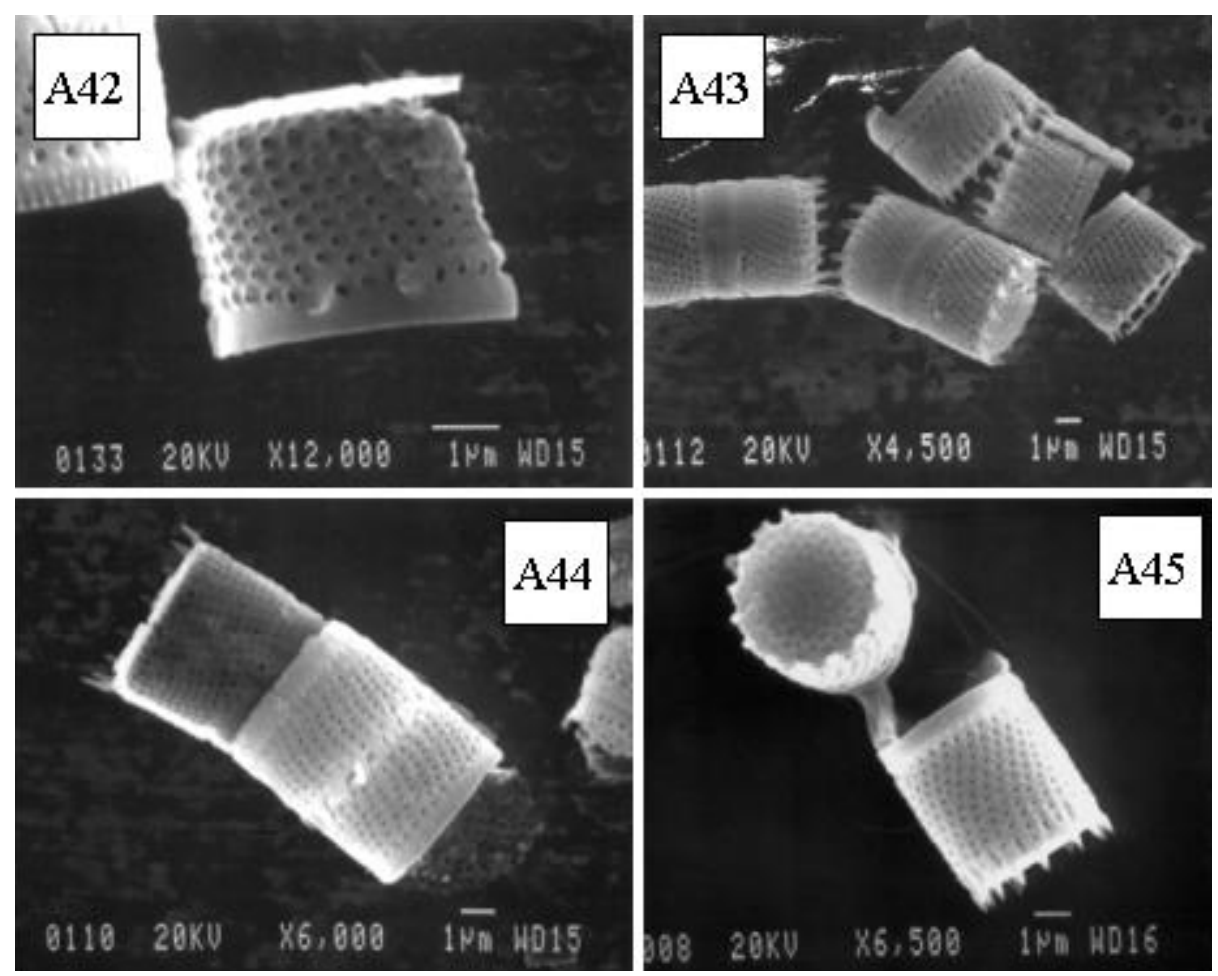

Figures A42-A45. Scanning electron micrographs of valves of an unidentified Aulacoseira species (A. sp. 3). 

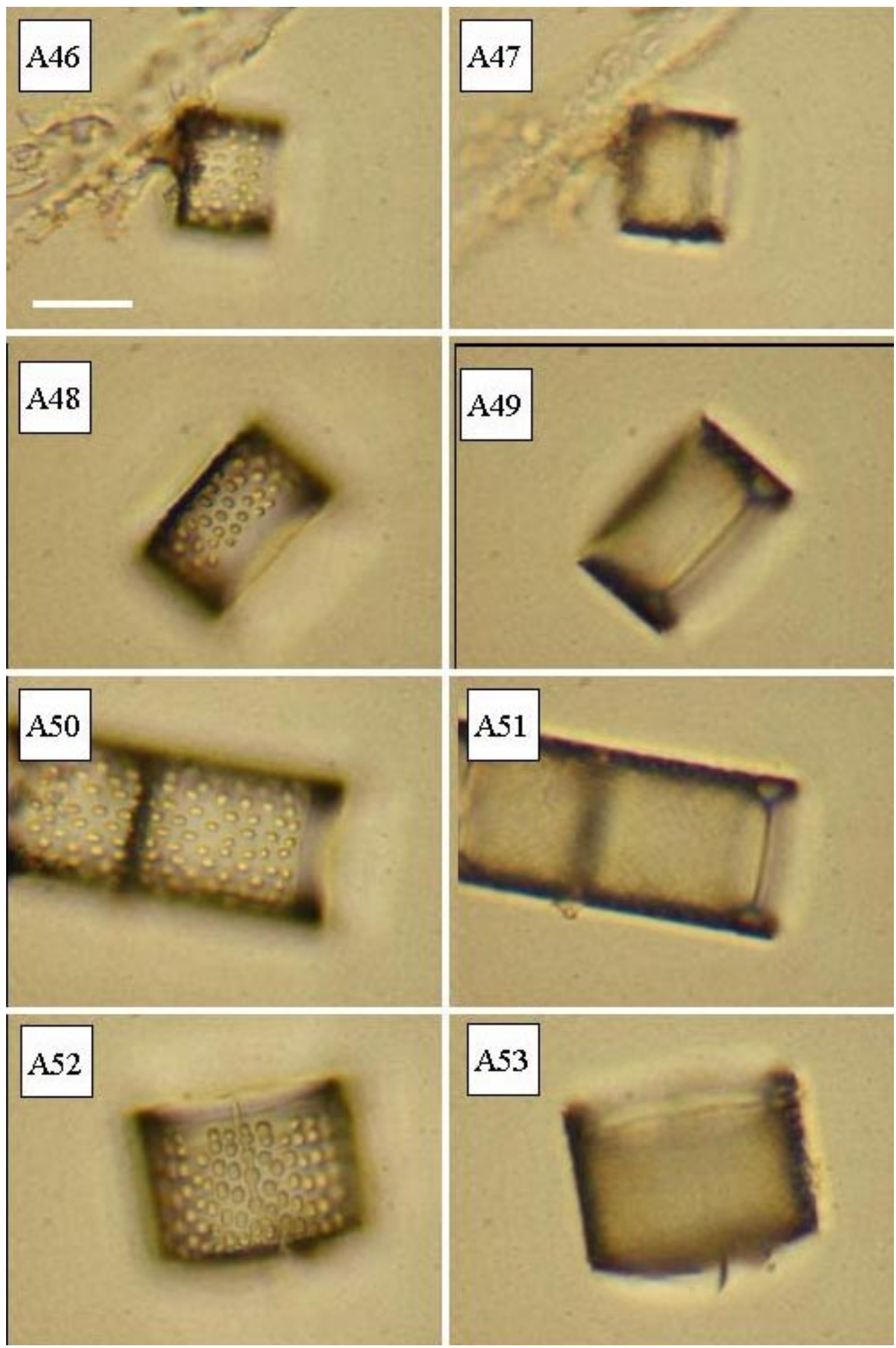

Figures A46-A53. Representative valves of an unidentified Aulacoseira species from New Zealand (A. sp. 4) showing the size range for the species (scale bar $=10 \mu \mathrm{m}$ ). 

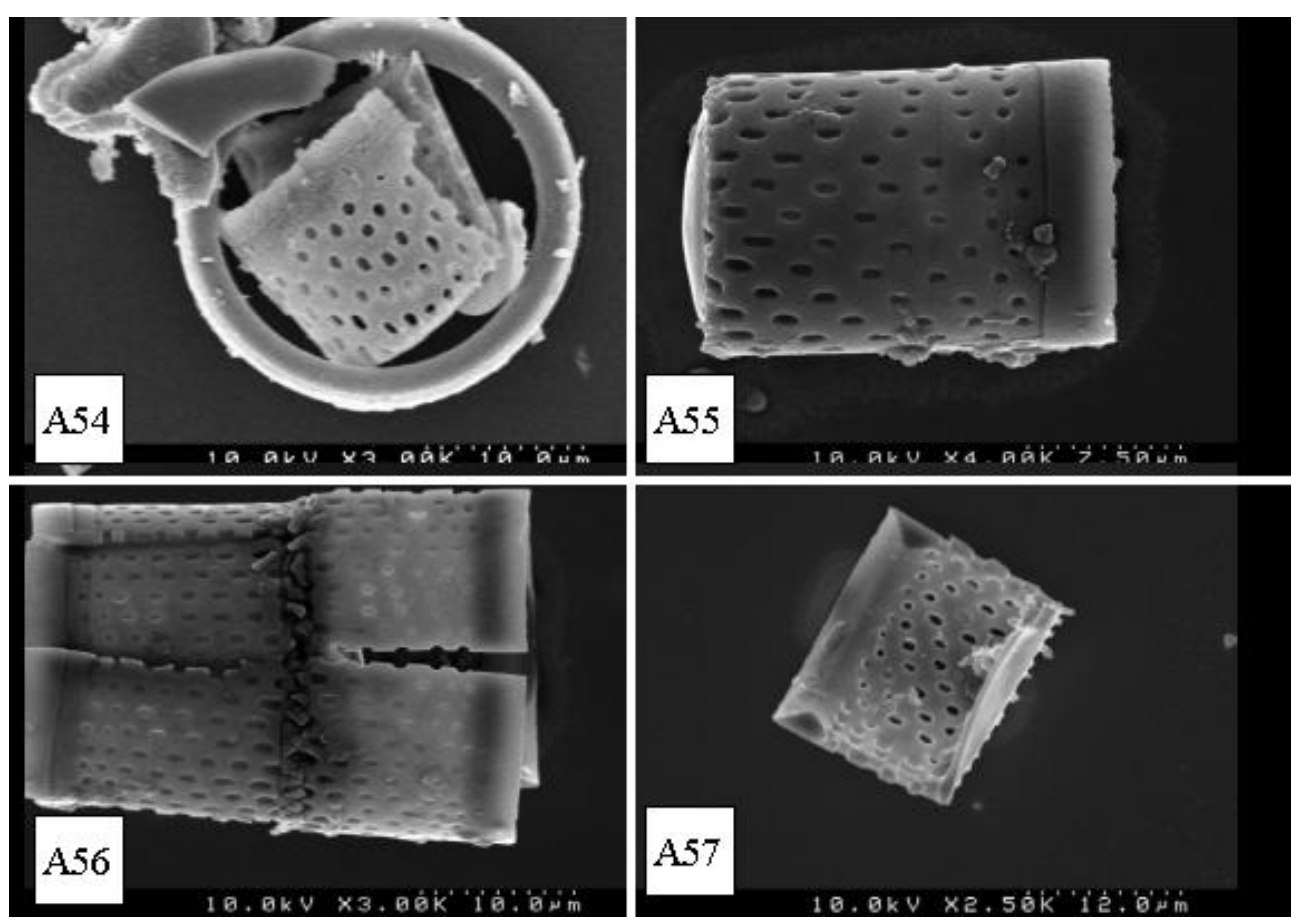

Figures A54-A57. Scanning electron micrographs of valves of an unidentified Aulacoseira species (A. sp. 4) from New Zealand. 

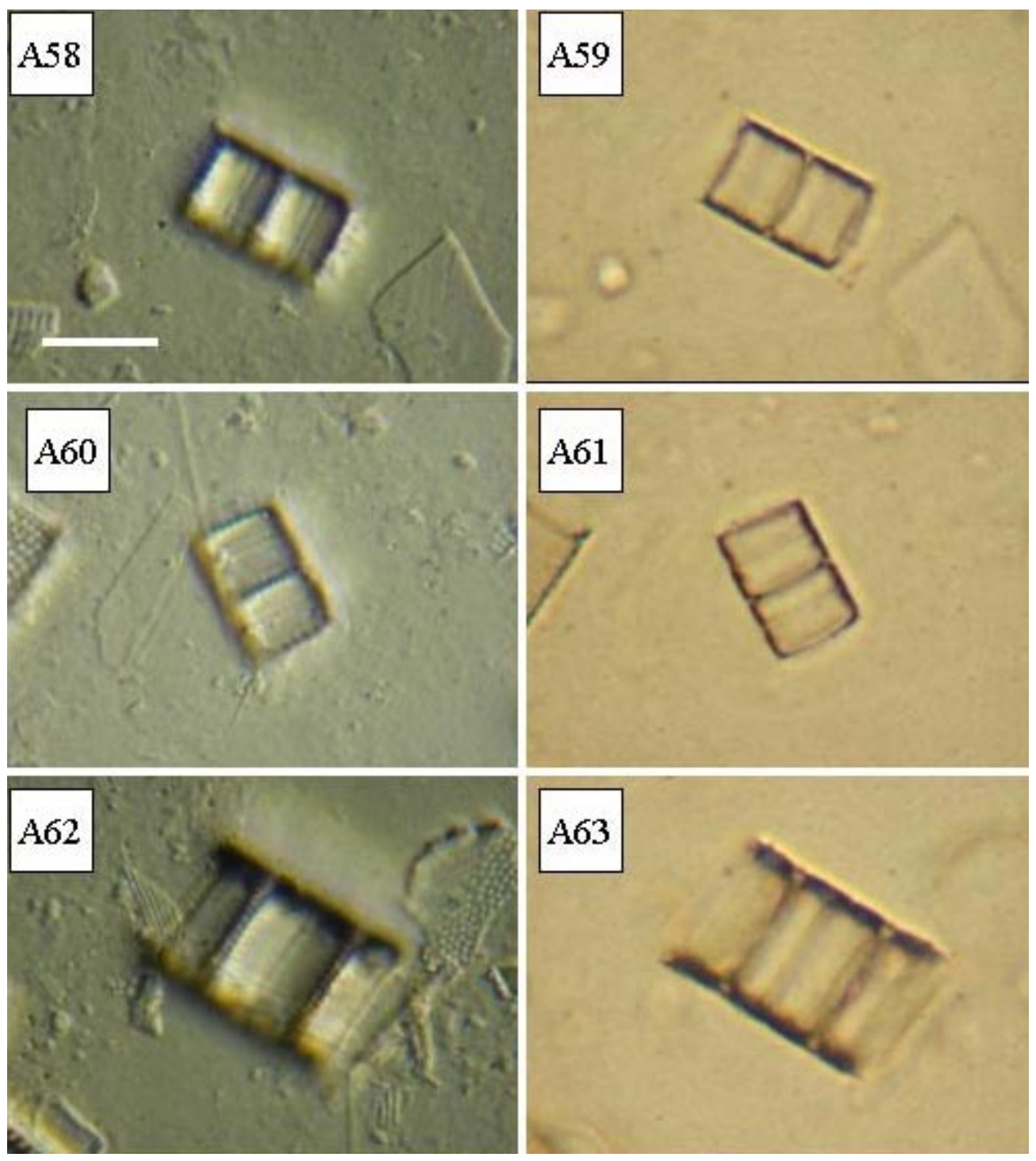

Figures A58-A63. Representative valves of an unidentified Aulacoseira species from Ecuador are shown $(A$. sp. 5 ; scale bar $=10 \mu \mathrm{m})$. 

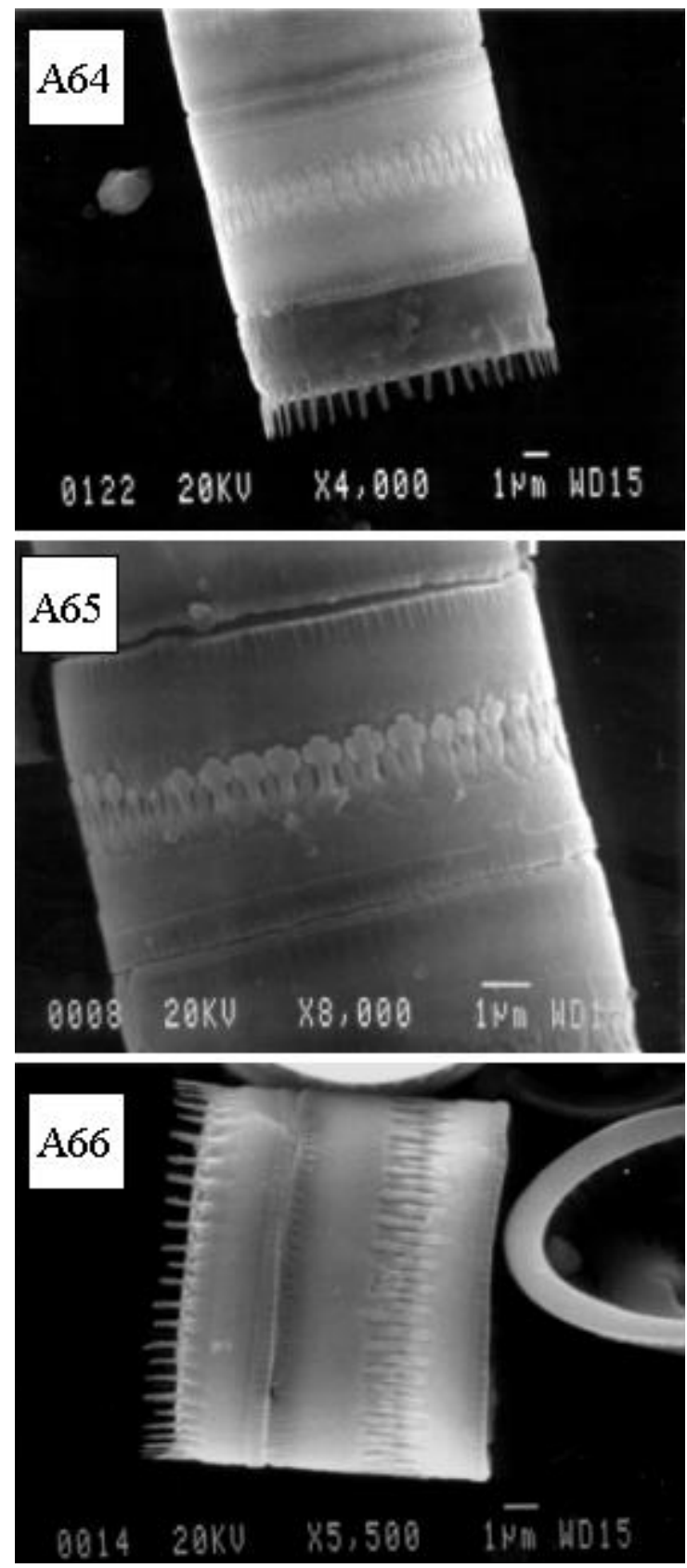

Figures A64-A66. Scanning electron micrographs of valves of an unidentified Aulacoseira species (A. sp. 5) from Ecuador. 

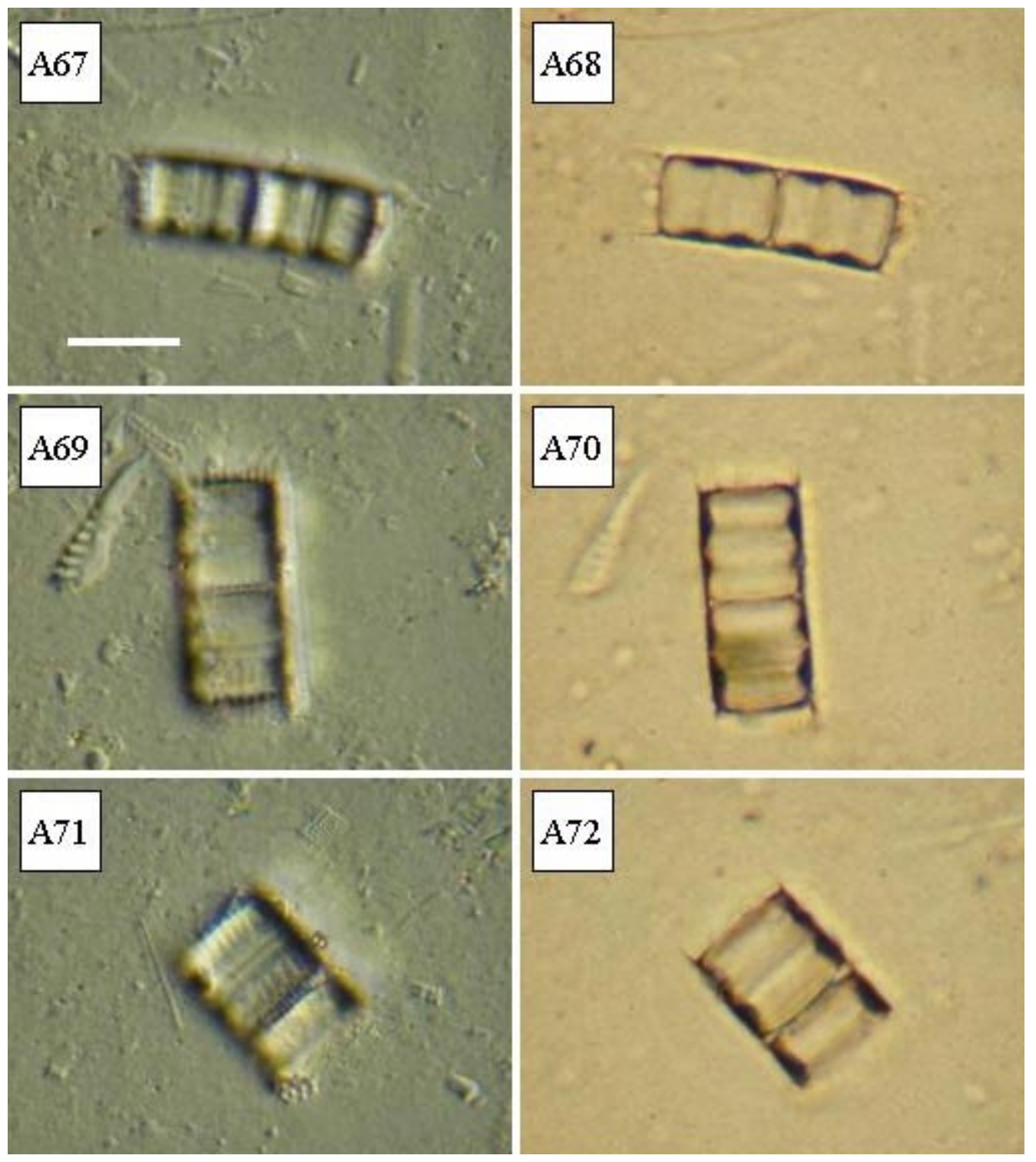

Figures A67-A72. Representative valves of an unidentified Aulacoseira species from Ecuador are shown $(A$. sp. 6 ; scale bar $=10 \mu \mathrm{m})$. 

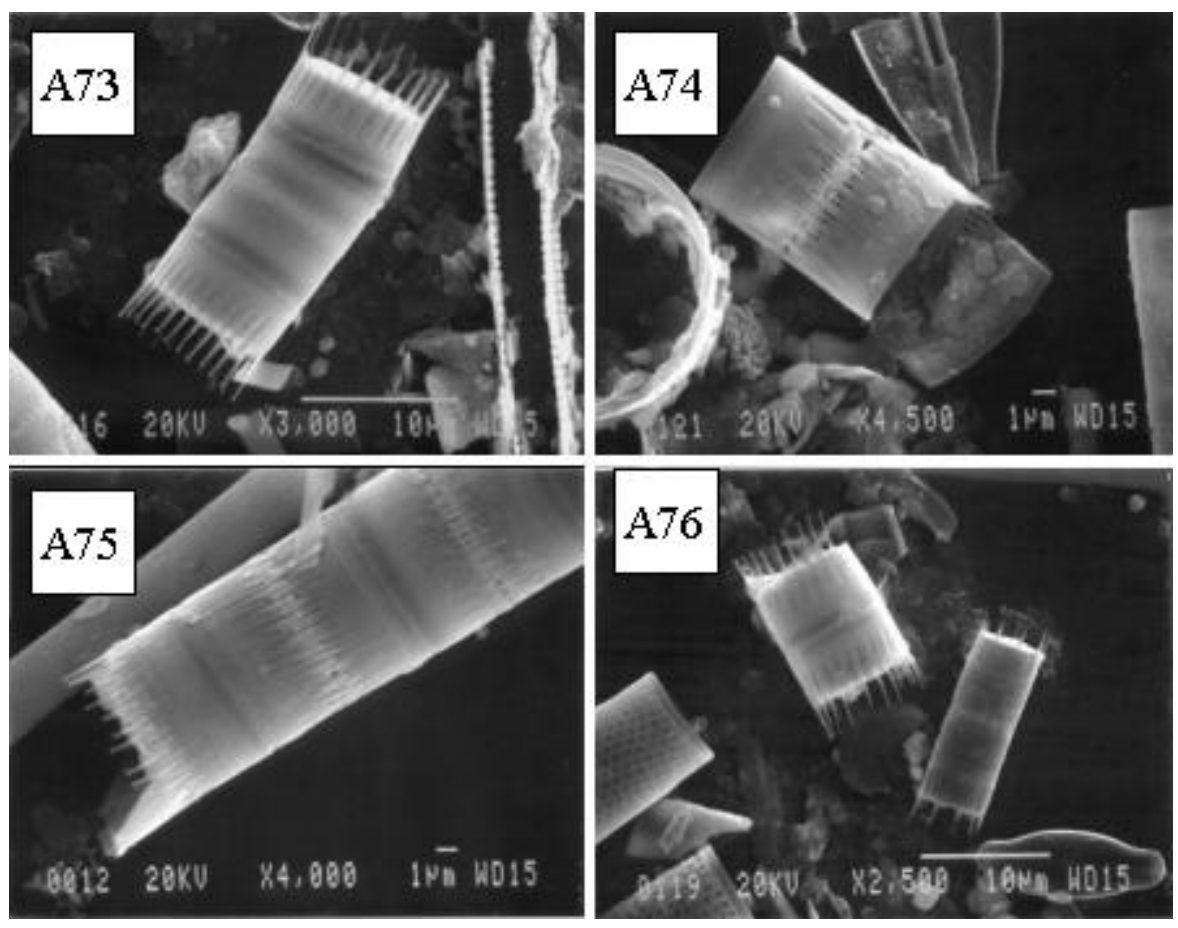

Figures A73-A76. Scanning electron micrographs of valves of an unidentified Aulacoseira species (A. sp. 6) from Ecuador. 

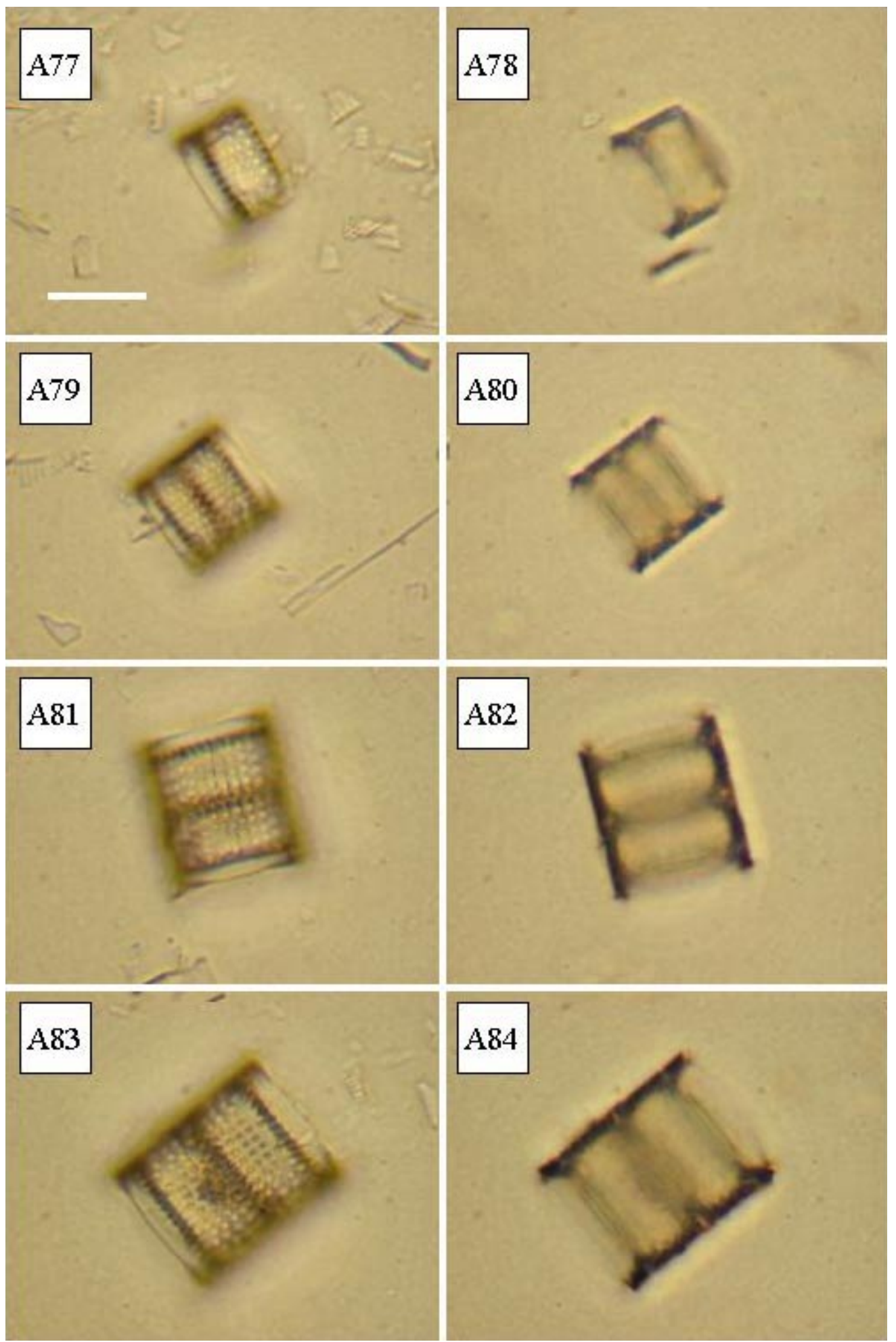

Figures A77-A84. Representative valves of Aulacoseira lirata (ADE 37) showing the size range for the species (scale bar $=10 \mu \mathrm{m}$ ). 

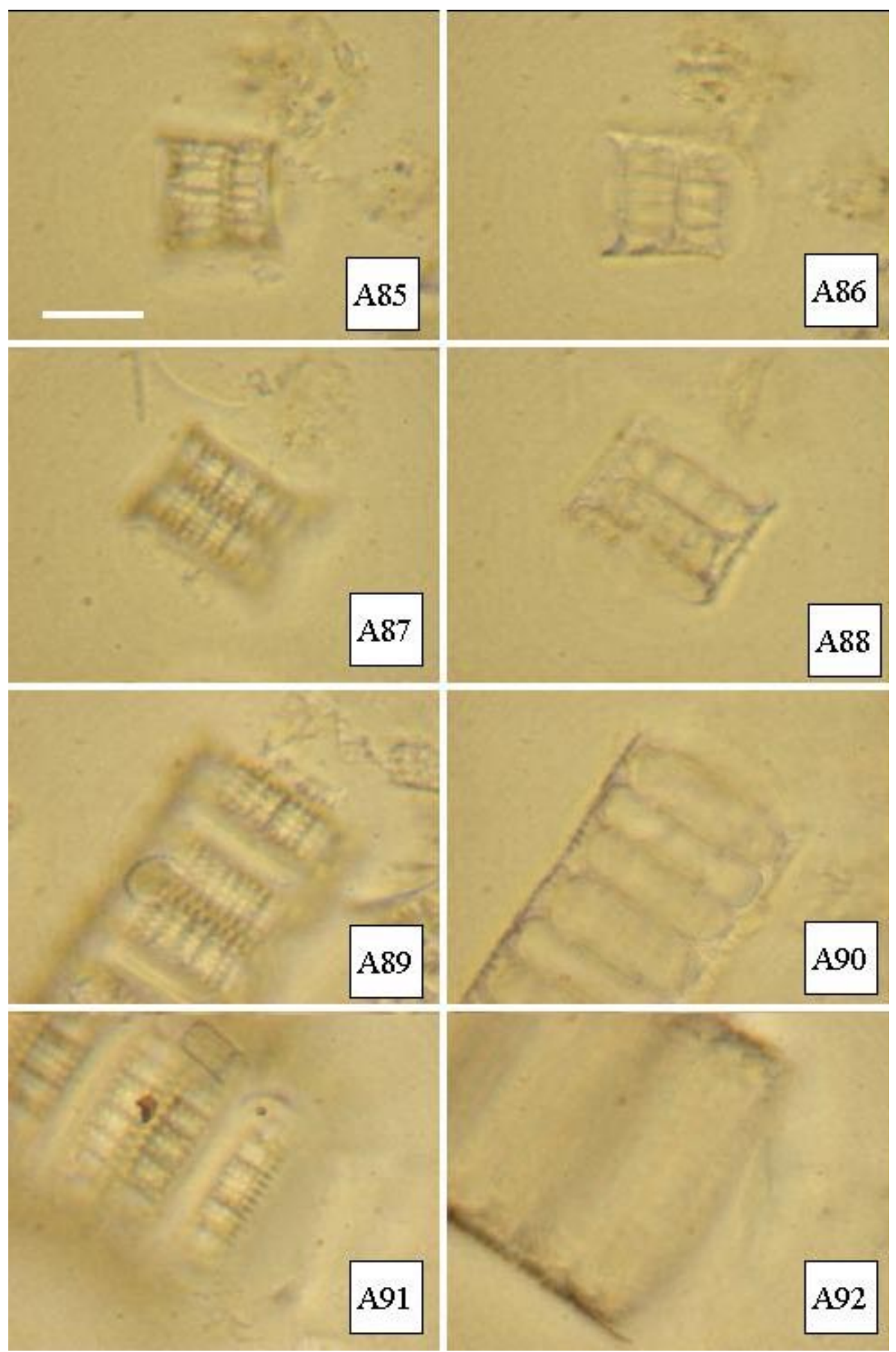

Figures A85-92. Representative valves of an unidentified Alveolophora showing the size range for the species (scale bar $=10 \mu \mathrm{m}$ ). 


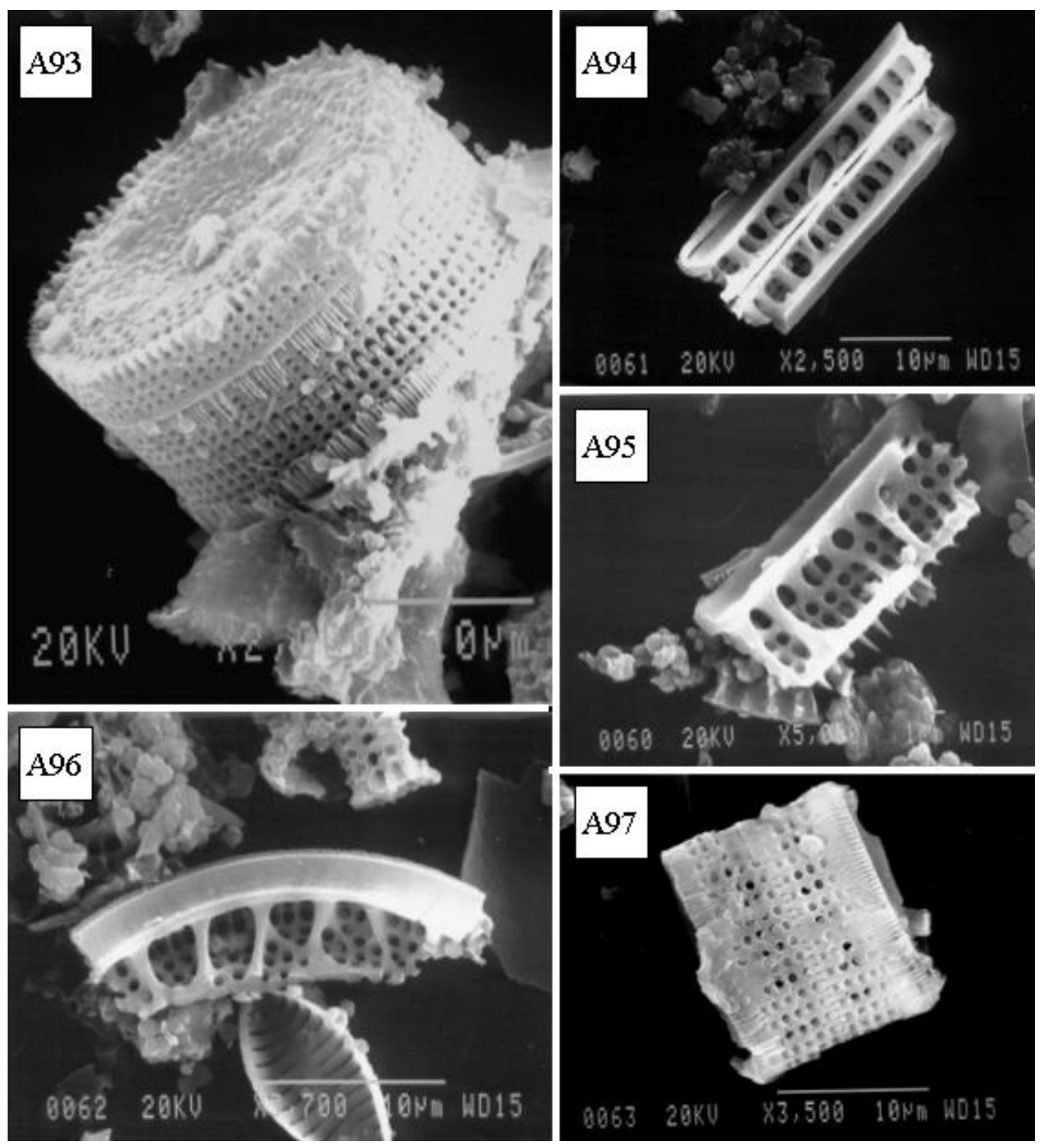

Figures A93-A97. Scanning electron micrographs of valves of an unidentified Alveolophora species. 

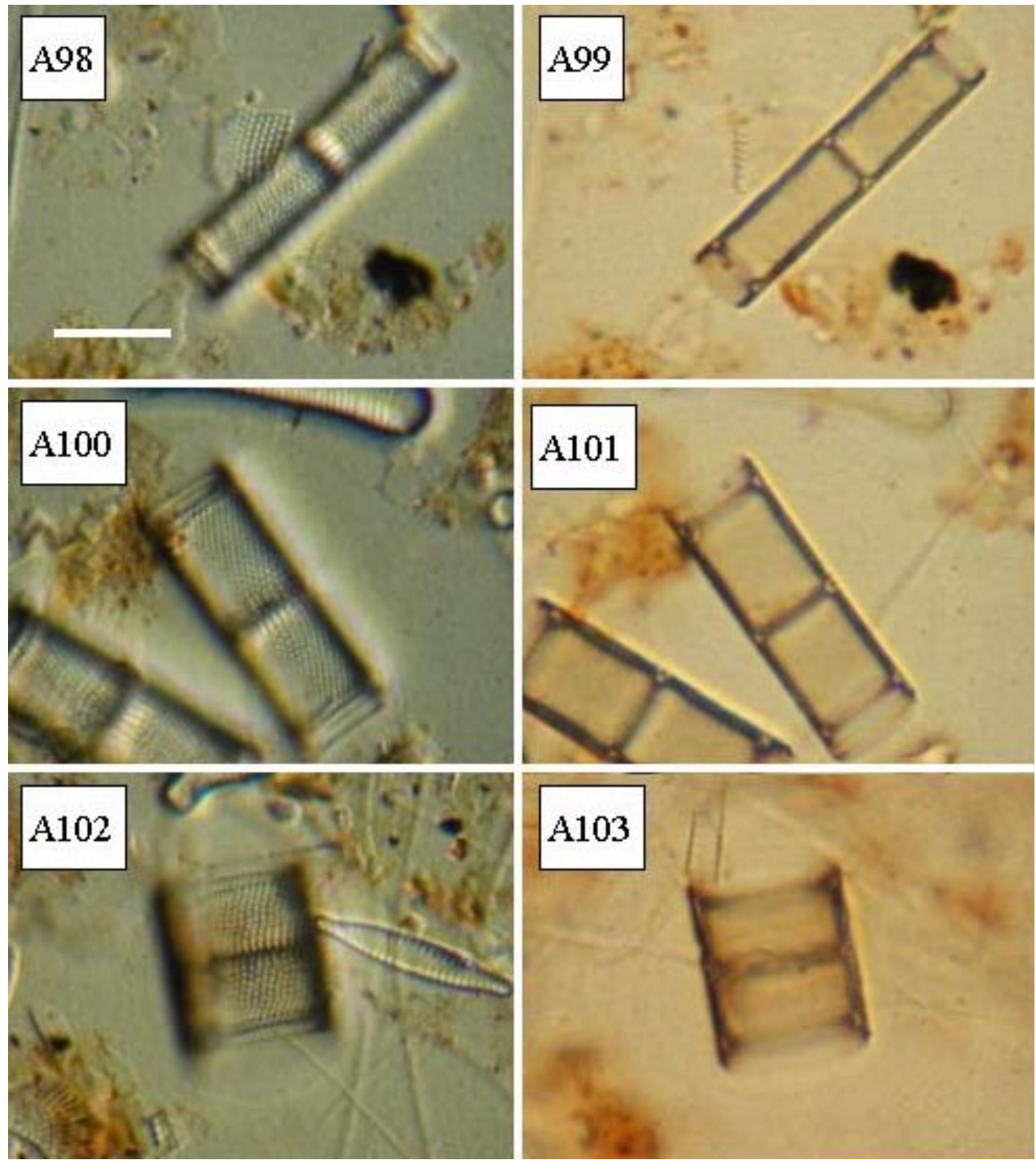

Figures A98-A103. Representative valves of Aulacoseira ambigua showing the size range for the species (scale bar $=10 \mu \mathrm{m}$ ). 

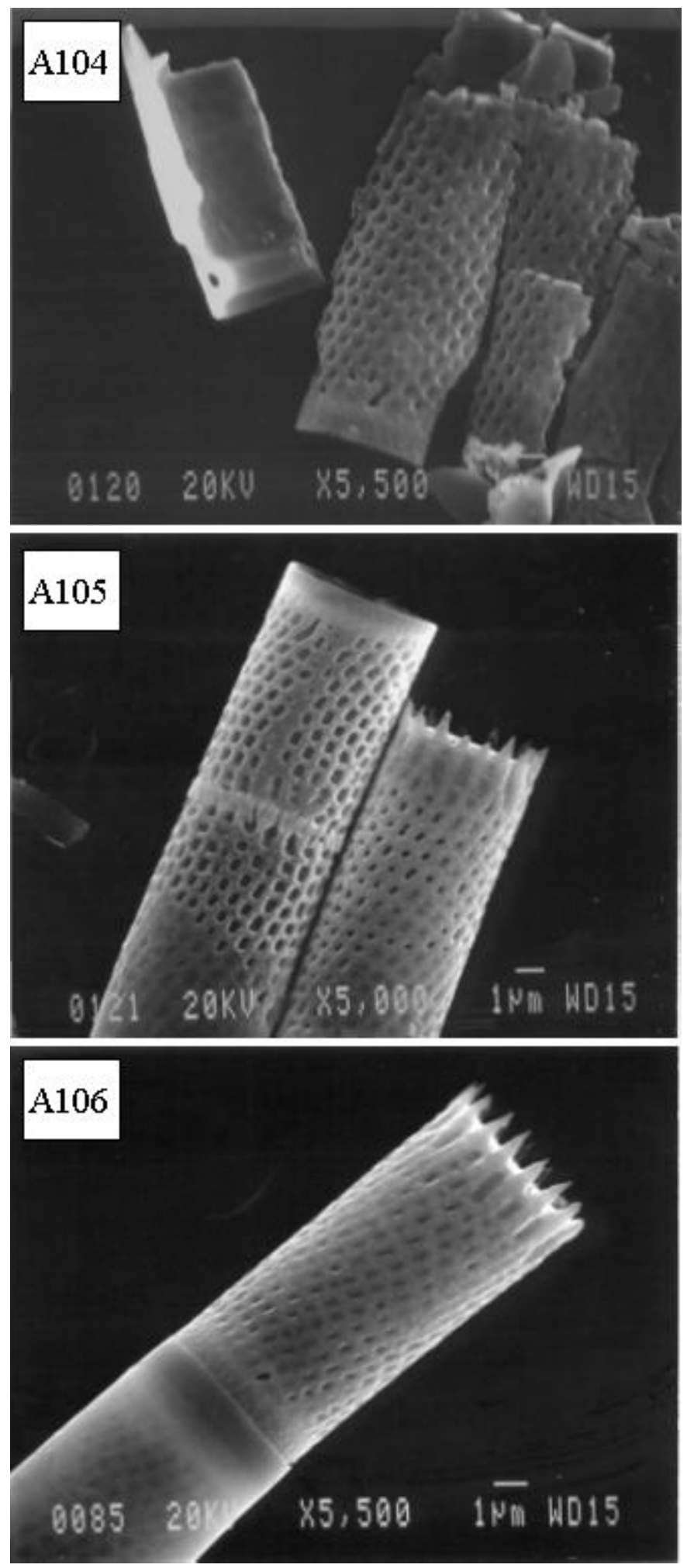

Figures A104-A106. Scanning electron micrographs of valves of Aulacoseira ambigua. 

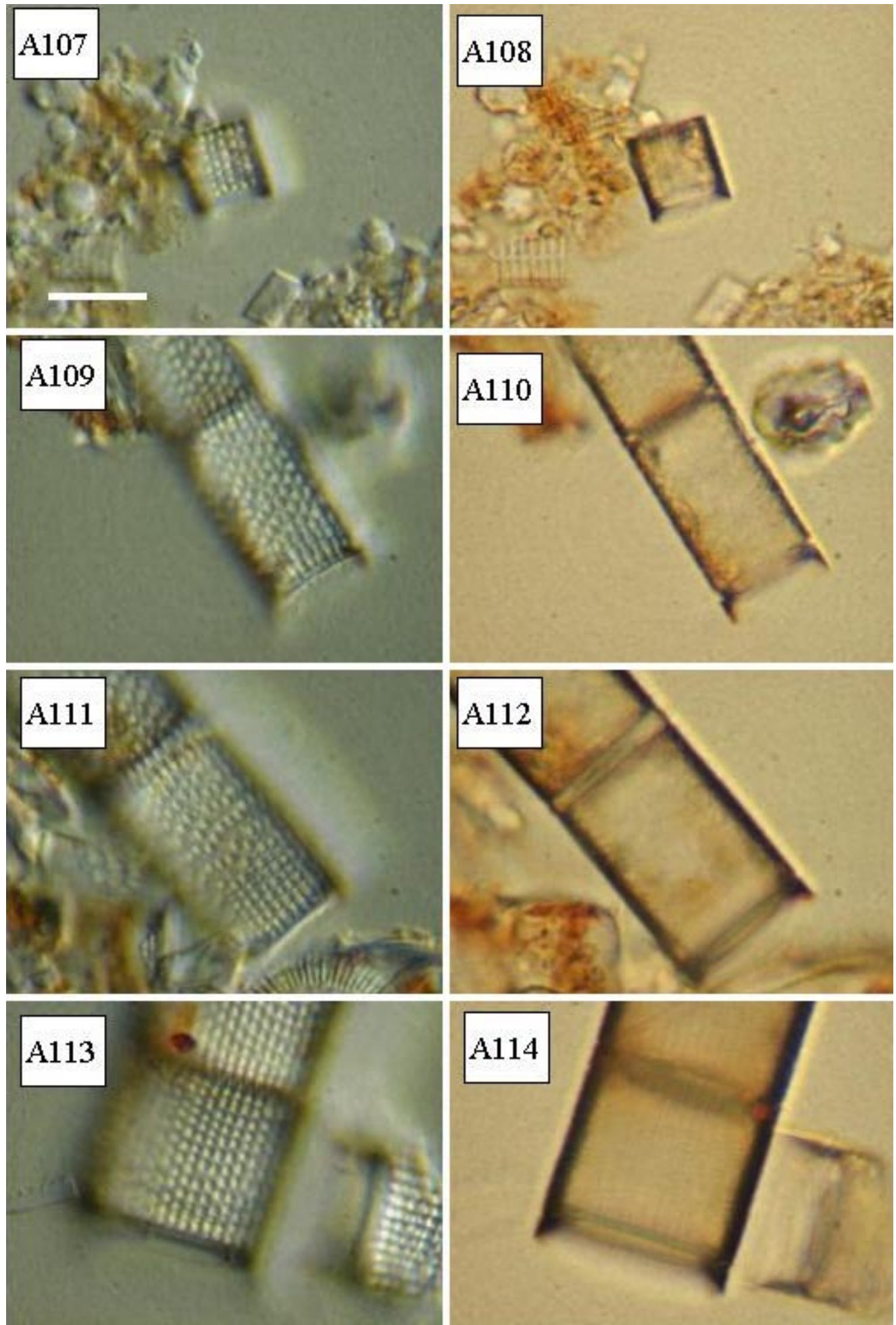

Figures A107-A114. Representative valves of Aulacoseira baicalensis showing the size range for the species (scale bar $=10 \mu \mathrm{m})$. 

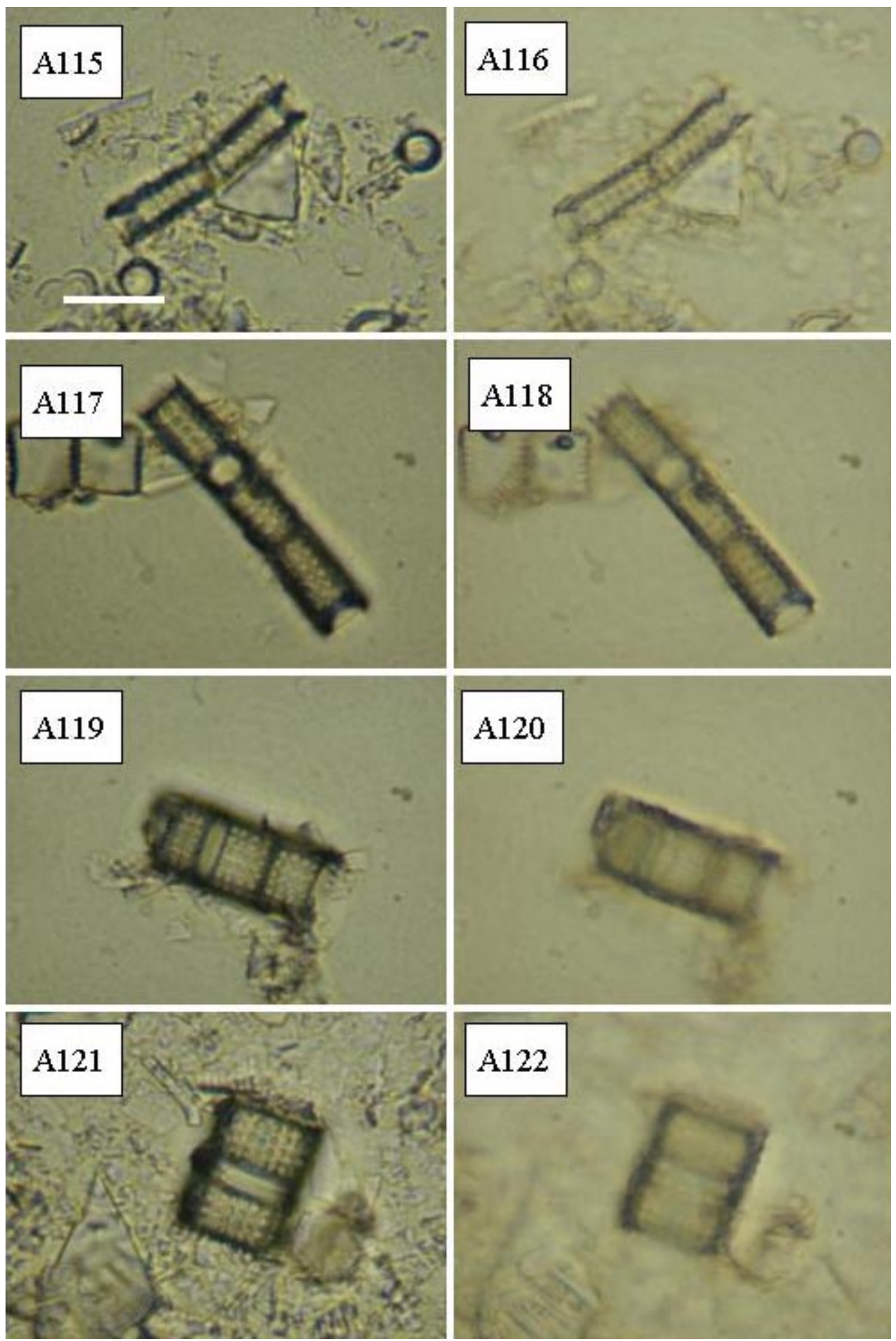

Figures A115-A122. Representative valves of Aulacoseira canadensis showing the size range for the species (scale $b a r=10 \mu \mathrm{m})$. 

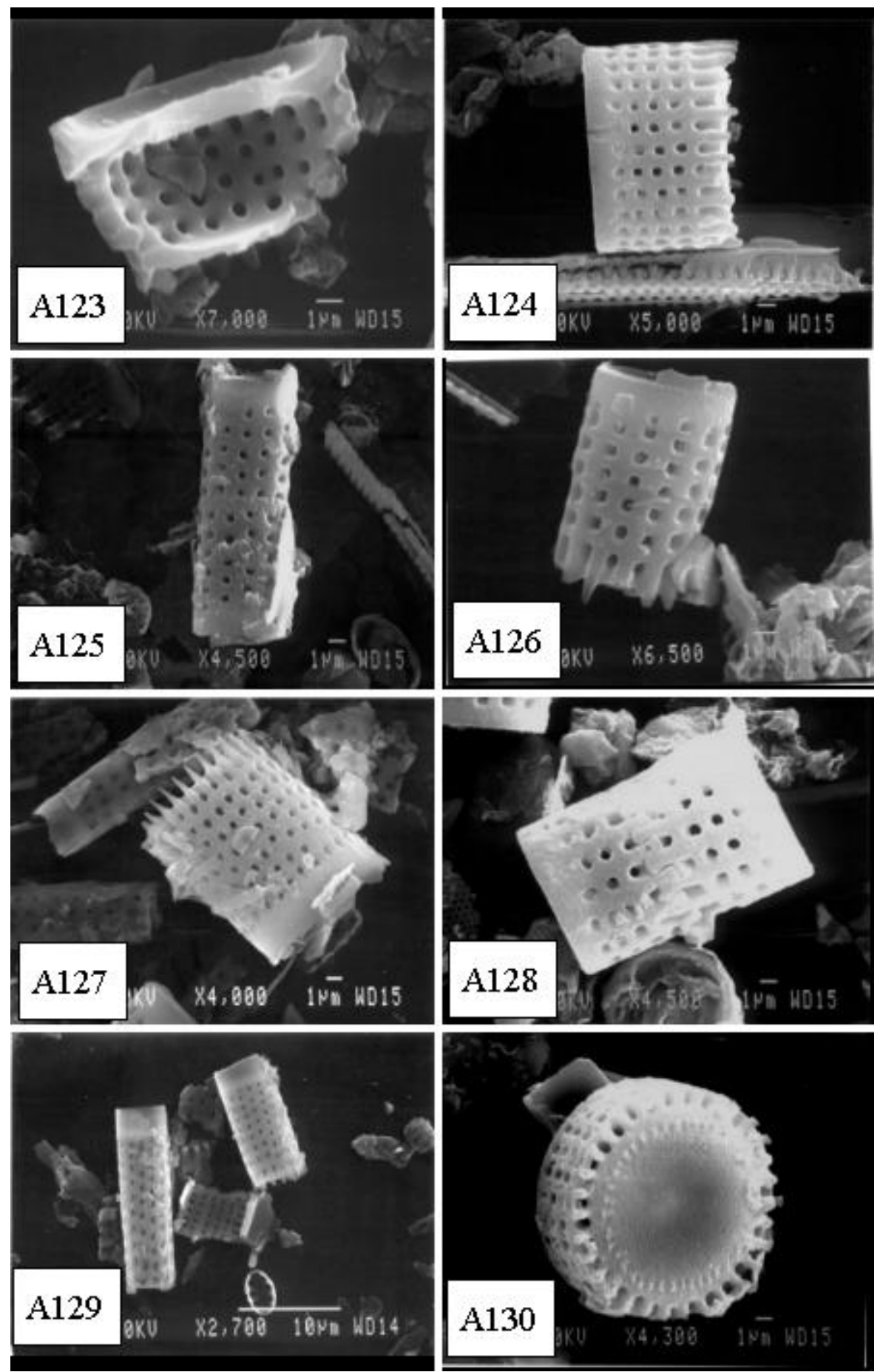

Figures A123-A130. Scanning electron micrographs of valves of Aulacoseira canadensis. 

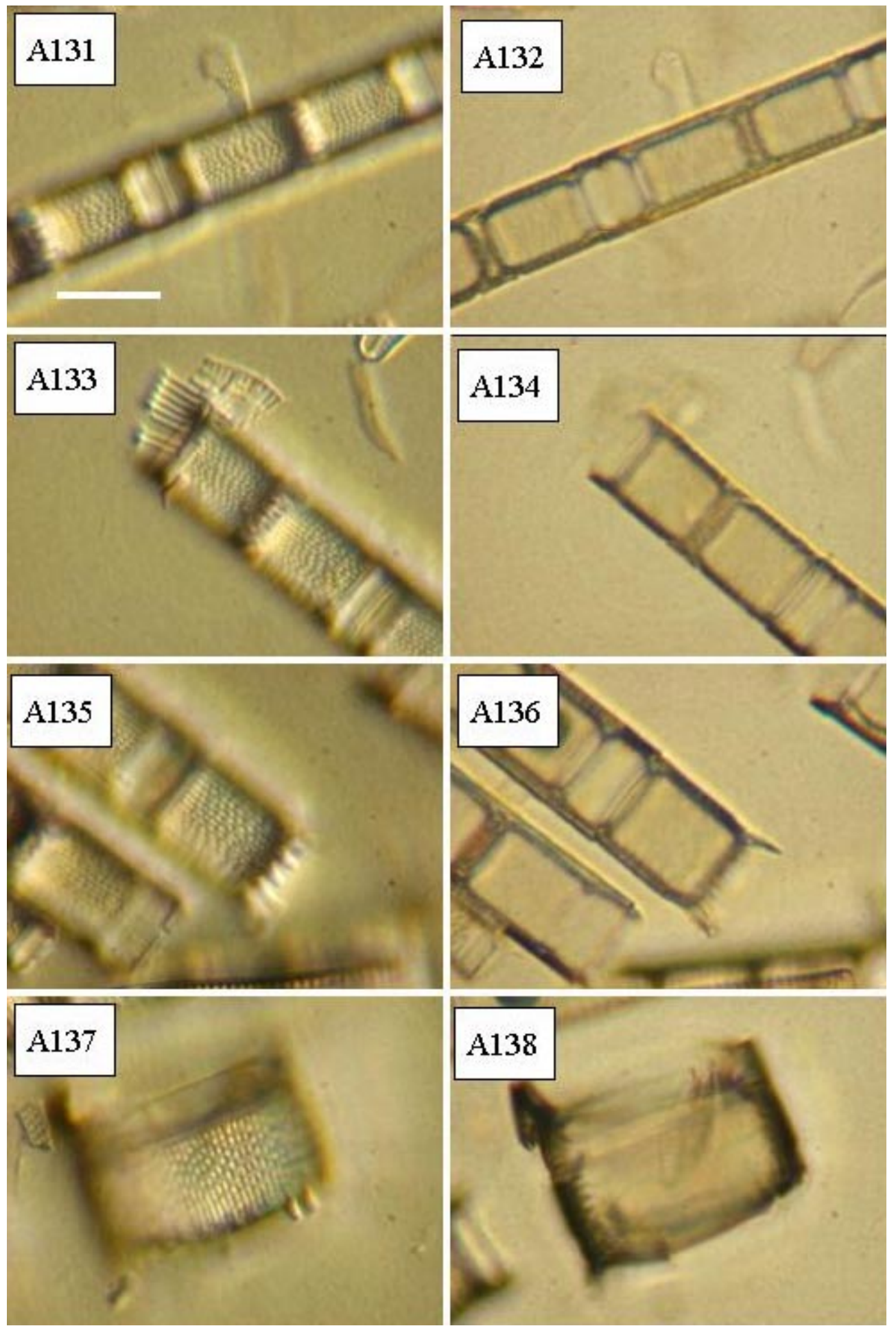

Figures A131-A138. Representative valves of Aulacoseira crenulata showing the size range for the species (scale bar $=10 \mu \mathrm{m})$. 

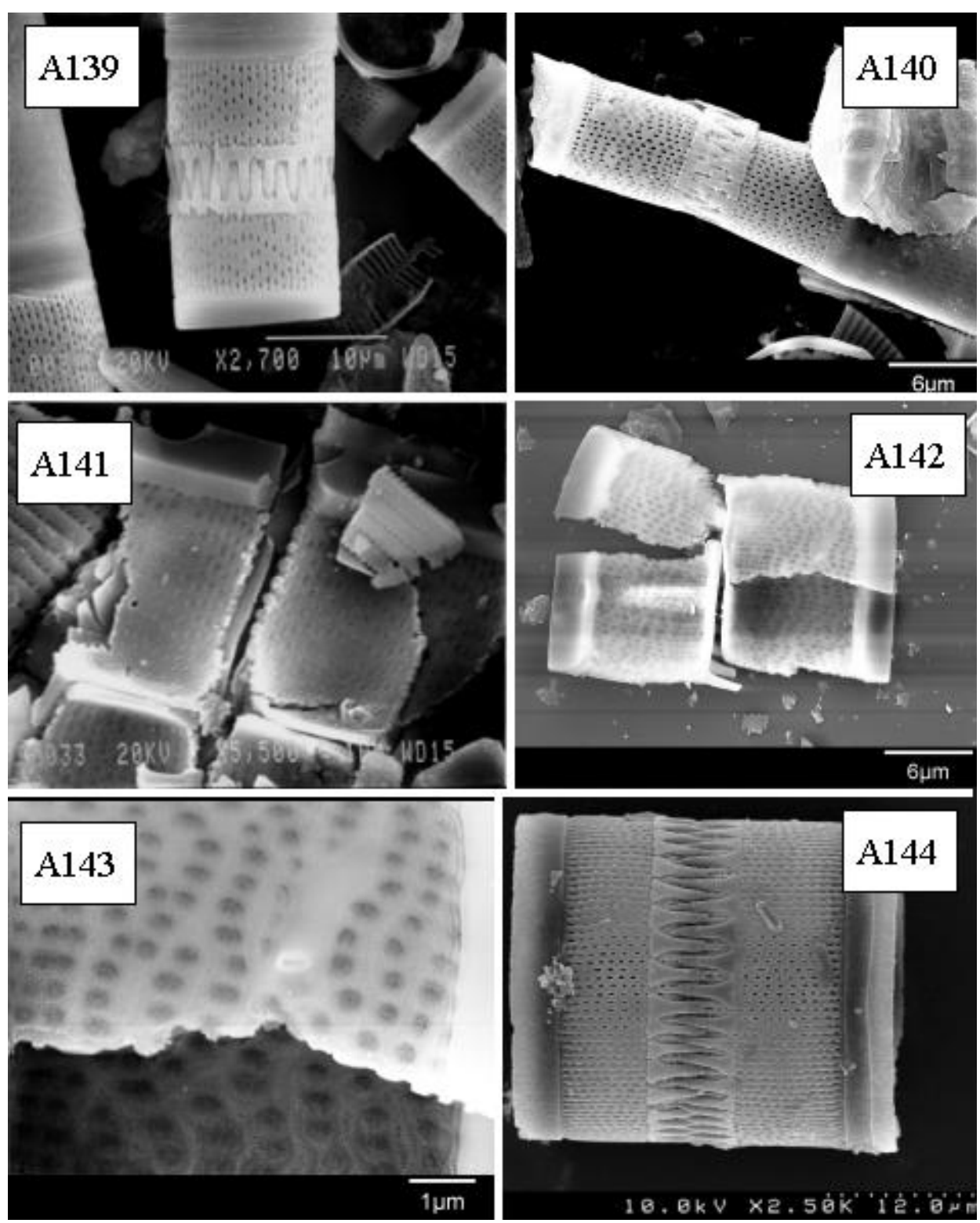

Figures A139-A144. Scanning electron micrographs of valves of Aulacoseira crenulata. 

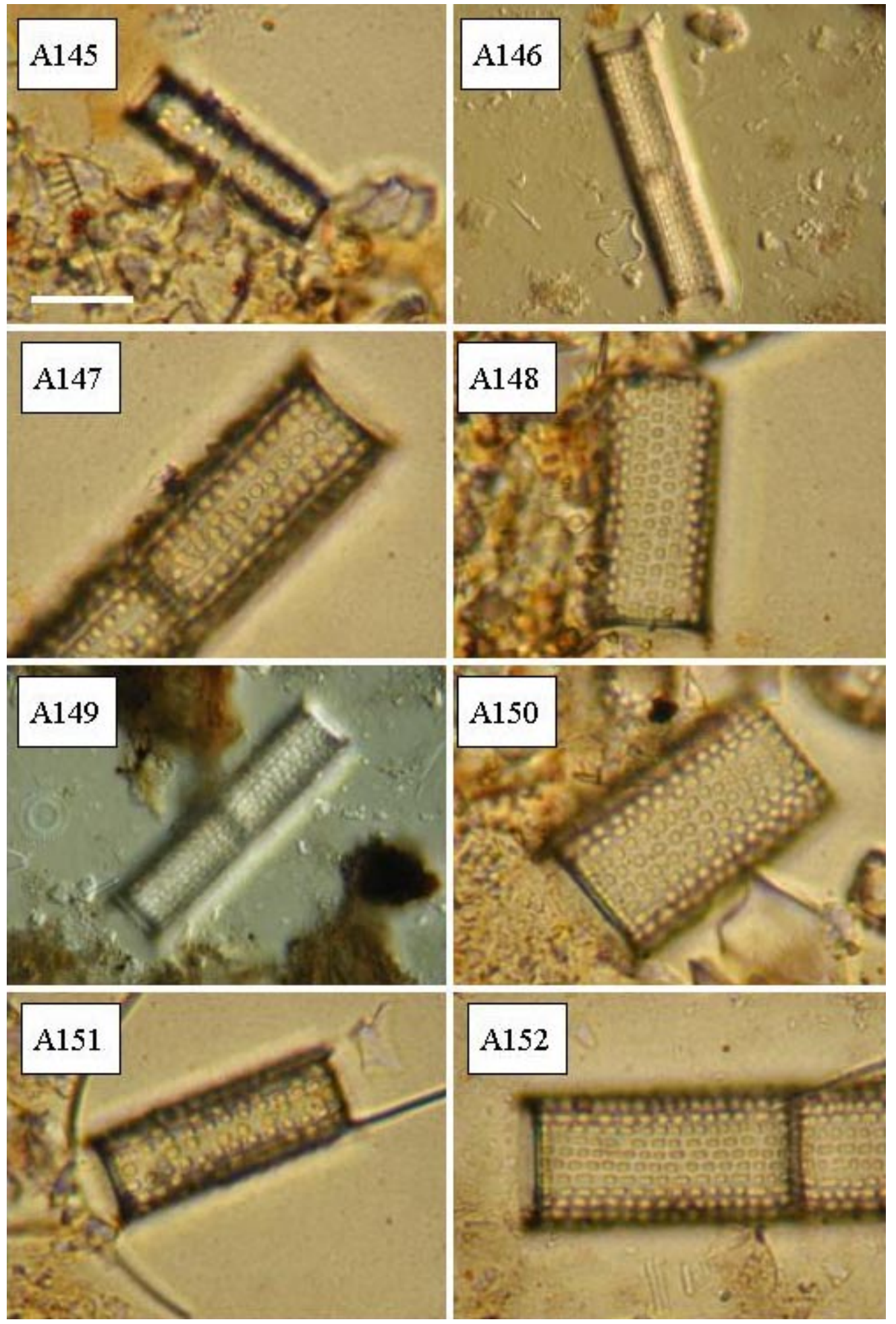

Figures A145-A152. Representative valves of Aulacoseira dianchiensis showing the size 
range for the species (scale bar $=10 \mu \mathrm{m})$.
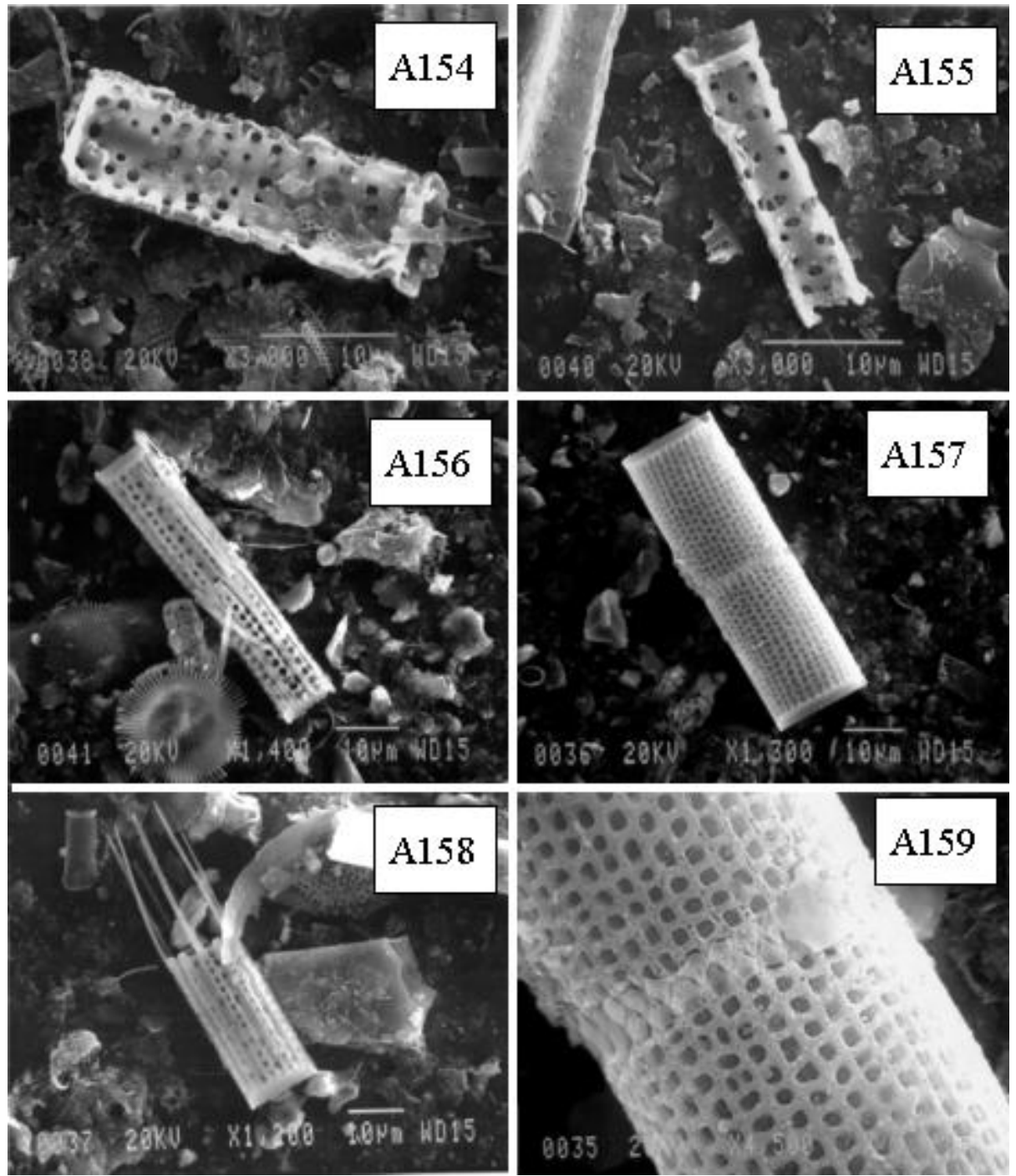

Figures A154-A159. Scanning electron micrographs of valves of Aulacoseira dianchiensis. 

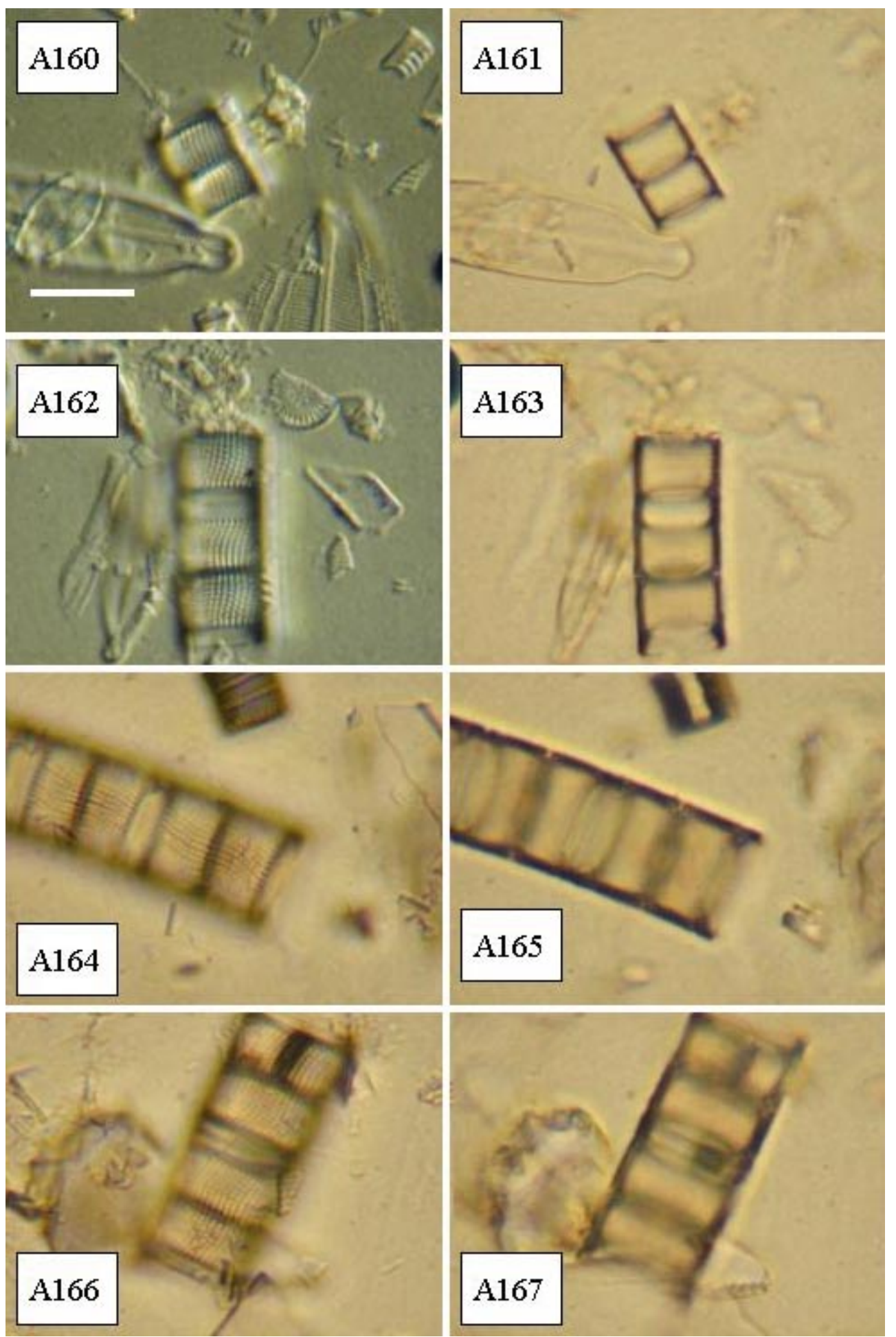
Figures A160-A167. Representative valves of Aulacoseira distans fide ADE showing the size range for the species (scale bar $=10 \mu \mathrm{m}$ ).
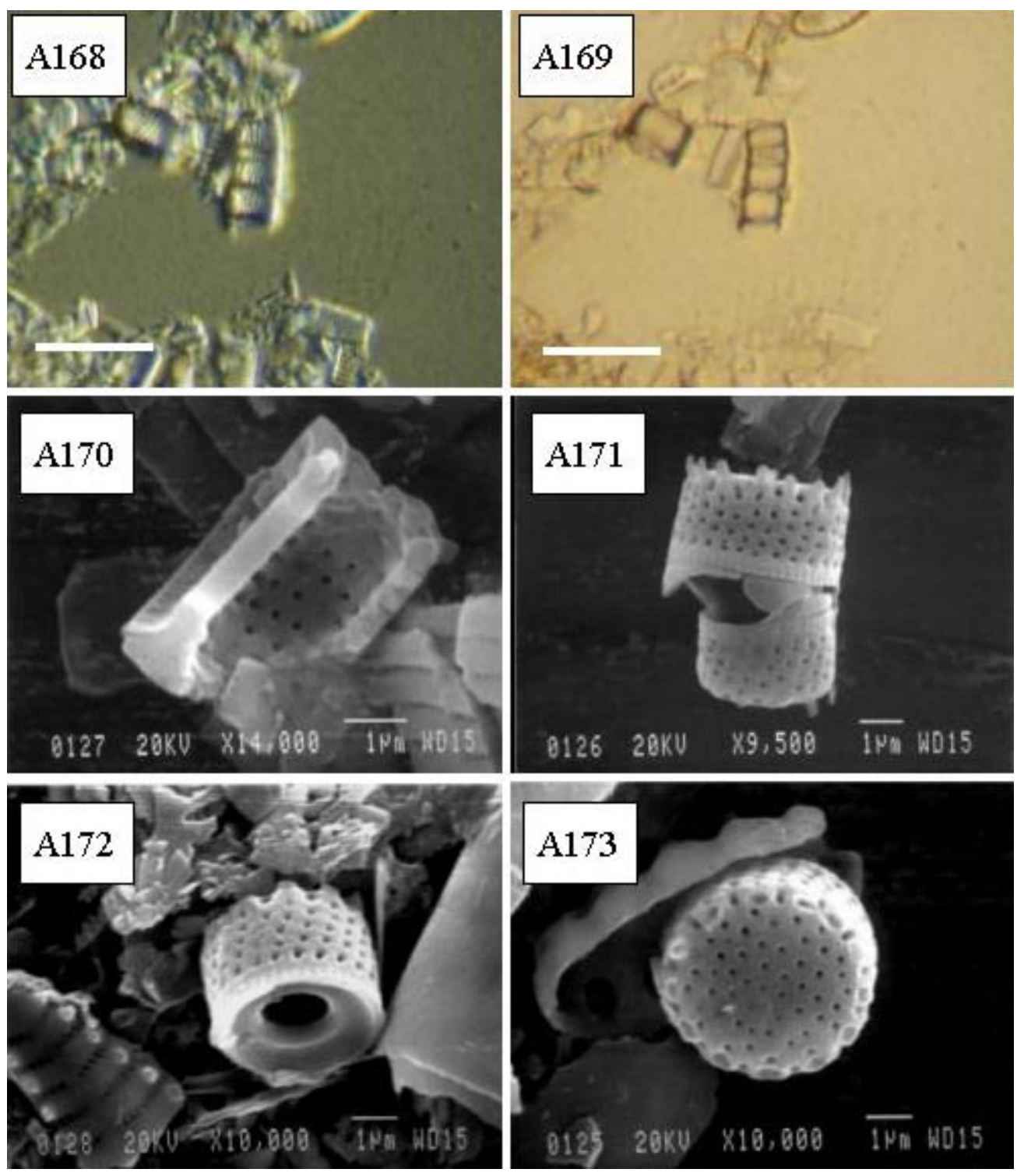

Figures A168-A173. Aulacoseira cf. distans. Figs. A168 \& A169. Light micrographs of A. cf. distans (scale bars $=10 \mu \mathrm{m}$ ). Figs. A170-A173. Scanning electron micrographs of A. cf. distans. 

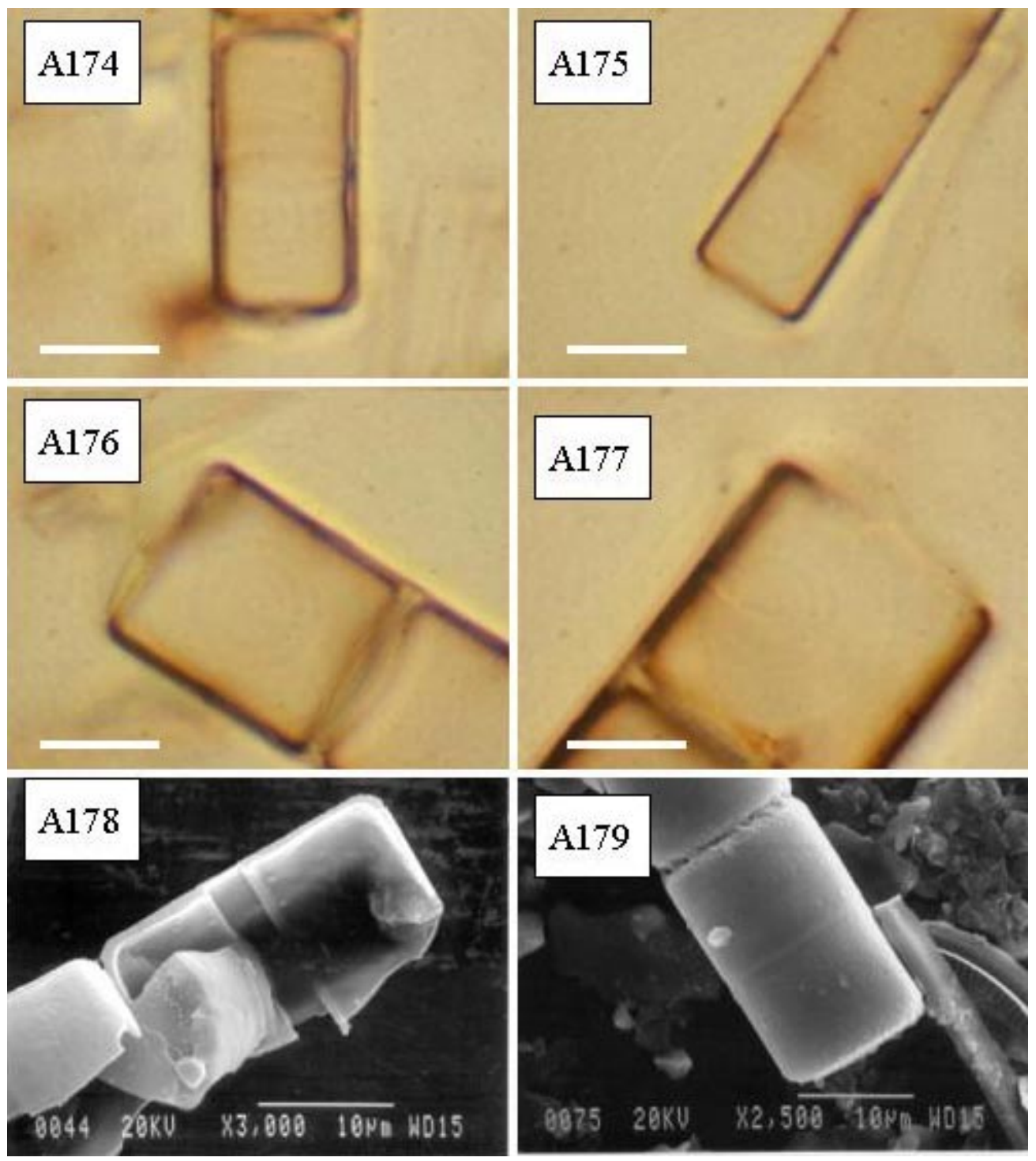

Figures A174-A179. Melosira varians. Figs. A174-A177. Light micrographs of M. varians (scale bars $=10 \mu \mathrm{m}$ ). Figs. A178 \& A179. Scanning electron micrographs of M. varians. 

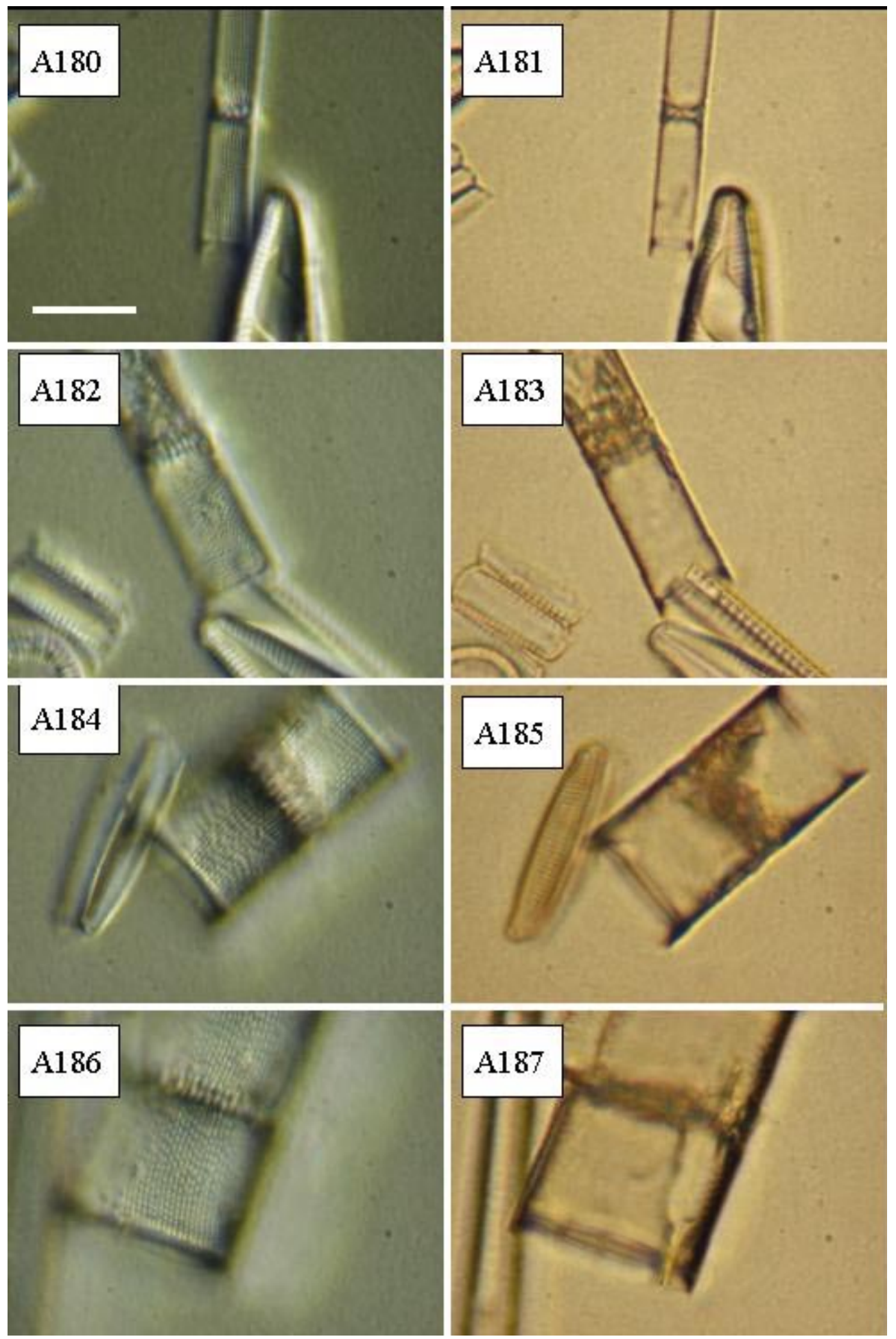
Figures A180-A187. Representative valves of Aulacoseira italica showing the size range for the species (scale bar $=10 \mu \mathrm{m})$.
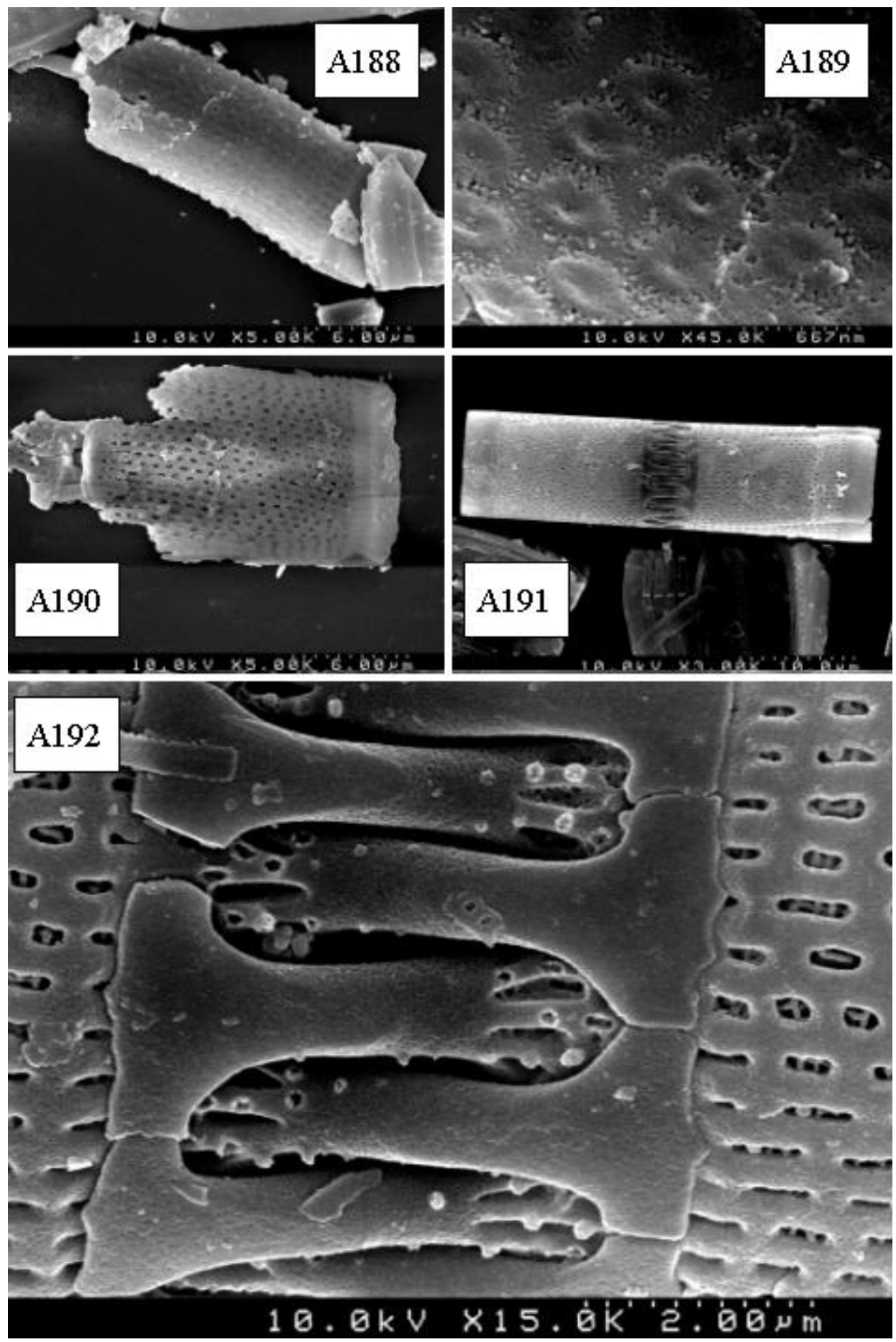

Figures A188-A192. Scanning electron micrographs of valves of Aulacoseira italica. 

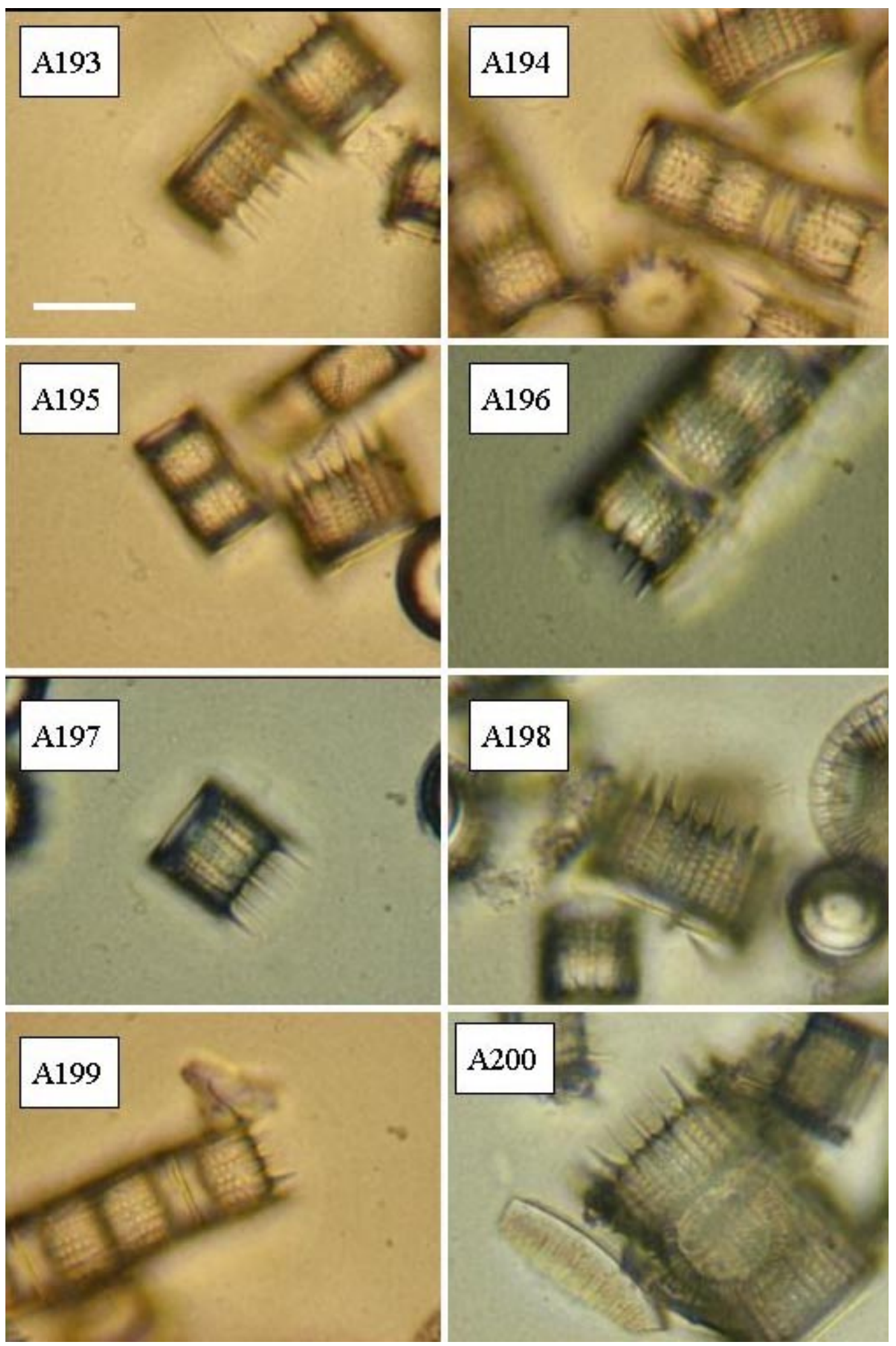

Figures A193-A200. Representative valves of Aulacoseira solida showing the size range for the species (scale bar $=10 \mu \mathrm{m}$ ). 

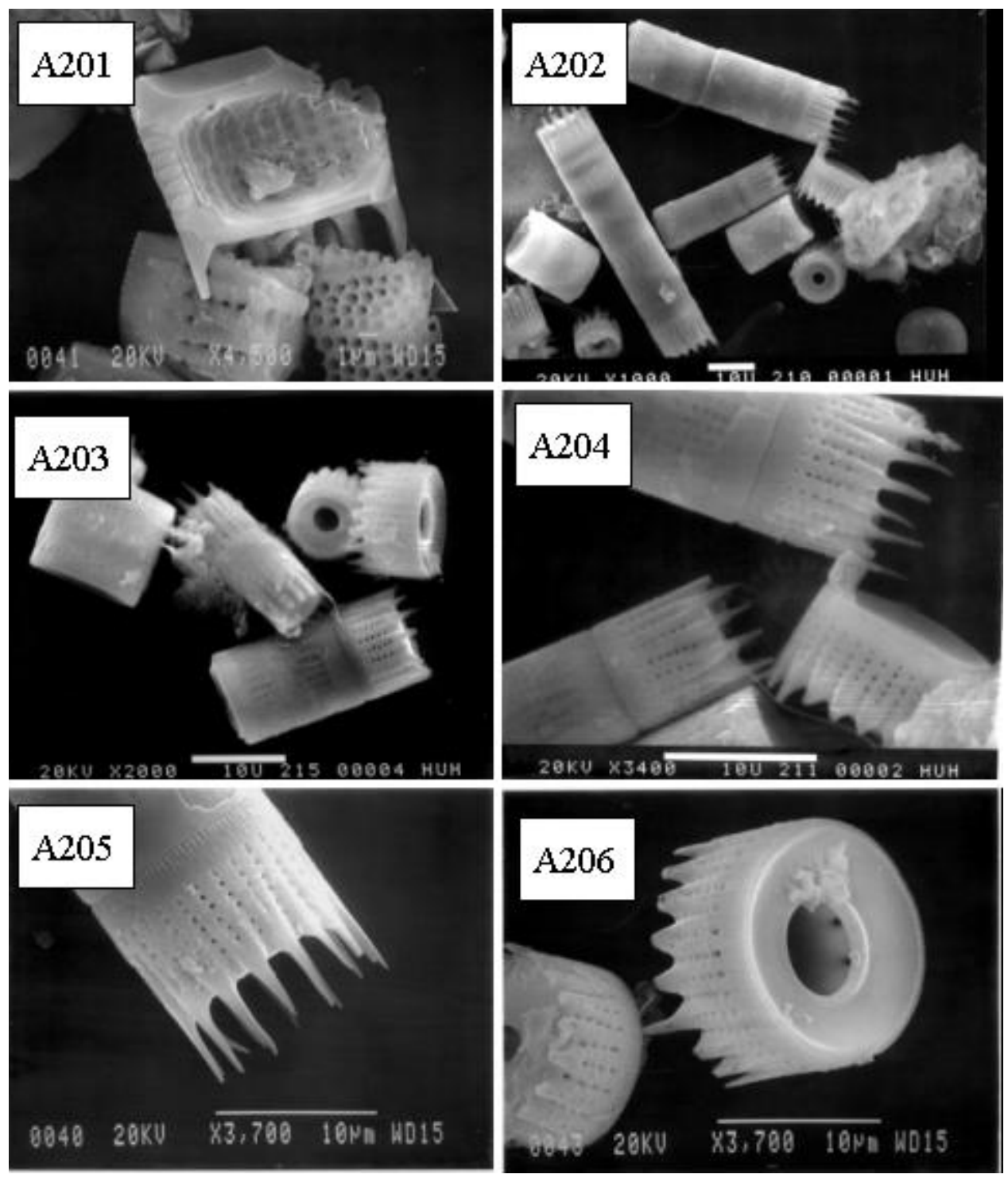

Figures A201-A206. Scanning electron micrographs of valves of Aulacoseira solida. 

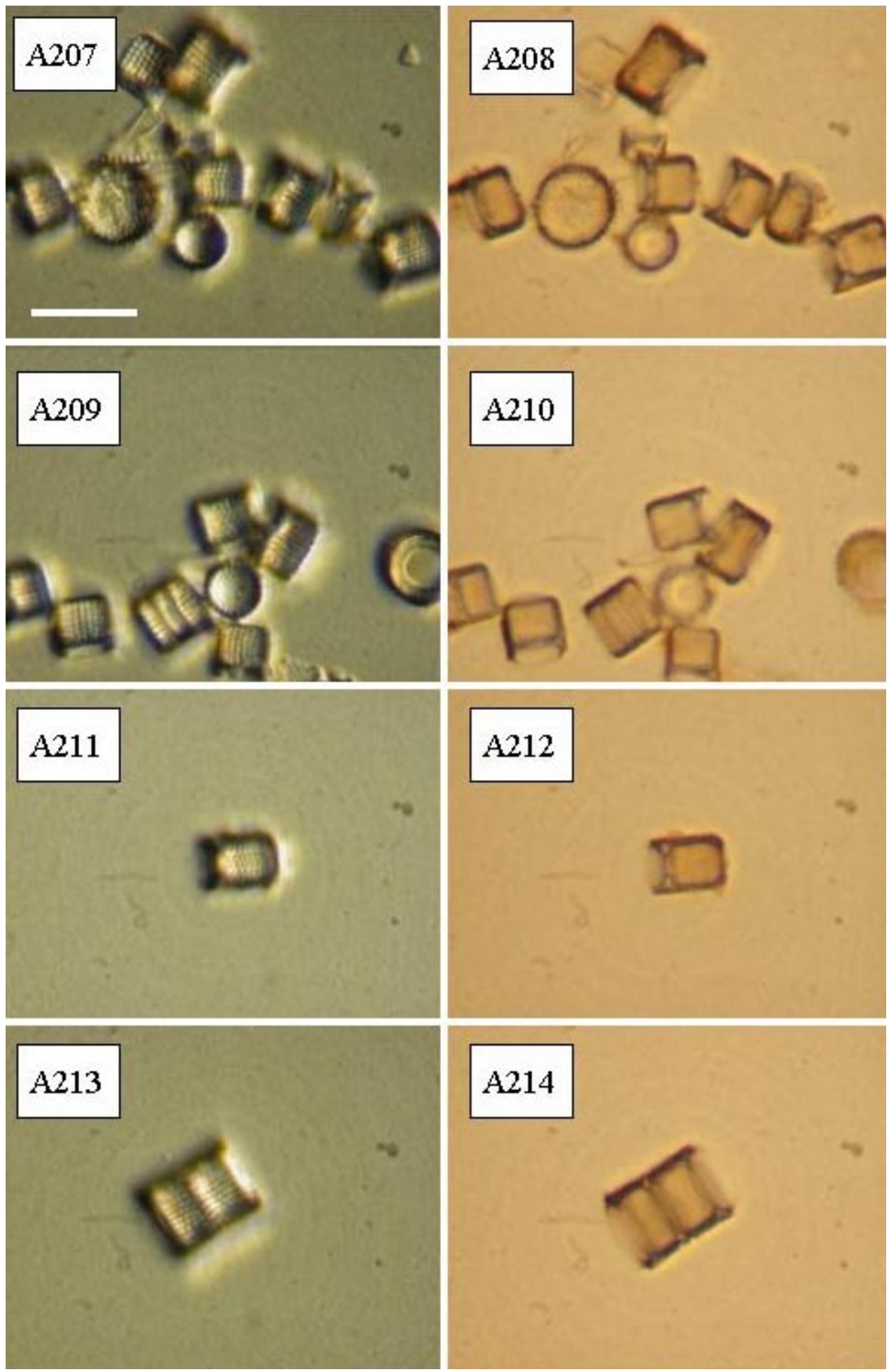

Figures A207-A214. Representative valves of Aulacoseira distans FH34 (Bilin material) showing the size range for the species (scale $b a r=10 \mu \mathrm{m}$ ). 

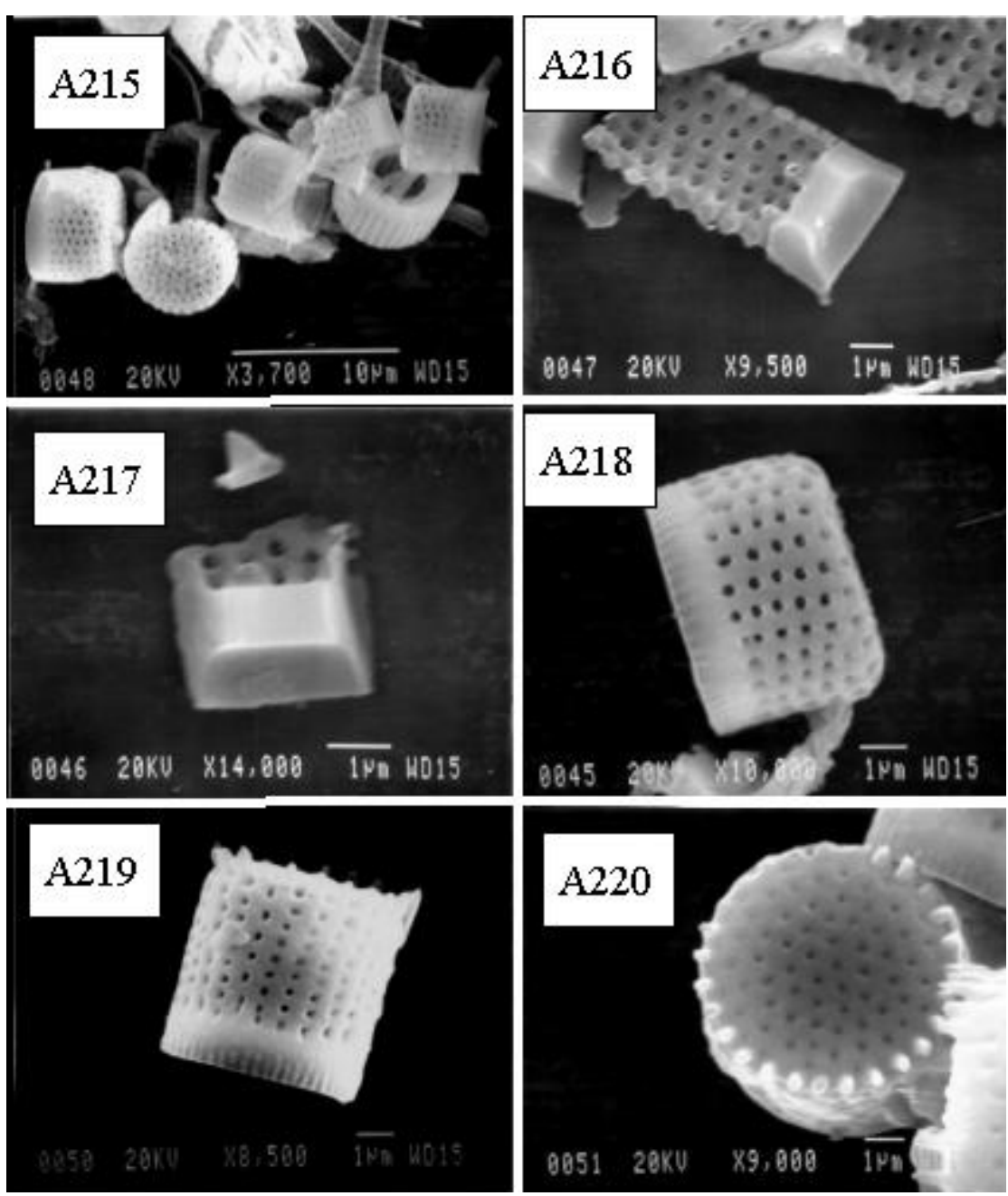

Figures A215-A220. Scanning electron micrographs of valves of Aulacoseira distans FH34 (Bilin material). 

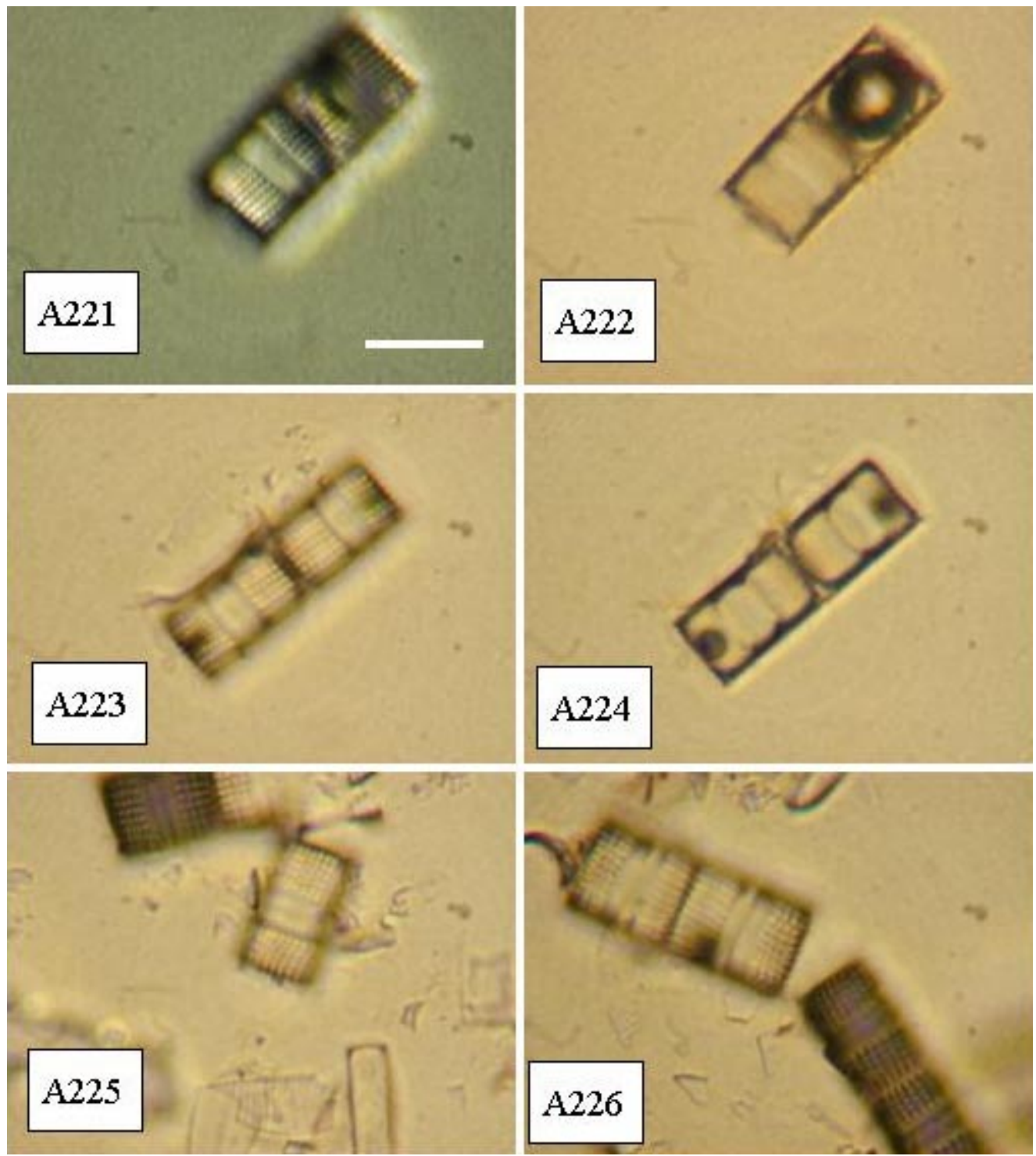

Figures A221-A226. Representative valves of Aulacoseira pfaffiana showing the size range for the species (scale bar $=10 \mu \mathrm{m}$ ). 


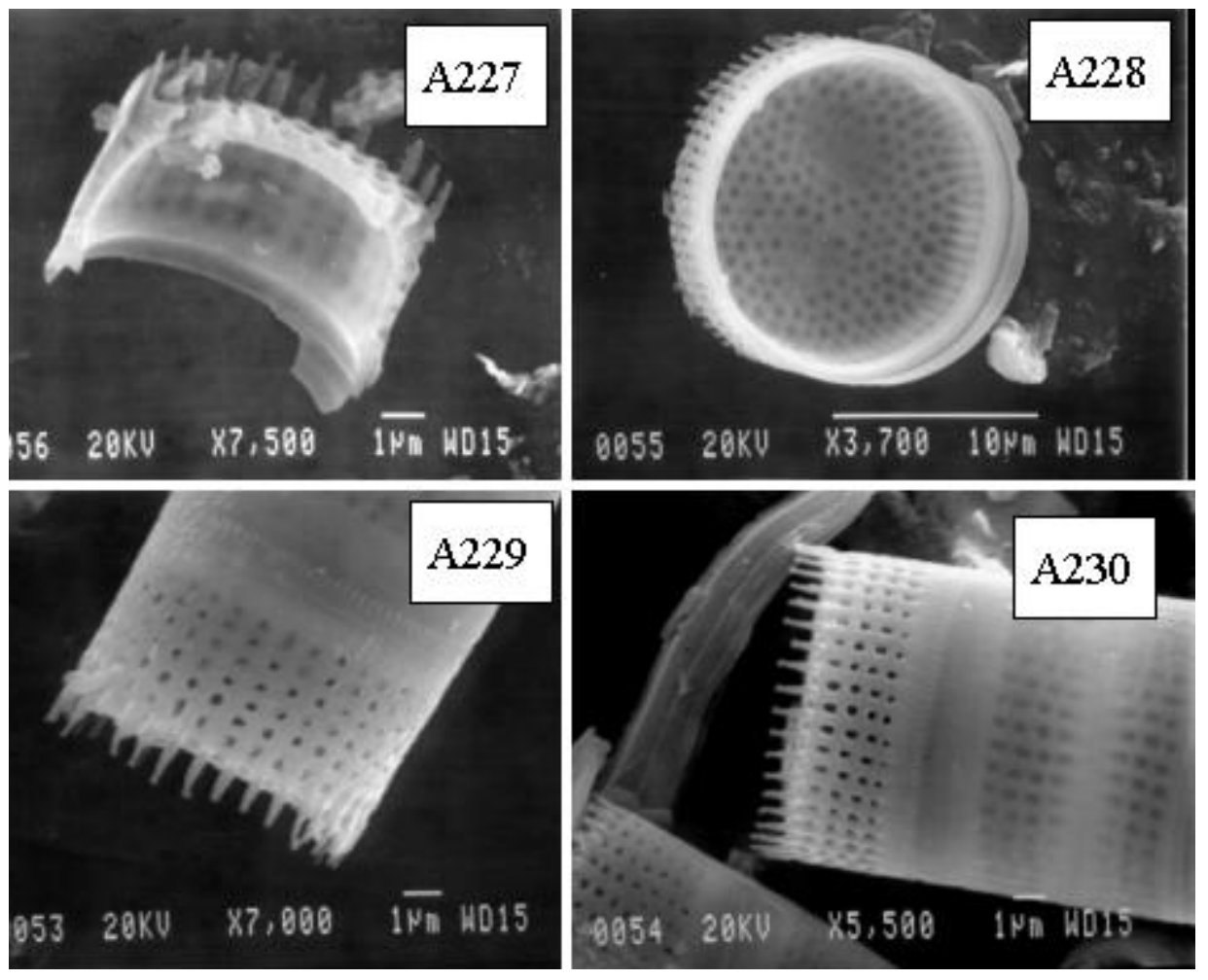

Figures A227-A230. Scanning electron micrographs of valves of Aulacoseira pfaffiana. 

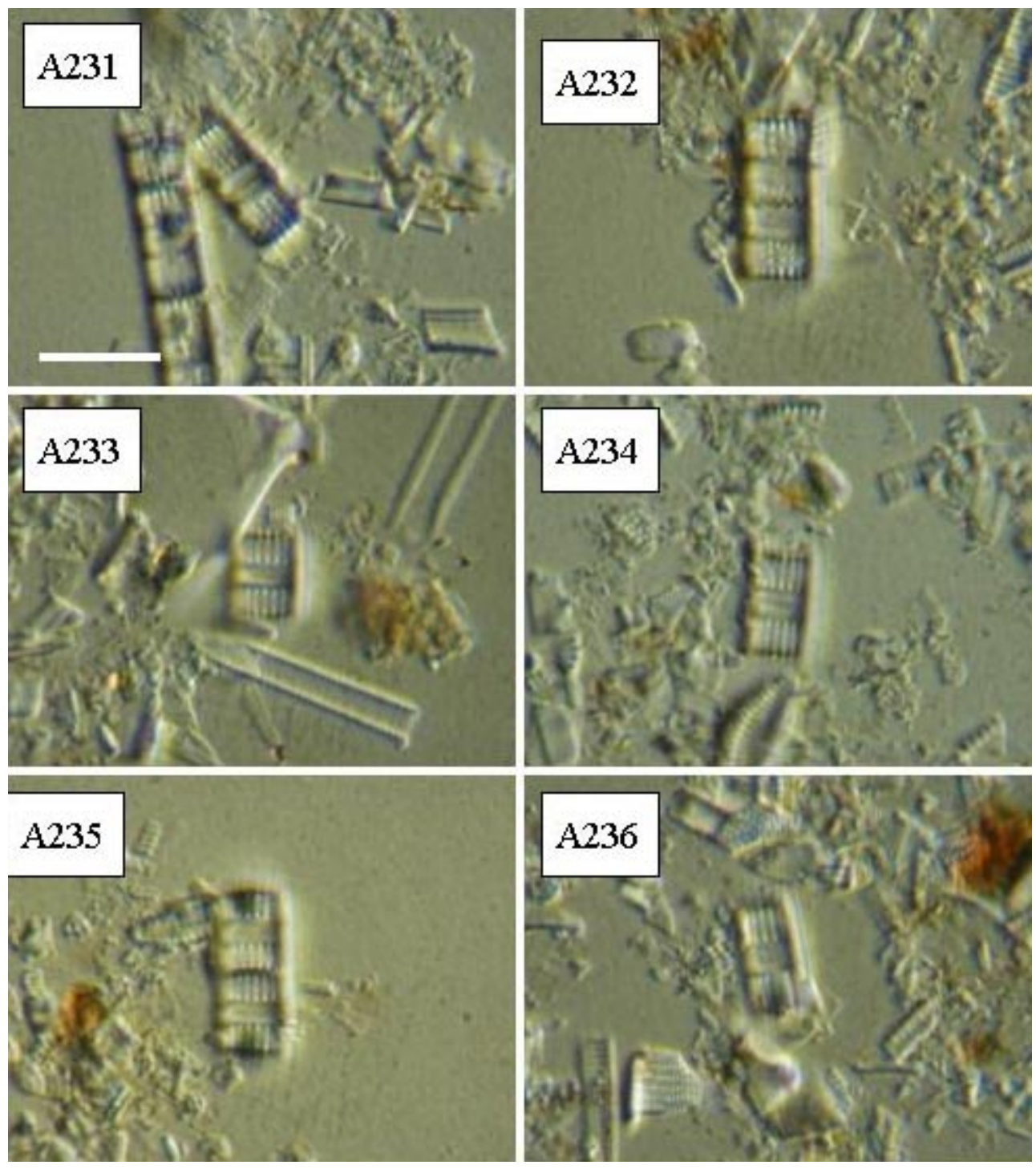

Figures A231-A236. Representative valves of Aulacoseira humilis showing the size range for the species (scale bar $=10 \mu \mathrm{m}$ ). 

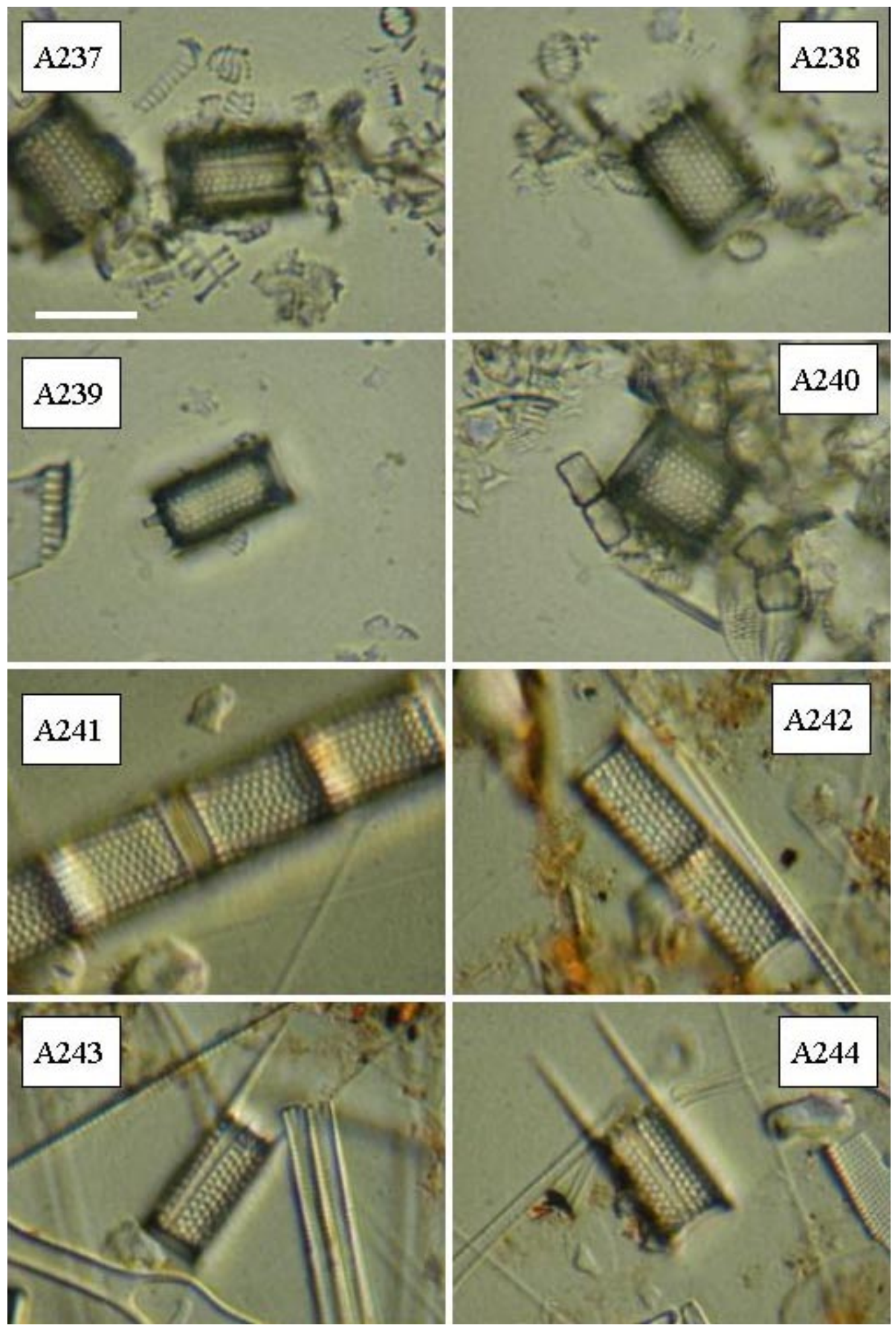

Figures A237-A244. Aulacoseira granulata. Figs. A237-A240. ECT1941 collection. Figs. A241-A244. MFP collection $($ scale bar $=10 \mu \mathrm{m})$. 

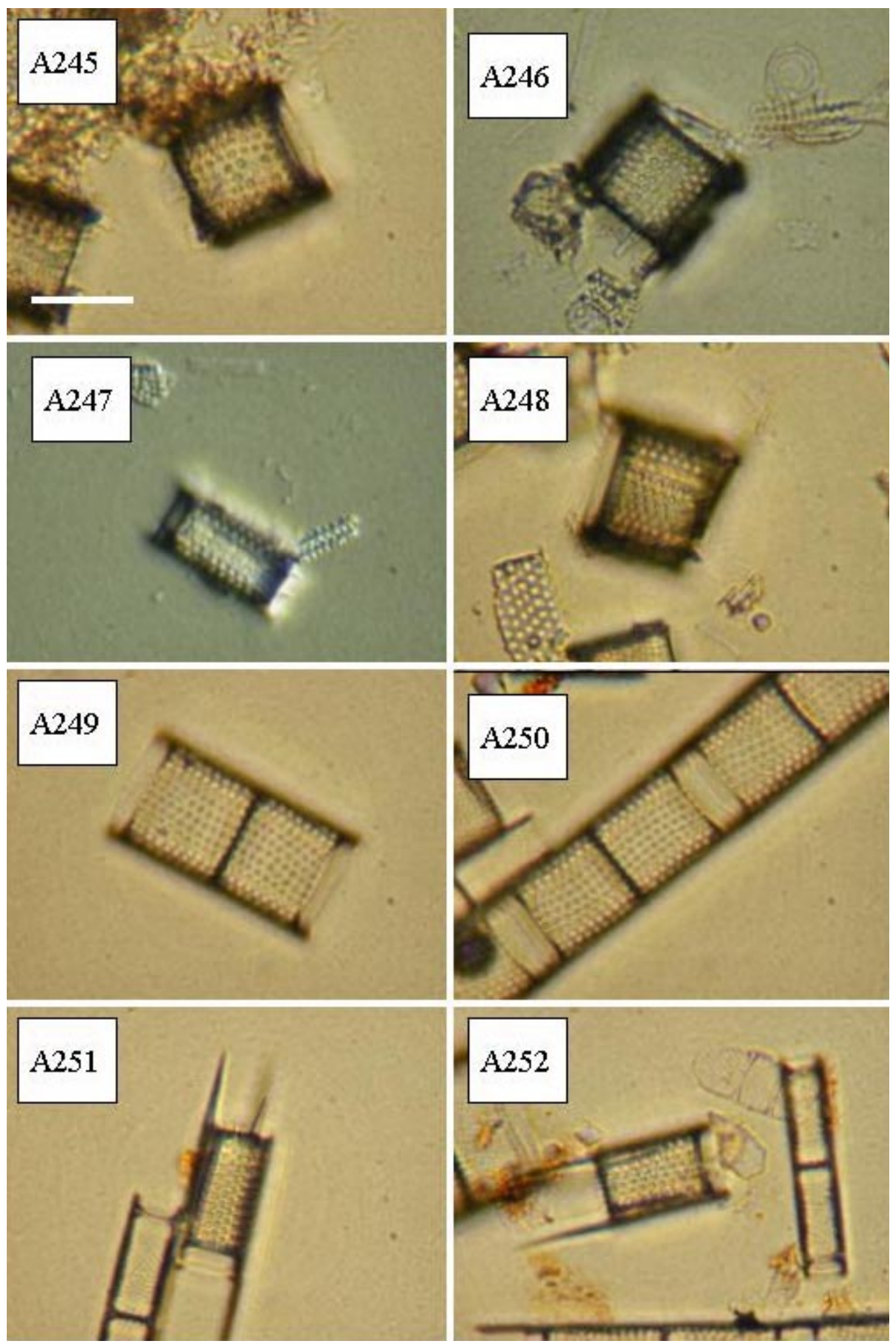

Figures A245-A252. Aulacoseira granulata. Figs. A245-A248. FH38 collection. Figs. A249-A252. MFH42 collection (scale bar $=10 \mu \mathrm{m}$ ). 

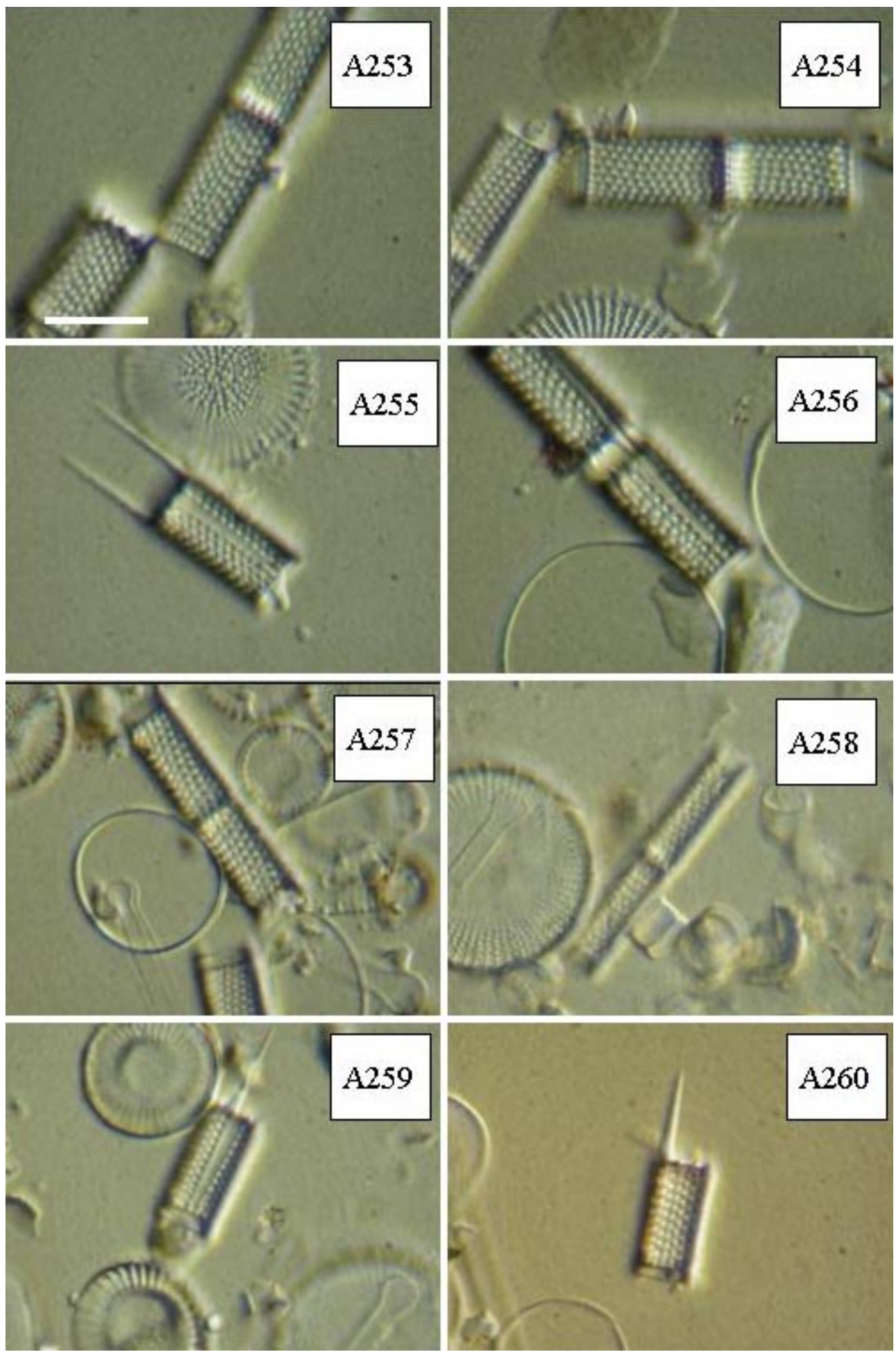

Figures A253-A260. Aulacoseira granulata. Figs. A253-A256. LB collection. Figs. A257-A260. ROR collection (scale bar $=10 \mu \mathrm{m}$ ). 

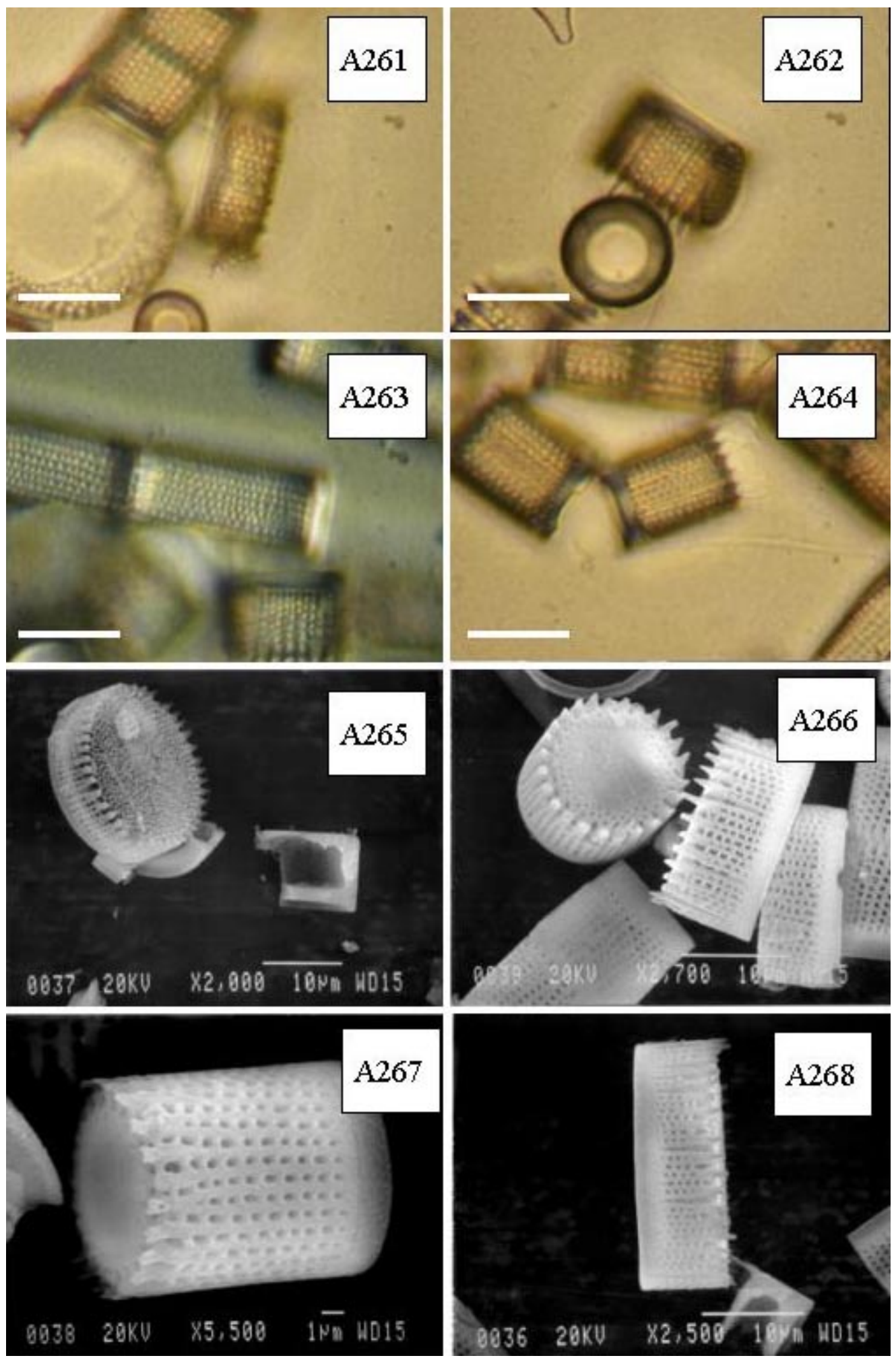

Figures A261-A268. Aulacoseira agassizii. Figs. A261-A264. Light micrographs (scale bars $=10 \mu \mathrm{m})$. Figs. A265-A268. Scanning electron micrographs. 

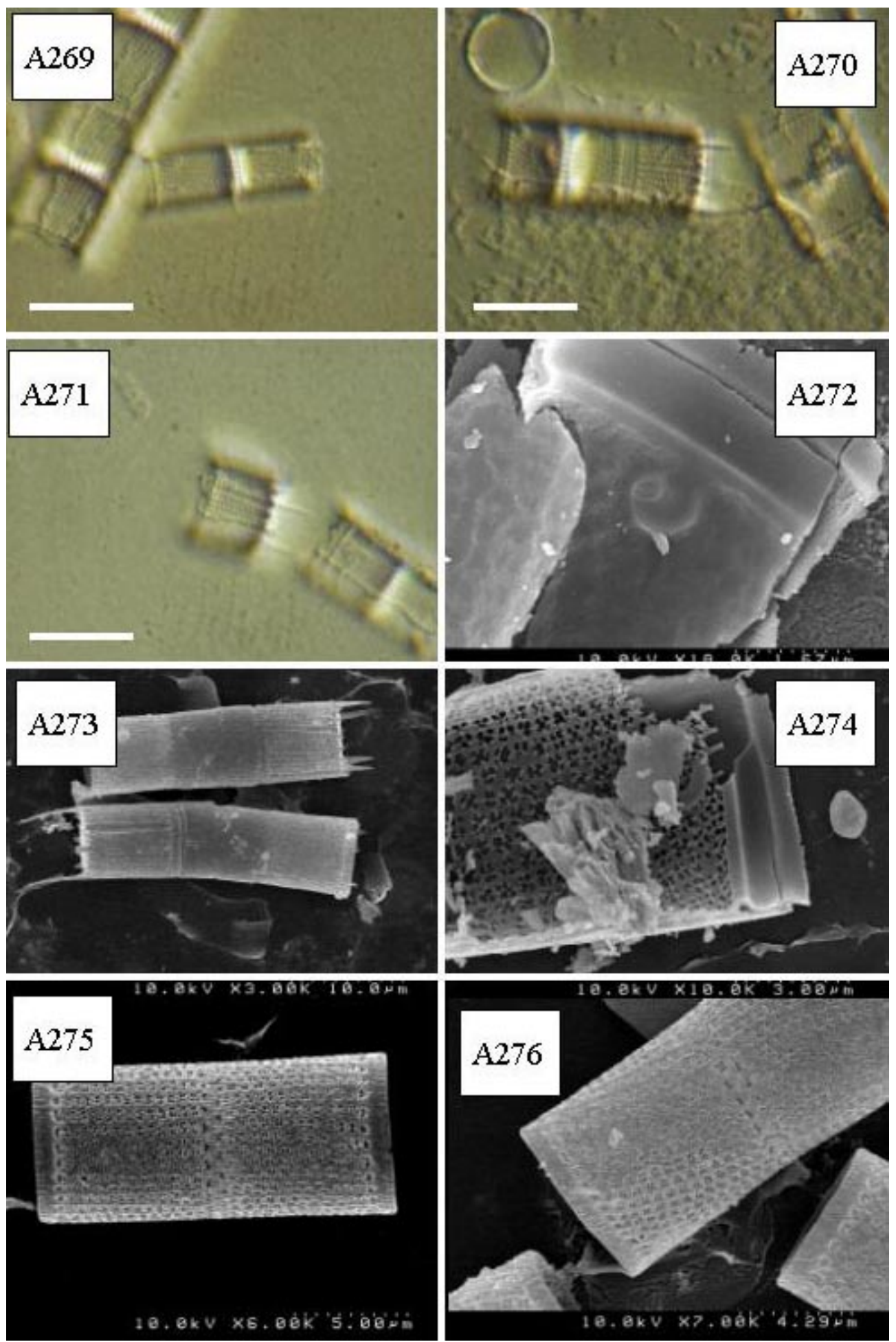

Figures A269-A276. Aulacoseira granulata var. jonensis. Figs. A269-A271. Light micrographs $($ scale bars $=10 \mu \mathrm{m})$. Figs. A272-A276. Scanning electron micrographs. 

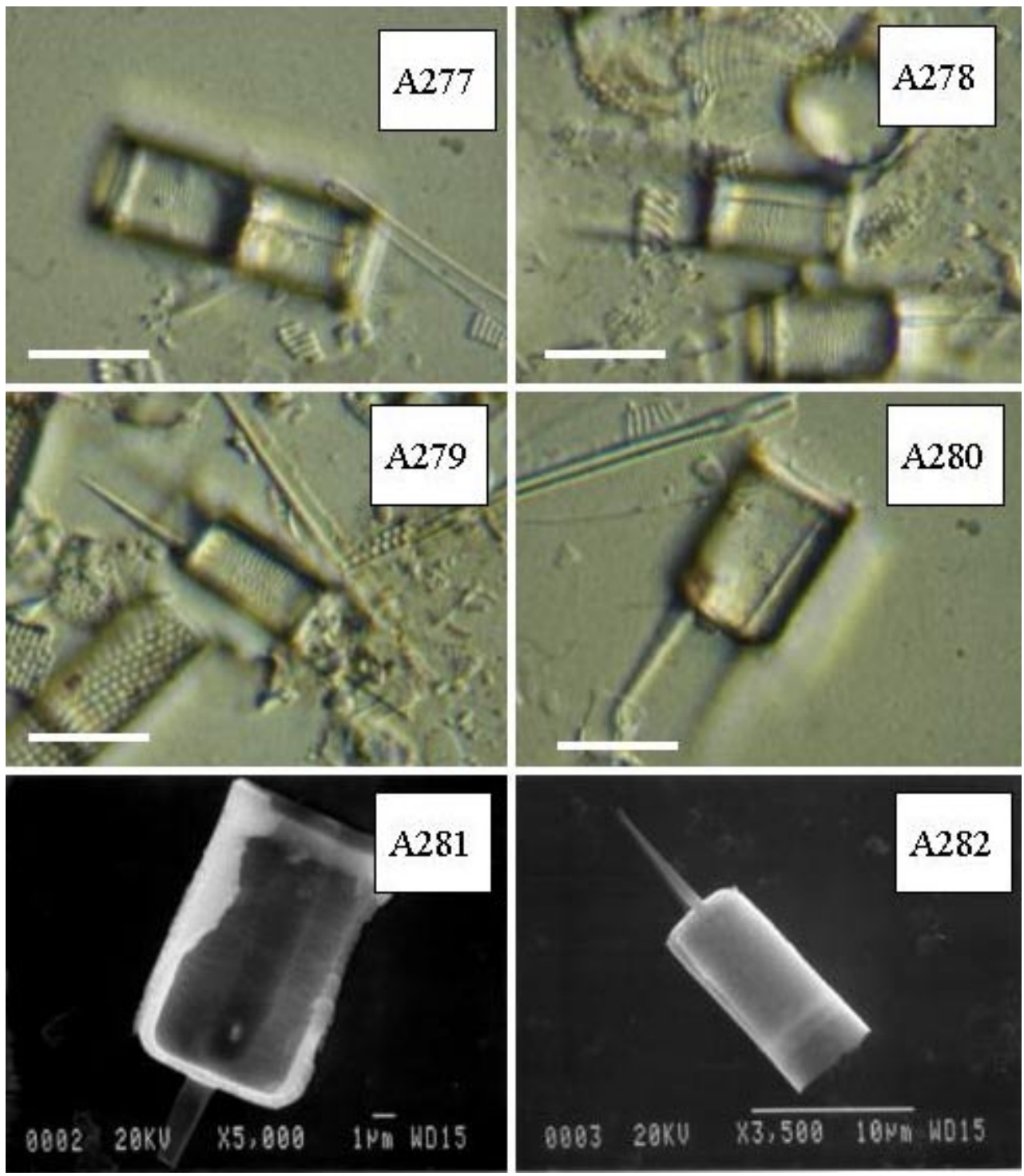

Figures A277-A282. Aulacoseira herzogii. Figs. A277-A280. Light micrographs (scale bars $=10 \mu \mathrm{m})$. Figs. A281-A282. Scanning electron micrographs. 


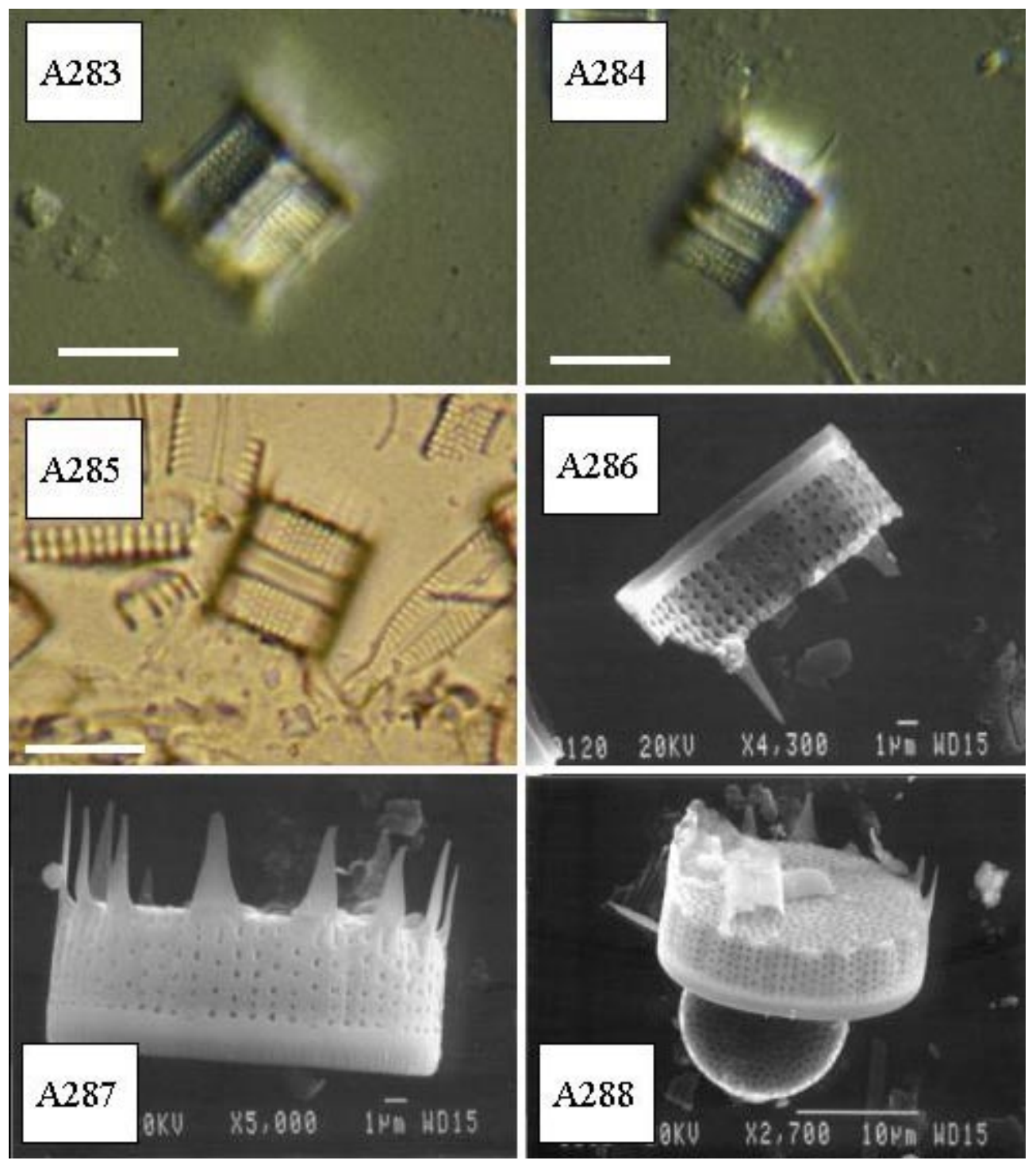

Figures A283-A288. Aulacoseira laevissima. Figs. A283-A285. Light micrographs (scale bars $=10 \mu \mathrm{m})$. Figs. A286-A288. Scanning electron micrographs. 

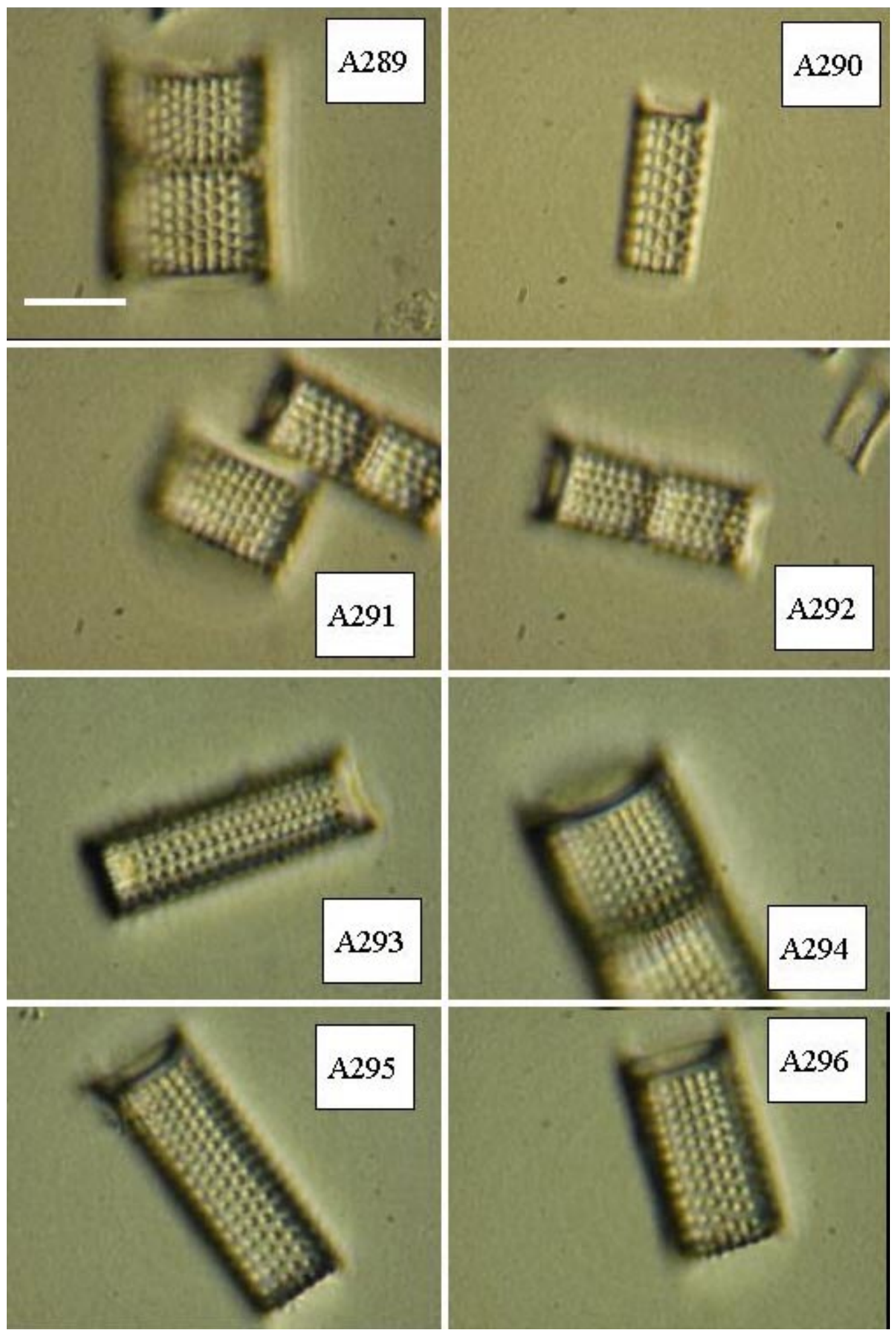

Figures A289-A292. Aulacoseira granulata fide H. L. Smith (scale bar $=10 \mu \mathrm{m}$ ). Figures. A293-A296. Aulacoseira granulata fide Van Heurck. 

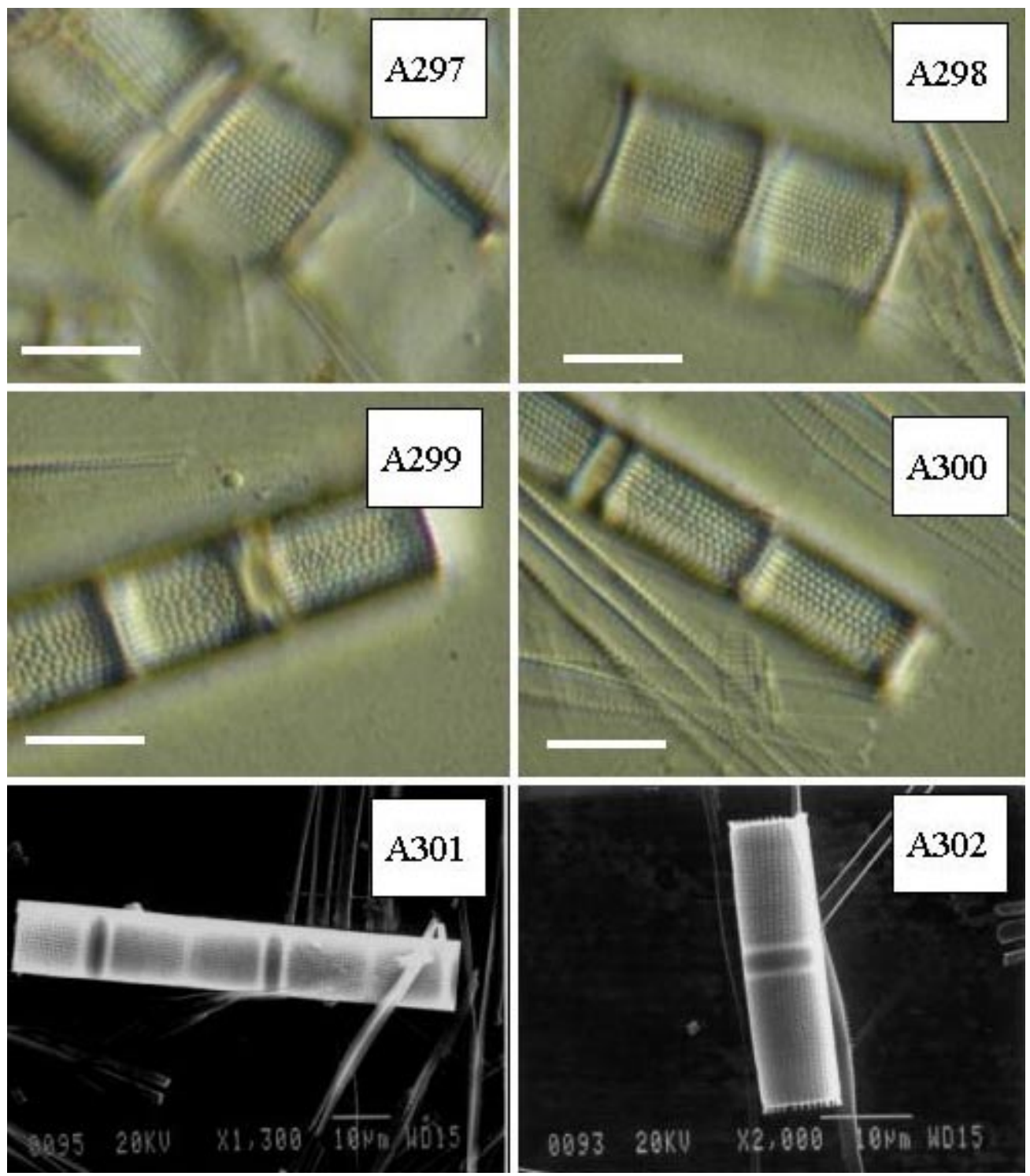

Figures A297-302. Aulacoseira islandica. Figs. A297-300. Light micrographs (scale bars $=10 \mu \mathrm{m})$. Figs. A301-A302. Scanning electron micrographs. 

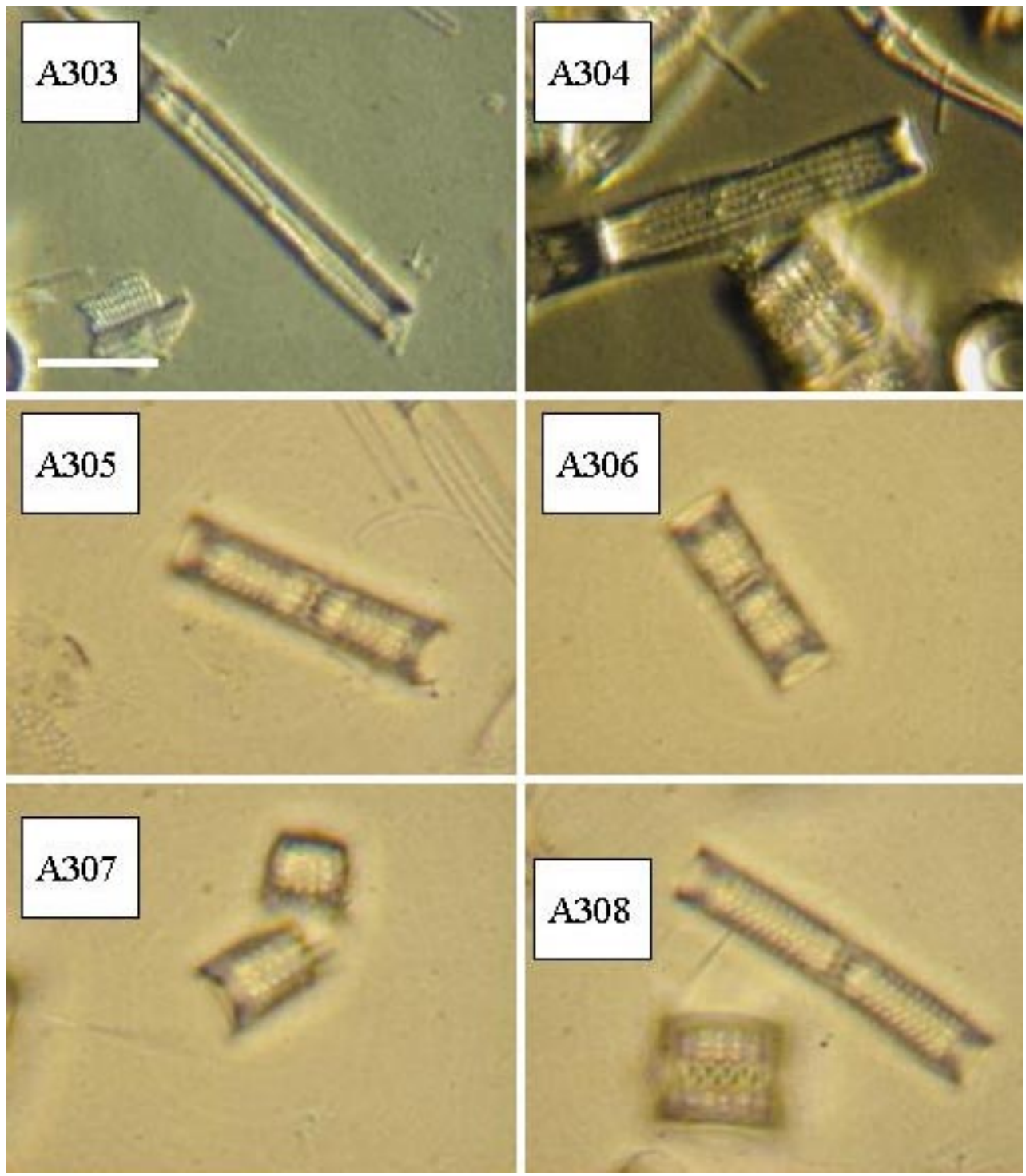

Figures A303-A308. Representative valves of Aulacoseira krammeri nom. prov. showing the size range for the species (scale bar $=10 \mu \mathrm{m}$ ). 

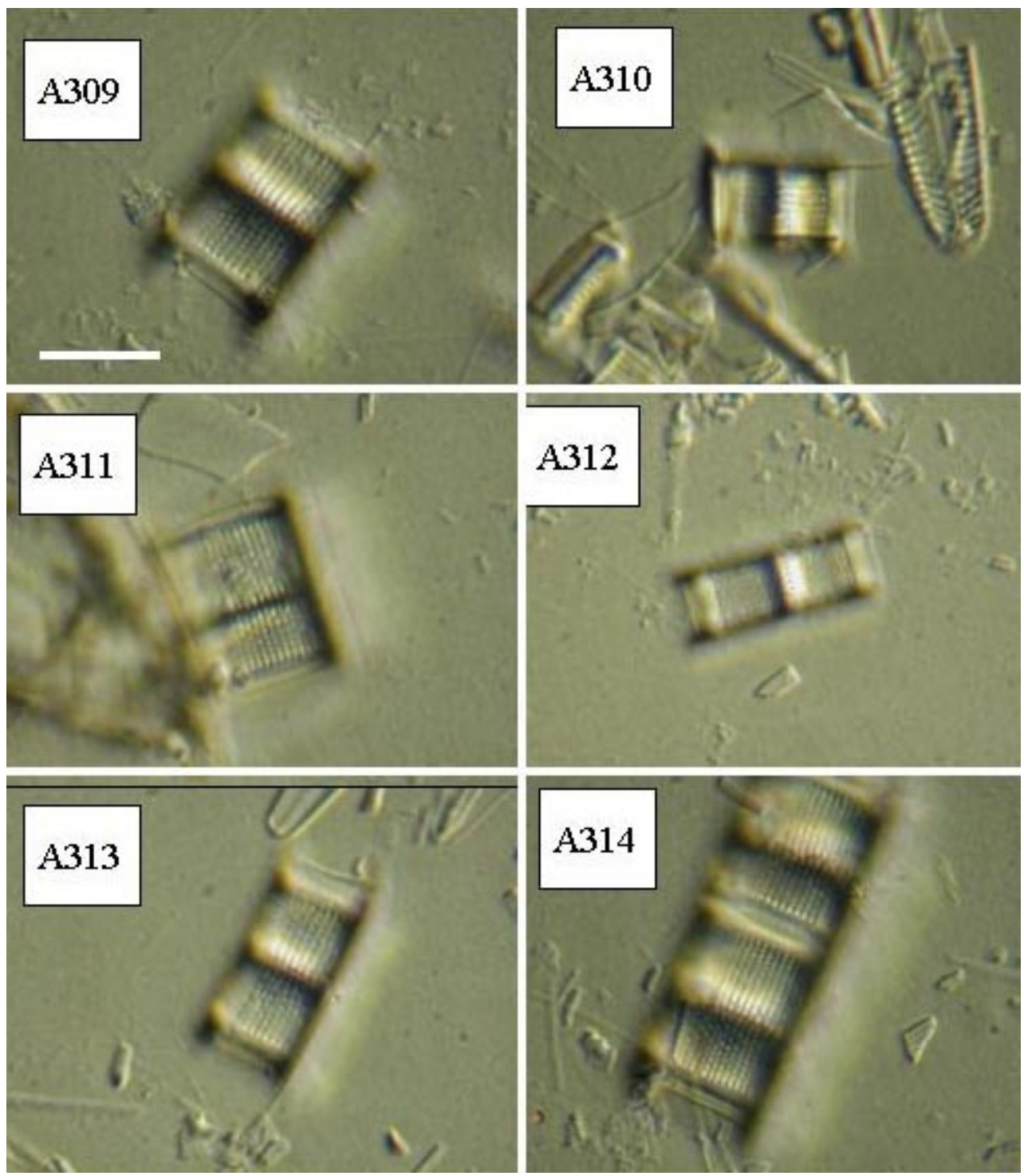

Figures A309-A314. Representative valves of Aulacoseira lacustris showing the size range for the species (scale bar $=10 \mu \mathrm{m})$. 

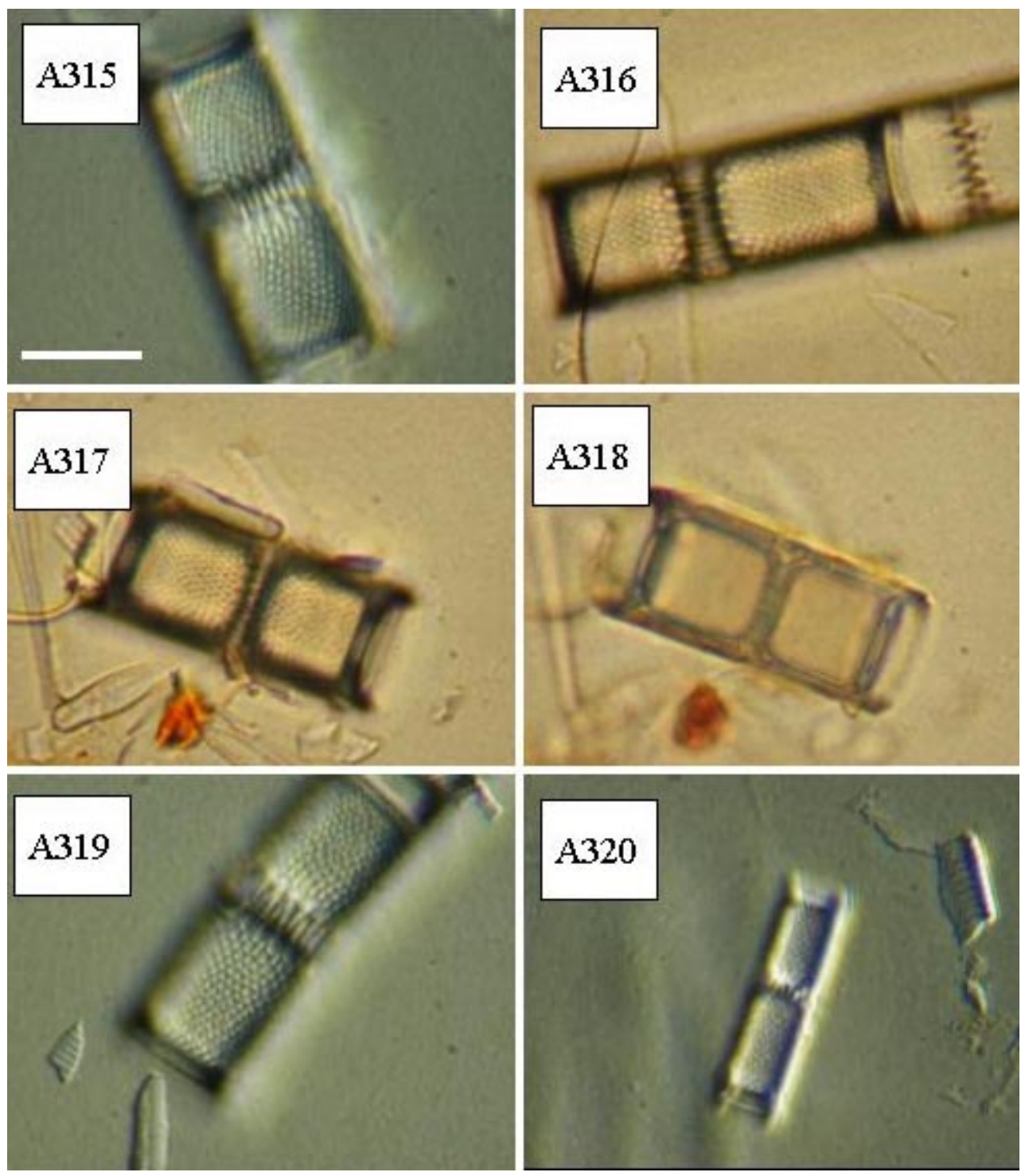

Figures A315-A320. Representative valves of Aulacoseira valida showing the size range for the species (scale bar $=10 \mu \mathrm{m})$. 

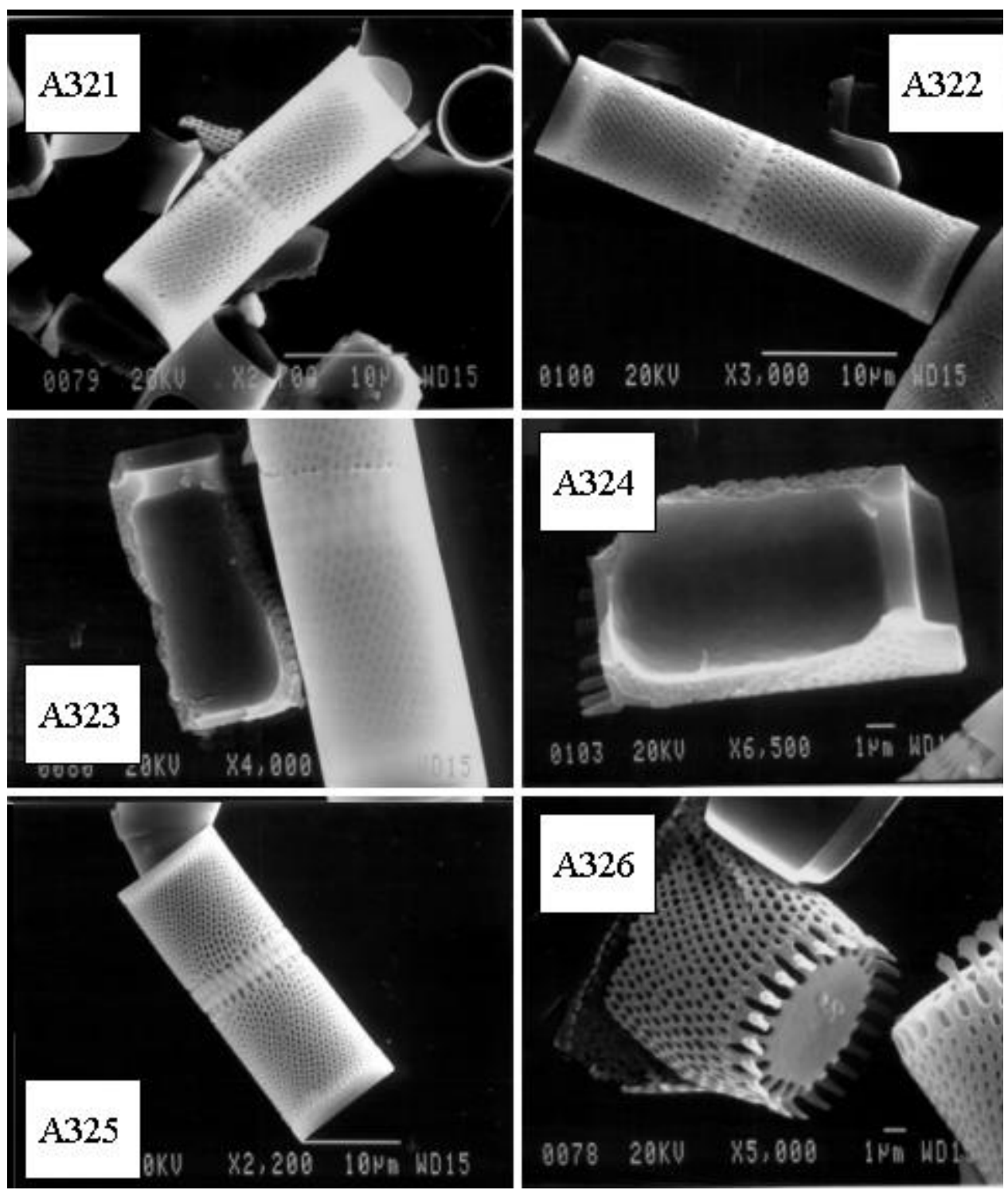

Figures A321-A326. Scanning electron micrographs of Aulacoseira valida. 


\section{REFERENCES}

Almeida, M. T. \& Bisby, F. A. 1984. A simple method for establishing taxonomic characters from measurement data. Taxon 33(3):405-409.

Archie, J. W. 1985. Methods for coding variable morphological features for numerical taxonomic analysis. Systematic Zoology 34:326-345.

Archie, J. W. 1989. A randomization test for phylogenetic information in systematic data. Systematic Zoology 38:239-252.

Battarbee, R. W. 1986. Diatom Analysis. In: Handbook of Holocene Paleoecology and Paleohydrology (B. E. Berglund, ed.). J. Wiley and Sons, London. pp. 527-571.

Bremer, K. 1994. Branch support and tree stability. Cladistics 10:295-304.

Bull, J. J., Huelsenbeck, J. P., Cunningham, C. W., Swofford, D. L., \& Waddell, P. J. 1993. Partitioning and combining data in phylogenetic analysis. Systematic Biology 42:384-397.

Camburn, K. E. \& Charles, D. F. 2000. Diatoms of low-alkalinity lakes in the Northeastern United States. Academy of Natural Sciences of Philadelphia Special Publication 18. $152 \mathrm{pp}$. 
Camburn, K. E. \& Kingston, J. C. 1986. The genus Melosira from soft-water lakes with special reference to northern Michigan, Wisconsin and Minnesota. In: Diatoms and Lake Acidity (Smol, J., Batterbee, R., Davis, R. \& Meriläinen, J., eds.), Junk, Dordrecht, the Netherlands, pp. 17-34.

Cannatella, D. C., Hillis, D. M., Chippindale, P. T., Weigt, L., Rand, A. S., \& Ryan, M. J. 1998. Phylogeny of frogs of the Physalaemus pustulosus species group, with an examination of data incongruence. Systematic Biology 47:311-355.

Chappill, J. A. 1989. Quantitative characters in phylogenetic analysis. Cladistics 5:217234.

Charles, D. F., Smol, J. P., \& Engstrom, D. R. 1994. Paleolimnological Approaches to Biological Monitoring. In: Biological Monitoring of Aquatic Systems (Loeb, S. L. \& Spacie, A., eds.). CRC Press, Boca Raton, Florida, p.233-293.

Chippindale, P. T. \& Wiens, J. J. 1994. Weighting, partitioning, and combining characters in phylogenetic analysis. Systematic Biology 43:278-287.

Colless, D. H. 1980. Congruence between morphometric and allozyme data for Menidia species: A reappraisal. Systematic Zoology 29:288-299. 
Cox, E. J. \& Williams, D. M. 2000. Systematics of naviculoid diatoms: the interrelationships of some taxa with a stauros. European Journal of Phycology 35:273282.

Cracraft, J. 1981. The use of functional and adaptive criteria in phylogenetic systematics. American Zoologist 21:21-36.

Cranston, P. S. \& Humphries, C. J. 1988. Cladistics and computers: a chironomid conundrum? Cladistics 4:72-92.

Crawford, R. M. 1971. The fine structure of the frustule of Melosira varians C. A. Agardh. British Phycological Journal 6:175-186.

Crawford, R. M. 1978. The taxonomy and classification of the diatom genus Melosira C. A. Agardh. III. Melosira lineata (Dillw.) C. A. Ag. and M. varians C. A. Ag. Phycologia 17:237-250.

Crawford, R. M. 1988. A reconsideration of Melosira arenaria and M. teres resulting in a proposed new genus Ellerbeckia. In: Algae and the Aquatic Environment (Round, F. E., ed.). Biopress, Bristol pp. 413-433.

Crawford, R. M. \& Likhoshway, Y. 1999. The frustule structure of original material of Aulacoseira distans (Ehrenberg) Simonsen. Diatom Research 14:239-250. 
Crawford, R. M. \& Likhoshway, Y. 2002. The velum of species of the diatom genus Aulacoseira Thwaites. In: Proceedings of the $15^{\text {th }}$ International Diatom Symposium, Perth, 1998 (J. John, ed.), Koeltz Scientific Books, Koenigstein, pp. 275-287.

Cronin, T. M. \& Schneider, C. E. 1990. Climatic influence on species: evidence from the fossil record. Trends in Ecology and Evolution 5: 275-279.

Darwin, C. 1859 (1981). On the Origin of Species. A Facsimile of the First Edition with an Introduction by Ernst Mayr. Harvard University Press (Cambridge, MA), $513 \mathrm{pp}$.

Daugbjerg, N. \& Andersen, R. A. 1997. A molecular phylogeny of the heterokont algae based on analyses of chloroplast-encoded $r b c L$ sequence data. Journal of Phycology 33:1031-1041.

de Queiroz, A. 1993. For consensus (sometimes). Systematic Biology 42:368-372.

Doyle, J. J. \& Doyle, J. L. 1987. A rapid isolation procedure for small quantities of fresh leaf material. Phytochemical Bulletin 19: 11-15.

Drebes, G. 1977. Sexuality. In The Biology of Diatoms (D. Werner, ed.), pp. 250-283. Oxford: Blackwell Scientific Publications. 
Edgar, R. K. \& Kociolek, J. P. MS. submitted 2002. Life Cycle-Associated Character Variation in Aulacoseira krammeri, sp. nov., a new Miocene species from Oregon (USA). Diatom Research.

Edgar, S. M. \& Theriot, E. C. 2003 . Heritability of mantle areolar characters in Aulacoseira subarctica (Bacillariophyta). Journal of Phycology (MS. accepted 2002).

Edlund, M. B. \& Stoermer, E. F. 1997. Ecological, evolutionary, and systematic significance of diatom life histories. Journal of Phycology 33:897-918.

Edlund, M. B., Stoermer, E. F., \& Taylor, C. M. 1996. Aulacoseira skvortzowii sp. nov. (Bacillariophyta), a poorly understood diatom from Lake Baikal, Russia, Journal of Phycology 32:165-175.

Faith, D. P. 1991. Cladistic permutation tests for monophyly and non-monophyly. Systematic Zoology 40: 366-375.

Faith, D. P. \& Cranston, P. S. 1991. Could a cladogram this short have arisen by chance alone? On permutation tests for cladistic structure. Cladistics 7: 1-28.

Falconer, D. S. 1981. Introduction to Quantitative Genetics. Second Edition. Longman Group Limited, London. 340 pp. 
Farris, J. S. 1989. Hennig86: a PC-DOS program for phylogenetic analysis. Cladistics 5: 163.

Farris, J. S. 1990. Phenetics in camouflage. Cladistics 6: 91-100.

Florin, M-B. 1981. The taxonomy of some Melosira species. A comparative morphological study. II. In: Proceedings of the $6^{\text {th }}$ International Diatom Symposium, Budapest, 1980. Koeltz, Koenigstein, pp. 43-74.

Forey, P. L. \& Kitching, I. J. 2000. Experiments in coding multistate characters. In: Homology and Systematics: Coding characters for phylogenetic systematics (eds. R. Scotland \& R. T. Pennington). Chapter 3. The Systematics Association Special Volume Series 58, Taylor \& Francis, London.

Freeman, S. \& Herron, J. C. 2001. Evolutionary Analysis. Second edition, Prentice Hall, New Jersey, 704 pp.

Fritsch, F. E. 1935. The structure and reproduction of the algae. Volume I. Introduction, Chlorophyceae, Xanthophyceae, Chrysophyceae, Bacillariophyceae, Cryptophyceae, Dinophyceae, Cloromonadineae, Euglenineae, Colourless Flegellate. Cambridge University Press, London, 791pp. 
Gassé, F. 1980. Les diatomées lacustres Plio-Pléistocenes du Gadeb (Éthiopie).

Systématique, paléoécologie, biostratigraphie. Revue Algolgique, Mem. hors-ser. no. 3, $249 \mathrm{pp}$.

Gift, N. \& Stevens, P. F. 1997. Vagaries in the delimitation of character states in quantitative variation - an experimental study. Systematic Biology 46:112-125.

Guillard, R. R. L. 1975. Culture of Phytoplankton for feeding marine invertebrates. In: Culture of Marine Invertebrate Animals (W. L. Smith \& M. H. Chanley, eds.), Plenum Press, New York. pp. 29-60.

Hamilton, P. B., Poulin, M., Charles, D. F., \& Angell, M. 1992. Americanarum Diatomarum Exsiccata: CANA, voucher slides from eight acidic lakes in northeastern North America. Diatom Research 7:25-36.

Hartl, D. L. \& Clark, A. G. 1989. Principles of Population Genetics. Second edition. Sinauer, Sunderland, Massachusetts, $682 \mathrm{pp}$.

Hawkins, J. A. 2000. A survey of primary homology assessment: different botanists perceive and define characters differently. In: Homology and Systematics: Coding characters for phylogenetic systematics (eds. R. Scotland \& R. T. Pennington). Chapter 2. The Systematics Association Special Volume Series 58, Taylor \& Francis, London. 
Haworth, E. 1988. Distribution of taxa in the old genus Melosira (now mainly Aulacoseira) in Cumbrian waters, In Round, F. [Ed.], Algae and Aquatic Environment. Biopress, Bristol, pp. 135-164.

Haworth, E. \& Sabater, S. 1993. A new Miocene Aulacoseira species in diatomite from the ancient lake in La Cerdanya (NE Spain). In: Progress in Diatom Studies:

contributions to taxonomy, ecology, and nomenclature (Sims, P. A., ed.). pp. 227-242. Nova Hedwigia 106.

Humphries, C. J. 2002. Homology, characters and continuous variables. In Morphology, Shape and Phylogeny (ed. MacLeod, N. \& Forey, P. L.). Chapter 5. pp. 8-26. Taylor \& Francis. London.

Hustedt, F. 1942. Diatomeae. In Huber-Pestalozzi, G. [Ed.] Das Phytoplankton des Süßwassers: Systematik und Biologie, Teil 2, Stuttgart, E. Schweizerbart'sche Verlagsbuchhandlung (Erwin Nägele), 549 pp.

Hutchinson, G. E. 1957. A Treatise on Limnology. Volume 1. Geography, Physics and Chemistry. J. Wiley \& Sons, New York. 1015 pp.

Hutchinson, G. E. 1967. A Treatise on Limnology. Volume II. Introduction to Lake Biology and the Limnoplankton. John Wiley and Sons, New York. 1115 pp. 
Julius, M. L. 2000. Phylogeny of the cyclostephanoid diatoms: an investigation of their morphology and stratigraphy. Ph.D. Dissertation (Natural Resources and Environment), University of Michigan, 209pp. UMI Microfilm 9977188.

Kaczmarska, I. 1985. The diatom flora of Miocene lacustrine diatomites from the Harper Basin, Oregon, U. S. A. Acata Palaeobotanica XXV(1,2):22-100.

Khursevich, G. K. 1995. Evolution of freshwater centric diatoms within the Euroasian continent. In: Proceedings of the Thirteenth International Diatom Symposium, Maratea, Italy. (Marino, D. \& Montresor, M., eds.), Biopress, Bristol, p. 507-520.

Kilham, P. 1990. Ecology of Melosira species in the Great lakes of Africa. In: Large Lakes: Ecological Structure and Function (eds. Tilzer, M. M. \& Serruya, C.). SpringerVerlag, Berlin 403 pp.

Kilham, S. S. \& Kilham, P. 1975. Melosira granulata (Ehr.) Ralfs: morphology and ecology of a cosmopolitan freshwater diatom. Verhandlungen Internationale Vereinigung für Theoretische und Angewandte Limnologie 19:2716-2721.

Klee, R. \& Houk, V. 1996. Morphology and ultrastructure of Cyclotella woltereckii Hustedt (Bacillariophyceae). Archiv für Protistenkunde 147:19-27. 
Kluge, A. 1989. A concern for evidence and a phylogenetic hypothesis of relationships among Epicrates (Boidae, Serpentes). Systematic Zoology 38:7-25.

Kluge, A. \& Farris, J. 1969. Quantitative phyletics and the evolution of anurans. Systematic Zoology 18:1-32.

Kociolek, J. P. \& Stoermer, E. F. 1988. A preliminary investigation of the phylogenetic relationships among the freshwater, apical pore field-bearing cymbelloid and gomphonemoid diatoms (Bacillariophyceae). Journal of Phycology 24:377-385.

Kooistra,W. \& Medlin, L. 1996. Evolution of the diatoms (Bacillariophyta). IV. A reconstruction of their age from small subunit rRNA coding regions and the fossil record. Molecular Phylogenetics and Evolution 6:391-407.

Krammer, K. 1991a. Morphology and taxonomy of some taxa in the genus Aulacoseira Thwaites (Bacillariophyceae): I. Aulacoseira distans and similar taxa. Nova Hedwigia 52 $: 89-112$.

Krammer, K. 1991b. Morphology and taxonomy of some taxa in the genus Aulacoseira Thwaites (Bacillariophyceae): II. Taxa in the A. granulata-, italica- and lirata-groups. Nova Hedwigia 53:477-496. 
Krammer, K. \& Lange-Bertalot, H. 1991. Bacillariophyceae, Teil 3: Centrales, Fragilariaceae, Eunotiaceae. - Die Süßwasserflora von Mitteleuropa (founded by A. Pascher) Fischer, Stuttgart. 2/3:1-576, 166 pl.

Krammer, K., Lange-Bertalot, H., \& Schiller, W. 1997. Miosira rhoehana n. gen. n. sp. (Bacillariophyceae) aus miozänen limnischen Sedimenten der Rhön im Vergleich mit anderen zentrischen Diatomeen. Paläontologische Zeitschrift 71:5-18.

Krebs, W. N. 1994. The biochronology of freshwater planktonic diatom communities in western North America. In: Proceedings of the $11^{\text {th }}$ International Diatom Symposium, San Francisco, California, 1990. (Kociolek, J. P., ed.). Memoirs of the California Academy of Sciences, Number 17, San Francisco. p. 485-499.

Likhoshway, Y. V. \& Crawford, R. M. 2001. The rimoportula - a neglected feature in the systematics of Aulacoseira. In: Proceedings of the $16^{\text {th }}$ International Diatom Symposium, Athens, (Economou-Amilli, A., ed.). pp. 33-47.

Lipscomb, D. L. 1992. Parsimony, homology and the analysis of multistate characters. Cladistics 8: 45-65.

Maddison, W. P. \& Maddison, D. R. 1992. MacClade: Analysis of Phylogeny and Character Evolution. Sinauer, Sunderland, Massachusetts. 
McBride, S. A. \& Edgar, R. K. 1998. Janus cells unveiled: frustular morphometric variability in Gomphonema angustatum. Diatom Research 13:293-310.

Medlin, L., Williams, D. M., \& Sims, P. A. 1993. The evolution of the diatoms (Bacillariophyta). I. Origin of the group and assessment of the monophyly of its divisions. European Journal of Phycology 28:261-275.

Medlin, L., Elwood, H. J., Stickel, S. \& Sogin, M. L. 1988. The characterization of enzymatically amplified eukaryotic 16S-like rRNA-coding regions. Gene 71:491-499.

Medlin, L.K., Kooistra, W.H.C.F., Gersonde, R. \& Wellbrock, U. 1996a. Evolution of the diatoms (Bacillariophyta). II. Nuclear-encoded small subunit rRNA sequence comparisons confirm a paraphyletic origin for the centric diatoms. Molecular Biology and Evolution 13:67-75.

Medlin, L.K., Kooistra, W.H.C.F., Gersonde, R. \& Wellbrock, U. 1996b. Evolution of the diatoms (Bacillariophyta): III. Molecular evidence for the origin of the Thalassiosirales. Nova Hedwigia 112:221-234.

Mickevich, M. F. 1978. Taxonomic congruence. Systematic Zoology 27:143-158. 
Mickevich, M. F. \& Johnson, M. S. 1976. Congruence between morphological and allozyme data in evolutionary inference and character evolution. Systematic Zoology $25: 260-270$.

Miyamoto, M. M. \& Fitch, W. M. 1995. Testing species phylogenies and phylogenetic methods with congruence. Systematic Biology 44:64-76.

Moiseeva, A. I. 1971. Atlas of Neogene Diatom Algae of Primorsky Kray. Nidra, Leningrad, $152 \mathrm{pp}$.

Moiseeva, A. I. \& Nevretdinova, T. L. 1990. The new family and genus of freshwater diatom algae (Bacillariophyta). Botanicheski Zhurnal. 75:539-543.

Nelson, G. 1979. Cladistic analysis and synthesis: principles and definitions, with a historical note on Adanson's "Familles des plantes" (1763-1764). Systematic Zoology 28: $1-21$.

Nixon, K. C. 1999. Winclada (BETA) ver. 0.9.9 PUBLISHED BY THE AUTHOR, ITHACA, NY.

Omland, K. E. 1994. Character congruence between a molecular and morphological phylogeny for dabbling ducks (Anas). Systematic Biology 43:369-387. 
Patterson, C. 1982. Morphological characters and homology. In: Problems of

Phylogenetic Reconstruction (Joysey, K. A. \& Friday, A. E., ed.). Chapter 2, pp. 21-74. Systematic Association Special Volume No. 21, Academic Press, London.

Patterson, C. 1988. Homology in classical and molecular biology. Molecular Biology and Evolution 5:603-625.

Pimentel, R. A. \& Riggins, R. 1987. The nature of cladistic data. Cladistics 3:201-209.

Poe, S. 1996. Data set incongruence and the phylogeny of crocodilians. Systematic Biology 45:393-414.

Poe, S. \& Wiens, J. J. 2000. Character selection and the methodology of morphological phylogenetics, In: Phylogenetic Analysis of Morphological Data. (Wiens, J. J., ed.), Chapter 2. Smithsonian Institute Press, Washington.

Pogue, M. G. \& Mickevich, M. F. 1990. Character definitions and character state delineation: the bête noire of phylogenetic inference, Cladistics 6:319-361.

Rae, T. C. 1998. The logical basis for the use of continuous characters in phylogenetic systematics. Cladistics 14:221-228. 
Reid, G. \& Sidwell, K. 2002. Overlapping variables in botanical systematics. In Morphology, Shape and Phylogeny (MacLeod, N. \& Forey, P. L., eds.). Chapter 5. Taylor \& Francis. London.

Ridley, M. 1993. Evolution. Blackwell Scientific Publications, Oxford, 670pp.

Rohlf, F. J. 2001. TPSDIG software program, vers. 1.30, http://life.bio.sunysb.edu/morph.

Round, F. E. 1981. The ecology of algae. Cambridge University Press, Cambridge. $653 \mathrm{pp}$.

Round, F. E., Crawford, R. M., \& Mann, D. G. 1990. The Diatoms: Biology \& Morphology of the Genera. Cambridge University Press, Cambridge. 747 pp.

Shcherbakova, T. A., Kiril'chik, S. V., Likhoshvai, E. V., \& Grachev, M. A. 1998. Phylogenetic position of diatom algae of the genus Aulacoseira from Lake Baikal based on the results of the comparison of 18S rRNA gene nucleotide sequences. Molekulyarnaya Biologiya Moscow 32:735-740.

Simmons, N. B. \& Geisler, J. H. 2002. Sensitivity analysis of different methods of coding taxonomic polymorphism: an example from higher level bat phylogeny. Cladistics 18:571-584. 
Simmons, M. P. \& Ochoterena, H. 2000. Gaps as characters in sequence-based phylogenetic analyses. Systematic Biology 49:369-381.

Simon, C. 1983. A new coding procedure for morphometric data with an example from periodical cicada wing veins. In: Numerical Taxonomy (ed. J. Felsenstein). pp. 378-283. Springer-Verlag, Berlin.

Simonsen, R. 1979. The diatom system: ideas on phylogeny. Bacillaria 2:9-71.

Siver, P. A. \& Kling, H. 1997. Morphological observations of Aulacoseira using scanning electron microscopy, Canadian Journal of Botany 75:1807-1835.

Sokal, R. \& Rohlf, F. J. 1995. Biometry: The principles and practice of statistics in biological research. Third Edition. W. H. Freeman and Company, New York, 887 pp.

Sorenson, M. D. 1999. TreeRot version 2. Boston University, Boston, Massachusetts.

Sorhannus, U., Gasse, F., Perasso, R., \& Baroin Tourancheau, A. 1995. A preliminary phylogeny of diatoms based on 28S ribosomal RNA sequence data. Phycologia 34:65-73.

Stearns, S. C. 1992. The Evolution of Life Histories. Oxford University Press, Oxford. 249 pp. 
Stevens, P. F. 1991. Character states, morphological variation, and phylogenetic analysis: a review. Systematic Botany 16:553-583.

Stevens, P. F. 2000. On characters and character states: do overlapping and nonoverlapping variation, morphology and molecules all yield data of the same value? In: Homology and Systematics: Coding characters for phylogenetic systematics (Scotland, R. \& Pennington, R. T., eds.). Chapter 4. The Systematics Association Special Volume Series 58, Taylor \& Francis, London.

Stoermer, E. F. 1967. Polymorphism in Mastagloia. Journal of Phycology 3:73-77.

Stoermer, E. F. \& Smol, J., eds. 2001. The Diatoms: Applications for the Environmental and Earth Sciences. Cambridge University Press, Cambridge. 482 pp.

Strait, D., Moniz, M. \& Strait, P. 1996. Finite mixture coding: a new approach to coding continuous characters. Systematic Biology 45: 67-78

Strong, E. E., \& Lipscomb, D. 1999. Character coding and inapplicable data. Cladistics 15:363-371. 
Swiderski, D. L., Zelditch, M. L., \& Fink, W. L. 1998. Why morphometrics is not special: coding quantitative data for phylogenetic analysis. Systematic Biology 47: 508519.

Swofford, D. L. 2000. PAUP*, Phylogenetic Analysis Using Parsimony (*and other methods), version 4.0, Beta version 10. Sinauer Associates, Sunderland, Massachusetts. Teubner, K. 1995. A light microscopical investigation and multivariate statistical analyses of heterovalvar cells of Cyclotella-species (Bacillariophyceae) from lakes of Berlin-Brandenburg region. Diatom Research 10:191-205.

Theriot, E. 1988. An empirically based model of variation in rotational elements in centric diatoms with comments on ratios in phycology. Journal of Phycology 24:400-407.

Theriot, E. 1992. Clusters, species concepts, and morphological evolution of diatoms. Systematic Biology 41:141-157.

Theriot, E. \& Serieyssol, K. 1994. Phylogenetic systematics as a guide to understanding features and potential morphological characters of the centric family Thalassiosiraceae. Diatom Research 9:429-450.

Thiele, K. 1993. The Holy Grail of the perfect character: the cladistic treatment of morphometric data. Cladistics 9:275-304. 
Thorpe, R. S. 1984. Coding morphometric characters for constructing distance wagner networks. Evolution 38:244-255.

Tilzer, M. M. \& Serruya, C., eds. 1990. Large Lakes: Ecological Structure and Function. Springer-Verlag, Berlin. 403 pp.

VanLandingham, S. L. 1964. Miocene non-marine diatoms from the Yakima region in South Central Washington. Beihefte zür Nova Hedwigia 14:1-78, 56 pls.

VanLandingham, S. L. 1967. Paleoecology and microfloristics of Miocene Diatomites from the Otis-Juntura Region of Harney and Malheur Counties, Oregon. Beihefte zür Nova Hedwigia 26:1-77, 25 pls.

VanLandingham, S. L. 1971. Catalogue of the Fossil and Recent Genera and Species of Diatoms and their Synonyms. Part IV. Fragilaria through Naunema. J. Cramer, Lehre, pp. xi-xiv, 1757-2385.

VanLandingham, S. L. 1979. Catalogue of the Fossil and Recent Genera and Species of Diatoms and their Synonyms. Part VIII. Supplementary Taxa (through 1964), Supplementary References, Synonym Addendum, Corrections, Additions. J. Cramer, Lehre, pp. 4241-4654. 
Wiens, J. J. 1998a. Combining data sets with different phylogenetic histories. Systematic Biology 47:568-581.

Wiens, J. J. 1998b. Does adding characters with missing data increase or decrease phylogenetic accuracy? Systematic Biology 47:625-640.

Wiens, J. J. 2001. Character analysis in morphological phylogenetics: problems and solutions. Systematic Biology 50:689-699.

Wiens, J. J. \& Reeder, T. W. 1995. Combining data sets with different numbers of taxa for phylogenetic analysis. Systematic Biology 44:548-558.

Williams, D. M. 1990. Cladistic analysis of some freshwater araphid diatoms (Bacillariophyta) with particular reference to Diatoma and Meridion. Plant Systematics and Evolution 171: 89-97.

Williams, D. M. \& Round, F. E. 1988. Phylogenetic systematics of Synedra. In: Proceedings of the $9^{\text {th }}$ International Diatom Symposium, Bristol (ed. F. E. Round). Koeltz, Koenigstein, pp. 305-315.

Wood, A.M., Lande, R., \& Fryxell, G.A. 1987. Quantitative genetic analysis of morphological variation in an antarctic diatom grown at two light intensities. Journal of Phycololgy 23:42-54. 


\section{Vita}

Stacy McBride Edgar was born in Canton, Ohio on April 16, 1973, the daughter of Peggy Anne McBride and Thomas Dwayne McBride. After completing her work at Shrewsbury High School, Shrewsbury, Massachusetts, she entered The University of Massachusetts Dartmouth, Massachusetts. She received a Bachelor of Science from The University of Massachusetts Dartmouth in May of 1995 and continued on there in pursuit of a Master of Science degree, which she received in May 1998. In September of 1998 she entered the Graduate School of The University of Texas at Austin.

Permanent Address: 1072 Clark Road, Danville, Vermont 05828-9515

This dissertation was typed by the author. 